\title{
DATA DEVELOPMENT TECHNICAL SUPPORT DOCUMENT FOR THE AIRCRAFT CRASH RISK ANALYSIS METHODOLOGY (ACRAM) STANDARD
}

Chris Y. Kimura

Ronald E. Glaser

Richard W. Mensing

Tom Lin

Timothy A. Haley

Andrew B. Barto

Martin A. Stutzke

August 1, 1996

This is an informal report intended primarily for internal or limited external distribution. The opinions and conclusions stated are those of the author and may or may not be those of the Laboratory.

Work performed under the auspices of the Department of Energy by the Lawrence Livermore National Laboratory under Contract W-7405-Eng-48.

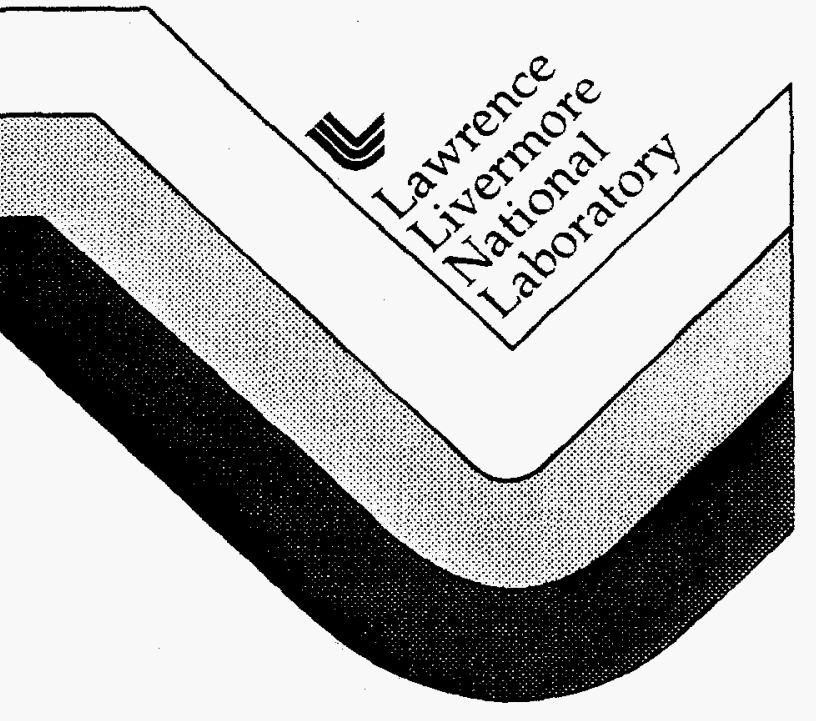




\section{DISCLAIMER}

This document was prepared as an account of work sponsored by an agency of the United States Govemment. Neither the United States Government nor the University of Califomia nor any of their employees, makes any warranty, express or implied, or assumes any legal liability or responsibility for the accuracy, completeness, or usefulness of any information, apparatus, product, or process disclosed, or represents that iis use would not infringe privately owned rights. Reference herein to any specific commercial product, process, or service by trade name, trademark, manufacturer, or otherwise, does not necessarily constitute or imply its endorsement, recommendation, or favoring by the United States Government or the University of California. The views and coinions of authors expressed herein do not necessarily state or reflect those of the United States Govemment or the University of Califomia, and shall not be used for advertising or product endorsement purposes.

This repon has been reproduced directly from the best avalable copy.

Available to DOE and DOE contractors from the Office of Scientific and Technical Information P.O. Box 62, Oak Ridge, TN 37831

Prices available from (615) 576-8401, FTS 626-8401

Available to the public from the

National Technical Information Service

U.S. Department of Commetce 5285 Pon Royal Rd.

Springfield, VA 22161 


\section{DISCLAIMER}

Portions of this document may be illegible in electronic image products. Images are produced from the best available original document. 


\begin{abstract}
The Aircratt Crash Risk Analysis Methodology (ACRAM) Panel has been formed by the U.S. Department of Energy Office of Defense Programs (DOE/DP) for the purpose of developing a standard methodology for determining the risk from aircratt crashes onto DOE ground facilities. In order to accomplish this goal, the ACRAM Panel has been divided into four teams, the data development team, the model evaluation team, the structural analysis team, and the consequence team. Each team, consisting of at least one member of the ACRAM plus additional DOE and DOE contractor personnel, specializes in the development of the methodology assigned to that team. This report documents the work performed by the data development team and provides the technical basis for the data used by the ACRAM Standard for determining the aircraft crash frequency.

This report should be used to provide the generic data needed to calculated the aircratt crash frequency into the facility under consideration as part of the process for determining the aircraft crash risk to ground facilities as given by the DOE Standard Aircratt Crash Risk Assessment Methodology (ACRAM). Some broad guidance is presented on how to obtain the needed site-specific and facility specific data but this data is not be provided by this document.
\end{abstract}




\section{DATA DEVELOPMENT TECHNICAL SUPPORT DOCUMENT FOR THE AIRCRAFT CRASH RISK ANALYSIS METHODOLOGY (ACRAM) STANDARD \\ TABLE OF CONTENTS}

SECTION

PAGE

ABSTRACT

LIST OF FIGURES

iii/in

LIST OF TABLES

vii

ix

1. INTRODUCTION $1-1$

1.1 Definitions of Terms $1-2$

1.2 How to Use This Report $1-11$

$\begin{array}{lll}1.3 & \text { References } & 1-13\end{array}$

2. COMMERCIAL AVIATION

2.1 Introduction 2-1

2.2 Air Carrier (14 CFR 121) Accident and Crash Rates

2.3 Air Taxi (14 CFR 135) Accident and Crash Rates 2-23

2.4 Commercial Aviation Crash Parameter Distributions 2-31

2.4.1 Off-Rumway Impact Location Distributions $2-32$

2.4.2 Off-Runway Heading Distributions 2-36

2.4.3 Off-Rumway Impact Velocity, Impact Angle, and Deceleration Distributions 2-38

2.5 Commercial Aviation Aircrat Characteristics $2-45$

2.6 Commercial Aviation References $\quad 2-52$

Appendix

A2.1 National Transportation Safety Board (NTSB) 14 CFR 121 Accident Database Used in the ACRAM Data Development Technical Support Document

A2-1

3. GENERAL AVIATION

3.1 Introduction 3-1

3.2 General Aviation Crashes 3-3

3.3 General Aviation Operations 3-33

3.4 General Aviation Crash Rates 3-58

3.5 General Aviation In-Flight Non-Ainway Crash Parameters 3-71

3.6 General Aviation Crash Parameter Distributions 3.72

3.6.1 General Aviation Crash Location 3-72

3.6.2 Impact Velocity Distribution 3.79

3.6.3 Impact Angle Distribution $3-80$

3.6.4 Skid Distance Distribution 3-81

3.7 General Aviation Aircraft Characteristics 3-84

3.8 General Aviation References $\quad 3-98$

Appendix

A3.1 General Aviation In Flight Crash Locations A3-1

4. MILITARY AVIATION

4.1 Introduction 4-1

4.2 Military Aviation Crash Frequencies 4-2

4.2.1 Estimates of Crash Frequencies Based on an Analysis of Impact
Accidents 
TABLE OF CONTENTS (Continued)

SECTION

4.2.2 Estimates of Crash Frequencies Based on an Analysis of the Mishap Database Developed for the Minuteman III WSSA

4.3 Military Aviation Crash Parameter Distributions

4.3.1 Crash Location Distributions

4.3.2 Impact Angle Distributions

4-14

4-15

4.3.3 Crash Velocity Distributions

4-23

4.3.4 Skid Distance Distributions

4-25

4-27

4.4 Military Aviation Aircraft Characteristics

4-29

4.5 Military Aviation References

4-41

5. AIRCRAFT OPERATIONS

5.1 Introduction

5.2 Near Airport Environment

5.3 Enroute/In-Flight Environment

5-1

5-4

5.4 TRACON Environment

5.5 References

5-10

5-12 


\section{LIST OF FIGURES}

EIGURE

PAGE

2. COMMERCIAL AVIATION

2.1 Coordinate System and Direction of Travel of Ref. 2.32

2.2 The Truncated Lognormal PDF for the X-Coordinate of Off-Rumway Take-Off Crashes

2.3 The Median Y-Coordinate of Impact for Off-Rumway Take-off Crashes Above the Rumway

2.4 The Truncated Lognormal PDF for the X-Coordinate of Off-Rumway Landing Crashes

2.5 The Median Y-Coordinate of Impact for Off-Rumway Landing Crashes Above the Runway

2.6 The Heading $\theta$ for the Aircraft on Take-off is Typically Greater Than the Arc Tangent $\theta_{0}=\tan ^{-1}\left(y / x^{\prime}\right)$ of its Position in the $\left(x^{\prime}, y\right)$ Coordinate System

2.7 The Mean Heading, in Degrees, at Impact for Off-Runway Take-off Crashes as a Function of the Nominal Heading, in Degrees

2.8 The Beta PDF of Heading, in Degress, for Off-Runway Landing Crashes

2.9 The Truncated Weibull PDF of Impact Velocity for Off-Rumway Take-off or Landing Crashes

2.10 The Median Impact Angle in Degrees as a Function of Impact Velocity for OffRunway Take-off or Landing Crashes

2.11 The Truncated Weibull PDF of Deceleration for Off-Runway Take-off or Landing Crashes

3. GENERAL AVIATION

3.1 General Aviation Empirical Skid Distance Distribution 3-83

3.2 Normal Probability Plots of Residuals

4. MILITARY AVIATION

4.1 USAF Class AB Impact Destroyed Mishaps On and OH Runway (1979-1993) 4-7

4.2 Crash Location Distribution for Large Military Aircraft During Landing

4.3 Crash Location Distribution for Large Military Aircraft During Takeoft

4.4 Crash Location Distribution for Small Military Aircraft During Landing

4-18

4.5 Crash Location Distribution for Small Military Aircraft During Takeoff

4-19

5. AIRCRAFT OPERATIONS

5.1 ARTCCs and Airspace Boundaries within the Continental United States 


\section{LIST OF TABLES}

TABLE

PAGE

1. INTRODUCTION

1.1 Frequency Screening Analysis Near Airport Environment

2. COMMERCIAL AVIATION

2.1 Scheduled U.S. Air Carriers Operating Under 14 CFR 121

2.2 Nonscheduled U.S. Air Carriers Operating Under 14 CFR 121

$2-8$

2.3 Total (Scheduled and Nonscheduled) U.S. Air Carriers Operating Under 14 CFP 121

2.4 Total 14 CFR 121 Aircraft Imvolved in Accidents by Flight Phase

2.5 Total (Scheduled and Nonscheduled) 14 CFR 121 Aircraft Involved in Accident Rate by Flight Phase

2.6 14 CFR 121 Crashes (Accidents with Destroyed or Major Damage to Airframe) by Flight Phase

2.7 14 CFR 121 Crash (Accidents with Destroyed or Major Damage to Airframe) Rates by Flight Phase

2.8 14 CFR 121 Crashes with Destroyed/Major-W.O. Airframes by Flight Phase

$2-14$

2.9 14 CFR 121 Crashes with Destroyed/Major-W.O. Airframe Rates by Flight Phase

2.10 Total Off Airport Only 14 CFR 121 Accidents by Flight Phase

2.11 Total (Scheduled and Nonscheduled) Off Airport Only 14 CFR 121 Accident Rates by Flight Phase

$2-15$

2-16

2.12 Off Aiport Only 14 CFR 121 Crashes (Accidents with Destroyed or Major Damage to Airframe), by Flight Phase

2.13 Off Airpon Only 14 CFR 121 Crash (Accidents with Destroyed or Major Damage to Airframe) Rates by Flight Phase

2.14 Off Airport Only 14 CFR 121 Crashes with Destroyed or Major-W.O. Airframes by Flight Phase

2.15 Off Aiport Only 14 CFR 121 Crashes with Destroyed or Major-W.O. Airtrame Rates by Flight Phase

2.16 Comparison of All 14 CFR 121 Aircraft Involved in Accidents/Crashes with 14 CFR 121 Aircraft Involved in Off Airport Accidents/Crashes

2.17 Scheduled U.S. Air Carriers Operating Under 14 CFR 135 (Commuters)

2.18 Nonscheduled U.S. Air Carriers Operating Under 14 CFR 135 (Air Taxis)

2.19 Total (Scheduled and Nonscheduled) U.S. Air Carriers Operating Under 14 CFR 135

2.20 Scheduled 14 CFR 135 Aircraft Accidemts by Damage and Flight Phase

2.21 Scheduled 14 CFR 135 Crash Rates by Flight Phase

2.22 Conditional Probabilities of Impact Location, Given a Crash on Take-off

2.23 Conditional Probabilities of Impact Location, Given a Crash on Landing

2.24 Impact Angle Distribution Properties

2.25 Selected Skid Distance Percentiles, in Feet

2.26 Characteristics of Air Carrier (14 CFR 121) Aircraft

2-27 Characteristics of Scheduled Air Taxi (14 CFR 135) Aircraft

3. GENERAL AVIATION

3.1 General Aviation Accidents by Phase of Flight Operation, Single EngineReciprocating, Fixed Wing

3.2 General Aviation Accidents by Phase of Flight Operation, Multi-Engine

Reciprocating, Fixed Wing

3.3 General Aviation Accidents by Phase of Flight Operation, Turboprops, Fixed Wing

3.4 General Aviation Accidents by Phase of Flight Operation, Turbojets, Fixed Wing

3.5 General Aviation Accidents by Phase of Flight Operation, Total, Fixed Wing 
3.6 General Aviation Accidents by Phase of Flight Operation, Reciprocating Engine, Rotary Wing

3.7 General Aviation Accidents by Phase of Flight Operation, Turbine Engine, Rotary Wing General Aviation Accidents by Phase of Flight Operation, Total, Rotary Wing General Aviation Accidents by Phase of Flight Operation, Total Powered Aircraft General Aviation Accidents by Phase of Flight Operation, Gliders General Aviation Accidents by Phase of Flight Operation, Balloons General Aviation Accidents by Phase of Flight Operation, Other Aircraft General Aviation Accidents by Phase of Flight Operation, Total Aircraft General Aviation Accidents by Flight Phases and by Aircraft Subcategories Summary

3.15 General Aviation Accidents by Flight Phases and by Aircratt Subcategories Summary, Manuevering Accidents and Accidents During Standing, Taxi, and Other Flight Phases Deleted General Aviation Operations, Single-Engine Reciprocating, Fixed Wing General Aviation Operations, Multi-Engine Reciprocating, Fixed Wing General Aviation Operations, Turboprop, Fixed Wing

$3-37$ General Aviation Operations, Turbojet, Fixed Wing General Aviation Operations, Total, Fixed Wing General Aviation Operations, Reciprocating, Rotary Wing General Aviation Operations, Total, Rotary Wing General Aviation Operations, Total Powered Aircraft General Aviation Operations, Oher Aircraft General Aviation Operations, Total Aircraft General Aviation Single-Engine Reciprocating, Fixed Wing Crash Rates by Flight Phase

General Aviation Multi-Engine Reciprocating, Fixed Wing Crash Rates by Flight

General Aviation Fixed Wing Crash Rates by Aircraft Subcategories and Flight

Phase, Adjusted for off Runway Crashes

Constants Required to Fomulate Location

\subsection{0}

3.41

3.42

3.43

3.44

3.45

3.46

3.47 General Aviation Takeoff Crash Locations

General Aviation Landing Crash Locations

Crash Location Probability $f(x, y)$ for General Aviation Takeotfs

Distribution of impact Velocities

Distribution of Impact Angles

Multiple Regression Analysis for Skid Distance

Characteristics of Single-Engine Reciprocating, Fixed Wing General Aviation

3.48 Characteristics of Single-Engine Agricultural, Fixed Wing General Aviation Aircraft 3-88

3.49 Characteristics of Multi-Engine Reciprocating, Fixed Wing General Aviation Aircraft 3-90

3.50 


\section{LIST OF TABLES (Continued)}

\section{TABLE}

3.51 Characteristics of Turbojet, Fixed Wing General Aviation Aircraft

3.52 Characteristics of Rotary Wing General Aviation Aircraft

4. MILITARY AVIATION

4.1 Summary of U.S. Air Force Crash Mishap History 4-8

4.2 Summany of Small (High-Performance) Aircraft Mishap History 4-9

4.3 Summary of Large (Bomber and Cargo) Aircratt Mishap History

4.4 Summany of Helicopter Aircraft Mishap History 4-10

4.5 Mishaps and Estimates of Crash Frequencies Based on Impact Mishaps,

Disregarding Damage Condition of the Crashing Aircraft

4.6 Mishaps and Estimates of Crash Frequencies Based on Impact Mishaps in Which the Crashing Aircratt Was Destroyed

4.7 Takeoff and Landing Crash Data and Estimates of Crash Frequencies Based on the Minuteman III Mishap Database

4.8 In-Flight Crash Data and Estimates of Crash Frequencies Based on the Minuteman III Mishap Database.

4.9 Per Square Mile Crash Location Probability, Given a Crash, for Large Military

4.10 Per Square Mile Crash Location Probability, Given a Crash, for Large Military Aircratt During Takeott

4.11 Per Square Mile Crash Location Probability, Given a Crash, for Small Military Aircratt During Landing

4.12 Per Square Mile Crash Location Probability, Given a Crash, for Small Military Aircraft During

4.13 Estimated Cumulative Distributions for Impact Angle

4.14 Estimated Cumulative Distributions for Cotangent of Impact Angle

4.15 Estimated Cumulative Distributions for Airspeed at Principal Impact

4.16 Estimated Cumulative Distributions for Horizontal Velocity at Principal Impact

4.17 Estimated Cumulative Distributions for Skjd Distance

4.18 Characteristics of Large Military Aviation Aircraft

4.19 Characteristics of Small High Performance Military Aviation Aircraft

4.20 Characteristics of Small Low Performance Military Aviation Aircraft

4.21 Characteristics of Military Helicopters

5. AIRCRAFT OPERATIONS

5.1 Survey of Major DOE Sites and Air Traffic Control Facilities

5.2 Continental United States ARTCC Air Camier and Air Taxi Aircraft Handled for 


\section{DATA DEVELOPMENT TECHNICAL SUPPORT DOCUMENT FOR}

THE AIRCRAFT CRASH RISK ANALYSIS METHODOLOGY (ACRAM) STANDARD

by

Chris Y. Kimura, Ronald E. Glaser, Lawrence Livermore National Laboratory (LLNL)

Richard W. Mensing, Logicon RDA

Tom Lin, Sandia National Laboratories (SNL)

Timothy A. Haley, Andrew B. Barto, Martin A. Stutzke, Science Application International Corporation (SAIC)

\section{INTRODUCTION}

The Aircratt Crash Risk Analysis Methodology (ACRAM) Panel has been formed by the U.S. Department of Energy Office of Defense Programs (DOENP) for the purpose of developing a standard methodology for determining the risk from aircraft crashes onto DOE ground facilities. In order to accomplish this goal, the ACRAM Panel has been divided into four teams, the data development team, the model evaluation team, the structural analysis team, and the consequence team. Each team, consisting of at least one member of the ACRAM Panel plus additional DOE and DOE contractor personnel, specializes in the development of the methodology assigned to that team. This report documents the work performed by the data development team and provides the technical basis for the data used by the ACRAM Standard [Ref. 1.1] for determining the aircratt crash frequency.

Aircraft crash risk analysis, like all transportation risk analyses, is highly dependent on the initiating event frequency, that is the liklihood of the accident happening. This is because transportation risk analyses, unlike nuclear power plant Probablistic Risk Assessments (PRAs) do not analyze the mitigating features on the transportation system which might serve to prevent or mitigate the effects of the transportation accident. Therefore, the development of accident data, operational data, and characterization of the accident becomes paramount in the transportation risk analyses. Fortunately, for aircraft crash risk analysis, the availability and quality of accident data and operational data, at least for the general aviation and commercial aviation sector, are relatively good due to the efforts of the National Transportation Safety Board (NTSB) and the Federal Aviation Administration (FAA).

This document is divided imto the following chapters. Chapter One presents introductory material. Chapter Two presents the accident rates and the crash parameter distributions developed for Commercial Aviation (Air Carriers and Air Taxi). Chapter Three presents the accident rates and the crash parameter distributions developed for General Aviation. Chapter Four presents the accident rates and the crash parameter distributions for Military Aviation. Chapter Five discusses the development of Aircraft Operation data. Each chapter is followed by applicable appendices.

This chapter presents the definition of important terms used throughout this document in Section 1.1. It also discusses how to use this report in Section 1.2 Section 1.3 presents the references used in this chapter. 


\subsection{DEFINITIONS OF TERMS}

As the first step in the development of data for the ACRAM Standard [Ref. 1.1], it is important that common definitions are understood for several significamt terms which are used throughout this report. The defined terms are listed in boldface and in alphabetical order. Where possible, the source of the definition has been identified. In addition, where a defined term has been used as part of a definition, it is capitalized.

Active Alrcraft: As defined by the Federal Aviation Administration (FAA), all legally registered civil aircratt which flew one or more hours during the time period under consideration.

Alr Carrier: As defined by the Federal Aviation Administration (FAA), the commercial system of air transportation consisting of the Certificated Air Carriers, Air Taxis (including commuters), Supplemental Air Carriers, Commercial Operators of Large Aircratt, and Air Travel Clubs. As defined by air traftic controllers in tabulating air trattic activity, aircratt in general commercial service capable of carrying 70 or more passengers irrespective of the the aviation flight nules under which the flight occurs.

Alrcraft Accident: As defined by the National Transportation Safety Board (NTSB), an occurrence associated with the operation of an aircraft which takes place between the time any person boards the aircraft with the intention of flight until such time as all such persons have disembarked, and in which any person sutfers a fatal or serious injury as a result of being in or upon the aircraft or by direct contact with the aircraft or anything attached thereto, or in which the aircratt receives substantial damage.

Alrcraft Crash: For Commercial Aviation, see Commercial Aircraft Crash. For General Aviation, see General Aviation Crash. For Military Aviation, see Military Aircraft Crash.

Alrcraft/Avlation Category: The broadest, most general classification of aircraft and aviation used in the ACRAM Standard [Ref. 1.1]. These categories are: 1) Air Carrier, 2) Air Taxi, 3) General Aviation, and 4) Military Aviation.

Alrcraft Operation: As defined by the Federal Aviation Administration (FAA) near an airport, the number of arrivals and departures from the airport at which an airport traffic control tower is located. Operations include aircraft which: 1) operate in the local traffic pattem or within sight of the airport, 2) are known to be departing for, arriving from flight in the local practice areas located within a 20-mile radius of the airport, or 3) execute simulated instrument approaches or low passes at the airport. See also Airport Operation.

Alrcraft/Aviation Subcategory: The next level of classification for aircraft and aviation used by the ACRAM Standard [Ref. 1.1]. For Air Carriers and Air Taxis, no subcategories are defined. For General Aviation, seven subcategories of aircratt/aviation are defined: 1) Single-engine piston powered, fixedwing, 2) Multi-engine piston powered, fixed wing, 3) Turboprop powered, fixed-wing, 4) Turbojetturbotan powered, fixed-wing, 5) Piston-engine powered, rotary wing, 6) Turboshaft powered, rotary wing, and 7) Other. For Military Aviation, three subcategories of aircraft/aviation are defined: 1) Large (which includes most Bombers, Cargo or transports), 2) Small (which includes most Fighters, Attack aircraft, and Trainers), and 3) Helicopters.

Alrcraft Type: As defined by 14 CFR 1.1 [Ref. 1.2], a specific make and basic model of aircraft, including modifications that do not change its handling or flight characteristics. As used with respect to the certification of aircraft, those aircraft which are similar in design. Examples include the Douglas DC-7 and the DC-7C, the Lockheed 1049G and 1049H, and the Folkker F-27 and F-27F.

Alport: As defined by the Federal Aviation Administration (FAA), an area of land or water that is used or intended to be used for the landing and takeoff of aircraft, and includes its buildings and facilities, if any. Airports include heliports, STOLPorts, and seaplane bases.

Aiport Approach Fllght Phase: The Flight Phase which consists of the time the aircraft intercepts the initial navigation fix defined by U.S. terminal approach procedures for that specific airport, numway and 
approach, until the aircraft crosses the outer marker detined by U.S. terminal approach procedures for that specific airport, nuway and approach.

Alport Operation: For the ACRAM Standard [Ref. 1.11, an Aircraft Operation which occurs in the Near Airport Environment. As defined by the Federal Aviation Administration (FAA), the number of arrivals and departures from the airport at which the Airport Traffic Control Tower is located. Aiport Operations are classified as either Local or Itinerant Operations. See also Aircraft Operation.

Airport Trafflc: As defined by the Federal Aviation Administration (FAA), aircraft operating in the air or on an airport surface exclusive of loading ramps and parking areas.

Alport Traffic Control Tower (ATCT): As defined by the Federal Aviation Administration (FAA), a central operations facility in the terminal air traffic control system, which consists of a tower cab structure, including an associated IFR room if radar equipped, and uses air/ground communications, radar, visual signaling, and other services to provide safe and expeditious movement of terminal air tratfic.

Alr Route Traffic Control Center (ARTCC): The FAA air traffic control facility responsible for providing air tratfic control service to aircratt operating on IFR flight plans in controlled airspace, primarily Class $A$ Airspace. ARTCCs are the central authority for issuing IFR clearances, and provide nationwide monitoring of each IFR flight, usually during the Enroute Flight Phase.

Alr Taxl: As defined by the Federal Aviation Administration (FAA), a classification of air carriers which transports in accordance with 14 CFR 135 [Ref. 1.12], persons, property, and mail using small aircraft (under 30 seats or a maximum payload capacity of less than 7,500 pounds). As defined by air traffic controllers in tabulating air traffic activity, aircraft in general commercial senvice that carry less than 70 passengers. As this classification includes small single-engine aircraft, the flight must usually be identified as an air taxi flight in its flight plan or the aircraft carries a commercial livery in order for the flight to be counted as an Air Taxi flight.

Alnway: The system of designated routes between VOR or VORTAC stations. Included within the definition of Ainway are High Altitude (Jet) Routes, Low Attitude (Victor) Routes, and Military Training Routes.

Armed Forces: As defined by 14 CFR 1.1 [Ref. 1.2], the United States Air Force (USAF), United States Army (USA), United States Navy (USN), United States Marine Corps (USMC), and the United States Coast Guard (USCG), including their regular and reserve component and members serving without component status.

Certiflcated Air Carrier: As delined by the Federal Aviation Administration (FAA), an Air Carrier holding a Certificate of Public Convenience and Necessity issued by the U.S. Department of Transportation (DOT) to conduct scheduled services interstate. Nonscheduled or charter operations may also be conducted by these carriers. These carriers operate large aircraft ( 30 seats or more or a maximum payload of 7,500 pounds or more) in accordance with 14 CFR 121 [Ref. 1.7].

Class A AIrspace: As defined by by 14 CFR 71.33 [Ref. 1.3], that airspace of the United States, including that airspace overtying the waters within 12 nautical miles of the coast of the 48 contiguous States, from 18,000 feet Mean Sea Level to and inctuding FL600 (60,000 feet mean sea level) excluding the states of Alaska and Hawaii, Santa Barbara Island, Faralion Island, and the airspace south of latitude $25^{\circ} 04^{\prime} 00^{\prime \prime}$ North. All operations are conducted under instrument flight rules (IFR) and are subject to ARTCC clearances and instructions. Aircraft separation and safety advisories are provided by the ARTCC. Formerly called Positive Control Areas (PCAs).

Class A Mishap: As defined by the United States Air Force (USAF), any mishap incidental to flight which results in the total cost of property damage, injury and/or illness of $\$ 1,000,000$ or more, or USAF aircratt destruction, or fatality.

Class B Airspace: Generally the airspace from the surface to 10,000 feet mean sea level (MSL) surrounding U.S. aiports with more than 150,000 annual instrument flight rule (IFR) operations or 
650,000 annual passenger enplanements. The configuration of each Class B Airspace is individually tailored and consists of a surtace area, usually 5 nautical miles in radius from the primary airport, and two or more layers extending laterally from the core at 3 to 10 nautical mile increments (typically) to a radius of approximately 20 nautical miles, with each layer having a prescribed altitude floor. Class B Airspace is designed to contain all published instrument procedures once an aircratt enters the airspace. Operations may be under either IFR or visual flight rules (VFR) with all aircraft subject to air traffic control clearances and instructions. Air traffic control provides aircraft separation and safety advisories. Formerly called Terminal Control Areas (TCAs).

Class B Mlshap: As detined by the United States Air Force (USAF), any mishap incidental to flight which results in the total cost of property damage, injury and/or illness of $\$ 200,000$ or more, but less than $\$ 1,000,000$.

Class C Alropece: Generally, the airspace from the surface to approximately 4,000 feet mean sea level (MSL) surrounding U.S. airports with more than 75,000 annual instrument flight rule (IFR) operations at the primary airport, or 100,000 annual IFR operations at the primary and secondary airports in the terminal hub area, or 250,000 annual passenger enplanements at the primary airport. The configuration of each Class C Airspace is individually tailored and consists of a surface area of 5 nautical miles radius from the primary airport, and another layer with a prescribed ahitude floor, extending laterally from the core to a radius of 10 nautical miles. Operations may be under either instrument flight rules (IFR) or visual flight rules (VFR), with all aircraft subject to air traffic control clearances and instructions. Air traffic control provides aircraft separation between IFR/FR and IFR/special VFR (SVFR) aircraft. VFR operations are given traffic advisories and, upon request, collision resolution instructions. Formerly called Aiport Radar Service Areas (ARSAs).

Class C Mishap: As defined by the United States Air Force (USAF), any mishap incidental to flight which results in the total cost of property damage of $\$ 10,000$ or more, but less than $\$ 200,000$ or injury or occupational illness resulted in a lost workday case involving days away from work.

Class D Alrspace: The airspace from the surface to 2,500 feet above ground level (AGL) within a 5 statute mile ( 4.3 nautical mile) radius of the center of the airport. Operations may be under either instrument flight rules (IFR) or visual flight rules (VFR), with all aircratt subject to air traffic control clearances and instructions. Air traffic control separation service is provided to IFR aircraft only, but all aircraft will be given traffic advisories and, upon request, conflict resolution instructions. Formerly called Airport Traffic Areas (ATAs) and Control Zones (CZs).

Climb to Crulse Flight Phase: The Flight Phase which consists of the time after the Takeoff Flight Phase when the aircraft landing gear is retracted and all aircraft flaps are in their normal cruise configuration until the aircraft has reached its initial assigned cruising or enroute altitude.

Commercial Alrcraft Crash: Any Aircraft Accident involving an aircraft type generally used for commercial senvice during flight operations (defined as the beginning of takeoff roll to landing touchdown) which results in the destruction of or substantial damage to the aircraft. Fatal or serious injury sustained as a result of the Aircraft Accident itself, without related destnction or substantial damage to the aircraft is not a criterion for consideration as a Commercial Aircraft Crash.

Commerclal Alport: As defined by the Federal Aviation Administration (FAA), a Public Airport which is determined to enplane 2,500 or more passengers annually and receive scheduled passenger service of aircratt.

Commerclal Avlation: All aspects of aviation involving aircraft operated under 14 CFR 121 [Ref. 1.7], 125 [Ref. 1.8], 127 [Ref. 1.9], 129 [Ref. 1.10], and 135 [Ref. 1.12] regulations.

Commerclal Operator: As defined by the Federal Aviation Administration (FAA), a person who, for compensation or hire, engages in the intrastate carriage of aircraft in air commerce of persons or property other than as an air camier or foreign air carrier. 
Commerclal Operator of Large Alrcraft: As defined by the Federal Aviation Administration (FAA), a Commercial Operator operating aircratt with 30 seats or more or a maximum payload capacity of 7,500 pounds or more.

Commuter Alr Carrier: As defined by the Federal Aviation Administration (FAA), an Air Taxi operator which performs at least five round trips per week between two or more points and publishes flight schedules which specity the times, days of the week, and points between which such flights are performed. Aso called Scheduled Air Taxis.

Crash Location Distribution: The normalized conditional probability distribution (i.e., given that the aircraft crash occurs) in terms of $x, y$ coordinates as a function of the location of the facility relative to the airport.

\section{Cruise Fllght Phase: See Enroute Flight Phase.}

Descem from Crulse Flight Phase: The Flight Phase which consists of the time when the aircraft begins its descent from its final assigned cruising or enroute altitude until the aircraft intercepts the initial navigation fix defined by U.S. terminal approach procedures for that specific airpon, numway and approach,

Destroyed: As defined by the United States Air Force (USAF), sufficient damage sustained by the aircraft in the accident such that it is uneconomical to repair. It is further defined by the number of manhours estimated as need to repair the aircraft to flight status and depends on the aircraft type.

Demand Alr Taxi: As defined by the Federal Aviation Administration (FAA), an Air Taxi operator which performs charter air service or less than five round trips per week between two or more points. Also called Nonscheduled Air Taxis.

Enroute: For the ACRAM Standard [Ref. 1.1], Enroute includes the Climb to Altitude Flight Phase, Enroute or Cruise Flight Phase, and the Descent from Cruise Flight Phase.

Enroute Analysis: The aircratt crash frequency analysis for Aircraft Operations outside of the Near Aipont and TRACON Environments. See also in-Flight Analysis.

Enroute Environment: The airspace outside of the Near Airport and TRACON Environments. See also In-Flight Environment.

Enroute Flight Phase: For Air Carriers and Air Taxis, the Flight Phase which consists of the time when the aircraft has reached its initial assigned cruising or enroute altitude until the time the aircraft begins its descent from its final assigned cruising or enroute attitude. Minor adjustments in assigned cnuising or enroute attitude are considered to be part of the Enroute Flight Phase. For Military Aviation, the Flight Phase from the point where the aircratt attitude is not affected by its proximity to the nunway. For Military Aviation, also called in-Flight Phase.

Fatal Injury: As defined by the National Transportation Safety Board (NTSB), any injury which results in death within 30 days of the accident.

Flight Mishap: As defined by the United States Air Force (USAF), a mishap involving a U.S. Air Force aircraft when intent for flight exists.

Fllght Phase: The parts of flight which are distinctly different for each due to the configuration of the aircraft and/or the conditions under which the flight is taking place. The following flight phases are generally defined: Ground operations, Taxi, Takeoff, Initial Climb, Climb to Altitude, Enroute or Cruise, Descent from Cruise, Airport Approach, Runway Approach, Landing, and Other.

Fllght Related: As defined by the United States Air Force (USAF), a mishap with little or no damage to the aircraft and which does not affect the Mishap Rate. 
14 CFR 91: Flight operations conducted under Title 14 (Aeronautics and Space) of the Code of Federal Regulations, Subchapter F, Air Traffic and General Operating Rules, Part 91, General Operating and Flight Rules [Ref. 1.4].

14 CFR 103: Flight operations conducted under Title 14 (Aeronautics and Space) of the Code of Federal Regulations, Subchapter F, Air Traffic and General Operating Rules, Part 103, Ultralight Vehicles [Ref. 1.5].

14 CFR 105: Flight operations conducted under Title 14 (Aeronautics and Space) of the Code of Federal Regulations, Subchapter F, Air Traftic and General Operating Rules, Part 105, Parachute Jumping [Ref. 1.6].

14 CFR 121: Flight operations conducted under Title 14 (Aeronautics and Space) of the Code of Federal Regulations, Subchapter G, Air Carriers, Air Travel Clubs, and Operators for Compensation or Hire: Certification and Operations, Part 121, Certification and Operations: Domestic, Flag, and Supplemental Air Carriers and Commercial Operators of Large Aircratt [Ref. 1.7].

14 CFR 125: Flight operations conducted under Title 14 (Aeronautics and Space) of the Code of Federal Regulations, Subchapter G, Air Carriers, Air Travel Clubs, and Operators for Compensation or Hire: Certification and Operations, Part 125, Certification and Operations: Airplanes Having a Seating Capacity of 20 or More Passengers or a Maximum Payload Capacity of 6,000 Pounds or More [Ref. 1.8].

14 CFR 127: Flight operations conducted under Title 14 (Aeronautics and Space) of the Code of Federal Regulations, Subchapter G, Air Carriers, Air Travel Clubs, and Operators for Compensation or Hire: Certification and Operations, Part 127, Certification and Operations of Scheduled Air Carriers with Helicopters [Ref. 1.9].

14 CFR 129: Flight operations conducted under Title 14 (Aeronautics and Space) of the Code of Federal Regulations, Subchapter G, Air Carriers, Air Travel Clubs, and Operators for Compensation or Hire: Certification and Operations, Part 129, Operations: Foreign Air Carriers and Foreign Operators of U.S.Registered Aircraft Engaged in Common Carriage [Ret. 1.10].

14 CFR 133: Flight operations conducted under Title 14 (Aeronautics and Space) of the Code of Federal Regulations, Subchapter G, Air Carriers, Air Travel Clubs, and Operators for Compensation or Hire: Certification and Operations, Part 133, Rotorcraft External-Load Operations [Ref. 1.11].

14 CFR 135: Flight operations conducted under Title 14 (Aeronautics and Space) of the Code of Federal Regulations, Subchapter G, Air Carriers, Air Travel Clubs, and Operators for Compensation or Hire: Certification and Operations, Part 135, Air Taxi Operators and Commercial Operators [Ref. 1.12].

14 CFR 137: Flight operations conducted under Title 14 (Aeronautics and Space) of the Code of Federal Regulations, Subchapter G, Air Carriers, Air Travel Clubs, and Operators for Compensation or Hire: Certification and Operations, Pan 137, Agricultural Aircratt Operations [Ref. 1.13].

General Aviation: All aspects of aviation imvolving aircraft operated under 14 CFR 91 [Ref. 1.4], 103 [Ref. 1.5], 105 [Ref. 1.6], 133 [Ref. 1.11], and 137 [Ret. 1.13] regulations.

General Aviation Alrport: As defined by the Federal Aviation Administration (FAA), any Airport which is used or to be used for public purposes, under the control of a public agency, the landing area of which is publicly owned.

General Avlation Crash: As defined by the NTSB for Aircraft Accidents.

High Alttude (Jet) Routes: The designated air routes based on radials from VOR or VORTAC stations extending from 18,000 feet Mean Sea Level (MSL) and up to and including 45,000 feet Mean Sea Level (MSL) (Flight Level 450). 
IFR Alrcraft Handled: As defined by the Federal Aviation Administration (FAA), the number of IFR Departures multiplied by two plus the number of IFR Overs. Assumes that the number of departures (acceptances, extensions, and originations of IFR flight plans) is equal to the number of landings (IFR flight plans closed).

IFR Departures: As defined by the Federal Aviation Administration (FAA), IFR Flights originating in the ARTCC's area, accepted by the ARTCC under the Sole Enroute clearance procedures, and extended by the ARTCC.

IFR Fllght: As defined by the Federal Aviation Administration (FAA), flight conducted in accordance with instrument tlight rules.

IFR Overs: As defined by the Federal Aviation Administration (FAA), IFR Flights that originate outside the ARTCC area and passes through the area without landing.

ILS: Instument Landing System.

In-Flight: For the ACRAM Standard [Ref. 1.1], In-Flight includes the Climb to Altitude Flight Phase, Enroute or Cruise Flight Phase, and the Descent from Cruise Flight Phase. For Military Aviation only, the Flight Phase where the aircraft altitude is not affected by its proximity to the runway. See also Enroute.

In-Filght Analysis: The aircraft crash frequency analysis for Aircraft Operations outside of the Near Airport and the TRACON Environments. See also Enroute Analysis.

In-Filght Environment: The airspace outside of the Near Airport and TRACON Environments. See also Enroute Environment.

Initlal Climb Fllght Phase: The Flight Phase which consists of the time the aircraft wheels leave the ground until the aircraft landing gear is retracted and all aircraft flaps are in their normal cruise configuration.

Instrument Landing System: A precision instrument approach system which normally consists of a localizer to provide course guidance to the numway, a glide stope to provide vertical guidance during the approach, and a marker beacon to provide aural and/or visual identification of a specific position along an instrument approach landing.

Instrument Operation: An aircraft operation in accordance with an IFR flight plan or an operation where IFR separation between aircraft is provided by a terminal control facility or an Air Route Traffic Control Center.

Ittneram Operation: As defined by the Federal Aviation Administration (FAA), all aircraft operations other than Local Operations. See Aiport Operations.

Jet Routes: See High Altitude Routes.

Landing: For ACRAM Standard [Ref. 1.1] for Air Carriers, Air Taxis, and General Aviation, Landing includes the Airport Approach Flight Phase, the Rumway Approach Flight Phase, and the Landing Flight Phase. For Military Aviation, Landing will be considered as the Flight Phase from the point where the aircraft altitude is atfected by its proximity from the approach numway to its departure from the runway under a controlled taxi.

Landing Flight Phase: The Flight Phase which consists of the time the aircraft wheels initially make contact with the rumway or landing surface until the aircraft leaves the numway or landing surface onto the designated taxiway.

Local Operation: As defined by the Federal Aviation Administration (FAA), operations performed by aircraft which 1) operate in the local traffic pattern or within sight of the airport;2) are known to be 
departing for, or arriving from flight in local practice areas located within a 20-mile radius of the airport; or 3) execute simulated instrument approaches or low passes at the aiport. See Aipont Operations.

Low Altitude (Victor) Alnway: The designated routes based on radials from VOR or VORTAC stations extending from a minimum of 1200 feet above ground level, up to but not including 18,000 feet Mean Sea Level (MSL).

Milltary Alrcraft Crash: All Class A and Class B Mishaps.

Mlltary Avlation: All aspects of aviation involving the Armed Forces of the United States. Military Aviation does not include chanter flights performed for the Armed Forces.

Milltary Training Routes: The system of designated routes used by the Armed Forces of the United States.

Mishap Rate: As defined by the United States Air Force (USAF), the number of Flight Mishaps per 100,000 flying hours.

Near Alrport Analysis: The aircraft crash frequency analysis for Aircraft Operations in the Near Airport Environment.

Near Alport Environment: The airspace area extending from the center of the airport outwards to a radius of approximately 5 miles to 35 miles depending on the presence (or absence) of a TRACON and other air traffic control considerations. The airspace near an Airport which encompasses the local airport traffic pattem, the airport numway approach pattern(s), and the airport initial climb departure pattern(s). This is generally the airspace near an airport where the aircraft are in the Rurway Approach Flight Phase, Landing Flight Phase, the Takeoff Flight Phase, and the Initial Climb Flight Phase.

Nonaliport Environment: The TRACON and the Enroute/In-Flight Environments. The airspace outside of the Near Airport Environment.

Nonprecision Approach Procedure: As defined by 14 CFR 1.1 [Ref. 1.2], a standard instrument approach procedure in which no electronic glide slope is provided.

Nonscheduled Alr Taxi: See Demand Air Taxi.

PAR: Precision Approach Radar.

Precision Approach Procedure: As defined by 14 CFR 1.1 [Ref. 1.2], a standard instrument approach procedure in which an electronic glide slope is provided, such as ILS and PAR.

Public Aircraft: As defined by 14 CFR 1.1 [Ref. 1.2], aircraft used only in the service of a government, or a political subdivision. It does not include any government-owned aircratt engaged in carrying persons or property for commercial purposes.

Public Alport: See General Aviation Aiport.

Operator: As defined by the National Transportation Safety Board (NTSB), any person who causes or authorizes the operation of an aircraft, such as the owner, leasee, or bailee of an aircraft.

Rotary Wing Alrcraft (Rotorcraft): As defined by 14 CFR 1.1 [Ref. 1.2], a heavier-than-air aircraft that depends principally for its support in flight on the litt generated by one or more rotors. More commonly known as helicopters.

Runway Approach Fllght Phase: The Flight Phase which consists of the time the aircraft crosses the outer marker defined by U.S. terminal approach procedures for that specific airport, runway and approach, until the aircraft wheels initially make contact with the nunway. 
Scheduled Alr Taxi: See Commuter Air Carrier.

Serlous In]ury: As defined by the National Transportation Safety Board (NTSB) in 49 CFR 830.2 [Ref. 1.13], any injury which 1) requires hospitalization for more than 48 hours, commencing within 7 days from the date the injury was received; 2) results in a fracture of any bone (except simple fractures of fingers, toes, or nose); 3) involves lacerations which cause severe hemorrhages, nerve, muscle, or tendon damage; 4) imvolves injury to any internal organ; or 5) involves second- or third-degree burns, or any burns affecting more than 5 percent of body surface.

Substantlal Damage: As defined by the National Transportation Safety Board (NTSB) in 49 CFR 830.2 [Ret. 1.13].

(1) Except as provided in subparagraph (2) of this paragraph, substantial damage means damage or structural failure which adversely affects the structural strength, perfomance or flight characteristics of the aircraft and which would normally require major repair or replacement of the affected component.

(2) Engine failure, damage limited to an engine, bent fairings or cowling, dented skin, small punctured holes in the skin of fabric, ground damage to rotor or propeller blades, damage to landing gear, wheels, tires, flaps, engine accessories, brakes, or wingtips are not considered "substantial damage".

Supplemental Alr Carrier (Charter): As defined by the Federal Aviation Administration (FAA), an Air Carrier which holds Certificates of Public Convenience and Necessity issued by the U.S. Department of Transportation (DOT), authorizing performance of passenger and cargo interstate charter services supplementing the scheduled service of the Certificated Air Carriers. The authority of Supplemental Air Carriers to engage in military charters is of an indelinite period. In Addition, they can perform on an emergency basis, as may be authorized by the DOT, scheduled operations including the transportation of individually ticketed passengers and individually waybilled cargo.

TACAN: Tactical Air Navigation. A UHF electronic mo (distance)-theta (azimuth) air navigation aid which provides suitably equipped aircraft (primarily military) with a continuous indication of bearing and distance to the TACAN racility.

Takeotf: For the ACRAM Standard [Ret. 1.1] for Air Carriers, Air Taxis and General Aviation, Takeoff includes the Takeoff Flight Phase, and the Initial Climb Flight Phase. For Military Aviation, Takeoff will be considered as the Flight Phase from the application of takeoff power on the numay to the point where the aircraft attitude is not affected by its proximity from the departure runway.

Takeoff Flight Phase: The Flight Phase which consists of the beginning of the aircraft takeoff roll to the moment the aircraft wheels leave the ground.

TRACON: Terminal Radar Control. The air traffic control tacility responsible for providing approachdeparture aircraft separation, especially for IFR operations, for the airspace area approximately 5 miles radius from the primary airport extending outwards to approximately $\mathbf{3 5}$ miles from the primary airport.

TRACON Analysis: The aircraft crash frequency analysis for Aircraft Operations in the TRACON Environment.

TAACON Environment: The airspace outside of the Near Airport Environment and inside and beneath the Enroute/In-Flight Environment in which all air traffic is under the control of the local TRACON.

VFR Fllght: As defined by the Federal Aviation Administration (FAA), flight conducted in accordance with visual flight rules.

Victor Airway: See Low Altitude Ainway. 
VOR: Very high frequency Omnidirectional Radio range. Used as a basis for navigation in the national airspace system.

VORTAC: Colocated VOR and TACAN navigation aids. An air navigation aid providing azimuth and distance measuring equipment at one site. 


\subsection{HOW TO USE THIS REPORT}

This report should be used to provide the generic data needed to calculated the aircraft crash frequency into the facility under consideration as part of the process for determining the aircraft crash risk to ground facilities as given by the DOE Standard Aircratt Crash Risk Assessment Methodology (ACRAM) [Ref. 1.1]. Some broad guidance will be offered on how to obtain the needed site-specific and facility specific data but this data will not be provided by this document.

The frequency that an aircraft will hit a particular building or facility is generally calculated using the the following formula which incorporates the following four factors: (1) the number of flights or operations near the area of interest, (2) the probability that an aircratt will crash or the expected crash rate, (3) the liklihood that the aircraft will hit the particular location where the building or facility is located (crash density function), and finally (4) the effective area of the building or facility. This expression is given as:

$$
\begin{aligned}
& F=\sum_{i j k} N_{i j k} \bullet P_{i j k} \bullet f(x, y)_{i j k} \bullet A_{j} \\
& \text { where: } \\
& F=\text { estimated total annual aircraft crash impact frequency for the facility of interest } \\
& \text { (no./year); } \\
& \sum_{i j k}=\text { site-specific summation over each applicable flight phase, " } " \text {, aircraft category or } \\
& \text { subcategory, "I", and flight source, "k"; } \\
& N_{i j k}=\text { annual number of estimated site-specific aircraft operations near or affecting the } \\
& \text { building or facility for each applicable summation parameter (no./year); } \\
& P_{i j k}=\text { generic aircraft crash probability during landing, takeoff, and enroute phases for } \\
& \text { each applicable category/subcategory and flight source; } \\
& f(x, y) \text { ijk }=\text { generic aircraft crash location probability density function (pdf) evaluated at } \\
& \text { the facility location for each applicable summation parameter; } \\
& \text { AF = the site-specific effective target area for facility of interest that includes both the } \\
& \text { skid and structural areas. }
\end{aligned}
$$

Tables 1.1 correlates the data needed for the factors of the four-factor formula with the data tables of this document. While guidance is given as to the general bcation of needed data both in this document and in the Modeling Technical Support Document [Ref. 1.15], the analyst is cautioned that interpretation and understanding of the data is required in order to assure that the correct data is being used for the correct purposes. 
TABLE 1.1

FREQUENCY SCREENING ANALYSIS, NEAR AIRPORT ENVIRONMENT

\begin{tabular}{|c|c|c|c|c|}
\hline CATEGORIES & N, Operations & $\begin{array}{l}\text { P, Alrcraft Crash } \\
\text { Probabllty/Fllght }\end{array}$ & $\begin{array}{l}f(x, y), \text { Alreraft } \\
\text { Crash Location } \\
\text { Distribution }\end{array}$ & $\begin{array}{l}\text { AF, Fecillty } \\
\text { Effectlve Target } \\
\text { Area }\end{array}$ \\
\hline $\begin{array}{l}\text { Alr Carrier } \\
\text { (AC) }\end{array}$ & $\begin{array}{l}\text { NACK } \\
\text { Airport Specific } \\
\text { Section } 1.3\end{array}$ & $\begin{array}{l}\text { PAC, TO } \\
\text { PAC, L } \\
\text { Section 2.2, } \\
\text { Table 2.8 }\end{array}$ & $\begin{array}{l}f(x, y) \text { Com., TOK } \\
f(x, y) \text { Com., Lk } \\
\text { Rumway Specific } \\
\text { Section } 2.3, \\
\text { Tables } 2.10,2.11\end{array}$ & $\begin{array}{l}\text { AFAC } \\
\text { Facility Specific } \\
\text { LSKCom. } \\
\text { Section } 2.3 \\
\text { Table } 2.12\end{array}$ \\
\hline Alr Taxi (AT) & $\begin{array}{l}\text { NATK } \\
\text { Airport Specific } \\
\text { Section } 1.3\end{array}$ & $\begin{array}{l}\text { PAT, TO } \\
\text { PAT, L } \\
\text { Section 2.2, } \\
\text { Table } 2.9\end{array}$ & $\begin{array}{l}f(x, y) \text { Com., TOK } \\
f(x, y) \text { Com., Lk } \\
\text { Rumway Specific } \\
\text { Section } 2.3, \\
\text { Tables } 2.10,2.11\end{array}$ & $\begin{array}{l}\text { AFAT } \\
\text { Facility Specific } \\
\text { LSKCom. } \\
\text { Section } 2.3 \text {, } \\
\text { Table } 2.12\end{array}$ \\
\hline $\begin{array}{l}\text { General } \\
\text { Aviation (GA) }\end{array}$ & $\begin{array}{l}\text { NGAK } \\
\text { Airpont Specific } \\
\text { Section } 1.3\end{array}$ & $\begin{array}{l}\text { PGA, TO } \\
\text { PGA, L } \\
\text { Section 3.4, } \\
\text { Table 3.37 }\end{array}$ & $\begin{array}{l}f(x, y) G A, \text { TOK } \\
f(x . y) G A, \text { LK } \\
\text { Rumway Specific } \\
\text { Section } 3.6, \\
\text { Tables } 3.45,3.46\end{array}$ & $\begin{array}{l}\text { AFGA } \\
\text { Facility Specific } \\
\text { LSKGA } \\
\text { Section } 3.6, \\
\text { Figure } 3.1\end{array}$ \\
\hline $\begin{array}{l}\text { Milltary } \\
\text { Aviation (MA) }\end{array}$ & $\begin{array}{l}\text { NMAK } \\
\text { Airport Specitic } \\
\text { Section } 1.3\end{array}$ & $\begin{array}{l}\text { PMA, TO } \\
\text { PMA, L } \\
\\
\text { Section 4.2, } \\
\text { Table 4.7 }\end{array}$ & $\begin{array}{l}f(x, y) M A, \text { TOK } \\
f(x, y) M A, \text { LK } \\
\text { Rumway Specific } \\
\text { Section } 4.3, \\
\text { Tables } 4.9-4.12\end{array}$ & $\begin{array}{l}\text { AFMA } \\
\text { Facility Specific } \\
\text { LSKMA } \\
\text { Section } 4.3 \\
\text { Table } 4.20\end{array}$ \\
\hline
\end{tabular}

Notes:

TO = Takeoff

$L=$ Landing

Com. = Commercial Aviation $=$ Air Carriers (ACs) and Air Taxis (ATs)

Each parameter with the subscript " $k$ " must be determined specifically for that site with respect to the $k$ th airport. 


\subsection{REFERENCES}

[1.1] DQE Standard Accident Analusis for Aircratt Crash into Hazardous Facilities. DOE-STD-3014-96, U.S. Department of Energy, Washington, DC, July 1996.

[1.2] "Definitions and Abbreviations", Ittle 14 Aeronautics and Space. Code of Federal Beoulations. Part 1 (14 CFB 1), U.S. National Archives and Record Administration, Washington, DC, January 1, 1995.

[1.3] Designation of Class A, Class B, Class C, Class D, and Class E Airspace Areas; Ainways; Routes; and Reponting Points, ", Inle 14 Aeronautics and Space, Code of Federal Requlations. Subchapter E-Airspace. Part 71 (14 CER 711, L U.S. National Archives and Record Administration, Washington, DC, January 1, 1995.

[1.4] "Air Traftic and General Operating and Flight Rules", Ittle 14 Aeronautics and Space. Code of Eederal Requlations, Chapter 1-Federal Aviation Administration. Department of Transportation Subchapter F. Air Traffic and General Operatino Rules. Part 91 (14 CFR 91). U.S. National Archives and Record Administration, Washington, DC, January 1, 1995.

[1.5] "Ultralight Vehicles", Ittle 14 Aeronautics and Soace Code of Federal Bequlations, Chapter L Eederal Aviation Administration. Department of Transoortation. Subchapter F. Air Traffic and General Operating Rules. Part 103 (14 CFR 103), U.S. National Archives and Record Administration, Washington, DC, January 1, 1995.

[1.6] "Parachute Jumping", Intle 14 Aeronautics and Space, Code of Federal Requlations. Chapter L Eederal Aviation Administration. Department of Iranspontation. Subchapter F. Air Traffic and General Ooeratino Rules. Part 105 (14 CFR 105), U.S. National Archives and Record Administration, Washington, DC, January 1, 1995.

[1.7] "Certification and Operations: Domestic, Flag, and Supplemental Air Carriers and Commercial Operators of Large Aircraft", Iitle 14 Aeronautics and Space. Code of Federal Requlations. Chapter L-Federal Aviation Administration. Department of Transportation Subchapter G-Air Carriers. Air Travel Clubs. and Operators for Compensation or Hire: Centification and Operations. Pan 121 (14 CFB 121), U.S. National Archives and Record Administration, Washington, DC, January 1, 1995.

[1.8] "Certification and Operations: Airplanes Having a Seating Capacity of 20 or More Passengers or a Maximum Payload of 6,000 Pounds or More", Iitle 14 Aeronautics and Soace. Code of Federal Bequlations, Chapter L-Federa L Aviation Administration. Department of Transportation. Subchapter G-Air Carriers. Air Travel Clubs, and Operators for Comoensation or Hire: Centification and Operations. Part 125 (14 CFR 125), U.S. National Archives and Record Administration, Washington, DC, January 1, 1995.

[1.9] "Certification and Operations of Scheduled Air Carriers with Helicopters", Title 14 Aeronautics and Space, Code of Federal Requlations, Chapter L-Federal Aviation Administration. Department of Iransportation Subchapter G-Air Carriers, Air Travel Clubs, and Operators for Comoensation or Hire: Certification and Operations. Part 127 (14 CFR 127), U.S. National Archives and Record Administration, Washington, DC, January 1, 1995.

[1.10] "Operations: Foreign Air Carriers and Foreign Operators of U.S.-Registered Aircraft Engaged in Common Carriage". Itlle 14 Aeronautics and Space. Code of Federal Requlations. Chapter. 
Eederal Aviation Administration. Department of Transportation. Subchapter G-Air Carmiers. Air Iravel Clubs, and Operators for Compensation or Hire: Certification and Operations. Part 129114 CFR 1291, U.S. National Archives and Record Administration, Washington, DC, January 1, 1995.

[1.11] "Rotorcraft External-Load Operations", Ittle 14 Aeronautics and Space. Code of Federal Bequlations, Chapter L-Federal Aviation Administration. Department of Transportation. Subchapter G-Air Carriers, Air Travel Clubs, and Operators for Compensation or Hire: Centification and Qeerations. Part 133 (14 CFR 133) U.S. National Archives and Record Administration, Washington, DC, January 1, 1995.

[1.12] "Air Taxi Operators and Commercial Operators", Intle 14 Aeronautics and Space, Code of Federal Beoulations, Chapter LEederal Aviation Administration. Department of Transoortation.

Subchapter G-Air Camiers, Air Travel Clubs, and Oeverators for Compensation or Hire: Centification and Operations. Pant 135 (14 CER 135). U.S. National Archives and Record Administration, Washington, DC, January 1, 1995.

[1.13] "Agricultural Aircratt Operations", Ittle 14 Aeronautics and Soace Code of Federal Regulations. Chapter L-Federal Aviation Administration. Department of Iranspontation. Subchapter G-Air Camiers. Air Travel Clubs, and Operators for Compensation or Hire: Certification and Operations. Pan 137 (14 CFR 137D. U.S. National Archives and Record Administration, Washington, DC, January 1, 1995.

[1.14] "Notification Reporting of Aircraft Accidents or Incidents and Overdue Aircraft, and Preservation of Aircraft Wreckage, Mail, Cargo, and Records", Ittle 49 Transportation. Chapter VIll-National Iransportation Safety Board. Part 830 (49 CER 830) U.S. National Archives and Record Administration, Washington, DC, October 1, 1995.

[1.15] ACRAM Modeling Technical Suppont Document, LA-UB-96-2460/TSA-11-95-B12, C. Bolig, D. Sanzo, D. Stack, R. Mensing, and R. Glaser, Los Alamos National Laboratory, Los Alamos, NM, July 1996. 


\section{COMMERCIAL AVIATION}

\subsection{INTRODUCTION}

Commercial aviation is the broad categorization of aviation activities which are performed for commercial purposes. Such activities include passengers flights performed by scheduled airlines, cargo flights by freight airlines, charter fights by nonscheduled airlines, etc. Because of the evolutionary development of aviation, and the related evolutionary development of the Federal aviation regulations, several classification schemes are currently in existence for classifying commercial aviation activities.

The Federal Aviation Administration (FAA) tabulates operations data for U.S. Air Carriers according to the economic classification under which the operator of the aircraft is classified. Under this classification arrangement, U.S. Air Carriers includes Certificated Air Carriers, Supplemental Air Carriers, Commercial Operators of Large Aircraft, and Air Taxis (inchuding commuters) [Ret. 2.1]. This classification arrangement can usually be correlated with the flights conducted under 14 CFR 121 [Ref. 2.1], and 14 CFR 135 [Ref. 2.2], respectively. The definition of these terms will be expanded in Section 2.2 for 14 CFR 121 Air Carriers, and in Section 2.3 for 14 CFR 135 Air Carriers.

Air Traffic Controllers use another classitication scheme in tabulating their data on aiport operations. As discussed in Ref. 2.3 and from discussions with the FAA staff which compiles the data on airport traftic activity, air carriers are defined as aircratt in general commercial service capable of carrying 70 or more passengers, regardless of whether that particular flight is being flown under 14 CFR 121 or 14 CFR 91 rules [Ref. 2.4]. All other aircraft in general commercial service that carny less than 70 passengers are classified as air taxies. However, since many air taxis operate small single-engine aircraft, air controllers often count these flights as general aviation flights unless the flight is identified by a flight plan or a commercial livery is carried by the aircraft.

To estimate an accident rate, one must obtain the number of accidents that occurred during the performance of some number of operational measures. The National Transportation Safety Board (NTSB) defines an aircraft accident as an occurrence associated with the operation of an aircratt which takes place between the time any person boards the aircraft with the intemtion of flight until such time as all such persons have disembarked, and in which any person suffers a fatal or serious injury as a result of being in or upon the aircraft or by direct contact with the aircraft or anything attached thereto, or in which the aircraft receives substantial damage. Operational measures could be defined in terms of number of departures or flights, number of aircraft hours flown, or the distance the aircrafts have flown (in terms of miles, nautical miles, kilometers, etc.).

The National Transportation Safety Board (NTSB) tabulates accident data for U.S. Air Carriers according to the regulations under which the flight was conducted. Under this classification arrangement, U.S. Air Carriers include flights conducted under 14 CFR 121 [Ref. 2.1], and 135 [Ref. 2.2].

For the ACRAM standard, the aircratt accidents of interest are those accidents which result in the destruction or substantial (major) damage to the aircraft. The reasoning behind this is that unless the aircraft suffered destruction or major damage, then the impact forces imposed on the aircraft were probably not very substantial, and therefore, the impact forces imposed on anything that aircraft hit, such as a building, would not be very substantial. For commercial aviation, i.e. air carriers and air taxis, a substantial portion of the accidents involve tatal or serious injuries but minor or no damage to the aircratt. Examples of such accidents include ground accidents involving ramp personnel, or severe air turbulence accidents involving passengers or crew members. Therefore, for the ACRAM standard, it is necessary to define commercial aircraft crashes differently from commercial aircraft accidents. More formally, a commercial aircratt crash is defined as any aircraft accident involving an aircraft type generally used for commercial service during flight operations (defined as the beginning of takeoff roll to landing touchdown) which results in the destruction or substantial damage to the aircraft. Fatal or serious injury sustained as a result of the accident itself, without related destruction or substantial damage to the aircraft is not a criterion for consideration as a commercial aircraft crash. Based on this definition for commercial aircraft crashes, a crash rate for air carriers and air taxis can be calculated based on the number of aircratt destroyed or substantially damaged per operational measure. 
For the ACRAM Standard [Ref. 2.5], commercial aviation will be considered to consist of two categories of aviation: 1) Air Carriers and 2) Air Taxis. Air Carriers and Air Taxis will be defined in accordance with the general practice used by air traffic controllers in classifying Air Carrier and Air Taxis operations.

The development of commercial aviation data for the ACRAM Standard is discussed in the following sections: 2.2 Air Carrier (14 CFR 121) Accident and Crash Rates, 2.3 Air Taxi (14 CFR 135) Accident and Crash Rates, 2.4 Commercial Aviation Crash Parameter Distributions, 2.5 Commercial Aviation Aircraft Characteristics, and 2.6 References. 


\subsection{AIR CARRIER (14 CFR 121) ACCIDENT AND CRASH RATES}

A Certificated Air Carrier is defined as an Air Carrier holding a Certificate of Public Convenience and Necessity issued by the U.S. Department of Transportation (DOT) to conduct scheduled services interstate. Nonscheduled or charter operations may also be conducted by these carriers. These carriers operate large aircraft ( 30 seats or more or a maximum payload of 7,500 pounds or more) in accordance with 14 CFR 121 [Ret. 2.1].

A Commercial Operator of Large Aircraft is defined as a Commercial Operator operating aircraft with 30 seats or more or a maximum paybad capacity of 7,500 pounds or more [Ret. 2.1].

A Supplemental Air Cartier or a charter Air Carrier is defined as an Air Carrier which holds Certificates of Public Convenience and Necessity issued by the U.S. Department of Transportation (DOT), authorizing performance of passenger and cargo interstate charter services supplementing the scheduled service of the Certificated Air Carriers. The authority of Supplememtal Air Carriers to engage in military charters is of an indefinite period. In addition, they can perform on an emergency basis, as may be authorized by the DOT, scheduled operations including the transportation of individually ticketed passengers and individually waybilled cargo [Ref. 2.1]

The NTSB has been granted the authority to investigate all aviation accidents that occur in the United States except for those involving militany aircraft only and for certain public-use aircraft only. In addition, the NTSB investigates other accidents as requested, e.g. U.S. air camier accidents in foreign airspace, foreign air carriers in foreign airspace involving U.S. buith aircraft and involving special circumstances, etc. The NTSB tabulates accident data annually for U.S. Air Carriers according to the regulations or part under which the flight was conducted, i.e. 14 CFR 121 [Ref. 2.1], and 14 CFR 135 [Ref. 2.2]. Flights conducted 14 CFR 121 involved aircratt capable of carrying 30 or more passengers or a maximum payload of 7,500 pounds or more. Tables 2.1 to 2.3 taken from the NTSB Annual Review of Aircraft Accident Data, U.S. Air Carrier Operations [Rel. 2.6 to 2.26] and the NTSB News Annual Press Release on U.S. Airline Accidents [Ref. 2.27 and 2.28] presents the following information for the years 1973 to 1995:

1. The number of fatal accidents,

2. The total number of accidents,

3. The number of passenger fatalities aboard the aircraft flying under that part,

4. The number of crew fatalities aboard the aircraft flying under that part,

5. Other fatalities associated with the accident including ground fatalities and fatalities aboard aircratt involved in the accident but not flying under that part, i.e. collisions,

6. The total number of fatalities,

7. The number of aircraft flying under that part involved in the accidents that were destroyed,

8. The number of aircraft flying under that part involved in the accidents that were substantially (major) damaged,

9. The number of aircraft flying under that part involved in the accidents that suffered minor or no damage,

10. The total number of aircraft flying under that part involved in the accidents,

11. The number of aircratt hours flown by aircraft flying under that part,

12. The number of aircratt miles by thousands flown by aircraft flying under that part,

13. The number of aircraft departures performed by aircraft flying under that part,

14. The average speed of aircraft flying under that part, calculated by dividing the number of aircraft miles flown by the number of aircraft hours flown,

15. The average number of aircraft miles flown by aircraft flying under that part per flight or departure, calculated by dividing the number of aircratt miles flown by the number of departures,

16. The average amount of time flown by aircraft flying under that part per flight or departure, calculated by dividing the number of aircraft hours flown by the number of departures,

17. The fatal accident rate per aircratt hour, aircraft mile, and departure, determined by dividing the number of fatal accidents by the number of aircratt hours, aircraft miles, and departures, respectively, 
18. The total accident rate per aircratt hour, aircraft mile, and departure, determined by dividing the total number of accidents by the number of aircraft hours, aircratt miles, and departures, respectively.

19. The crash rate per aircraft hour, aircratt mile, and departure, determined by dividing the number of crashes (defined as aircraft flying under that part involved in the accident that were destroyed or substantially damaged) by the number of aircratt hours, aircraft miles, and departures, respectively.

The total, average, and standard deviation for each of the above categories of information were calculated for those years in which information was available, except for the calculated average speed, distance and time per departure (14. to 16.) and calculated fatal accident, total accident and crash rates (17. to 19.) which had only the average and standard deviation calculated. Table 2.1 presents data for the scheduled U.S. air carriers operating under 14 CFR 121. Table 2.2 presents data for the nonscheduled or charter U.S. air carriers operating under 14 CFR 121. Table 2.3 presents data for total U.S. air carriers operating under 14 CFR 121 and is the sum of Tables 2.1 and 2.2.

While Tables 2.1 to 2.3 were taken from NTSB documents [Refs. 2.6 to 2.28], only the accident data and the aircraft damage data were compiled by the NTSB. All operations data are tabulated by the F.A.A. It should be emphasized that all of the accidents, aircrafts imvolvement, and operations given in Tables 2.1 to 2.3 were tabulated on the basis of the part or regulation under which the flight was conducted and that the accident and crash rates are calculated on a consistent basis.

Because the data of Tables 2.1 to 2.3 were tabulated on the basis of the part or regulation under which the flight was conducted, certain caveats should be associated with these tables when used in conjunction with the ACRAM standard. As the final result desired by the frequency analysis of the ACRAM standard is the aircraft crash frequency into the facility, the analysis should be based on the total number of aircraft operations near the facility, regardless of the part or regulations under which the flight is conducted. This is exactly how air traffic controllers tabulate aircraft operations near airports and is consistent with the requirements of the ACRAM Standard [Ref. 2.5]. The crash rate needed by the crash frequency analysis however, is determined by the part under which the tlight is conducted which is inconsistent with the requirements of the ACRAM Standard. The result of using crash rate based solely on the part or regulation under which the flight is conducted is that other flights such as repositioning tlights and training flights, flown by the same aircraft are conducted under Part 91 rules and are not counted in the NTSB tables. The resultant error introduced by this inconsistency is probably not large, but it is there nevertheless.

Another caveat associated with Tables 2.1 to 2.3 is that accidents by foreign air carriers in U.S. airspace are not included. This is because foreign air carriers conduct their flights in U.S. airspace under 14 CFR Pant 129 rules [Ret. 2.29]. As the foreign air carrier operations in U.S. airspace are also not counted in Tables 2.1 to 2.3, no emor is introduced. However, for the ACRAM Standard [Ref. 2.5], an aircraft crash is equally damaging to a facility whether by a U.S. air carrier or toreign air carrier. This factor is compensated to some degree by the fact that accidents by U.S. air carriers in foreign airspace are counted by the NTSB and the operations by U.S. air carriers in foreign airspace are included. Again, this is because these flights are conducted under Part 121 nules even while in foreign airspace. Whether these two factors are comparable and therefore one can consider them to cancel each other out is unknown at this time.

The solution to this dilemma posed by these caveats would be to determine the crash rate regardless of the part or regulation under which that the flight is conducted. This would required determining the number of U.S. and foreign air carrier accidents and operations which occurTed in U.S. airspace only, under all flight nules. It would be possible to develop this information but this would require additional research.

Accident data compiled by the NTSB and operations data compiled by the FAA prior to 1980 were gathered on the basis of the economic classification of the air carrier, i.e. certificated route air carriers, supplemental air carriers, commercial operators of large aircraft, and all cargo air service. Flights performed by these air carriers were further broken down into scheduled and nonscheduled service, domestic and intermational service, passenger and other operations. The change in the way the NTSB 
and the FAA compiled their accident and operation data was due to the deregulation of the U.S. airline industry in 1978. One of the result of the deregulation of the U.S. airline industry in 1978 was that many of the economic classifications were now obsolete and that classification of operations and accidents under the previous classification system was no longer desired. This is the main reason for the changeover in 1980 by the NTSB and FAA to gather accident and operations data on the basis of the economic classification of the air carriers to flight nules under which the flight was conducted. The result of this changeover is that accident and operations data compiled prior to 1980 may be inconsistent with accident and operations data compiled after 1980. Certain assumptions must be made regarding accident and operations data compiled betore 1980 if this data is included with accident and operations data compiled after 1980. One of these assumptions is that the operations of U.S. Air Carriers operating on scheduled certificated route operations are comparable to the Scheduled U.S. Air Carriers operated under 14 CFR 121 for the 1973-1979 time period. Another assumption is that the operations of U.S. Air Carriers operating on nonscheduled certificated route operations are comparable to the Nonscheduled U.S. Air Carriers operated under 14 CFR 121 for the 1973-1979 time period.

In order to calculate an accident and crash rate that is reasonably conservative and yet incorporates recent data, it is necessary to inchude data over a reasonably long period of time that is complete and current. For U.S. air carriers operating under 14 CFR 121, the years 1973 to 1994 were selected. This enabled the accident and crash rates for 121 air carriers to be determined for a 22 year periods.

The accident and crash rates given by Tables 2.1 to 2.3 for 121 air carriers represent the accident and crash rates over the entire flight. As experience and numerous past studies on commercial aviation safety has shown, accidents do not occur with equal probability during the course of the flight. Certain flight phases, e.g. landing and takeoff, experience significantly higher number of accidents and crashes. To determine accident and crash rates by flight phase, it is necessary to determine the number of accidents and crashes that occur in each flight phase.

Table 2.4 presents the total number of aircraft flying under 14 CFR 121 regulations involved in accidents by flight phase for the 1973-1994 time period. From the number of 14 CFR 121 aircraft involved in accidents given by Table 2.4, and assuming 1.4 hour average flight duration, 570 miles average distance flown per flight, and 407 miles/hour average velocity for each flight, for the 140,872,105 departures (flights) flown by all U.S. Air Carriers (scheduled and nonscheduled) for 1973 to 1994, the accident rates by flight phases are determined in Table 2.5. The adjusted number of accidents by flight phase in Table 2.5 (the 4th column from the left) are the number of accidents minus the accidents which occurred in the taxi, ground accident, and unknown flight phases. The adjusted percentage by flight phase in Table 2.5 (the 5th column from the left is the adjusted number of accidents for that flight phase divided by the total adjusted number of accidents. The ACRAM Standard [Ref. 2.5] defines three flight phases, takeoff, landing and in-flight or enroute. Takeoff is defined as encompassing the actual takeoff roll and the initial climb phase. Landing is defined as including the landing approach, and the actual landing roll. Enroute or in-flight is defined as including the climb to cruise, cruise or enroute, and the descent from cruise flight phases. To meet the needs of the ACRAM Standard, crash rates for the flight phases used by the ACRAM Standard must be determined. This is also done by combining the accidents occurring in the takeoff and initial climb phases into the ACRAM takeoff accident rate. The ACRAM in-flight accident rate was determined by combining the accidentss occuming during the climb to cruise, cruise/enroute, and descent from cruise flight phases. The ACRAM landing accident rate was determined by combining the accidents occurring during the landing approach and landing flight phases.

Table 2.6 presents the number of aircraft flying under 14 CFR 121 regulations involved in crashes (accidents resulting in destruction or major damage to the airframe) by flight phase for the 1973-1994 time period. The crash rates of Table 2.7 are calculated from the number of crashes determined in Table 2.6 by the procedure used in Table 2.5. Similar operations assumptions are also used in the calculation. Crash rates by ACRAM defined flight phases are determined in the last four columns of Table 2.7.

Table 2.8 presents the number of aircraft flying under 14 CFF 121 regulations involved in crashes which resulted in destruction or such major damage to the airframe that the aircraft is written off (major-w.0.) by flight phase for the 1973-1994 time period. The crash rates of Table 2.9 are calulated from the number of crashes determined in Table 2.8 by the procedure used in Table 2.5. Similar operations assumptions are 
also used in the calculation. Crash rates by ACRAM defined flight phases are also determined in the last four columns of Table. 2.9.

Among the primary assumptions used by the ACRAM Standard [Ref. 2.5] is that the facility under consideration is located far enough from the aipport that only aircraft impacts (crashes) located off the nunway need be considered. Unfortunately, the information available on 14 CFR 121 Air Carrier accidents in many instances allows only the identification of whether the accident occurred on or off the airport. In most cases, the accident which occurs on the airport also occurs on the runway. For these reasons, a sort was performed on the 14 CFR 121 Air Carrier accidents to screen those events which occurred on the airport.

Table 2.10 presents the total number of aircraft flying under 14 CFR 121 regulations imvolved in accidents which occurred off the airport by flight phase for the 1973-1994 time period. The accident rates of Table 2.11 are calculated from the number of accidents determined in Table 2.10 by the procedure used in Table 2.5. Similar operations assumptions are also used in the calculation. Crash rates by ACRAM defined flight phases are determined in the last four columns of Table 2.11.

Table 2.12 presents the number of aircraft thing under 14 CFR 121 regulations involved in crashes (accidents resulting in destruction or major damage to the aiframe) which occurred off the airport by flight phase for the 1973-1994 time period. The accidem rates of Table 2.13 are calculated from the number of crashes determined in Table 2.12 by the procedure used in Table 2.5. Similar operations assumptions are also used in the calculation. Crash rates by ACRAM defined flight phases are determined in the last four columns of Table 2.13.

Table 2.14 presents the number of aircraft flying under 14 CFR 121 regulations involved in crashes which result in destruction or such major damage to the aiframe that the aircraft is written off (major-w.o.) which occurred off the airport by flight phase for the 1973-1994 time period. The accident rates of Table 2.15 are calculated from the number of crashes determined in Table 2.14 by the procedure used in Table 2.5. Similar operations assumptions are also used in the calculation. Crash rates by ACRAM defined flight phases are determined in the last four columns of Table 2.15.

Finally, Table 2.16 presents a comparison of the total number of aircraft flying under 14 CFR 121 regulations involved in accidents/crashes with the number of aircraft flying under 14 CFA 121 regulations involved in off airport accidents/crashes. Note that for the taxi, takeoff, landing, and ground flight phases, the number of 14 CFR 121 aircratt involved in off airport accidents/crashes are, be definition, zero. 
Table 2.1

Scheduled U.S. Ar Corriers Opareting Undor 14 CFA 121 [References 2.6 to 2.28]

\begin{tabular}{|c|c|c|c|c|c|c|c|c|c|c|c|c|c|c|c|}
\hline Yoar & $\begin{array}{l}\text { Fas } \\
\text { Aosa }\end{array}$ & $\begin{array}{l}121 \\
P \infty 20\end{array}$ & $\begin{array}{c}\text { Fetaliven } \\
121 \\
\text { Crow }\end{array}$ & Other & Tat & $\begin{array}{l}\text { Toud } \\
\text { Aona }\end{array}$ & Den. & Mapor & 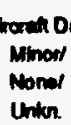 & Tow & $\begin{array}{l}121 \text { Arron } \\
\text { Hours } \\
\text { Flown }\end{array}$ & $\begin{array}{l}121 \text { Arroruth } \\
\text { Mim } \\
(000)\end{array}$ & $\begin{array}{l}121 \text { Nracen } \\
\text { Dopportures: }\end{array}$ & 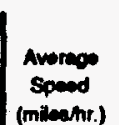 & Ac Millow \\
\hline 1972 & & & & & & & & & & & & & & & \\
\hline $\begin{array}{l}1973 \\
1974\end{array}$ & of & $\begin{array}{l}100 \\
410\end{array}$ & 29 & 1 & $\begin{array}{l}222 \\
457\end{array}$ & 30 & $?$ & 10 & 10 & 95 & $\begin{array}{l}5,000,575 \\
5,474,485\end{array}$ & $\begin{array}{l}2,446,114 \\
2,250,136\end{array}$ & $\begin{array}{l}5.133,810 \\
4,725,703\end{array}$ & $\begin{array}{l}415.03 \\
412.40\end{array}$ & 476.60 \\
\hline 1075 & 2 & 118 & 9 & 0 & 125 & 31 & - & 10 & 15 & 31 & $5,122,665$ & $2.220,505$ & $4,704,052$ & 413.17 & 478.20 \\
\hline 1978 & 2 & 30 & 2 & 0 & & 2 & 4 & 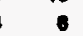 & 12 & 22 & $5,507,601$ & $2,319,967$ & $4,836,130$ & 415.20 & 470.1 \\
\hline 1977 & 3 & $\infty$ & 3 & : & 70 & 10 & 2 & : & 12 & 22 & $5,800,843$ & $2,418,652$ & $4,934,004$ & 416.95 & 400.10 \\
\hline pere & 5) & tat & 11 & 14 & 100 & 20 & - & 2 & 12 & 20 & 0.001 .740 & $2,520,185$ & $3,015,000$ & 417.02 & 502.40 \\
\hline 1070 & 4) & 323 & 25 & 3 & 351 & 23 & 4 & $n$ & 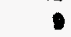 & 24 & $8,713,004$ & $2,701,120$ & $5,300,052$ & 415.77 & 516.91 \\
\hline 1000 & o & 0 & 0 & 0 & & 15. & 4 & 4 & 7 & 15 & $7,060,000$ & $2.929,000$ & $5,587,000$ & 414.34 & 528.14 \\
\hline 1901 & 4) & i & i & 2 & & 25 & i & 11 & 13 & 25 & 8.034 .000 & $2,011,000$ & $5,420,000$ & 411.33 & 518.63 \\
\hline 1902 & 3] & 210 & 11 & 12 & 233 & 15. & 9 & 7 & 5 & 15 & $0,607,770$ & $2,008,085$ & $5,162,340$ & 419.36 & $\sin 11$ \\
\hline 1863 & a & 8 & 6 & 1 & 15 & 22 & 4 & 10 & 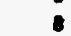 & 22 & $8,014,000$ & $2,020,900$ & $5,235,232$ & $\$ 22.40$ & 357.23 \\
\hline 1804 & 11 & 1 & 3 & 0 & & 19] & 2 & 4 & 7 & 13 & $7,736,037$ & $3,258,910$ & $5,068,078$ & 421.26 & 575.10 \\
\hline 1905 & 4 & 174 & 22 & 1 & 197 & 17 & 6 & . & 3 & 17 & 1205.332 & $3,452,750$ & $8,060.003$ & 417.74 & 3600 \\
\hline 1006 & 1 & 0 & 1 & 0 & & 201 & 2 & - & 12 & 20 & 905,150 & $3,820,120$ & $0.928,103$ & 400.27 & 552.70 \\
\hline 1007 & & 219 & 17 & 2 & 232 & 32 & 5 & 15 & 13 & 95 & $10.115,407$ & $4.125,674$ & $7,200,005$ & 407.00 & 585.75 \\
\hline 1008 & 3] & 256 & is & 11 & 205 & 20 & 7 & 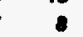 & 13 & 20 & $10,521,052$ & $\$, 200,785$ & $7,347,575$ & 200.08 & 570.00 \\
\hline 1000 & of & 122 & 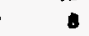 & 1 & 131 & 24] & ; & 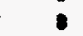 & 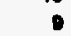 & 24 & $10,597,022$ & $4,037,234$ & $7,267,341$ & 400.25 & 39681 \\
\hline 90 & 5 & . & 5 & 24 & 37 & 2 & 4 & 8 & 13 & 23 & $11,524,720$ & $4,711,542$ & $7,028,357$ & 406.02 & $\operatorname{sen} 26$ \\
\hline 1001 & $\mathrm{~d}$ & so & 11 & 1 & 62 & 25 & 5 & - & 12 & 20 & $11,258,579$ & $4,584,562$ & $7,072,307$ & 407.21 & 307.54 \\
\hline 1002 & 4 & 20 & 5 & 2 & 33 & 17 & 4 & 0 & 19 & 17 & $11,000,020$ & $4,015,632$ & $7,717,406$ & 406.32 & 624.00 \\
\hline 1003 & 1 & 0 & 0 & i & & 21) & i & 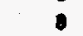 & 13 & 22 & $12,189,525$ & $4,060,025$ & $7,035,749$ & 100.04 & 820.30 \\
\hline 1004 & 4 & 220 & 11 & 0 & 200 & 10) & 3 & 5 & 10 & 10) & $12,472.010$ & 5.102 .114 & $0.041,709$ & 400.12 & 034.54 \\
\hline & 2 & 152 & a & - & 160 & 33 & NA & M.A & N.A & N.A. & $12,040,000$ & $5,269,000$ & $8,220,000$ & 418.59 & Bas.00 \\
\hline Toxal & B2 & 10 & 241 & 91 & 3078 & 3501 & 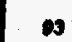 & 150 & 200 & 314 & $197.150,232$ & $8 x, 200,713$ & 144210,709 & 411.87 & 500.00 \\
\hline$y$ & 3.57 & 110.30 & 10.48 & 3.96 & 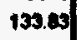 & 20.30 & 4.23 & 7.18 & 11.85 & 23.30 & $8,371,740$ & $3.530,406$ & $0,270,025$ & 412.80 & $\mathbf{5 3 3 . 3 0}$ \\
\hline std $>$, & & & 9.69 & 6.11 & & 6.80 & 1.00 & 3.38 & 5.21 & 6.89 & $2,597,410$ & $1,043,691$ & $1,260,010$ & 5.36 & 54.20 \\
\hline
\end{tabular}

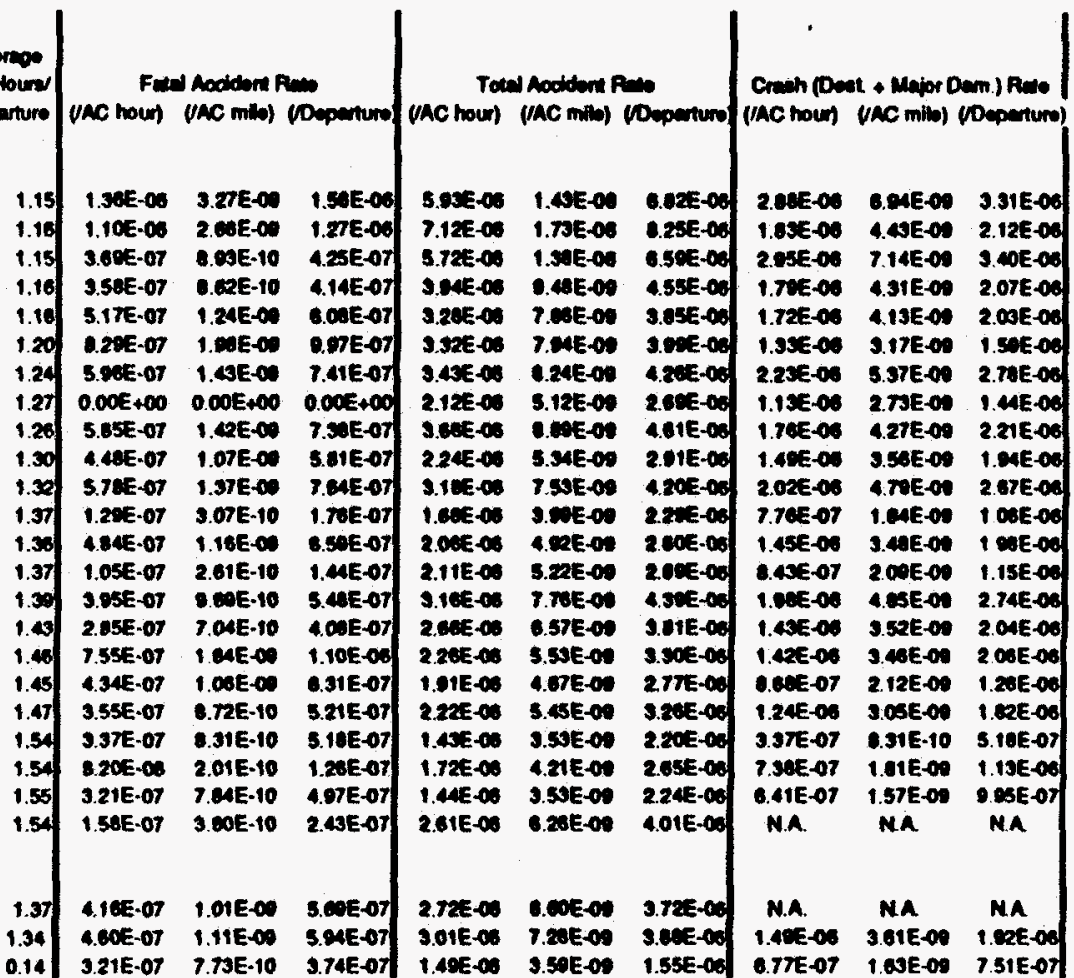

P- Proliminary Datn

NA - Mormation not aveileblo whon Lbblo properw.

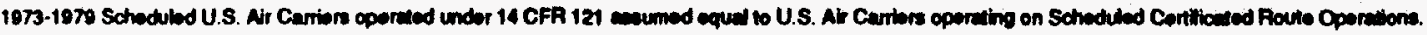

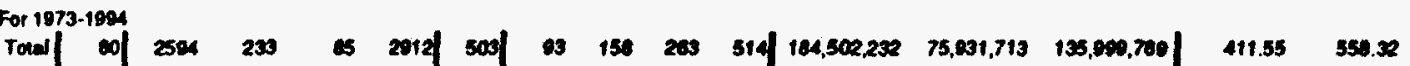

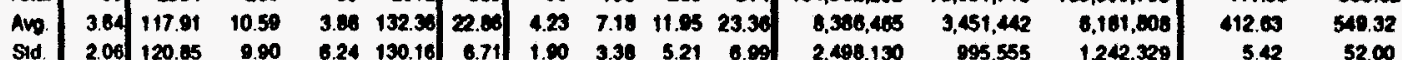

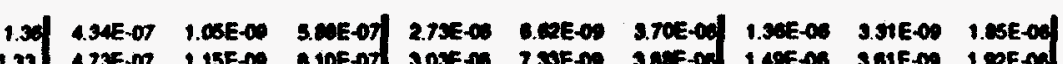

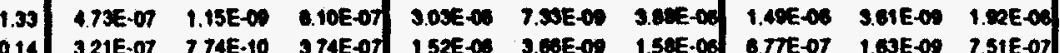


Tablo 2.2

Nonschedulod U.S. Air Carriers Operating Under 14 CFA 121

[Roferences 2.6 to 2.28]

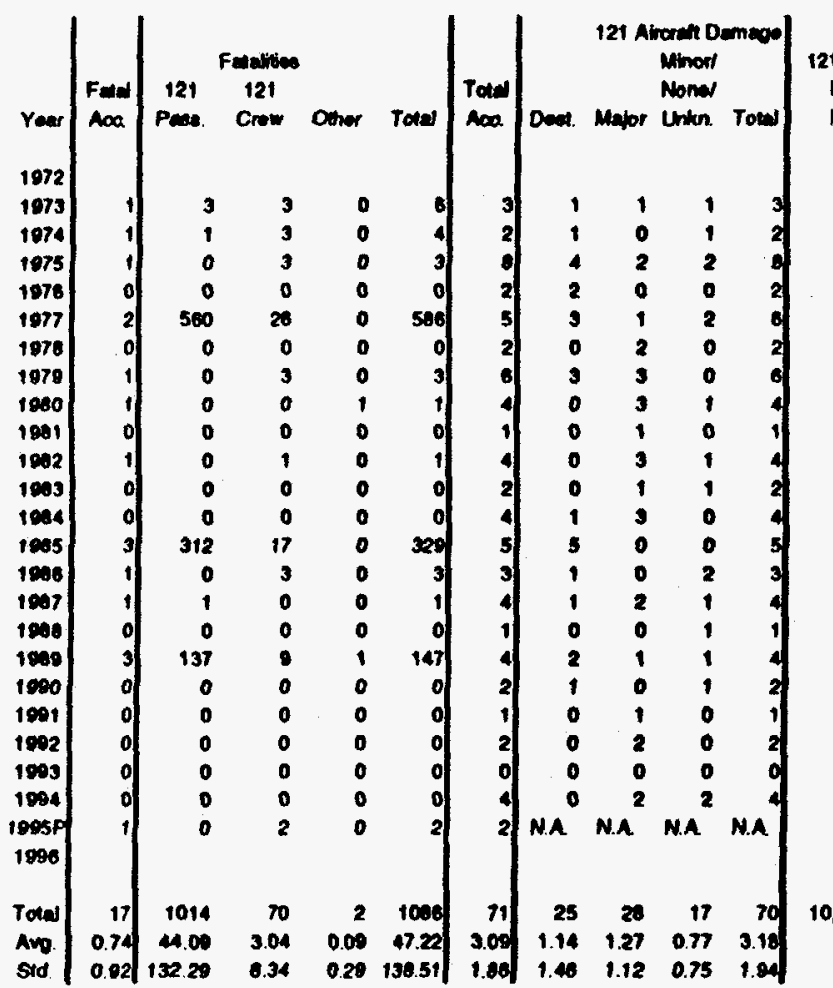

121 Arros 121 Airoreth

Foum Miles 121 nimeren

\begin{tabular}{lll|l}
$231,800 \quad 107,618$ & 24,181 & 464.00 & 1278.72
\end{tabular}

\begin{tabular}{lll|ll}
202,042 & 92,670 & 76,003 & 450.06 & 1218.30
\end{tabular}

\begin{tabular}{llll|ll}
184,794 & 84.128 & 72.132 & 458.24 & 1166.20 \\
218,126 & 0.192 & 87,363 & 454.75 & 1135.40
\end{tabular}

\begin{tabular}{rrr|rr}
218,126 & 09.192 & 87,363 & 454,75 & 1135.40 \\
240,840 & 108,451 & 108,440 & 41.98 & 981.60
\end{tabular}

\begin{tabular}{lll|ll}
202,403 & 06,197 & 06,351 & 434.72 & 015.37
\end{tabular}

\begin{tabular}{lll|ll}
165,017 & 06,018 & 06,311 & 410.20 & 760.06
\end{tabular}

\begin{tabular}{lll|ll}
310,000 & 115,000 & 182,000 & 370.97 & 70000 \\
\hline 292,000 & 109,000 & 155,000 & 37320 & 70323
\end{tabular}

\begin{tabular}{lll|ll}
292,000 & 109.000 & 155,000 & 373.20 & 703.23 \\
342,555 & 131,028 & 180,787 & 342.25 & 507.23
\end{tabular}

\begin{tabular}{lll|ll}
342,555 & 131,628 & 180,787 & 344.25 & 607.23 \\
\hline 393,630 & 140,409 & 300,112 & 360.85 & 70971
\end{tabular}

\begin{tabular}{|lll|ll}
383,830 & 140,400 & 209.112 & 360.85 & 700.71
\end{tabular}

\begin{tabular}{lll|ll}
429,007 & 100.153 & 232.778 & 394.22 & 720.66 \\
441592 & 178.264 & 237.868 & 400.90 & 760.49
\end{tabular}

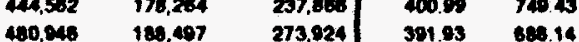

\begin{tabular}{|ll|lll}
520,705 & 234,047 & 273,924 & 391.83 & 300.14
\end{tabular}

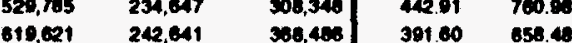

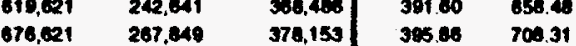

\begin{tabular}{lll|ll}
625,300 & 256,545 & 200,545 & 365.68 & 700.31 \\
\hline 13,41 & 871.06
\end{tabular}

\begin{tabular}{lll|ll}
641,444 & 288,287 & 313,203 & 415.14 & 850.04
\end{tabular}

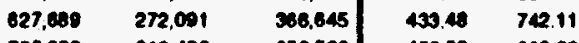

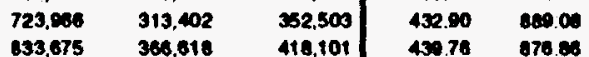

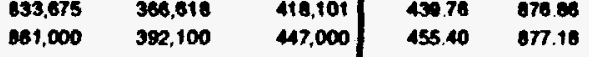

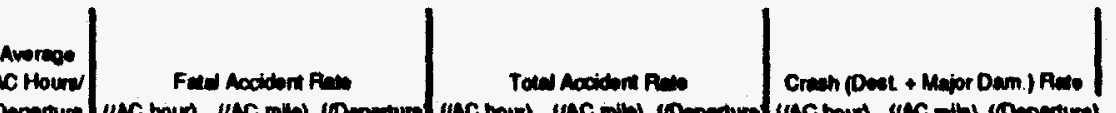

$40,260,372 \quad 4,300,400$

\begin{tabular}{ll|ll}
$4,300,403$ & $5.319,336$ & 418.70 & 000.45 \\
\hline
\end{tabular}

\begin{tabular}{l|ll}
231,275 & 418.20 & 000.45 \\
118.16 & 056.65
\end{tabular}

$219.154 \quad 95.578$

P. Pralminary Data

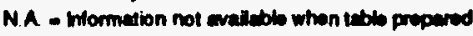

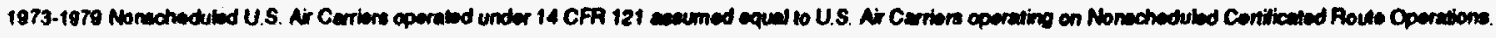

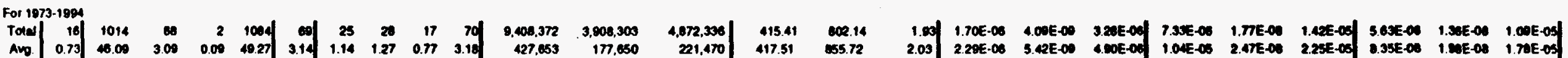

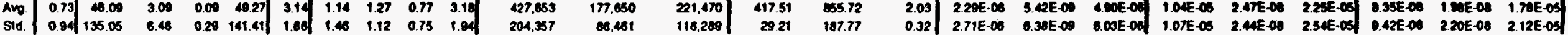

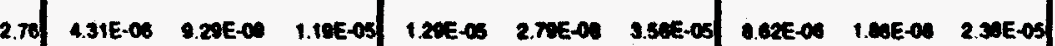
2.67 1.93E-06 1.00E-00 1.31E-05

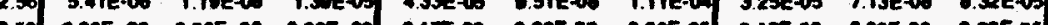

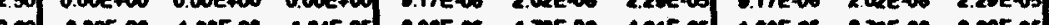

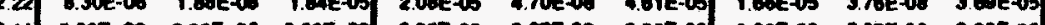
o.

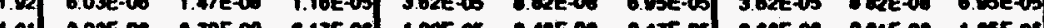

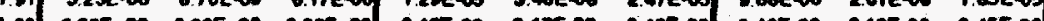

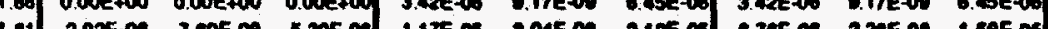

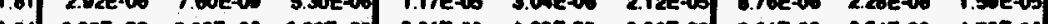

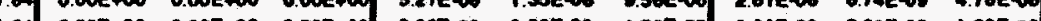
(..4)

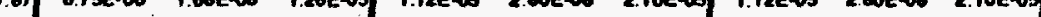
2.0E-

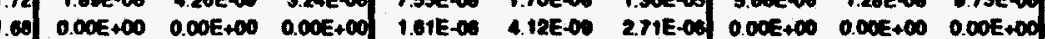

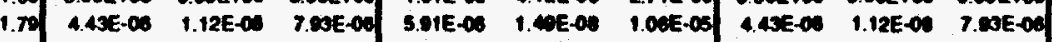

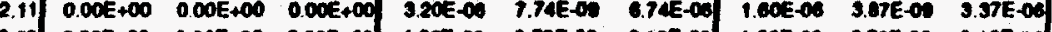

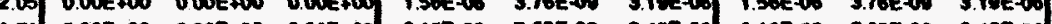

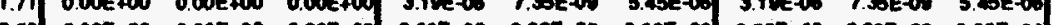

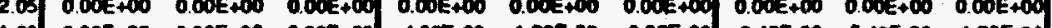

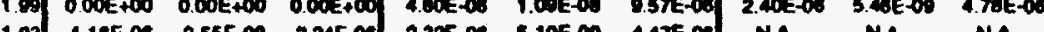

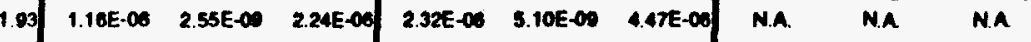

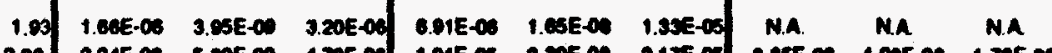

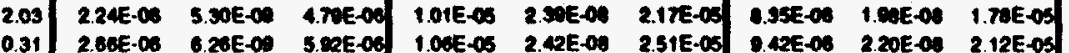

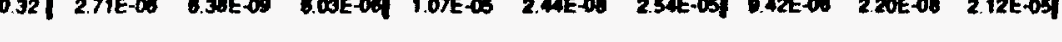


Toble 2.3

Total (Scheduled and Nonscheduled) U.S. Air Carriors Operating Under 14 CFR 121 [Rotorences 2.6 to 2.28]

\begin{tabular}{|c|c|c|c|c|c|c|c|c|c|c|c|c|c|c|c|}
\hline Year & $\begin{array}{l}\text { Fatal } \\
\text { Aoc. }\end{array}$ & $\begin{array}{l}121 \\
\text { Pess. }\end{array}$ & $\begin{array}{l}\text { Fandion } \\
\text { Crow }\end{array}$ & Other & Total & $\begin{array}{l}\text { Tout } \\
\text { Aoc. }\end{array}$ & Dewt. & Major & $\begin{array}{l}\text { Momon De } \\
\text { Mhort } \\
\text { Noned } \\
\text { Unken. }\end{array}$ & Towal & $\begin{array}{l}121 \text { Arcran } \\
\text { Houn } \\
\text { Flown }\end{array}$ & $\begin{array}{l}121 \text { Arcraft } \\
\text { Miles } \\
(000)\end{array}$ & $\begin{array}{l}121 \text { Arocian } \\
\text { Deppertureses }\end{array}$ & $\begin{array}{l}\text { Avereos } \\
\text { Spend } \\
\text { (milosetr.) }\end{array}$ & $\begin{array}{l}\text { Averaos } \\
\text { AC milear } \\
\text { Deperture }\end{array}$ \\
\hline 1973 & 9) & 201 & 20 & 1 & 220 & 30 & . & 11 & 10 & 30 & $0.130,465$ & $2.555,732$ & $3.217,077$ & 418.69 & 79 \\
\hline 1974 & 7 & 410 & 42 & 0 & 401 & 41 & 7 & 4 & 31 & 42 & $5,677.937$ & $2,350,008$ & $4,801,046$ & 414.07 & 409.50 \\
\hline 1975 & 3 & 110 & 12 & 0 & 120 & 30 & 10 & 12 & 17 & 30 & $5,007,450$ & $2,324,631$ & $4,778,984$ & 114.56 & 486.71 \\
\hline 1976 & 2 & 30 & 2 & 0 & 30 & 24 & 6 & 6 & 12 & 24 & $5,005,727$ & $2,419,150$ & $4,022,501$ & 418.60 & 491.45 \\
\hline on & 5 & 625 & 31 & $\bullet$ & 605 & 24| & 5 & - & 14 & 28 . & 0.041 .602 & $2,525,103$ & $5,042,540$ & 417.95 & 500.76 \\
\hline 1870 & ग) & 141 & 11 & 14 & 100 & 22 & 6 & 4 & 12 & 22 & B.,234,626 & $2,608,362$ & $5,112,290$ & 118.37 & 510.21 \\
\hline 1970 & 가 & 323 & 20 & 3 & 354 & 29) & 7 & 14 & - & 30 & $8,078,911$ & $2,059,136$ & $5,465,063$ & 41564 & 521.17 \\
\hline 1080 & & 0 & 0 & 1 & & 19] & 4 & 7 & - & 19 & $7,979.000$ & $3.044,000$ & $5,729,000$ & 412.52 & 531.33 \\
\hline 1901 & 4 & 1 & 1 & 2 & 4 & 20 & 1 & 12 & 13 & 26 & $7,128,000$ & $2,920,000$ & $5,575,000$ & 400.77 & $523 . \pi$ \\
\hline 1802 & 4 & 210 & 12 & 12 & 234 & 10 & 3 & 10 & 6 & 19 & $7,040,325$ & $2,940,513$ & $5,351,133$ & 417.87 & 540.51 \\
\hline 1803 & 4 & . & 8 & 1 & 15 & 24 & 4 & 11 & . & 24 & $7,290,790$ & $3,009,318$ & $5,444,374$ & 420.52 & 503.78 \\
\hline 1004 & 1 & 1 & 3 & 0 & & 17 & 3 & 7 & 7 & 17 & $8,105,124$ & $3,428,003$ & $5,800,852$ & 118.64 & 581.14 \\
\hline 1005 & ? & 400 & 30 & 1 & 520 & 22. & 11 & • & 5 & 22 & $8,709,000$ & $3,031,017$ & $6,300,750$ & 416.60 & 575.73 \\
\hline 1006 & 2 & 0 & 4 & - & & 23] & 3 & 6 & 14 & 23 & $0.978,104$ & $4,017,026$ & $7,202,027$ & 402.72 & 557.05 \\
\hline 1907 & 5 & 214 & 17 & 2 & 233 & 30 & 6 & 17 & 16 & 39 & $10,645,192$ & $4,360,521$ & $7,601,373$ & 400.62 & 573.05 \\
\hline 1000 & 3 & 256 & 10 & 11 & 205 & 20 & 7 & - & 14 & 29 & $11,140,673$ & $0,503,426$ & $7,716,001$ & 404.23 & 503.04 \\
\hline 1900 & 11 & 250 & 17 & 2 & 278 & 20 & 9 & ? & 10 & 28 & $11,274,543$ & $4,005,009$ & $1,045,404$ & 400.45 & 602.33 \\
\hline 1000 & 5 & - & 5 & 24 & 37) & 24 & 5 & 6 & 14 & 25 & $12,150,116$ & $4,970,007$ & $0,224,802$ & 400.00 & 804.27 \\
\hline 1001 & & 50 & 11 & 1 & 62 & 26 & 3 & 10 & 12 & 27 & $11,900,023$ & $4,050,840$ & 7.005 .030 & 407.63 & 607.45 \\
\hline 1002 & & 20 & 5 & 2 & 33 & 10 & 4 & 2 & 13 & 19 & $12,500,610$ & $3,007,723$ & $8,004,053$ & 406.74 & 629.36 \\
\hline 1003 & & 0 & 0 & 1 & & 21! & 1 & o & 13 & 22 & $12,013,401$ & $3,290,427$ & $0,280,246$ & 410.38 & 630.30 \\
\hline 1994 & & 220 & 11 & 0 & $230^{\circ}$ & 22 & 3 & 7 & 12 & 22 & $13,306,405$ & 5.409 .452 & $8.450,900$ & 411.04 & 648.51 \\
\hline $85 \mathrm{P}$ & 3 & 152 & 10 & B & 188 & 35 & N.A & N.A & N.R & N.A & $13,508,000$ & $5,061,100$ & $0,667,000$ & 418.06 & 053.18 \\
\hline Total & 9. & 60 & 311 & 93 & 184 & 307 & 110 & 106 & 200 & sed & $207,419,004$ & $85,501,116$ & $149,530,105$ & 412.21 & 571.70 \\
\hline Awg. & & 160.40 & 13.52 & 4.04 & 1.04 & 26.30 & 5.36 & 0.45 & 12.73 & 26.55 & $0,010,24$ & 3.717 .460 & $8.501,700$ & 413.08 & 561.41 \\
\hline sid. & & 25 & 12.19 & 6.06 & & 5 & 2.68 & 3.51 & 5.37 & 7.16 & $2,810,564$ & $1.138,705$ & $1,402,483$ & 5.17 & 5410 \\
\hline
\end{tabular}

P- Proliminary Deale

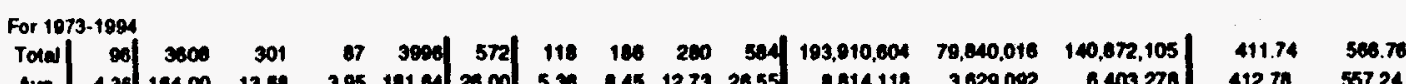

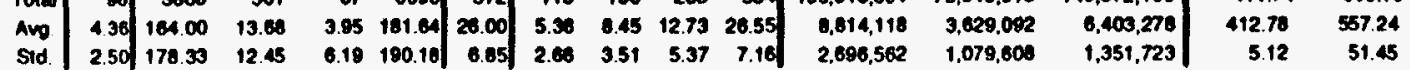

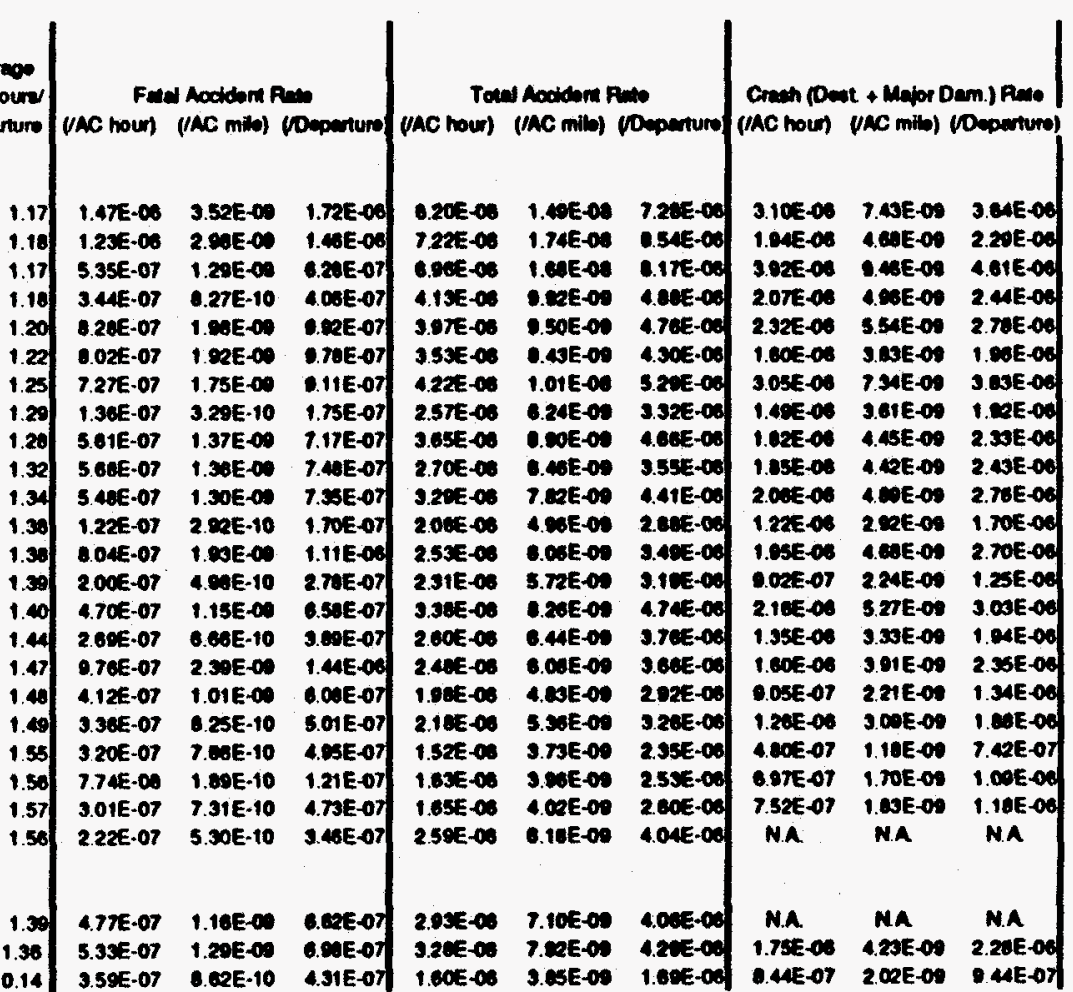

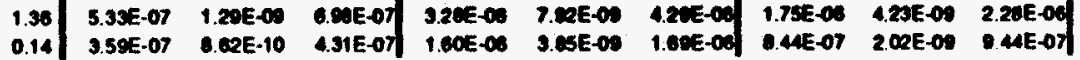

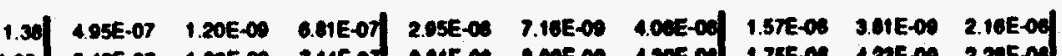

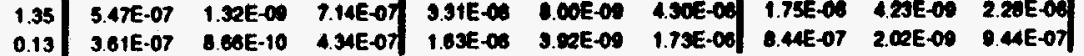




\section{Table 2.4}

TOTAL 14 CFP 121 AIACAAFT INVOLVED IN ACCIDENTS BY FLIGHT PHASE

[Refs. 2.610 2.28]

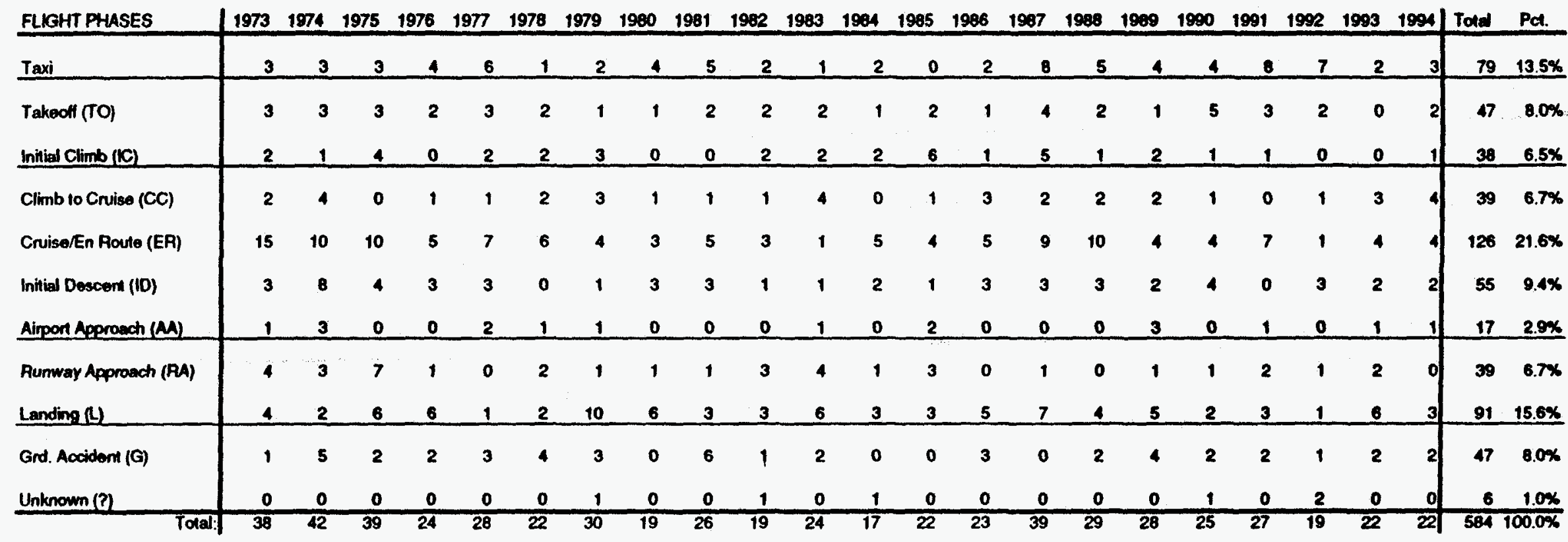




\section{Table 2.5}

Total (Scheduled and Nonscheduled) 14 CFR 121 Alrcraft Involved In Accident Rate by Filght Phase

\begin{tabular}{|c|c|c|c|c|c|c|c|c|c|c|c|c|c|c|c|c|c|c|}
\hline Flight Phases & $\begin{array}{c}\text { No. of } \\
\text { Accidents }\end{array}$ & Pat. & \multicolumn{2}{|c|}{$\begin{array}{l}\text { Adjusted } \\
\text { No. of Acquited } \\
\text { Aocidem Pct. }\end{array}$} & $\begin{array}{l}\text { Average } \\
\text { Pct. } \\
\text { Fi. Time }\end{array}$ & $\begin{array}{l}\text { Average } \\
\text { Fit. Time } \\
\text { (hours) }\end{array}$ & \multicolumn{2}{|c|}{$\begin{array}{l}\text { Assumed } \\
\text { Average Velocity }\end{array}$} & \multicolumn{2}{|c|}{$\begin{array}{l}\text { Calculated } \\
\text { Average Distance }\end{array}$} & $\begin{array}{l}\text { Accident Rate } \\
\text { Accidents } \\
\text { Oeparture } \\
\end{array}$ & $\begin{array}{l}\text { by Flight Ph } \\
\text { Accidents } \\
\text { IAC Hour }\end{array}$ & $\begin{array}{l}\text { hases } \\
\text { Accidents } \\
\text { Mibs }\end{array}$ & $\begin{array}{l}\text { Accidents } \\
\text { Knol }\end{array}$ & $\begin{array}{l}\text { Combined A } \\
\text { Accidents } \\
\text { Depanture }\end{array}$ & $\begin{array}{l}\text { ACRAM Fligh } \\
\text { Accidents } \\
\text { ACHour }\end{array}$ & $\begin{array}{l}\text { Al Phases Aoc } \\
\text { Accidents } \\
\text { Mile }\end{array}$ & $\begin{array}{l}\text { scident Rates } \\
\text { Accidents } \\
\text { Knot }\end{array}$ \\
\hline$\underline{T a x i}$ & 79 & $13.53 \%$ & & & & & & & & & & & & & & & & \\
\hline $\begin{array}{l}\text { Takeoff (TO) } \\
\text { Initial Climb (KC }\end{array}$ & $\begin{array}{r}47 \\
38 \\
\end{array}$ & $\begin{array}{l}8.05 \% \\
6.51 \% \\
\end{array}$ & $\begin{array}{l}47 \\
39 \\
\end{array}$ & $\begin{array}{r}10.40 \% \\
8.41 \% \\
\end{array}$ & $\begin{array}{l}1.00 \% \\
1.00 \% \\
\end{array}$ & $\begin{array}{l}0.014 \\
0.014 \\
\end{array}$ & $\begin{array}{l}100.00 \\
230.16 \\
\end{array}$ & $\begin{array}{r}86.90 \\
\quad 200.00 \\
\end{array}$ & $\begin{array}{r}1.40 \\
3.22 \\
\end{array}$ & $\begin{array}{r}1.22 \\
2.80 \\
\end{array}$ & $\begin{array}{r}3.336 E-07 \\
2.697 E-07 \\
\end{array}$ & $\begin{array}{r}\text { 2.383E-05 } \\
1.927 E-05 \\
\end{array}$ & $\begin{array}{l}2.323 E-07 \\
8.372 E-08\end{array}$ & $\begin{array}{l}2.742 E-07 \\
9.634 E-08 \\
\end{array}$ & 6.034E-07 & 7 2.155E-05 & $1.305 E-07$ & 1.502E-07 \\
\hline $\begin{array}{l}\text { Climb to } \\
\text { Cruise (CC) }\end{array}$ & 39 & $6.68 \%$ & 39 & 8.63\% & $13.00 \%$ & 0.182 & 356.74 & 310.00 & 64.93 & 56.42 & $2.768 E-07$ & $1.521 E-06$ & 4.264E-09 & $4.907 E-09$ & & & & \\
\hline $\begin{array}{l}\text { Cruisel } \\
\text { Enroute (ER) }\end{array}$ & 126 & $21.58 \%$ & 126 & $27.68 \%$ & $60.00 \%$ & 0.840 & 477.57 & 415.00 & 401.16 & 348.60 & 8.944E-07 & $1.065 E-06$ & $2.230 E-09$ & $2.568 E-09$ & $1.682 E-06$ & $1.292 E-06$ & $3.055 E-09$ & $3.516 E-09$ \\
\hline $\begin{array}{l}\text { Initival } \\
\text { Descent (ID) }\end{array}$ & 55 & $9.42 \%$ & 55 & $12.17 \%$ & $10.00 \%$ & 0.140 & 356.74 & 310.00 & 49.94 & 43.40 & 3.904E-07 & 2.789E-06 & 7.817E-0s & $0.996 E-09$ & & & & \\
\hline $\begin{array}{l}\text { Aipon } \\
\text { Approach (AA) }\end{array}$ & 17 & $2.91 \%$ & 17 & $3.76 \%$ & $10.00 x$ & 0.140 & 247.42 & 215.00 & 34.64 & 30.10 & 1.207E-07 & 8.620E-07 & 3.494E-09 & $4.009 E-09$ & & & & \\
\hline $\begin{array}{l}\text { Runway } \\
\text { Approach (RA) }\end{array}$ & 39 & $6.68 \%$ & & $8.63 \%$ & $4.00 \%$ & 0.056 & 247.42 & 215.00 & & 12.04 & 2.768E-07 & 4.944E-06 & $1.998 E-08$ & $2.209 E-00$ & & & & \\
\hline Landing (L) & 91 & $15.58 \%$ & 91 & $20.13 \%$ & $1.00 \%$ & 0.014 & 100.00 & 86.90 & 1.40 & 1.22 & $6.460 \mathrm{E}-07$ & 4.614E-05 & 4.614E-07 & $5.310 E-07$ & $0.220 E-07$ & $1.318 E-05$ & $6.049 E-00$ & 6.961E-00 \\
\hline $\begin{array}{l}\text { Ground } \\
\text { Accident (G) }\end{array}$ & 47 & $8.05 \%$ & & & & & & & & & & & & & & & & \\
\hline Unknown (?) & 6 & $1.03 \%$ & & & & & & & & & & & & & & & & \\
\hline TOTAL: & 584 & $100.00 \%$ & 452 & $100.00 \%$ & $100.00 \%$ & 1.400 & 407.53 & 354.14 & $\mathbf{5 7 0 . 5 5}$ & 495.79 & $3.209 E-06$ & 2.292E-06 & 5.624E-09 & $6.472 E-09$ & 3.209E-06 & $2.202 E-06$ & 5.624E-09 & 6.472E-09 \\
\hline Bases for Total & 14 CFR 12 & 1 Aociden & it Rales by & Fight Phe & lase, 1973- & -1994 Tola & & & & & $\begin{array}{r}1.4 \\
570 \\
407.14 \\
140,872,105\end{array}$ & $\begin{array}{l}\text { Hours Avera } \\
\text { Miles Averas } \\
\text { Miles/Hour A } \\
\text { Departures }\end{array}$ & $\begin{array}{l}\text { age Fligh D } \\
\text { ge Flight Dis } \\
\text { Average Flig }\end{array}$ & $\begin{array}{l}\text { uration } \\
\text { itance } \\
\text { ht Velocity }\end{array}$ & & & & \\
\hline
\end{tabular}


Table 2.6

14 CFR 121 CRASHES (ACCIDENTS WTH DESTROYED OR MAJOR DAMAGE TO AIRFRAME)

BY FUGHT PHASE

[Ref3. 2.6 to 2.28]

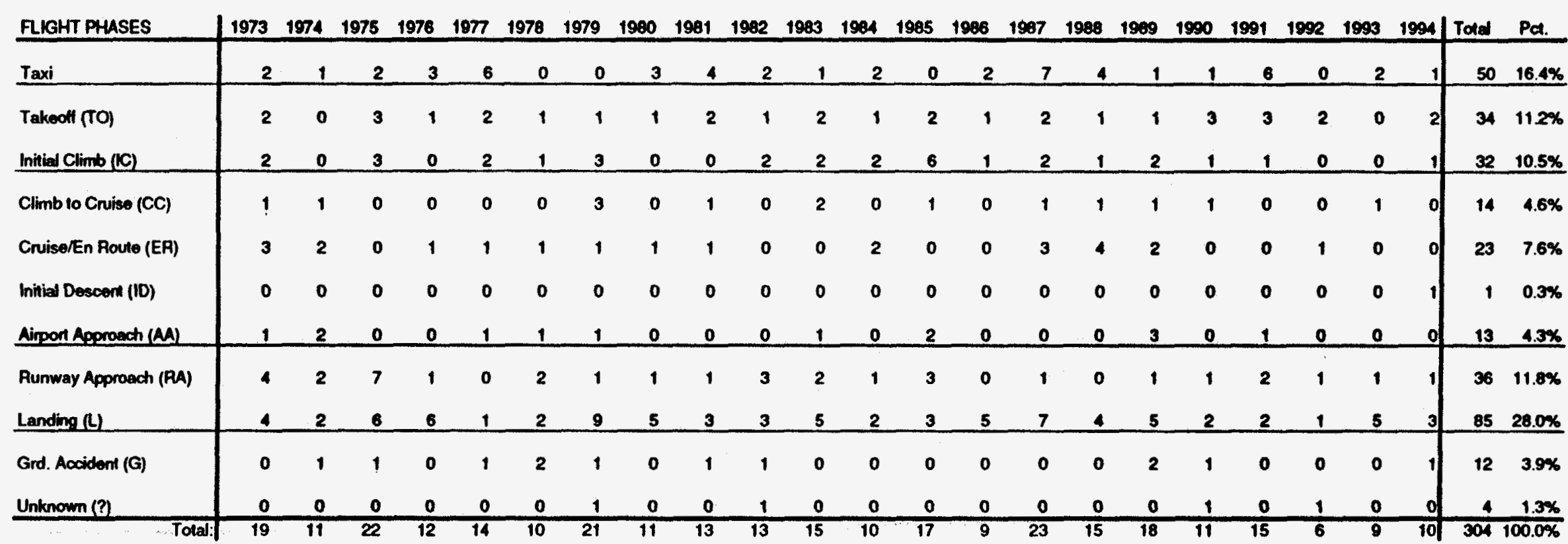


Table 2.7

14 CFR 121 Crash (Accldents with Destroyed or Major Damage to Alrtrame) Rates by Flight Phase

\begin{tabular}{|c|c|c|c|c|c|c|c|c|c|c|c|c|c|c|c|c|c|c|}
\hline Flight Phases & $\begin{array}{l}\text { No. of } \\
\text { Crashes }\end{array}$ & Pet. & $\begin{array}{c}\text { Adjusted } \\
\text { No. of } \\
\text { Crashes }\end{array}$ & $\begin{array}{c}\text { Acjuated } \\
\text { Pct. }\end{array}$ & $\begin{array}{l}\text { Average } \\
\text { Pct. } \\
\text { Fn. Time }\end{array}$ & $\begin{array}{l}\text { Average } \\
\text { FI. Time } \\
\text { (hours) }\end{array}$ & \multicolumn{2}{|c|}{$\begin{array}{l}\text { Assumed } \\
\text { Average Velocily }\end{array}$} & \multicolumn{2}{|c|}{ Aalculated } & $\begin{array}{l}\text { Crash Rate by } \\
\text { Crashes } \\
\text { Departure }\end{array}$ & $\begin{array}{l}\text { Flight Phas } \\
\text { Crashes } \\
\text { IAC Hour }\end{array}$ & $\begin{array}{l}\text { ses } \\
\text { Crashes } \\
\text { Milo }\end{array}$ & $\begin{array}{l}\text { Crashes } \\
\text { Mnot }\end{array}$ & $\begin{array}{l}\text { Combined A } \\
\text { Crashes } \\
\text { Oeparture } \\
\end{array}$ & $\begin{array}{l}\text { ACPAM Fligh } \\
\text { Crashos } \\
\text { ACHour }\end{array}$ & $\begin{array}{l}\text { A Phases Cra } \\
\text { Crashes } \\
\text { Mile }\end{array}$ & $\begin{array}{l}\text { ash Rates } \\
\text { Crashes } \\
\text { Knot }\end{array}$ \\
\hline Taxi & 50 & $16.45 \%$ & & & & & & & & & & & & & & & & \\
\hline $\begin{array}{l}\text { Takeoff (TO) } \\
\text { Initial Climb (1C }\end{array}$ & $\begin{array}{r}34 \\
32 \\
\end{array}$ & $\begin{array}{l}11.18 \% \\
10.53 \% \\
\end{array}$ & $\begin{array}{r}34 \\
32 \\
\end{array}$ & $\begin{array}{l}14.29 \% \\
13.45 \% \\
\end{array}$ & $\begin{array}{l}1.00 \% \\
1.00 \% \\
\end{array}$ & $\begin{array}{l}0.014 \\
0.014\end{array}$ & $\begin{array}{l}100.00 \\
230.16 \\
\end{array}$ & $\begin{array}{r}86.90 \\
\quad 200.00 \\
\end{array}$ & $\begin{array}{l}1.40 \\
3.22 \\
\end{array}$ & $\begin{array}{r}1.22 \\
2.80 \\
\end{array}$ & $\begin{array}{l}2.414 E-07 \\
2.272 E-07 \\
\end{array}$ & $\begin{array}{l}1.724 E-05 \\
1.023 E-05 \\
\end{array}$ & $\begin{array}{l}1.724 E-07 \\
7.050 E-08 \\
\end{array}$ & $\begin{array}{l}1.984 E-07 \\
8.113 E-08 \\
\end{array}$ & 4.685E-07 & 1.673E-05 & 1.014E-07 & $1.166 \mathrm{E}-07$ \\
\hline $\begin{array}{l}\text { Climb to } \\
\text { Cruise (CC) }\end{array}$ & 14 & $4.61 \%$ & 14 & $5.88 \%$ & $13.00 \%$ & 0.182 & 356.74 & 310.00 & 64.93 & 56.42 & $9.938 E-08$ & $5.460 E-07$ & $1.531 E-09$ & $1.761 E-09$ & & & & \\
\hline $\begin{array}{l}\text { Cruisel } \\
\text { Enroute (ER) }\end{array}$ & 23 & $7.57 \%$ & 23 & $9.66 \%$ & $60.00 \%$ & 0.840 & 477.57 & $\quad 415.00$ & 401.16 & 348.60 & $1.633 E-07$ & $1.944 E-07$ & $4.070 E-10$ & 4.684E-10 & $3.620 \mathrm{E}-07$ & 2.781E-07 & 6.574E-10 & $7.566 \mathrm{E}-10$ \\
\hline $\begin{array}{l}\text { Initial } \\
\text { Descent (ID) }\end{array}$ & 1 & $0.33 \%$ & 1 & $0.42 \%$ & $10.00 \%$ & 0.140 & 356.74 & 310.00 & 49.94 & 43.40 & $7.099 E-09$ & $5.070 E-08$ & $1.421 E-10$ & $1.636 E-10$ & & & & \\
\hline $\begin{array}{l}\text { Aiport } \\
\text { Approach (AA) }\end{array}$ & 13 & $428 \%$ & 13 & $5.46 \%$ & $10.00 \%$ & 0.140 & 247.42 & 215.00 & 34.64 & 30.10 & $9.228 E-08$ & 6.592E-07 & 2.664E-09 & $3.066 E-09$ & & & & \\
\hline $\begin{array}{l}\text { Runway } \\
\text { Approach (RA) }\end{array}$ & 36 & $11.84 \%$ & 36 & $15.13 \%$ & $4.00 \%$ & 0.056 & & $\quad 215.00$ & & 12.04 & & & 1.844E-08 & $2.123 E-08$ & & & & \\
\hline Landing $(L)$ & 85 & $27.96 \%$ & 85 & $35.71 \%$ & $1.00 \%$ & 0.014 & 100.00 & 86.90 & 1.40 & 1.22 & 6.034E-07 & 4.310E.05 & 4.310E-07 & $4.960 E-07$ & 8.589E-07 & $1.227 E-05$ & $5.630 E-\infty 0$ & 6.479E-08 \\
\hline $\begin{array}{l}\text { Ground } \\
\text { Accident (G) }\end{array}$ & 12 & $3.95 \%$ & & & & & & & & & & & & & & & & \\
\hline Unknown (?) & 4 & $1.32 \%$ & & & & & & & & & & & & & & & & \\
\hline TOTAL: & 304 & $100.00 \%$ & 238 & $100.00 \%$ & $100.00 \%$ & 1.400 & 407.53 & 354.14 & 570.55 & 495.79 & $1.689 \mathrm{E}-06$ & $1.207 E-06$ & 2.961E-09 & $3.400 E-09$ & 1.609E-06 & 1.207E-06 & 2.961E-09 & 3.4 \\
\hline
\end{tabular}

Bases for Total 14 CFR 121 Crash Rates by Flight Phase, 1973-1994 Totals:

1.4 Hours Average Flight Duration

570 Miles Average Fligh Distanco

407.14 Miles/Hour Average Flight Volocity

140,872,105 Departures 
Table 2.8

14 CFA 121 CAASHES WTH DESTROYEDMAJOR-W.O. AIRFRAMES

BY FLGHT PHASE

[Rofs. 2.6 to 2.28]

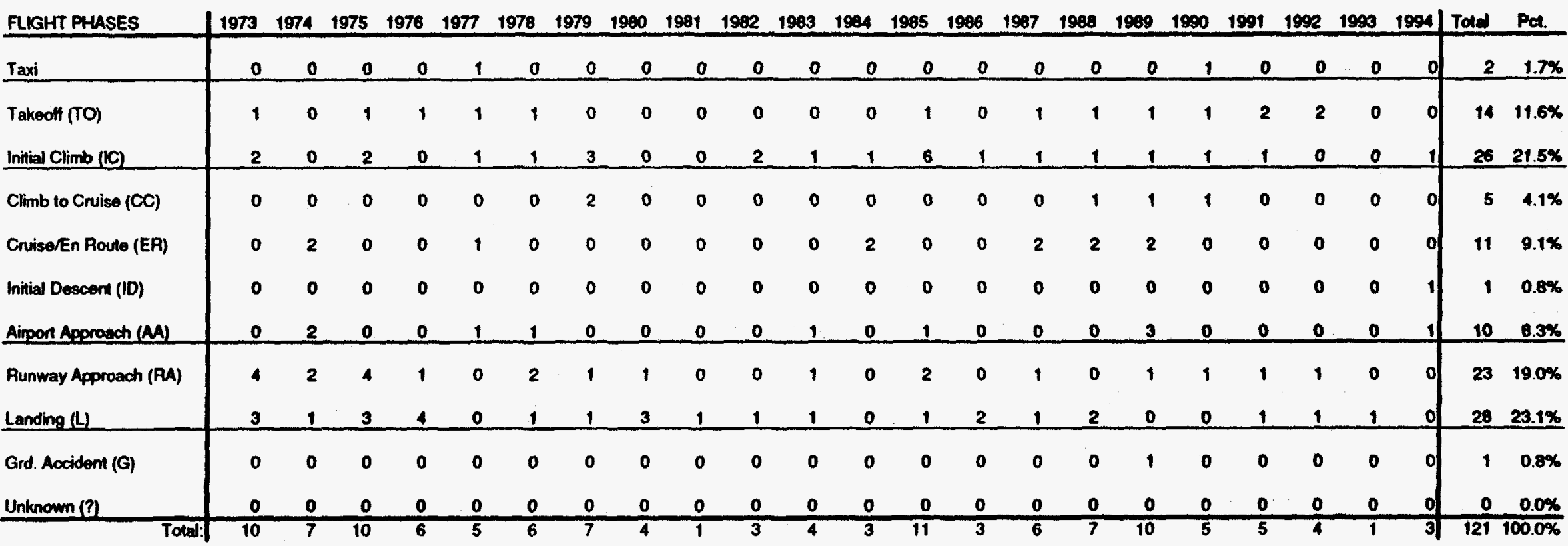


Table 2.9

14 CFR 121 Crashes with Destroyed/Major-W.O. Alrirame Rates by Flight Phase

\begin{tabular}{|c|c|c|c|c|c|c|c|c|c|c|c|c|c|c|c|c|c|c|}
\hline Flight Phases & $\begin{array}{c}\text { No. of } \\
\text { Crashos }\end{array}$ & Pat. & $\begin{array}{l}\text { Acjusted } \\
\text { No. of } \\
\text { Creshes }\end{array}$ & $\begin{array}{c}\text { Adjunted } \\
\text { Pat. }\end{array}$ & $\begin{array}{l}\text { Average } \\
\text { Pct. } \\
\text { Fh. Time }\end{array}$ & $\begin{array}{l}\text { Average } \\
\text { Fit. Time } \\
\text { (hours) }\end{array}$ & \multicolumn{2}{|c|}{$\begin{array}{l}\text { Assumed } \\
\text { Average Velocily }\end{array}$} & \multicolumn{2}{|c|}{$\begin{array}{l}\text { Caloulated } \\
\text { Average Distance } \\
\text { Miles Knots }\end{array}$} & \begin{tabular}{|l|} 
Crash Rate b \\
Crashes \\
Departure
\end{tabular} & $\begin{array}{l}\text { Flight Phas } \\
\text { Crashes } \\
\text { AC Hour }\end{array}$ & $\begin{array}{l}\text { ses } \\
\text { Crashes } \\
\text { Mile }\end{array}$ & $\begin{array}{l}\text { Crashes } \\
\text { Knol }\end{array}$ & $\begin{array}{l}\text { Combined A } \\
\text { Creshes } \\
\text { Depenture }\end{array}$ & $\begin{array}{l}\text { CRAM Fligh } \\
\text { Crashes } \\
\text { ACHow }\end{array}$ & $\begin{array}{l}\text { Phases Cro } \\
\text { Crashes } \\
\text { Milo }\end{array}$ & $\begin{array}{l}\text { ash Pates } \\
\text { Crashes } \\
\text { Knol }\end{array}$ \\
\hline Taxi & 2 & $1.65 \%$ & & & & & & & & & & & & & & & & \\
\hline $\begin{array}{l}\text { Takeofl (TO) } \\
\text { Initial Climb (K }\end{array}$ & $\begin{array}{l}14 \\
26 \\
\end{array}$ & $\begin{array}{r}11.57 \% \\
21.49 \% \\
\end{array}$ & $\begin{array}{l}14 \\
26 \\
\end{array}$ & $\begin{array}{l}11.06 \% \\
22.03 \% \\
\end{array}$ & $\begin{array}{r}1.00 \% \\
1.00 \% \\
\end{array}$ & $\begin{array}{l}0.014 \\
0.014 \\
\end{array}$ & $\begin{array}{l}100.00 \\
230.16 \\
\end{array}$ & $\begin{array}{r}86.90 \\
\quad 200.00 \\
\end{array}$ & $\begin{array}{r}1.40 \\
3.22 \\
\end{array}$ & $\begin{array}{r}1.22 \\
2.80 \\
\end{array}$ & $\begin{array}{r}9.938 E-08 \\
1.846 E-07 \\
\end{array}$ & $\begin{array}{l}7.099 E-06 \\
1.318 E-05 \\
\end{array}$ & $\begin{array}{l}7.099 E-08 \\
5.728 E-08 \\
\end{array}$ & $\begin{array}{r}6.169 E-08 \\
6.592 E-08 \\
\end{array}$ & 3) $2.639 E-07$ & 1.014E-05 & ; 6.143E-08 & $7.069 E-00$ \\
\hline $\begin{array}{l}\text { Climb to } \\
\text { Cnuise (CC) }\end{array}$ & 5 & $4.13 \%$ & 5 & $4.24 \%$ & $13.00 \%$ & 0.182 & 356.74 & 310.00 & 64.93 & 56.42 & $3.549 E-08$ & $1.950 \mathrm{E}-07$ & 5.467E-10 & $6.291 E-10$ & & & & \\
\hline $\begin{array}{l}\text { Cruisel } \\
\text { Enroure (EA) }\end{array}$ & 11 & $9.09 \%$ & 11 & $9.32 \%$ & $60.00 \%$ & 0.840 & 477.57 & 415.00 & 401.16 & 348.60 & $7.809 E-08$ & $9.206 E-08$ & 1.946E-10 & $2.240 E-10$ & 1.917E-07 & 1.472E-07 & 3.481E-10 & $4.005 E-10$ \\
\hline $\begin{array}{l}\text { Intial } \\
\text { Descont (ID) }\end{array}$ & 1 & $0.83 \%$ & 1 & $0.85 \%$ & $10.00 \%$ & 0.140 & 356.74 & 310.00 & 49.94 & 43.40 & $7.099 E-09$ & $5.070 E-08$ & 1.421E-10 & $1.050-10$ & & & & \\
\hline $\begin{array}{l}\text { Aiport } \\
\text { Approeach (A) }\end{array}$ & 10 & $8.26 \%$ & 10 & $8.47 \%$ & $10.00 \%$ & 0.140 & 247.42 & 215.00 & 34.64 & 30.10 & 7.099E-08 & $5.070 E-07$ & 2.049E-09 & $2.358 E-09$ & & & & \\
\hline $\begin{array}{l}\text { Punway } \\
\text { Approach (RA) }\end{array}$ & 23 & $19.01 \%$ & 23 & $19.49 \%$ & $4.00 \%$ & 0.056 & 247.42 & 215.00 & 13.86 & 12.04 & & $2.916 E-06$ & $1.178 E-08$ & $1.356 E-08$ & & & & \\
\hline Landing (L) & 28 & $23.14 \%$ & 28 & $23.73 \%$ & $1.00 \%$ & 0.014 & 100.00 & 86.90 & 1.40 & 1.22 & 1.998E-07 & 1.420E-05 & 1.420E-07 & $1.634 E-07$ & 3.620 E- 07 & 5.172E-06 & $2.373 E-00$ & $2.731 E-00$ \\
\hline $\begin{array}{l}\text { Ground } \\
\text { Accident (G) }\end{array}$ & 1 & $0.83 \%$ & & & & & & & & & & & & & & & & \\
\hline Unknown (?) & 0 & $0.00 \%$ & & & & & & & & & & & & & & & & \\
\hline TOTAL: & 121 & $100.00 \%$ & 118 & $100.00 \%$ & $100.00 \%$ & 1.400 & 407.53 & 354.14 & 570.55 & 495.79 & $8.376 E-07$ & 5.983E-07 & $1.468 E-09$ & $1.609 E-09$ & 8. $876 E-07$ & 5.983E-07 & $1.468 E-09$ & 1.6 \\
\hline
\end{tabular}

Bases for Total 14 CFR 121 Crash Rates by Flight Phase, 1973-1994 Tolals: 


\section{Table 2.10}

TOTAL OFF AIRPORT ONLY 14 CFR 121 ACCIDENTS BY FUGHT PHASE

[Rels. 2.6 to 2.28]

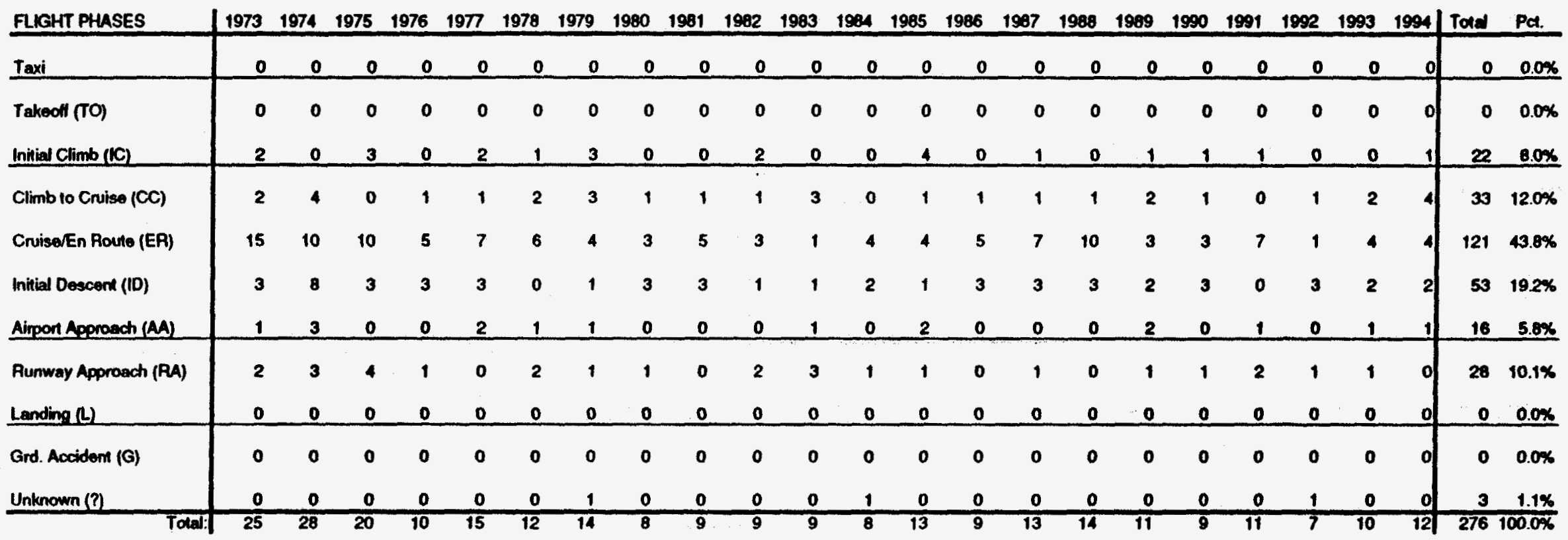


Table 2.11

Total (Scheduled and Nonscheduled) Off Airport Oniy 14 CFR 121 Accident Rates by Fllght Phase

\begin{tabular}{|c|c|c|c|c|c|c|c|c|c|c|c|c|c|c|c|c|c|c|}
\hline Flight Phoses & $\begin{array}{c}\text { No. of } \\
\text { Accidents }\end{array}$ & Pat. & \multicolumn{2}{|c|}{$\begin{array}{l}\text { Adjuated } \\
\text { No. of Adjusted } \\
\text { Accidents Pa. }\end{array}$} & \multicolumn{2}{|c|}{$\begin{array}{|ll|}\text { Average } & \text { Average } \\
\text { Pct. } & \text { Fh. Time } \\
\text { Fi. Time } & \text { (hours) } \\
\end{array}$} & \multicolumn{2}{|c|}{\begin{tabular}{|l} 
Assumed \\
Average Velocily \\
Miles Knots \\
\end{tabular}} & \multicolumn{2}{|c|}{$\begin{array}{l}\text { Calculated } \\
\text { Average Distance } \\
\text { Miles Knots } \\
\end{array}$} & $\begin{array}{l}\text { Accident Rate } \\
\text { Accidents } \\
\text { Departure }\end{array}$ & $\begin{array}{l}\text { by Flight Ph } \\
\text { Accidents } \\
\text { AAC Hour }\end{array}$ & $\begin{array}{l}\text { hases } \\
\text { Accidents } \\
\text { Mib }\end{array}$ & $\begin{array}{l}\text { Accidents } \\
\text { Knot }\end{array}$ & $\begin{array}{l}\text { Combined A } \\
\text { Accidents } \\
\text { Departure }\end{array}$ & $\begin{array}{l}\text { ACPAM Flight } \\
\text { Accidents } \\
\text { IAC Hour }\end{array}$ & $\begin{array}{l}\text { Phases Aoc } \\
\text { Accidents } \\
\text { Mile }\end{array}$ & $\begin{array}{l}\text { cident Rales } \\
\text { Aocidents } \\
\text { Knot } \\
\end{array}$ \\
\hline Taxi & 0 & $0.00 \%$ & & & & & & & & & & & & & & & & \\
\hline $\begin{array}{l}\text { Takeof (TO) } \\
\text { Inhial Climb (KC) }\end{array}$ & $\begin{array}{r}0 \\
22 \\
\end{array}$ & $\begin{array}{l}0.00 \% \\
7.97 \% \\
\end{array}$ & $\begin{array}{r}0 \\
22 \\
\end{array}$ & $\begin{array}{l}0.00 \% \\
8.00 \% \\
\end{array}$ & $\begin{array}{l}1.00 \% \\
1.00 \% \\
\end{array}$ & $\begin{array}{l}0.014 \\
0.014 \\
\end{array}$ & $\begin{array}{l}100.00 \\
230.16 \\
\end{array}$ & $\begin{array}{r}86.90 \\
\quad 200.00 \\
\end{array}$ & $\begin{array}{r}1.40 \\
3.22 \\
\end{array}$ & $\begin{array}{r}1.22 \\
2.80 \\
\end{array}$ & $\begin{array}{l}0.000 E+00 \\
1.562 E-07 \\
\end{array}$ & $\begin{array}{r}0.000 E+00 \\
1.116 E-05 \\
\end{array}$ & $\begin{array}{r}0.000 E+00 \\
4.847 E-08 \\
\end{array}$ & $\begin{array}{l}0.000 E+00 \\
5.578 E-00 \\
\end{array}$ & 1.562E-07 & S.578E-06 & $3.379 E-08$ & $3.888 E-08$ \\
\hline $\begin{array}{l}\text { Climb to } \\
\text { Cruise (CC) }\end{array}$ & 33 & $11.96 \%$ & 33 & $12.09 \%$ & $13.00 \%$ & 0.182 & 356.74 & 310.00 & 64.93 & 56.42 & 2.343E-07 & 1.287E-06 & $3.608 E-09$ & $4.152 E-09$ & & & & \\
\hline $\begin{array}{l}\text { Cruisel } \\
\text { Enroute (EA) }\end{array}$ & 121 & $43.84 \%$ & 121 & $4.32 \%$ & $60.00 \%$ & 0.840 & 477.57 & 415.00 & 401.16 & 348.60 & 8.589E-07 & 1.023E-06 & 2.141E-09 & $2.464 E-09$ & 1.503E-00 & 1.216E-08 & 2.875E-09 & $3.300 E-09$ \\
\hline $\begin{array}{l}\text { Initial } \\
\text { Descom (ID) }\end{array}$ & 53 & $19.20 \%$ & 53 & $19.41 \%$ & $10.00 \%$ & 0.140 & 356.74 & 310.00 & 49.94 & 43.40 & 3.762E-07 & 2.687E-06 & $7.533 E-09$ & $8.669 E-09$ & & & & \\
\hline $\begin{array}{l}\text { Aipport } \\
\text { Approach }(A \mathrm{~A})\end{array}$ & 16 & $5.80 \%$ & 16 & $5.86 \%$ & $10.00 \%$ & 0.140 & 247.42 & 215.00 & 34.64 & 30.10 & 1.136E-07 & 8.113E-07 & 3.279E-09 & $3.773 E-09$ & & & & \\
\hline $\begin{array}{l}\text { Rumway } \\
\text { Approach (RA) }\end{array}$ & 28 & $10.14 \%$ & 28 & $10.26 \%$ & $4.00 \%$ & 0.056 & 247.42 & 215.00 & & 12.04 & $1.988 E-07$ & $3.549 E-06$ & $1.435 E-08$ & $1.65 t E-00$ & & & & \\
\hline Landing (ㄴ) & $\underline{0}$ & $0.00 \%$ & 0 & $0.00 \%$ & $1.00 \%$ & 0.014 & 100.00 & 86.90 & 1.40 & 1.22 & $0.000 E+\infty 0$ & $0.000 E+00$ & $0.000 E+00$ & $0.000 E+00$ & 1.988E-07 & $2.059 E-03$ & $1.303 E-03$ & 1.499E-00 \\
\hline $\begin{array}{l}\text { Ground } \\
\text { Accident (G) }\end{array}$ & 0 & $0.00 \%$ & & & & & & & & & & & & & & & & \\
\hline Unknown (?) & 3 & $1.09 \%$ & & & & & & & & & & & & & & & & \\
\hline TOTAL: & 276 & $100.00 \%$ & 2731 & $100.00 \%$ & $100.00 \%$ & 1.400 & 407.53 & 354.14 & 570.55 & 495.79 & $1.938 E-06$ & 1.384E-06 & 3.397E-09 & $3.909 E-09$ & 1.938E-06 & $1.394 E-08$ & 3.397E-09 & 3.9 \\
\hline
\end{tabular}

Bases for Total 14 CFR 121 Accidem Rates by Flight Phase, 1973-1994 Totals:

1.4 Hours Average Fligh Duration

570 Miles Average Fligh Distance

407.14 Miles/Hour Average Flight Velocity

140,872,105 Departures 
Table 2.12

OFF AIRPORT ONLY 14 CFR 121 CRASHES (ACCIDENTS WITH DESTROYED OR MAJOR DAMAGE TO AIRFRAME, BY FUGHT PHASE

[Refs. 2.6 to 2.28]

\begin{tabular}{|c|c|c|c|c|c|c|c|c|c|c|c|c|c|c|c|c|c|c|c|c|c|c|c|c|c|}
\hline FLIGHT PHASES & 1973 & 1974 & 197 & & 1976 & 1977 & 1978 & 1979 & 1980 & 1901 & 1962 & 1983 & 1904 & 1985 & 1986 & 1987 & 1988 & 1909 & 1990 & 1991 & 1992 & 1993 & 1994 & Totel & Pa. \\
\hline Taxi & 0 & D & & 0 & 0 & 0 & 0 & 0 & 0 & $\mathbf{0}$ & 0 & 0 & 0 & 0 & 0 & $\mathbf{0}$ & 0 & 0 & 0 & 0 & 0 & 0 & of & 0 & $0.0 \%$ \\
\hline Takeof (TO) & 0 & 0 & & 0 & 0 & 0 & 0 & o & 0 & o & o & 0 & 0 & 0 & 0 & 0 & 0 & 0 & 0 & o & 0 & 0 & 0 & 0 & $0.0 \%$ \\
\hline Initial Climb (K) & 2 & 0 & & 2 & 0 & 2 & 1 & 3 & 0 & 0 & 2 & 0 & 0 & 4 & 0 & 0 & 0 & 1 & 1 & 1 & 0 & 0 & 1 & 20 & $22.2 \%$ \\
\hline Climb to Cruise (CC) & 1 & 1 & & 0 & 0 & 0 & 0 & 3 & 0 & 1 & 0 & 1 & 0 & 1 & 0 & 1 & o & 1 & 1 & 0 & 0 & 0 & 이 & 11 & $12.2 \%$ \\
\hline Cruise/En Route (ER) & 3 & 2 & & 0 & 1 & 1 & 1 & 1 & 1 & 1 & 0 & 0 & 1 & 0 & 0 & 2 & 4 & 1 & 0 & 0 & 1 & 0 & 0 & 20 & $22.2 \%$ \\
\hline Initial Descent (ID) & 0 & 0 & & 0 & 0 & 0 & 0 & 0 & o & 0 & 0 & 0 & 0 & 0 & 0 & 0 & 0 & 0 & 0 & $\mathbf{0}$ & 0 & 0 & 1) & 1 & $1.1 \%$ \\
\hline Airpon Approach $(A)$ & 1 & 2 & & 0 & 0 & 1 & 1 & 1 & 0 & 0 & 0 & 1 & 0 & 2 & 0 & 0 & 0 & 2 & 0 & 1 & 0 & 0 & 1 & 13 & $14.4 \%$ \\
\hline Runway Approach (RA) & 2 & 2 & & 4 & 1 & 0 & 2 & 1 & 1 & o & 2 & 1 & 1 & 1 & $\mathbf{0}$ & 1 & 0 & 1 & 1 & 2 & 1 & 0 & o) & 24 & $26.7 \%$ \\
\hline Landing (L) & o & 0 & & 0 & 0 & 0 & 0 & 0 & 0 & 0 & $\underline{0}$ & 0 & $\mathbf{0}$ & 0 & 0 & o & 0 & 0 & 0 & 0 & 0 & 0 & 0 & 0 & $0.0 \%$ \\
\hline Grd. Accidem (G) & 0 & $\mathbf{0}$ & & 0 & 0 & o & 0 & 0 & 0 & 0 & $\mathbf{0}$ & 0 & 0 & $\mathbf{0}$ & o & o & 0 & 0 & 0 & 0 & 0 & $\mathbf{0}$ & o) & 0 & $0.0 \%$ \\
\hline Unknown (?) & 0 & 0 & & 0 & 0 & 0 & 0 & 1 & 0 & o & 0 & 0 & 0 & 0 & 0 & 0 & 0 & 0 & 0 & 0 & $\mathbf{0}$ & 0 & of & 1 & $1.1 \%$ \\
\hline Tota & 9 & 7 & & 6 & 2 & 4 & 5 & 10 & 2 & 2 & 4 & 3 & 2 & 8 & 0 & 4 & 4 & 6 & 3 & 7 & 2 & 0 & 3 & & $100.0 \%$ \\
\hline
\end{tabular}


Table 2.13

Off Aliport Only 14 CFR 121 Crash (Accidents with Destroyed or Major Damage to Alrframe) Rates by Flight Phase

\begin{tabular}{|c|c|c|c|c|c|c|c|c|c|c|c|c|c|c|c|c|c|c|}
\hline Flight Phasos & $\begin{array}{c}\text { No. of } \\
\text { Crashos }\end{array}$ & Pat. & $\begin{array}{c}\text { Adjusted } \\
\text { No. of } \\
\text { Creshes }\end{array}$ & $\begin{array}{c}\text { Adputed } \\
\text { Pat. }\end{array}$ & $\begin{array}{l}\text { Average } \\
\text { Pct. } \\
\text { Fi. Time }\end{array}$ & $\begin{array}{l}\text { Average } \\
\text { Fh. Time } \\
\text { (hours) }\end{array}$ & \multicolumn{2}{|c|}{$\begin{array}{l}\text { Assumed } \\
\text { Average Velociky } \\
\text { Miles Knots }\end{array}$} & \multicolumn{2}{|c|}{\begin{tabular}{|l|} 
Calculated \\
Average Distance \\
Miles Knots
\end{tabular}} & $\begin{array}{l}\text { Crash Rate by } \\
\text { Crashes } \\
\text { Oeparture }\end{array}$ & $\begin{array}{l}\text { Fligh Phas } \\
\text { Crashes } \\
\text { AC Hour }\end{array}$ & $\begin{array}{l}\text { ses } \\
\text { Crashes } \\
\text { Mib } \\
\end{array}$ & $\begin{array}{l}\text { Craches } \\
\text { Mnot }\end{array}$ & $\begin{array}{l}\text { Combined A } \\
\text { Crashes } \\
\text { Oeparture }\end{array}$ & $\begin{array}{l}\text { ACRAM Fligh } \\
\text { Crashes } \\
\text { ACHour }\end{array}$ & $\begin{array}{l}\text { Phases Cra } \\
\text { Crashes } \\
\text { Mile }\end{array}$ & $\begin{array}{l}\text { ash Pates } \\
\text { Crashes } \\
\text { Knot }\end{array}$ \\
\hline Taxi & 0 & $0.00 \%$ & & & & & & & & & & & & & & & & \\
\hline $\begin{array}{l}\text { Takeoff (TO) } \\
\text { Intial Climb (K }\end{array}$ & $\begin{array}{r}0 \\
20 \\
\end{array}$ & $\begin{array}{r}0.00 \% \\
22.22 \% \\
\end{array}$ & $\begin{array}{r}0 \\
20 \\
\end{array}$ & $\begin{array}{r}0.00 \% \\
22.47 \% \\
\end{array}$ & $\begin{array}{l}1.00 \% \\
1.00 \% \\
\end{array}$ & $\begin{array}{l}0.014 \\
0.014 \\
\end{array}$ & $\begin{array}{r}100.00 \\
230.16 \\
\end{array}$ & $\begin{array}{r}86.90 \\
5 \quad 200.00 \\
\end{array}$ & $\begin{array}{l}1.40 \\
3.22 \\
\end{array}$ & $\begin{array}{r}1.22 \\
\quad \quad 2.80 \\
\end{array}$ & $\begin{array}{l}0.000 E+\infty 0 \\
1.420 E-07 \\
\end{array}$ & $\begin{array}{l}0.000 E+00 \\
1.014 E-05 \\
\end{array}$ & $\begin{array}{r}0.000 E+00 \\
4.406 E-08 \\
\end{array}$ & $\begin{array}{l}0.000 E+00 \\
5.070 E-08 \\
\end{array}$ & 1.420E-07 & $5.070 E-06$ & $3.072 E-08$ & $3.535 E-08$ \\
\hline $\begin{array}{l}\text { Climb to } \\
\text { Cruise (CC) }\end{array}$ & 11 & $12.22 \%$ & 11 & $12.36 \%$ & $13.00 \%$ & 0.182 & 356.74 & 310.00 & 64.93 & $\quad 56.42$ & 7.809E-08 & $4.290 E-07$ & 1.203E-09 & 1.384E-09 & & & & \\
\hline $\begin{array}{l}\text { Cruise/ } \\
\text { Enroute (ER) }\end{array}$ & 20 & $2222 \%$ & 20 & $22.47 \%$ & $60.00 \%$ & 0.840 & 477.57 & 415.00 & 401.16 & 348.60 & $1.420 E-07$ & $1.690 \mathrm{E}-07$ & $3.539 E-10$ & 4.073E-10 & 3.194E-07 & $2.453 E-07$ & $5.801 E-10$ & $6.676 E-10$ \\
\hline $\begin{array}{l}\text { Initial } \\
\text { Descent (ID) }\end{array}$ & 1 & $1.11 \%$ & 1 & $1.12 \%$ & $10.00 \%$ & 0.140 & 356.74 & 310.00 & 49.94 & 43.40 & $7.099 \mathrm{E}-09$ & $5.070 E-08$ & $1.421 E-10$ & $1.006 \mathrm{E}-10$ & & & & \\
\hline $\begin{array}{l}\text { Aipport } \\
\text { Approach (AA) }\end{array}$ & 13 & $14.44 \%$ & 13 & $14.61 \%$ & $10.00 \%$ & 0.140 & 247.42 & 215.00 & 34.64 & 30.10 & 9.228E-08 & $6.592 E-07$ & 2.664E-09 & $3.066 E-09$ & & & & \\
\hline $\begin{array}{l}\text { Aunway } \\
\text { Approach (RA) }\end{array}$ & 24 & $26.67 \%$ & 24 & $26.97 \%$ & $4.00 \%$ & 0.056 & 247.42 & 215.00 & & 12.04 & & & & 1.415E-08 & & & & \\
\hline Landing (ㄴ) & 0 & $0.00 \%$ & 0 & $0.00 \%$ & $1.00 \%$ & 0.014 & 100.00 & 66.90 & 1.40 & 1.22 & $0.000 E+\infty 0$ & $0.000 E+00$ & $0.000 E+00$ & $0.000 E+\infty$ & 1.704E-07 & 2.434E-06 & 1.117E-00 & 1.205E-00 \\
\hline $\begin{array}{l}\text { Ground } \\
\text { Accidenl (G) }\end{array}$ & 0 & $0.00 \%$ & & & & & & & & & & & & & & & & \\
\hline Unknown (?) & 1 & $1.11 \%$ & & & & & & & & & & & & & & & & \\
\hline TOTAL: & 90 & $100.00 \%$ & 89 & $100.00 \%$ & $100.00 \%$ & 1.400 & 407.53 & \begin{tabular}{|l|}
354.14 \\
\end{tabular} & 570.55 & 495.79 & 6.318E-07 & 4.513E-07 & 1.107E-09 & 1.274E-D9 & 6.318E-07 & 4.513E-07 & 1.107E-09 & 1. \\
\hline
\end{tabular}

Bases for Total 14 CFR 121 Crash Rates by Flight Phase, 1973-1994 Totals:

1.4 Hours Average Flight Duration

570 Miles Average Flight Distanco

407.143 Miles/Hour Average Flight Velocity

$140,872,105$ Departures 
Table 2.14

OFF AIRPORT ONLY 14 CFR 121 CRASHES WITH DESTROYED OR MAJOR-W.O. AIAFRAMES

BY FUGHT PHASE

[Refs. 2.6 to 2.28]

\begin{tabular}{|c|c|c|c|c|c|c|c|c|c|c|c|c|c|c|c|c|c|c|c|c|c|c|c|c|}
\hline FLKGHT PHASES & 1973 & 1974 & 1975 & 1976 & 1977 & 1978 & 1979 & 1980 & 1981 & 1982 & 1903 & 1984 & 1985 & 1986 & 1987 & 1988 & 1909 & 1990 & 1991 & 1992 & 1993 & 1994 & Total & Pat. \\
\hline Taxi & 0 & 0 & 0 & 0 & 0 & 0 & 0 & $\mathbf{0}$ & 0 & 0 & 0 & 0 & 0 & 0 & 0 & 0 & 0 & 0 & 0 & 0 & 0 & 0 & 0 & $0.0 \%$ \\
\hline Takeoll (TO) & 0 & o & 0 & 0 & 0 & 0 & 0 & 0 & 0 & 0 & 0 & 0 & 0 & 0 & 0 & 0 & 0 & 0 & 0 & o & 0 & o) & o & $0.0 \%$ \\
\hline Initial Climb (IC) & 2 & 0 & 2 & 0 & 1 & 1 & 3 & 0 & 0 & 2 & 0 & 0 & 4 & 0 & 0 & 0 & 1 & 1 & 1 & 0 & 0 & 1 & 19 & $30.6 \%$ \\
\hline Climb to Cruise (CC) & 0 & 0 & o & o & 0 & 0 & 2 & 0 & 0 & 0 & $\mathbf{0}$ & 0 & 0 & 0 & 0 & 0 & 1 & 1 & 0 & 0 & 0 & 0 & 4 & $6.5 \%$ \\
\hline Cruise/En Route (ER) & 0 & 2 & o & o & 1 & 0 & 0 & 0 & 0 & 0 & 0 & 2 & 0 & 0 & 1 & 2 & 2 & 0 & 0 & 0 & $\mathbf{0}$ & 0) & 10 & $16.1 \%$ \\
\hline Initial Descent (ID) & 0 & 0 & $\mathbf{0}$ & o & 0 & $\mathbf{0}$ & $\mathbf{0}$ & 0 & 0 & 0 & $\mathbf{0}$ & 0 & $\mathbf{0}$ & 0 & 0 & 0 & 0 & 0 & o & o & 0 & 1 & 1 & $1.6 \%$ \\
\hline Aippon Approach (M) & 0 & 2 & o & 0 & 1. & 1 & $\underline{0}$ & 0 & o & 0 & 1 & 0 & 1 & 0 & 0 & 0 & 2 & 0 & $\underline{0}$ & 0 & 0 & 1 & 9 & $14.5 \%$ \\
\hline Runway Approach (RA) & 3 & 2 & 3 & 1 & 0 & 2 & 1 & 1 & $\mathbf{0}$ & 0 & 1 & 0 & 1 & 0 & 1 & 0 & 1 & 0 & 1 & 1 & 0 & o) & 19 & $30.6 \%$ \\
\hline Landing (L) & 0 & 0 & o & 0 & 0 & $\mathbf{0}$ & o & 0 & o & 0 & $\mathbf{0}$ & 0 & 0 & 0 & 0 & 0 & 0 & 0 & $\underline{0}$ & 0 & 0 & of & o & $0.0 \%$ \\
\hline Grd. Accident (G) & 0 & 0 & o & 0 & 0 & 0 & o & 0 & $\mathbf{0}$ & o & 0 & 0 & 0 & 0 & 0 & 0 & 0 & 0 & 0 & 0 & 0 & 0 & $\mathbf{0}$ & $0.0 \%$ \\
\hline Unknown (?) & 0 & 0 & 0 & 0 & 0 & 0 & 0 & 0 & $\mathbf{0}$ & 0 & 0 & 0 & 0 & 0 & 0 & 0 & 0 & 0 & 0 & 0 & 0 & o) & 0 & $0.0 \%$ \\
\hline Tota & 5 & 6 & 5 & 1 & 3 & 4 & 6 & 1 & 0 & 2 & 2 & 2 & 6 & $\overline{0}$ & 2 & 2 & 7 & 2 & 2 & 1 & 0 & 3 & & $100.0 \%$ \\
\hline
\end{tabular}


Table 2.15

Off Airpont Only 14 CFR 121 Crashes with Destroyed/Major-W.O. Alrframe Rates by Filght Phase

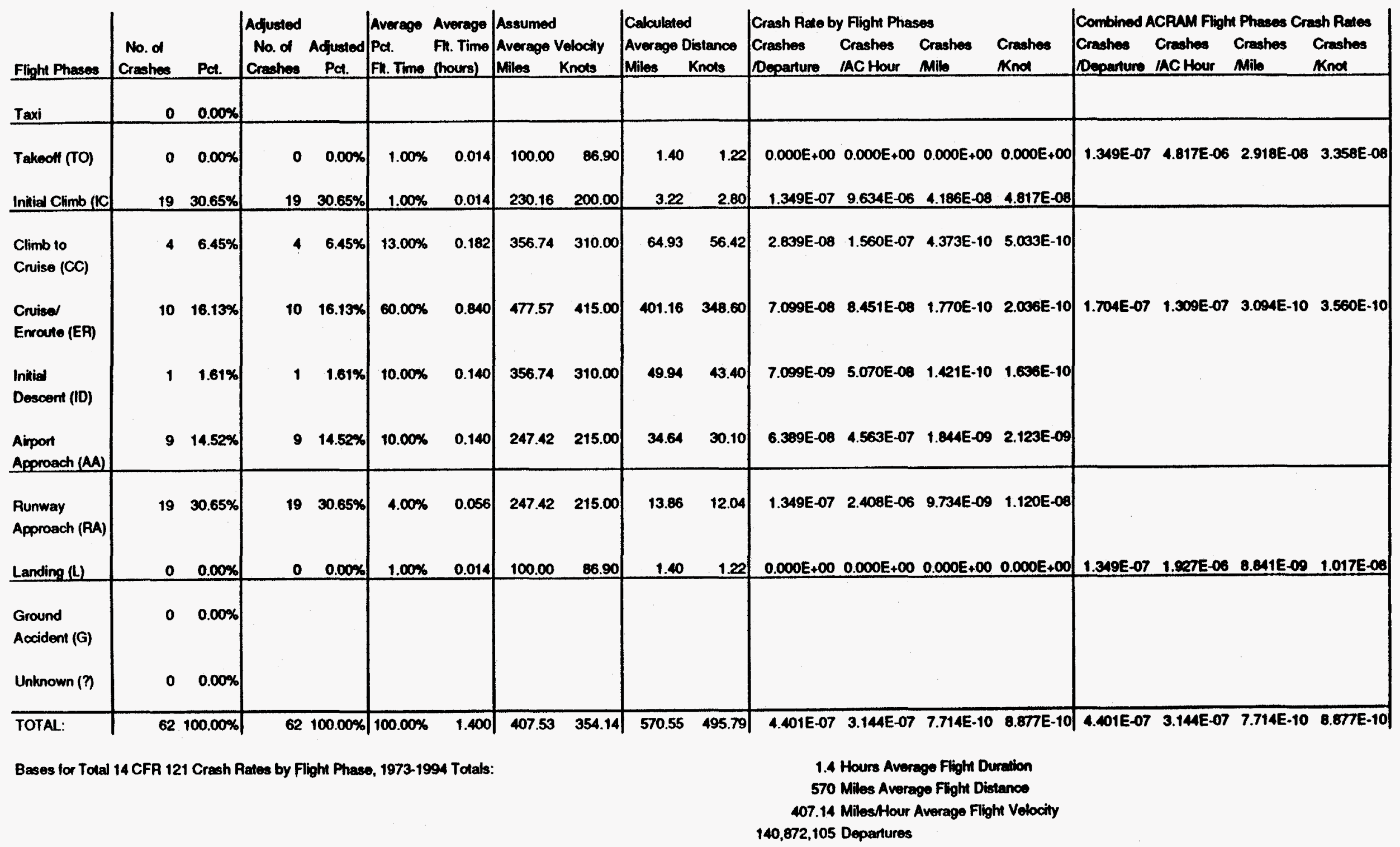


Table 2.16

\section{Comparison of All 14 CFR 121 Alrcraft Involved In Accidents/Crashes with} 14 CFR 121 Alrcraft Involved in Off Airport Accidents/Crashes

\begin{tabular}{|c|c|c|c|c|c|c|c|c|c|}
\hline Flight Phases & $\begin{array}{c}\text { Total } \\
\text { All } \\
\text { Accidents } \\
\text { No. of } \\
\text { Accidents }\end{array}$ & $\begin{array}{l}\text { Total } \\
\text { Off } \\
\text { Aippon } \\
\text { No. of } \\
\text { Accidents }\end{array}$ & $\begin{array}{c}\text { Pct. } \\
\text { Off Airport } \\
\text { All } A \infty c .\end{array}$ & $\begin{array}{c}\text { All } \\
\text { Accidents } \\
\text { Destroyed } \\
\text { or Major } \\
\text { Damage } \\
\text { No. of } \\
\text { Crashes } \\
\end{array}$ & $\begin{array}{c}\text { Oft } \\
\text { Aiport } \\
\text { Destroyed } \\
\text { or Major } \\
\text { Damge } \\
\text { No. of } \\
\text { Crashes } \\
\end{array}$ & $\begin{array}{c}\text { Pct. } \\
\text { Off Airport } \\
\text { All Acc. }\end{array}$ & $\begin{array}{c}\text { All } \\
\text { Accidents } \\
\text { Destroyed } \\
\text { Major-W.O. } \\
\text { Airframe } \\
\text { No. of } \\
\text { Crashes } \\
\end{array}$ & $\begin{array}{c}\text { Off } \\
\text { Airport } \\
\text { Destroyed/ } \\
\text { Major-W.O. } \\
\text { Airframe } \\
\text { No. of } \\
\text { Crashes } \\
\end{array}$ & $\begin{array}{l}\text { Pct. } \\
\text { Off Airport } \\
\text { All Acc. }\end{array}$ \\
\hline Taxi & 79 & 0 & $0.0 \%$ & 50 & 0 & $0.0 \%$ & 2 & 0 & $0.0 \%$ \\
\hline Takeoff (TO) & 47 & 0 & $0.0 \%$ & 34 & 0 & $0.0 \%$ & 14 & 0 & $0.0 \%$ \\
\hline Initial Climb (IC) & 38 & 22 & $57.9 \%$ & 32 & 20 & $62.5 \%$ & 26 & 19 & $73.1 \%$ \\
\hline $\begin{array}{l}\text { Climb to } \\
\text { Cruise (CC) }\end{array}$ & 39 & 33 & $84.6 \%$ & 14 & 11 & $78.6 \%$ & 5 & 4 & $80.0 \%$ \\
\hline $\begin{array}{l}\text { Cruise/ } \\
\text { Enroute (ER) }\end{array}$ & 126 & 121 & $96.0 \%$ & 23 & 20 & $87.0 \%$ & 11 & 10 & $90.9 \%$ \\
\hline $\begin{array}{l}\text { Initial } \\
\text { Descent (ID) }\end{array}$ & 55 & 53 & $96.4 \%$ & 1 & 1 & $100.0 \%$ & 1 & 1 & $100.0 \%$ \\
\hline $\begin{array}{l}\text { Airport } \\
\text { Approach (AA) }\end{array}$ & 17 & 16 & $94.1 \%$ & 13 & 13 & $100.0 \%$ & 10 & 9 & $90.0 \%$ \\
\hline $\begin{array}{l}\text { Rumway } \\
\text { Approach (RA) }\end{array}$ & 39 & 28 & $71.8 \%$ & 36 & 24 & $66.7 \%$ & 23 & 19 & $82.6 \%$ \\
\hline Landing (L) & 91 & 0 & $0.0 \%$ & 85 & 0 & $0.0 \%$ & 28 & 0 & $0.0 \%$ \\
\hline $\begin{array}{l}\text { Ground } \\
\text { Accident (G) }\end{array}$ & 47 & 0 & $0.0 \%$ & 12 & 0 & $0.0 \%$ & 1 & 0 & $0.0 \%$ \\
\hline Unknown (?) & 6 & 3 & $50.0 \%$ & 4 & 1 & $25.0 \%$ & 0 & 0 & $0.0 \%$ \\
\hline TOTAL: & 584 & 276 & $47.3 \%$ & 304 & 90 & $29.6 \%$ & 121 & 62 & $51.2 \%$ \\
\hline
\end{tabular}




\subsection{AIR TAXI (14 CFR 135) ACCIDENT AND CRASH RATES}

An Air Taxi is defined as a classification of Air Carriers which transports in accordance with 14 CFR 135 [Ref. 2.2], persons, property, and mail using small aircraft (under 30 seats or a maximum payload capacity of less than 7,500 pounds). A Commuter Air Carrier is defined as an Air Taxi operator which performs at least five round trips per week between two or more points and publishes flight schedules which specify the times, days of the week, and points between which such flights are performed.

Tables 2.16 to 2.18 taken from the NTSB Annual Review of Aircratt Accident Data, U.S. Air Carrier Operations [Ref. 2.6 to 2.26] and the NTSB News Annual Press Release on U.S. Airline Accidents [Ref. 2.27 and 2.28] presents the following information for the years 1975 to 1995 :

1. The number of tatal accidents,

2. The total number of accidents,

3. The number of passenger fatalities aboard the aircraft flying under that part,

4. The number of crew fatalities aboard the aircraft flying under that part,

5. Other fatalities associated with the accident including ground fatalities and fatalities aboard aircraft involved in the accident but not flying under that part, i.e. collisions,

6. The total number of fatalities,

7. The number of aircraft flying under that part involved in the accidents that were destroyed,

8. The number of aircratt flying under that part involved in the accidents that were substantially (major) damaged,

9. The number of aircraft flying under that part involved in the accidents that suffered minor or no damage,

10. The total number of aircraft flying under that part involved in the accidents,

11. The number of aircraft hours flown by aircraft flying under that part,

12. The number of aircraft miles by thousands flown by aircraft flying under that part,

13. The number of aircraft departures performed by aircraft flying under that part,

14. The average speed of aircraft flying under that part, calculated by dividing the number of aircraft miles flown by the number of aircrat hours flown,

15. The average number of aircraft miles flown by aircraft flying under that part per flight or departure, calculated by dividing the number of aircratt miles flown by the number of departures,

16. The average amount of time flown by aircratt flying under that part per flight or departure, calculated by dividing the number of aircraft hours flown by the number of departures,

17. The fatal accident rate per aircraft hour, aircraft mile, and departure, determined by dividing the number of fatal accidents by the number of aircraft hours, aircraft miles, and departures, respectively,

18. The total accident rate per aircraft hour, aircraft mile, and departure, determined by dividing the total number of accidents by the number of aircraft hours, aircraft miles, and departures, respectively.

19. The crash rate per aircratt hour, aircraft mile, and departure, determined by dividing the number of crashes (defined as aircraft flying under that part involved in the accident that were destroyed or substantially damaged) by the number of aircraft hours, aircraft miles, and departures, respectively.

The total, average, and standard deviation for each of the above categories of information were calculated for those years in which information was available, except for the calculated average speed, distance and time per departure (14. to 16.) and calculated fatal accident, total accident and crash rates (17. to 19.) which had only the average and standard deviation calculated. Table 2.17 presents data for the scheduled or commuter U.S. air carriers operating under 14 CFR 135. Table 2.18 presents data for the nonscheduled or air taxi U.S. air carriers operating under 14 CFR 135 . Table 2.19 presents for total U.S. air carriers operating under 14 CFR 135 and is the sum of Tables 2.17 and 2.18.

While Tables 2.17 to 2.19 were taken from NTSB documents [Refs. 2.11 to 2.28], only the accident data and the aircraft damage data were compiled by the NTSB. All operations data are tabulated by the F.A.A. It should be emphasized that all of the accidents, aircratt involvment, and operations given in Tables 2.17 to 2.19 were tabulated on the basis of the part or regulation under which the flight was conducted and that 
the accident and crash rates are conculated on a consistent basis.

The caveats stated for 14 CFR 121 Air Carriers in Section 2.2 generally apply to the 14 CFR 135 Air Carriers (Air Taxis), i.e., repositioning or ferry tights, and training flights are not counted as part of the operations, and operations by foreign air carriers in U.S. airspace are not counted. The errors introduced by these restrictions on the data are not known but are assumed to be small. The number of air taxi flights by foreign air carriers in U.S. airspace are expected to be particularty small because of the distances involved and the range limitation of air taxi aircraft types.

In order to calculate an accident and crash rate that is reasonably conservative and yet incorporates recent data, it is necessary to include data over a reasonably long period of time that is complete and current. For U.S. air carriers operating under 14 CFR 135, the years 1980 to 1993 were selected. This enabled the accidemt and crash rates for 135 air carriers to be determined for a 14 year periods.

The accident and crash rates given by Tables 2.17 to 2.19 for 135 air carriers represent the accidemt and crash rates over the entire flight. As experience and numerous past studies on commercial aviation safety has shown, accidents do not occur with equal probability during the course of the flight. Certain flight phases, e.g. landing and takeoff, experience significantly higher number of accidents and crashes. To determine accident and crash rates by flight phase, it is necessary to determine the number of accidents and crashes that occur in each flight phase.

Table 2.20 presents the total number of accidents involving aircraft flying under scheduled 14 CFR 135 regulations by flight phase and by aircrath damage state for the 1980 to 1993 time period except for 1981 [Refs. 2.13 to 2.26]. From the number of accidents involving Scheduled 14 CFR 135 aircraft given by Table 2.20, and assuming 0.7 hour average flight duration, 127.5 miles average distance flown per flight, and 179.5 miles/hour average velocity for each flight, for the $36,582,419$ departures (flights) flown by Scheduled 14 CFR 135 Air Carriers for 1980 to 1993, the accident and crash rates are determined in Table 2.21. The ACRAM Standard [Ref. 2.5] defines three flight phases, takeoff, landing and in-flight or enroute. Takeoff is defined as encompassing the actual takeoff roll and the initial climb phase. Landing is defined as including the landing approach, and the actual landing roll. Enroute or in-flight is defined as including the climb to cruise, cruise or enroute, and the descent from cruise flight phases. To meet the needs of the ACRAM Standard, crash rates for the flight phases used by the ACRAM Standard must be determined. This is also done by combining the crashes occurring in the takeoff and initial climb phases into the ACRAM takeott crash rate. The ACRAM in-flight crash rate was determined by combining the crashes occurring during the climb to cruise, cruise/enroute, and descent from cruise flight phases. The ACRAM landing crash rate was determined by combining the crashes occurring during the landing approach and landing flight phases.

Table 2.21 has a column (2nd from the left) which lists the calculated number of accidents. This was determined by taking the flight phase percentages determined in Table 2.20 and applying the percentages to the total number of Scheduled 14 CFR 135 accidents for the 1980-1993 time period determined from Table 2.17. This was done because the number for Scheduled 14 CFR 135 accidents by flight phase for 1981 was not available at the time Table 2.20 was prepared. It is hope that this data can be obtained and that these tables can be revised to present the actual 1981 data in the calculations.

Finally, applying the assumption used by the ACRAM Standard [Ref. 2.5] that facilities considered by the ACRAM Standard are located far enough from the aimport that only aircraft impacts (crashes) located off the rumway need be considered, cannot be done for 14 CFR 135 aircraft due to unavailability of that data. Therefore, the crash rates presented by Table 2.21 represent all Scheduled 14 CFR 135 accidents, both on and off the airport. A possible factor which could be applied to the crash rates of Table 2.21 is to apply the off airportthotal accident percentages of 14 CFR 121 Air Carriers determined in Tables 2.16 to the

Scheduled 14 CFR 135 Air Carrier crash rates. It should be noted that there are significant differences in the type of aircrafts used in 14 CFR 121 operations versus Scheduled 14 CFR 135 operations. In addition, the type of operations performed under 14 CFR 121 differ significantly from the type of operations performed under 14 CFR 135 except for the largest Scheduled 14 CFR 135 Air Carriers flying the larger aircraft types. Another possible factor which could be applied to the crash rates of Table 2.21 is to apply the off rumway/otal accident percentages for General Aviation determined in Chapter 3 to the

2. COMMERCIAL AVIATION 
Scheduled 14 CFR 135 Air Carrier crash rates. 
Table 2.17

Schodulod U.S. Air Comiors Operating Under 14 CFA 135 (Commutors) [Roferences 2.11 to 2.28]

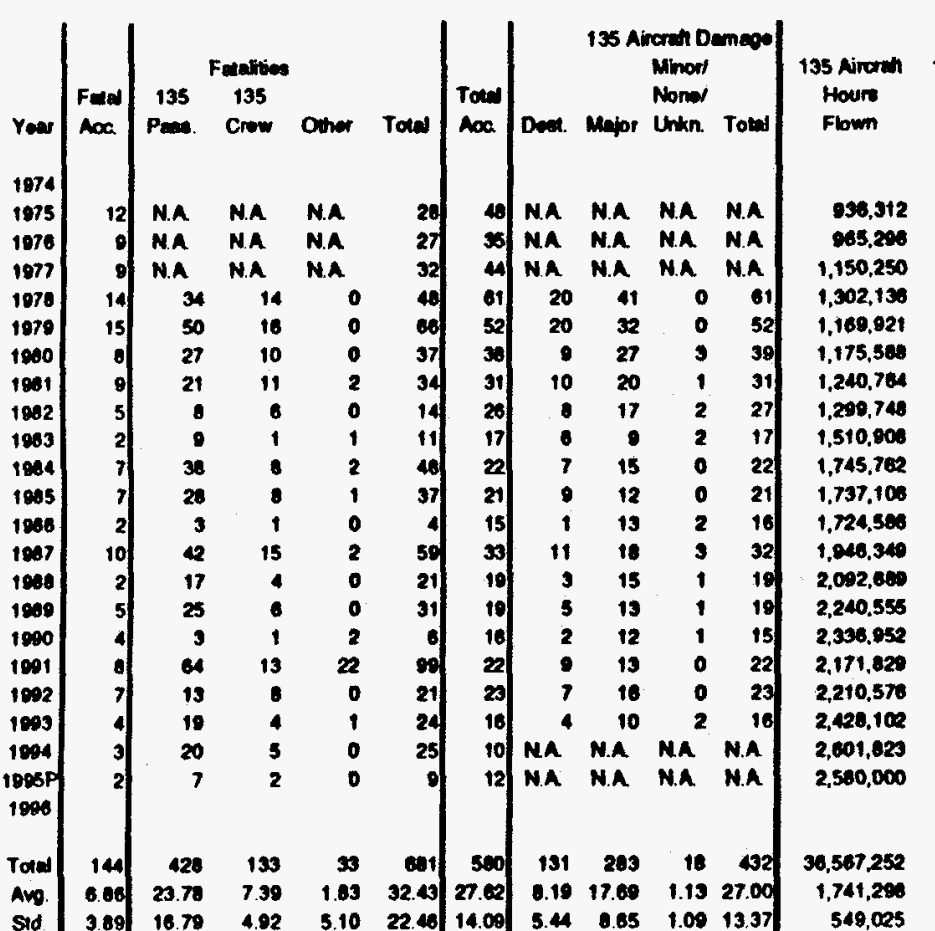

P. Proliminary Data

NA - Intormation not availablo when teblo propored.

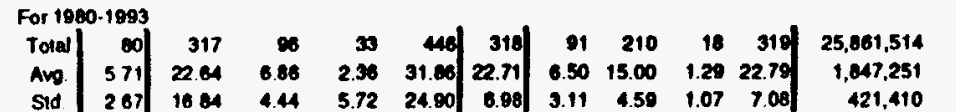


Toble 2.18

Nonscheduled U.S. Air Carriors Operating Under 14 CFR 135 (Ar Taxis) [Aotorences 2.11 to 2.28]

\begin{tabular}{|c|c|c|c|c|c|c|c|c|c|c|c|c|c|c|c|c|c|c|c|c|c|c|c|c|c|}
\hline \multirow{2}{*}{$\begin{array}{l}\text { Your } \\
1974 \\
1075\end{array}$} & \multirow{2}{*}{$\left|\begin{array}{c}F a n \\
n \infty a \\
24\end{array}\right|$} & \multirow{2}{*}{$\begin{array}{l}\text { 135 } \\
\text { Pane. } \\
\text { Na }\end{array}$} & \multicolumn{3}{|l|}{ Fatalition } & Tow & Den. & \multicolumn{3}{|c|}{ 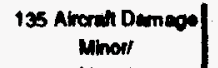 } & $\begin{array}{l}135 \text { Arrorint } \\
\text { Hours } \\
\text { Flown }\end{array}$ & $\begin{array}{l}135 \text { Aircrah } \\
\text { Milive } \\
(000)\end{array}$ & $\begin{array}{l}135 \text { Airesent } \\
\text { Dopenturat }\end{array}$ & $\begin{array}{l}\text { Averace } \\
\text { Spend } \\
\text { (milow/wr.) }\end{array}$ & $\begin{array}{l}\text { Averesos } \\
\text { AC Menes } \\
\text { Depertures }\end{array}$ & $\begin{array}{c}\text { Averease } \\
\text { AC Hoines } \\
\text { Departurs }\end{array}$ & \multicolumn{3}{|c|}{ 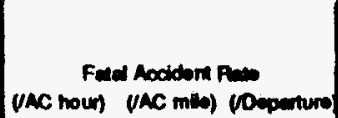 } & \multicolumn{3}{|c|}{ 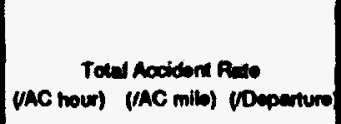 } & \multicolumn{3}{|c|}{ 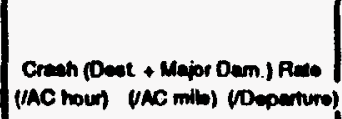 } \\
\hline & & & $\begin{array}{l}\text { N.A } \\
\text { N.A. }\end{array}$ & $\begin{array}{l}\text { Na } \\
\text { Nan }\end{array}$ & of & 152 & MA & $\begin{array}{l}\text { N.A } \\
\text { N.A }\end{array}$ & $\begin{array}{l}\text { N.A. } \\
\text { N.A. }\end{array}$ & $\begin{array}{l}\text { N.A } \\
\text { N.A. }\end{array}$ & 2.528.271 & N.A & NA & Na & N.A. & N.A & $9.50 E-\infty 8$ & Ma & N.A. & c.02E-05 & Na & N.A & N.A. & Na & N.A \\
\hline 1977 & 31 & N.A & NA & N.A & 110 & 158 & NA & N.A & M.A & N.R & $\begin{array}{l}2,03,203 \\
3,304,220\end{array}$ & $\begin{array}{l}\text { N.A. } \\
\text { N.A. }\end{array}$ & $\begin{array}{l}\text { N.A } \\
\text { N.A. }\end{array}$ & N.A & $\begin{array}{l}\text { N.A. } \\
\text { N.A. }\end{array}$ & $\begin{array}{l}\text { N.A. } \\
\text { N.A }\end{array}$ & $\begin{array}{l}1.15 E-05 \\
9.30 E-06\end{array}$ & N.A. & $\begin{array}{l}\text { W.A. } \\
\text { N.A. }\end{array}$ & 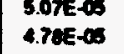 & $\begin{array}{l}\text { NaR } \\
\text { MA }\end{array}$ & $\begin{array}{l}\text { N.M } \\
\text { N.A. }\end{array}$ & $\begin{array}{l}\text { N.A. } \\
\text { N.A. }\end{array}$ & $\begin{array}{l}\text { N.A } \\
\text { N.A }\end{array}$ & $\begin{array}{l}\text { NA } \\
\text { N.A }\end{array}$ \\
\hline 1978 & 54 & $\infty$ & s3 & 3 & 15s: & 190 & 74 & 125 & 1 & 200 & $3,545,753$ & N.A & N.A & N.A & N.A. & NA & $1.52 E-06$ & M.M & M.A. & S.SeE- $-\infty$ & $\mathrm{NA}$ & N.A & s.01E-0s & MA & NA. \\
\hline 1079 & 30 & 30 & 34 & 4 & 77 & 160 & 40 & 118 & 3 & 161 & $3,604,321$ & N.A & N.A & N.A & N.A. & N.A & $0.14 E-\infty 6$ & N.A & N.A. & A.SUE-W6 & MA & NA & $420 E-05$ & NA & N.A. \\
\hline 1900 & 48 & 02 & 41 & 2 & 105 & 171 & 52 & 110 & 2 & 172 & $3,617,724$ & N.A. & N.A. & N.A & N.A. & N.A. & $1.27 E-\infty 6$ & N.A. & N.A. & 4.73E- & NA & N.A & $4.70 E-05$ & N.A & NA \\
\hline 001 & 40 & 57 & 35 & 2 & 04 & 157) & 34 & 102 & 2 & 150 of & $2,005,027$ & N.A & N.A & Na & NA. & NA & $1.30 E-08$ & NA & $\mathrm{NA}$ & 5.42E- -6 & NA & $\mathrm{Na}$ & 5. 39E-05 & NA & N.A. \\
\hline 1902 & 31 & 45 & 27 & 0 & 72 & 132 & 47 & 63 & 4 & 134 & $3,000,000$ & N.A & N.A & N.A & N.A. & N.A & $1.03 E-05$ & NA & M.A. & $4.30 E-\infty 6$ & M.A & N.A & 4.32E-05 & $\mathbf{N A}$ & NA \\
\hline $2 * 3$ & 27. & 27 & 30 & 5 & 62 & 141 & 33 & 106 & 4 & 143 & 2.370 .000 & N.A & N.A & N.A & N.A. & N.A & $1.14 E-06$ & N.A & N.A. & 5.83E- -6 & N.A & N.A & S.85E-05 & $\mathbf{N . A}$ & N.A. \\
\hline 964 & 23 & 22 & 30 & 0 & 52 & 100 & 40 & 104 & 3 & 147 & $2,043,000$ & N.A. & N.A. & NA. & N.A. & NA & BOSE- $\infty 6$ & NA & N.A. & 3.14E-06 & M.A & NA & $5.07 E-05$ & N.A & N.A. \\
\hline 2085 & 35 & 30 & 36 & 1 & 78 & 154 & 30 & 104 & 3 & 157 & $2,570,000$ & N.A & N.A. & N.A & N.A. & NA & $1.36 E-05$ & N.A & N.A. & 5.90E- -6 & N.A & N.A & 3. $.00 E=03$ & N.A & NA. \\
\hline 1000 & 31 & 20 & 35 & 4 & 65. & 117 & 30 & $n$ & 3 & 118 & $2,090,000$ & N.A & N.A. & N.A & N.A. & N.A & $1.15 E-05$ & N.A. & N.A. & 4.35E-05 & $M A$ & N.A & $4.20 \leq-05$ & N.A & N.A \\
\hline 1967 & 30 & 31 & 32 & 2 & 65 & $\infty$ & 34 & 02 & 4 & 100 & $2,657,000$ & N.A & NA & N.A & NA. & N.A. & $1.13 E-\infty 5$ & NA & N.A. & S.81E-16 & MA & $\mathrm{NA}$ & 3.61E.05 & MA & NA \\
\hline 968 & 20) & 22 & 33 & 4 & 50. & 101 & 37 & 62 & 2 & 101 & $2,632,000$ & N.A & N.A & N.A & N.A. & NA & $1.06 E-06$ & Na & N.a & 3.0AE $\infty$ & Ma & M.A & $3.76 E-03$ & $\mathrm{Na}$ & NA. \\
\hline 60 & 25 & 46 & 36 & 2 & 03 & 110 & 32 & $\infty$ & 0 & 112 & $3,020,000$ & M.A. & NA & N.A & N.A. & M.A & $0.20 E-06$ & N.A & N.A. & J.64E- -6 & MA & N.A & 3.71E-05 & N.A & M.A. \\
\hline 000 & 28) & 20 & 20 & 2 & 50 & 108 & 30 & 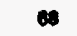 & 2 & 108 & $2,240,000$ & N.A & NA & NA & NA. & N.A & $124 E-05$ & N.A & N.A. & 4.71E-65 & MA & N.A & $4.71 E-05$ & NA & N.A. \\
\hline 1001 & 27) & 36 & 31 & 4 & 70 & 67 & 31 & 53 & 4 & 86. & $2,241.000$ & N.A & N.A & N.A & N.A. & M.A & $1.20 E-05$ & N.A & N.A. & $3.606-\infty$ & Na & N.A. & 3.75E-05 & N.A & N.A. \\
\hline 1892 & 24 & 43 & 22 & $\mathbf{3}$ & of & 78 & 26 & $\infty$ & 1 & 78 & 2.000 .000 & NA & N.A & N.A & NA. & N.A & $1.10 E-05$ & N.A & N.A. & $3.70 E-05$ & $\mathrm{MA}$ & NA & $3.73 \in-05$ & N.A & N.A. \\
\hline 1003 & 10. & 20 & 22 & o & 42 & $\infty$ & 20 & 4 & 0 & 70 & $1,000,000$ & N.A & NA & NA & NA. & NA & $1.05 E-05$ & NA & N.A. & 3.81E-05 & NA & NA & 3.e7E.05 & N.A & N.A. \\
\hline 1004 & 26 & N.A & N.A & M.A & 62 & 85 & N.A & N.A. & $\mathbf{M a}$ & N.A & $1,003,000$ & N.A & N.A & N.A & N.A. & $\mathrm{NaA}$ & $1.300-05$ & N.A. & M.A. & $420 E-05$ & $\mathrm{Ma}$ & N.A & NA. & N.A & N.A. \\
\hline $\begin{array}{c}1905 f \\
1896\end{array}$ & 24) & NA & MA & N.A & 52 & 76 & N.A & N.A & N.A. & N.A & $2,000,000$ & N.A & N.A & N.A & NA. & NA & $1.20 E-05$ & M.A. & N.A. & 3.EOE-65 & Na & N.A & NA & Na & N.A \\
\hline Total & 634 & 630 & 524 & 30 & 1500 & 2029 & 052 & 1355 & 30 & 2005 & $56,376,319$ & N.A & N.A & N.A. & N.A. & N.A. & $1.12 E-05$ & N.A & N.A. & 4.806-05 & $\mathrm{NA}$ & N.A & NA. & $\mathrm{Na}$ & N.A. \\
\hline Avo & 30.10 & 30.56 & 32.75 & 2.36 & 7600 & 125.2 & 40.75 & 400 & 2.39 & 127.6 & $2,064,587$ & NA & N.A & NA & N.A. & NA & $1.13 E-05$ & N.A & N.A. & $4.02 E-05$ & Ma & N.A & 4.SAE-05 & N.a & N.A \\
\hline Sid. & 8.00 & 20.40 & 7.35 & 1.50 & 28.33 : & $|36.28|$ & 12.32 & 26.67 & 1.30 & 36.96| & 546,509 & N.A & NA & NA & N.A. & N.A. & $1.02 E-\infty 0$ & N.A & N.A. & $8.10 E-08$ & N.A. & N.A & $0.201-08$ & N.A & NA. \\
\hline
\end{tabular}

P-Protiminery Data

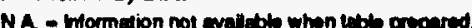

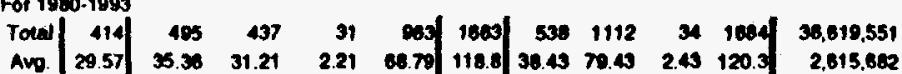

N.

\begin{tabular}{l|l} 
N.A & N.A \\
N.A & N.A \\
N.A & N.A
\end{tabular}

\begin{tabular}{ll|ll} 
N.A. & N.A & $1.13 E-05$ & N.A \\
N.A. & N.A & $1.13 E-05$ & N.A \\
N.A. & N.A & $1.70 E-\infty 6$ & N.A
\end{tabular}

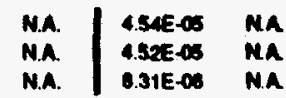

\begin{tabular}{l|l} 
M.A & 4.51E.05 \\
N.A & L.46E-05
\end{tabular}

\begin{tabular}{l|ll} 
N.A & 1.4BE-0S \\
N.A & M.23E-06
\end{tabular}

\begin{tabular}{l|l} 
N.A \\
NA \\
N.A.
\end{tabular} 
Table 2.19

Totel (Scheduled and Nonscheduled) U.S. Air Carriers Operating Under 14 CFR 135 [Roforences 2.11 to 2.28]

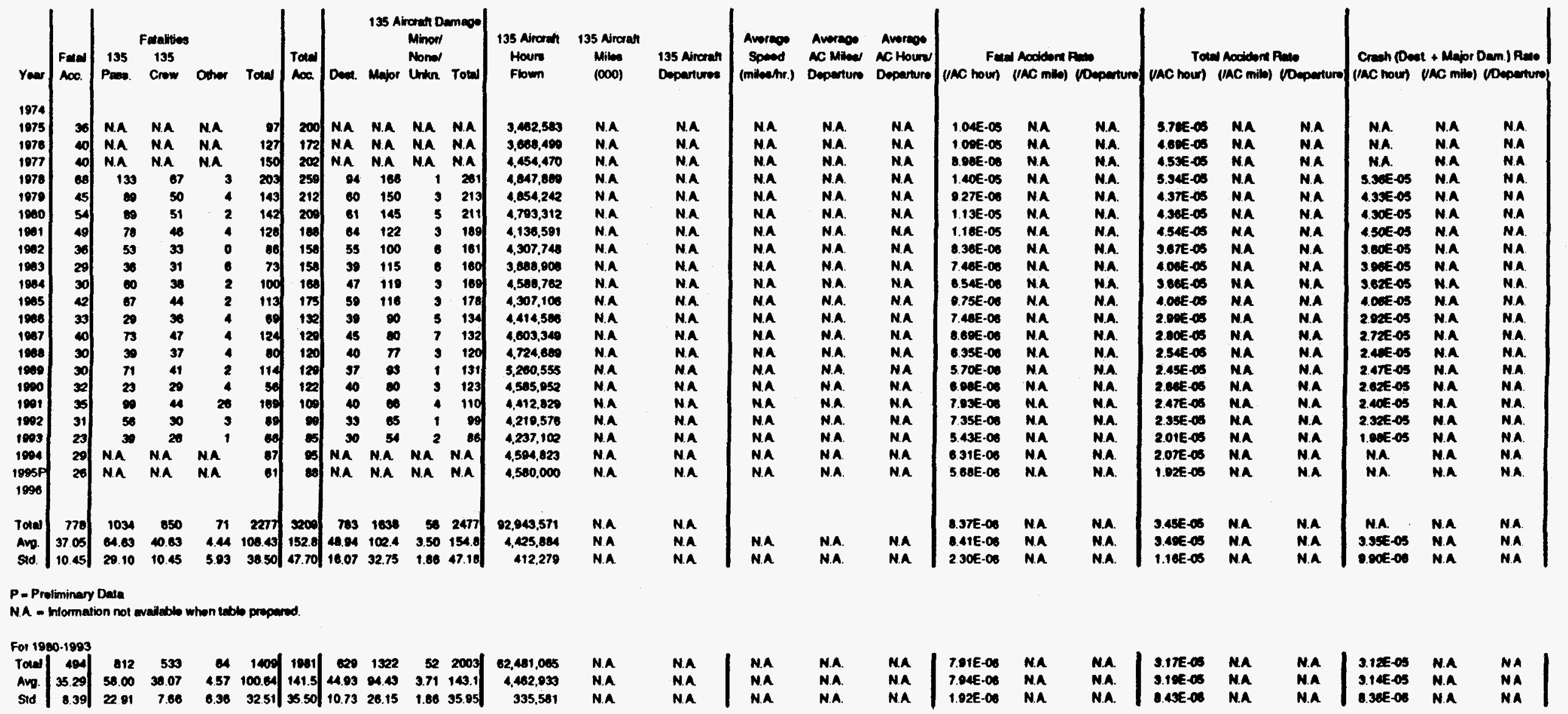


Tabte 2.20

Schodulod 14 CFR 135 Alroreh Aooldente by Damngo and Flight Phace [Rolo. 2.13-2.20]

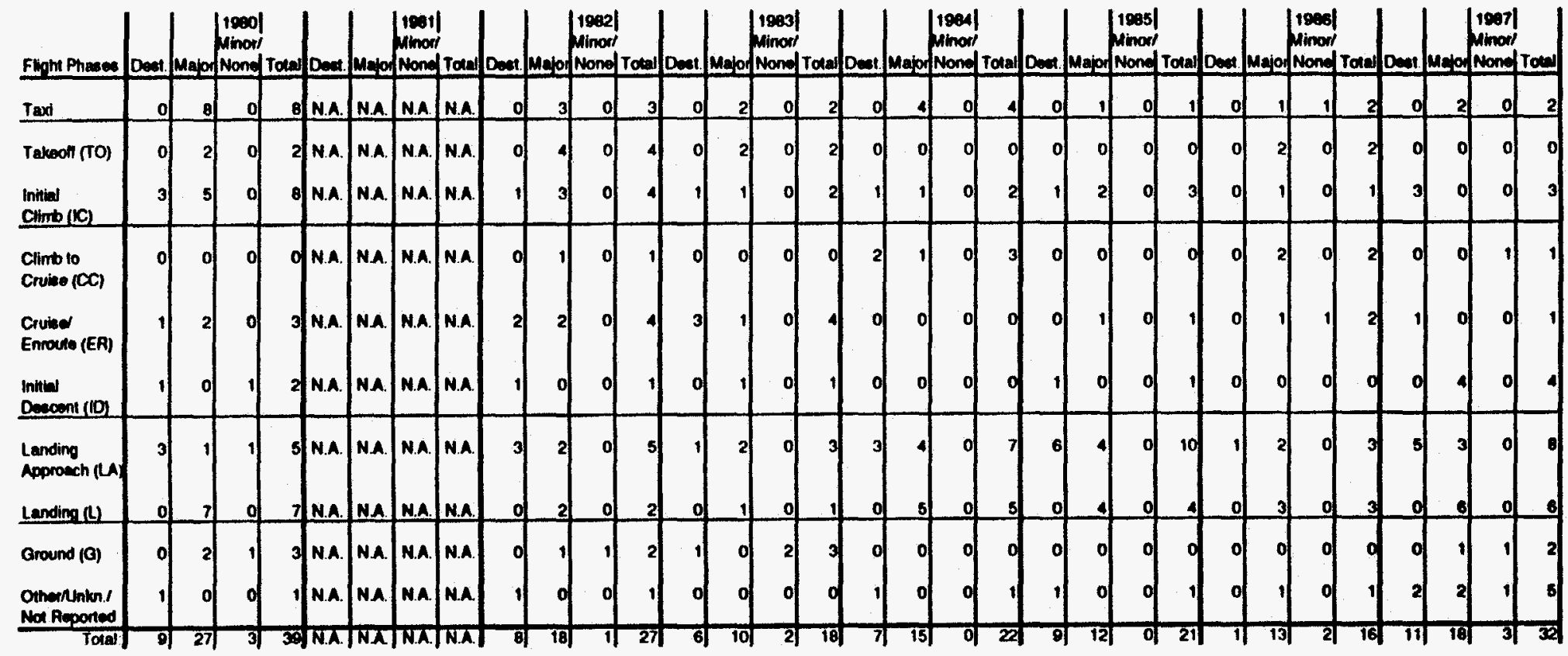

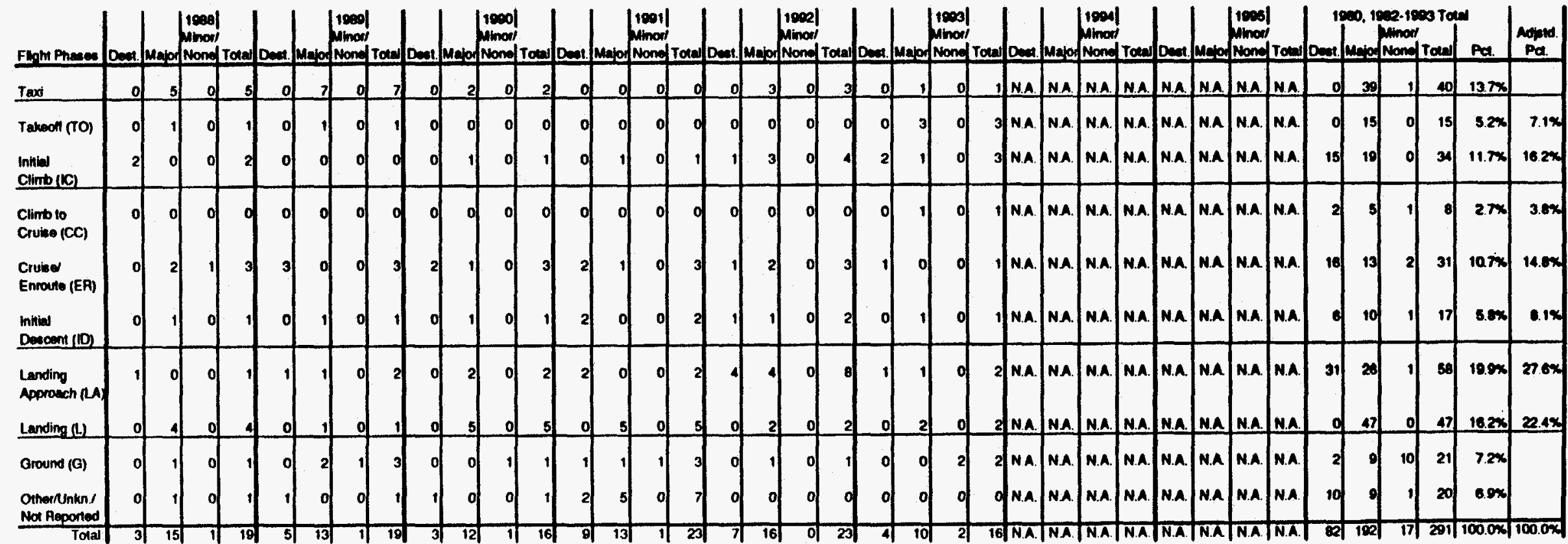




\section{Tablo 2.21}

Scheduled 14 CFR 135 Crash Ratea by Fight Phaso

\begin{tabular}{|c|c|c|c|c|c|c|c|c|c|c|c|c|c|c|c|c|c|c|}
\hline Flight Phases & \multicolumn{2}{|l|}{$\begin{array}{l}\text { Calc. } \\
\text { No. of } \\
\text { Acos. }\end{array}$} & \multirow[t]{2}{*}{$\begin{array}{l}\text { Cale. } \\
\text { No. of } \\
\text { Acos. }\end{array}$} & \multirow[t]{2}{*}{$\begin{array}{l}\text { Adjusied } \\
\text { Pat. }\end{array}$} & \multirow[t]{2}{*}{$\begin{array}{l}\text { Average } \\
\text { Pct. } \\
\text { Fh. Time }\end{array}$} & \multirow[t]{2}{*}{$\begin{array}{l}\text { Average } \\
\text { Fit. Time } \\
\text { (hours) }\end{array}$} & \multicolumn{2}{|c|}{$\begin{array}{l}\text { Assumed } \\
\text { Average Velocity } \\
\text { Miles Knots } \\
\end{array}$} & \multicolumn{2}{|c|}{$\begin{array}{l}\text { Calculated } \\
\text { Average Distances } \\
\text { Miles Knols } \\
\end{array}$} & \multicolumn{3}{|c|}{$\begin{array}{l}\text { Crash Rate by Fligh Pheses } \\
\text { Crashes Crashes Crashes } \\
\text { Departure ACHour Mile }\end{array}$} & \multirow[t]{2}{*}{$\begin{array}{l}\text { Crashes } \\
\text { Knot }\end{array}$} & \multicolumn{4}{|c|}{$\begin{array}{l}\text { Combined ACPAM Fight Pheses Crash Aatos } \\
\text { Creshos Creshes Crashes Crashes } \\
\text { Oegarture ACHour Mile }\end{array}$} \\
\hline Taxi & & $13.70 \%$ & & & & & & & & & & & & & & & & \\
\hline $\begin{array}{l}\text { Takeoff } \\
\text { Initial Climb }\end{array}$ & $\begin{array}{l}17 \\
37 \\
\end{array}$ & $\begin{array}{r}5.20 \% \\
11.70 \% \\
\end{array}$ & $\begin{array}{l}17 \\
37 \\
\end{array}$ & $\begin{array}{r}7.10 \% \\
16.20 \% \\
\end{array}$ & $\begin{array}{l}1.13 \% \\
2.25 \% \\
\end{array}$ & $\begin{array}{l}0.008 \\
0.016 \\
\end{array}$ & $\begin{array}{r}86.31 \\
126.59 \\
\end{array}$ & $\begin{array}{r}75.00 \\
110.00 \\
\end{array}$ & $\begin{array}{l}0.69 \\
2.03 \\
\end{array}$ & $\begin{array}{l}0.60 \\
1.76 \\
\end{array}$ & $\begin{array}{l}4.520 \mathrm{E}-07 \\
1.017 \mathrm{E}-06 \\
\end{array}$ & $\begin{array}{l}5.650 \mathrm{E}-05 \\
6.357 \mathrm{E}-05 \\
\end{array}$ & $\begin{array}{l}6.547 E-07 \\
5.022 E-07 \\
\end{array}$ & $\begin{array}{l}7.534 E-07 \\
5.779 E-07 \\
\end{array}$ & 1.469E-06 & 6.121E-05 & $5.409 E-07$ & $6.225 E-07$ \\
\hline $\begin{array}{l}\text { Climb to } \\
\text { Cruise }\end{array}$ & 9 & $2.70 \%$ & 9 & $3.80 \%$ & $18.73 \%$ & 0.133 & 155.35 & 135.00 & 20.66 & 17.96 & 2.347E-07 & $1.765 E-06$ & $1.136 E-08$ & $1.307 E-\infty$ & & & & \\
\hline $\begin{array}{l}\text { Cruised } \\
\text { Enroute }\end{array}$ & 34 & $10.70 \%$ & 34 & $14.80 \%$ & $31.41 \%$ & 0.223 & 224.40 & 195.00 & 50.04 & 43.49 & 9.301E-07 & $4.171 E-06$ & $1.859 E-00$ & $2.139 E-00$ & $1.669 E-06$ & $3.406 E-06$ & $1.553 E-00$ & $1.536 \mathrm{E}-00$ \\
\hline $\begin{array}{l}\text { Descent } \\
\text { Irom Cruise }\end{array}$ & 18 & $5.80 \%$ & 18 & $8.10 \%$ & $26.48 \%$ & 0.188 & 195.63 & 170.00 & 36.78 & 31.96 & 5.042E-07 & 2.682E-06 & 1.371E-08 & $1.578 E-08$ & & & & \\
\hline $\begin{array}{l}\text { Landing } \\
\text { Approach }\end{array}$ & 63 & $19.90 \%$ & 63 & $27.60 \%$ & $18.87 \%$ & 0.134 & 126.59 & 110.00 & 16.96 & 14.74 & $1.730 E-06$ & $1.291 E-05$ & $1.020 E-07$ & 1.174E-07 & & & & \\
\hline Landing & & $16.20 \%$ & & $22.40 \%$ & $1.13 \%$ & 0.008 & 46.03 & 40.00 & 0.37 & 0.32 & $1.408 E-06$ & $1.760 E-04$ & $3.824 E-08$ & 4.401E-06 & 3.130E-06 & $2.210 E-05$ & 1.811E-07 & 2.004E-07 \\
\hline $\begin{array}{l}\text { Ground } \\
\text { OherNunkn./ } \\
\text { Not Reported }\end{array}$ & $\begin{array}{l}23 \\
22\end{array}$ & $\begin{array}{l}7.20 \% \\
6.90 \%\end{array}$ & & & & & & & & & & & & & & & & \\
\hline $\begin{array}{l}\text { TOTAL: } \\
\text { Bases for Sch }\end{array}$ & $\begin{array}{r}318 \\
\text { eduled } 14\end{array}$ & $\begin{array}{l}100.00 \% \\
\text { CFR } 135 \mathrm{C}\end{array}$ & $\begin{array}{r}230 \\
\text { Crash Rate }\end{array}$ & $\begin{array}{l}100.00 \% \\
\text { es by Figh }\end{array}$ & $\begin{array}{l}100.00 \% \\
\text { If Phase, } 1\end{array}$ & $\begin{array}{r}0.710 \\
1960-1993\end{array}$ & $\begin{array}{l}179.62 \\
\text { Totals: }\end{array}$ & 156.09 & 127.53 & 110.82 & $\begin{array}{r}6.276 E-06 \\
0.71 \\
127.5 \\
179.58 \\
36,582,419\end{array}$ & $\begin{array}{l}\text { 8.840E-06 } \\
\text { Mours Avera } \\
\text { Miles Avorag } \\
\text { Miles/Hour A } \\
\text { Departures }\end{array}$ & $\begin{array}{l}\text { 4.921E-08 } \\
\text { go Flight Dur } \\
\text { Fighth Dist } \\
\text { Average Fligh }\end{array}$ & $\begin{array}{l}\text { 5.663E-00 } \\
\text { reation } \\
\text { tanco } \\
\text { ht Velocity }\end{array}$ & 6.276E-08 & $8.840 E-08$ & $4.921 E-08$ & $5.663 E-08$ \\
\hline
\end{tabular}




\subsection{COMMERCIAL AVIATION CRASH PARAMETER DISTRIBUTIONS.}

Calculation of the impact frequency for near airport crashes by means of the four factor methodology given by the ACRAM Standard [Ref. 2.5] requires the probability distribution of impact location, as well as representative values of impact angle and skid distance. These distributional quamities may readily be extracted from results from a more general methodology derived in LLNL report UCAL-ID-122512 [Ref. 2.32], which provides frequency estimates of hitting a facility at or above a specified velocity, and models both off-rumway impacts and numway run-ofts and overuns. Since in DOE applications the facility of interest will by assumption be located sufficiently far from any numway that the comtribution from numay nun-offs or overruns is negligible, the relevant probability distributions for the LLNL evaluation are those which model the off-runway impact location, angle, and velocity, and the heading and deceleration after impact. Reasonable parametric families of distributions and associated parameter settings have been obtained from the examination and analysis of a large data base extracted from published reports of commercial aircraft accidents. The data base is essentially complete for accidents that occurred between 1950 and 1990 and which involved large commercial jets. The rumway is a contributor to the model only through its role in orienting the coordinate system. Locations are measured in reference to the rumway; only the rumway's length, $q$, and width, 2l, are pertinent. The prototypical numway has length $q=10000$ feet and width $21=150$ feet. The origin in the LLNL development, unlike the Standard's origin which is at the center of the numway, is at the center of the threshold end of the runway for a landing; the point $(-q, 0)$ is at the center of the departure end for a takeoff. Successful takeotts and landings are assumed to go from right to left. [in the Standard the intended direction of travel is the opposite: left to right.] See Figure 2.1.

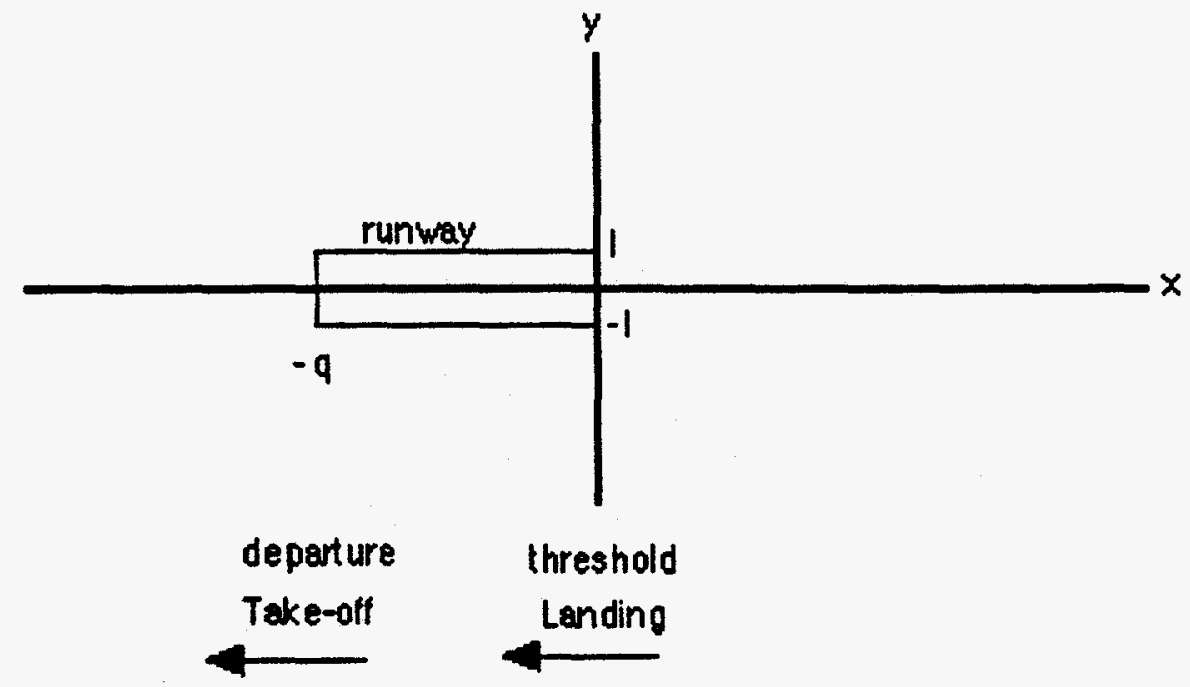

Figure 2.1 Coordinate system and direction of travel of Ref. 2.32.

Most quantities modeled have finite ranges. Beta distributions have been successfully applied in cases of limited ranges, e.g. heading angles. In cases of finite but essentially open-ended variables, e.g. impact locations, velocities, and decelerations, truncated versions of common distributions with infinite range (lognormal and Weibull) have been successfully fitted to the data.

All results should be viewed as preliminary. Additions and revisions of data will undoubtedly lead to improved parameter estimates. More detail than required by the Standard is presented here for the sake of faithfulness to the LLNL approach.

The units used are (feet, seconds, radians) for (distance, time, angle). 


\subsubsection{OFF-RUNWAY IMPACT LOCATION DISTRIBUTIONS.}

Consider the location of an impact caused by a crash on takeoff. The $x$-coordinate measures the position along the extended rumway center line, the intended direction of travel is right to left, and the origin is at the right extreme (threshold end) of the numway. Since the data base shows a dependency between $x$ and $y$, the joint poff $f X, Y(x, y)$ is derived as

$$
f_{X}, Y(X, y)=f_{X}(x) f_{Y \mid X}(y \mid X)
$$

The data base shows no accidents with $x$-coordinate larger than 0 , as this would require the aircraft to impact at a point behind where it commenced its takeoff nu down the runway. In addition, all impacts have $x$-coordinates greater than -90000 (about 15 miles beyond the departure end of the numay), since impacts less than this figure would occur only if the aircraft is beyond the takeoff/climb phase of light. A truncated lognormal distribution has been found to adequately fit the $x$-coordinate of takeoff impacts. Specifically, the potf $f(x)$ is given by

$$
f_{x}(x)=\frac{h(x)}{1-H\left(x_{1}\right)}
$$

where $h(x)=\frac{1}{-x \sqrt{2 \pi} \sigma} \exp \left(\frac{1}{2 \sigma^{2}}[\ln (-x)-\mu]^{2}\right),-\infty<x<0$,

and $H(t)=\int_{-}^{t} h(x) d x$,

with $x \mid=-90000, \mu=9.3663$, and $\sigma=0.4913$. Essentially $\ln (-X)$ is normally distributed with mean 9.3663 and standard deviation 0.4913 , with truncation on the left at $x=-90000$. The mean of this distribution is around -13200 , implying that a typical impact occurs about 3200 feet beyond the departure end of the runway. See Figure 2.2. The distribution of the y-coordinate of impact depends on the value of the $x$ coordinate, since the distance from the extended numway center line tends to increase as the flight progresses and a jetway is followed toward a destination. A Weibull log linear regression that employs maximum likelihood techniques on exact, censored, and interval data has produced a satisfactory fit. The conditional poff $Y \mid X(y \mid x)$ is determined as follows:

- $Y \mid X$ is distributed as $W_{X}+75$ with probability $1 / 2$, and as $-W_{X}-75$ with probability $1 / 2$, if $-10000 \leq x \leq 0$

- $Y \mid X$ is distributed as $W_{X}$ with probability $1 / 2$, and as $-W_{X}$ with probability $1 / 2$, if $-90000 \leq x<-10000$

- $W_{X}$ is Weibull with scale parameter

$\alpha(x)=\exp (4.9576-0.00009955 x)$ and shape parameter $c=0.5381$,

i.e., $W_{\mathbf{X}}$ has potf

$$
g(w)=\frac{c}{\alpha(x)}\left(\frac{w}{\alpha(x)}\right)^{c-1} \exp \left(-\left[\frac{w}{\alpha(x)}\right]^{c}\right)
$$




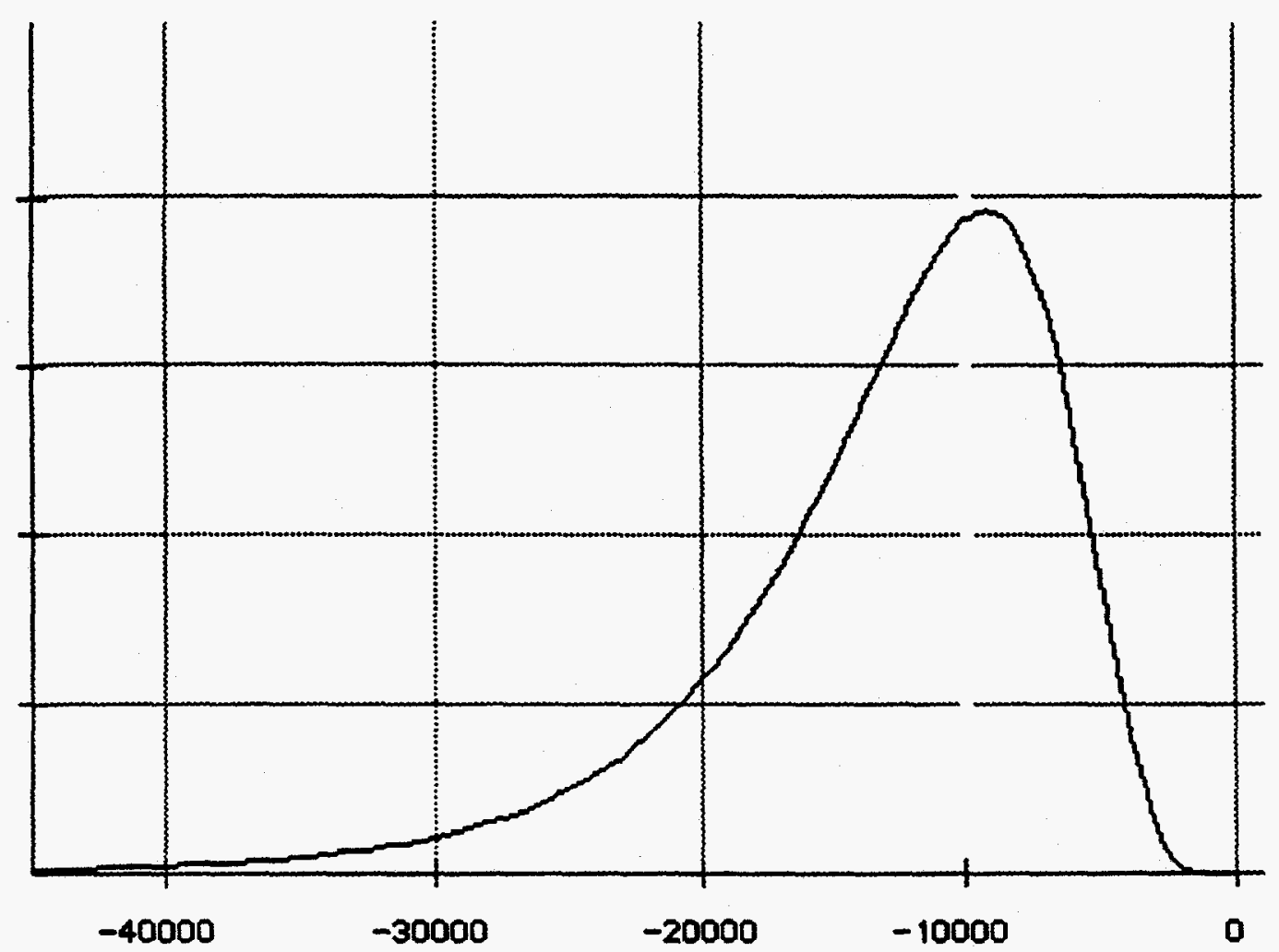

Figure 2.2 The truncated lognormal poff for the $x$-coordinate of off-nunway takeoff crashes.

A feature of the dependency of $Y$ on $x$ is shown in Figure 2.3 in which the median $y$-coordinate is plotted against $x$ for impacts above the rurway. The mirror image is valid for impacts below the rurway.

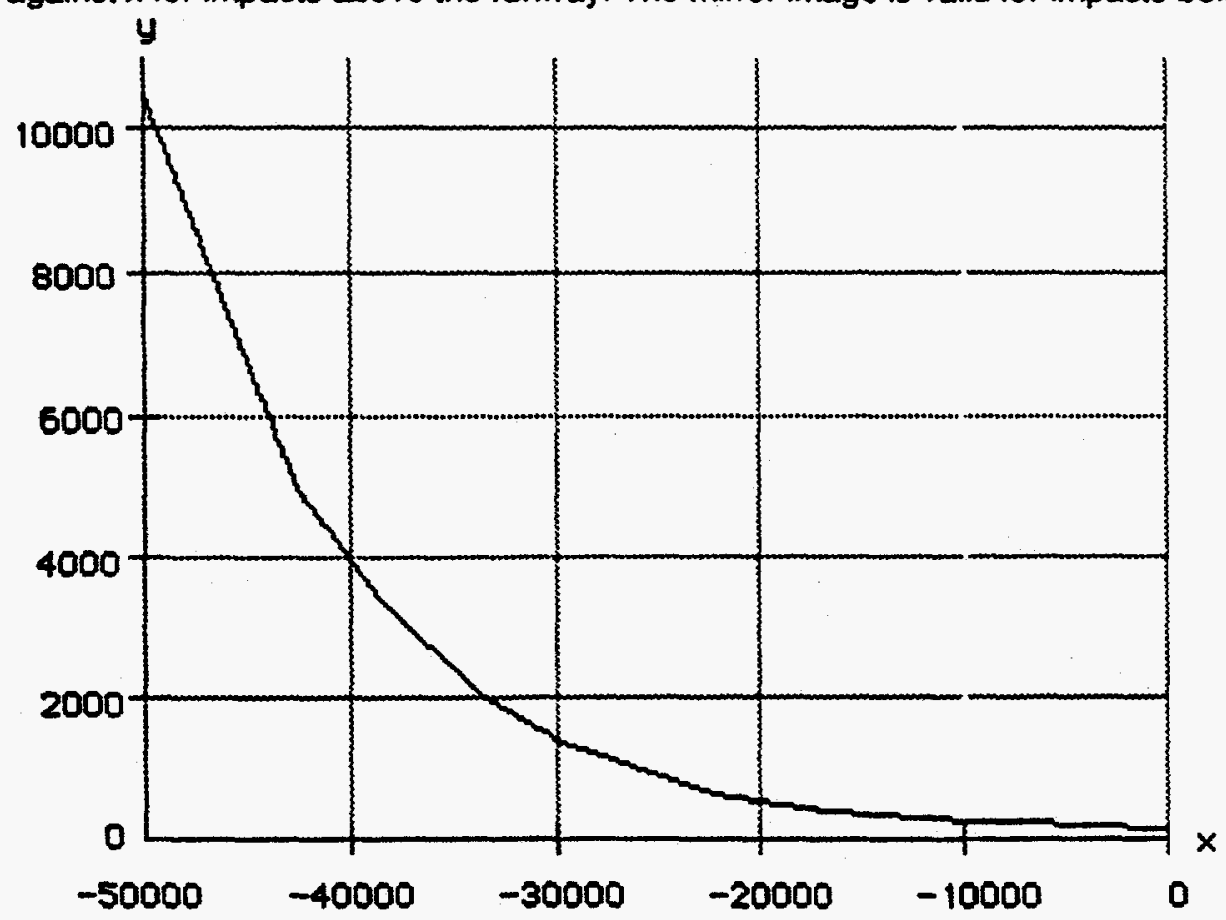

Figure 2.3 The median y-coordinate of impact for off-runway takeoff crashes above the runway. 
A similar approach can be used to model the location of off-rumway landing impacts. Landing phase crashes tend to occur short of the numway. Since no data base crashes occurred beyond the rumway departure end, and crashes with $x$-coordinate greater than 105000 (about 20 miles before the numway threshold) are not viewed as landinglanding approach phase accidents, an $x$-coordinate range from -10000 to 105000 seems appropriate. A truncated lognormal distribution provides an adequate fit for the $x$-coordinate of landing impacts. Specifically, the poff $f(x)$ is given by

$$
f_{x}(x)=\frac{k(x)}{K\left(x_{u}\right)} \text {, }
$$

where $k(x)=\frac{1}{\left(x-x_{0}\right) \sqrt{2 \pi} \sigma} \exp \left(-\frac{1}{2 \sigma^{2}}\left\{\ln \left[x-x_{0}\right]-\mu\right)^{2}\right), x>x_{0}$,

and $K(t)=\int_{x_{0}}^{t} k(x) d x$,

with $x_{u}=105000, x_{0}=-10000, \mu=9.6607$, and $\sigma=0.4947$. Thus $\ln (x+10000)$ is normally distributed with mean 9.6607 and standard deviation 0.4947 , with truncation on the right at $x=105000$. The mean of this distribution is around 7600 , implying that a typical impact occurs about 7600 feet short of the threshold end of the numway. See Figure 2.4. The distribution of the y-coordinate of impact depends on the $x$-coordinate, since typical landing pattems involve turning toward the extended runway center line. The Weibull log linear regression methodology employed for the takeoff data has been used to fit the landing data with comparable success. Landing phase impacts tend to fall closer to the extended rumway center line as the flight progresses and the approach to the rumway is executed.

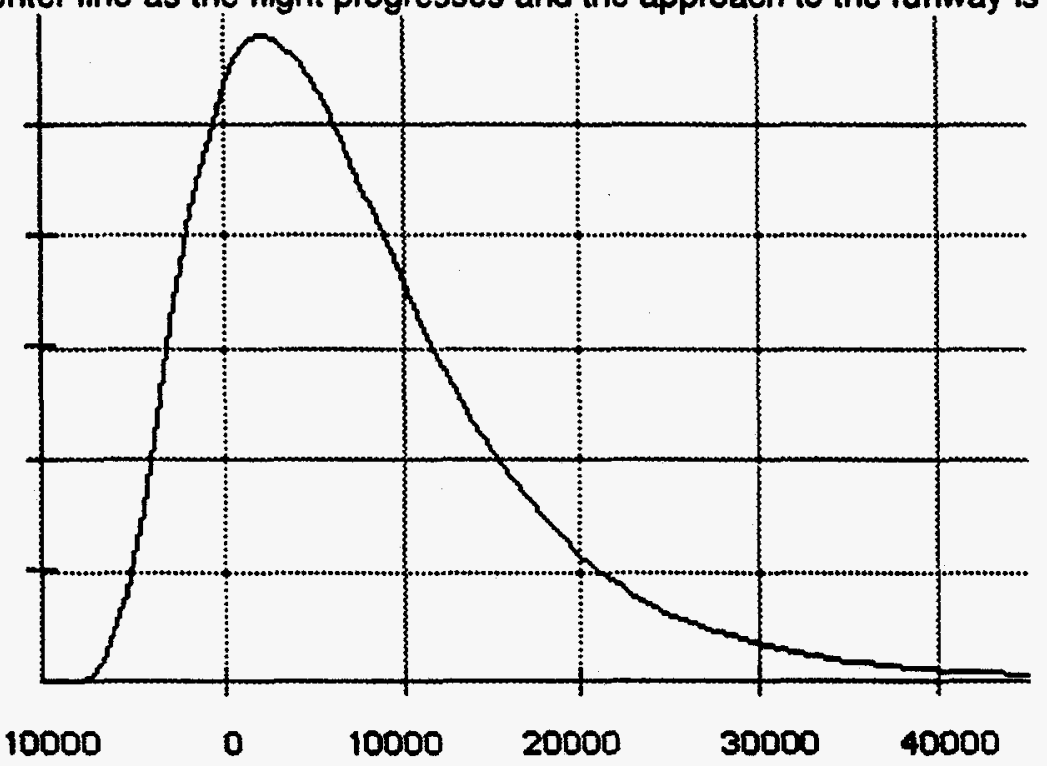

Figure 2.4 The truncated lognomal poff for the $x$-coordinate of off-rumway landing crashes.

The conditional pdt $f \mid x(y \mid x)$ is determined as follows:

- $Y \mid x$ is distributed as $W_{X}$ with probability $1 / 2$, and as $-W_{X}$ with probability $1 / 2$, if $0 \leq x \leq 105000$

- $Y \mid x$ is distributed as $W_{X}+75$ with probability $1 / 2$, and as

$-W_{X}-75$ with probability $1 / 2$, if $-10000 \leq x \leq 0$

- $W_{x}$ is Weibull with scale parameter

$\alpha(x)=\exp (5.8542+0.000056063 x)$ and shape parameter $c=0.82406$,

i.e., $W_{X}$ has poff (2.5). 
In Figure 2.5 the median $y$-coordinate is plotted against $x$ for impacts above the runway. The mirror image is valid for impacts below the rumway.

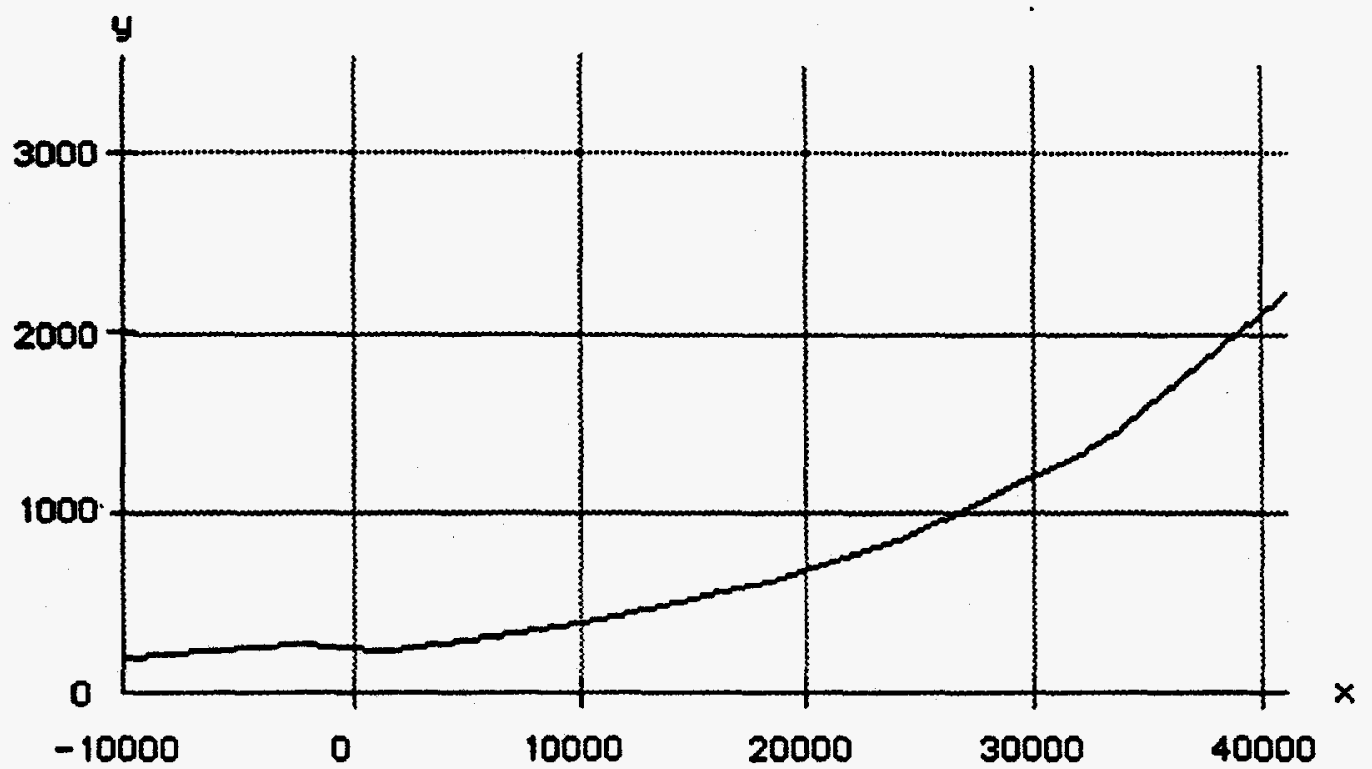

Figure 2.5 The median y-coordinate of impact for off-rumway landing crashes above the rumway.

Crash frequency screening requires a grid of impact location probabilities for takeoff and landing crashes. Such grids are provided in Table 2.22 (Takeofts) and Table 2.23 (Landings). Abbreviated versions are given in the ACRAM Standard [Ref. 2.5]. It is important to note that the ACRAM Standard screening uses a different origin, namely the center of the rumway, rather than the threshold end of the runway used in the ULNL report. Moreover, the direction of flight is left to right in the Standard, as opposed to right to left in the LLNL report. The entry in Table 2.22, $(x \in[2,3], y \in[1,2])$, is seen to be 3.818E-03. This number is the conditional probability, given a takeoff crash occurs, that the impact will take place within the one square mile area characterized by $\{(x, y): 2 \leq x \leq 3,1 \leq y \leq 2\}$, where $(x, y)$ denotes the impact coordinates based on the origin situated at the runway center and the intended direction of travel being left to right. [For a runway of length 2 miles, the corresponding region in the coordinate system having origin at the runway threshold and flight from right to left would be $\{(x, y):-4 \leq x \leq-3,1 \leq y \leq 2\}$.] The entries in Tables 2.22 and 2.23 were computed by integrating the appropriate joint densities (2.1). 


\subsubsection{OFF-RUNWAY HEADING DISTRIBUTIONS.}

The aircraft's heading after an off-rurway impact is not explicitly given in accident reports. The resting location of the wreckage is however reported, and the heading is estimated by calculating the angle, relative to the extended numay center line, of the line from the impact point to the center of the main wreckage segment. The heading distribution is not used in the ACRAM Standard [Ref. 2.5].

The heading of the aircraft after a takeoff crash depends on the impact location. Because typical takeoff tlight patterns tend to require a turn away from the extended rumway center line, the heading is usually greater than the arc tangent of the position of the aircraft. See Figure 2.6.

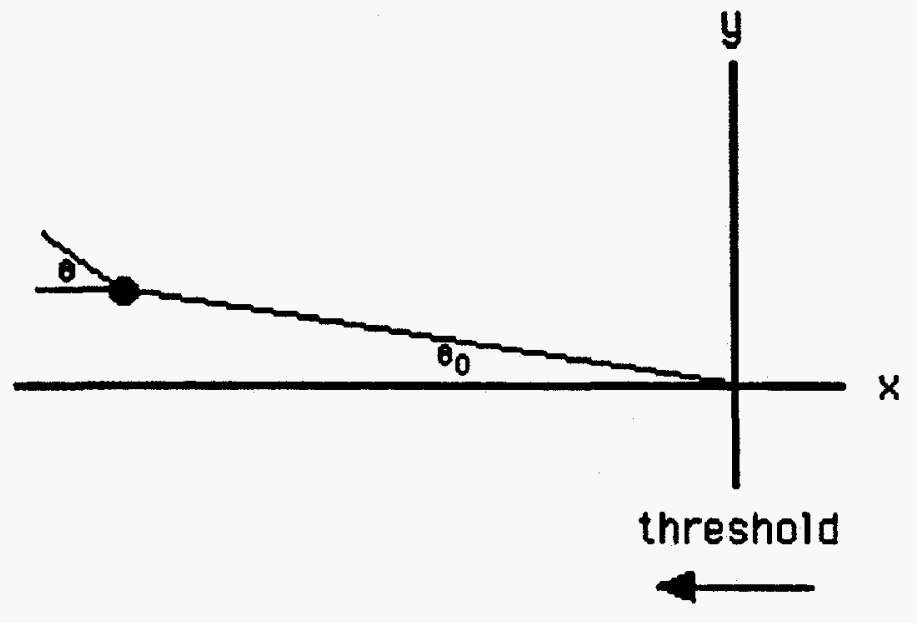

Figure 2.6 The heading $\theta$ of the aircratt on takeoff is typically greater than the arc tangent $\theta_{0}=\tan ^{-1}(|y| /|x|)$ of its position.

A regression on headings in off-runway takeoff impacts has found the mean heading $\theta_{0}^{*}$ to satisfy the log linear relation

$$
\dot{\theta_{0}}=0.97126+0.16906 \ln \left(\theta_{0}\right)
$$

where $\theta_{0}$ marks the nominal heading on impact, i.e.,

$$
\theta_{0}=\tan ^{-1}(|y| /|x|) \text {, }
$$

and the heading itself to be fit adequately as

$$
\Theta=\theta_{0}^{*}+2 \pi(B-1 / 2)
$$

where $B$ denotes a symmetric beta distributed random variable with parameter 132 . That is, $B$ has pdf

$$
g_{B}(x ; a, b)=\frac{\Gamma(a+b)}{\Gamma(a) \Gamma(b)} x^{a-1}(1-x)^{b-1}, 0<x<1
$$

with $a=b=132$. Therefore the conditional poff $f \Theta \mid X, Y$ used in calculations of crash frequency is given by

$$
G_{\theta \mid X, Y}(\theta \mid x, y)=\frac{1}{2 \pi} g_{B}\left(\frac{\theta-\theta_{0}^{*}+\pi}{2 \pi} ; 132,132\right), \quad \theta_{0}^{*}-\pi<\theta<\theta_{0}^{*}+\pi \text {. }
$$

The mean heading $\theta_{0}^{*}$ as a function of nominal heading $\theta_{0}$ is shown in Figure 2.7.

The headings in off-rumway landing impacts do not appear from the data base to depend on the location. $A$ beta distribution with a modest tendency for headings away from the extended runway center line 


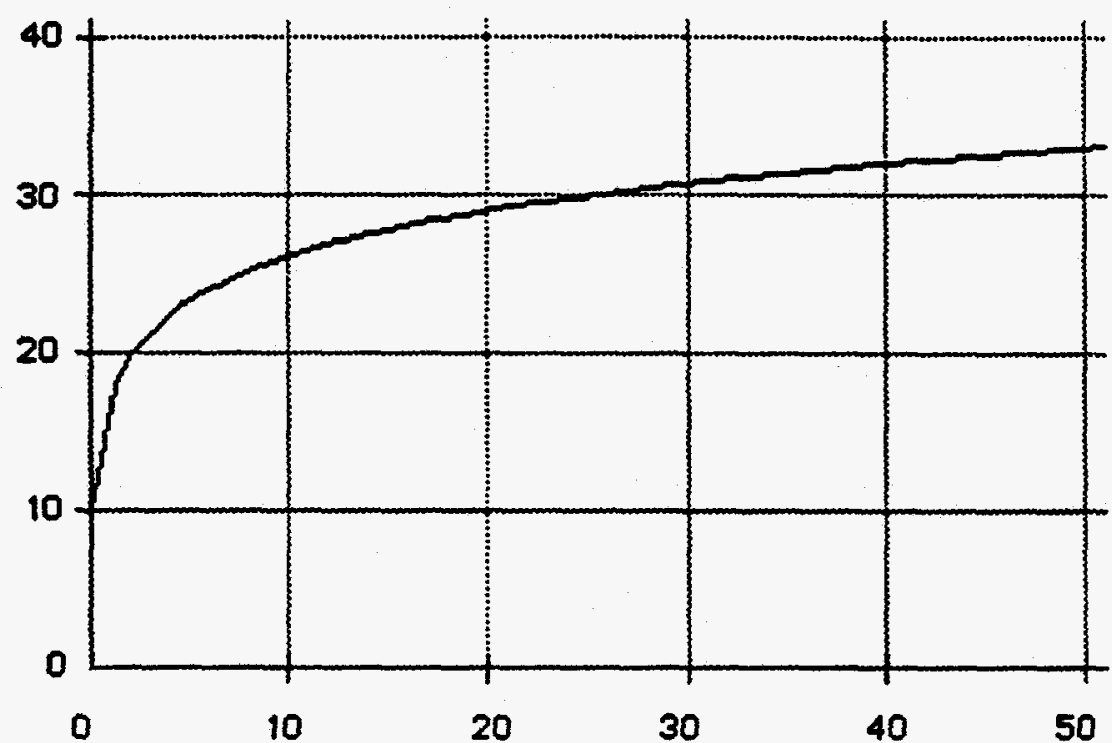

Figure 2.7 The mean heading, in degrees, at impact for off-numay takeoff crashes as a function of the nominal heading, in degrees.

provides a satisfactory fit. Specifically, $\theta$ is distributed as

$$
\Theta=2 \pi\left(B-\frac{1}{2}\right)
$$

where $B$ has pdf (2.12) with $a=61.18$ and $b=56.76$, so that the conditional pdf $f \Theta \mid X, Y$ used in calculations is given by

$$
t_{\theta \mid X, Y}(\theta \mid x, y)=\frac{1}{2 \pi} g_{B}\left(\frac{\theta+\pi}{2 \pi} ; 61.18,56.76\right), \quad-\pi<\theta<\pi .
$$

This density is plotted in Figure 2.8. The mean value is 6.75 degrees.

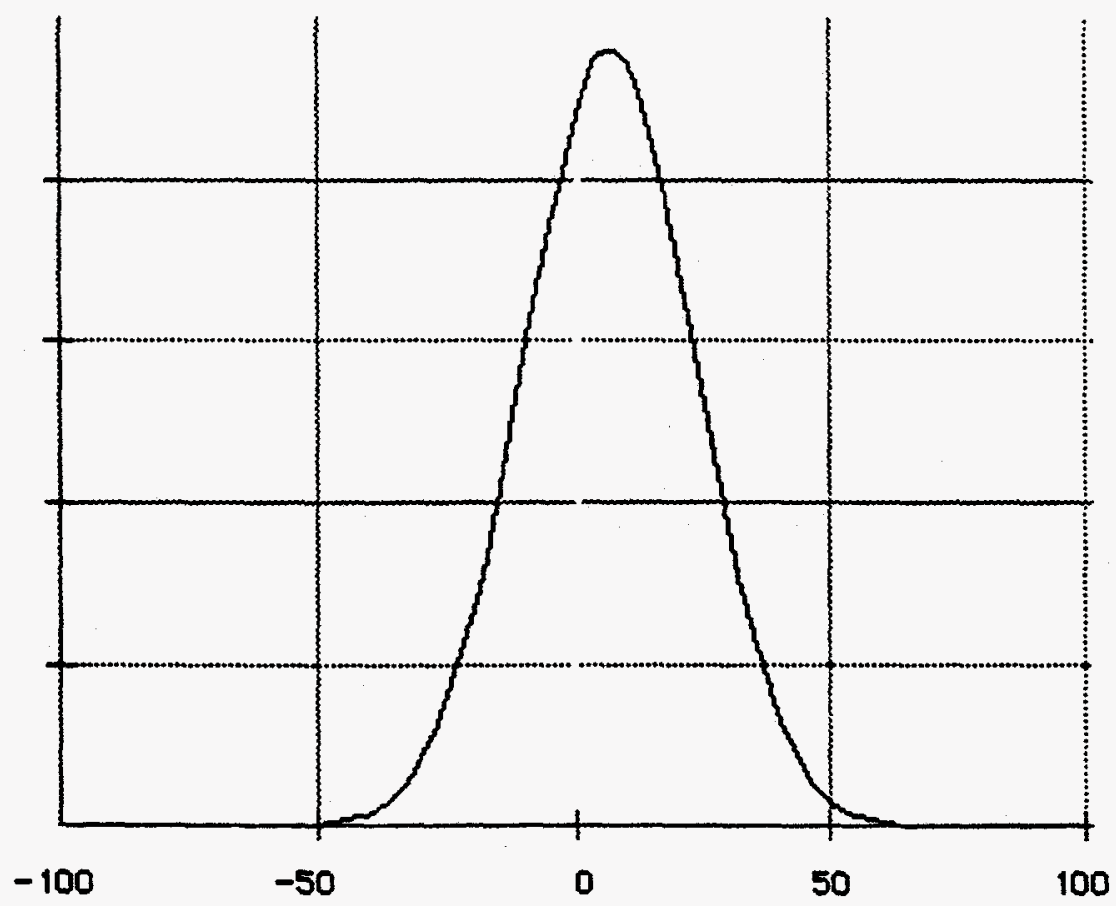

Figure 2.8 The beta potf of heading, in degrees, for off-runway landing crashes. 


\subsubsection{OFF-RUNWAY IMPACT VELOCITY, IMPACT ANGLE, AND DECELERATION DISTRIBUTIONS.}

The ACRAM Standard [Ref. 2.5] requires the mean cotangent of the impact angle and the mean skid distance in its impact frequency calculation. The cumulative distribution function of impact velocity is used in the structural response calculations. The LLNL development models deceleration after impact, rather than skid distance, because of the apparent independence of deceleration, a ground related quantity, and impact velocity/angle, pilot related quantities.

Although impact velocities and angles are provided in accident reports, decelerations after impact are not. Nonetheless, since the resting point of the main wreckage is reported, the deceleration rate may be calculated from Newtonian principles. The deceleration, assuming a constant rate along the straight line segment of length s from the impact point to the resting point, is given by

$$
a=\frac{v_{k}^{2} \cos ^{2} \phi}{2 s}
$$

Impact velocities and angles as well as decelerations do not appear to depend on whether the accidents are takeoff or landing related. The impact velocities are adequately modeled by a truncated Weibuil distribution. Specifically, the off-nunway impact velocity poff $h_{k}$ is given by

$\left.f_{v_{k}}(v)=\frac{c}{\alpha}\left[\frac{\left(v-\underline{v}_{k}\right)}{\alpha}\right]^{c-1} \exp \left(-\left[\frac{\left(v-\underline{v}_{k}\right)}{\alpha}\right]^{c}\right) / 1-\exp \left(-\left[\frac{\bar{v}_{k}-\underline{v}_{k}}{\alpha}\right]^{c}\right)\right], \underline{v}_{k}<v<\bar{v}_{k}$,

where $\alpha=161.7, c=1.951$, and $\left(\underline{v}_{k}, \bar{v}_{k}\right)=(100 ., 500$.). The mean of this distribution is about 243 . See Figure 2.9. The cumulative distribution function associated with this potf is used in the Standard's structural response calculations.

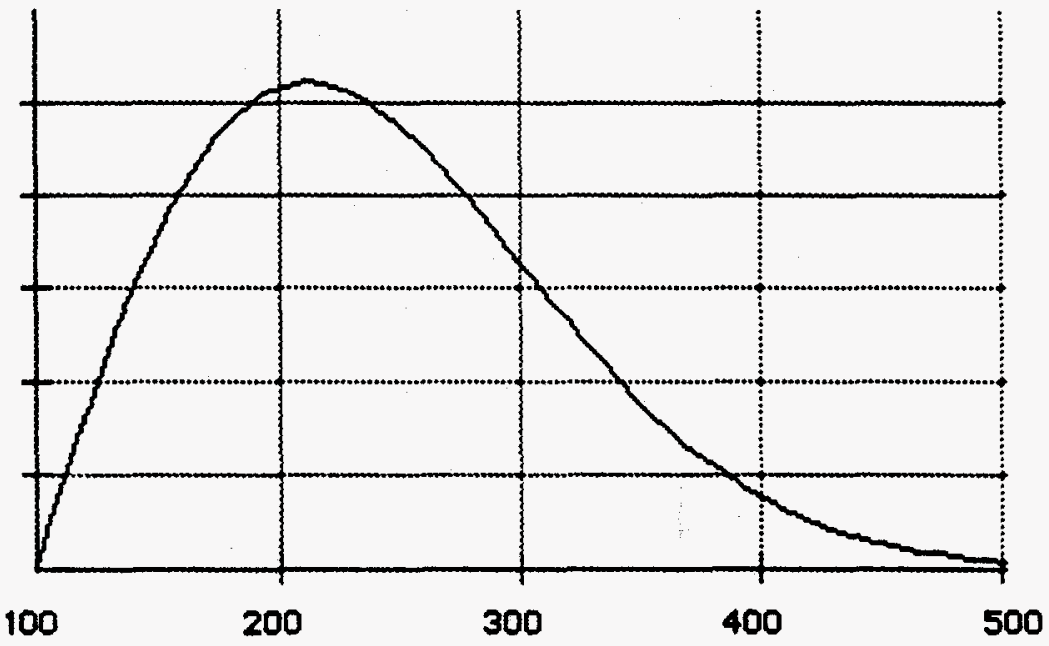

Figure 2.9 The truncated Weibull pdf of impact velocity for off-rumway takeoft or landing crashes.

Impact angles depend on impact velocities as larger angles are associated with both smaller and larger velocities. Weibull log linear regression provides a satisfactory fit to allow evaluation of the conditional CDF $F_{\Phi} \mid V_{k}$ required in calculations. In fact, $\Phi \mid V_{k}$ is distributed as a truncated Weibull with petf

$$
\left.\left.g(\phi \mid v)=\frac{c}{\alpha(v)}\left(\frac{\phi}{\alpha(v)}\right)^{c-1} \exp \left(-\frac{\phi}{\alpha(v)}\right]^{c}\right)\left[\exp \left(-\left[\frac{\phi}{\alpha(v)}\right]^{c}\right)-\exp \left(-\frac{\bar{\phi}}{\alpha(v)}\right]^{c}\right)\right], \phi<\phi<\bar{\phi},
$$

with $c=1.08931, \alpha(v)=\exp \left(1.8580-0.022191 v+0.000036529 v^{2}\right)$, and $(\phi, \bar{\phi})=\left(\frac{\pi}{360}, \frac{\pi}{2}\right)$, i.e., the range is from $1 / 2$ to 90 degrees. The median impact angle as a function of impact velocity is shown in Figure 2.10. The mean cotangent of impact angle is used by the Standard in effective area calculations. 
This quantity, obtainable in principle from the marginal distribution of impact angle, which is a function of (2.17) and (2.18), was computed in fact by Monte Carlo simulation based on two million realizations of $\left(V_{k}, \Phi \mid V_{k}\right)$. See Table 2.24.

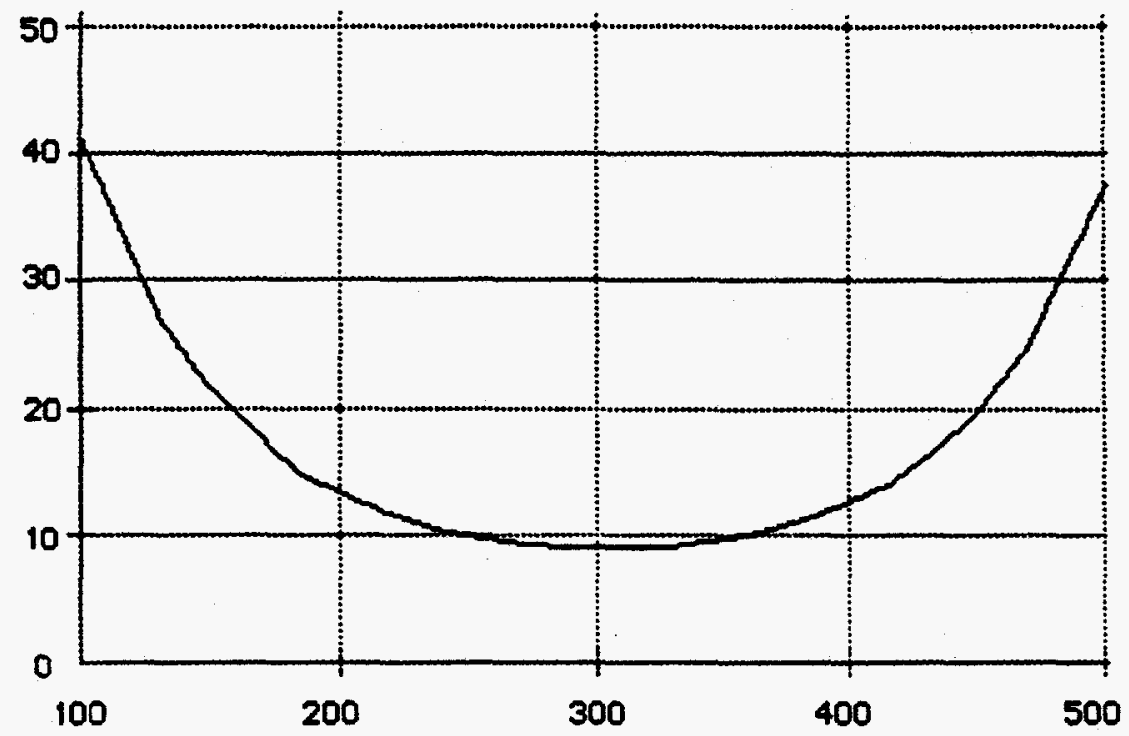

Figure 2.10 The median impact angle in degrees as a function of impact velocity for off-runway takeoff or landing crashes.

The calculated decelerations are adequately modeled by a truncated lognomal distribution. Specifically, the off-rumway deceleration poff $f_{A}$ is given by

$t_{A}(a)=\frac{\frac{1}{a \sqrt{2 \pi} \sigma} \exp \left[-\frac{1}{2 \sigma^{2}}(\ln a-\mu)^{2}\right]}{\Phi([\ln \bar{a}-\mu] / \sigma)-\Phi([\ln \underline{a}-\mu] / \sigma)}, \quad \underline{a} \leq a \leq \bar{a}$,

where $\mu=3.0390, \sigma=0.6770,(\underline{a}, \bar{a})=(1 ., 150$.$) , and \Phi$ is the standard nomal CDF,

$$
\Phi(z)=\int_{-}^{z} \frac{1}{\sqrt{2 \pi}} \exp \left(-\frac{1}{2} t^{2}\right) d t
$$

The mean deceleration is approximately 26. See Figure 2.11 .

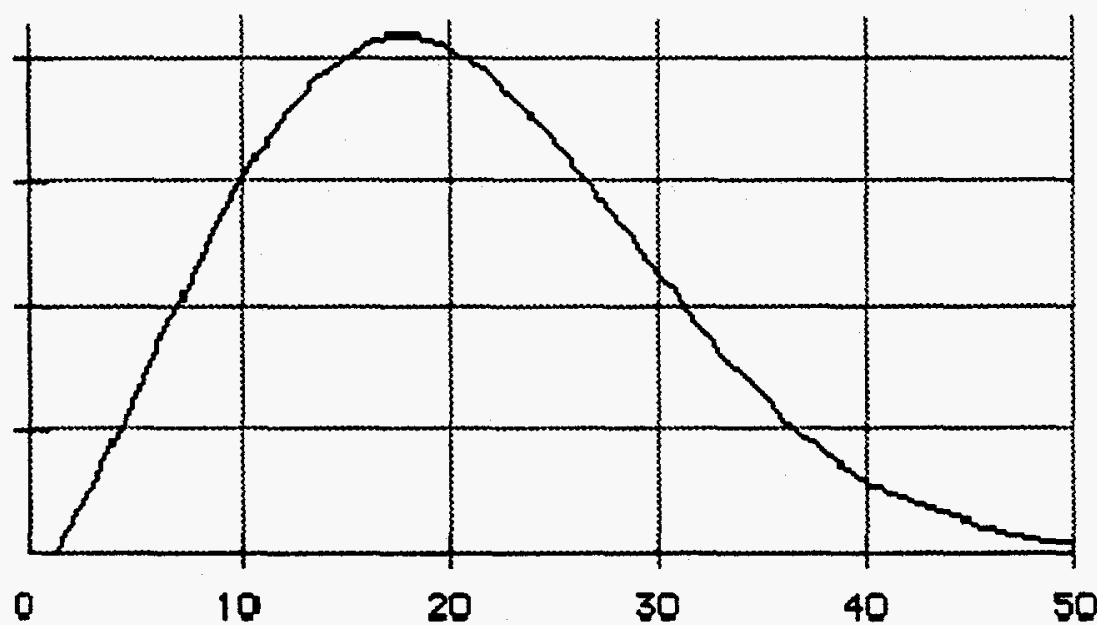

Figure 2.11 The inuncated Weibull pot of deceleration for off-runway takeoff or landing crashes. 
The LLNL commercial model uses the deceleration distribution in conjunction with the ground impact velocity and angle distributions to characterize the sliding, or skidding, into a structure at a given velocity. Alternative models, such as the Standard's, which do not stochastically address the aircraft's velocity at the moment of skidding impact with the structure, make use of the skid distance distribution. The distance $S$ that the aircraft slides, or skids, until it comes to rest is given by the Newtonian relation

$$
S=\frac{V_{k}^{2} \cos ^{2} \Phi}{2 A}
$$

The pof of $S$ can be derived in principle from the distributions obtained eartier for $V_{k}, \Phi / V_{k}$, and $A$. It is easier, however, to determine the distribution by Monte Carlo computer simulation. In Table 2.25 selected percentiles of the skid distance distribution are presented, based on Monte Carbo simulation with two million realizations of $\left(V_{k}, \Phi \mid V_{k}, A\right)$. To construct effective areas in crash frequency screening the Standard employs the mean skid distance. 


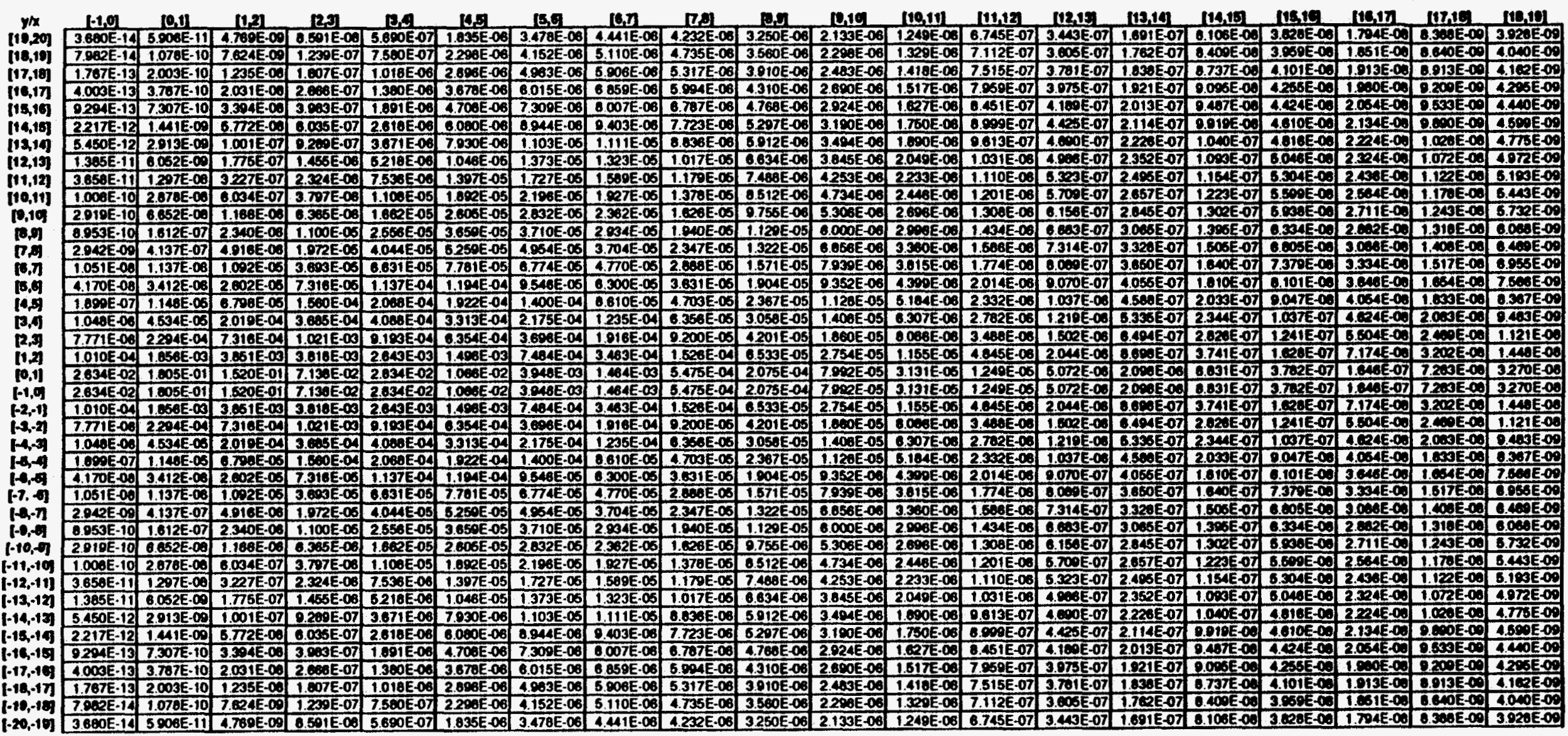

imended drection of traved in ien to right

origitn is conter of rumway

unils are miles

Table 2.22

Conditional Probabilities of Impact Location, Given a Crash on Takeoft 


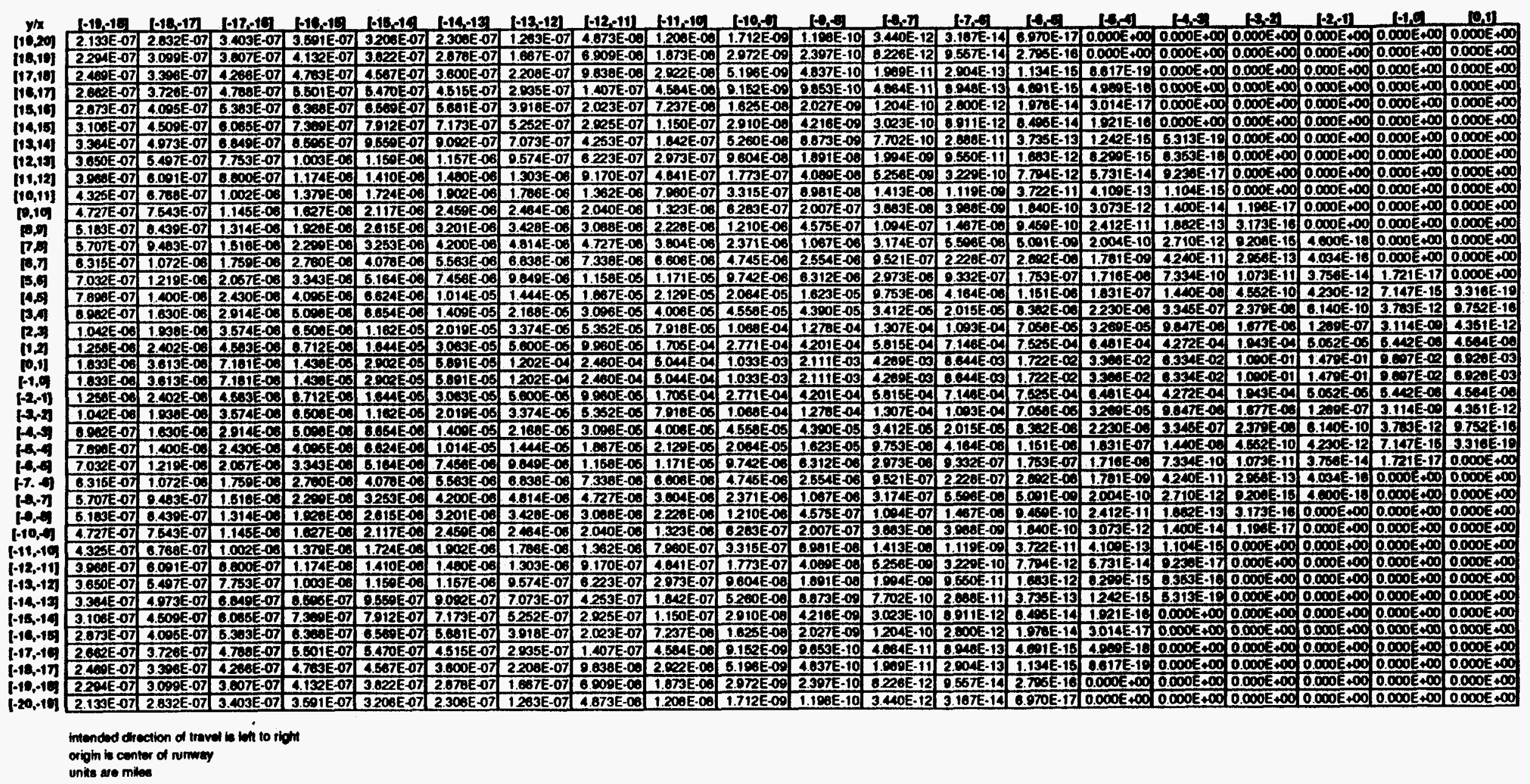

Table 2.23

Conditional Probabilitios of Impact Location, Givan a Crash on Landing 


\begin{tabular}{|c|c|}
\hline percentile & angle \\
\hline 5 & 1.5 \\
\hline 10 & 2.5 \\
\hline 15 & 3.6 \\
\hline 20 & 4.6 \\
\hline 25 & 5.8 \\
\hline 30 & 7.0 \\
\hline 35 & 8.2 \\
\hline 40 & 9.6 \\
\hline 45 & 11.0 \\
\hline 50 & 12.6 \\
\hline 55 & 14.4 \\
\hline 60 & 16.4 \\
\hline 65 & 18.6 \\
\hline 70 & 21.2 \\
\hline 75 & 24.3 \\
\hline 80 & 28.1 \\
\hline 85 & 33.0 \\
\hline 90 & 40.0 \\
\hline 95 & 52.0 \\
\hline
\end{tabular}

mean: $\quad 17.64$

mean cotangent: $\quad \mathbf{9 . 5 5 4}$

\begin{tabular}{|c|c|}
\hline angle & CDF \\
\hline 5 & 0.2160 \\
\hline 10 & 0.4150 \\
\hline 15 & 0.5660 \\
\hline 20 & 0.6780 \\
\hline 25 & 0.7610 \\
\hline 30 & 0.8220 \\
\hline 35 & 0.8670 \\
\hline 40 & 0.9000 \\
\hline 45 & 0.9245 \\
\hline 50 & 0.9436 \\
\hline 55 & 0.9580 \\
\hline 60 & 0.9690 \\
\hline 65 & 0.9778 \\
\hline 70 & 0.9846 \\
\hline 75 & 0.9899 \\
\hline 80 & 0.9941 \\
\hline 85 & 0.9974 \\
\hline 90 & 1.0000 \\
\hline
\end{tabular}

Table 2.24

Impact Angle Distribution Properties 


\begin{tabular}{|c|c|}
\hline percentlle & $\begin{array}{c}\text { skid } \\
\text { distance }\end{array}$ \\
\hline 5 & 160 \\
\hline 10 & 260 \\
\hline 15 & 350 \\
\hline 20 & 440 \\
\hline 25 & 530 \\
\hline 30 & 620 \\
\hline 35 & 710 \\
\hline 40 & 820 \\
\hline 45 & 930 \\
\hline 50 & 1050 \\
\hline 55 & 1190 \\
\hline 60 & 1340 \\
\hline 65 & 1520 \\
\hline 70 & 1730 \\
\hline 75 & 1990 \\
\hline 80 & 2320 \\
\hline 85 & 2760 \\
\hline 90 & 3430 \\
\hline 95 & 4700 \\
\hline
\end{tabular}

Table 2.25

Selected Skid Distance Percentiles (ft) 


\subsection{COMMERCIAL AVIATION AIRCRAFT CHARACTERISTICS}

This section was prepared to provide to the analyst those characteristics of aircraft that could be important to determine the effect of the aircraft on the facility. Such aircraft characteristics which could be crucial in determine whether a facility could withstand an impact from an aircraft could be the weight of the aircraft, the amount of fuel aboard the aircraft, the speed of the aircraft, the dimensions of the aircraft, and potential missiles generated by the aircraft as its structure failed. Many of these parameters would vary greatly during the course of the flight, but the values of the characteristics given in this section could be considered the upper bound of these parameters. To determine the characteristics of an aircraft at the moment of impact in a particular accident, the analyst should refer to the specific accident report.

Tables 2.26 and 2.27 taken from References 2.33 and 2.34 presents the following information on commercial aviation aircraft characteristics:

1. Aircraft type,

2. Powerplant(s), number and type,

3. Nominal power rating of each powerplant,

4. Operating empty weight expressed in pounds, and kilograms,

5. Maximum allowed (or certificated) takeoff weight expressed in pounds, and kilograms,

6. Maximum allowed (or certificated) landing weight expressed in pounds, and kilograms,

7. Maximum zero-fuel weight expressed in pounds, and kilograms,

8. Maximum fuel-capacity expressed in imperial gallons, and liters,

9. Overall wing span expressed in feet and inches, and in meters,

10. Overall length expressed in feet and inches, and in meters,

11. Overall height expressed in feet and inches, and in meters,

12. Wing area expressed in $\mathrm{tt}^{2}$ and meters ${ }^{2}$,

13. Takeoff numway length according to Federal Air Regulations (FAR) requirements, expressed in feet,

14. Landing numay length according to Federal Air Regulations (FAR) requirements, expressed in feet,

15. Stall speed with gear flaps deployed, expressed in miles/hour.

Powerplant(s) were included as potential missile generators. Weights were given for mass during various points of the flight. Fuel capacity was included in order to provide the maximum fuel available for a fire following the crash. Aircraft dimensions will be needed when the facility effective area is determined. The takeoff nunway length and landing numway length were provided so that an analyst could screen certain aircraft trom certain airports due to their inability to operate from the aimport's runway(s). Stall speed was provided so that an estimate of the impact speed could be made as a large percentage of commercial aircraft crashes occur during the landing approach flight phase, and most commercial aircraft fly the approach phase at about 1.3 times the stall speed.

Table 2.26 presents the characteristics of Air Carrier aircraft that generally conduct flights under 14 CFR 121 regulations. The aircraft types are arranged in descending order of maximum takeoff weight with jetpowered aircraft listed first, followed by turboprop aircraft and pistonprop aircraft.

Table 2.27 presents the characteristics of Scheduled Air Taxi aircraft that generally conduct flights under 14 CFR 135 regulations. Again, the aircraft types are arranged in descending order of maximum takeotf weight. It should be noted that many general aviation aircratt types are also used for Air Taxi flights, particularly nonscheduled flights, but also for scheduled flights. These types have not been included due to lack of time but could be added in the future.

Finally, additional research is needed to characterize the missiles (projectiles) generated during the course of an aircraft's crash. Obvious examples of such missiles are the aircraft's engines, the auxiliary power units (APUs), and the landing gear. Much of the information on these aircraft components is not readily available and may have to be obtained directly from the aircraft or engine manufacturer or from the company that produces the component. 
Table 2.28

Chareandoties of Ar Cerriar (14 CFA 121) Aremat [Aloference 233 and 2.34]

\begin{tabular}{|c|c|c|c|c|c|c|c|c|c|c|c|}
\hline \multirow[b]{2}{*}{ Areren Typo } & \multicolumn{2}{|c|}{ Pomeplent(3) } & \multirow{2}{*}{ 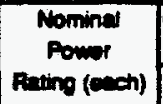 } & \multicolumn{2}{|c|}{$\begin{array}{l}\text { Operating } \\
\text { Enply Weinht }\end{array}$} & \multicolumn{2}{|c|}{$\begin{array}{l}\text { Maximum } \\
\text { rakeorf Woioh! }\end{array}$} & \multicolumn{2}{|c|}{$\begin{array}{l}\text { Maxinum } \\
\text { Lendin Weint }\end{array}$} & \multicolumn{2}{|c|}{$\begin{array}{l}\text { Maximum } \\
\text { Zero-fuel weipht }\end{array}$} \\
\hline & No. & Type & & Pound & Klogrem & Pound & Klooram & Pound 1 & Klooram & Pounds & Kiogram \\
\hline \multicolumn{12}{|l|}{ AR CARRIER (14 CFA 121) JETS } \\
\hline Bosin $747-400$ & 4 & RR. RB.211.524G METF & $89,000 \mathrm{be}$ & 401800 & 182255 & 870,000 & $\operatorname{sen}, 625$ & N.A. & N.A. & N.A. & N.A. \\
\hline Bowin $747-400$ & 4 & GE. CF6-EOC234 HETF & 57,000 be. 82 & 300,100 & 181,030 & 870,000 & 304.825 & N.A. & N.A. & N.A. & N.A. \\
\hline Bowin $747-400$ & 4 & PEW. PW4OSB HETF & $50,750 \mathrm{los}$. & 300,00 & 180,985 & 870,000 & 304,025 & N.A. & N.A. & N.A. & N.A. \\
\hline Bosing 747-300 & 4 & GE. CF6.50C2 HBTF & 50,000 at at & 384,480 & 174,400 & 033,000 & 37.840 & 000,000 & 295,765 & 585,000 & 258,280 \\
\hline Bowin $747-300$ & 4 & PEW JTOD-7A4G2 HBTF & 54,750 be. of & 394,480 & 174,400 & 830,000 & 37.84 & 200,000 & 285,785 & $565, \infty$ & 256,280 \\
\hline Boeving 747-300 & 4 & RR. RB.211-52404 HETF & 59,110 best. & 394,460 & 174,400 & 833,000 & $3 \pi, 800$ & 030.000 & 285,785 & 565,000 & 256,280 \\
\hline Booin 747-300 & 4 & GE. CF6-50E2 HBTF & $52,500 \mathrm{los} . \mathrm{ut}$ & 384,480 & 174,400 & 830,000 & 37,040 & 000,000 & 205,765 & 565,000 & 256,280 \\
\hline Bowing $747-200$ & 4 & PAW. JPD-7RAG2 HBTF & SA,750 be at & 375,170 & 170,180 & 003,000 & 37,840 & 600,000 & 205,765 & 526,500 & 233,815 \\
\hline Bowing 747-200 & 4 & RA. AB.211.524D4 HBTF & 59,110 be. at. & 375,170 & 170.180 & 833,000 & $3 \pi 840$ & 850,000 & 205,705 & 528,500 & 238,815 \\
\hline Bowin 747-200 & 4 & GE. CFQ-50E2 HBTF & 52,500 bs. 81. & 375.170 & 170,180 & 830,000 & 37840 & 850,000 & 205,785 & 526,500 & 238,815 \\
\hline MeDomell Dougles MD-11 & 3 & GE. CF $6-60 \mathrm{C2}$ & 0,200 b. 01 & 27,000 & 125,846 & $\infty$ & 273,300 & 430,000 & 195,047 & 400,000 & 181,439 \\
\hline MicDomnell Doungles MD-11 & 3 & PQW. PW4358 & 50,000 Bas. Al & 27,000 & 125.646 & 602,500 & 273,300 & 430,000 & 185,047 & 400,000 & 181,439 \\
\hline MeDonnell Dougtas DC-10-30 & 3 & GE. CFB-SOC2B HBTF & 5,000 thes. 11 & 287,197 & 121,198 & 500,000 & 260,085 & 403,000 & $182,766 \mid$ & 368,000 & 166,022 \\
\hline MeDonnell Douglas DC-10-30 & 3 & GE. CFB-S & 52,500 thes. 81. & 287,197 & 121,198 & 580,000 & 200,085 & 403,000 & 182,766 & 368,000 & 166,222 \\
\hline Noles DC-10-30 & 3 & GE. CF6-50 & $49,000 \mathrm{bs} .81$. & 287,197 & 121,180 & 500,000 & 263,085 & 403,000 & 182,768 & 368,000 & 166,922 \\
\hline Atous A.34 & 4 & CFMI. CF & 30,000 tbs. st. & 245,000 & 111,130 & 542,300 & 246,000 & N.A. & N.A. & N.A. & N.A. \\
\hline Artus 1.340-200 & 4 & ICFMI. CFMS & 30,000 tos. 81. & 245,000 & 111,130 & $\$ 42,300$ & 246,000 & N.A. & N.A. & N.A. & N.A. \\
\hline Loctroed L.1011-500 & 3 & AR. ABR2 & 50,00 & 245,000 & 111,312 & 510.000 & 231,330 & 368,000 & 168,820 & 338,000 & 153,315 \\
\hline Airtous A.330 & 2 & GE. CF6-D & 64,000 tos. st. & 245,000 & 111,130 & 450,000 & 204,000 & N.A & N.A. & N.A. & N.A. \\
\hline Ainteus A.330 & 2. & PEW. PW & $\infty, 0$ & 245,000 & 111,130 & 450,000 & 201,000 & N.A. & N.A. & N.A. & N.A. \\
\hline Aerospatiale BAC Concorde & 4 & RA OYmpe & Dos. st. & 173,500 & 78,700 & 400,000 & 185,085 & 245,000 & 111,130 & 2003,000 & 92,030 \\
\hline Arbus A.3. & 2 & IGE.CFG-8 & 1toes. 81. & 187,787 & 69,715 & 375,880 & 170,500 & NA. & N.A. & 296,600 & 130,000 \\
\hline Dougles DC & 4 & CFM. CFM & s. 1. & 186,500 & 75,500 & 355,000 & 161,025 & 258,000 & 117,000 & $231, \infty$ & 104,780 \\
\hline Bowing 767-300 & 2 & IPAW. JT9L & 50,000 tos st. & 188,000 & 85,638 & 352,200 & 159.755 & 300,000 & 136,078 & 278.000 & 126,008 \\
\hline Booin 767.300 & 2 & GE. CFE- & $50,000100.8 \mathrm{st}$ & 188,800 & 85,638 & 352,200 & 159,755 & 300,000 & 136,078 & 278,000 & 126,008 \\
\hline Airbes 1.310 & 2 & PaW. JT & 16.81. & 169,842 & 7,040 & 337,305 & 153,000 & 271,170 & 123,000 & 248.120 & 113,000 \\
\hline Booing $707.320 \mathrm{C}$ & 4. & P\&W. 5 & Hes. $8 \mathrm{st}$ & 146,400 & 66,408 & 333,600 & 151,315 & 247,000 & 112,037 & 230,000 & 104,330 \\
\hline Boving 767.200 & 2 & P\&W. & 8. 01.1 & 178,200 & 70,223 & 315,000 & 142,891 & 272,000 & 123,377 & 250,000 & 113,398 \\
\hline Booing 767.200 & 2 & GE. CF6-8 & 48,000 bes. 81. & 176,200 & 79,923 & 315,000 & 142,001 & $272, \infty 00$ & 123,377 & 250,000 & 113,398 \\
\hline Booin 757-200 & 2 & ARR. AB211-535E4 HBTF & 40,100 bes. $\mathrm{E}$. & 128,250 & 57,267 & 240,000 & 109,848 & 198,000 & 89,813 & $184,0 \infty$ & 83,462 \\
\hline Booing $757-200$ & 2 & PEW. PWZO & $39,200106.81$ & 128,250 & 57,267 & 240,000 & 108,846 & 198,000 & 89.813 & 184,000 & 83,462 \\
\hline Booing 727-200 & 3 & PEW JT8D-17RTF & $16,000 \mathrm{tbos} .11$ & 101,73 & 46,184 & 200,500 & 85,027 & 181,000 & 73,028 & 144,000 & 65,315 \\
\hline Airbuse A.321 & 2 & CFMI CFM 56-5B1 HBTF & 31,000 1be. st. & 104,746 & 47,512 & 182,034 & 80,000 & N.A. & N.A. & N.A. & N.A \\
\hline MCDonnell Douglas MD-80-55 & 2 & IIAE V2528-05 TF & 20,000 lbs. st. & 94455 & 42,844 & 172,500 & 78,245 & N.A. & N.A. & N.A & N.A \\
\hline Booing 737-800 & 2 & CFMI C & os. st.] & A. & N.A. & 168,500 & 78,450 & A. & A. & N.A & 1. \\
\hline Airtus A.320-200 & 2. & CFMI. CF & $25,000105.8 \mathrm{st}$ & 85,604 & 38,830 & 158,730 & 72,000 & 138,690 & 63,000 & 130,072 & 59,000 \\
\hline glas MD-80-30 & 2 & IAE. V25: & 25,000100 st. & 82,200 & 40,007 & 156,000 & 70,760 & N.A. & N.A. & N.A. & N.A. \\
\hline Booing $737-700$ & 2 & CFMI.CFM & 24,000 105. 8t. & N.A. & N.A. & 149,000 & 67,585 & N.A. & N.A. & N.A. & N.A. \\
\hline Airbus A $320-100$ & 2 & CFMI CFM56-5 HBTF & $25,000100.81$. & 84,171 & 38,180 & 145,503 & $68, \infty$ & 134,480 & 61,000 & 125,662 & 57,000 \\
\hline MeDomnell Dougles MD-81 & 2 & PaW. JT8D TF & 18,500106.81. & 78,420 & 35,570 & 140,000 & 63,503 & 128,000 & 58,030 & 118,000 & 53,524 \\
\hline Booing $737-400$ & 2 & CFMI. CFM56-38 & $22,000165.81$. & 73,710 & 33,435 & 138,500 & 62,820 & N.A. & N.A. & N.A & N.A. \\
\hline Booing $737-300$ & 2 & CFMI. CFMS & 22.000106 .81$. & 09,590 & 31,561 & 135,000 & 61,235 & 114,000 & 1,710 & 100,500 & 18,306 \\
\hline Booing 737-200 & 2 & PQW. JP & 18,400 tbes. s1. & $\infty, 210$ & 27,310 & 128,100 & 59,108 & 107,000 & E,534 & 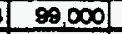 & 4,806 \\
\hline Cougles DC-930 & 2 & TPEW JT80-17 TF & 16,000 tos. 81 & 57,180 & 25,840 & 121,000 & $5,5,825$ & 110,000 & 48,895 & 98,500 & 44,678 \\
\hline SUD-EST SE.210 Ceravello 1083 & 2 & PEW J8D-7 TF & 14,000 10.81 & 66,259 & 30,055 & 119,048 & 34,000 & 109,127 & 49,500 & 87,081 & 39,500 \\
\hline BAC $1-11-500$ & 2 & IAA. Spek MkS12DW TF & 12,550 bes. 11 & 54,582 & 24,758 & 104,500 & 47,400 & $87,0,0$ & 39,462 & $81, \infty$ & 36,741 \\
\hline Britien Aarospeces BAC.146-200 & 1 & Aveo Lreoming ALF 502R- & 8.070 bes. 31 & 50,500 & 22,681 & 03,000 & \begin{tabular}{l|l|l|} 
& 42,184 \\
\end{tabular} & 81,000 & 36,741 & 73,500 & 33,339 \\
\hline Folkter 100 & 2 & AR. TeY MKE20-15 TF & $13,320100.81$ & 51,360 & 23,250 & 81,500 & 41,500 & 64,500 & 38,330 & 76,500 & 34,700 \\
\hline Fokker F.28 Fellowship & 2 & JAR. RB.183-2 Soer Mk555-15P T) & 8,000 tos. at. & 38,000 & 17,645 & $73,0 \infty$ & 33.113 & 3] 69,500 & 31,525 & 62,000 & 28,123 \\
\hline
\end{tabular}

N.A. - intormacion not aveilable whon able prepared.
HETF = High Bypes Tutbolan

TF - Turtotan

$T J=$ Turtojot
TP $=$ TUtooprop
PP = Pudial Piston
IP = In-Line Piston 
Toble 2.28 (continued)

Charectirtaties of Ar Corrior (14 CFA 121) Aroran

[Rolurences 2.33 and 2.34]

\begin{tabular}{|c|c|c|c|c|c|c|c|c|c|c|c|c|c|}
\hline \multirow[b]{2}{*}{ Aroran Typ } & \multicolumn{2}{|c|}{$\begin{array}{l}\text { Maximum } \\
\text { Fuel Cagon }\end{array}$} & \multicolumn{2}{|c|}{$\begin{array}{l}\text { Orerall } \\
\text { Win. Span }\end{array}$} & \multicolumn{2}{|c|}{ Oanet Lenoth } & \multicolumn{2}{|c|}{ loment Heinht } & \multicolumn{2}{|l|}{ Wing Area } & \multirow{2}{*}{$\begin{array}{c}\text { Terrofi } \\
\text { Dist. (n) } \\
\text { (FAA) }\end{array}$} & \multirow{2}{*}{$\begin{array}{l}\text { Unding } \\
\text { Dist. (t). } \\
\text { (FAF) }\end{array}$} & \multirow{2}{*}{$\begin{array}{l}\text { Stall Spoed } \\
\text { (oomrmaps } \\
\text { dom, mph) }\end{array}$} \\
\hline & mp. as & Liton & Fi. in. & Momes & $\mathrm{FI}$ in. & Molere & Ft. in. & Mons & FI."-2 & $M^{*+2}$ & & & \\
\hline $80 \sin 747-400$ & N.A. & N.A. & $219^{\circ} \circ$ & $04.31 \mathrm{~m}$ & $231^{\prime}+0$ & $70.08 \mathrm{~m}$ & $25^{\circ}$ & $10.33 \mathrm{~m}$ & 5.050 .0 & 524.9 & N.A. & N.A. & A. \\
\hline Eowin $747-400$ & N.A & N.A. & $211^{\circ} 0$ & $64.31 \mathrm{~m}$. & $231^{\prime} 10^{\circ}$ & $70.63 \mathrm{~m}$ & est 5 & $10.33 \mathrm{~m}$. & 5.650 .0 & 524.0 & N.A. & N.A. & N.A. \\
\hline Bowin $747-100$ & N.A. & N.A. & $211^{\prime} 0$ & $64.34 \mathrm{~m}$ & $231^{\circ} 10$ & $70.03 \mathrm{~m}$ & $655^{\circ}$ & $19.33 \mathrm{~m}$ & 5.050 .0 & 524.0 & N.A. & N.A. & NA \\
\hline Eowin 747.300 & 48,395 & 210,087 & 1056 & $50.64 \mathrm{~m}$ & $231^{\prime} 10$ & $70.68 \mathrm{~m}$ & $655^{\circ}$ & $10.33 \mathrm{~m}$ & 5.500 .0 & 511.0 & 10,450 & 0.020 & N.A. \\
\hline Bosin 747300 & 48,395 & 210.867 & 1858 & $59.04 \mathrm{~m}$ & $231 \cdot 10$ & $70.6 \mathrm{~m}$ & 255 & $10.33 \mathrm{~m}$ & $5,500.0$ & 511.0 & 10450 & 0,220 & N.A. \\
\hline Bowin 747300 & 48,335 & 210,087 & $105^{\circ} 0^{\circ}$ & $50.04 \mathrm{~m}$ & $231 \cdot 10$ & $70.08 \mathrm{~m}$ & $65^{2}$ & $10.33 \mathrm{~m}$ & $5,500.0$ & 511.0 & 10,450 & 0.920 & N.A. \\
\hline Bovin: 747.300 & 46,305 & 210,057 & 1858 & $59.64 \mathrm{~m}$ & $231^{\prime} 10$ & $7063 \mathrm{~m}$ & $655^{\circ}$ & $18.33 \mathrm{~m}$ & $5,500.0$ & 5110 & 10,450 & 8.920 & N.A. \\
\hline Bosin 747-200 & 40,345 & 210,857 & 1858 & $59.64 \mathrm{~m}$. & $231^{\prime} 10$ & $70.66 \mathrm{~m}$ & 835 & $10.35 \mathrm{~m}$ & $5,500.0$ & 511.0 & 10,400 & 8.950 & U. \\
\hline Dowin $747-200$ & 48,395 & 210,087 & $105^{\prime} 8$ & $59.84 \mathrm{~m}$. & $201 \cdot 10$ & $70.08 \mathrm{~m}$ & $655^{\circ}$ & $10.30 \mathrm{~m}$. & $5,500.0$ & 511.0 & 10,400 & 6.050 & N.A. \\
\hline Eovin. 747-200 & 48,335 & 210.87 & $185^{\prime} 8^{\circ}$ & $50.04 \mathrm{~m}$ & $231: 10$ & $70.65 \mathrm{~m}$ & $\cos 5$ & $18.30 \mathrm{~m}$ & $5,500.0$ & 511.0 & 10,400 & 6,050 & IA. \\
\hline MeDonnell Dough MD-11 & 82,165 & 146,300 & $160^{\prime} 6^{\circ}$ & $51.70 \mathrm{~m}$ & 1007 & $00.50 \mathrm{~m}$ & 379 & $17.00 \mathrm{~m}$ & 3.674 .0 & 341.3 & 10,100 & 6,470 & N.A. \\
\hline McDomnt Dounies MD-11 & 32,185 & 140,300 & 1096 & $51.70 \mathrm{~m}$ & 1067 & $00.50 \mathrm{~m}$ & 579 & $17.00 \mathrm{~m}$ & 3.674 .0 & 341.3 & 10,100 & 6,470 & N.A. \\
\hline MeDomell Dounles DC-10-30 & 30.050 & 150,250 & $165 ' 49$ & $50.40 \mathrm{~m}$ & 98210 & $5.50 \mathrm{~m}$ & 5819 & $17.70 \mathrm{~m}$ & 3.858 .0 & 367.7 & 0.269 & 5,870 & N.A. \\
\hline MCDomell Dourles DC-10-30 & 39,050 & 150,850 & $165^{\circ} 4^{\circ}$ & $50.40 \mathrm{~m}$ & 18217 & $55.50 \mathrm{~m}$ & 5819 & $17.70 \mathrm{~m}$ & 3.959 .0 & 367.7 & 0.289 & 5,870 & NA. \\
\hline McDonnell Dounles DC-10-30 & 39,850 & 150.850 & $185 \cdot 4$ & $50.40 \mathrm{~m}$ & 18217 & $55.50 \mathrm{~m}$ & $584^{4}$ & $17.70 \mathrm{~m}$ & 3.858 .0 & 387.7 & 0,289 & 5,870 & N.A. \\
\hline Arbu $1340-300$ & 24,500 & 111,380 & 1800 & $58.0 \mathrm{~m}$ & 20098 & $63.6 \mathrm{~m}$ & $55^{\prime} 1.5$ & $18.8 \mathrm{~m}$ & 3.500 .0 & 325.2 & N.A. & N.A. & N.A. \\
\hline Airbue $1340-200$ & 24,500 & 111,380 & 1000 & $58.0 \mathrm{~m}$ & $184^{\circ}+10$ & $59.39 \mathrm{~m}$ & $55 ' 1.5 \%$ & $18.8 \mathrm{~m}$. & $3,500,0$ & 325.2 & N.A. & N.A. & NA. \\
\hline Locitheed L.1011-500 & 26,347 & 118,774 & $164^{\prime} 4^{\prime}$ & $50.09 \mathrm{~m}$ & $164 \cdot 2.5$ & $50.05 \mathrm{~m}$ & 55.4 & $16.87 \mathrm{~m}$ & $3,541.0$ & 329.0 & 8,200 & 6.770 & N.A. \\
\hline Aitbus $\mathbf{1 . 3 3 0}$ & 24,500 & 111,590 & $180^{\circ} 0^{\circ}$ & $58.0 \mathrm{~m}$ & $205^{\prime} 4.5$ & $62.6 \mathrm{~m}$ & $55,1.59$ & $16.8 \mathrm{~m}$. & $3,500.0$ & 325.2 & N.A. & N.A. & N.A. \\
\hline Alitus $\mathbf{A} .330$ & 24,500 & 111,380 & $190^{\circ} 0^{\circ}$ & $58.0 \mathrm{~m}$ & $205^{\circ} 4.5^{\circ}$ & $62.8 \mathrm{~m}$ & $55^{\prime} 1.5^{\circ}$ & $18.8 \mathrm{~m}$ & $3,500.0$ & 325.2 & N.A. & N.A. & N.A. \\
\hline Aerospatiale BAC Con & 28,350 & 119,783 & 8310 & $25.56 \mathrm{~m}$ & 2039 & $62.10 \mathrm{~m}$ & 375 & $1140 \mathrm{~m}$ & $3,858.0$ & 358.3 & 11,200 & 7,300 & N.A. \\
\hline Airtous A.300-600 & 13,858 & 82,800 & $1471^{\circ}$ & $4484 \mathrm{~m}$ & $1775^{\circ}$ & $5408 \mathrm{~m}$ & SA' 6.5 & $16.62 \mathrm{~m}$ & $2,783.6$ & 260.0 & 7,600 & 5,025 & N.A. \\
\hline Dounles DC-8-73 & 20,213 & 01800 & $148^{\prime} 5$ & $45.20 \mathrm{~m}$ & 1875 & $57.12 \mathrm{~m}$ & 430 & $13.11 \mathrm{~m}$. & $2,827.0$ & 271.9 & 10,000 & 6,500 & N.A. \\
\hline Eocing 767-300 & 13,000 & 63,218 & $1571^{\circ}$ & $47.57 \mathrm{~m}$ & 1803 & $54.04 \mathrm{~m}$ & $520^{\circ}$ & $15.85 \mathrm{~m}$. & $3,050.0$ & 283.3 & 7,800 & 5,600 & N.A. \\
\hline Boping 767.300 & 13.000 & 63,216 & 1574 & $47.57 \mathrm{~m}$. & $180^{\circ}$ & $54.94 \mathrm{~m}$ & 520 & $15.85 \mathrm{~m}$ & $3,050.0$ & 283.3 & 7,800 & 5,600 & N.A. \\
\hline Airbus A $310-300$ & 15,016 & 68,200 & $144^{\prime} 0^{\circ}$ & $43.00 \mathrm{~m}$ & $153: 11$ & $48.68 \mathrm{~m}$ & $51 \div 10$ & $15.81 \mathrm{~m}$. & $2,357.0$ & 219.0 & 7,575 & 5,000 & $N A$ \\
\hline Boing $707-320 \mathrm{C}$ & 19.893 & $\infty, 290$ & $145^{\prime} \theta^{\circ}$ & $44.42 \mathrm{~m}$ & 15211 & $48.6 \mathrm{~m}$ & 425 & $12.85 \mathrm{~m}$. & 3.050 .0 & 283.4 & 10,020 & 6,400 & N.A. \\
\hline Booing $767-200$ & 13,200 & 83.216 & $150^{\prime} 10^{\circ}$ & $47.57 \mathrm{~m}$ & 1592 & $48.51 \mathrm{~m}$ & $520^{\circ}$ & $15.85 \mathrm{~m}$ & $3,050.0$ & 203.3 & 5,650 & 4750 & N.A. \\
\hline Bosing $767-200$ & 13,000 & 63.216 & $156^{\prime \prime}$ & $47.57 \mathrm{~m}$ & 1592 & $48.51 \mathrm{~m}$. & 520 & $15.85 \mathrm{~m}$. & $3,050.0$ & 289.3 & 5.850 & 4.750 & N.A. \\
\hline Booing $757-200$ & 9,370 & 42,597 & $124 \cdot 10^{\prime}$ & $39.05 \mathrm{~m}$ & $155: 3^{\circ}$ & $47.32 \mathrm{~m}$ & $44^{\prime} 8^{\prime}$ & $13.58 \mathrm{~m}$ & 1.004 .0 & 185.3 & 7,760 & 4,600 & N.A. \\
\hline Booing $757-200$ & 9,370 & 42,597 & $124^{\circ} 10^{\circ}$ & $38.05 \mathrm{~m}$ & $155 \cdot 3^{\circ}$ & $47.32 \mathrm{~m}$ & $44^{\prime} 6^{\prime \prime}$ & $13.56 \mathrm{~m}$ & 1.0040 & 185.3 & 7,760 & 4,600 & NA. \\
\hline Boging $727-200$ & 7,292 & 40,010 & $100^{\prime} 0^{\circ}$ & $32.22 \mathrm{~m}$ & 1532 & $46.69 \mathrm{~m}$ & $34^{\prime} 0^{\circ}$ & $10.36 \mathrm{~m}$ & $1,700.0$ & 157.9 & 9,950 & 4,900 & N.A \\
\hline Airouse A.321 & N.A. & N.A. & $111^{\prime} 9.5^{\circ}$ & $34.09 \mathrm{~m}$ & $146^{\circ} 0^{\circ}$ & $44.50 \mathrm{~m}$ & $38: 8.5$ & $11.00 \mathrm{~m}$ & $1,320.0$ & 123.0 & N.A. & N.A. & N.A. \\
\hline MeDonnell Dougles MD-80-55 & N.A. & N.A & $10710^{\circ}$ & $32.87 \mathrm{~m}$ & 1527 & $46.51 \mathrm{~m}$ & 307 & $8.33 \mathrm{~m}$ & $1,209.0$ & 112.3 & N.A. & N.A. & N.A. \\
\hline Boping $737-800$ & N.A. & N.A & $111 \cdot 2$ & $33.80 \mathrm{~m}$ & $128^{\circ} 8^{\circ}$ & $39.20 \mathrm{~m}$ & $38^{\prime} 6^{\prime}$ & $11.13 \mathrm{~m}$ & $1,344.0$ & 125.0 & N.A. & N.A. & N.A. \\
\hline Airbus $1.320-200$ & 5,150 & 23,410 & 1119.5 & $34.09 \mathrm{~m}$ & $123^{\prime} 3^{\prime}$ & $37.58 \mathrm{~m}$. & $38^{\prime} 8.5^{\prime}$ & $11.80 \mathrm{~m}$ & $1,320.0$ & 123.0 & 5,630 & 4,750 & N.A. \\
\hline MeDonnall Dougles I & N.A. & N.A & 10710 & $32.87 \mathrm{~m}$ & 1527 & $48.51 \mathrm{~m}$. & 307 & $9.33 \mathrm{~m}$ & $1,209.0$ & 112.3 & N.A. & N.A. & N.A. \\
\hline Booing $737-700$ & N.A. & N.A. & $111 \cdot 2$ & $33.80 \mathrm{~m}$ & $101^{\prime} 8^{\circ}$ & $31.00 \mathrm{~m}$ & $36^{\prime} 6^{\prime \prime}$ & $11.13 \mathrm{~m}$ & 1,3440 & 125.0 & N.A. & N.A & NA. \\
\hline Artous A.320-100 & 3,300 & 15,365 & $111+3$ & $33.91 \mathrm{~m}$ & $123^{\prime} 3^{\circ}$ & $37.58 \mathrm{~m}$ & 3877 & $11.76 \mathrm{~m}$ & 1.317 .5 & 122.4 & 5,630 & 4,750 & N.A. \\
\hline MeDonnell Dougles MD-81 & 4,812 & 21,876 & 10710 & $32.87 \mathrm{~m}$ & $14710^{\circ}$ & $45.08 \mathrm{~m}$ & $288^{\circ}$ & $9.04 \mathrm{~m}$ & $1,270.0$ & 118.0 & 7250 & 4,860 & N.A. \\
\hline Bogin $737-400$ & N.A & N.A. & $24^{\prime} 8^{\prime}$ & $28.88 \mathrm{~m}$ & 1187 & $38.45 \mathrm{~m}$ & $35^{\prime} 6^{\prime \prime}$ & $11.13 \mathrm{~m}$ & $1,135.0$ & 105.4 & N.A. & N.A. & NA \\
\hline Booing 737-300 & 5,000 & 23,470 & $94^{\prime} 9$ & $28.88 \mathrm{~m}$ & $100^{\prime} 7$ & $30.40 \mathrm{~m}$ & $38^{\circ} 6^{\prime}$ & $11.13 \mathrm{~m}$ & $1,135.0$ & 105.4 & 6,360 & 4,580 & N.A. \\
\hline Booing 737-200 & 4,971 & 22,598 & 250 & $28.35 \mathrm{~m}$ & 1002 & $30.53 \mathrm{~m}$. & 370 & $11.28 \mathrm{~m}$ & $1,000.0$ & 102.0 & 6,000 & 4,430 & N.A. \\
\hline Douplas DC-9 30 & 3,083 & 13,025 & $035^{\circ}$ & $28.47 \mathrm{~m}$ & $110^{\circ} 3.5^{\circ}$ & $33.37 \mathrm{~m}$ & $278^{\circ}$ & $8.38 \mathrm{~m}$ & $1,000.7$ & 23.0 & 5,530 & 4,290 & N.A. \\
\hline SUD-EST SE 210 Caravollo 1093 & 4,180 & 10,000 & $1128^{\prime}$ & $34.30 \mathrm{~m}$ & $100^{\prime} 3.5^{\circ}$ & $30.01 \mathrm{~m}$ & 287 & $8.72 \mathrm{~m}$ & $1,579.0$ & 148.7 & 8,850 & 5,180 & N.A. \\
\hline BAC 1-11.500 & 3,725 & 17,208 & $93^{\circ}$ & $28.50 \mathrm{~m}$ & 1070 & $32.61 \mathrm{~m}$. & $24^{\prime} 6^{\prime}$ & $7.47 \mathrm{~m}$ & $1,001.0$ & 85.8 & 7,300 & N.A. & N.A. \\
\hline Brtizh Aerospece BA. 148-200 & $2,8<: 8$ & 12,801 & 865 & $23.34 \mathrm{~m}$ & $83: 10^{\circ}$ & $28.60 \mathrm{~m}$ & $20^{\circ} 3^{\prime}$ & $6.61 \mathrm{~m}$ & 832.0 & 77.3 & 4,950 & 3,790 & N.A. \\
\hline Fokker 100 & 2,283 & 13,040 & 921.5 & $28.08 \mathrm{~m}$ & 1510.3 & $35.31 \mathrm{~m}$. & $27^{\prime} 10.5^{\circ}$ & $8.50 \mathrm{~m}$ & 1.014 .7 & 24.3 & 5,870 & 4,430 & N.A. \\
\hline Fokker F.28 Fellowship & 2,000 & 13,040 & $62^{2}$ & $25.07 \mathrm{~m}$. & 871.87 & $28.61 \mathrm{~m}$ & 278.5 & $6.47 \mathrm{~m}$ & 850.0 & 79.0 & 5,200 & 3,495 & N.A. \\
\hline
\end{tabular}

N.A. = Intormation not avilable whon thble prepared 
Toble 2.28 (continund)

Charectertsties of Nr Carrior (14 CFR 121) Arreratt

[Roturenes 233 end 2.34]

\begin{tabular}{|c|c|c|c|c|c|c|c|c|c|c|c|}
\hline \multirow[b]{2}{*}{ Airent Type } & \multicolumn{2}{|c|}{ Pownolant(s) } & \multirow{2}{*}{$\begin{array}{c}\text { Nomind } \\
\text { Power } \\
\text { Rating (each) }\end{array}$} & \multicolumn{2}{|c|}{$\begin{array}{l}\text { Openting } \\
\text { Empry Woinht }\end{array}$} & \multicolumn{2}{|c|}{$\begin{array}{l}\text { Maximum } \\
\text { Tekeoff Woight }\end{array}$} & \multicolumn{2}{|c|}{$\begin{array}{l}\text { Meximum } \\
\text { Lendin Woinh! }\end{array}$} & \multicolumn{2}{|c|}{$\begin{array}{l}\text { Maximum } \\
\text { Zero-Fuel Weight }\end{array}$} \\
\hline & No. & Type & & Pounds & Kloginm F & Pounds & Klogram & Founds & Wogrem & Pounds If & Klogmens \\
\hline \multicolumn{12}{|c|}{ AIA CARAIER (14 CFA 121) TUFEOPPOPS } \\
\hline Lectoneed L-100-30 Hercules & 4 & Alteon S01D-22A TP & $4600 \mathrm{chp}$ & 77.800 & 35,235 & 155,000 & 70,300 & 135,000 & 61,235 & 128,790 & 58,420 \\
\hline Loesheed L-18sa Electra & 4 & Alieon $5010-13$ TP & $3,750 \mathrm{cho}$ & 61,500 & 27,095 & 110,000 & 52,684 & 8.650 & 43,525 & 86,000 & 39,010 \\
\hline Vicker Viscount exo & 4 & AR. Dat MkS10 TP & 1740 chel & 41,000 & 18,000 & $64,5 \infty$ & 29200 & 58,500 & 28.585 & 54,000 & 24,404 \\
\hline Conver 580 & 2 & Ainon SOI-D1SH TP & $3,750 \mathrm{cho}$ & 30,275 & 13.732 & 80.140 & 28,371 & 50,070 & 2205 & N.A. & N.A. \\
\hline Bitlen AnOSPCO ATP & 2 & PEWC. PW124 TP & 2.150 enp & 29.070 & 13,504 & 49500 & 23.53 & 48,000 & 21.773 & 4300 & 20,009 \\
\hline Senb 2000 & 2 & Amin AE2100A TP & $4,125 \sin$ & 29,702 & 13.500 & 49.500 & 2000 & N.A. & N.A. & N.A. & N.A. \\
\hline ATA.72-200 & 2 & PEWC. PW1248 TP & $2,100 \operatorname{sh} 9$ & 27,558 & 12,500 & 47.00 & 21,500 & N.A. & N.A. & N.A. & N.A. \\
\hline Folver F.so & 2 & PEWC. PW124 TP & 2,150 shp & 27,850 & 12,023 & $45, \infty 00$ & 20,020 & 41,805 & 18, 80 & 40,350 & 18,300 \\
\hline Folder F.27 Friendahip & 2 & AR Dan MkS22 TP & $2,200 \mathrm{chp}$ & 22,006 & 10,205 & 45,000 & 20,110 & 41,000 & 18,000 & 30,500 & 17,817 \\
\hline DeHevillend Cen. DHC-7-100 & 4 & P\&W. PTEA-50 TP & $1,120 \mathrm{shp}$ & 27,600 & 12,530 & 4,000 & 18.058 & 4,000 & 18,058 & 39,000 & 17,690 \\
\hline ATA.42-200 & 2 & PAWC. PW120 TP & 1,000 shp & 21,808 & 8,973 & 34,722 & 15,750 & 34,171 & 15,500 & 31,866 & 14.500 \\
\hline DeHevilland Can. DHC-8-100 & 2 & PSW. PWIZOATP & 1800 ehp & 21,500 & 9,793 & 34,500 & 15.649 & 33,000 & 14,823 & 31,000 & 14,062 \\
\hline CASA-Numnio CN.235 & 2 & GE. CT7.7ATP & 1700 sho & 20.723 & 8,400 & 31,248 & 14,400 & 31,305 & 14,200 & 29,902 & 13,600 \\
\hline Dornier 328 & 2 & PEW. PW1199TP & 1,850 sho & 18,420 & 8,810 & 30,071 & 13,640 & N.A. & N.A. & N.A. & N.A. \\
\hline Seab Fainchild 340 & 2 & GE. CT7-5A2TP & $1,735 \operatorname{sh} 0$ & 17,215 & 7,608 & 27,275 & 12,371 & 28,500 & 12,020 & 25,000 & 11,340 \\
\hline Shorts 360 & 2 & PEW PTEA65ARTP & $1,424 \operatorname{shp}$ & 16.250 & 7,689 & 26,000 & 11,783 & 25,700 & 11,657 & N.A. & NA \\
\hline \multicolumn{12}{|c|}{ AIA CARAIEA (14 CFR 121) PISTONPAOPS } \\
\hline Douglas DC-7C & 4 & W. R3350-EA1 RP & 3,400 hp & 80,000 & 36,287 & 143,000 & 64,865 & N.A. & N.A. & N.A. & N.A \\
\hline Dougtas DC.7F & 4 & W. R3350-EA1 RP & $3100 \mathrm{hg}$ & 86,200 & $30,0,0$ & 126,000 & 57,153 & N.A. & N.A & N.A. & N.A \\
\hline Douplas DC-6B & 4 & PEW R2900-CB17 RP & $2.500 \mathrm{hp}$ & 62,000 & 26,123 & 107,000 & 48,534 & 88,200 & $10,0,0$ & 83,200 & 37,740 \\
\hline Dougles DC-4 & 4 & PEW A2000-2SO-BG AP & $1450 \mathrm{sp}$ & 43,500 & 18,480 & 73,000 & 33,112 & N.A. & N.A. & N.A. & N.A. \\
\hline Convair 440 & 2 & PEW R2800-CB17 RP & $2,500 \mathrm{hp}$ & 33,314 & 15,110 & 49,700 & 22,544 & 47,850 & 21,814 & 47,000 & 21,320 \\
\hline Curtiss $\mathrm{C}-46$ Commando & 2 & PQW R2800-34 RP & $2,000 \mathrm{shp}$ & 33,000 & 14,870 & 48,000 & 21,772 & 46,800 & 21,228 & 45,168 & 20488 \\
\hline Convair 340 & 2 & PEW R2800-CB16 RP & $2400 \mathrm{np}$ & 29,486 & 13,375 & 47,000 & 21,320 & N.A. & NA. & NA & NA \\
\hline Convair 240 & 2 & PEW R2800-CA18 RP & $2,000 \mathrm{hp}$ & 27,600 & 12,520 & 41,790 & 18,956 & N.A. & N.A. & N.A. & N.A. \\
\hline Dougles DC-3 & 2 & P\&W R1830-92 AP & $1,200 \mathrm{hp}$ & 17,720 & $8,0<0$ & $28, \infty 001$ & 12,700 & N.A. & N.A. & N.A. & N.A. \\
\hline
\end{tabular}

N.A. - informacion not avaitable when table preparod.
HBTF - High Eypass Turbolan

TF = Turbotan

TJ - Turbojet
TP = Turboprop

RP = Redial Piston

IP = In-Line Pivton 
Teble 2.23 (continued)

Charecteristies of Al Cartic (14 CFR 121) Mrerant

[Rotorence 2.33 and 2.34]

\begin{tabular}{|c|c|c|c|c|c|c|c|c|c|c|c|c|c|}
\hline \multirow[b]{2}{*}{ Areran Type } & \multicolumn{2}{|c|}{$\begin{array}{l}\text { Moximum } \\
\text { Fuel Capacivy }\end{array}$} & \multicolumn{2}{|c|}{$\begin{array}{l}\text { Overall } \\
\text { Wing Span }\end{array}$} & \multicolumn{2}{|c|}{ Overll Lengen } & \multicolumn{2}{|c|}{ Ooverll Height } & \multicolumn{2}{|l|}{ I Who Area } & \multirow{2}{*}{$\begin{array}{l}\text { Takeoft } \\
\text { Dint. (I.) } \\
\text { (FAR) }\end{array}$} & \multirow{2}{*}{$\begin{array}{l}\text { Lending } \\
\text { Dist. (t.) } \\
\text { (FAR) }\end{array}$} & \multirow{2}{*}{$\begin{array}{l}\text { Stull Spoed } \\
\text { (ouarmaps } \\
\text { down, mph) }\end{array}$} \\
\hline & imp. Gal & Liters & Ft. In. & Maten & Ft. In. & monen & Ft. In. & Moner & $F t^{.02}$ & $\overline{M-2} 2$ & & & \\
\hline & & & & & & & & & & & & & \\
\hline Lockined L-100-50 Heraing & Eeous & 37,770 & 1327 & $40.41 \mathrm{~m}$ & 1120 & $34.37 \mathrm{~m}$ & 30 & $11.08 \mathrm{~m}$ & $1,745.0$ & 102.1 & 0,200 & 4.850 & N.A. \\
\hline Locthed L-183A Electro & 4,590 & 20,005 & $98 \sigma^{\circ}$ & $30.18 \mathrm{~m}$ & $104^{\circ} 0^{\circ}$ & $31.81 \mathrm{~m}$ & $32 \cdot 10$ & $10.0 \mathrm{~m}$ & 1300.0 & 120.8 & 4.720 & 4,300 & N.A. \\
\hline Vickere Viecount 800 & 1.245 & 8,042 & $938^{\circ}$ & $28.55 \mathrm{~m}$ & $650^{\circ}$ & $28.11 \mathrm{~m}$ & 2000 & $8.15 \mathrm{~m}$. & $\cos 3.0$ & 024 & 5,310 & 4.050 & N.A. \\
\hline Conver seo & 2,300 & 10,801 & $105^{4} 4^{\circ}$ & $32.12 \mathrm{~m}$ & $81 \cdot 0$ & $24.64 \mathrm{~m}$. & $28 \%$ & $8.60 \mathrm{~m}$. & 90.0 & 05.5 & 4,700 & 4,100 & N.A. \\
\hline Bttigh Alocopes ATP & 1,400 & 6,305 & $1006^{\circ}$ & $30.63 \mathrm{~m}$ & 8540 & $21.01 \mathrm{~m}$ & $23^{\prime} 5^{4}$ & $7.14 \mathrm{~m}$. & 893.0 & 78.3 & N.A. & N.A. & N.A. \\
\hline Se00 2000 & N.A. & N.A. & $81^{\prime} 3^{\circ}$ & $24.76 \mathrm{~m}$ & $680^{\circ}$ & $27.00 \mathrm{~m}$ & $25^{\circ} 4^{4}$ & $7.73 \mathrm{~m}$ & $\infty 00$ & 55.7 & N.A. & N.A. & N.A. \\
\hline ATR. $72-200$ & N.A. & N.A. & $88 \theta^{\circ}$ & $27.05 \mathrm{~m}$ & 807 & $27.18 \mathrm{~m}$. & $25 \%$ & $7.65 \mathrm{~m}$. & 658.6 & 61.0 & N.A. & NA. & N.A. \\
\hline Foldker F.50 & 1,130 & 5,136 & $95^{\prime} 1.8^{\circ}$ & $20.00 \mathrm{~m}$ & $2 \cdot 10$ & $25.25 \mathrm{~m}$ & $287 \pi$ & $8.00 \mathrm{~m}$ & 754.0 & 70.0 & 5,770 & 3,480 & N.A. \\
\hline Folkker F.27 Friendatip & 1,034 & 7,425 & $95^{\circ} 2^{\circ}$ & $20.00 \mathrm{~m}$ & 77.3 .50 & $23.56 \mathrm{~m}$ & $27^{\circ} 117$ & $8.51 \mathrm{~m}$. & 753.5 & 70.0 & 3240 & 3,200 & N.A. \\
\hline Dettavilland Can. DHC-7-100 & 1,232 & 5,002 & $930^{\circ}$ & $28.35 \mathrm{~m}$ & 600 & $24.54 \mathrm{~m}$ & $20^{\circ} 2$ & $7.28 \mathrm{~m}$ & 800.0 & 79.9 & 2,250 & 2,160 & 76 \\
\hline ATA.42-200 & 1,254 & 5,700 & $800^{7} 75^{\circ}$ & $24.57 \mathrm{~m}$ & $74^{\prime} 4.5^{\circ}$ & $22.67 \mathrm{~m}$ & $24^{\prime} 14^{\prime}$ & $7.50 \mathrm{~m}$ & 508.6 & S4.5 & 3,575 & 3,150 & N.A. \\
\hline Dothavillend Cen. DHC-8-100 & 1,24 & 5,656 & $85^{\prime} 0^{\circ}$ & $25.91 \mathrm{~m}$ & $730^{\circ}$ & $22.25 \mathrm{~m}$ & 247 & $7.49 \mathrm{~m}$ & ses.0 & 544 & 3,110 & 3,150 & N.A. \\
\hline CASA-Nuranio CN.235 & 1,150 & 5,268 & $64^{\prime} 0^{\circ}$ & $25.81 \mathrm{~m}$ & $70^{\circ} 0.75^{\circ}$ & $21.35 \mathrm{~m}$ & $20^{\prime} 10^{\circ}$ & $8.18 \mathrm{~m}$ & 645.8 & 60.0 & 2,165 & 1,920 & N.A. \\
\hline Dornier 328 & N.A. & N.A. & $68^{\prime} 10^{\circ}$ & $20.98 \mathrm{~m}$ & $69^{\prime} 8^{\circ}$ & $21.22 \mathrm{~m}$ & $23^{\prime} 8^{4}$ & $7.24 \mathrm{~m}$ & N.A. & N.A. & N.A. & N.A. & N.A. \\
\hline Senob Fairchild 340 & 700 & 3,220 & $70^{\circ} 4^{\circ}$ & $21.44 \mathrm{~m}$ & $84^{\prime} 8^{\circ}$ & $18.72 \mathrm{~m}$ & $22^{\prime} 6.5^{\prime}$ & $6.87 \mathrm{~m}$ & 450.0 & 41.8 & 4,000 & 4,000 & N.A. \\
\hline Shorts $\mathbf{3 6 0}$ & 480 & 2,182 & $74 \cdot 10^{\prime}$ & $22.81 \mathrm{~m}$ & $70^{\prime} 10^{\circ}$ & $21.59 \mathrm{~m}$ & $23^{\prime} 8^{\circ}$ & $7.21 \mathrm{~m}$ & 453.0 & 42.1 & 4,200 & 4,020 & N.A. \\
\hline Douglas DC-7C & $\overline{N . A .}$ & N.A. & $1276^{\circ}$ & $39.80 \mathrm{~m}$ & $1123^{\circ}$ & $34.23 \mathrm{~m}$ & $31^{\prime} 8$ & $9.65 \mathrm{~m}$. & 1.637 .0 & 152.0 & NA. & N.A. & N.A. \\
\hline Dougias DC-7F & NA. & N.A. & $1176^{\circ}$ & $35.81 \mathrm{~m}$ & 108119 & $33.24 \mathrm{~m}$. & $29^{\prime} 3^{4}$ & $8.92 \mathrm{~m}$ & 1,4630 & 135.0 & N.A. & N.A. & N.A. \\
\hline Douglas DC-68 & 4,580 & 20,820 & $1176^{\circ}$ & $35.81 \mathrm{~m}$ & 105.7 & $32.18 \mathrm{~m}$ & $29^{\circ} 3^{4}$ & $8.92 \mathrm{~m}$ & $1,463.0$ & 135.9 & 6,150 & 5,000 & NA \\
\hline Douglas DC-4 & N.A. & N.A. & $1176^{\circ}$ & $35.81 \mathrm{~m}$ & $83^{\prime} 10^{\circ}$ & $28.60 \mathrm{~m}$ & $276^{\circ}$ & $8.38 \mathrm{~m}$ & $1,460.0$ & $\$ 35.6$ & N.A. & N.A. & NA. \\
\hline Convair 440 & 1440 & 8,548 & $105^{\circ} 4^{\circ}$ & $32.12 \mathrm{~m}$ & $81^{\prime} 6^{\circ}$ & $24.84 \mathrm{~m}$ & $28^{\prime} z^{\prime}$ & $8.59 \mathrm{~m}$. & 920.0 & 85.5 & N.A. & N.A. & N.A. \\
\hline Curtess $C-46$ Commando & 1400 US & 5,305 & $108^{\circ} 0^{\circ}$ & $32.22 \mathrm{~m}$ & $76^{\circ} 4^{\circ}$ & $23.27 \mathrm{~m}$ & $21^{\prime} B^{\prime \prime}$ & $6.60 \mathrm{~m}$. & $1,358.0$ & 126.2 & N.A. & NA. & N.A. \\
\hline Convair 340 & N.A. & N.A. & $105^{\circ} 4^{\circ}$ & $32.12 \mathrm{~m}$ & $79^{\circ} 2^{\circ}$ & $24.13 \mathrm{~m}$ & 282 & $8.50 \mathrm{~m}$ & 820.0 & 85.5 & N.A. & N.A. & N.A. \\
\hline Convair 240 & N.A. & N.A. & $81^{\prime} \theta^{\circ}$ & $27.97 m$ & $74^{\prime} 8^{\prime \prime}$ & $22.78 \mathrm{~m}$ & $20^{\prime} 119$ & $8.20 \mathrm{~m}$ & 817.0 & 75.0 & N.A. & N.A. & N.A. \\
\hline Douglas DC-3 & 670 & 3,046 & $95^{\circ} 0^{\circ}$ & $28.96 \mathrm{~m}$ & $64^{\circ} 6^{\circ}$ & $18.66 \mathrm{~m}$ & $16^{\circ} 11.5^{\circ}$ & $5.18 \mathrm{~m}$ & 897.0 & 91.7 & N.A. & N.A. & N.A \\
\hline
\end{tabular}

N.A. = intormation not avitabls when uble prepared. 
Table 2.27

Charecteristles of sechedulad Ar Tax (14 CFR 136) Nrenat [Raforence 233 and 2.34]

\begin{tabular}{|c|c|c|c|c|c|c|c|c|c|c|c|}
\hline \multirow[b]{2}{*}{ Aromen Typo } & \multicolumn{2}{|c|}{ Poweronit(s) } & \multirow{2}{*}{$\begin{array}{l}\text { Moning } \\
\text { Powner } \\
\text { Pand (onch) }\end{array}$} & \multicolumn{2}{|c|}{$\begin{array}{l}\text { Operating } \\
\text { Enpiy Weinh }\end{array}$} & \multicolumn{2}{|c|}{$\begin{array}{l}\text { Meximum } \\
\text { Tcyofl Weiont }\end{array}$} & \multicolumn{2}{|c|}{$\begin{array}{l}\text { Maximum } \\
\text { Lending Weinht }\end{array}$} & \multicolumn{2}{|c|}{$\begin{array}{l}\text { Maximum } \\
\text { Zero-Fue Weint }\end{array}$} \\
\hline & No. & Type & & Pound: & Koogen & Pound & Koeren & Pound & Klogran & Pounds & Kilogren: \\
\hline Enbrer EMB-120 Brisilia & 2 & PAWC PW118 TP & $1,000 \operatorname{sho}$ & 15,183 & 6.078 & 25,358 & 11500 & 24,202 & 11,250 & 23,148 & 10,500 \\
\hline 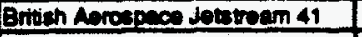 & 2 & Genot TPES31-14TP & 1,050 sho & 14,000 & 8,350 & $24, \infty$ & 10,ess & N.A. & N.A. & N.A. & N.A. \\
\hline 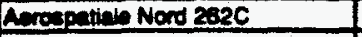 & 2 & Tutormen Begen VIA TP & $1.130 \mathrm{cho}$ & 15,920 & 7,205 & 20,810 & 10,000 & 23,040 & 10,450 & 22,710 & 10,300 \\
\hline Ehom $300-200$ & 2 & PEWC. PTEA-45RTP & $1,108 \operatorname{sen}$ & 14,727 & 6.00 & 22,000 & 10,337 & 22,000 & 10251 & N.A. & N.A. \\
\hline Brith Aorvenges destroem 31 & 2 & Gein TPES31-10UF TP & 90 chp & 0.570 & 4,341 & 15,212 & $0 . \infty$ & 14,500 & 800 & 13.899 & 0,300 \\
\hline Feinchild Swosingen Mevo III & 2 & GeNTI TPE-331-114-611G TP & $1,000 \mathrm{shg}$ & 8,797 & 3003 & 14,500 & 6.577 & 14,000 & 6.350 & 12,500 & 5.670 \\
\hline Eeconerin 1800 & 2 & PQWe PTeAeSB TP & 1,100 he & 8,700 & 3.24 & 18,000 & $7,5 \times 0$ & 18,100 & 7.300 & 14,000 & 6.350 \\
\hline Embrer EMB-1 10 Eandeinante & 2 & PQWC. PTOA-34TP & $750 \mathrm{ch}$ & 0.505 & 3.255 & 13,010 & 5,000 & 12,590 & 5,700 & 12,015 & 5,450 \\
\hline Dorniv 220 & 2 & Gamen TPE301-5-2520 TP & $715 \operatorname{chs}$ & 7.00 & 3,547 & 12,583 & 5,700 & 12.568 & 5,700 & 11,000 & 5,400 \\
\hline Dortervilind Can. OHC.0-500 & 2 & PAW, PTEA-27TP & 6xosho & 7.415 & 3,300 & 12500 & 5,070 & 12,500 & 5,670 & 12,300 & 5,579 \\
\hline Eecherencs & 2 & PQWC FTQA-36TP & $715 \operatorname{shp}$ & 8,494 & 2046 & 11,300 & 5,128 & 11,500 & 5,120 & N.A. & N.A. \\
\hline CAF Nomed $24 A$ Commusertiner & 2 & Dowoin Alisen 250-8179 TP & $400 \sin$ & 5,107 & N.A. & 9.00 & N.A. & N.A. & N.A. & N.A. & N.A. \\
\hline GAF Nomed N228 Commutortined & 2 & Alicon 250-B17C TP & 420 hos & 5,453 & 2448 & 8,500 & 3.25 & $8,5 \infty$ & 3,255 & 8,250 & 3.742 \\
\hline Pilatus BN.2T Turbo tslander & 2 & Dotroil Altison $250-B 17 C$ TP & 400 thp & 4,040 & 1.832 & 7,000 & 3,175 & 6.800 & 3,004 & 6,300 & 2.857 \\
\hline
\end{tabular}

HETF = High Eypese Tutbotan TF = Turbotan

$T J=$ Turbojot
TP = Turoprop RP = Rudid Piston

IP = In-Line Piston 
Table 2.27 (continued)

Charecteristies of Schodulad Air Taxi (14 CFR 135) Airemaft

[Roterence 2.33 and 2.34]

\begin{tabular}{|c|c|c|c|c|c|c|c|c|c|c|c|c|c|}
\hline \multirow[b]{2}{*}{ Aircran Type } & \multicolumn{2}{|c|}{$\begin{array}{l}\text { Maximum } \\
\text { Fuol Capacity }\end{array}$} & \multicolumn{2}{|c|}{$\begin{array}{l}\text { Overall } \\
\text { Wing Span }\end{array}$} & \multicolumn{2}{|c|}{ Overall Length } & \multicolumn{2}{|c|}{ Loverall Height } & \multicolumn{2}{|c|}{ Wing Area } & \multirow{2}{*}{$\begin{array}{c}\text { Takeoft } \\
\text { Dist. (ft.) } \\
\text { (FAR) }\end{array}$} & \multirow{2}{*}{$\begin{array}{c}\text { Landing } \\
\text { Dist. (ft.) } \\
\text { (FAR) }\end{array}$} & \multirow{2}{*}{$\begin{array}{l}\text { Stall Spoed } \\
\text { (gear/laps } \\
\text { down, mph) }\end{array}$} \\
\hline & Imp. Gal. & Liters & Ft. In. & Melers & $\mathrm{Ft} . \mathrm{In}$ & Moters & Ft. In. & Metars & $F t .{ }^{\infty} 2$ & $M^{*-2} 2$ & & & \\
\hline Embrear EMB-120 Brasilia & 734 & 3,340 & $64^{\prime} 10.8^{\prime \prime}$ & $19.78 \mathrm{~m}$ & $65^{\prime} 7$ & $20.00 \mathrm{~m}$ & $20^{\prime} 10^{\circ}$ & $6.35 \mathrm{~m}$. & 424.5 & 39.4 & 4,660 & 4,495 & N.A. \\
\hline British Aerospece Jotstream 41 & N.A. & N.A. & $60^{\circ} 0^{\circ}$ & $18.29 \mathrm{~m}$ & $63^{\circ} 2$ & $19.25 \mathrm{~m}$ & $18^{\prime} 10^{*}$ & $5.74 \mathrm{~m}$. & 350.8 & 32.6 & N.A. & N.A. & N.A. \\
\hline Acrospetiale Nord 262C & 585 & 2,570 & $74: 2$ & $22.60 \mathrm{~m}$ & $63^{\circ}$ & $18.28 \mathrm{~m}$ & $20^{\prime} 4^{\prime \prime}$ & $6.21 \mathrm{~m}$. & 592.0 & 55.0 & 2,690 & N.A. & N.A. \\
\hline Shorts $330-200$ & 560 & 2,546 & $74^{\prime} 8^{\prime}$ & $22.76 \mathrm{~m}$ & $58^{\prime} 0.5$ & $17.69 \mathrm{~m}$ & $163^{\circ}$ & $4.85 \mathrm{~m}$. & 453.0 & 42.1 & 3,420 & 3,380 & N.A. \\
\hline British Aerospece Jetstream 31 & 378 & 1.718 & $520^{\circ}$ & $15.85 \mathrm{~m}$ & $471.5^{n}$ & $14.37 \mathrm{~m}$ & $17^{\prime \prime} 5.5^{\circ}$ & $5.32 \mathrm{~m}$. & 271.3 & 25.2 & 3,200 & 3,820 & N.A. \\
\hline Farchild Swearingen Metro III & 540 & 2,452 & $5710^{\circ}$ & $17.37 \mathrm{~m}$ & 594.37 & $18.09 \mathrm{~m}$ & $188^{4}$ & $5.08 \mathrm{~m}$. & 300.0 & 28.7 & 3250 & 2,805 & 99 \\
\hline Beechoreh 1800 & s5e & 1,627 & $54^{\prime} 6^{\circ}$ & $16.61 \mathrm{~m}$ & $57^{\prime} 10^{\circ}$ & $17.63 \mathrm{~m}$ & $14^{\prime}+1^{\prime \prime}$ & $4.54 \mathrm{~m}$. & 303.0 & 28.2 & 3,260 & 2540 & N.A. \\
\hline Embraer EMB-110 Eandeirante & 378 & 1,720 & $50^{\prime} 3.5$ & $15.33 \mathrm{~m}$ & $48^{\prime} 6.5^{\prime}$ & $15.10 \mathrm{~m}$ & $16^{\prime} 1.8^{\prime \prime}$ & $4.92 \mathrm{~m}$ & 313.2 & 29.1 & 4,000 & 4,400 & N.A. \\
\hline Dornier 228 & 525 & 2,396 & $55^{\prime} 8^{\circ}$ & $16.87 \mathrm{~m}$ & $54 \cdot 3$ & $16.55 \mathrm{~m}$ & $15^{\prime} 11.5^{\circ}$ & $4.86 \mathrm{~m}$ & 344.3 & 32.0 & 1845 & 1,970 & N.A. \\
\hline Dotheviltand Can. DHC 6-300 & 393 & 1,783 & $65^{\prime} 0^{\circ}$ & $19.84 \mathrm{~m}$ & $51^{\circ} 9$ & $15.7 \mathrm{~m}$ & $10^{\prime} 6^{\circ}$ & $5.94 \mathrm{~m}$. & 420.0 & 39.0 & 1,500 & 1.940 & 66 \\
\hline Beecheraft 099 & 310 & 1,412 & $45^{\prime} 10.5$ & $13.89 \mathrm{~m}$ & $44^{\prime} 7^{\prime}$ & $13.58 \mathrm{~m}$ & $14^{\prime} 4^{\circ}$ & $4.37 \mathrm{~m}$ & 279.7 & 26.0 & N.A. & N.A. & N.A. \\
\hline GAF Nomed 24 A Commuteriner & 39 & 1,770 & N.A. & N.A. & N.A. & N.A. & N.A. & N.A. & N.A. & N.A. & 1,706 & 1,380 & 65 \\
\hline GAF Nomed N228 Commulartiner & 371 & 1,688 & $54^{\prime} 2^{\prime \prime}$ & $16.51 \mathrm{~m}$ & $41^{\prime} 3$ & $12.57 \mathrm{~m}$ & $18^{\prime} 2^{\circ}$ & $5.5 \mathrm{~m}$. & 324.0 & 30.1 & 1,180 & 1,340 & 62 \\
\hline Pilatus BN.2T Turbo lstander & 108 & 492 & $53^{\prime} 0^{-1}$ & $16.15 \mathrm{~m}$ & $36^{\prime} 38^{\prime \prime}$ & $11.07 \mathrm{~m}$ & $13^{\circ} 88^{\circ}$ & $4.18 \mathrm{~m}$ & 337.0 & 31.3 & 1,250 & 1,115 & NA. \\
\hline
\end{tabular}

N.A. = Intormation not available when rble prepared 


\subsection{COMMERCIAL AVIATION REFERENCES}

[2.1] "Cerification and Operations: Domestic, Flag, and Supplemental Air Carriers and Commercial Operators of Large Aircraft", Ittle 14 A Amnautics and Space. Code of Federal Bequlations. Chaoter L-Federal Aviation Administration. Department of Transpontation. Subchaoter G-Air Carriers. Air Travel Clubs, and Ooerators for Compensation or Hire: Certification and Operations. Part 121 (14 CFR 1211, U.S. National Archives and Record Administration, Washington, DC, January 1, 1995.

[2.2] "Air Taxi Operators and Commercial Operators", Itte 14 Aerenautics and Space. Code of Eederal Reoulations, Chaoter L-Federal Aviation Administration. Department of Iranspontation Subchapter G-Air Carriers, Air Travel Clubs, and Operators for Comoensation or Hire: Certification and Operations, Part 135 (14 CFR 135). U.S. National Archives and Record Administration, Washington, DC, January 1, 1995.

[2.3] Commercial Aviation Safety, Alexander T. Wells, TAB Books, Bhe Ridge Summit, PA, 1991.

[2.4] "Air Traffic and General Operating and Flight Rules". Iitle 14 Aeronautics and Space, Code of Eederal Requlations. Chaoter L. Federal Aviation Administration. Department of Transportation. Subchapter F. Air Trattic and General Operatino Bules. Part 91 (14 CFR 91), U.S. National Archives and Record Administration, Washington, DC, January 1, 1995.

[2.5] DOE Standard Accident Analysis for Aircratt Crash inte Hazardous Facilities DOE-STD-3014-96, U.S. Department of Energy, Washington, DC, July 1996.

[2.6] Annual Beviews of Aircratt Accident Data. U.S. Air Carrier Operations. 1973. NTSB/ARC-74-2 U.S. National Transportation Safety Board, Bureau of Aviation Safety, Washington, DC, October 24, 1974.

[2.7] Annual Review of Aircraft Accident Data, U.S. Air Carrier Operations 1974. NTSB/ARC-76-1 U.S. National Transportation Safety Board, Bureau of Aviation Safety, Washington, DC, December 30, 1975.

[2.8] Annual Review of Aircratt Accident Data. U.S. Air Carier Operations. 1975. NTSB/ARC-77-1 U.S. National Transportation Safety Board, Bureau of Technology, Washington, DC, January 25 , 1977.

[2.9] Annual Review of Aircraft Accident Data. US, Air Camier Operations, 1976. NTSB/ARC-78-1 U.S. National Transportation Safety Board, Bureau of Technology, Washington, DC, January 5 , 1978.

[2.10] Annual Review of Aircraft Accident Data U.S. Air Carrier Operations. 1977. NTSB/ARC-78-2 U.S. National Transportation Safety Board, Bureau of Tochnology, Washington, DC, September 6, 1978.

[2.11] Annua| Review of Aircraft Accident Data. U.S. Air Carrier Operations. 1978. NTSB/ARC-80-1 U.S. National Transportation Safety Board, Bureau of Technology, Washington, DC, October 6 , 1980.

[2.12] Annual Review of Aircratt Accident Data, U.S. Air Carrier Operations. 1979. NTSB/ARC-81-1 U.S. National Transportation Safety Board, Bureau of Technology, Washington, DC, November 16, 1981.

[2.13] Annual Review of Aircraft Accident Data. U.S. Air Carrier Operations Calendar Year 1980 NTSB/ARC-83/01, U.S. National Transportation Safety Board, Bureau of Safety Programs, Washington, DC, January 14, 1983. 
[2.14] Annual Review of Aircraft Accident Data. U.S. Air Camier Operations Calendar Year 1981 NTSB/ABC-85/01 U.S. National Transportation Safety Board, Bureau of Safety Programs, Washington, DC, February 1, 1985.

[2.15] Annual Review of Aircratt Accident Data. US. Air Camier Operations Calendar Year 1982 NTSB/ABC-86/01 U.S. National Transportation Safety Board, Bureau of Safety Programs, Washington, DC, (No Date).

[2.16] Annual Review of Aircraft Accident Data. U.S. Air Carrier Operations Calendar Year 1983. NTSB/ARC-B7/01 U.S. National Transportation Safety Board, Bureau of Safety Programs, Washington, DC, February 13, 1987.

[2.17] Annual Review of Aircraft Accident Data. US. Air Carrier Operations Calendar Year 1984 NTSB/ARC-87/02, U.S. National Transportation Safety Board, Bureau of Safety Programs, Washington, DC, April 15, 1987.

[2.18] Annual Review of Aircraft Accident Data. U.S. Air Carrier Operations Calendar Year 1985, NTSB/ARC-87/03, U.S. National Transportation Safety Board, Bureau of Safety Programs, Washington, DC, November 27, 1987.

[2.19] Annual Beview of Aircraft Accident Data U.S. Air Carrier Operations, Calendar Year 1986. NTSB/ARC-89/01, U.S. National Transportation Safety Board, Bureau of Safety Programs, Washington, DC, February 3, 1989.

[2.20] Annual Beview of Aircraft Accident Data. U.S. Air Carrier Operations. Calendar Year 1987 NTSB/ARC-90/01, U.S. National Transportation Safety Board, Washington, DC, November 29 , 1990.

[2.21] Annual Beview of Aircraft Accident Data. U.S. Air Carrier Operations. Calendar Year 1988 NTSB/ABC-91101, U.S. National Transportation Safety Board, Washington, DC, April 18, 1991.

[2.22] Annual Beview of Aircratt Accident Data. U.S. Air Camier Operations, Calendar Year 1989 NTSB/ARC-93/01, U.S. National Transportation Satety Board, Washington, DC, May 7, 1993.

[2.23] Annual Beview of Aircraft Accident Data. U.S. Air Carrier Operations. Calendar Year 1990 NTSB/ARC-93/02, U.S. National Transportation Safety Board, Washington, DC, October 4, 1993.

[2.24] Annual Beview of Aircraft Accident Data. U.S. Air Carrier Operations, Calendar Year 1991 NTSB/ABC-94101, U.S. National Transportation Safety Board, Washington, DC, April 15, 1994.

[2.25] Annual Beview of Aircraft Accident Data. U.S. Air Carrier Operations. Calendar Year 1992 NTSB/ARC-94-02 U.S. National Transportation Safety Board, Washington, DC, September 15, 1994.

[2.26] Annual Beview of Aircraft Accident Data. U.S. Air Carrier Oeerations. Calendar Year 1993. NTSB/ARC-95/01. U.S. National Transportation Safety Board, Washington, DC, September 8 , 1995.

[2.27] NTSB News Annual Press Release on U.S. Airtine Accidents, SB 95-03, January 19, 1995.

[2.28] NTSB News Annual Press Release on U.S. Airline Accidents, SB 96-03, January 25, 1996.

[2.29] "Operations: Foreign Air Carriers and Foreign Operators of U.S.-Registered Aircraft Engaged in Common Carriage", Iitle 14 Aeronautics and Space. Code of Federal Requlations Chapter I. Eederal Aviation Administration. Department of Iransportation. Subchapter G-Air Carriers. Air 
Iravel Clubs, and Operators for Compensation or Hire: Certification and Operations. Part 129 14.CFR 129】 U.S. National Archives and Record Administration, Washington, DC, January 1, 1994.

[2.30] Statistical Summary of Commercial Jet Aircraft Accidems Woridwide Operations 1959-1993, Boeing Commercial Aiplane Group, Seattle, WA, March 1994.

[2.31] ACRAM Modelino Technical Supoont Document. LA-UR-96-2460/TSA-11-95-B12 C. Bolig, D. Sanzo, D. Stack, R. Mensing, and R. Glasser, Los Alamos National Laboratory, Los Alamos, NM, July, 1996.

[2.32] Probabilistic Model. Analysis and Comouter Code for Take-off and Landing Related Aircraft Crashes imto a Sinucture, UCBI-10-122512, Ron Glaser,Lawrence Livermore National Laboratory, Livermore, CA, February 6, 1996.

[2.33] Modem Commercial Aircratt. William Green, Gordon Swanborough, and John Mowinski, Portland House, New York, NY, 1987.

[2.34] The Intemational Directory of Civil Aircraft 1995/1996, Gerard Frawley and Jim Thorn, Aerospace Publications Pty Ltd, Westron Creek, Australia, 1995. 
APPENDIX 2.1 NATIONAL TRANSPORTATION SAFETY BOARD (NTSB) 14 CFR 121 ACCIDENT DATABASE USED IN THE ACRAM DATA DEVELOPMENT TECHNICAL SUPPORT DOCUMENT

This appendix presents the database of aircrafts that suffered an accident while ftying under 14 CFR 121 regulations during the years 1973 to 1994. This database was used in the development of the ACRAM Data Development Technical Support Document and was derived primarily from the National Transportation Safety Board accident database and various other NTSB publications. The following information, listed in order of cotumns from left to right, is presented for each accident in the database:

1. Accident Date (ACC. DATE)

2. Airtine or Operator of Aircraft and Flight Number (OPERATOR FH. No.)

3. Type of Flight (FLT. TYPE)

4. AIRCRAFT TYPE

5. Registration Number (REGN. NO.)

6. AIRCRAFT DAMAGE

7. Flight Phase that Accident Occurred (FLT. PHS.)

8. Airport Proximity (ONoff)

9. ACCIDENT LOCATION

10. Degree of Injuries Associated with Accident (Injury Degree)

11. Crew FATALITIES

12. Passenger (Pass.) FATALITIES

13. Other FATALITIES

14. Total (Crew+Pass.+Other) FATALITIES

15. Number of Serious Injuries (Serious Injury)

16. Total Crew Aboard Aircraft (Crew Aboard AC)

17. Total Passengers Aboard Aircraft (Pass. Aboard AC)

18. NTSB Report Where Information Was Obtained (NTSB Report)

19. NTSB File Number

20. NTSB First Occurrence Classification 


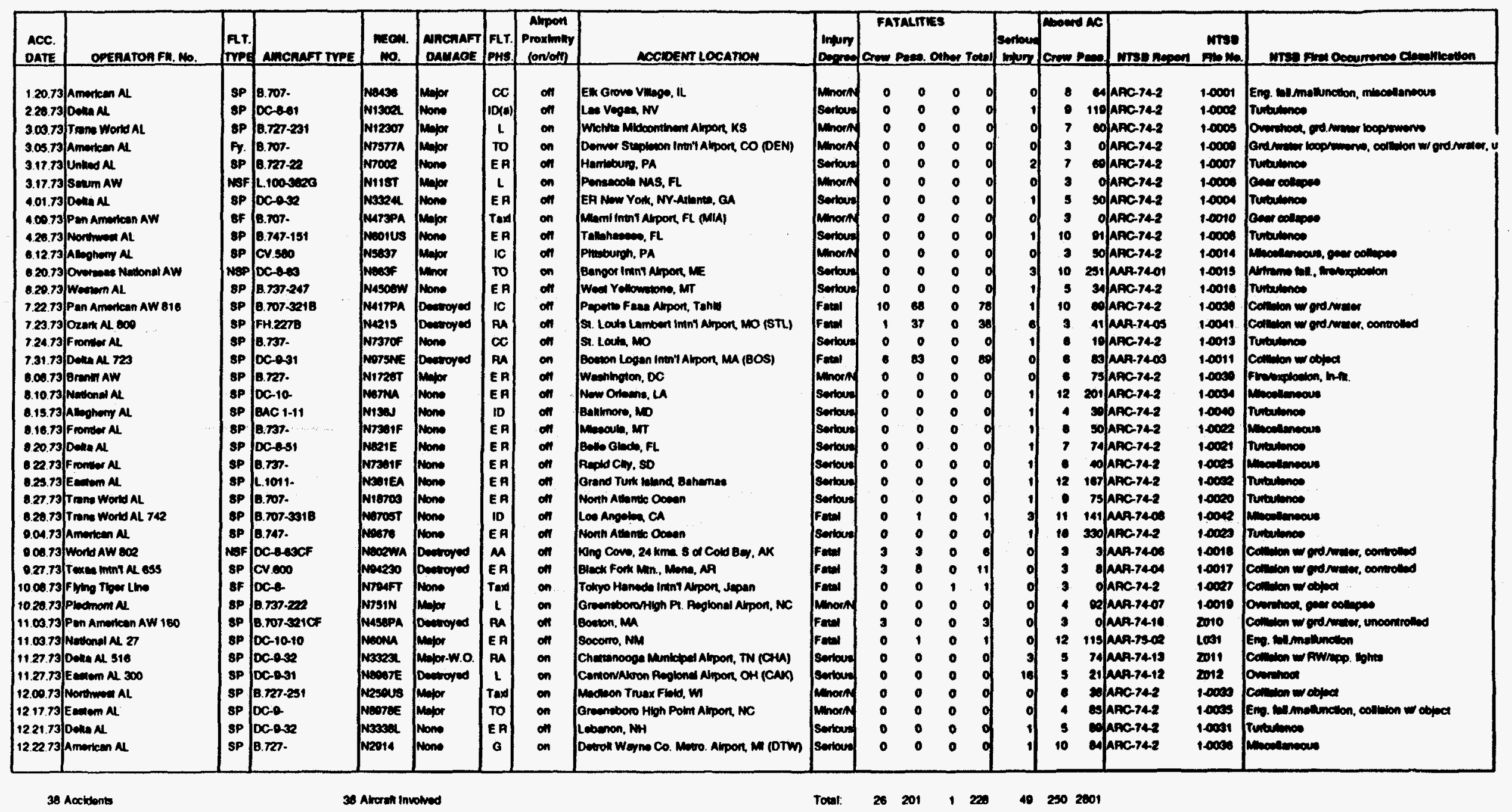




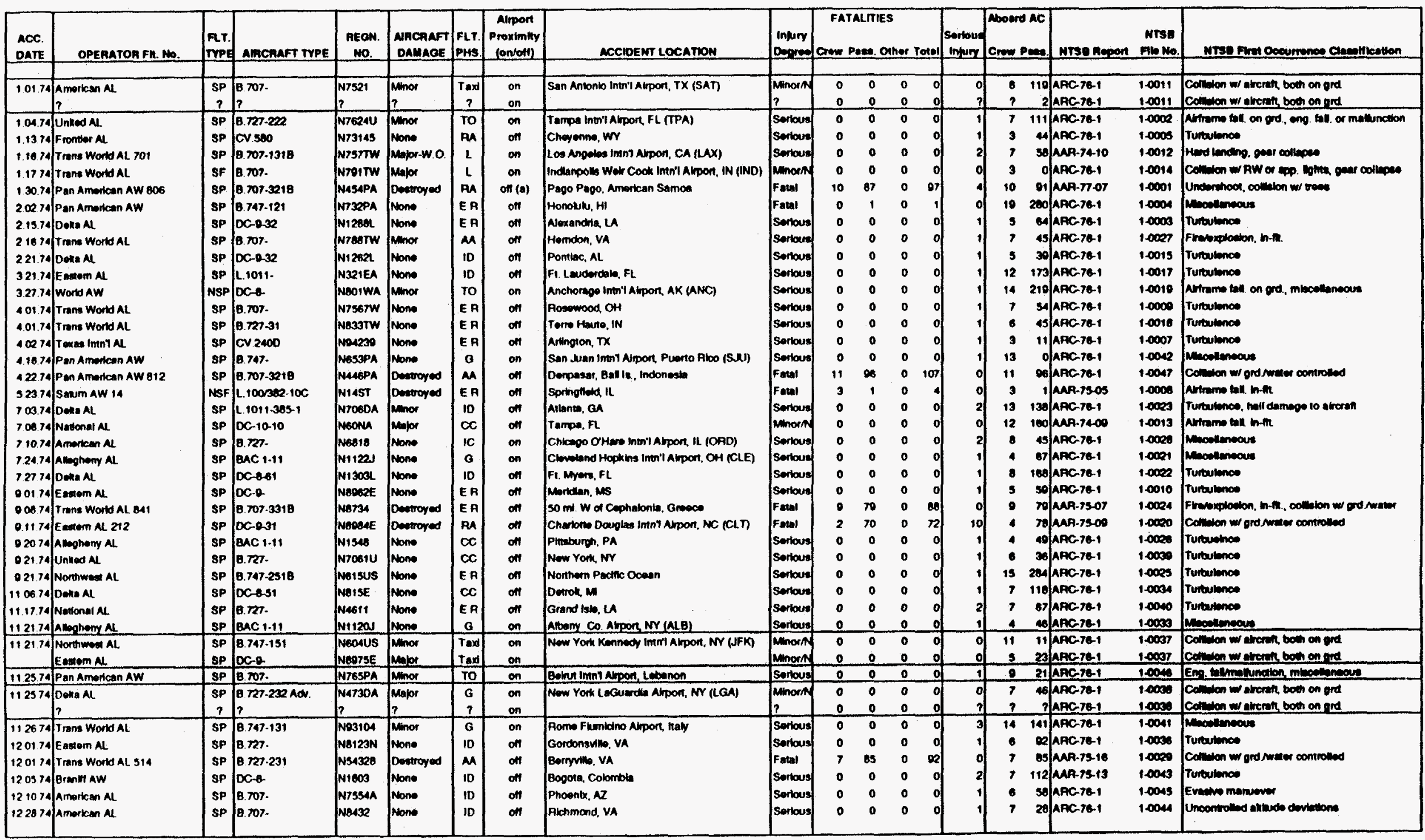




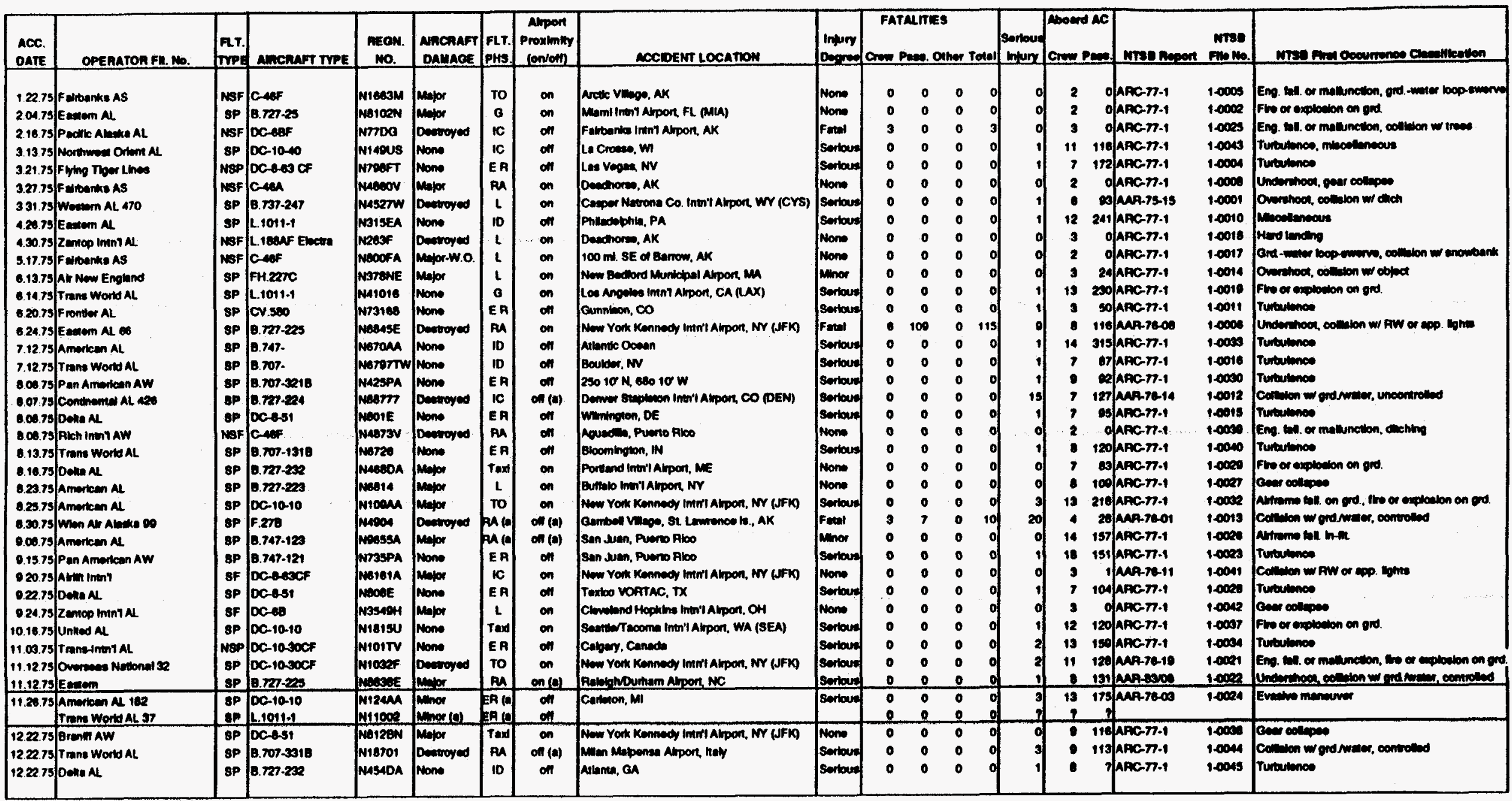




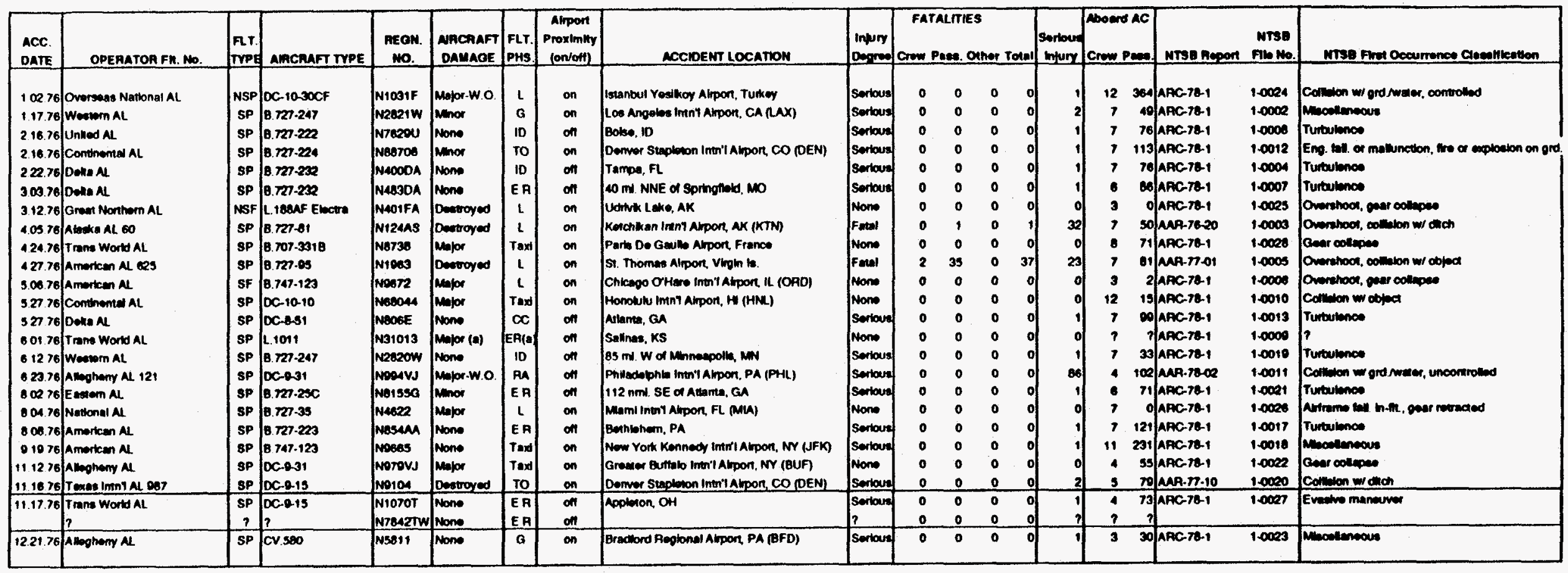




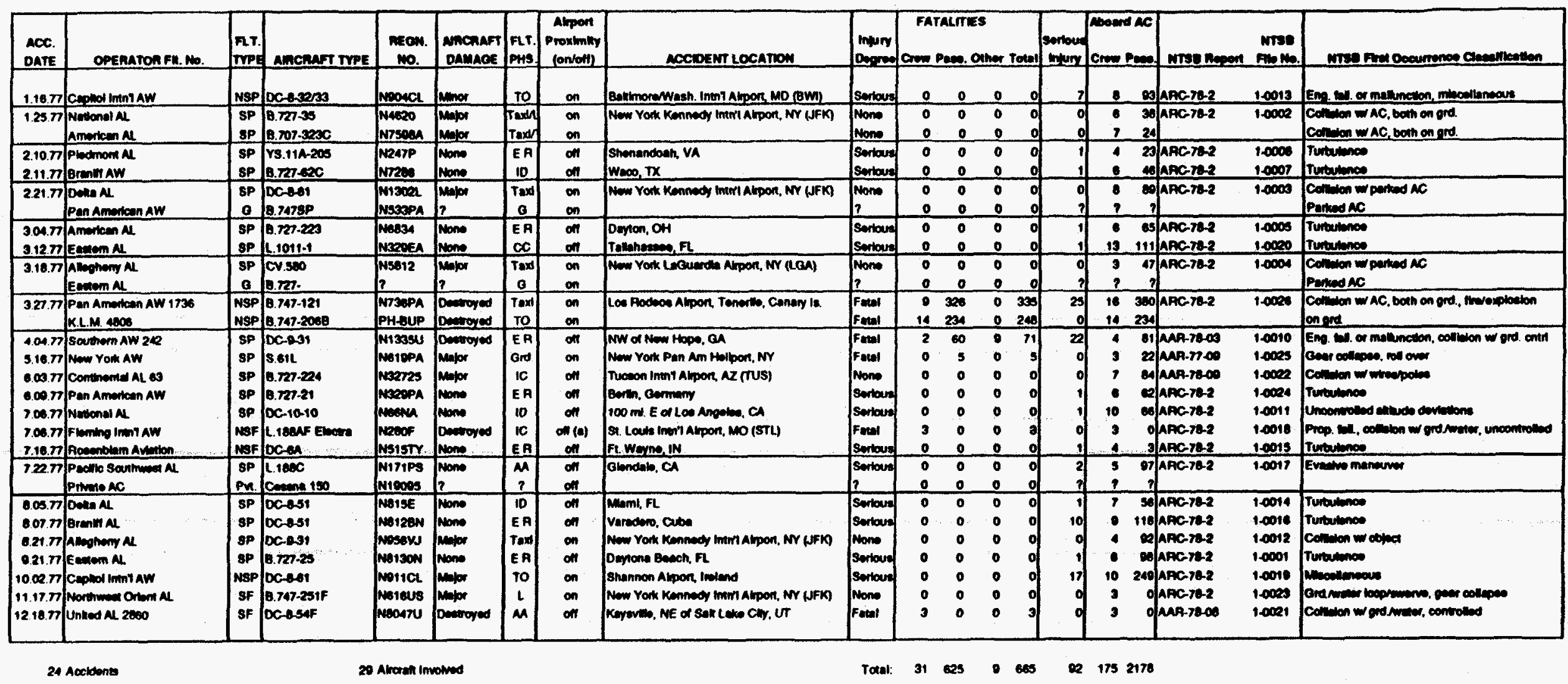




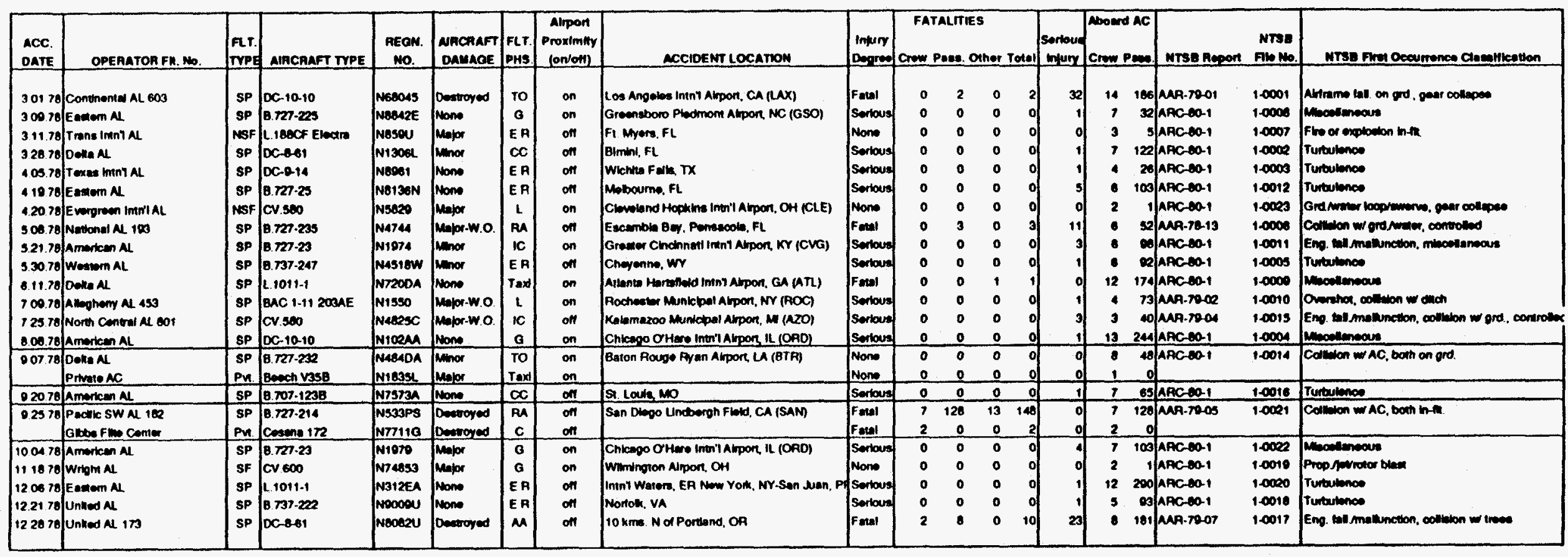

22 Aoctionts 


\begin{tabular}{|c|c|c|c|c|c|c|c|c|c|c|c|c|c|c|c|c|c|}
\hline $\begin{array}{l}\text { ACC. } \\
\text { OAIE }\end{array}$ & OPEAATOA FH. MO. & $\begin{array}{l}\text { Par. } \\
\text { rres: }\end{array}$ & AMCnaft TrPE & $\begin{array}{l}\text { mean. } \\
\text { No. }\end{array}$ & $\begin{array}{l}\text { NACMAFT } \\
\text { OAMAOE } \\
\end{array}$ & FLt. & $\begin{array}{c}\text { Alpont } \\
\text { Proxkmily } \\
\text { concofin }\end{array}$ & ACCIDEMT LOCATION & inguny & Lom $P$ & ALTKE: & & \begin{tabular}{|l|} 
sontoun \\
neveny
\end{tabular} & $\begin{array}{l}\text { abound ac } \\
\text { crom pen }\end{array}$ & Imserement & Finte. & 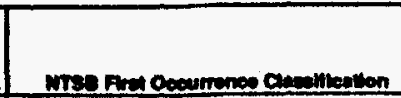 \\
\hline $\begin{array}{l}1.05 .70 \\
2.12 .70 \\
2.15 .70\end{array}$ & 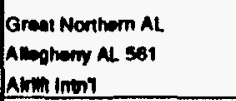 & $\begin{array}{c}\text { NSP } \\
\text { SP } \\
\text { SF } \\
\end{array}$ & 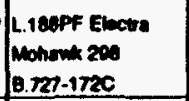 & $\begin{array}{l}\text { N4OOSGN } \\
\text { N20024 } \\
\text { N7274L }\end{array}$ & 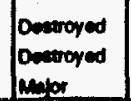 & $\begin{array}{c}\text { an } \\
\text { ic } \\
L\end{array}$ & $\begin{array}{l}\text { on } \\
\text { on } \\
\text { on }\end{array}$ & 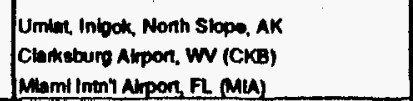 & $\begin{array}{l}\text { Innor } \\
\text { Fand } \\
\text { Nome }\end{array}$ & $\begin{array}{l}0 \\
1 \\
0\end{array}$ & $\begin{array}{l}0 \\
1 \\
0\end{array}$ & $\begin{array}{l}0 \\
0 \\
0\end{array}$ & & $\begin{array}{l}0 \\
3 \\
3\end{array}$ & $\begin{array}{l}\text { Aac-o101 } \\
\text { aAA-20-12 } \\
\text { ARC-2101 }\end{array}$ & $\begin{array}{l}10019 \\
1.0011 \\
1.0001\end{array}$ & 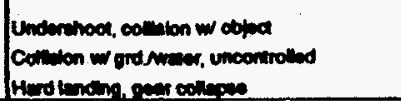 \\
\hline 2.15 .70 & Denta $N$ & $\begin{array}{l}\mathrm{SP} \\
\mathrm{SF}\end{array}$ & \begin{tabular}{|l}
$8.721-232$ \\
$B .747 .1325$
\end{tabular} & $\begin{array}{l}\text { NAG7DA } \\
\text { MEOAFT }\end{array}$ & Nomonos & $\begin{array}{c}T \text { Tax } \\
L\end{array}$ & on & 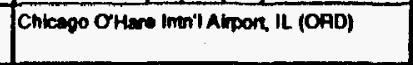 & Nome & 0 & $:$ & $\begin{array}{l}0 \\
0\end{array}$ & o & $\begin{array}{lr}0 & 100 \\
3 & 3 \\
\end{array}$ & ANA.72-11 & 1.0012 & $\begin{array}{l}\text { Collidion wAC, both on god } \\
\text { Codlen wAC, both on ord }\end{array}$ \\
\hline \begin{tabular}{|l|}
2.21 .70 \\
2.24 .70 \\
4.04 .70 \\
4.00 .70 \\
4.16 .70 \\
4.18 .70 \\
1.10 .70 \\
4.21 .70 \\
1.21 .70 \\
5.25 .70 \\
3.27 .70 \\
5.31 .70 \\
0.24 .70 \\
7.15 .70 \\
126.70 \\
1.07 .70 \\
0.16 .70 \\
0.12 .70 \\
0.18 .70 \\
10.14 .70 \\
10.31 .70 \\
11.16 .70 \\
11.18 .70 \\
11.24 .70 \\
12.27 .70
\end{tabular} & 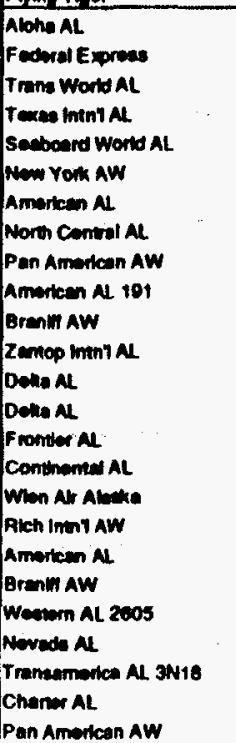 & 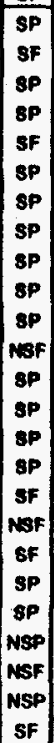 & 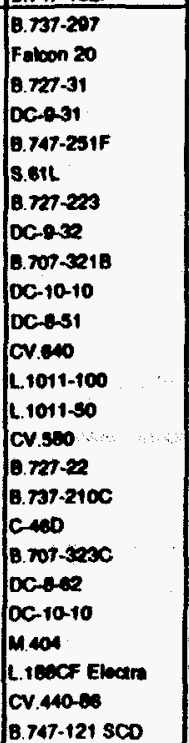 & 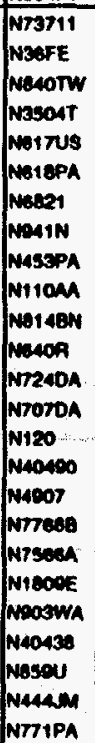 & 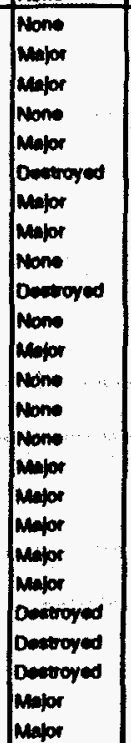 & \begin{tabular}{c|c|}
$E A$ \\
$L$ \\
$E A$ \\
$E A$ \\
$T O$ \\
$C C$ \\
$L$ \\
$L$ \\
10 \\
$1 C$ \\
0 \\
0 \\
$Q$ \\
$T a x$ \\
$E A$ \\
$L$ \\
$L$ \\
$D$ \\
$M$ \\
$L$ \\
$L$ \\
$L C$ \\
$\infty$ \\
$c$ \\
$L$
\end{tabular} & $\begin{array}{l}\text { of } \\
\text { on } \\
\text { of } \\
\text { on } \\
\text { on } \\
\text { of } \\
\text { on } \\
\text { on } \\
\text { of } \\
\text { of } \\
\text { on } \\
\text { on } \\
\text { on } \\
\text { on } \\
\text { on } \\
\text { on } \\
\text { on } \\
\text { of (n) } \\
\text { of } \\
\text { on } \\
\text { on } \\
\text { of } \\
\text { on } \\
\text { of } \\
\text { on }\end{array}$ & 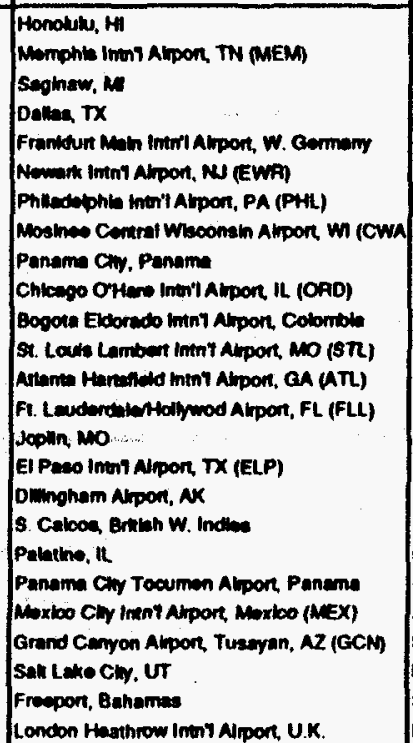 & 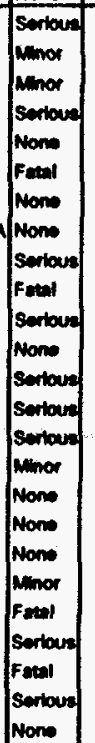 & $\begin{array}{c}0 \\
0 \\
0 \\
0 \\
0 \\
0 \\
0 \\
0 \\
0 \\
13 \\
0 \\
0 \\
0 \\
0 \\
0 \\
0 \\
0 \\
0 \\
0 \\
11 \\
0 \\
3 \\
0 \\
0\end{array}$ & $\begin{array}{l}0 \\
0 \\
0 \\
0 \\
0 \\
3 \\
0 \\
0 \\
0 \\
250 \\
0 \\
0 \\
0 \\
0 \\
0 \\
0 \\
0 \\
0 \\
0 \\
0 \\
01 \\
0 \\
0 \\
0 \\
0\end{array}$ & \begin{tabular}{cc|}
0 & 0 \\
0 & 0 \\
0 & 0 \\
0 & 0 \\
0 & 0 \\
0 & 0 \\
0 & 0 \\
0 & 0 \\
0 & 0 \\
2 & 273 \\
0 & 0 \\
0 & 0 \\
0 & 0 \\
0 & 0 \\
0 & 0 \\
0 & 0 \\
0 & 0 \\
0 & 0 \\
0 & 0 \\
0 & 0 \\
1 & 73 \\
0 & 0 \\
0 & 3 \\
0 & 0 \\
0 & 0
\end{tabular} & \begin{tabular}{r|}
2 \\
0 \\
0 \\
1 \\
0 \\
13 \\
0 \\
0 \\
1 \\
2 \\
4 \\
0 \\
2 \\
2 \\
0 \\
0 \\
0 \\
0 \\
0 \\
13 \\
10 \\
0 \\
1 \\
0
\end{tabular} & \begin{tabular}{|rr}
5 & 72 \\
3 & 0 \\
7 & 02 \\
3 & 113 \\
1 & 0 \\
3 & 19 \\
0 & 05 \\
1 & 37 \\
1 & 70 \\
13 & 250 \\
0 & 120 \\
1 & 0 \\
12 & 87 \\
12 & 200 \\
1 & 30 \\
7 & 36 \\
2 & 2 \\
2 & 1 \\
3 & 0 \\
0 & 130 \\
13 & 70 \\
3 & 41 \\
3 & 0 \\
3 & 43 \\
3 & 0
\end{tabular} & 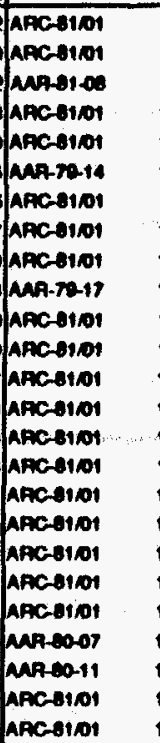 & 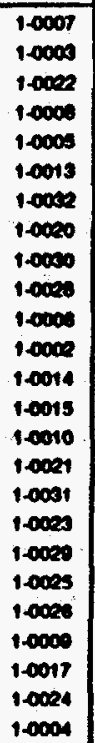 & 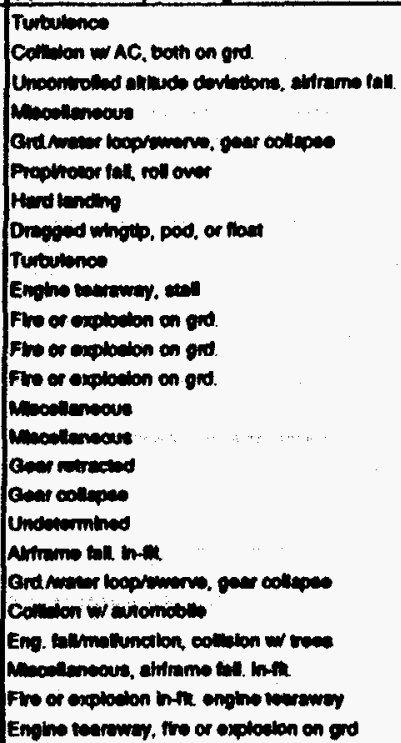 \\
\hline
\end{tabular}




\begin{tabular}{|c|c|c|c|c|c|c|c|c|c|c|c|c|c|c|c|c|c|}
\hline $\begin{array}{l}\text { ACC. } \\
\text { DATE }\end{array}$ & OPEAATOA FH. NO. & $\begin{array}{l}\text { FL, } \\
\text { TYPS }\end{array}$ & AMCAAFT TYPE & $\begin{array}{c}\text { AEOM. } \\
\text { NO. }\end{array}$ & $\begin{array}{l}\text { NACAAFT } \\
\text { DAMAOE } \\
\end{array}$ & $\begin{array}{l}\text { FLT. } \\
\text { PHS }\end{array}$ & $\begin{array}{c}\text { Ahport } \\
\text { Proxtmily } \\
\text { (onjoty) }\end{array}$ & ACCIDENT LOCATION & $\begin{array}{l}\text { Innury } \\
\text { Donneof }\end{array}$ & $\begin{array}{l}\text { FATI } \\
\text { Crom P }\end{array}$ & ALTTE: & Other Toded & 4 Sortove & $\begin{array}{l}\text { Abourd AC } \\
\text { Crom Peose }\end{array}$ & Irsenepon & Fum. & 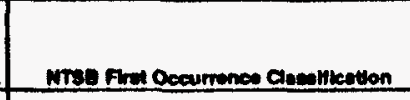 \\
\hline $\begin{array}{l}1.1860 \\
11080 \\
12380 \\
3.1780 \\
10480 \\
421.80\end{array}$ & 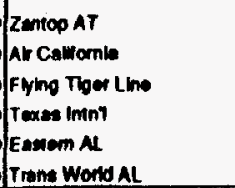 & \begin{tabular}{|} 
NSF \\
SP \\
SF \\
SP \\
SP \\
SP
\end{tabular} & $\begin{array}{l}0.1000 x \\
0.737-203 \\
0 C-8-634 F \\
0 c-0.14 \\
D C-0.31 \\
8.707-3318\end{array}$ & 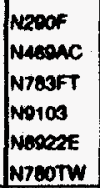 & $\begin{array}{l}\text { Major } \\
\text { Major } \\
\text { Mapor } \\
\text { Mapor-w.O. } \\
\text { Nono } \\
\text { None }\end{array}$ & $\begin{array}{c}E A \\
\text { Taxd } \\
\text { Toxi } \\
L \\
\text { EA } \\
10 \\
\end{array}$ & $\begin{array}{l}\text { on } \\
\text { on } \\
\text { on } \\
\text { on } \\
\text { on } \\
\text { on }\end{array}$ & 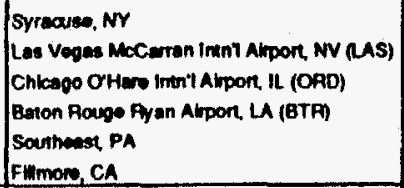 & 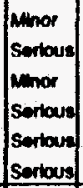 & $\begin{array}{l}0 \\
0 \\
0 \\
0 \\
0 \\
0\end{array}$ & $\begin{array}{l}0 \\
0 \\
0 \\
0 \\
0 \\
0\end{array}$ & $\begin{array}{l}0 \\
0 \\
0 \\
0 \\
0 \\
0\end{array}$ & $\begin{array}{l}0 \\
0 \\
0 \\
0\end{array}$ & \begin{tabular}{rr|}
4 & 0 \\
5 & 20 \\
4 & 0 \\
4 & 4 \\
5 & 63 \\
0 & 115 \\
\end{tabular} & $\begin{array}{l}\text { ARC-20201 } \\
\text { APC-2301 } \\
\text { APC-2301 } \\
\text { APC-6301 } \\
\text { APC-6201 } \\
\text { ARC-6301 }\end{array}$ & $\begin{array}{l}1.0000 \\
1.0007 \\
1.0005 \\
1.0015 \\
1.0000 \\
1.0010 \\
\end{array}$ & 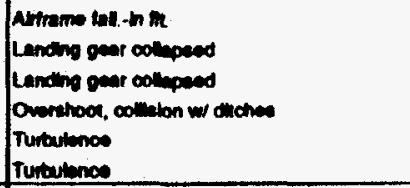 \\
\hline 60780 & $\begin{array}{l}\text { Heghoe Ahmeat } \\
\text { Privero AC }\end{array}$ & \begin{tabular}{|l} 
SP \\
$\mathrm{Pu}$ \\
\end{tabular} & $\begin{array}{l}D C-0.31 \\
C \operatorname{cosen} 1152\end{array}$ & $\begin{array}{l}\text { NoitsaW } \\
\text { Ne67640 }\end{array}$ & Mnnor & \begin{tabular}{|l}
$\operatorname{tanx}$ \\
$\operatorname{Taxd}$
\end{tabular} & $\begin{array}{l}\text { on } \\
\text { on }\end{array}$ & Oranpe County John Wayno Arpor, CA (SN) & Nono & : & $\begin{array}{l}0 \\
0 \\
\end{array}$ & 0 & & & ARC-0301 & 1.0000 & 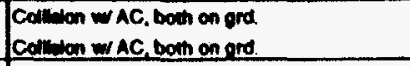 \\
\hline 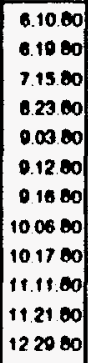 & 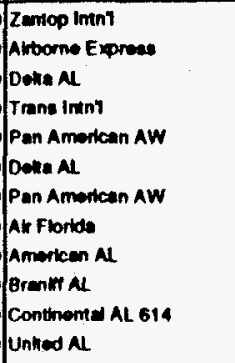 & \begin{tabular}{|l} 
NSF \\
$S F$ \\
$S P$ \\
NSF \\
$S P$ \\
$S P$ \\
$S P$ \\
$S P$ \\
$S P$ \\
$S P$ \\
SP \\
NSP
\end{tabular} & 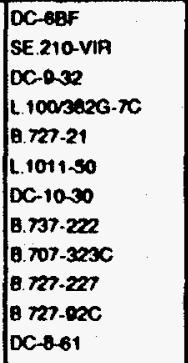 & 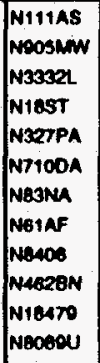 & 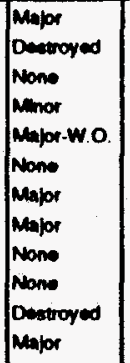 & $\begin{array}{c}1 \\
L \\
10 \\
1 D \\
P M \\
E R \\
T O \\
L \\
C C \\
L \\
L \\
T=x\end{array}$ & $\begin{array}{l}\text { on } \\
\text { on } \\
\text { of } \\
\text { of } \\
\text { on } \\
\text { of } \\
\text { on } \\
\text { on } \\
\text { of } \\
\text { on } \\
\text { on } \\
\text { on }\end{array}$ & 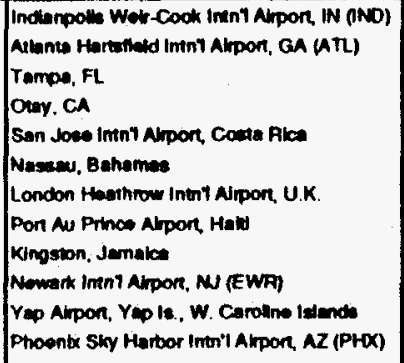 & 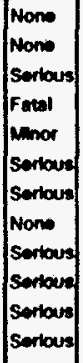 & $\begin{array}{l}0 \\
0 \\
0 \\
0 \\
0 \\
0 \\
0 \\
0 \\
0 \\
0 \\
0 \\
0\end{array}$ & $\begin{array}{l}0 \\
0 \\
0 \\
0 \\
0 \\
0 \\
0 \\
0 \\
0 \\
0 \\
0 \\
0\end{array}$ & $\begin{array}{l}0 \\
0 \\
0 \\
1 \\
0 \\
0 \\
0 \\
0 \\
0 \\
0 \\
0 \\
0\end{array}$ & $\begin{array}{l}0 \\
0 \\
0 \\
0 \\
0 \\
0 \\
0 \\
0\end{array}$ & $\begin{array}{rr}3 & 0 \\
3 & 1 \\
3 & 31 \\
1 & 0 \\
6 & 67 \\
12 & 37 \\
17 & 217 \\
6 & 73 \\
7 & 03 \\
7 & 30 \\
1 & 67 \\
0 & 230\end{array}$ & 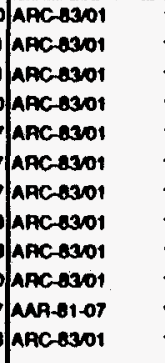 & $\begin{array}{l}1.0008 \\
1.0011 \\
1.0001 \\
1.0001 \\
1.0017 \\
1.0010 \\
1.0016 \\
1.0018 \\
10012 \\
1.0013 \\
1.0002 \\
1.0014\end{array}$ & 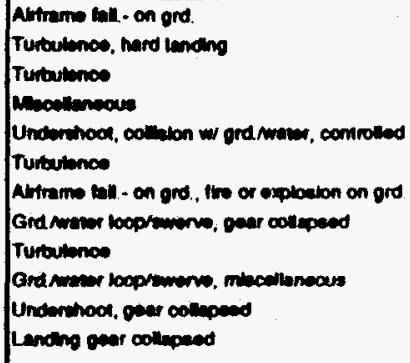 \\
\hline
\end{tabular}




\begin{tabular}{|c|c|c|c|c|c|c|c|c|c|c|c|c|c|c|c|c|c|}
\hline $\begin{array}{l}\text { ACC. } \\
\text { OATE }\end{array}$ & OPERATOA FR. MO. & rrt: & AnCnAFT TME & $\begin{array}{c}\text { neom. } \\
\text { mo. }\end{array}$ & $\begin{array}{l}\text { NACANAFT } \\
\text { OAMAGE }\end{array}$ & $\begin{array}{l}\text { FLT. } \\
\text { PHAS. }\end{array}$ & $\begin{array}{c}\text { Alpoort } \\
\text { Proxtminy } \\
\text { confotit }\end{array}$ & ACCIDERT LOCATIOM & Impury & $\begin{array}{l}\text { FATA } \\
\text { Com Po }\end{array}$ & LITES & ther roud & semoun & 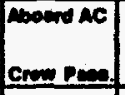 & Eermepon & $\begin{array}{c}\text { Nises } \\
\text { mus } \\
\end{array}$ & 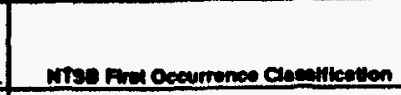 \\
\hline 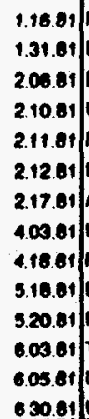 & 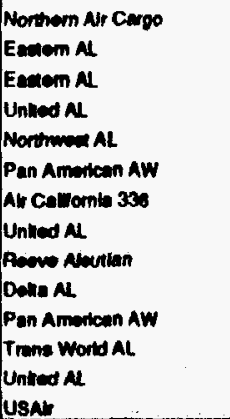 & $\begin{array}{l}S F \\
S P \\
S P \\
S P \\
S P \\
S P \\
S P \\
S P \\
8 P \\
S P \\
S P \\
8 P \\
8 P \\
S P\end{array}$ & 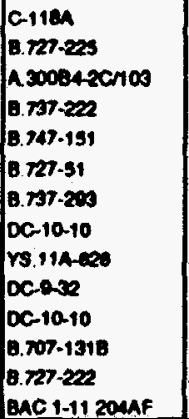 & 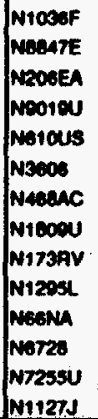 & 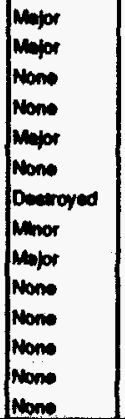 & $\begin{array}{c}E A \\
L \\
G \\
10 \\
T a x \\
0 \\
L \\
E A \\
R A \\
E A \\
T E d \\
G \\
E A \\
10\end{array}$ & $\begin{array}{l}\text { on } \\
\text { on } \\
\text { on } \\
\text { on } \\
\text { on } \\
\text { on } \\
\text { on } \\
\text { on } \\
\text { on } \\
\text { on } \\
\text { on } \\
\text { on } \\
\text { on } \\
\text { on }\end{array}$ & 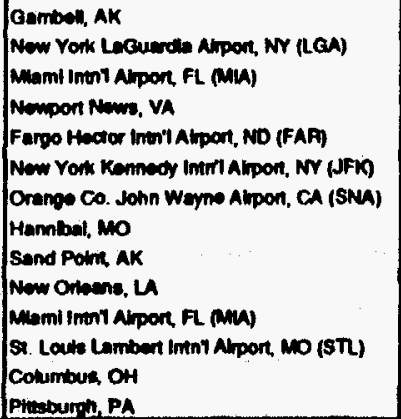 & 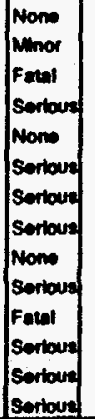 & $\begin{array}{l}0 \\
0 \\
0 \\
0 \\
0 \\
0 \\
0 \\
0 \\
0 \\
0 \\
0 \\
0 \\
0\end{array}$ & $\begin{array}{l}0 \\
0 \\
0 \\
0 \\
0 \\
0 \\
0 \\
0 \\
0 \\
0 \\
0 \\
0 \\
0 \\
0\end{array}$ & $\begin{array}{l}0 \\
\vdots \\
0 \\
0 \\
0 \\
0 \\
\vdots \\
\vdots \\
\vdots \\
0\end{array}$ & $\begin{array}{l}0 \\
0 \\
0 \\
1 \\
0 \\
1 \\
4 \\
4 \\
0 \\
0 \\
1 \\
0 \\
0 \\
1\end{array}$ & 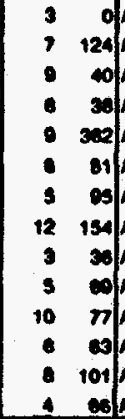 & 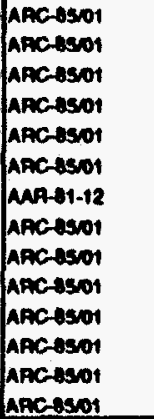 & $\begin{array}{l}10000 \\
10014 \\
10007 \\
10000 \\
10000 \\
10000 \\
10024 \\
10010 \\
10001 \\
10002 \\
10000 \\
10004 \\
10010 \\
10000\end{array}$ & 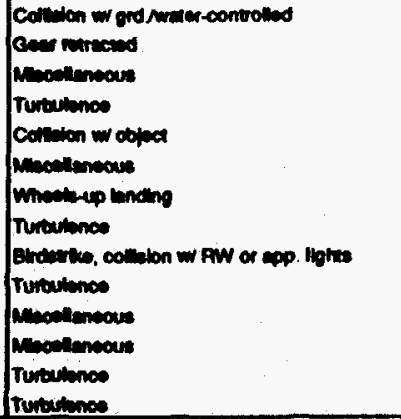 \\
\hline 8.13 .81 & $\begin{array}{l}\text { Orank N } \\
\text { Avpon Tomitul Serves }\end{array}$ & $\begin{array}{c}8 P \\
?\end{array}$ & $\begin{array}{l}\text { DC-2.20 } \\
\sin 20 . T C\end{array}$ & $\begin{array}{l}\text { Nopal } \\
\text { nsezen }\end{array}$ & Mapror & $\begin{array}{ll}0 \\
0\end{array}$ & on & S. Louk Lamben ImnT Ahpor, MO (ST) & Sortoun & 0 & $\begin{array}{l}0 \\
0\end{array}$ & $\begin{array}{ll}0 & 0 \\
0 & 0 \\
\end{array}$ & & \begin{tabular}{ll|}
3 & 54 \\
1 & 1
\end{tabular} & AAC-OSO1 & 10015 & 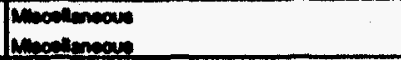 \\
\hline $\begin{array}{l}0.17 .81 \\
0.25 .81 \\
0.03 .81 \\
0.07 .61 \\
0.20 .81 \\
0.22 .81 \\
0.2281 \\
10.12 .81 \\
10.13 .81 \\
10.23 .01 \\
12.30 .81\end{array}$ & 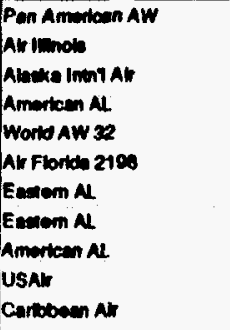 & \begin{tabular}{l|} 
SP \\
SP \\
NSF \\
SP \\
SP \\
SP \\
SP \\
SP \\
SP \\
SP \\
SF
\end{tabular} & 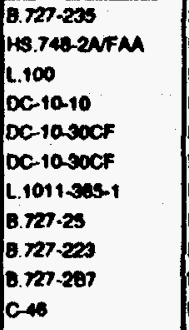 & 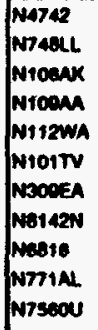 & 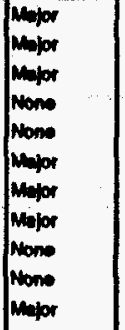 & 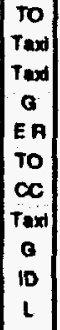 & $\begin{array}{l}\text { on } \\
\text { on } \\
\text { on } \\
\text { on } \\
\text { on } \\
\text { on } \\
\text { on } \\
\text { on } \\
\text { on } \\
\text { on } \\
\text { on }\end{array}$ & 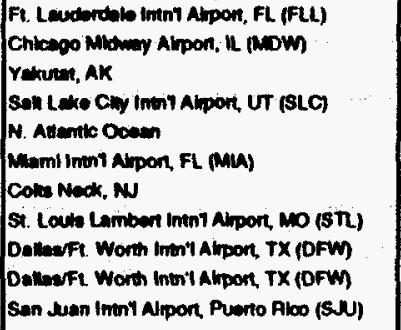 & 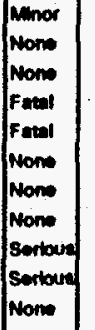 & $\begin{array}{l}0 \\
0 \\
0 \\
0 \\
1 \\
0 \\
0 \\
0 \\
0 \\
0 \\
0\end{array}$ & $\begin{array}{l}0 \\
0 \\
1 \\
0 \\
0 \\
0 \\
0 \\
0 \\
0\end{array}$ & $\begin{array}{l}0 \\
0 \\
0 \\
0 \\
0 \\
0 \\
0 \\
0 \\
0 \\
0 \\
0\end{array}$ & of & 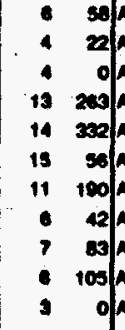 & 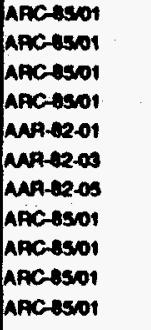 & $\begin{array}{l}1+003 \\
1+0014 \\
10012 \\
1+013 \\
1+002 \\
10003 \\
1+0028 \\
1+0021 \\
10017 \\
10018 \\
1+0010\end{array}$ & 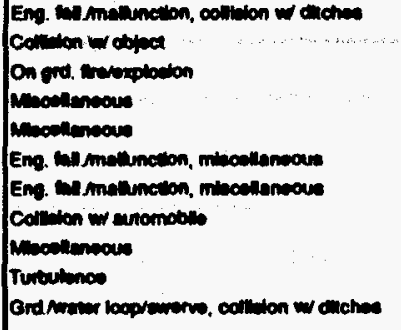 \\
\hline
\end{tabular}

26 Aockdons

27 Arrem imolnod

Tat: 1,24211072511 
APPENDIX A2.1 NTSB 14 CFR 121 ACCIDENT DATABASE

\begin{tabular}{|c|c|c|c|c|c|c|c|c|c|c|c|c|c|c|c|c|c|c|}
\hline $\begin{array}{l}\text { ACC. } \\
\text { DATE }\end{array}$ & OPERATOR FH. HO. & $\mid \begin{array}{ll}\mathrm{nt} \\
\mathrm{mpP}\end{array}$ & AMCAART TPPE & $\begin{array}{c}\text { REON. } \\
\text { NO. }\end{array}$ & $\begin{array}{l}\text { MACMAFT } \\
\text { DAMAOE }\end{array}$ & $\begin{array}{l}\text { FLT. } \\
\text { PHS }\end{array}$ & $\begin{array}{c}\text { Alpoon } \\
\text { Proxhmily } \\
\text { Conuoti) }\end{array}$ & ACCIDENT LOCATION & $\left|\begin{array}{l}\text { Inpory } \\
\text { Dompon }\end{array}\right|$ & $\begin{array}{l}\text { FATAL } \\
\text { Crom Pas }\end{array}$ & LTIES & ther Tot: & & infury & $\begin{array}{l}\text { Aboond AC } \\
\text { Crew Pane }\end{array}$ & MTse nepon & Fiboto. & NTse Fret Ocaumence Quentlketton \\
\hline 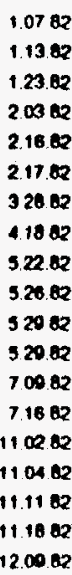 & 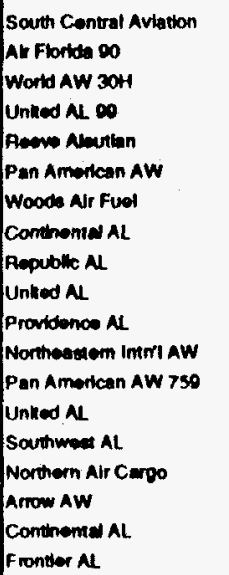 & \begin{tabular}{|c|} 
NSF \\
SP \\
SP \\
$S P$ \\
$S P$ \\
NSF \\
$S P$ \\
$S P$ \\
$S P$ \\
$S F$ \\
SP \\
SP \\
SP \\
SP \\
NSF \\
NSF \\
SP \\
SP
\end{tabular} & 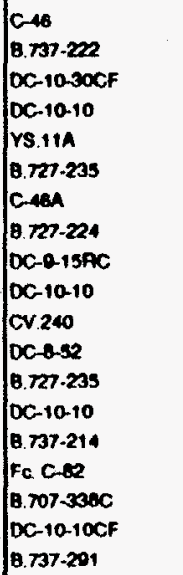 & 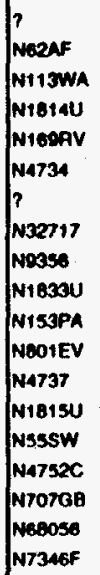 & 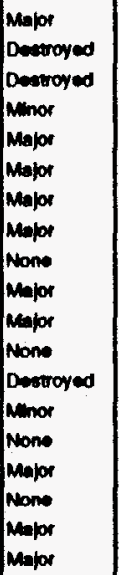 & 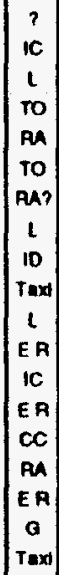 & $\begin{array}{l}\text { on (a) } \\
\text { of } \\
\text { on } \\
\text { on } \\
\text { of } \\
\text { on } \\
\text { of (a) } \\
\text { on } \\
\text { of } \\
\text { on } \\
\text { on } \\
\text { of } \\
\text { of } \\
\text { of } \\
\text { of } \\
\text { on } \\
\text { oft } \\
\text { on } \\
\text { on }\end{array}$ & 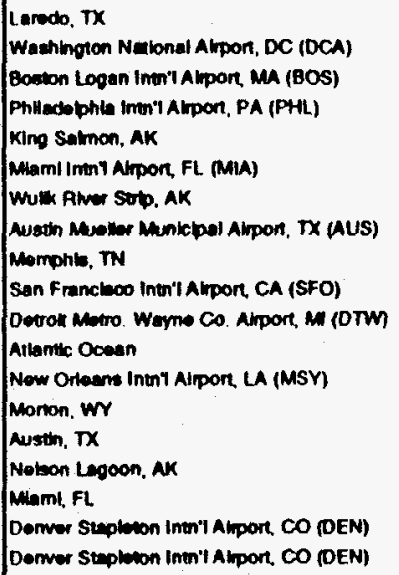 & 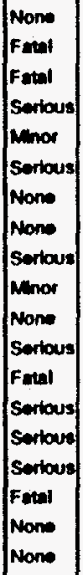 & $\begin{array}{l}0 \\
1 \\
0 \\
0 \\
0 \\
0 \\
0 \\
0 \\
0 \\
0 \\
0 \\
0 \\
7 \\
0 \\
0 \\
0 \\
0 \\
1 \\
0 \\
0\end{array}$ & $\begin{array}{c}0 \\
70 \\
2 \\
0 \\
0 \\
0 \\
0 \\
0 \\
0 \\
0 \\
0 \\
0 \\
36 \\
0 \\
0 \\
0 \\
0 \\
0 \\
0\end{array}$ & $\begin{array}{l}0 \\
0 \\
0 \\
0 \\
0 \\
0 \\
0 \\
0 \\
0 \\
0 \\
0 \\
0 \\
0 \\
0 \\
0 \\
0 \\
0 \\
0 \\
0 \\
0\end{array}$ & $\begin{array}{r}0 \\
78 \\
2 \\
0 \\
0 \\
0 \\
0 \\
0 \\
0 \\
0 \\
0 \\
0 \\
130\end{array}$ & \begin{tabular}{l|}
0 \\
6 \\
1 \\
1 \\
0 \\
1 \\
0 \\
0 \\
1 \\
0 \\
0 \\
1 \\
0 \\
7 \\
1 \\
2 \\
0 \\
0
\end{tabular} & 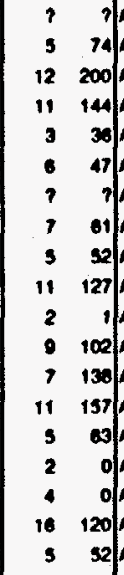 & 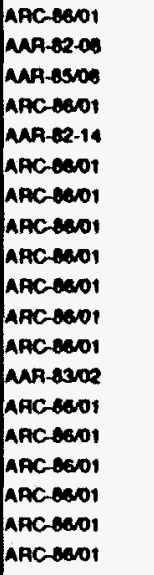 & $\begin{array}{l}1600 \\
0050 \\
2000 \\
1100 \\
2170 \\
3140 \\
3300 \\
2400 \\
3200 \\
2000 \\
3413 \\
2027\end{array}$ & 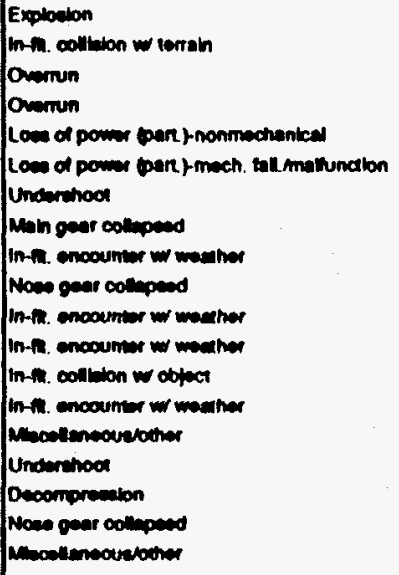 \\
\hline
\end{tabular}

10 Aocidenens

10 Arcrant Involved

Tan: $\begin{array}{lllllll}12 & 210 & 12 & 234 & 33 & 121 & 1374\end{array}$ 


\begin{tabular}{|c|c|c|c|c|c|c|c|c|c|c|c|c|c|c|c|c|c|}
\hline $\begin{array}{l}\text { ACC. } \\
\text { DATE }\end{array}$ & OPEAATOAFR. NO. & rir. & OARCANFT TYPE & $\begin{array}{l}\text { neon. } \\
\text { mo. }\end{array}$ & $\begin{array}{c}\text { Anchart } \\
\text { OAMAOE }\end{array}$ & $\begin{array}{l}\text { FLT. } \\
\text { PHAS }\end{array}$ & $\begin{array}{c}\text { Alpoent } \\
\text { Proximny } \\
\text { convelf }\end{array}$ & ACCDENT LOCATIOH & $\begin{array}{l}\text { Inquy } \\
\text { Dompen }\end{array}$ & anemp $p$ & LLTHE & 1 & antouns & $\begin{array}{l}\text { Abound AC } \\
\text { Crnow Pene }\end{array}$ & ITsenepont & NTSO & 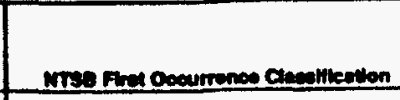 \\
\hline 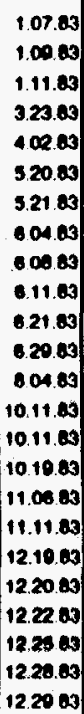 & 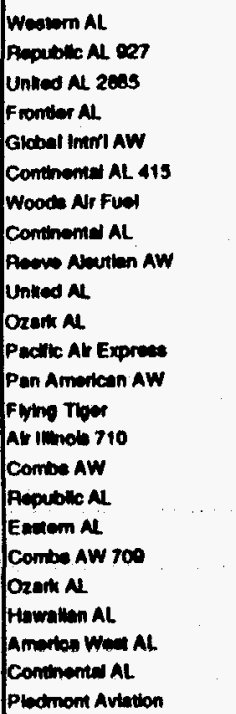 & \begin{tabular}{|l}
$S P$ \\
$S P$ \\
$S P$ \\
$S P$ \\
$N S P$ \\
$S P$ \\
$N S F$ \\
$S P$ \\
$S P$ \\
$S P$ \\
$S P$ \\
$S P$ \\
$S P$ \\
$S P$ \\
$S P$ \\
$S F$ \\
$S P$ \\
$S P$ \\
$S P$ \\
$S P$ \\
$S P$ \\
$S P$ \\
$S P$ \\
$S P$
\end{tabular} & 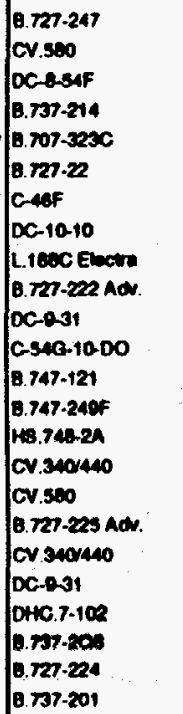 & 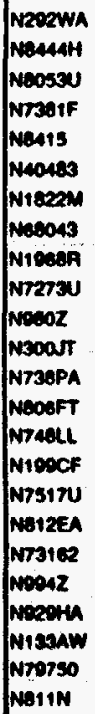 & 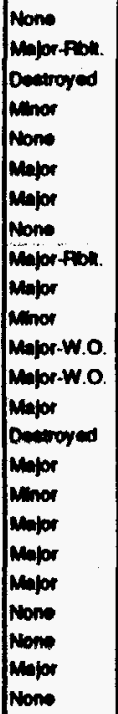 & 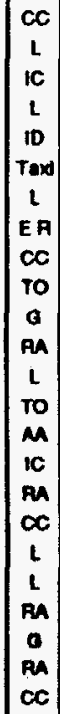 & $\begin{array}{l}\text { on } \\
\text { on } \\
\text { on } \\
\text { on } \\
\text { on } \\
\text { on } \\
\text { on } \\
\text { of } \\
\text { on } \\
\text { on } \\
\text { on } \\
\text { of } \\
\text { on } \\
\text { on } \\
\text { on } \\
\text { on } \\
\text { on } \\
\text { on } \\
\text { on } \\
\text { on } \\
\text { of } \\
\text { on } \\
\text { on } \\
\text { of }\end{array}$ & 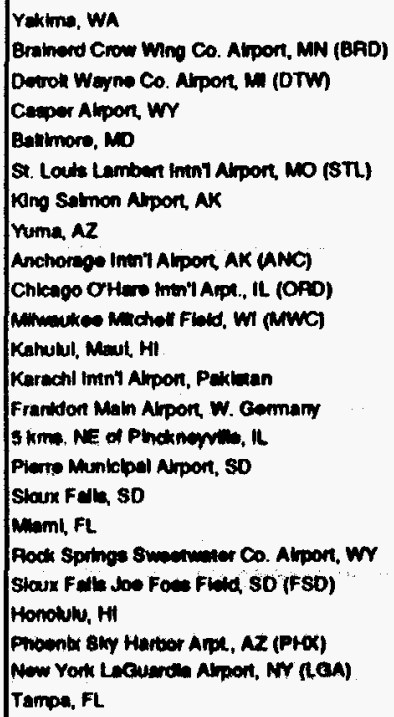 & 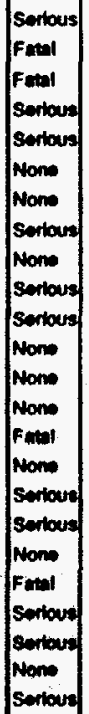 & $\begin{array}{l}0 \\
0 \\
3 \\
0 \\
0 \\
0 \\
0 \\
0 \\
0 \\
0 \\
0 \\
0 \\
0 \\
0 \\
3 \\
0 \\
0 \\
0 \\
0 \\
0 \\
0 \\
0 \\
0 \\
0\end{array}$ & $\begin{array}{l}0 \\
1 \\
0 \\
0 \\
0 \\
0 \\
0 \\
0 \\
0 \\
0 \\
0 \\
0 \\
0 \\
0 \\
0 \\
0 \\
0 \\
0 \\
0 \\
0 \\
0 \\
0 \\
0\end{array}$ & $\begin{array}{l}0 \\
0 \\
0 \\
0 \\
0 \\
0 \\
0 \\
0 \\
0 \\
0 \\
0 \\
0 \\
0 \\
0 \\
0 \\
0 \\
0 \\
1 \\
0 \\
0 \\
0\end{array}$ & $\begin{array}{l}0 \\
0 \\
0 \\
0 \\
0\end{array}$ & 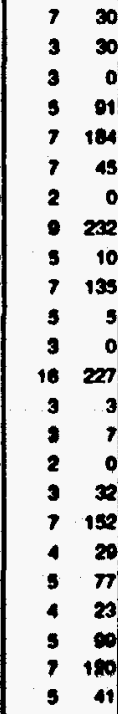 & 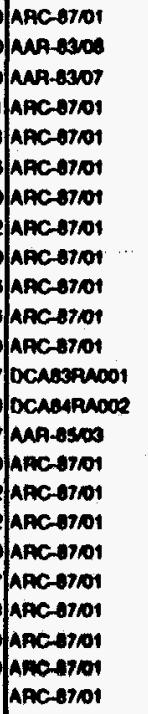 & 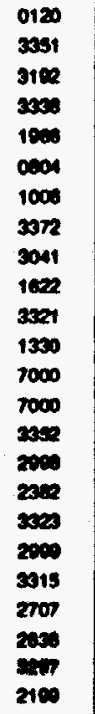 & 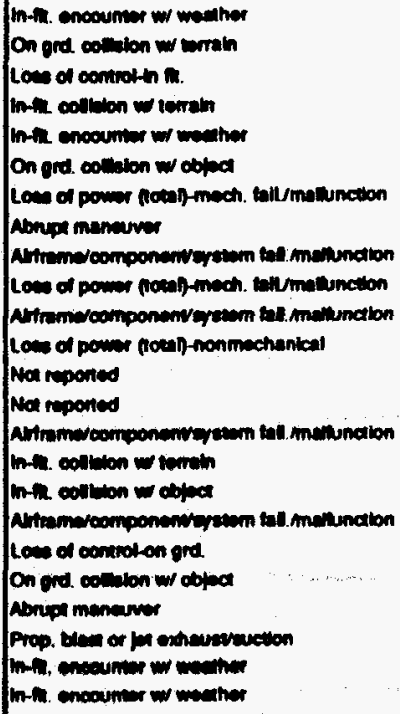 \\
\hline
\end{tabular}


APPENDIX A2.1 NTSB 14 CFA 121 ACCIDENT DATABASE

\begin{tabular}{|c|c|c|c|c|c|c|c|c|c|c|c|c|c|c|c|c|c|}
\hline $\begin{array}{l}\text { ACC. } \\
\text { DATE }\end{array}$ & OPEAATOAFL. No. & Int: & AMCANAT TYPE & $\begin{array}{c}\text { MEON. } \\
\text { No. }\end{array}$ & $\begin{array}{l}\text { AMCMART } \\
\text { DAMAOE }\end{array}$ & $\begin{array}{l}\text { FLT. } \\
\text { PHS. }\end{array}$ & $\begin{array}{c}\text { Alpport } \\
\text { Proximiny } \\
\text { condotil }\end{array}$ & ACCRDENT LOCATION & 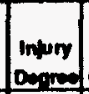 & $\begin{array}{l}\text { FATA } \\
\text { Con P P }\end{array}$ & LITES & nner rotal & sombere & $\begin{array}{l}\text { noper AC } \\
\text { Crow pons }\end{array}$ & ursenerent & $\begin{array}{c}\text { Nrse } \\
\text { Pib }\end{array}$ & 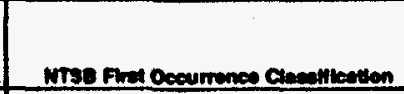 \\
\hline 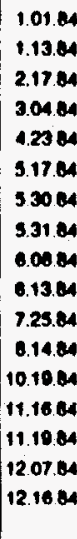 & 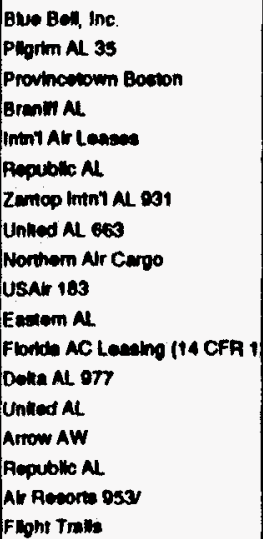 & \begin{tabular}{|l|}
$\mathrm{NSF}$ \\
$\mathrm{SP}$ \\
$\mathrm{SP}$ \\
$\mathrm{SP}$ \\
$\mathrm{SP}$ \\
$\mathrm{SP}$ \\
$\mathrm{SF}$ \\
$\mathrm{SP}$ \\
$\mathrm{NSF}$ \\
$\mathrm{SP}$ \\
$\mathrm{SP}$ \\
$\mathrm{NSF}$ \\
$\mathrm{SP}$ \\
$\mathrm{SP}$ \\
$\mathrm{SP}$ \\
$\mathrm{SP}$ \\
$\mathrm{NSP}$
\end{tabular} & 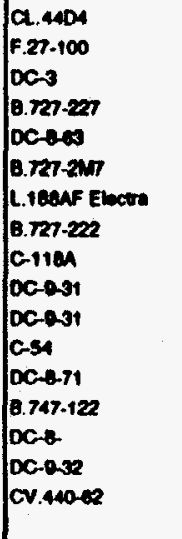 & 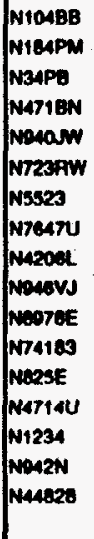 & 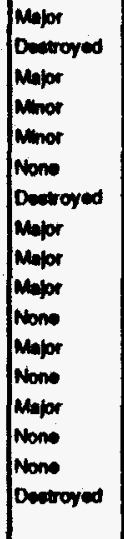 & 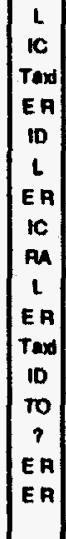 & $\begin{array}{l}\text { on } \\
\text { on } \\
\text { on } \\
\text { on } \\
\text { of } \\
\text { on } \\
\text { of } \\
\text { on } \\
\text { of } \\
\text { on } \\
\text { of } \\
\text { on } \\
\text { of } \\
\text { on } \\
\text { ontal } \\
\text { of } \\
\text { on }\end{array}$ & 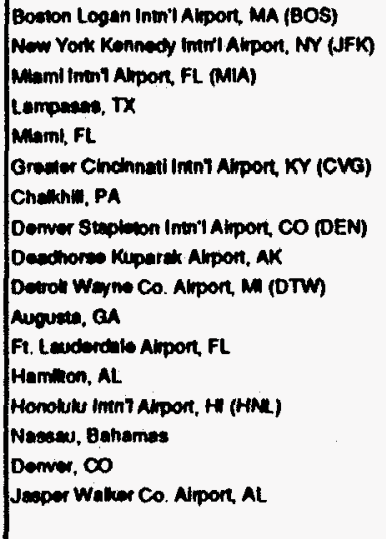 & 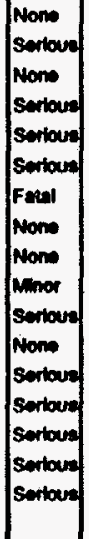 & $\begin{array}{l}0 \\
0 \\
0 \\
0 \\
0 \\
0 \\
3 \\
0 \\
0 \\
0 \\
0 \\
0 \\
0 \\
0 \\
0 \\
0\end{array}$ & $\begin{array}{l}0 \\
0 \\
0 \\
0 \\
0 \\
0 \\
1 \\
0 \\
0 \\
0 \\
0 \\
0 \\
0 \\
0 \\
0 \\
0 \\
0\end{array}$ & $\begin{array}{l}0 \\
0 \\
0 \\
0 \\
0 \\
0 \\
0 \\
0 \\
0 \\
0 \\
0 \\
0 \\
0 \\
0 \\
0 \\
0 \\
0\end{array}$ & $\begin{array}{l}1 \\
0 \\
0 \\
0 \\
0 \\
1 \\
0 \\
1 \\
2 \\
1\end{array}$ & 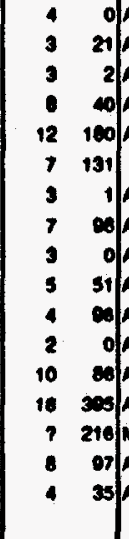 & 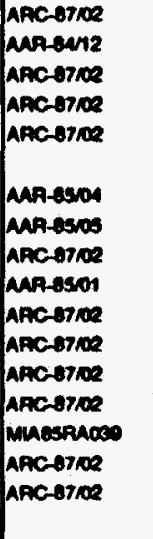 & $\begin{array}{l}0504 \\
1907 \\
0000 \\
1000 \\
0737 \\
2003 \\
1074 \\
2500 \\
3011 \\
1070 \\
1000 \\
1004 \\
3106 \\
3020 \\
7000 \\
2000 \\
2001\end{array}$ & 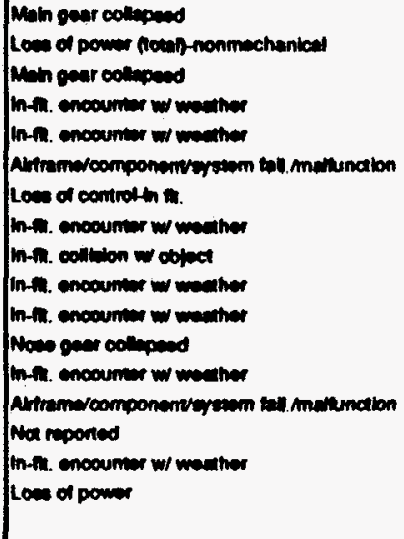 \\
\hline
\end{tabular}




\begin{tabular}{|c|c|c|c|c|c|c|c|c|c|c|c|c|c|c|c|c|c|c|}
\hline $\begin{array}{l}\text { Acc. } \\
\text { DATE }\end{array}$ & OPERATOHFL. No. & rit & AMCNATT IVPE & $\begin{array}{l}\text { AECW. } \\
\text { Mo. }\end{array}$ & $\begin{array}{l}\text { Minchaft } \\
\text { DaunaE }\end{array}$ & $\begin{array}{l}\text { Flt. } \\
\text { pats. }\end{array}$ & $\begin{array}{c}\text { Alpon } \\
\text { Proximiny } \\
\text { Coniofl] }\end{array}$ & MCCTOENT LOCATION & 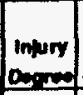 & $\begin{array}{l}\text { FAT } \\
\text { Crow } \mathrm{P}\end{array}$ & ALITEE & & Tolal & $\begin{array}{l}\text { Sortones } \\
\text { menns }\end{array}$ & $\begin{array}{l}\text { Arowo ac } \\
\text { crese peos }\end{array}$ & Tep Reen & & 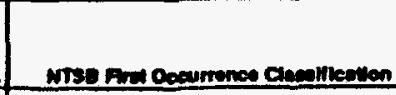 \\
\hline 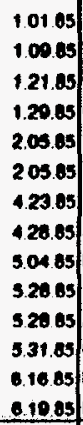 & 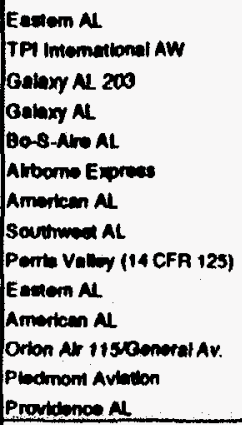 & \begin{tabular}{|c|}
$S P$ \\
NSF \\
NSP \\
$S F$ \\
$S F$ \\
$S F$ \\
$S P$ \\
$S P$ \\
NSP \\
$S P$ \\
$S P$ \\
$S F$ \\
$S P$ \\
PSF \\
\end{tabular} & 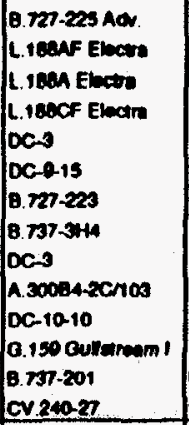 & 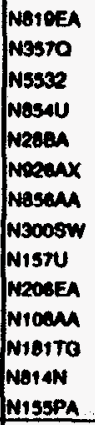 & 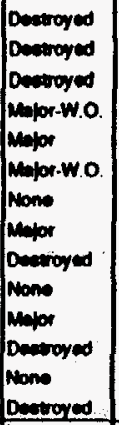 & 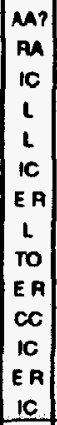 & $\begin{array}{l}\text { of } \\
\text { of } \\
\text { of } \\
\text { on } \\
\text { on } \\
\text { on } \\
\text { on } \\
\text { on } \\
\text { on } \\
\text { on } \\
\text { on } \\
\text { on } \\
\text { of } \\
\text { on }\end{array}$ & 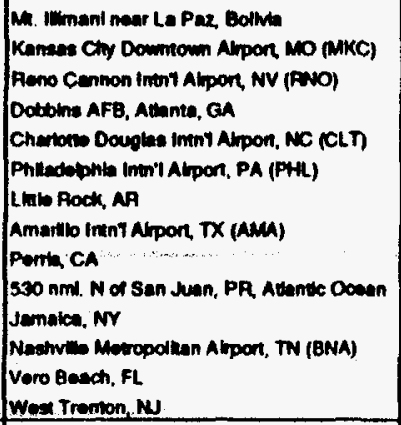 & 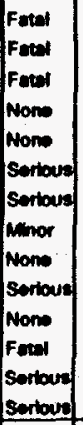 & $\begin{array}{l}0 \\
3 \\
0 \\
0 \\
0 \\
0 \\
0 \\
0 \\
0 \\
0 \\
2 \\
0 \\
0\end{array}$ & $\begin{array}{c}21 \\
0 \\
0 \\
0 \\
0 \\
0 \\
0 \\
0 \\
0 \\
0 \\
0 \\
0 \\
0 \\
0 \\
0\end{array}$ & $\begin{array}{l}0 \\
0 \\
0 \\
0 \\
0 \\
0 \\
0 \\
0 \\
0 \\
0 \\
0 \\
0 \\
0 \\
0\end{array}$ & \begin{tabular}{c|}
20 \\
3 \\
70 \\
0 \\
0 \\
0 \\
0 \\
0 \\
0 \\
0 \\
0 \\
2 \\
0 \\
0 \\
0
\end{tabular} & $\begin{array}{l}0 \\
2 \\
1 \\
0 \\
0 \\
2 \\
0 \\
0\end{array}$ & $\begin{array}{rr}0 & 21 \\
3 & 0 \\
0 & 05 \\
3 & 0 \\
2 & 0 \\
2 & 0 \\
7 & 05 \\
5 & 120 \\
2 & 31 \\
0 & 89 \\
14 & 100 \\
2 & 0 \\
3 & 45 \\
2 & 0 \\
\end{array}$ & 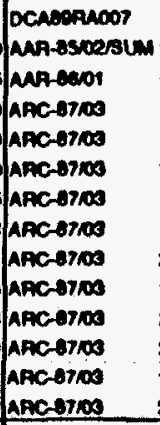 & $\begin{array}{r}7000 \\
10011 \\
0212 \\
1131 \\
12003 \\
2000 \\
2001 \\
1007 \\
2002 \\
1200 \\
2000 \\
2000 \\
1700 \\
2000 \\
\end{array}$ & 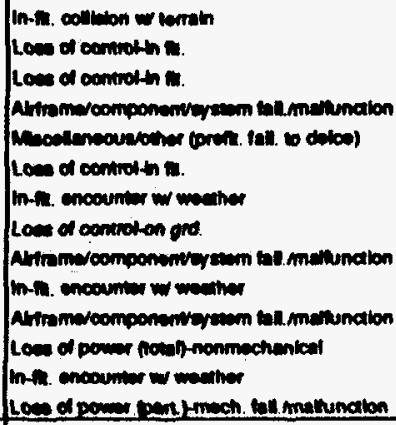 \\
\hline .023 .85 & $\begin{array}{l}\text { Amortan AL } 1003 \\
\text { Priteme AC }\end{array}$ & SP. & $\begin{array}{l}.727 .123 \\
\text { comin } 421 \mathrm{C}\end{array}$ & $\begin{array}{l}\text { Nices } \\
\text { Nizool }\end{array}$ & Nome & \begin{tabular}{|l|} 
ID \\
EA \\
\end{tabular} & on & Sount Haven, Ma & $\begin{array}{l}\text { Sorbong } \\
\text { Menorat }\end{array}$ & $\begin{array}{l}0 \\
0 \\
0\end{array}$ & $\begin{array}{l}0 \\
0\end{array}$ & $\begin{array}{l}0 \\
0\end{array}$ & & 2 & 103 & AAC- 0700 & 2474 & 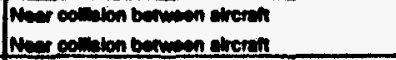 \\
\hline \begin{tabular}{c|}
0.27 .05 \\
0.02 .05 \\
0.06 .05 \\
0.25 .05 \\
11.00 .05 \\
11.07 .05 \\
12.12 .05
\end{tabular} & 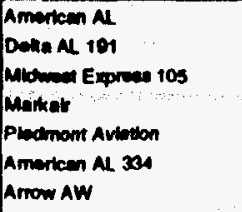 & \begin{tabular}{|l|} 
SP \\
$S P$ \\
$S P$ \\
$S P$ \\
$S P$ \\
SP \\
NSP \\
\end{tabular} & 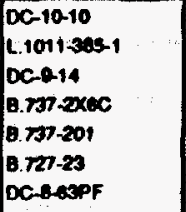 & 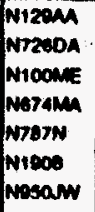 & 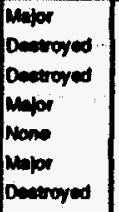 & $\begin{array}{l}T O \\
R A \\
1 C \\
R A \\
E A \\
M A \\
C\end{array}$ & $\begin{array}{l}\text { on } \\
\text { on } \\
\text { of } \\
\text { on } \\
\text { on } \\
\text { of } \\
\text { of }\end{array}$ & 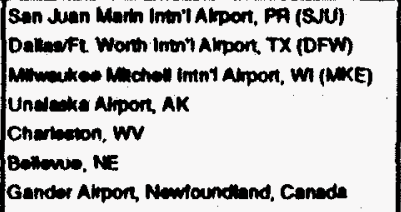 & 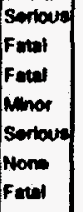 & $\begin{array}{l}: \\
: \\
: \\
: \\
:\end{array}$ & $\begin{array}{r}0 \\
126 \\
27 \\
0 \\
0 \\
0 \\
240\end{array}$ & $\begin{array}{l}0 \\
1 \\
0 \\
0 \\
0 \\
0 \\
0\end{array}$ & $\begin{array}{r}0 \\
135 \\
34 \\
0 \\
0 \\
0 \\
236\end{array}$ & 13 & $\begin{array}{rr}13 & 257 \\
11 & 152 \\
4 & 27 \\
1 & 17 \\
1 & 52 \\
7 & 240\end{array}$ & 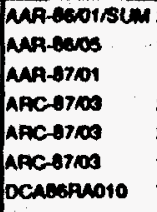 & $\begin{array}{l}2400 \\
1572 \\
1147 \\
2060 \\
2304 \\
1001 \\
7000\end{array}$ & 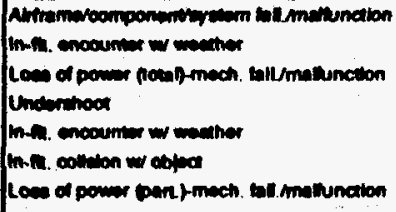 \\
\hline
\end{tabular}

22 Aocidents

23 Akroun involued

Tal: $30406,526 \quad 30 \quad 1319304$ 
APPENDIX A2.1 NTSB 14 CFA 121 ACCIDENT DATABASE

\begin{tabular}{|c|c|c|c|c|c|c|c|c|c|c|c|c|c|c|c|c|c|}
\hline $\begin{array}{l}\text { ACC. } \\
\text { oATE }\end{array}$ & OPERAYOA FW. No. & Frt & AnCWATT TPPE & $\begin{array}{c}\text { mean. } \\
\text { No. }\end{array}$ & $\begin{array}{l}\text { MRCMAFT } \\
\text { DAMAOE }\end{array}$ & $\begin{array}{l}\text { FLt. } \\
\text { PHS. }\end{array}$ & \begin{tabular}{|c|}
$\begin{array}{c}\text { Alppon } \\
\text { Proximily } \\
\text { (onvoff) }\end{array}$ \\
\end{tabular} & MCCIOENT LOCATIOM & inpury & Con po & LITIES & ener total & sorrous & $\begin{array}{l}\text { Aboerd AC } \\
\text { crim peen }\end{array}$ & Mrsenepen & $\begin{array}{c}\text { Mrsse } \\
\text { Mabne. }\end{array}$ & 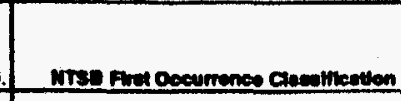 \\
\hline 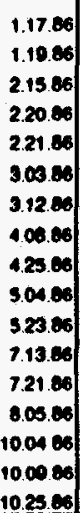 & 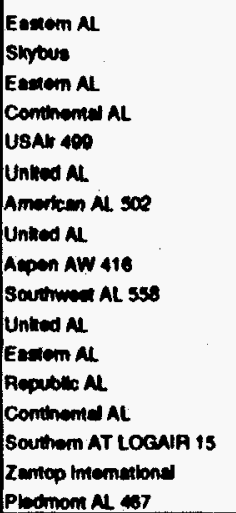 & \begin{tabular}{|l|}
$S P$ \\
NSP \\
SP \\
$S P$ \\
$S P$ \\
$S P$ \\
$S P$ \\
$S P$ \\
$S P$ \\
$S P$ \\
$S P$ \\
$S P$ \\
$S P$ \\
$S P$ \\
NSF \\
NSF \\
SP \\
\end{tabular} & 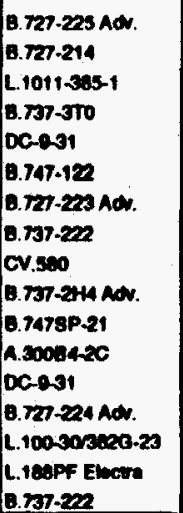 & 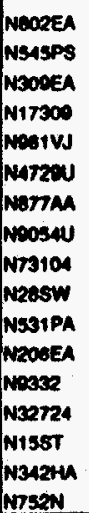 & 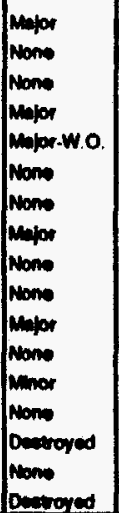 & $\begin{array}{c}L \\
C C \\
G \\
T O \\
L \\
E A \\
C C \\
L \\
1 D \\
E A \\
L \\
D \\
E A \\
C C \\
C \\
Q \\
L\end{array}$ & $\begin{array}{l}\text { on } \\
\text { on } \\
\text { on } \\
\text { on } \\
\text { on } \\
\text { of } \\
\text { on } \\
\text { on } \\
\text { on } \\
\text { on } \\
\text { on (a) } \\
\text { of } \\
\text { of } \\
\text { of } \\
\text { on } \\
\text { on } \\
\text { on }\end{array}$ & 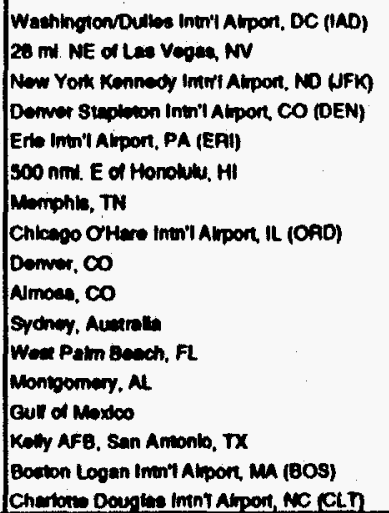 & 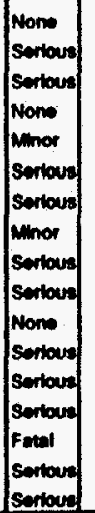 & $\begin{array}{l}0 \\
0 \\
0 \\
0 \\
0 \\
0 \\
0 \\
0 \\
0 \\
0 \\
0 \\
0 \\
0 \\
0 \\
3 \\
0\end{array}$ & $\begin{array}{l}0 \\
0 \\
0 \\
0 \\
0 \\
0 \\
0 \\
0 \\
0 \\
0 \\
0 \\
0 \\
0 \\
0\end{array}$ & $\begin{array}{l}0 \\
0 \\
0 \\
0 \\
0 \\
0 \\
0 \\
0 \\
0 \\
0 \\
0 \\
0 \\
0 \\
0 \\
0 \\
0 \\
0\end{array}$ & $\begin{array}{l}0 \\
0 \\
2\end{array}$ & 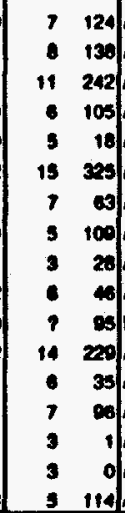 & 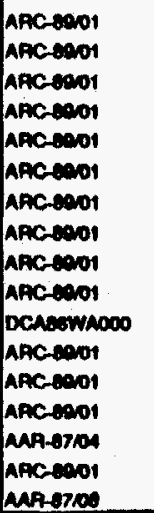 & $\begin{array}{l}2740 \\
0016 \\
1170 \\
1107 \\
1307 \\
2000 \\
0000 \\
2000 \\
2000 \\
1700 \\
7000 \\
1305 \\
1530 \\
1100 \\
1001 \\
2501 \\
1000\end{array}$ & 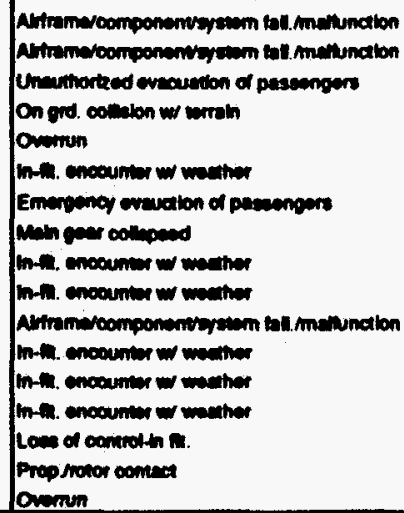 \\
\hline 1106.06 & $\begin{array}{l}\text { Pen Ammerican AW } \\
\text { Privelo Nireren }\end{array}$ & \begin{tabular}{|l|} 
SP \\
Pn \\
\end{tabular} & $\begin{array}{l}8.727-235 \\
\text { Pper PA20-190 }\end{array}$ & $\begin{array}{l}\text { N4743 } \\
\text { N210sp }\end{array}$ & $\begin{array}{l}\text { Mator } \\
\text { Deneroyed }\end{array}$ & $\begin{array}{c}T=0 \\
L\end{array}$ & $\begin{array}{l}\text { on } \\
\text { on }\end{array}$ & Tamos inn'Alapor, FL (TPA) & $\begin{array}{l}\text { Sortoun } \\
\text { Foral }\end{array}$ & $\begin{array}{l}0 \\
1\end{array}$ & $\begin{array}{l}0 \\
0\end{array}$ & $\begin{array}{l}0 \\
0 \\
\end{array}$ & 7 & $\begin{array}{r}17 \\
1 \quad 0 \\
\end{array}$ & AAP-8706 & 1000 & 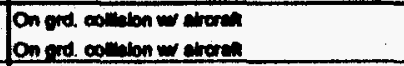 \\
\hline $\begin{array}{l}11.07 .86 \\
11.13 .06 \\
12.1506 \\
122286 \\
123086\end{array}$ & 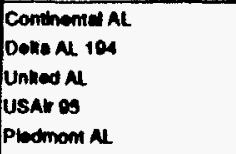 & \begin{tabular}{|l|}
$S P$ \\
SP \\
SP \\
SP
\end{tabular} & $\begin{array}{l}\text { DC. } 10-10 \\
\text { L.1011-305-1 } \\
\text { B.r27-222 } \\
\text { B.r37-397 } \\
\text { F. } 26-4000\end{array}$ & 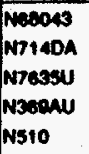 & 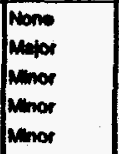 & \begin{tabular}{c|c|}
$E R$ \\
Tan \\
10 \\
ER \\
0
\end{tabular} & $\begin{array}{l}\text { of } \\
\text { on } \\
\text { of } \\
\text { of } \\
\text { on }\end{array}$ & 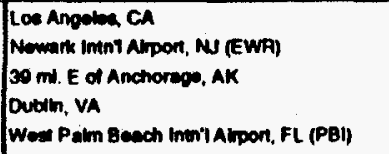 & $\begin{array}{l}\text { Sertoun } \\
\text { Nono } \\
\text { semtoun } \\
\text { sertown } \\
\text { sertoun }\end{array}$ & $\begin{array}{l}0 \\
0 \\
0 \\
0 \\
0\end{array}$ & $\begin{array}{l}0 \\
0 \\
0 \\
0 \\
0\end{array}$ & $\begin{array}{l}0 \\
0 \\
0 \\
0 \\
0\end{array}$ & 1 & $\begin{array}{rr}14 & 100 \\
11 & 110 \\
7 & 95 \\
7 & 120 \\
4 & 75\end{array}$ & 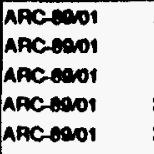 & $\begin{array}{l}2407 \\
2001 \\
1500 \\
2300 \\
2015\end{array}$ & 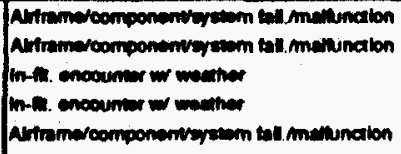 \\
\hline
\end{tabular}

23 Aocidonts

24 Areran involves

Total: $4 \quad 0 \quad 0 \quad 4 \quad 23$ 164 2307 


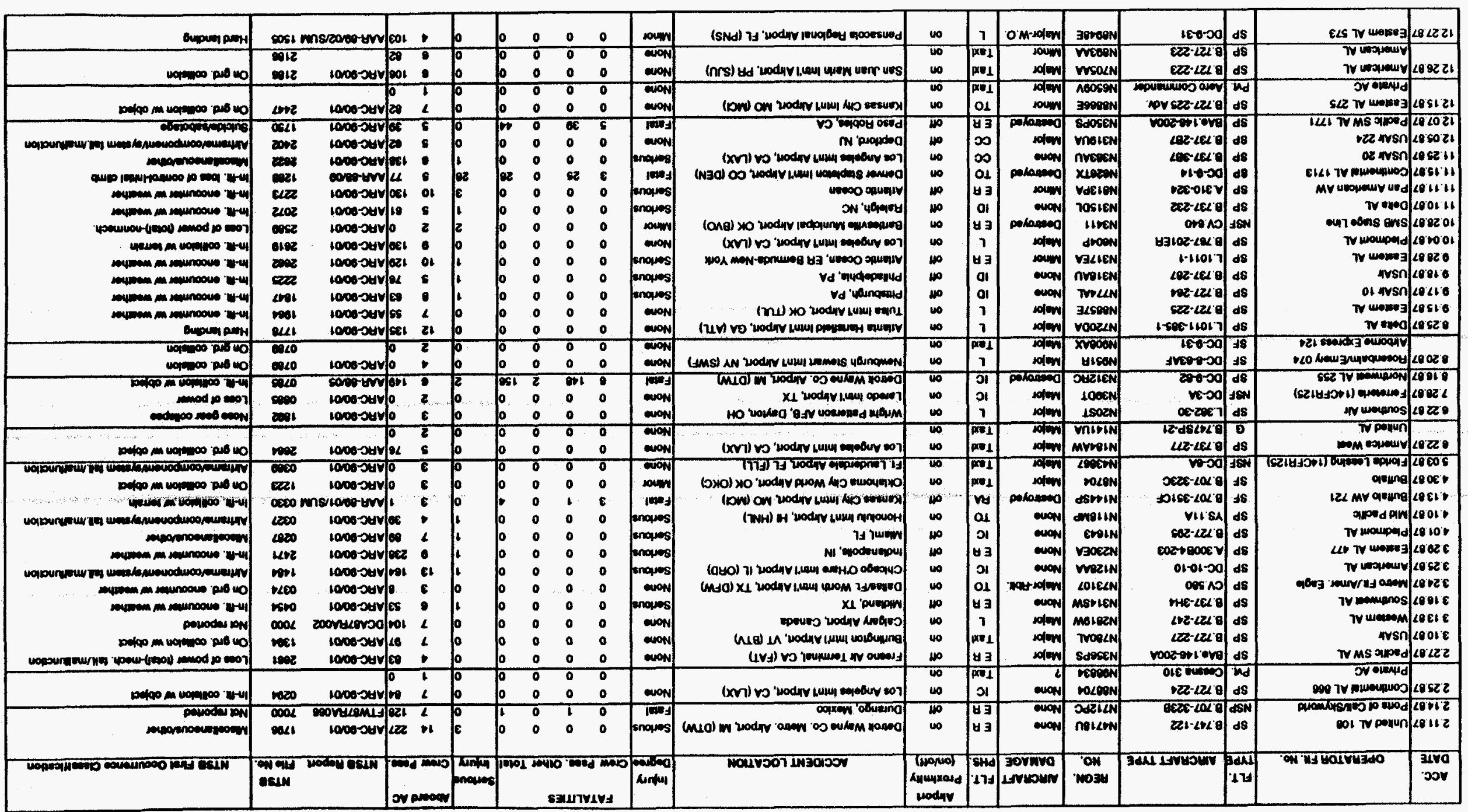




\begin{tabular}{|c|c|c|c|c|c|c|c|c|c|c|c|c|c|c|c|c|c|c|}
\hline $\begin{array}{l}\text { ACC. } \\
\text { DAIE }\end{array}$ & OPEAATOMFL. No. & tr. & AmCraft TPPE & $\begin{array}{c}\text { meON. } \\
\text { mo. }\end{array}$ & $\begin{array}{l}\text { MACTAFT } \\
\text { DAMAGE }\end{array}$ & $\begin{array}{l}\text { FLT. } \\
\text { PHS }\end{array}$ & $\begin{array}{c}\text { Alpoent } \\
\text { Proximly } \\
\text { convelin }\end{array}$ & MCCDEENTLOCATION & 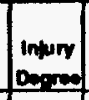 & $\begin{array}{l}\text { FATAL } \\
\text { crom pes }\end{array}$ & 20.0 & oner to & & mours & 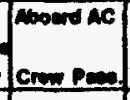 & Mrsenepent & An & 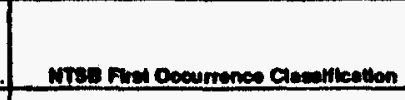 \\
\hline $\begin{array}{l}1.10 .68 \\
1.13 .80 \\
1.19 .8 \\
1.10 .40 \\
1.25 .60 \\
1.27 .60 \\
2.08 .68\end{array}$ & 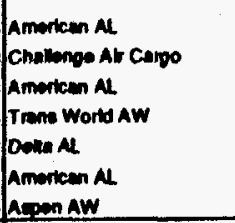 & $\begin{array}{l}\text { SP } \\
\text { SF } \\
\text { SP } \\
\text { SP } \\
\text { SP } \\
\text { SP } \\
\text { SP } \\
\end{array}$ & 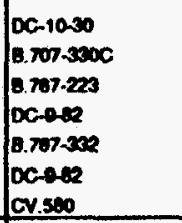 & 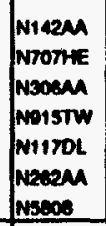 & 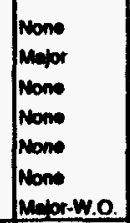 & $\begin{array}{c}G \\
L \\
10 \\
E A \\
10 \\
E R \\
L\end{array}$ & $\begin{array}{l}\text { on } \\
\text { on (a) } \\
\text { of } \\
\text { of } \\
\text { on } \\
\text { on } \\
\text { on }\end{array}$ & 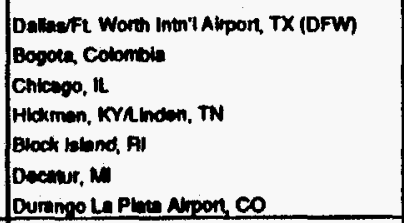 & 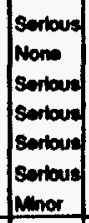 & $\begin{array}{l}0 \\
0 \\
0 \\
0 \\
0 \\
0 \\
0 \\
\end{array}$ & $\begin{array}{l}0 \\
0 \\
0 \\
0 \\
0 \\
0\end{array}$ & $\begin{array}{l}0 \\
0 \\
0 \\
0 \\
0 \\
0 \\
0\end{array}$ & 0 & & \begin{tabular}{rr|}
13 & 270 \\
3 & 0 \\
0 & 130 \\
0 & 91 \\
0 & 100 \\
1 & 70 \\
3 & 30 \\
\end{tabular} & 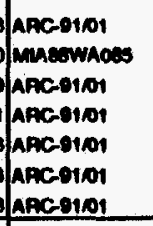 & $\begin{array}{l}22000 \\
7000 \\
15000 \\
0800 \\
00372 \\
0114 \\
0100\end{array}$ & 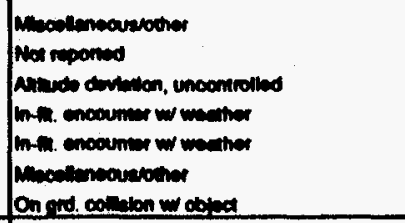 \\
\hline 3.2068 & $\begin{array}{l}\text { Aowle Trame } \\
7\end{array}$ & $\begin{array}{l}\text { NSF } \\
\text { FY. }\end{array}$ & 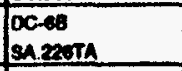 & $\begin{array}{l}\text { moed } \\
\text { nsooke }\end{array}$ & mor & $\begin{array}{c}T=x \\
a\end{array}$ & $\begin{array}{l}\text { on } \\
\text { on }\end{array}$ & Mami Intrit Alpor, FL (MLA) & $\begin{array}{l}\text { Nome } \\
\text { Nome }\end{array}$ & $\begin{array}{l}0 \\
0\end{array}$ & $\begin{array}{l}0 \\
0\end{array}$ & : & & 7 & $\begin{array}{l}3 \\
2 \\
\end{array}$ & & oces & 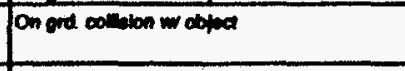 \\
\hline 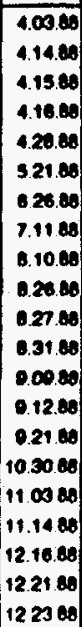 & 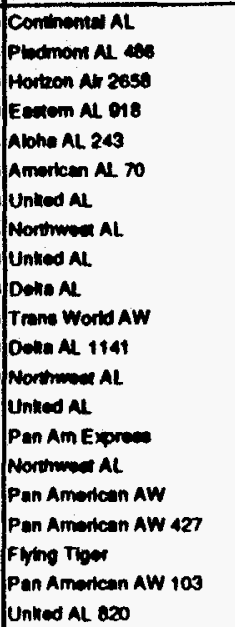 & \begin{tabular}{|l|}
$S P$ \\
$S P$ \\
$S P$ \\
$S P$ \\
$S P$ \\
$S P$ \\
$S P$ \\
$S P$ \\
$S P$ \\
$S P$ \\
$S P$ \\
$S P$ \\
$S P$ \\
$S P$ \\
$S P$ \\
$S P$ \\
$S P$ \\
$S P$ \\
$S P$ \\
$S P$
\end{tabular} & 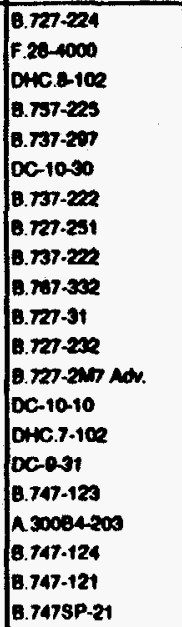 & 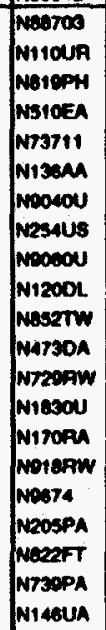 & 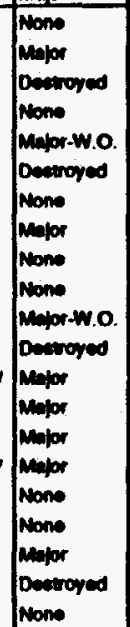 & 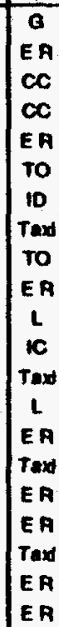 & $\begin{array}{l}\text { on } \\
\text { or } \\
\text { on } \\
\text { on (o) } \\
\text { of } \\
\text { on } \\
\text { on } \\
\text { on } \\
\text { on } \\
\text { of } \\
\text { on } \\
\text { on } \\
\text { on } \\
\text { on } \\
\text { of } \\
\text { on } \\
\text { on } \\
\text { on (1) } \\
\text { on } \\
\text { of } \\
\text { of }\end{array}$ & 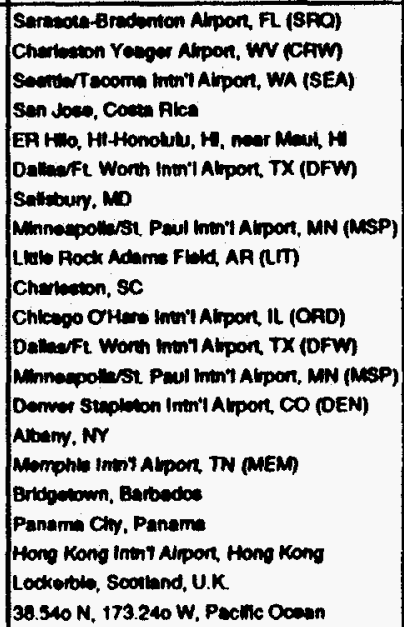 & 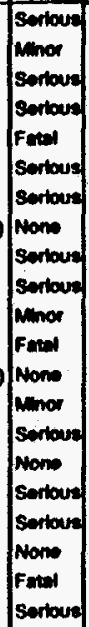 & $\begin{array}{l}0 \\
0 \\
0 \\
0 \\
0 \\
0 \\
0 \\
0 \\
0 \\
0 \\
2 \\
0 \\
0 \\
0 \\
0 \\
0 \\
0 \\
0 \\
1 \\
0\end{array}$ & $\begin{array}{l}0 \\
0 \\
0 \\
0 \\
0 \\
0 \\
0 \\
0 \\
0 \\
0 \\
0 \\
0 \\
0\end{array}$ & $\begin{array}{l}0 \\
0 \\
0 \\
0 \\
0 \\
0 \\
0 \\
0 \\
0 \\
0 \\
0 \\
0 \\
0 \\
0 \\
0 \\
0 \\
0 \\
0 \\
0 \\
11\end{array}$ & $\begin{array}{r}0 \\
0 \\
0 \\
0 \\
1 \\
0 \\
0 \\
0 \\
0 \\
0 \\
0 \\
14 \\
0 \\
0 \\
0 \\
0 \\
0\end{array}$ & 0 & 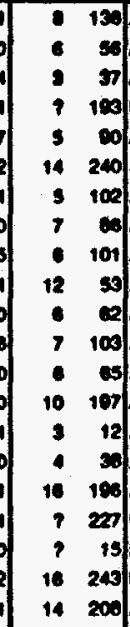 & 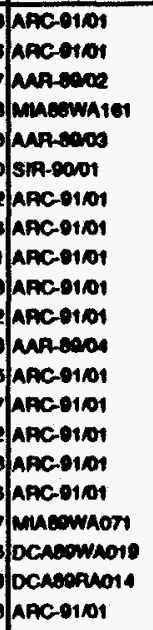 & 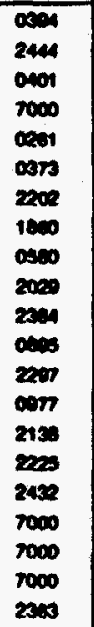 & 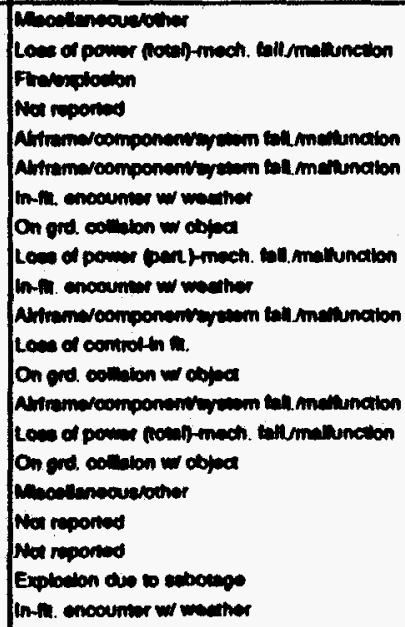 \\
\hline
\end{tabular}

20 Accidents 


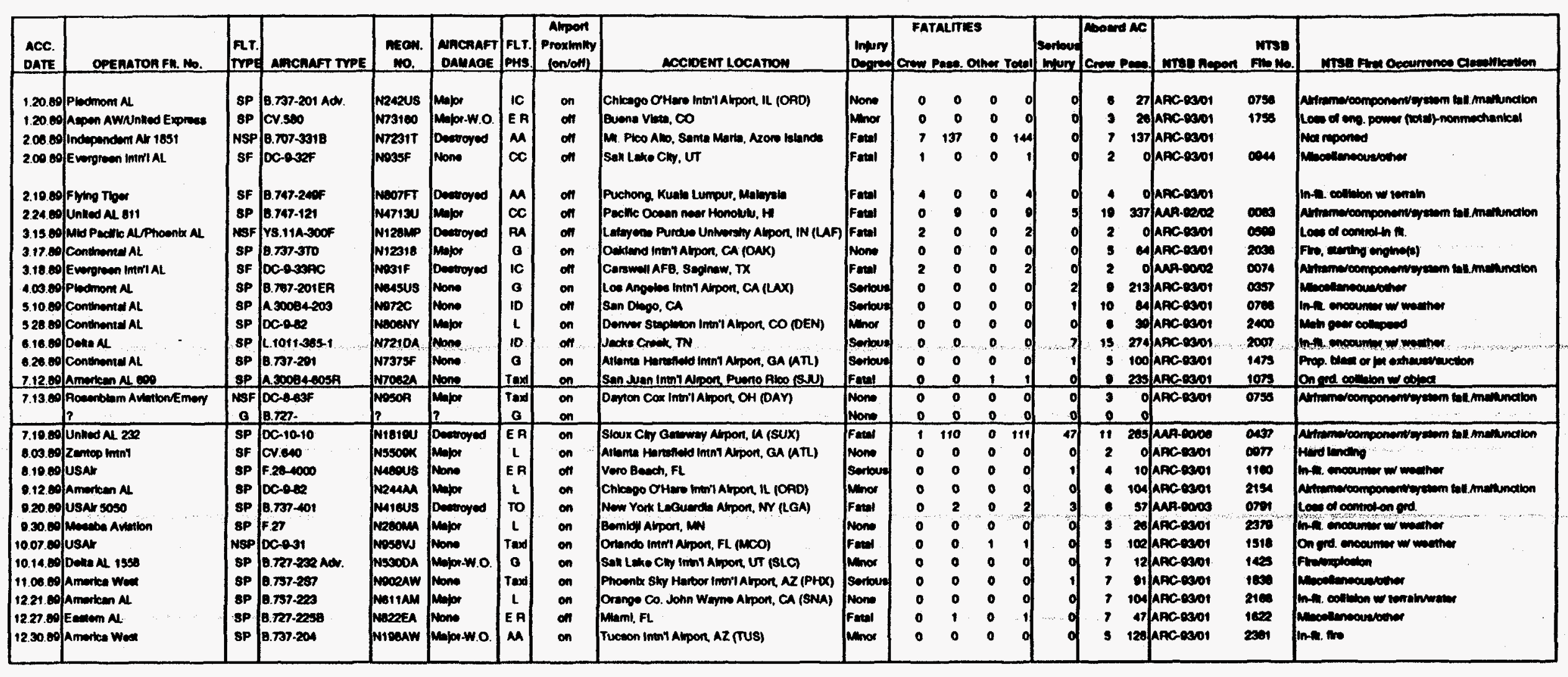

28 Aocidones

20 Akeram involvod

Tat: $17250 \quad 2 \quad 270 \quad 6172500$ 


\begin{tabular}{|c|c|c|c|c|c|c|c|c|c|c|c|c|c|c|c|c|c|}
\hline $\begin{array}{l}\text { ACC. } \\
\text { OAIE }\end{array}$ & OPEATAOA FH. No. & Fir. & Ancrast TrPE & $\begin{array}{l}\text { wean. } \\
\text { no. }\end{array}$ & $\begin{array}{l}\text { MACMAFT } \\
\text { DAMAOE }\end{array}$ & $\begin{array}{l}\text { FLT. } \\
\text { PHS. }\end{array}$ & $\begin{array}{c}\text { Alport } \\
\text { Proximily } \\
\text { confoefly }\end{array}$ & ACCDEEMT LOCATION & Inminy & $\begin{array}{l}\text { FATA } \\
\text { Cane P }\end{array}$ & LiTIE: & & Sortous & $\begin{array}{ll}\text { Anowd AC } \\
\text { Crow Pene }\end{array}$ & NTBS Repen & $\begin{array}{c}\text { MTses } \\
\text { rmo No. }\end{array}$ & 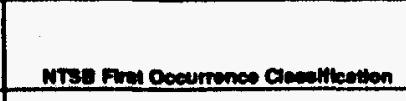 \\
\hline $\begin{array}{l}1.02 .00 \\
1.18 .00\end{array}$ & $\begin{array}{l}\text { Amertan N } \\
\text { Unted AL }\end{array}$ & $\begin{array}{l}\mathrm{sp} \\
\mathrm{sp} \\
\end{array}$ & $\begin{array}{l}\text { DC-10-10 } \\
\text { B.757-2m }\end{array}$ & $\begin{array}{l}\text { Nitoon } \\
\text { usosuA }\end{array}$ & Nomo & $\begin{array}{l}\text { E R } \\
\text { ro }\end{array}$ & on & 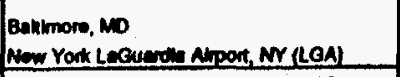 & $\begin{array}{l}\text { Sertouns } \\
\text { Nome }\end{array}$ & $\begin{array}{l}0 \\
0 \\
\end{array}$ & $\begin{array}{l}0 \\
0 \\
\end{array}$ & $\begin{array}{l}0 \\
0 \\
\end{array}$ & & $\begin{array}{rr}10 & 247 \\
7 & 100 \\
\end{array}$ & $\begin{array}{l}\text { AAC-BODR } \\
\text { AACDOOR }\end{array}$ & 1000 & 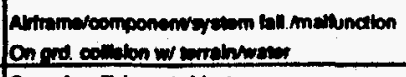 \\
\hline 1.18 .00 & 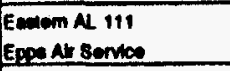 & SP. & \begin{tabular}{|l|} 
B.727-225 Adr. \\
Bech. A100 KMg Arr
\end{tabular} & \begin{tabular}{|l|} 
NGE67E \\
ManUE \\
\end{tabular} & Merior.w.O. & \begin{tabular}{|l|} 
\\
Text \\
\end{tabular} & on & 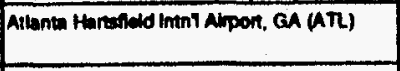 & Nons & $i$ & $\begin{array}{l}0 \\
0\end{array}$ & 0 & 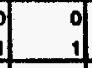 & 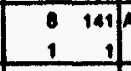 & MA-9100 & 9 & On god collelon w/ atjeat \\
\hline $\begin{array}{l}1.20 .00 \\
1.31 .00 \\
3.16 .00 \\
3.31 .00 \\
.11000 \\
3.05 .00 \\
5.11 .00 \\
.02 .00 \\
\end{array}$ & 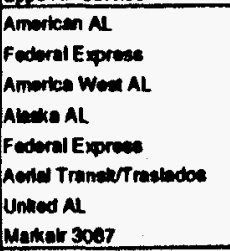 & \begin{tabular}{|l|}
$S P$ \\
$S F$ \\
$S P$ \\
$S P$ \\
$S F$ \\
$S F$ \\
$S P$ \\
$S P F$
\end{tabular} & 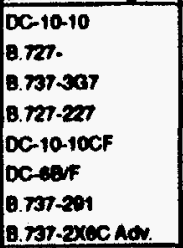 & 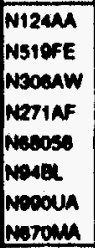 & 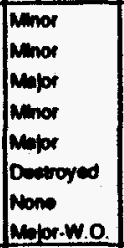 & \begin{tabular}{c|}
10 \\
0 \\
$?$ \\
10 \\
1 \\
10 \\
10 \\
104 \\
\end{tabular} & $\begin{array}{l}\text { on } \\
\text { on } \\
\text { on } \\
\text { on } \\
\text { on } \\
\text { on } \\
\text { on } \\
\text { on }\end{array}$ & 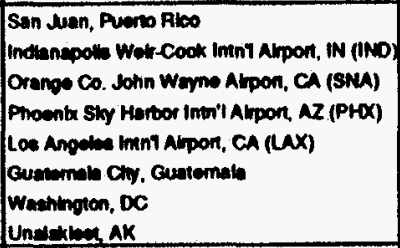 & 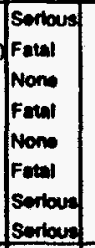 & $\begin{array}{l}0 \\
0 \\
0 \\
0 \\
0 \\
3 \\
0 \\
0\end{array}$ & $\begin{array}{l}0 \\
0 \\
0 \\
0 \\
0 \\
0 \\
0 \\
0\end{array}$ & $\begin{array}{cc}0 & 0 \\
1 & 1 \\
0 & 0 \\
1 & 1 \\
0 & 0 \\
22 & 25 \\
0 & 0 \\
0 & 0 \\
\end{array}$ & . & \begin{tabular}{rr|r|}
11 & 140 \\
3 & 3 \\
$?$ & 110 \\
6 & 35 \\
3 & 0 \\
3 & 0 \\
3 & 2 \\
4 & 0 & 0 \\
\end{tabular} & 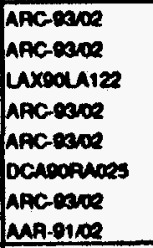 & $\begin{array}{l}1123 \\
1000 \\
1001 \\
1050 \\
1432 \\
7000 \\
0100 \\
0274 \\
\end{array}$ & 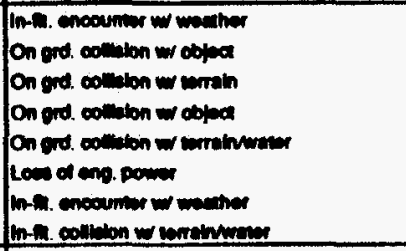 \\
\hline 0.18 .00 & $\begin{array}{l}\text { USAN } 1946 \\
\text { Ameln } 600\end{array}$ & $\begin{array}{ll}s p \\
P p\end{array}$ & $\begin{array}{l}F .20-1000 \\
8.247 .2439 \text { complo }\end{array}$ & $\begin{array}{l}\text { Mussous } \\
\text { 1-DEWC }\end{array}$ & mavor & \begin{tabular}{|c|} 
\\
Taxi \\
\end{tabular} & on & Now Yort Kennedy Iment Alpon, NY (JFK) & $\begin{array}{l}\text { None } \\
\text { Mone }\end{array}$ & 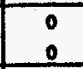 & 0 & 0 & 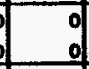 & $i$ & ARC-0208 & 1207 & On prod colletion wo obpat \\
\hline $\begin{array}{l}.21 .00 \\
7.14 .00 \\
7.18 .00 \\
7.22 .00 \\
1.27 .00 \\
1.05 .00 \\
0.00 .00 \\
0.12 .00 \\
0.20 .80 \\
10.03 .00\end{array}$ & 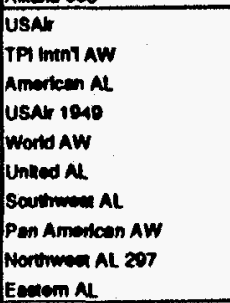 & \begin{tabular}{|l|}
$\mathrm{SP}$ \\
$\mathrm{NBF}$ \\
$\mathrm{SP}$ \\
$\mathrm{SP}$ \\
$\mathrm{NBP}$ \\
$\mathrm{SP}$ \\
$\mathrm{SP}$ \\
$\mathrm{SP}$ \\
$\mathrm{SP}$ \\
$\mathrm{SP}$
\end{tabular} & 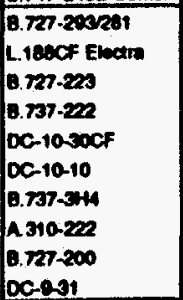 & 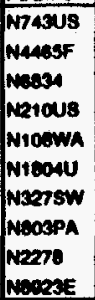 & 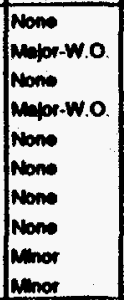 & \begin{tabular}{|l|}
10 \\
$\infty$ \\
10 \\
To \\
Taw \\
E A \\
E A \\
TO \\
Tax \\
EA \\
\end{tabular} & $\begin{array}{l}\text { on } \\
\text { on } \\
\text { on } \\
\text { on } \\
\text { on } \\
\text { on } \\
\text { on } \\
\text { on } \\
\text { on } \\
\text { on }\end{array}$ & 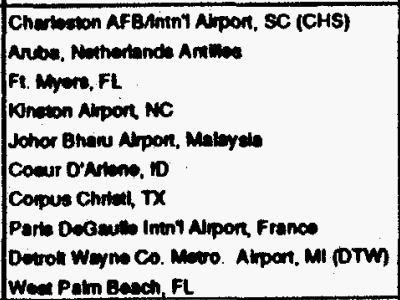 & 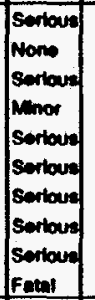 & $\begin{array}{l}0 \\
0 \\
0 \\
0 \\
0 \\
0 \\
0 \\
0 \\
0 \\
0\end{array}$ & $\begin{array}{l}0 \\
0 \\
0 \\
0 \\
0 \\
0 \\
0 \\
0 \\
0 \\
1\end{array}$ & $\begin{array}{l}0 \\
0 \\
0 \\
0 \\
0 \\
0 \\
0 \\
0 \\
0 \\
0\end{array}$ & 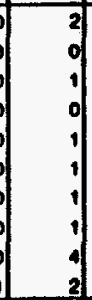 & 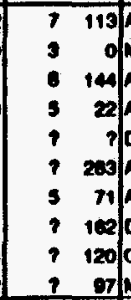 & 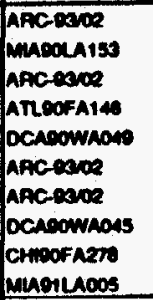 & $\begin{array}{l}1409 \\
2110 \\
0010 \\
2300 \\
7000 \\
1167 \\
1500 \\
1000 \\
1000 \\
2130 \\
\end{array}$ & 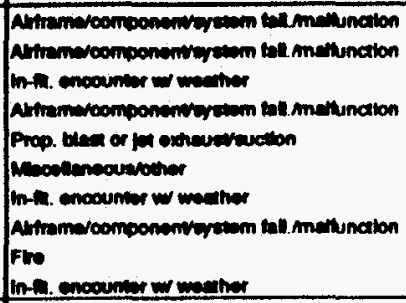 \\
\hline 10.20 .00 & 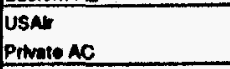 & PP. & $\begin{array}{l}\text { DC-R31 } \\
\operatorname{cosens} 310\end{array}$ & Mor2VJ & miner & \begin{tabular}{|l|} 
Taxd \\
Taxd \\
\end{tabular} & on & Pon Columberalment Aipon, OH (CNAH) & $\begin{array}{l}\text { None } \\
?\end{array}$ & 0 & 0 & : & & $?$ & AAC:S002 & 1780 & On gred colletion we obind \\
\hline 12.0300 & 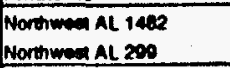 & $\begin{array}{l}\mathbf{S P} \\
\mathbf{3 P} \\
\end{array}$ & $\begin{array}{l}0 C-0-14 \\
\text { B.727-251 Aov. }\end{array}$ & $\begin{array}{l}\text { N3013L } \\
\text { N270us }\end{array}$ & $\begin{array}{l}\text { Destroved } \\
\text { never }\end{array}$ & \begin{tabular}{|c|} 
Tax \\
To \\
\end{tabular} & $\begin{array}{l}\text { on } \\
\text { on }\end{array}$ & Detrot Weyno Co. Nevo. Ahpor, Mil (DTM) & $\begin{array}{l}\text { Fonas } \\
\text { Nonse }\end{array}$ & i & $\begin{array}{l}7 \\
0\end{array}$ & 0 & 10 & \begin{tabular}{|r|}
100 \\
$\quad 146$ \\
\end{tabular} & MA-0105 & 1300 & $\begin{array}{l}\text { On ord collition } \\
\text { Onned. comition }\end{array}$ \\
\hline & & & & & & & & & & & & & & & & & \\
\hline
\end{tabular}




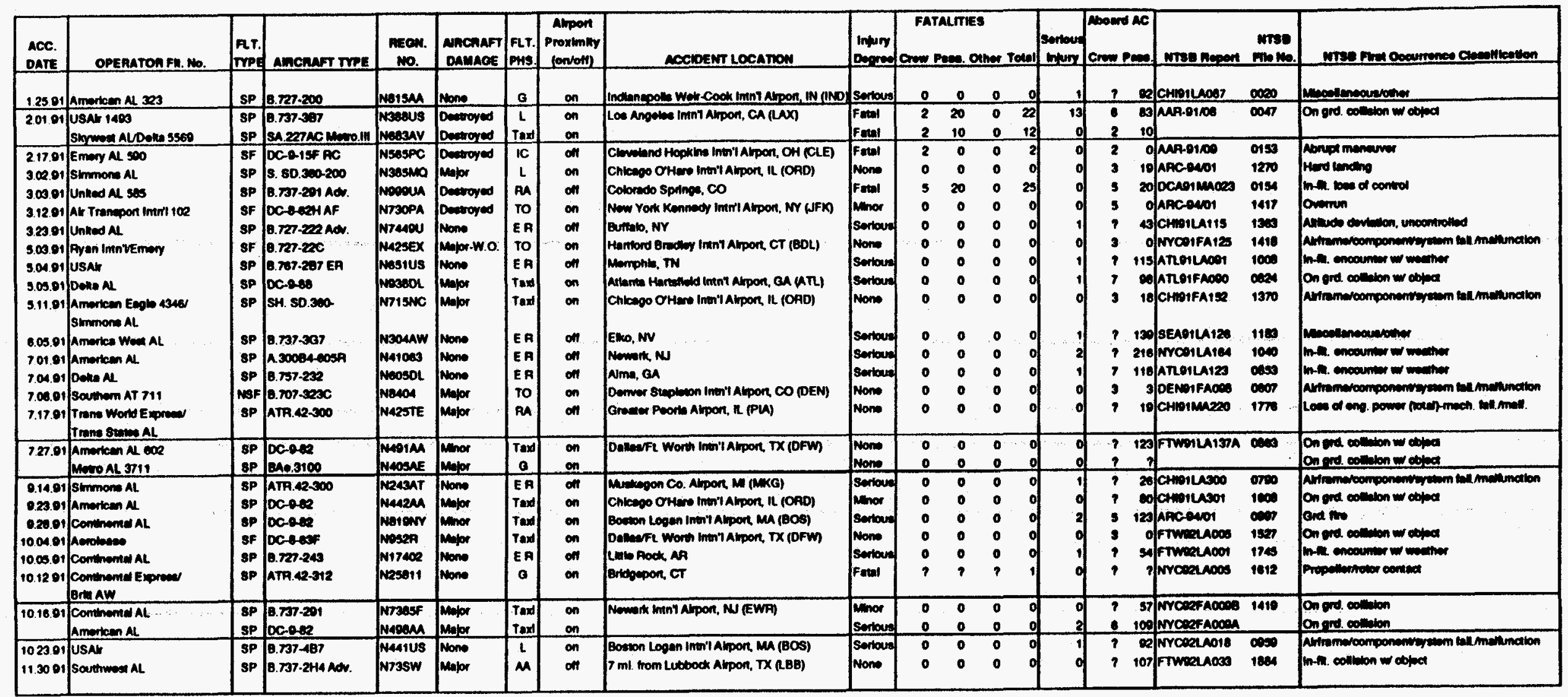


APPENDIX A2.1 NTSB 14 CFR 121 ACCIDENT DATABASE

\begin{tabular}{|c|c|c|c|c|c|c|c|c|c|c|c|c|c|c|c|c|c|}
\hline $\begin{array}{l}\text { acc. } \\
\text { DaIIE }\end{array}$ & OPEAATORFH. MO. & $\begin{array}{l}\text { ret. } \\
\text { rregt }\end{array}$ & AncenafT TYPE & $\begin{array}{c}\text { Mean. } \\
\text { No. }\end{array}$ & $\begin{array}{l}\text { NACMAFT } \\
\text { DAUAOE }\end{array}$ & $\begin{array}{l}\text { FLT. } \\
\text { PHS. }\end{array}$ & \begin{tabular}{|c|} 
Alpoon \\
Proximuly \\
(onvoli)
\end{tabular} & ACCIOENT LOCATION & 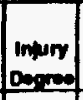 & $\begin{array}{l}\text { FATA } \\
\text { Canew Pe. }\end{array}$ & & & 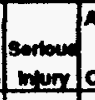 & $\begin{array}{l}\text { Abourd AC } \\
\text { Crow Pen }\end{array}$ & mrserement. & $\begin{array}{c}\text { Nrse } \\
\text { mon Ma. }\end{array}$ & 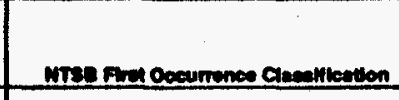 \\
\hline 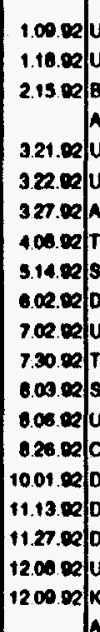 & 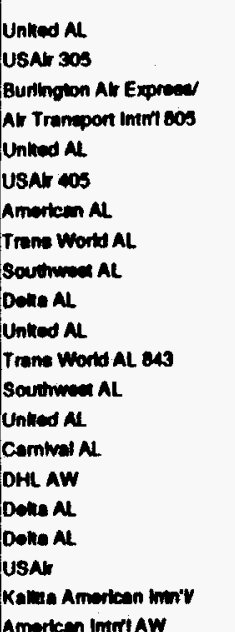 & $\begin{array}{l}\text { SP } \\
S P \\
S F \\
S P \\
S P \\
S P \\
S P \\
S P \\
S P \\
S P \\
S P \\
S P \\
S P \\
S P \\
N S F \\
S P \\
S P \\
S P \\
N S F\end{array}$ & 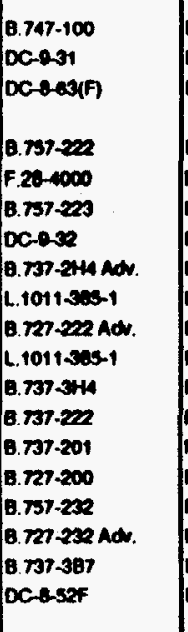 & 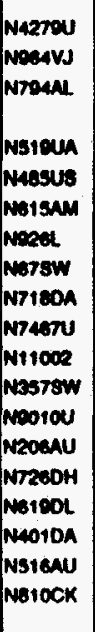 & 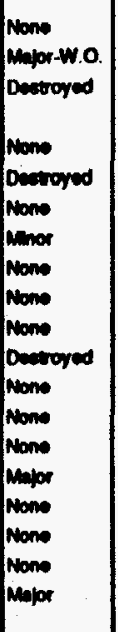 & 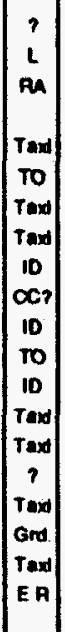 & $\begin{array}{l}\text { of } \\
\text { on } \\
\text { of } \\
\text { on } \\
\text { on } \\
\text { on } \\
\text { on } \\
\text { of } \\
\text { of } \\
\text { of } \\
\text { on } \\
\text { of } \\
\text { on } \\
\text { on } \\
\text { on (a) } \\
\text { on } \\
\text { on } \\
\text { on } \\
\text { of }\end{array}$ & 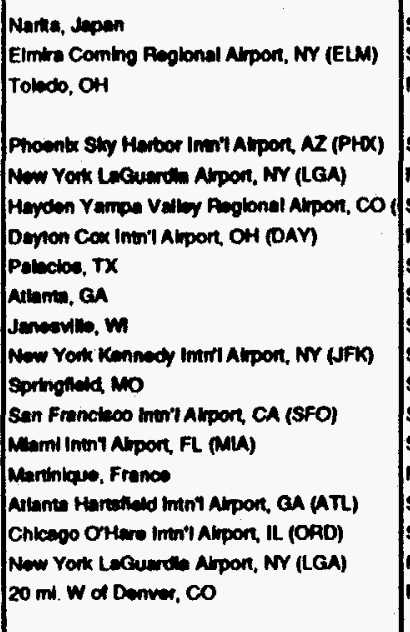 & 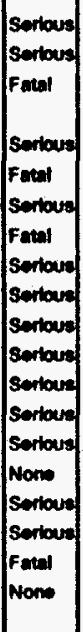 & $\begin{array}{l}0 \\
0 \\
3 \\
0 \\
2 \\
0 \\
0 \\
0 \\
0 \\
0 \\
0 \\
0 \\
0 \\
0 \\
0 \\
0 \\
0 \\
0 \\
0\end{array}$ & $\begin{array}{l}0 \\
0 \\
1 \\
0 \\
29 \\
0 \\
0 \\
0 \\
0 \\
0 \\
0 \\
0 \\
0 \\
0 \\
0 \\
0 \\
0 \\
0 \\
0\end{array}$ & $\begin{array}{ll}0 & 0 \\
0 & 0 \\
0 & 1 \\
0 & 0 \\
0 & 27 \\
0 & 0 \\
1 & 1 \\
0 & 0 \\
0 & 0 \\
0 & 0 \\
0 & 0 \\
0 & 0 \\
0 & 0 \\
0 & 0 \\
0 & 0 \\
0 & 0 \\
0 & 0 \\
1 & 1 \\
0 & 0\end{array}$ & $\begin{array}{r}1 \\
24 \\
1 \\
0 \\
1 \\
1 \\
1 \\
1 \\
1 \\
1 \\
1 \\
1 \\
0 \\
1 \\
1 \\
1 \\
0 \\
0\end{array}$ & 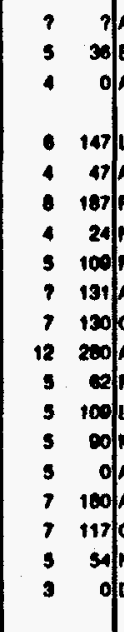 & 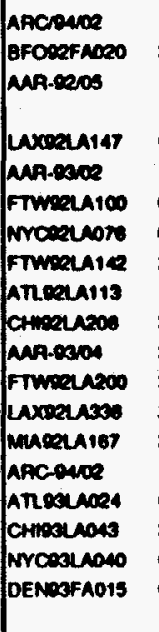 & $\begin{array}{l}? \\
20000 \\
1002 \\
0000 \\
1000 \\
00011 \\
0011 \\
20000 \\
2000 \\
2000 \\
2000 \\
2000 \\
2000 \\
? \\
0000 \\
2000 \\
0004 \\
0000\end{array}$ & 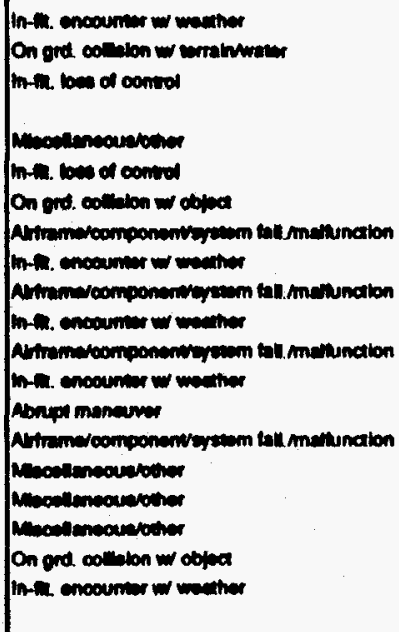 \\
\hline
\end{tabular}




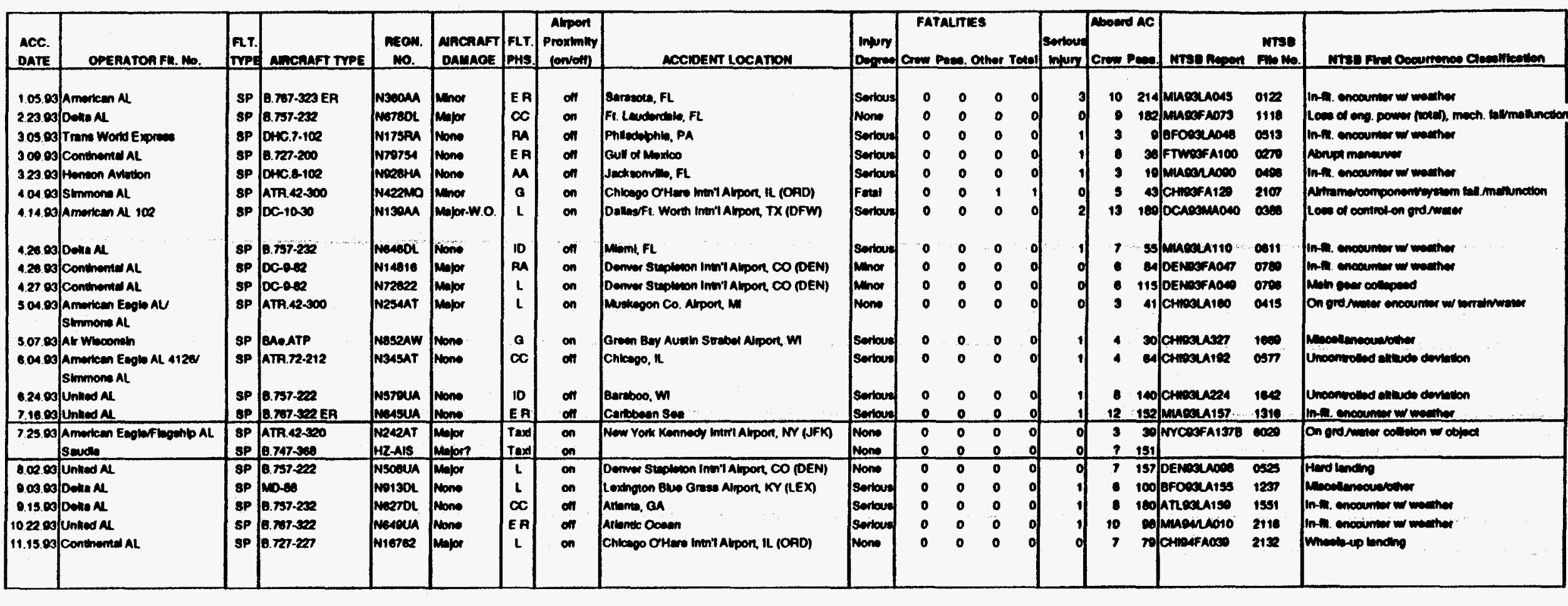




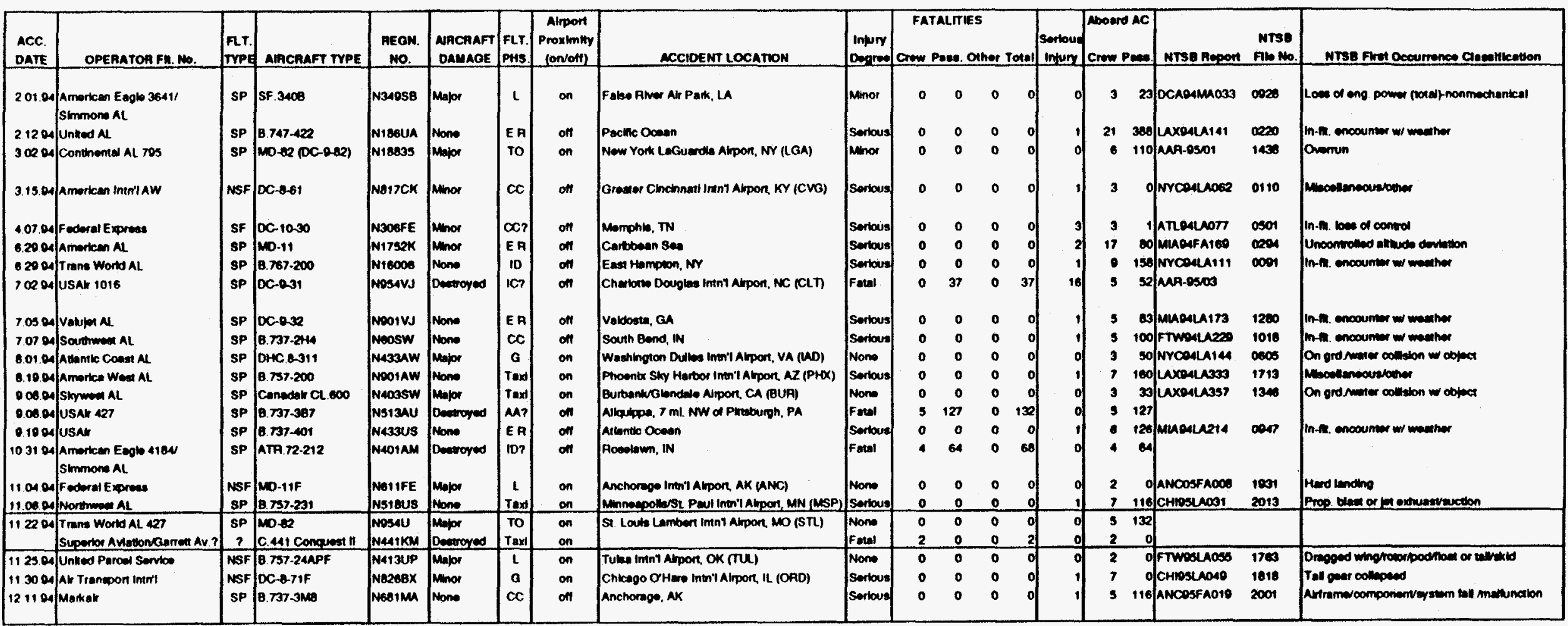




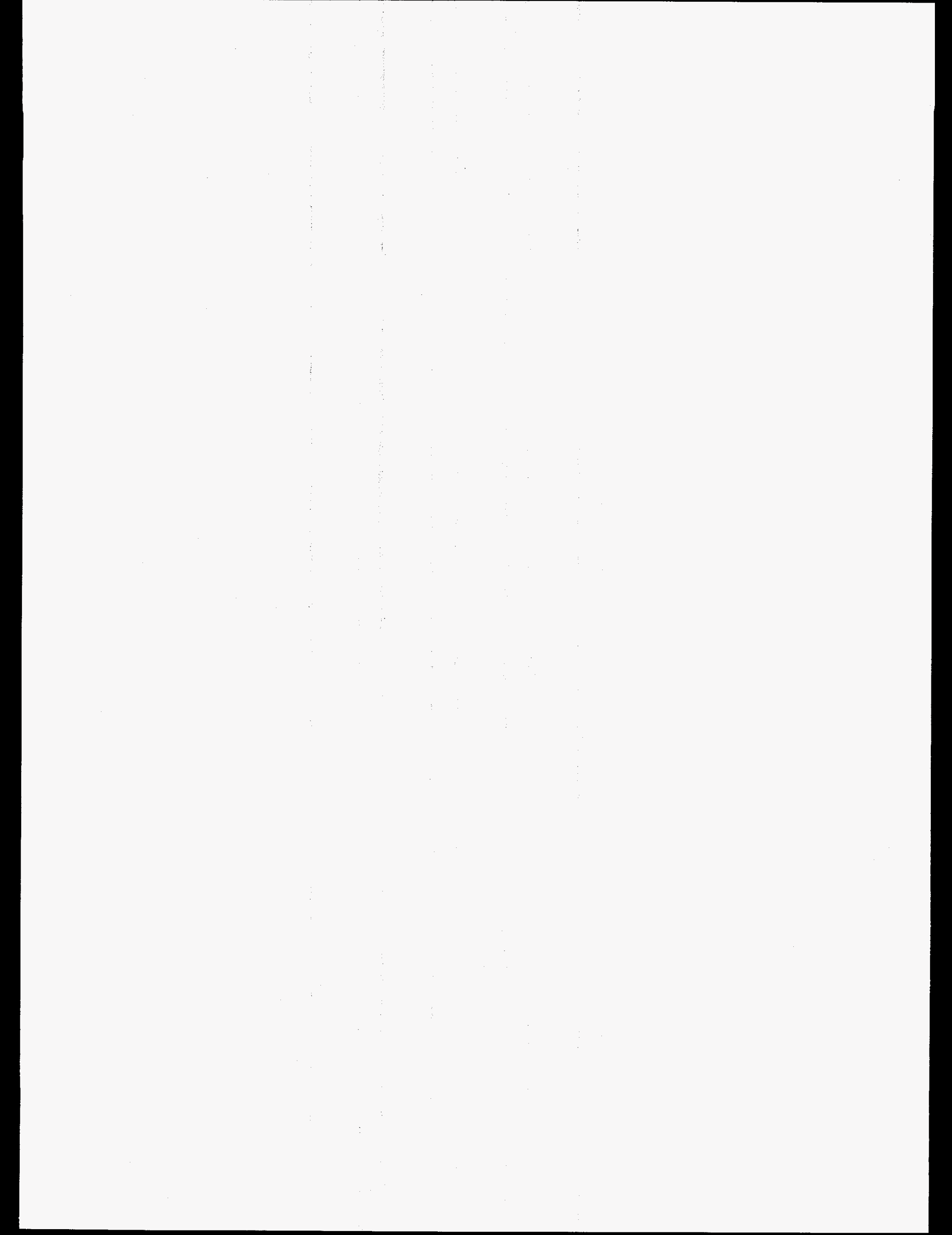




\section{GENERAL AVIATION}

\subsection{INTRODUCTION}

General aviation is the broad categorization of all aviation activities which do not fall under the categories of commercial aviation and military aviation. It includes diverse and dissimilar aviation activities such as personal flying, business flying, corporate/executive flights, aerial (spray) application, and instructional or training flights. As the first step in compiling the data necessary to determine the risk to facilities from general aviation aircraft crashes, the term general aviation must be more formally and precisely defined. This definition will determine which aircraft accidents and operations (tlights, departures, landings, aircraft hours, aircraft miles, etc.) will be tabulated to determine the aircraft crash rate relevant to the risk analysis.

The Federal Aviation Administration (FAA) in their General Aviation Activity Survey [Refs. 3.1 to 3.8 ], defines general aviation as all aircraft in the U.S. civil air fleet except those operated under 14 CFR 121 [Ref. 3.9] and 14 CFR 127 [Ref. 3.10]. Thus, under the FAA definition, general aviation includes all aircraft of the U.S. civil air fleet operated under 14 CFR 91 [Ref. 3.11], 103 [Ref. 3.12], 105 [Ret. 3.13], 125 [Ref. 3.14], 133 [Ref. 3.15], 135 [Ref. 3.16], and 137 [Ref. 3.17]. Note that this definition excludes all foreign owned and foreign operated aircraft . Those foreign aircratt which operate as air carriers in U.S. airspace and foreign operators of U.S. registered aircratt engaged in common carriage operate under 14 CFR 129 [Ref. 3.18].

As defined by the National Transportation Safety Board (NTSB) in their compilation of accident data [Refs. 3.19 to 3.25], general aviation is considered as all accidents involving U.S. registered aircraft that were not conducting air carrier revenue operations under 14 CFR 121, 125, 127 or 135. So, under the NTSB definition, general aviation includes aircraft operated under 14 CFR 91, 103, 105, 133, and 137. Note that the difference between the FAA's definition and the NTSB's definition of general aviation is the inclusion or exclusion of aircraft operating under 14 CFR 125 and 135.

For the purpose of developing the ACRAM Standard [Ref. 3.19], general aviation will be as defined by the NTSB in their compilation of general aviation accident data, that is all accidents involving U.S. registered aircraft that were not conducting air carrier revenue operations under 14 CFR 121, 125, 127, or 135.

The reason for adopting the NTSB for general aviation is because of the method that the FAA uses to tabulate their estimate of the general aviation operations. In conversations with the FAA staff which prepares the General Aviation Activity Survey [Refs. 3.1 to 3.8 ] and reviewing the operational data in those documents, it was discovered that contrary to their definition given in their introductory section, no 14 CFR 125 operators, and those operators that were known to be scheduled 14 CFR 135 operators were not included in the survey. Nevertheless, despite a conscious effort to exclude these operators, some scheduled 14 CFR 135 operators (commuter air carriers) were included in the survey along with nonscheduled 14 CFR 135 operators (air taxis).

If the FAA definition was used for general aviation, it would be necessary to determine the accidents of those particular scheduled 14 CFR 135 operators along with all of the nonscheduled 14 CFR 135 operators which were included in the General Aviation Activity Survey [Refs. 3.1 to 3.8]. This would require the identification of those scheduled 14 CFR 135 operators and the nonscheduled 14 CFR 135 operators which were included in the General Aviation Activity Survey, and determining the accidents that occurred to the particular aircratt operated by these operators while performing scheduled 14 CFR 135 flights. These accidents would then have to be added to the accidents compiled by the NTSB for general aviation. Then an accident rate could be determined using the combined accidents of the NTSB for general aviation and the scheduled 14 CFR 135 operators included in the General Aviation Activity Survey, divided by the total number of operations (landings, aircraft hours, aircraft miles, etc.) as estimated by the General Aviation Activity Survey.

Instead, we have chosen to subtract the operations of the scheduled and nonscheduled14 CFR 135 operators included in the General Aviation Activity Survey from the total number of general aviation operations estimated. It is then possible to determine the general aviation accident rate by dividing the total general aviation accidents tabulated by the NTSB by general aviation operations (total-scheduled 
135 and nonscheduled 135 operators) calculated from the General Aviation Activity Survey. This method was chosen because it was judged to be easier to accomplish.

The major category of general aviation has been divided further into seven subcategories. This was done because the general aviation covers a wide array of dissimilar aircraft types, accident and operational data was available for the subcategories, and by determining the general aviation accident rates by subcategory would enable certain subcategories of general aviation aircraft to be screened from further analysis due to facility structural considerations, i.e. the facility is buit such that it could withstand the direct impact with smaller general aviation aircratt subcategories. For general aviation, seven subcategories are defined: 1) single-engine piston, fixed wing, 2) multiple-engine piston, fixed wing, 3) turboprop, fixed wing, 4) turbojet, fixed wing, 5) piston, rotary wing, 6) turbine, rotary wing, and 7) other. The seventh subcategory, other, developed only for the purpose of completing the accident and operations table such that it could be screened from further consideration. Aircraft included in the "other" subcategory include hanggliders, gliders, balloons, and any other aircratt which did not fit the previous six subcategories.

The development of general aviation data for the ACRAM Standard is discussed in the following sections: 3.2 General Aviation Crashes, 3.3 General Aviation Operations, 3.4 General Aviation Crash Rates, 3.5 General Aviation In-Flight Non-Airway Crash Rate, 3.6 General Aviation Crash Parameter Distributions, 3.7 General Aviation Aircraft Characteristics, and 3.8 References. 


\subsection{GENERAL AVIATION CRASHES}

Tables 3.1 to 3.13 taken from the NTSB Annual Review of Aircraft Accident Data, U.S. General Aviation [Ref. 3.20 to 3.27] presents the following information by years for the years 1986 to 1993:

1. The number of fatal accidents by phase of operation for each year,

2. The number of injury accidents by phase of operation for each year,

3. The number of property damage accidents by phase of operation for each year, and

4. The total number of accidents by phase of operation for each year.

The tables listed below gives the general aviation subcategories for which the general aviation accidents by phase of flight operation table was created.

Table 3.1 Single-engine reciprocating (piston), fixed wing

Table 3.2 Multiple-engine reciprocating (piston), fixed wing

Table 3.3 Turboprop, fixed wing

Table 3.4 Turbojet, fixed wing

Table 3.5 Total, fixed wing (summation of Tables 3.1 to 3.4)

Table 3.6 Reciprocating (piston), rotary wing

Table 3.7 Turbine, rotary wing

Table 3.8 Total, rotary wing (summation of Tables 3.6 and 3.7)

Tab.e 3.9 Total powered aircraft (summation of Tables 3.1 to 3.4, 3.6 and 3.7)

Table 3.10 Gliders

Table 3.11 Balloons

Table 3.12 Other aircraft

Table 3.13 Total aircraft (summation of Tables 3.1 to $3.4,3.6$ to $3.7,3.10$ to 3.12 )

The years 1986 to 1993 were chosen as the period of interest because it is the most current and complete. General aviation accident data compiled by the NTSB prior to 1985 were tabulated by on the bases of aircraft destruction, aircraft substantial damage, or minor or no aircratt damage. It would be possible to tabulate NTSB general aviation accident data prior to 1985 and to combine it with the current tables. However, the utility of that exercise would be limited due to limitations on the operations data compiled by the FAA prior to 1985 . See Section 3.3 for further discussion on the general aviation operations data prior to 1985.

Because of the number of phases of operation used by the NTSB in their general aviation accident summary documents [Ref. 3.20 to 3.27 ], and because the ACRAM standard defines only three flight phases for its frequency analysis, it was judge necessary to combine the phases of operation into a smaller set of flight phases that could be more easily matched with the ACRAM flight phases. The phases of operation called standing, stand-preflight, standing-standing engine(s), standing-engine(s) operating, standing-engine(s) not operating, and standing-idling rotors were combined into the standing flight phase. The phases of operation called taxi, taxi-to takeoff, take-from landing, and taxi-aerial were combined into the taxi flight phase. The phases of operation called takeoff, takeott-aborted, and takeotfground run were combined into the takeoff flight phase. The phase of operation called takeotf-initial climb was retained as the intial climb flight phase. The climb and climb-to-cruise phases of operation were combined into the climb (to cruise) flight phase. The phases of operation called cruise, cruise-normal, and cruise-holding (IFR) were combined into the cruise (enroute) flight phase. The Phases of operation called descent, descent-nomal, descent-emergency, and descent-uncomtrolled were combined into the descent (from cruise) flight phase. The flight phases called approach, approach-VFR pattern-downwind, approach-VFR pattern-base turn, approach-VFR pattern-base to final, approach-VFR pattern-final approach, approach-go around (VFR), approach-IAF to FAR/outer marker (IFR), approach-FAR/outer marker to threshold, approach-circling (IFR), and approach-missed approach (IFR) were combined in the landing approach flight phase. The phases of operation called landing-aborted, landing, landingflare/touchdown, and landing/roll were combined in the landing flight phase. The phases of operation called maneuvering, manuevering-aerial application, manuevering-turn to reverse direction, manueveringturn to landing area (emer.), and hover were combined into the manuevering flight phase. The other, unknown, and not reported phases of operation were combined into the other/?/NR flight phase. The manuevering and other/?/NR flight phases were created for the sole purpose of tabulating the accidents 
to assure that the numbers in Tables 3.1 to 3.13 were consistent with Refs. 3.20 to 3.27 . These flight phases were not included in determining the accident percentages by flight phase.

One additional feature of Table 3.13 is the inclusion of aircraft damage for each year. The NTSB did not tabulate accident data according to aircraft damage by flight phase after 1986 but did include totals on aircratt damage. These data are included at the bottom of Table 3.13. An noteworthy feature is that of the 18.418 general aviation accidents tabulated by the NTSB for 1986 to 1993 , only 236 accidents or $1.28 \%$ of the total resulted in minor or no damage to the aircraft. From this, it was determined that for general aviation, different definitions for aircraft accidents and aircraft crashes were not necessary since virtually all general aviation accidents resulted in destruction or substantial damage to the aircraft. Therefore, for general aviation only, the terms aircraft accidents and aircraft crashes are used synonymously.

Table 3.14 summarizes the general aviation accidents by phase of flight operation and by aircraft subcategories as presented by Tables 3.1 to 3.13. Table 3.15 presents the same information as Table 3.14 (General aviation accidents by phase of flight operation and by aircraft subcategories) except that accidents during the standing, and taxi flight phases and accidents classified as either other, unknown, or not recorded flight phases have been excluded. This was done in order the develop the aircraft accident percentages occurring in the flights phases applicable to the ACRAM standard frequency analysis. 
General Aviation Aceldents by Phase of Flight Operation

Single Engine-Aeclprocaling. Fixed Wing

References 3.20 to 3.27

Single-Engine Reciprocating, Fixod Wing

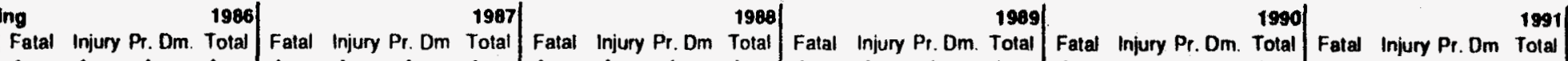

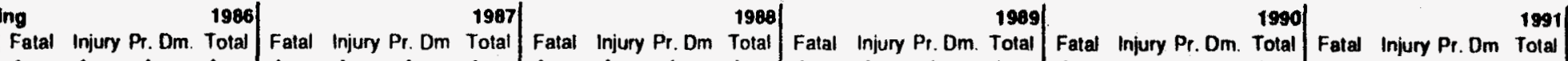
Phase of Operation Acc. ACC. ACC. ACC

Standing

Standing-prellight

Standing-starting engine(s)

Standing-engine(s) operating

Standing-engine(s) not operating

Standing-kdling rotors

Taxi

Texi-to takeolf

Taxi-trom landing

Taxi-gerial

Takeoll-abortad

Takeofl-ground run

Takeoll -initial climb

Climb

Climb-to cruise

Cruise

Cruise-normal

Cruise-holding (IFR)

Descent

Descent-nomal

Descent-emergency

Descent-uncontrolled

Approach

Approact-VFR pattern-downwind

Approsch. VFA pattern-base turn

Approach-VFA pattern-base to final

Approach-VFR pattern-final approach

Approach-go-around (VFR)

Approach-IAF to FAFlouter marker (IFA)

Approach-FAF/outer marker to thresholr

Approach-circling (IFA)

Approach-missed approach (IFP)

Landing-aborted

Landing

Landing-flare/touchdown

Landing-roll

Maneuvering

Manouvering-aerial application

Maneuvering-turn to reverse direction

Maneuvering turn to lending ares lemer

Hover

Unknown

Not roported

\begin{tabular}{ll}
0 & 0 \\
1 & 0 \\
0 & 5 \\
1 & 0 \\
0 & 0 \\
0 & 0 \\
\hline 1 & 0 \\
\hline
\end{tabular}

ACC. ACC. ACC. ACC ACC. ACC. ACC. ACC.

Total:

\begin{tabular}{rr|rrrr|rrrr|r}
0 & 0 & 0 & 0 & 0 & 0 & 0 & 0 & 1 & 1 & 0 \\
0 & 1 & 0 & 1 & 0 & 1 & 0 & 0 & 1 & 1 & 0 \\
16 & 21 & 1 & 2 & 17 & 20 & 0 & 3 & 15 & 18 & 0 \\
4 & 5 & 2 & 2 & 7 & 11 & 1 & 1 & 6 & 8 & 1 \\
0 & 0 & 0 & 0 & 1 & 1 & 0 & 0 & 0 & 0 & 0 \\
0 & 0 & 0 & 0 & 0 & 0 & 0 & 0 & 0 & 0 & 0 \\
\hline 2 & 13 & 0 & 0 & 3 & 3 & 1 & 0 & 5 & 6 & 0
\end{tabular}

\begin{tabular}{ll}
0 & 0 \\
0 & 0 \\
0 & 0 \\
1 & 4 \\
0 & 0 \\
0 & 0 \\
\hline 0 & 0
\end{tabular}

\begin{tabular}{rr}
0 & 1 \\
0 & 0 \\
0 & 13 \\
4 & 9 \\
0 & 0 \\
0 & 0 \\
\hline 0 & 5
\end{tabular}

\begin{tabular}{r|rrrr|r} 
& ACC. & ACC. & ACC. & ACC. & ACC \\
1 & 0 & 1 & 0 & 1 & \\
0 & 0 & 0 & 0 & 0 & \\
13 & 0 & 1 & 7 & 8 & \\
14 & 0 & 1 & 6 & 7 & \\
0 & 0 & 0 & 0 & 0 & \\
0 & 0 & 0 & 0 & 0 & \\
\hline 5 & 1 & 0 & 9 & 10 &
\end{tabular}

\begin{tabular}{ll}
0 & 0 \\
1 & 1 \\
0 & 2 \\
2 & 1 \\
0 & 0 \\
0 & 0 \\
\hline 0 & 1
\end{tabular}

2
0
4
0
0

\begin{tabular}{rr|}
2 & 2 \\
0 & 2 \\
4 & 6 \\
0 & 11 \\
0 & 0 \\
0 & 0 \\
\hline
\end{tabular}

$\begin{array}{llll}0 & 0 & 28 & 28\end{array}$

\begin{tabular}{rrrr|rrrr}
0 & 0 & 0 & 0 & 0 & 0 & 0 & 0 \\
\hline 6 & 5 & 19 & 30 & 1 & 6 & 15 & 22 \\
0 & 0 & 0 & 0 & 0 & 0 & 0 & 0
\end{tabular}

$\begin{array}{lll}5 & 20 \\ 1 & 15 & 16\end{array}$

\begin{tabular}{rrrr}
0 & 0 & 23 & 23 \\
0 & 0 & 0 & 0 \\
\hline
\end{tabular}

\begin{tabular}{rrrr|rrrr|rrr|r}
4 & 6 & 100 & 110 & 1 & 7 & 99 & 107 & 1 & 2 & 70 & 73 \\
\hline 54 & 52 & 204 & 310 & 42 & 63 & 195 & 300 & 56 & 64 & 167 & 287 \\
\hline 11 & 5 & 18 & 34 & 4 & 4 & 10 & 18 & 3 & 2 & 13 & 18
\end{tabular}

\begin{tabular}{rrrr}
18 & 5 & 22 & 45 \\
\hline 29 & 13 & 96 & 138 \\
36 & 32 & 143 & 211
\end{tabular}

\begin{tabular}{rrr}
0 & 0 \\
2 & 1 \\
\hline 48 & 48 & 185 \\
\hline 6 & 1
\end{tabular}

\begin{tabular}{r|rrr}
\hline 0 & 0 & 7 & 21 \\
70 & 3 & 3 & 6 \\
\hline
\end{tabular}

\begin{tabular}{rrrr|rrrr}
12 & 6 & 27 & 45 & 21 & 4 & 23 & 48 \\
\hline 30 & 8 & 73 & 111 & 33 & 16 & 85 & 134 \\
0 & 15 & 134 & 197 & 45 & 27 & 143 & 215
\end{tabular}

$\begin{array}{r}43 \\ 0 \\ \hline\end{array}$

\begin{tabular}{rr}
2 & 16 \\
17 & 121 \\
0 & 1 \\
\hline 1 &
\end{tabular}

\begin{tabular}{r|r}
14 & 64 \\
\hline 149 & \\
187 & 29
\end{tabular}

\begin{tabular}{rr|rrrr|rr}
7 & 16 & 8 & 1 & 5 & 14 & 1 & 1 \\
34 & 39 & 5 & 2 & 18 & 25 & 9 & 5 \\
1 & 2 & 0 & 0 & 2 & 2 & 0 & 0 \\
0 & 1 & 1 & 0 & 2 & 3 & 2 & 0 \\
\hline 19 & 33 & 11 & 7 & 29 & 47 & 8 & 2
\end{tabular}

\begin{tabular}{rrrr|rrrr|rrrr|rrrrrr}
3 & 1 & 1 & 5 & 2 & 0 & 2 & 4 & 0 & 1 & 0 & 1 & 1 & 0 & 2 & 3 & 2 & 0 \\
\hline 9 & 5 & 17 & 31 & 5 & 6 & 21 & 32 & 9 & 5 & 19 & 39 & 11 & 7 & 29 & 47 & 8 & 2 \\
4 & 2 & 15 & 21 & 9 & 4 & 10 & 23 & 3 & 3 & 10 & 16 & 5 & 2 & 8 & 15 & 11 & 1 \\
4 & 0 & 5 & 9 & 1 & 2 & 3 & 6 & 2 & 2 & 5 & 9 & 3 & 1 & 4 & 8 & 4 & 1
\end{tabular}

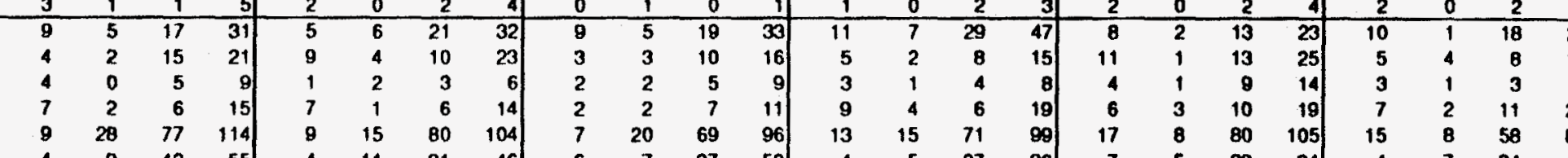

14

$\begin{array}{ll}4 & 9 \\ 1 & 2\end{array}$

\begin{tabular}{rrrr|r}
1 & 3 & 6 & 23 & 6 \\
1 & 0 & 1 & 2 & 1 \\
1 & 0 & 3 & 4 & 3 \\
\hline 0 & 0 & 0 & 0 & 0
\end{tabular}

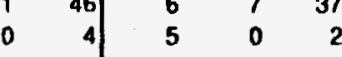

\begin{tabular}{r|r}
50 & 4 \\
7 & 4
\end{tabular}


Table 3.1 (Continued)

General Aviation Aceldent by Pheee of Flight Operation

Single Engine-Poclprocating, Fixed Wing

Referencese 3.20103 .27

\begin{tabular}{|c|c|c|c|c|c|c|c|c|c|c|c|c|c|c|c|}
\hline $\begin{array}{c}\text { SIngle-Engine Reclprocating, Fixed Wir } \\
\text { Phase of Operation }\end{array}$ & $\begin{array}{l}\text { Ing } \\
\text { Fatal } \\
\text { Aoc. }\end{array}$ & \multicolumn{2}{|c|}{$\begin{array}{l}\text { Injury } \mathrm{Pr} \text {. Drn } \\
\text { Acc. AC. }\end{array}$} & $\begin{array}{c}1992 \\
\text { Total } \\
\text { Acc. }\end{array}$ & $\begin{array}{l}\text { Fatal } \\
\text { Acc. }\end{array}$ & \multicolumn{2}{|c|}{$\begin{array}{l}\text { Injury Pr. Dm } \\
\text { Acc. Acc. }\end{array}$} & $\begin{array}{c}1993 \\
\text { Total } \\
\text { Acc. }\end{array}$ & $\begin{array}{l}\text { Fatal } \\
\text { Acc. }\end{array}$ & $\begin{array}{l}\text { Iniury } \\
\text { Acc. }\end{array}$ & Pr. Dm. & $\begin{array}{c}\text { TOTAL } \\
\text { Total } \\
\text { Acc. } \\
\end{array}$ & $\begin{array}{l}\text { Flight } \\
\text { Phase }\end{array}$ & No. & Pct. \\
\hline Standing & 0 & 0 & 0 & 0 & 0 & 1 & 1 & 2 & 0 & 2 & 5 & 7 & & & \\
\hline Standing-preflight & 0 & 1 & 0 & 1 & 0 & 0 & 0 & 0 & 2 & 3 & 1 & 6 & & & \\
\hline Standing-starting ongino(s) & 0 & 1 & 3 & 4 & 1 & 0 & 8 & 9 & 2 & 14 & 83 & 99 & & & \\
\hline Standing-engine(s) operating & 2 & $\mathbf{0}$ & 1 & 3] & 3 & $\mathbf{3}$ & 2 & 8 & 12 & 12 & 43 & 67 & & & \\
\hline Standing-engine(s) not operating & 0 & 0 & 0 & o] & 0 & 0 & 0 & 0 & 0 & 0 & 1 & 1 & & & \\
\hline Standing-idling rotors & 0 & 0 & a & 0 & 0 & o & 0 & 0 & 0 & 0 & 0 & 0 & Standing & 180 & $1.22 \%$ \\
\hline$\overline{\text { Taxi }}$ & 0 & $\mathbf{0}$ & 4 & 4 & 0 & 0 & 6 & 6 & 3 & 1 & 48 & 52 & & & \\
\hline Taxi-to takeoft & $\mathbf{0}$ & 2 & 15 & 17 & 0 & 1 & 17 & 18 & 0 & 4 & 152 & 156 & & & \\
\hline Taxi-trom landing & 0 & 1 & 13 & 14 & 0 & 1 & 14 & 15 & 0 & 5 & 150 & 155 & & & \\
\hline Toxi-aerial & 0 & 0 & 0 & of & 0 & 0 & 0 & 0 & 0 & 0 & 1 & 1 & Taxi & 364 & $2.48 \%$ \\
\hline Takeoff & 4 & 2 & 11 & 17 & 4 & 2 & 13 & 19 & 33 & 34 & 140 & 207 & & & \\
\hline Takeolf-aborted & 0 & 0 & 0 & o & 0 & 0 & 0 & 0 & 0 & 1 & 15 & 16 & & & \\
\hline Takeoff-ground run & 0 & 4 & 67 & 71 & 2 & $\mathbf{0}$ & 70 & 72 & 14 & 28 & 620 & 662 & Takoolf & 885 & $6.02 \%$ \\
\hline Takeoff-initial dimb & 46 & 46 & 153 & 245 & 40 & 44 & 147 & 231 & 383 & 425 & 1377 & 2185 & Initial Climb & 2185 & $14.86 x$ \\
\hline$\overline{\text { Climb }}$ & 5 & $T$ & 14 & 20 & $\overline{8}$ & 3 & 17 & 28 & 56 & 30 & 104 & 180 & & & \\
\hline Climb-to cruise & 4 & 3 & 16 & 23 & 5 & 5 & 14 & 24 & 88 & 34 & 145 & 267 & Climb & 457 & $3.11 \%$ \\
\hline Cruiso & 39 & 13 & 73 & 125 & 39 & 21 & 97 & 157 & 290 & 122 & 687 & 1099 & & & \\
\hline Cruise-normal & 35 & 11 & 105 & 151 & 27 & 10 & 89 & 126 & 298 & 144 & 955 & 1397 & & & \\
\hline Cruise-holding (IFA) & 0 & 0 & 0 & of $1-3-1-1-1$ & 0 & 0 & 0 & of & 0 & 0 & 4 & 4 & Cruise & 2500 & $17.00 \%$ \\
\hline Descent & 4 & 2 & 2 & 8) & 10 & 0 & 6 & 16 & 49 & 10 & 46 & 105 & & & \\
\hline Descent-normal & 10 & 2 & 24 & 36 & $\mathbf{3}$ & 0 & 24 & 27 & 50 & 24 & 191 & 265 & & & \\
\hline Descent-emergency & 1 & o & 2 & 3) & 1 & 1 & 3 & 5 & 7 & 4 & 19 & 30] & & & \\
\hline Descent-uncontrolled & 1 & 2 & 2 & 5 & 0 & 1 & 2 & 3 & 11 & 5 & 13 & 29 & Descent & 429 & $2.82 \%$ \\
\hline Approach & 9 & 7 & 5 & 21 & 6 & 2 & 15 & 23 & 67 & 35 & 137 & 239 & & & \\
\hline Approach-VFR pattern-downwind & 2 & 1. & 14 & 17 & 2 & 3 & 4 & 9 & 41 & 20 & 82 & 143 & & & \\
\hline Approach-VFA pattern-base turn & 2 & 1 & 6 & 9) & 3 & 2 & 3 & 8] & 22 & 10 & 38 & 70 & & & \\
\hline Approach-VFA pattern-base to final & 6 & $\mathbf{3}$ & 6 & 15 & 5 & 0 & 10 & 15 & 49 & 17 & 62 & 128 & & & \\
\hline Approach-VFR pattern-final approach & 12 & 11 & 61 & 84 & 10 & 9 & 63 & 82 & 92 & 114 & 559 & 765 & & & \\
\hline Approach-po-around (VFR) & 6 & 3 & 23 & 32 & 3 & 6 & 34 & 43 & 38 & 53 & 250 & 341 & & & \\
\hline Approach-iAF to FAFlouter marker (IFA) & $\mathbf{3}$ & 0 & 1 & 4. & 1 & 0 & 1 & 2 & 23 & 4 & B & 35 & & & \\
\hline Approach-FAFlouter marker to threshold & 7 & 1 & 4 & 12 & 4 & 2 & 4 & 10 & 44 & 15 & 29 & 88) & & & \\
\hline Approach-circling (IFA) & $\mathbf{0}$ & 0 & o & 0 & 2 & 0 & 0 & 2 & 4 & 2 & 2 & 8] & & & \\
\hline Approach-missed approach (IFA) & 2 & 0 & 1 & 3) & 0 & 2 & 0 & 2 & 14 & 3 & 6 & 23 & Approach & 1840 & $12.51 \%$ \\
\hline Landing-aborted & $\mathbf{0}$ & $\mathbf{0}$ & 0 & 0 & 0 & 0 & $\mathbf{0}$ & 0 & 3 & 4 & 18 & 25 & & & \\
\hline Landing & 3 & 2 & 32 & 37) & 0 & 3 & 31 & 34 & 13 & 23 & 229 & 265 & & & \\
\hline Landing-flare/louchiown & 2 & 7 & 139 & 148 & 2 & 3 & 123 & 128 & 15 & 48 & 1231 & 1294 & & & \\
\hline Landing-rolf & 3 & 4 & 209 & 216 & 1 & 5 & 209 & 215 & 15 & 44 & 1857 & 1916 & Landing & 3500 & $23.80 x$ \\
\hline Manouvering & $\mathbf{B O}$ & 18 & 44 & 142 & 94 & 25 & 41 & 160 & 706 & 174 & 395 & 1275 & & & \\
\hline Manouvering-agrial application & 6 & B & 34 & 48 & 8 & 7 & 27 & 42 & 67 & 68 & 303 & 438 & & & \\
\hline Maneuvering-turn to reverse direction & 9 & 5 & 9 & 23 & 3 & 3 & 7 & 1.3 & 58 & 28 & 68 & 154 & & & \\
\hline Manouvering-turn to landing area (emer. & 1 & 1 & 0 & 2 & 0 & $\mathbf{0}$ & 1 & 1 & 5 & 1 & 12 & 18 & & & \\
\hline Hover & 0 & 0 & 0 & of & $\underline{0}$ & 0 & 0 & of & o & $\underline{0}$ & 0 & of & Manouvering & 1885 & $12.82 x_{0}$ \\
\hline Other & 10 & 5 & 39 & 54 & 2 & 3 & 42 & 47 & 34 & 23 & 248 & 305 & & & \\
\hline Unknown & 13 & 0 & 6 & 19 & 12 & 2 & 1 & 15 & 101 & 6 & 15 & 122 & & & \\
\hline Not reported & 4 & 0 & 7 & 11 & 4 & 2 & 5 & 11 & 23 & 2 & 30 & 55 & Other/?/NR & 482 & $3.28 \%$ \\
\hline Total: & 331 & 168 & 1145 & $1644 \mid$ & 305 & 772 & 1151 & 1628| & 2732 & 1596 & 10379 & 14707 & & 14707 & $100.00 \%$ \\
\hline
\end{tabular}


General Avtation Accldents by Phmee of Filght Operation Multi-Engine Aeciprocating, Flxed Wing

Reterences 3.20103 .27

Mulli-Englne Reclprocaling, Flxed Wing

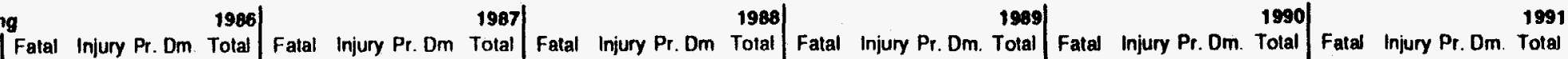

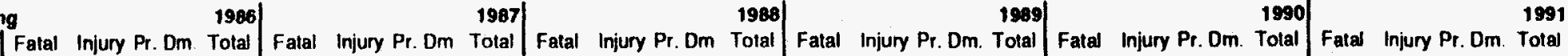

Phase of Operation ACc. \begin{tabular}{l} 
Fatal Injury Pr. Dm. Total \\
Acc. Fatal Injury Pr. Dm. Total \\
\hline
\end{tabular}

Standing

Standing-prellight

Slanding-starting engine(s)

Standing-engine(s) operating

Standing-engine(s) not operating

Slanding-ddling rotors

Taxi-to lakeolf

Taxi-trom landing

Taxi-gerial

Takeoff

Takeofl-aborted

Takeoll-ground run

Takooff-initial climb

Climb

Climb. 10 cruise

Cruise

Cruise-normal

Cruise-holding (IFP)

Descent

Descent-nomal

Descent-emergency

Descent-uncontrofled

Approach

Approach-VFA pattern-downwind

Approach-VFR pattern-base hirn

Approach-VFR pattern-base to final

Approach-VFR pattern-linal approach

Approach-go-around (VFR)

Approact. IAF to FAF/outer marker (IFA)

Approach-FAF/outer marker to threshold

Approach-circling (IFP)

Approach-missed approach (IFB)

Landing-aborted

Landing

L. anding flare/touchdown

Landing-roll

Maneuvering

Maneuvering-aerial application

Maneuvering turn to reverse direction

Maneuvering-turn to landing area (emer.

Hover

Unknown

Not reported 
Tablo 3.2 (Continued)

General Aviation Aceldent by Phese of Fllght Operation

Multi-Engine Reciprocalling, Flxed Wing

Reterences 3.20 to 3.27

\begin{tabular}{|c|c|c|c|c|c|c|c|c|c|c|c|c|c|c|c|}
\hline $\begin{array}{c}\text { Multt-Englne Aoclprocating, Fixed Wir } \\
\text { Phase of Operation }\end{array}$ & $\begin{array}{l}9 \\
\text { Fatal } \\
\text { Acc. }\end{array}$ & $\begin{array}{l}\text { Injury } \\
\text { ACC. }\end{array}$ & $\begin{array}{l}\text { y } \mathrm{Pr} . \mathrm{Dr} \\
\mathrm{ACC} \\
\end{array}$ & $\begin{array}{r}1992 \\
\text { Total } \\
\text { Acc } \\
\end{array}$ & $\begin{array}{l}\text { Fatal } \\
\text { ACC. }\end{array}$ & $\begin{array}{l}\text { Injur } \\
\mathbf{A C x} \text {. }\end{array}$ & $\begin{array}{l}\text { Pr. Dm } \\
\text { Acc. }\end{array}$ & $\begin{array}{l}1993 \\
\text { Total } \\
\text { Acc. } \\
\end{array}$ & $\begin{array}{l}\text { Fatal } \\
\text { Acc. }\end{array}$ & $\begin{array}{c}\text { Injury } \\
\text { Acc. }\end{array}$ & $\begin{array}{c}\text { Pr. Dm. } \\
\text { Acc. }\end{array}$ & $\begin{array}{l}\text { TOTAL } \\
\text { Total } \\
\text { Acc. } \\
\end{array}$ & $\begin{array}{c}\text { Flight } \\
\text { Phase } \\
\end{array}$ & No. & Pct. \\
\hline Standing & 0 & & 0 & 0 & 0 & r & 0 & o) & 0 & 0 & 2 & 2 & & & \\
\hline Standing-prellight & 0 & & 0 & 0 & 0 & ( & 0 & 0 & 0 & 0 & 1 & 1 & & & \\
\hline Standing-starting engine(s) & 0 & & 0 & 1 & 0 & c & 2 & 2 & 0 & 0 & 5 & 5 & & & \\
\hline Standing-engine(s) operating & o & & 0 & 0 & 0 & c & 0 & 0 & 0 & 0 & 3 & 3 & & & \\
\hline Standing-engine(s) not operating & o & c & 0 & 0 & o & ( & 1 & 1 & 0 & 0 & 2 & 2 & & & \\
\hline Standing-idling rotors & 0 & $\mathrm{c}$ & 0 & 0 & $\underline{0}$ & 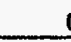 & 0 & of & 0 & 0 & 0 & of & Standing & 13 & $1.00 \%$ \\
\hline Taxi & 1 & & 0 & 2 & $\overline{0}$ & 7 & 0 & 0 & 1 & $\overline{0}$ & 4 & 5 & & & \\
\hline Taxi-to takeoff & 0 & & 0 & 0 & 0 & ( & 2 & 2 & 0 & 0 & 20 & 20 & & & \\
\hline Taxi-from landing & o & c & 0 & 1 & 0 & c & 0 & 0 & 1 & $\mathbf{0}$ & 12 & 13 & & & \\
\hline Taxi-aerial & 0 & c & 0 & 0 & 0 & 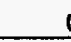 & 0 & of & 0 & 0 & 0 & 0 & Taxd & 38 & $3.00 \%$ \\
\hline Takeoff & 0 & c & $\overline{0}$ & 1 & 1 & 7 & 1 & 2 & 11 & 1 & 15 & 27 & & & \\
\hline Takeoff-aborted & 0 & & 0 & 0 & 0 & c & 0 & o. & 0 & 0 & 0 & o) & & & \\
\hline Takeotf-ground run & D & & 1 & 4 & 1 & 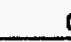 & 3 & 4 & 2 & 4 & 38 & 44] & Takooff & 71 & $5.60 \%$ \\
\hline Takeotf-initial dimb & 4 & c & 0 & 11 & 8 & 3 & 16 & 27 & 57 & 16 & $8 B$ & 161 & Initial Climb & 161 & $12.70 \%$ \\
\hline Climb & 1 & & 0 & 1 & $\overline{2}$ & $\bar{T}$ & $T$ & 3 & 9 & 4 & 7 & 20 & & & \\
\hline Climb-to cruise & 4 & c & 0 & 5 & 2 & 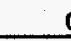 & 0 & 2 & 20 & 3 & 13 & 36 & Climb & 56 & $4.42 \%$ \\
\hline Cruise & $\overline{4}$ & & 2 & 12 & 6 & 7 & B & 18 & 39 & 11 & 39 & 89 & & & \\
\hline Cruise-normal & 12 & & 5 & 24 & 3 & c & 5 & 8 & 64 & 14 & 71 & 149 & & & \\
\hline Cruise-holding (IFA) & $\underline{0}$ & c & 0 & 0 & 0 & 1 & 0 & of & 0 & 0 & 1 & 1 & Cruise & 239 & $18.85 \%$ \\
\hline Descent & 1 & & 0 & 2 & 0 & 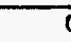 & 0 & 0 & 6 & 0 & 2 & 8 & & & \\
\hline Descent-normal & 2 & & 0 & 6 & 1 & c & 0 & 1 & 4 & 2 & 13 & 19 & & & \\
\hline Descent-emergency & 0 & & 0 & o) & 0 & $\mathrm{c}$ & $\mathbf{0}$ & 0 & 0 & $\mathbf{0}$ & 1 & 1 & & & \\
\hline Descent-uncontrolled & o & c & 0 & of & $\mathbf{0}$ & 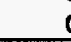 & 0 & of & 3 & $\underline{0}$ & 0 & 3] & Descent & 31 & $2.44 \%$ \\
\hline Approach & 3 & & 0 & 8 & $\overline{3}$ & 7 & 1 & 4 & 21 & 2 & 19 & 42 & & & \\
\hline Approach-VFR pattern-downwind & 1 & & 0 & 1 & 0 & c & 4 & 4 & 3 & 2 & 14 & 19 & & & \\
\hline Approach-VFR pattern-base turn & 0 & & 0 & 0 & 0 & c & 0 & 0 & 3 & 1 & 0 & 4) & & & \\
\hline Approach-VFA pattern-base to inal & 0 & & 0 & of & 2 & c & 0 & 2 & 8 & 0 & 4 & 12 & & & \\
\hline Approach-VFR pattern-final approach & o & & 1 & 6) & 0 & 3 & 4 & 7 & 6 & 13 & 39 & 58 & & & \\
\hline Approach-go-around (VFA) & 1 & & 0 & 2 & 0 & c & 3 & 3) & 6 & 4 & 16 & 26 & & & \\
\hline Approach-IAF to FAF/outer marker (IFR) & o & & 0 & 0 & 0 & 1 & 0 & 1 & B & 3 & 0 & 11 & & & \\
\hline Approach-FAF/outer marker to threshold & 1 & 1 & 1 & 3 & o & c & $\mathbf{3}$ & 3 & 11 & 7 & 15 & 33 & & & \\
\hline Approach-circling (IFA) & 1 & 1 & 1 & 3 & 1 & 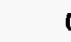 & $\mathbf{0}$ & 1 & 9 & 2 & 4 & 15 & & & \\
\hline Approach-missed approach (IFA) & 2 & & 1 & 4 & 1 & 1 & 1 & 2 & 8 & 3 & 5 & 16 & Approach & 236 & $18.61 \%$ \\
\hline Landing-aborted & 0 & & 0 & 0 & 0 & t & 0 & 0 & $\mathbf{0}$ & 0 & 0 & 0 & & & \\
\hline Landing & 0 & & 0 & 2 & 0 & 2 & 2 & 4 & 1 & 2 & 19 & 22 & & & \\
\hline Landing-flare/touchiown & 0 & c & 0 & 14 & 1 & ( & 12 & 13 & 3 & 1 & 112 & 116 & & & \\
\hline Landing-roll & D & c & 0 & 7 & 1 & c & 25 & 26 & 2 & 2 & 158 & 162 & Landing & 300 & $23,66 \%$ \\
\hline Maneuvering & 8 & & 0 & 10 & $\overline{6}$ & 7 & 0 & 6 & 41 & 3 & 9 & 53 & & & \\
\hline Maneuvering-aerial application & 0 & c & 0 & 0 & 0 & ( & 0 & 0 & 2 & 0 & 0 & 2 & & & \\
\hline Maneuvering-turn to reverse direction & 1 & & 0 & 1 & 1 & 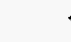 & 0 & 2 & 6 & 2 & 1 & 9 & & & \\
\hline Maneuvering-turn to landing area (emer. & 0 & & 0 & 0 & 0 & ( & 0 & 0 & $\mathbf{0}$ & 0 & $\mathbf{0}$ & 0] & & & \\
\hline Hover & $\underline{0}$ & & 0 & 0 & o & 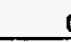 & 0 & 0 & 0 & 0 & 0 & of & Maneuvering & 64 & $5.05 \%$ \\
\hline Other & 0 & & 0 & 3 & 0 & 7 & 1 & 1 & 4 & 0 & 11 & 15 & & & \\
\hline Unknown & 1 & & 0 & 2 & 2 & 1 & 0 & 3 & 9 & 1 & 4 & 14 & & & \\
\hline Not reported & 1 & 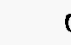 & 0 & 5 & 4 & 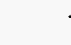 & 5 & 10 & 12 & 2 & 16 & 30 & Other/?NR & 59 & $4.65 \%$ \\
\hline Tota & 49 & 12 & 2 & 41 & $\overline{46}$ & 11 & 100 & 162 & 380 & 105 & 783 & 1268 & & 1268 & .00 \\
\hline
\end{tabular}




\begin{tabular}{|c|c|c|c|c|c|c|c|c|c|c|c|c|}
\hline 3 & 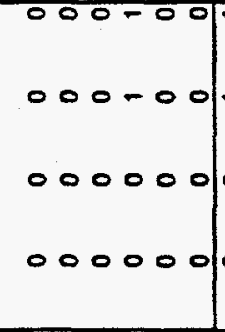 & {$\left[\begin{array}{llll}-0 & 0 & 0 & 0 \\
-0 & 0 & 0 \\
0 & 0 & 0 & 0 \\
0 & 0 & 0 & 0\end{array}\right.$} & $\left|\begin{array}{lll}0 & 0 & 0 \\
0 & 0 & 0 \\
0 & 0 & 0 \\
0 & 0 & 0\end{array}\right|^{2}$ & 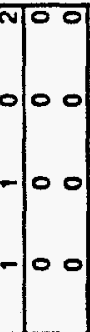 & 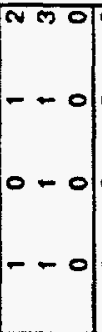 & 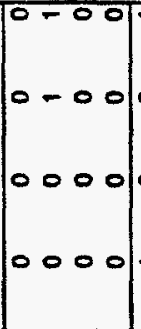 & {$\left[\begin{array}{l}-00-00-00 \\
000-000-0 \\
0000000000 \\
-000000--0\end{array}\right]$} & $\left|\begin{array}{llll}0 & 0 & 0 & 0 \\
0 & 0 & 0 & 0 \\
0 & 0 & 0 & 0 \\
0 & 0 & 0 & 0\end{array}\right|$ & 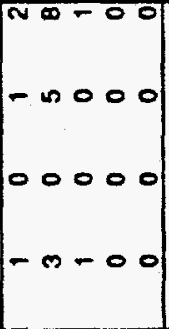 & {$\left[\begin{array}{lll}0 & 0 & 0 \\
0 & 0 & - \\
0 & 0 & 0\end{array}\right.$} & ov & 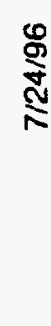 \\
\hline 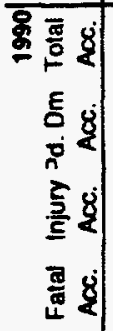 & 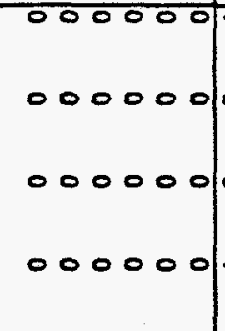 & 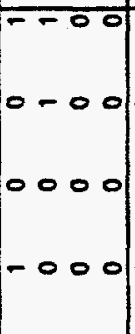 & $\left|\begin{array}{ccc}- & 0 & 0 \\
0 & 0 & 0\end{array}\right|^{-10}$ & जo & 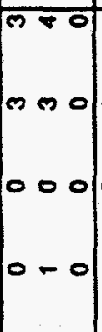 & 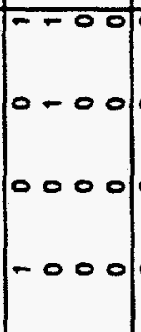 & 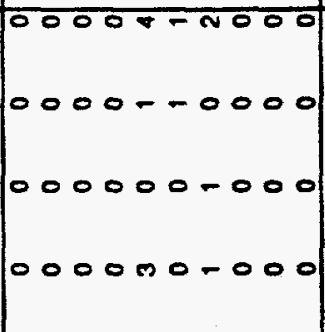 & 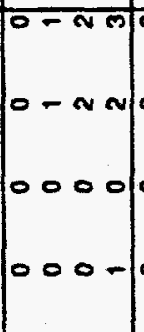 & 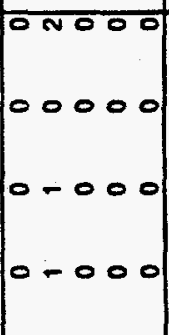 & $\begin{array}{l}-=- \\
-\infty \\
00 \\
00\end{array}$ & $\begin{array}{l}\bar{m} \\
\bar{\sim}\end{array}$ & \\
\hline 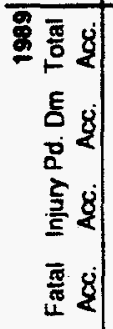 & 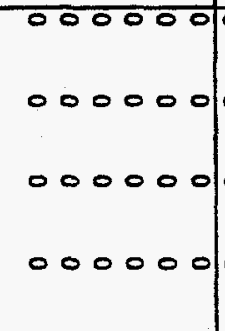 & $\left|\begin{array}{cccc}0 & 0 & - & 0 \\
0 & 0 & 0 & 0 \\
0 & 0 & 0 & 0 \\
0 & 0 & - & 0\end{array}\right|$ & $\left|\begin{array}{ccc}- & 0 & 0 \\
-0 & 0 \\
0 & 0 & 0 \\
0 & 0 & 0\end{array}\right|$ & \begin{tabular}{c|cc}
0 & 0 & 0 \\
0 & 0 \\
0 & 0 & 0
\end{tabular} & {$\left[\begin{array}{ll}- & 0 \\
-0 & 0 \\
- & 0\end{array} \mid\right.$} & $\left|\begin{array}{llll}0 & 0 & 0 & 0 \\
0 & 0 & 0 & 0 \\
0 & 0 & 0 & 0 \\
0 & 0 & 0 & 0\end{array}\right|$ & $\begin{array}{l}-00 n-F, 000 \\
00000-0000 \\
000-000000\end{array}$ & $\left|\begin{array}{cccc}0 & 0 & - & -1 \\
0 & 0 & - & 0 \\
0 & 0 & 0 & 0 \\
0 & 0 & 0 & 0\end{array}\right|$ & 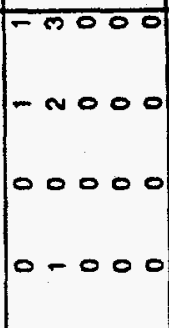 & $\mid \begin{array}{lll}0 & 0 & 0 \\
0 & 0 & 0 \\
0 & 0 & 0 \\
0 & 0 & 0\end{array}$ & $\int_{0}^{\infty}$ & \\
\hline 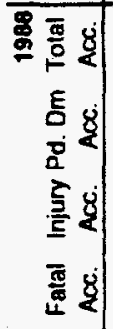 & 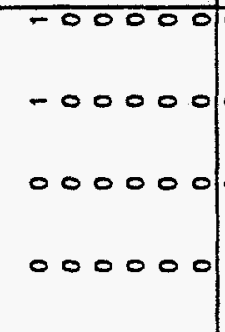 & $\left|\begin{array}{llll}0 & - & 0 & 0 \\
0 & - & 0 & 0 \\
0 & 0 & 0 & 0 \\
0 & 0 & 0 & 0\end{array}\right|$ & $\left|\begin{array}{lll}0 & 0 & 0 \\
0 & 0 & 0 \\
0 & 0 & 0 \\
0 & 0 & 0\end{array}\right|$ & mo- & $\left|\begin{array}{lll}0 & 0 & 0 \\
0 & 0 & 0 \\
0 & 0 & 0 \\
0 & 0 & 0\end{array}\right|$ & 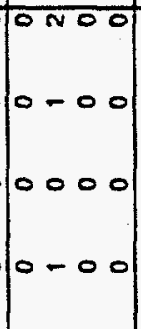 & $\begin{array}{l}0000-7-00- \\
0000-00-00 \\
0000000-00 \\
00000-1.0-\end{array}$ & 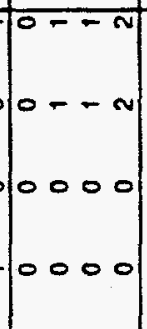 & 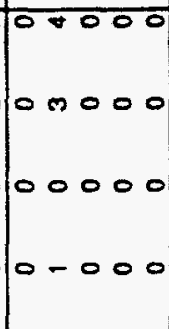 & $\mid \begin{array}{lll}0 & 0 & 0 \\
0 & 0 & 0 \\
0 & 0 & 0 \\
0 & 0 & 0\end{array}$ & $\int_{0}^{2}$ & 吕 \\
\hline 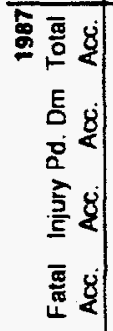 & 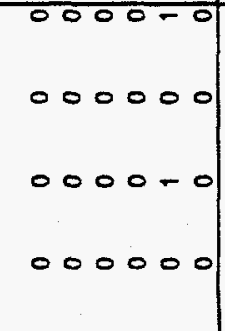 & $\left|\begin{array}{llll}0 & - & - & 0 \\
0 & - & - & 0 \\
0 & 0 & 0 & 0 \\
0 & 0 & 0 & 0\end{array}\right|$ & $\left|\begin{array}{lll}0 & 0 & n \\
0 & 0 & - \\
0 & 0 & - \\
0 & 0 & 0\end{array}\right|$ & $-\begin{array}{cc}0 & 0 \\
- & 0 \\
0 & -0\end{array}$ & $\begin{array}{lll}0 & 0 & 0 \\
0 & 0 & 0 \\
0 & 0 & 0 \\
0 & 0 & 0\end{array}$ & 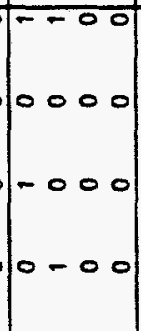 & $\begin{array}{l}-000-7-0-0 \\
-000-00000 \\
0000000000 \\
00000--0-0\end{array}$ & 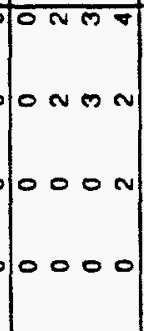 & 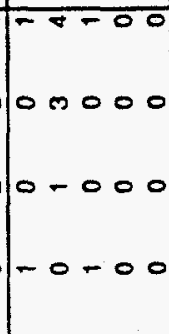 & $\mid \begin{array}{lll}0 & - & - \\
0 & 0 & 0 \\
0 & 0 & 0 \\
0 & - & -\end{array}$ & $\int_{\infty}^{\infty}$ & \\
\hline 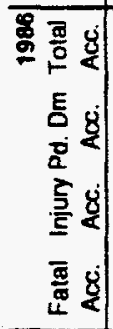 & 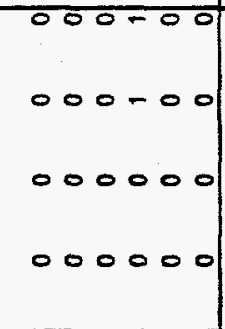 & 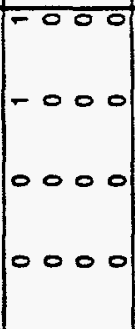 & $\left|\begin{array}{lll}0 & 0 & - \\
0 & 0 & 0 \\
0 & 0 & 0\end{array}\right|$ & $\begin{array}{lll}0 & 0 & 0 \\
- & 0 & 0 \\
-0 & 0\end{array}$ & 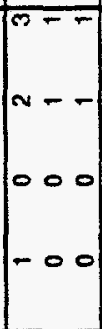 & 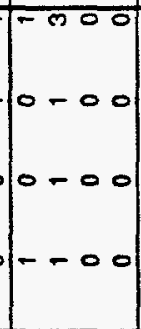 & $\begin{array}{l}n=00 n-=n 00 \\
-000--0000 \\
-000000000\end{array}$ & $\left|\begin{array}{llll}0 & w & - & - \\
0 & w & - & - \\
0 & 0 & 0 & 0 \\
0 & 0 & 0 & 0\end{array}\right|$ & $\begin{array}{l}-0=0 \\
0--0 \\
000 \\
-2000 \\
-200\end{array}$ & $\mid \begin{array}{lll}0 & 0 & 0 \\
0 & 0 & 0 \\
0 & 0 & 0 \\
0 & 0 & 0\end{array}$ & $\int_{\infty}^{\frac{1}{\infty}}$ & \\
\hline 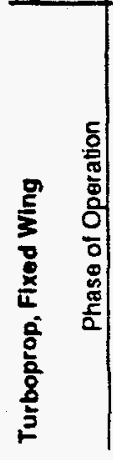 & 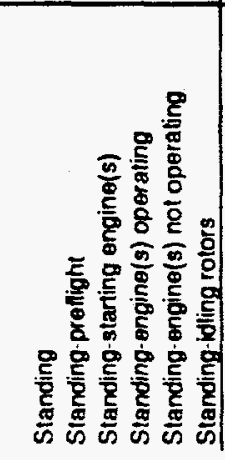 & 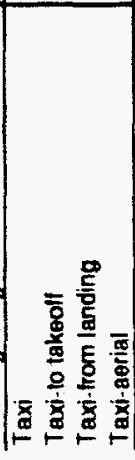 & 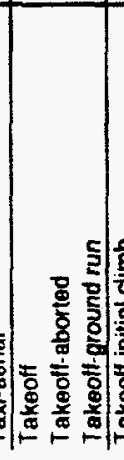 & 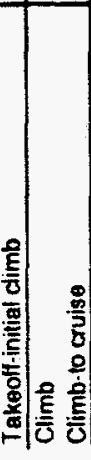 & 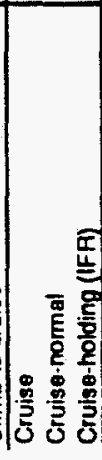 & 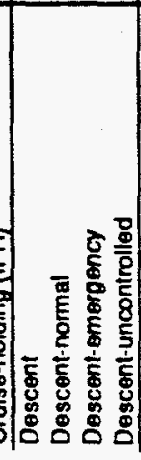 & 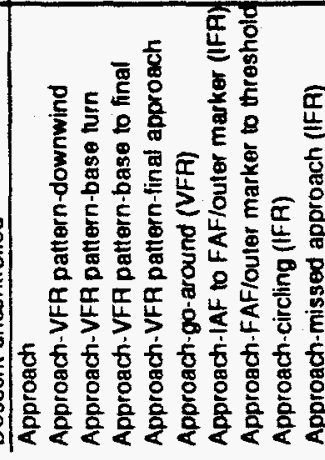 & 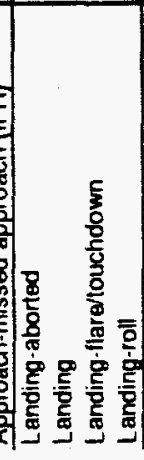 & 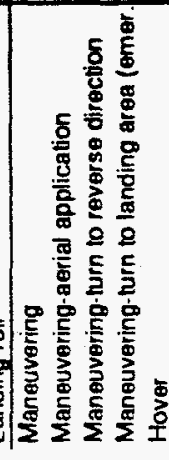 & $\mid$ & 焉 & 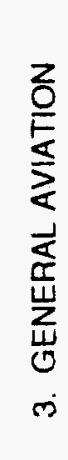 \\
\hline
\end{tabular}


Table 3.3 (Continued)

General Aviation Aceldents by Pheses of Fllght Operation

Turboprop, Fixed Wing

Reterences 3.20 to 3.27

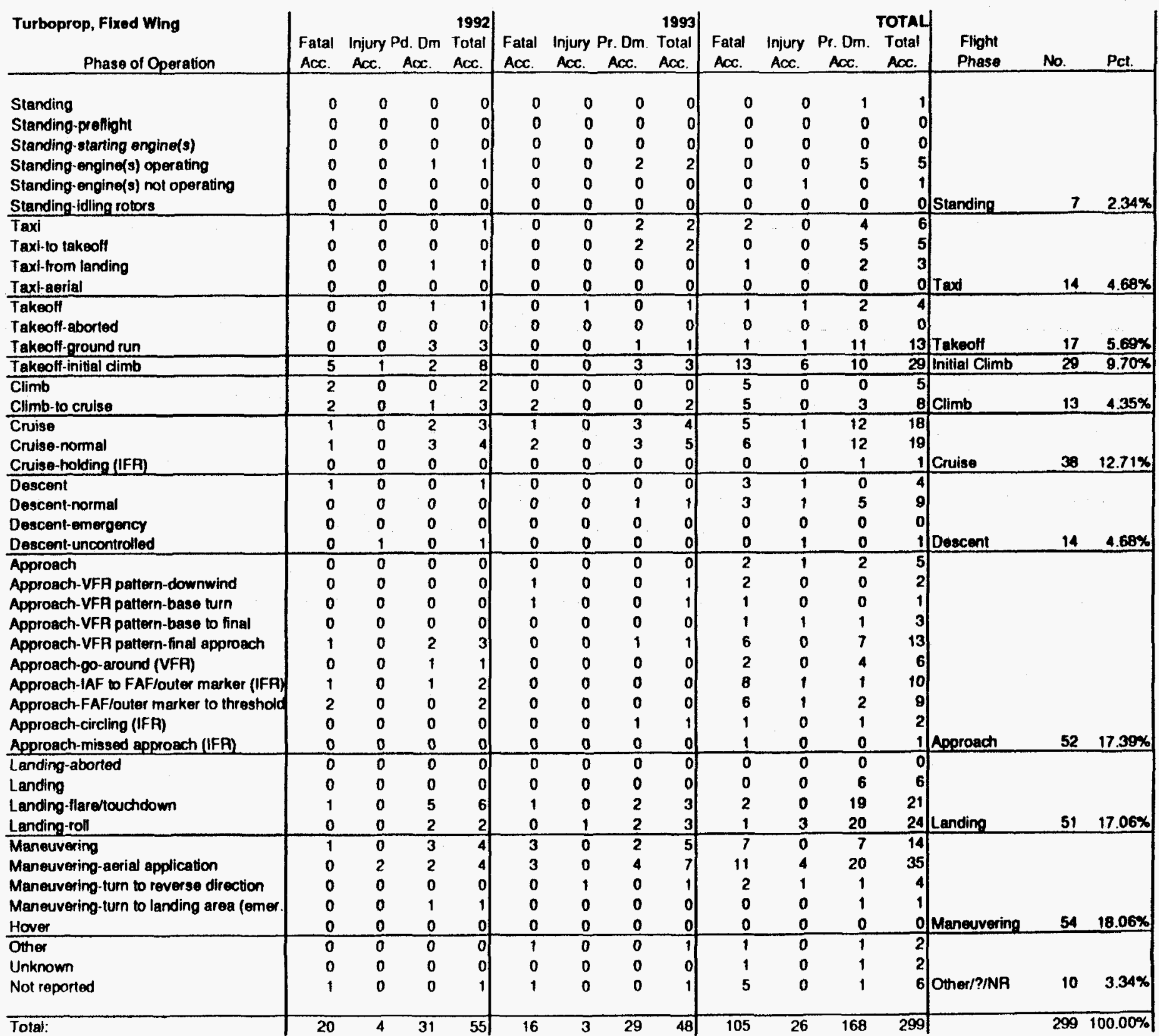




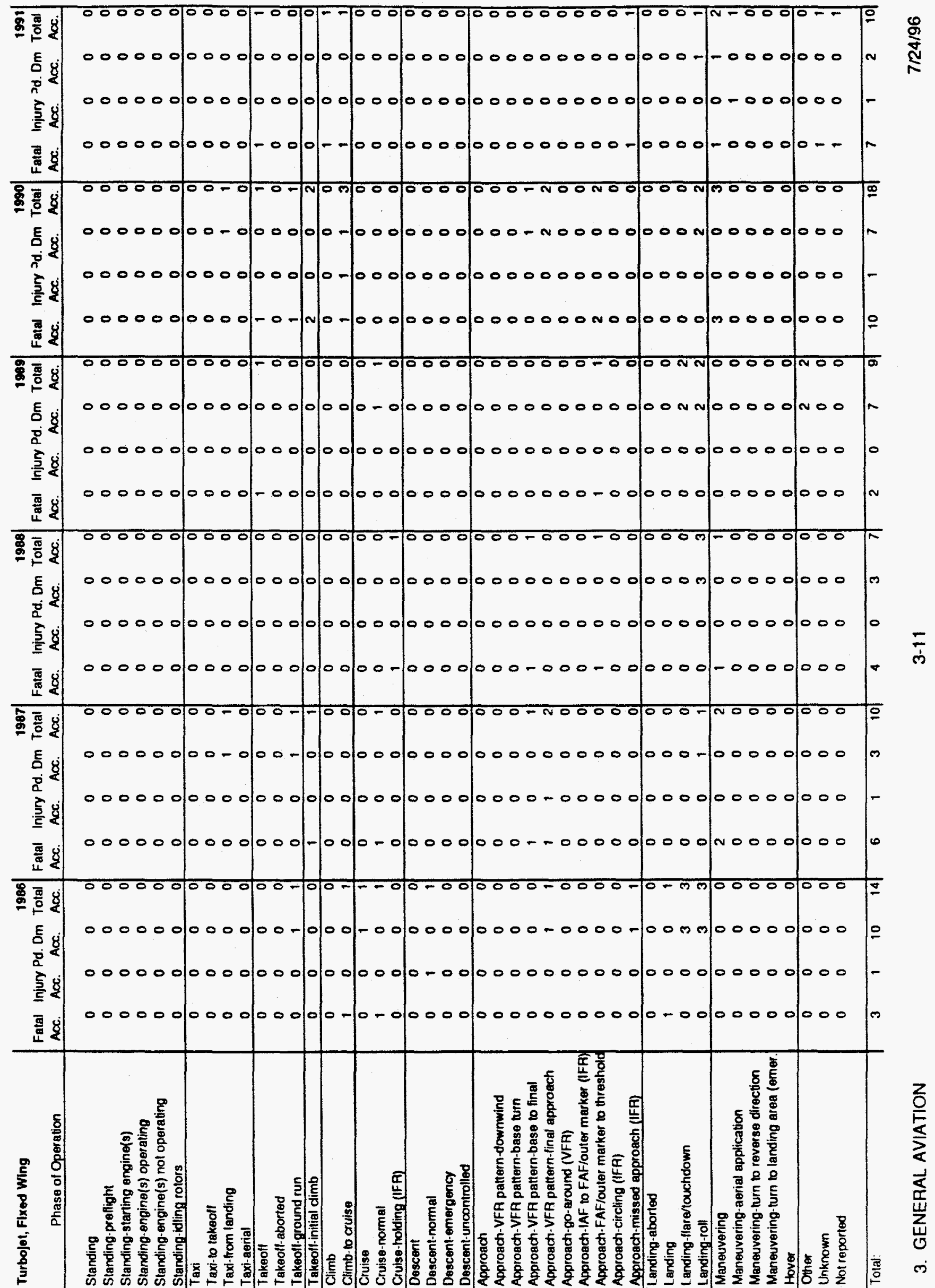


Table 3.4 (Continued)

General Aviation Accidents by Phese of Flight Operation

Turbojel, Fixed Wing

References 3.20103 .27

\begin{tabular}{|c|c|c|c|c|c|c|c|c|c|c|c|c|c|c|c|c|}
\hline $\begin{array}{l}\text { Turbolet, Flxed Wing } \\
\text { Phase of Operation }\end{array}$ & $\begin{array}{l}\text { Fatal } \\
\text { Acc. }\end{array}$ & $\begin{array}{l}\text { Injury } \\
\text { ACC. }\end{array}$ & $\begin{array}{r}\text { Pd. D } \\
A \propto C\end{array}$ & & $\begin{array}{l}1992 \\
\text { otal } \\
\text { cc. }\end{array}$ & $\begin{array}{l}\text { Fatal } \\
\text { Acc. }\end{array}$ & $\begin{array}{l}\text { Injury } \\
\text { Acc. }\end{array}$ & $\begin{array}{l}\text { Pr. Dm } \\
\text { ACc. }\end{array}$ & $\begin{array}{l}1993 \\
\text { Total } \\
\text { ACc. }\end{array}$ & $\begin{array}{l}\text { Fatal } \\
\text { Acc. }\end{array}$ & $\begin{array}{l}\text { Injury } \\
\text { ACC. }\end{array}$ & $\begin{array}{l}\text { Pr. Dm. } \\
\text { Acc. }\end{array}$ & $\begin{array}{c}\text { Total } \\
\text { Total } \\
\text { Acc. }\end{array}$ & $\begin{array}{l}\text { Flight } \\
\text { Phase }\end{array}$ & No. & Pct. \\
\hline Standing & 0 & 0 & 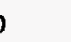 & 0 & 이 & 0 & 0 & 0 & 0 & 0 & 0 & 0 & 0 & & & \\
\hline Standing-preflight & $\mathbf{0}$ & 0 & 0 & 0 & 0 & 0 & 0 & 0 & 0 & $\mathbf{0}$ & 0 & 0 & 0 & & & \\
\hline Standing-starting engine(s) & 0 & 0 & 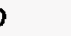 & 0 & of & o & $\mathbf{0}$ & 0 & 0 & o & 0 & 0 & of & & & \\
\hline Standing-ongine(s) operating & 0 & 0 & 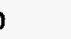 & 0 & o) & 0 & 0 & 0 & 0 & 0 & 0 & 0 & of & & & \\
\hline Standing-engine(s) not operating & 0 & 0 & 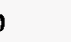 & 0 & 0 & 0 & 0 & 0 & 0 & 0 & 0 & 0 & o. & & & \\
\hline Standing-idling rotors & 0 & 0 & 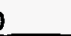 & 0 & o & 0 & 0 & 0 & 0 & 0 & 0 & 0 & 0 & Standing & 0 & $0.00 x$ \\
\hline Taxi & $\overline{0}$ & $\overline{0}$ & 5 & 0 & 0 & $\overline{0}$ & 0 & 0 & 0 & 0 & 0 & 0 & 0 & & & \\
\hline Taxi-to takeoff & 0 & 0 & 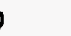 & 0 & 0 & 0 & 0 & 0 & 0 & 0 & 0 & 0 & of & & & \\
\hline Taxi-from landing & 0 & 0 & 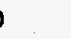 & 0 & 0 & 0 & 0 & $\mathbf{0}$ & 0 & 0 & 0 & 2 & 2 & & & \\
\hline Taxi-aerial & 0 & 0 & 2 & 0 & of & 0 & 0 & 0 & 0 & 0 & 0 & 0 & of & Taxj & 2 & $2.27 \%$ \\
\hline Takeoff & 1 & 0 & ) & 0 & T1 & 0 & 0 & $T$ & 1 & 4 & 0 & 1 & 5 & & & \\
\hline Takeoff-aborted & 0 & 0 & & 0 & o) & 0 & 0 & 0 & 0 & 0 & 0 & 0 & 0 & & & \\
\hline Takeotf-ground run & 0 & 1 & 1 & 0 & 1 & 0 & 0 & 0 & 0 & 1 & 1 & 2 & 4 & Takoolf & 9 & $10.23 \%$ \\
\hline Takeolf-initial dimb & 0 & 0 & 5 & 0 & 0 & 0 & 0 & 1 & 1 & 3 & $\overline{0}$ & 1 & 4 & Initial Climb & $\frac{7}{4}$ & $4.55 \%$ \\
\hline Climb & $\overline{0}$ & 0 & 5 & 0 & 0 & $\overline{0}$ & 0 & 0 & 0 & 1 & $\overline{0}$ & 0 & 1) & & & \\
\hline Climb-to cruise & 0 & 0 & & 0 & D. & 0 & 0 & 0 & 0 & 3 & 1 & 1 & 5 & Climb & 6 & $6.82 \%$ \\
\hline Cruise & 0 & $\overline{0}$ & & $\overline{0}$ & of & $\overline{0}$ & 0 & $\overline{0}$ & 0 & 0 & 0 & 1 & T) & & & \\
\hline Cruise-normal & 0 & 0 & 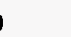 & 0 & o) & 0 & 0 & 1 & i] & 2 & 0 & 2 & 4 & & & \\
\hline Cruise-holding (IFR) & 0 & 0 & & 0 & 0 & 0 & 0 & 0 & o & 1 & 0 & 0 & 1 & Cruise & 6 & $6.82 \%$ \\
\hline Descent & 0 & $\overline{0}$ & 5 & 0 & 0 & $\overline{0}$ & 0 & 0 & 0 & 0 & 0 & $\mathbf{0}$ & 0 & & & \\
\hline Descent-normal & 0 & 0 & & o & of & 0 & 0 & 0 & o) & 0 & 1 & 0 & 1 & & & \\
\hline Descent-emergency & 0 & 0 & & 0 & 0 & 0 & 0 & 0 & D & 0 & 0 & $\mathbf{0}$ & o) & & & \\
\hline Descent-uncontrolled & 0 & 0 & & $\mathbf{0}$ & o) & 0 & 0 & 0 & 0 & 0 & 0 & 0 & ol & Descent & 1 & $1.14 \%$ \\
\hline Approach & $T$ & 0 & & $T$ & 2 & 1 & 0 & 0 & 1 & 2 & $\overline{0}$ & 1 & 3 & & & \\
\hline Approach-VFA pattern-downwind & 0 & $\mathbf{0}$ & & $\mathbf{0}$ & 0 & 0 & 0 & 0 & o) & 0 & 0 & 0 & 0) & & & \\
\hline Approach-VFR pattern-base turn & $\mathbf{0}$ & $\mathbf{0}$ & & 0 & of & 0 & 0 & 0 & of & 0 & 0 & 0 & 0 & & & \\
\hline Approach-VFA pattern-base to final & 0 & 0 & & 0 & o] & 0 & 0 & 0 & 0 & 2 & 0 & 1 & 3) & & & \\
\hline Approach-VFA pattern-final approach & 0 & 0 & & o & o) & 0 & 0 & 0 & of & 1 & 1 & 3 & 5 & & & \\
\hline Approach-go-around (VFF) & 0 & 0 & & 0 & 이 & 0 & 0 & 0 & of & $\mathbf{0}$ & $\mathbf{0}$ & $\mathbf{0}$ & of & & & \\
\hline Approach-IAF to FAF/outer marker (IFR) & 0 & 0 & & 0 & o) & 0 & 0 & 0 & o) & 0 & 0 & 0 & of & & & \\
\hline Approach-FAF/outer marker to threshold & 0 & 0 & & 0 & o) & 0 & 0 & 0 & of & 4 & 0 & 0 & 4 & & & \\
\hline Approach-circling (IFA) & 0 & 0 & & 0 & o) & o & 0 & $\mathbf{0}$ & of & 0 & $\mathbf{0}$ & 0 & of & & & \\
\hline Approach-missed approach (IFA) & 0 & 0 & & o & ol & 0 & 0 & 0 & D. & 1 & 0 & 1 & 2. & Appraach & 17 & $19.32 \%$ \\
\hline Landing-aborted & 0 & 0 & & o & of & D & 0 & D & 0 & 0 & 0 & 0 & of & & & \\
\hline Landing & 0 & $\mathbf{0}$ & & 0 & 0 & 0 & 0 & 0 & o) & 1 & 0 & 0 & 1 & & & \\
\hline Landing-flara/touchidown & 0 & $\mathbf{0}$ & & $\mathbf{0}$ & o) & 0 & $\mathbf{0}$ & 0 & o & $\mathbf{0}$ & $\mathbf{0}$ & 5 & 5 & & & \\
\hline Landing-roll & 0 & 0 & & 4 & 4 & 0 & 0 & 2 & 2 & 0 & 0 & 18 & 18 & Landing & 24 & $27.27 \%$ \\
\hline Manouvering & $T$ & $\overline{0}$ & & 0 & 1 & 2 & 0 & 0 & 2 & 10 & $\overline{0}$ & $T$ & 11 & & & \\
\hline Maneuvering-aerial application & 0 & 0 & & 0 & of & 0 & 0 & 0 & o] & 0 & 1 & $\mathbf{0}$ & 1 & & & \\
\hline Maneuvering-turn to reverse direction & 0 & 0 & & 0 & 0 & 0 & 0 & 0 & o) & 0 & $\mathbf{0}$ & 0 & of & & & \\
\hline Maneuvering-turn to landing area (emer. & 0 & 0 & & 0 & 0 & 0 & 0 & 0 & 0 & 0 & 0 & 0 & o) & & & \\
\hline Hover & 0 & 0 & & 0 & of & 0 & 0 & 0 & 0 & 0 & 0 & 0 & of & Meneuvering & 12 & $13.64 \%$ \\
\hline Other & 0 & 0 & & 0 & 0 & 0 & 0 & 0 & 0 & 0 & 0 & 2 & 2 & & & \\
\hline Unknown & 0 & 0 & & 0 & o. & 0 & 0 & 0 & o. & 1 & 0 & 0 & 1 & & & \\
\hline Not reported & 0 & 0 & & 2 & 2 & 0 & 0 & 1 & 1 & 1 & 0 & 3 & 4) & Other/?/NR & 7 & $7.95 \%$ \\
\hline Total: & 3 & 1 & & 7 & 11 & 3 & 0 & 6 & 9] & 38 & 5 & 45 & 88 & & 88 & $100.00 \%$ \\
\hline
\end{tabular}




\section{Standing}

Standing-prellight

Standing-starting engine(s)

Standing-engine(s) operating

Slanding-engine(s) not operating

Standing-kdling rotors

Taxi

Taxi-to takeolf

Taxi-hom landing

Taxi-aerial

Takeolf

Takeoff-aborted

Takeoff-ground run

Takeof initiol dimb

Climb

Climb-to cruise

Cruise-normal

Cruise-holding (IFP)

Descent

Descent-normal

Descent-emergency

Descent-uncontrolled

Approach

Approach-VFR pattern-downwind

Approach-VFA paftern-base turn

Approach-VFA pattern-base to final

Approach-VFF pattern-final approech

Approach-oo-around (VFA)

Approach-1AF D FAF/outer marker (IFA)

Approach-FAF/outer marker to threshold

Approach-circling (IFA)

Approach-missed approach (IFF)

Landing-aborted

Landing

Landing-llaratouchdown

Landing-roll

Manouvering

Manouvering-aerial application

Maneuvering-turn to reverse direction

Maneuvering-turn to landing ares (emer.

Hover

Other

Unknown

Not reported

Total:

\begin{tabular}{|c|c|c|c|c|c|c|c|c|c|c|c|c|c|c|c|c|c|c|c|c|c|c|c|}
\hline 0 & 0 & 1 & 1 & 0 & 0 & 0 & 0 & 0 & 0 & 3 & 3 & 0 & 0 & 1 & 1) & 0 & 1 & 0 & 1 & 0 & 0 & 2 & 2 \\
\hline 1 & 0 & 0 & 1 & 0 & 1 & 0 & 1 & o & D & 1 & 1 & 0 & 0 & 0 & 0 & 0 & 0 & 0 & 이 & 1 & 1 & 1 & 3 \\
\hline 0 & 5 & 17 & 22 & 1 & 2 & 17 & 20 & 0 & 3 & 15 & 18 & 0 & 0 & 13 & 13 & 0 & 1 & 7 & B & 0 & 2 & 5 & 7 \\
\hline 1 & 0 & 5 & 6 & 2 & 2 & 9 & 13 & 1 & 1 & 6 & 8) & 1 & 4 & 9 & 14 & 0 & 1 & 6 & 7 & 2 & 1 & 10 & 13 \\
\hline 0 & 0 & 0 & 0 & 0 & 1 & 1 & 2 & 0 & 0 & 0 & 0 & 0 & 0 & 1 & 1) & 0 & 0 & 0 & 0) & 0 & 0 & 0 & 0 \\
\hline 0 & 0 & 0 & 0 & 0 & 0 & 0 & 0 & 0 & 0 & 0 & o) & 0 & 0 & 0 & o) & 0 & 0 & 0 & o & 0 & 0 & 0 & D) \\
\hline 1 & 0 & 13 & 14 & 0 & 0 & 5 & 5 & 1 & 0 & 6 & 7 & 0 & $\overline{0}$ & 5 & 5 & 2 & 0 & 9 & 11 & 0 & 1 & 5 & 6 \\
\hline 0 & 0 & 21 & 21 & 0 & 0 & 32 & 32 & 0 & 1 & 24 & 25 & 0 & 0 & 22 & 22 & 0 & 0 & 26 & 26 & 0 & 0 & 16 & 16 \\
\hline 0 & 1 & 18 & 19 & 0 & 0 & 28 & 28 & 1 & 0 & 30 & 31 & 1 & 0 & 26 & 27 & 0 & 0 & 19 & 19 & 0 & 2 & 16 & 18 \\
\hline 0 & 0 & 0 & 0 & 0 & 0 & 0 & of & 0 & 0 & 0 & of & 0 & 0 & 0 & of & 0 & 0 & 1 & 1 & 0 & 0 & $\underline{0}$ & 의 \\
\hline 8 & 5 & 24 & 37 & 2 & 6 & 18 & 26 & 5 & 5 & 20 & 30 & 4 & 3 & 21 & 28 & 8 & $\bar{B}$ & 22 & 38 & 12 & 4 & 25 & 41 \\
\hline 0 & 0 & 0 & 0 & 0 & 0 & 0 & 0 & 0 & 1 & 15 & 16 & 0 & 0 & 0 & 0 & 0 & 0 & 0 & o) & 0 & 0 & 0 & o) \\
\hline 4 & 6 & 107 & 117 & 2 & 9 & 109 & 120 & 1 & 2 & 77 & 80 & 2 & 1 & 77 & 80 & 5 & 3 & 73 & $\theta 1$ & 1 & 7 & 81 & 89 \\
\hline 68 & 54 & 217 & 339 & 48 & 67 & 209 & 324 & 66 & 64 & 175 & 305 & 61 & 51 & 194 & 306 & 61 & 60 & 173 & 294 & 49 & 57 & 179 & 285 \\
\hline 12 & 6 & 19 & 37 & 5 & 4 & 12 & 21 & 5 & 3 & 14 & 22 & 9 & 2 & 7 & 18 & 7 & 5 & 8 & 20 & 15 & 10 & 19 & 44 \\
\hline 21 & 5 & 26 & 52 & 14 & $\theta$ & 29 & 51 & 25 & 4 & 25 & 54 & 11 & 2 & 14 & 27 & 13 & 7 & 14 & 34 & 13 & 4 & 22 & 39 \\
\hline 34 & 15 & 105 & 154 & 33 & 8 & 78 & 119 & 41 & 16 & 88 & 145 & 48 & 17 & 95 & 160 & 38 & 16 & 79 & 133 & 50 & 22 & 105 & 177 \\
\hline 44 & 35 & 158 & 237 & 59 & 18 & 145 & 222 & 59 & 27 & 150 & 236 & 60 & 17 & 135 & 212 & 34 & 13 & 129 & 176 & 34 & 23 & 110 & 167 \\
\hline 0 & 0 & 1 & 1 & 0 & 0 & 0 & 0 & 1 & 0 & 3 & 4 & 0 & 0 & 1 & 1 & 0 & 0 & 0 & 0 & 0 & 0 & 1 & 1 \\
\hline 12 & 0 & 9 & 21 & 4 & 1 & 4 & 9 & 8 & 1 & 7 & 17 & 9 & 1 & 5 & 15 & 5 & 1 & 8 & 14 & 3 & 5 & 6 & 14 \\
\hline 10 & 7 & 25 & 42 & B & 6 & 30 & 44 & 5 & 1 & 38 & 44 & 5 & 2 & 19 & 26 & 9 & 5 & 21 & 35 & 4 & 5 & 23 & 32 \\
\hline 2 & 2 & 4 & B & 0 & 0 & 5 & 5 & 1 & 0 & 1 & 2 & 0 & 0 & 2 & 2 & 0 & 0 & 1 & 1) & 2 & 1 & 2 & 5 \\
\hline 3 & 1 & 1 & 5 & 4 & 0 & 2 & 6 & 0 & 1 & 0 & 1 & 1 & 0 & 2 & 3 & 2 & 0 & 2 & 4 & 3 & 0 & 2 & 5 \\
\hline 11 & 6 & 20 & 37 & 6 & 6 & 22 & 34 & 11 & 6 & 21 & 38 & 15 & 7 & 33 & 55 & 13 & 3 & 15 & 31 & 13 & 1 & 21 & 35 \\
\hline 5 & 2 & 16 & 23 & 9 & 4 & 11 & 24 & 3 & 4 & 12 & 19 & 6 & 2 & 8 & 16 & 12 & 2 & 16 & 30 & 5 & 4 & 11 & 20 \\
\hline 5 & 0 & 5 & 10 & 1 & 3 & 3 & 7 & 3 & 2 & 5 & 10 & 3 & 1 & 4 & 8 & 5 & 1 & 9 & 15 & 3 & 1 & 3 & 7 \\
\hline 9 & 2 & 7 & 18 & 9 & 1 & 7 & 17 & 5 & 2 & 7 & 14 & 10 & 5 & 6 & 21 & 6 & 3 & 12 & 21 & B & 2 & 13 & 23 \\
\hline 13 & 30 & 84 & 127 & 11 & 17 & 87 & 115 & B & 23 & 72 & 1003 & 14 & 16 & 79 & 100 & 21 & $\theta$ & 88 & 118 & 15 & 9 & 62 & 86 \\
\hline 4 & 9 & 46 & 59 & 6 & 11 & 32 & 49 & 8 & 7 & 39 & 54 & 4 & 5 & 29 & 38 & 7 & B & 26 & 41 & 7 & 8 & 36 & 51 \\
\hline 3 & 3 & 1 & 7 & 5 & 0 & 0 & 5 & B & 1 & 2 & 11 & B & 1 & 0 & $\theta$ & 5 & 1 & 2 & 8) & 5 & 1 & 1 & 7 \\
\hline 18 & 6 & 8 & 32 & 7 & 4 & 5 & 16 & 7 & 5 & 5 & 17 & 4 & 0 & 4 & 8 & 9 & 1 & 6 & 16 & 6 & 3 & 6 & 15 \\
\hline 2 & 0 & 2 & 4 & 3 & 0 & 0 & 3 & 3 & 1 & 2 & 6 & 0 & 0 & 1 & 1 & 1 & 0 & 0 & 1 & 1 & 2 & 0 & 3 \\
\hline 2 & 2 & 4 & 8 & 4 & 0 & 1 & 5 & 5 & 0 & 2 & 7 & 1 & 0 & 0 & 1 & 4 & 0 & 1 & 5) & 3 & 1 & 1 & 5 \\
\hline 0 & 0 & 0 & 0 & $\overline{0}$ & 0 & 0 & 0 & 3 & 4 & 18 & 25 & 0 & 0 & 0 & 아 & 0 & 0 & 0 & 0 & $\overline{0}$ & 0 & $\overline{0}$ & 0 \\
\hline 4 & 7 & 40 & 51 & 4 & 3 & 34 & 41 & 0 & $i$ & 39 & 40 & 2 & 2 & 40 & 44 & 1 & 2 & 22 & 25 & 1 & 3 & 12 & 16 \\
\hline $\mathbf{3}$ & 9 & 210 & 222 & 2 & 9 & 223 & 234 & 2 & 5 & 166 & 173 & 3 & 8 & 161 & 172 & 3 & 6 & 153 & 162 & 0 & 2 & 150 & 161 \\
\hline 7 & 6 & 290 & 303 & 0 & 11 & 309 & 320 & 1 & $\mathbf{g}$ & 262 & 272 & 2 & 7 & 242 & 251 & 2 & 6 & 257 & 265 & 1 & 0 & 230 & 234 \\
\hline 95 & 30 & 55 & 180 & 119 & 20 & 49 & 188 & 104 & 21 & 56 & 181 & 69 & 23 & 61 & 153 & 81 & 13 & 43 & 147 & 81 & 27 & 56 & 174 \\
\hline B & 8 & 42 & 58 & 9 & 11 & 45 & 65 & 6 & 14 & 42 & 62 & 18 & 7 & 39 & 64 & 11 & 6 & 42 & 59 & 11 & 10 & 46 & 67 \\
\hline 10 & 2 & 18 & 31 & 5 & 8 & 8 & 21 & 10 & 2 & 9 & 21 & 7 & 2 & 0 & 9) & 11 & 4 & 8 & 24 & 9 & 3 & 9 & 21 \\
\hline 0 & 0 & 2 & 2 & 1 & 0 & 0 & 1 & 0 & 0 & 7 & 7 & 0 & 0 & 1 & 1 & 2 & 0 & 0 & 2) & 1 & D & 1 & 2 \\
\hline 0 & 0 & 0 & 0 & 0 & 0 & 0 & 0 & 0 & 0 & 0 & 0 & 0 & 0 & 0 & o] & 0 & 0 & 0 & 0 & 0 & 0 & 0 & 0 \\
\hline 3 & 0 & 3 & $\overline{6}$ & 4 & 1 & 21 & 26 & 0 & 0 & T & 1 & 4 & 6 & 56 & 66 & 6 & 5 & 55 & 66 & 9 & $\overline{3}$ & 41 & 53 \\
\hline 13 & 2 & 1 & 16 & 13 & 1 & 3 & 17) & 11 & 1 & 2 & 14 & 17 & 0 & 2 & 19 & 18 & 0 & 3 & 21 & 12 & 0 & 1 & 13 \\
\hline 1 & 0 & 1 & 2 & 7 & 0 & 2 & 9 & 2 & 0 & 4 & 6 & 1 & 0 & 0 & 1) & 6 & 1 & 10 & 17 & 9 & 0 & 9 & 18 \\
\hline 437 & 266 & 1627 & 2330 & 407 & 243 & 1595 & 2245 & 421 & 238 & 1471 & 2130 & 400 & 192 & 1415 & 2007 & 418 & 192 & 1367 & 1977 & 399 & 225 & 1351 & 1975 \\
\hline
\end{tabular}


Tablo 3.5 (Conilnued)

General Aviation Accidents by Phase of Filight Operation

Totel, Flxed Wing Aireraft

Relerences 3.20 to 3.27

\begin{tabular}{|c|c|c|c|c|c|c|c|c|c|c|c|c|c|c|c|}
\hline $\begin{array}{r}\text { Tolal, Flxod Wing Alreraft } \\
\text { Phase ol Operation }\end{array}$ & $\begin{array}{l}\text { Fatal } \\
\text { AcC. }\end{array}$ & $\begin{array}{l}\text { Injury ' } \\
A C C \text {. }\end{array}$ & $\begin{array}{l}\text { d. Dm } \\
\text { Aoc. }\end{array}$ & $\begin{array}{l}1992 \\
\text { Total } \\
\text { Acc } \\
\end{array}$ & $\begin{array}{l}\text { Fatal } \\
\text { ADC. }\end{array}$ & $\begin{array}{l}\text { Injury } F \\
\text { Acc. }\end{array}$ & $\begin{array}{l}\text { Pd. Dm } \\
\text { Aoc. }\end{array}$ & $\begin{array}{l}1993 \\
\text { Total } \\
\text { ACC. }\end{array}$ & $\begin{array}{l}\text { Fatal } \\
\text { Acc. }\end{array}$ & $\begin{array}{l}\text { Injury } \\
\text { Acc. }\end{array}$ & $\begin{array}{l}\text { Pr. Dm. } \\
\text { Ace. }\end{array}$ & $\begin{array}{c}\text { TOTAL } \\
\text { Total } \\
\text { ACc. }\end{array}$ & $\begin{array}{l}\text { Flight } \\
\text { Phase }\end{array}$ & No. & Pct. \\
\hline Standing & 0 & 0 & 0 & 0 & 0 & 1 & 1 & 2 & 0 & 2 & 8 & 10 & & & \\
\hline Standing-prenlight & 0 & 1 & 0 & 1 & 0 & 0 & 0 & of & 2 & 3 & 2 & 7 & & & \\
\hline Standing-starting engine(s) & 0 & 1 & 4 & 5 & 1 & 0 & 10 & 11) & 2 & 14 & 88 & 104 & & & \\
\hline Standing-engine(s) operating & 2 & 0 & 2 & 4 & 3 & 3 & 4 & 10 & 12 & 12 & 51 & 75 & & & \\
\hline Standing-ongino(s) not operating & 0 & $\mathbf{0}$ & 0 & o] & $\mathbf{0}$ & 0 & 1 & $1]$ & 0 & 1 & 3 & $4]$ & & & \\
\hline Standing-idling rotors & $\underline{0}$ & 0 & 0 & of & $\mathbf{0}$ & 0 & 0 & o) & $\underline{0}$ & 0 & 0 & o.: & Standing & 200 & $1.22 x$ \\
\hline Taxi & 2 & $\mathbf{0}$ & 5 & 7 & 0 & 0 & 8 & 8 & 6 & $T$ & 56 & 63 & & & \\
\hline Taxito takeoff & 0 & 2 & 15 & 17 & D & 1 & 21 & 22 & 0 & 4 & 177 & 181 & & & \\
\hline Taxt-fom landing & 0 & 1 & 15 & 16 & 0 & 1 & 14 & 15 & 2 & 5 & 166 & 173 & & & \\
\hline Toxt-aerial & o & 0 & 0 & of & 0 & 0 & 0 & of & 0 & 0 & 1 & 1 & $\operatorname{Tax}$ & 418 & $2.55 x$ \\
\hline Takeolf & 5 & $\frac{2}{2}$ & 13 & 20 & $\overline{5}$ & 3 & 15 & 23 & 49 & 36 & 158 & 243 & & & \\
\hline Takeoff-aborted & 0 & 0 & $\mathbf{0}$ & 0 & 0 & 0 & 0 & 0 & 0 & 1 & 15 & 16 & & & \\
\hline Takeolt-ground run & 0 & 6 & 73 & 79 & 3 & 0 & 74 & 77) & 18 & 34 & 671 & 723) & Takeoff & 802 & $6.00 x$ \\
\hline Takeoff-initial dimb & 55 & 47 & 162 & 264 & 48 & 47 & 167 & 262 & 456 & 447 & 1476 & 2379 & Initial Climb & 2378 & $14.54 x$ \\
\hline$\overline{C l i m b}$ & 8 & 1 & 14 & 23 & 10 & $\overline{3}$ & 18 & 31 & 71 & 34 & 111 & 216 & & & \\
\hline Climb-to cruise & 10 & 3 & 18 & 31 & 9 & 5 & 14 & 20 & 116 & 38 & 162 & 316 & Climb & 532 & $3.25 \%$ \\
\hline Cruise & $\overline{44}$ & 15 & 81 & 140 & 46 & 25 & 108 & 179 & 334 & 134 & 739 & 1207 & & & \\
\hline Cruise-nomal & 48 & 16 & 115 & 179 & 32 & 10 & 98 & 140 & 370 & 159 & 1040 & 1569 & & & \\
\hline Cruise-holding (IFR) & 0 & 0 & 0 & of & 0 & 0 & 0 & o & 1 & 0 & 6 & $7 \mathrm{c}$ & Crulse & 2783 & $17.01 \%$ \\
\hline Descent & 6 & 2 & 3 & 11 & 10 & $\overline{0}$ & 6 & 16 & 58 & 11 & 48 & 117 & & & \\
\hline Descent-normal & 12 & 2 & 28 & 42 & 4 & 0 & 25 & 29 & 57 & 28 & 209 & 294 & & & \\
\hline Descent-emergency & 1 & 0 & 2 & 3 & 1 & 1 & 3 & 5 & 7 & 4 & 20 & 31 & & & \\
\hline Descent-uncontrolled & 1 & 3 & 2 & 6. & 0 & 1 & 2 & 3 & 14 & 6 & 13 & 331 & Descent & 475 & $2.80 \%$ \\
\hline Approach & 13 & 7 & 11 & 31 & 10 & 2 & 16 & 28 & 92 & 38 & 159 & 260 & & & \\
\hline Approach-VFR pattern-downwind & 3 & 1 & 14 & 18 & $\mathbf{3}$ & 3 & 8 & 14 & 46 & 22 & 96 & 164 & & & \\
\hline Approach-VFA pattern-base hurn & 2 & 1 & 6 & 9] & 4 & 2 & $\mathbf{3}$ & 9 & 26 & 11 & 38 & 75 & & & \\
\hline Approach VFA pattorn-base to final & 6 & 3 & 6 & 15 & 7 & $\mathbf{0}$ & 10 & 17 & 60 & 18 & 68 & 146] & & & \\
\hline Approach-VFR pattern-linal approsch & 13 & 12 & 68 & 93. & 10 & 12 & 68 & 90 & 105 & 128 & 608 & B41 & & & \\
\hline Approach-go-around (VFR) & 7 & 3 & 25 & 35 & 3 & 6 & 37 & 46 & 46 & 57 & 270 & 373 & & & \\
\hline Approach-IAF to FAFlouter marker (IFR) & 4 & 0 & 2 & 6 & 1 & 1 & 1 & 3 & 39 & $\boldsymbol{8}$ & $\mathbf{9}$ & 56] & & & \\
\hline Approach-FAF/outer marker to threshold & 10 & 2 & 5 & 17. & 4 & 2 & 7 & 13 & 65 & 23 & 46 & 134) & & & \\
\hline Approach-circling (IFR) & 1 & 1 & 1 & 3 & $\mathbf{3}$ & $\mathbf{0}$ & 1 & 4 & 14 & 4 & 7 & 25 & & & \\
\hline Approach-missed approsch (IFB) & 4 & 1 & 2 & 7 & 1 & 2 & 1 & 4) & 24 & 6 & 12 & 42) & Approach & 2145 & $13.11 \%$ \\
\hline Landing-aborted & $\overline{0}$ & 0 & 0 & 0 & 0 & o & o & of & 3 & 4 & 18 & 25 & & & \\
\hline Landing & 3 & 2 & 34 & 39 & 0 & 5 & 33 & 38 & 15 & 25 & 254 & 284 & & & \\
\hline Landing-flarenouchdown & 3 & 7 & 158 & 168 & 4 & 3 & 137 & 144| & 20 & 49 & 1367 & 1436 & & & \\
\hline Landing-roll & 3 & 4 & 222 & 229 & 2 & 6 & 238 & 246. & 18 & 49 & 2053 & $2120] \mathrm{L}$ & Landing & 3975 & $23.68 \times$ \\
\hline Manouvering & 90 & 18 & 49 & 157 & 105 & 25 & 43 & 173 & 764 & 177 & 412 & 1353 & & & \\
\hline Maneuvering-aerial application & 6 & 10 & 36 & 52 & 11 & 7 & 31 & 49] & Bo & 73 & 323 & 476 & & & \\
\hline Maneuvering-turn to reverse direction & 10 & 5 & $\mathbf{9}$ & 24) & 4 & 5 & 7 & 16) & 66 & 31 & 70 & 167| & & & \\
\hline Maneuvering-turn to landing area (emer. & 1 & 1 & 1 & 3) & 0 & 0 & 1 & i) & 5 & 1 & 13 & 19 & & & \\
\hline Hover & $\mathbf{0}$ & 0 & 0 & of & $\mathbf{0}$ & 0 & $\mathbf{0}$ & of & $\mathbf{0}$ & $\mathbf{0}$ & 0 & of & Manouvering & 2015 & $12.32 \%$ \\
\hline Other & 10 & 5 & 42 & 57 & 3 & 3 & 43 & 49 & 39 & 23 & 262 & 324 & & & \\
\hline Unknown & 14 & 0 & 7 & 21 & 14 & 3 & 1 & 18 & 112 & 7 & 20 & 139 & & & \\
\hline Not reported & 6 & 0 & 13 & 19 & 9 & 3 & 11 & 23 & 41 & 4 & 50 & $95 \mathrm{C}-2-2-3$ & Other/?NA & 558 & $3.41 \%$ \\
\hline Total & 403 & 185 & 1263 & 1851 & 370 & 191 & 1286 & $1847 \mid$ & 3255 & 1732 & 11375 & 16362 & & 16362 & $100.00 \%$ \\
\hline
\end{tabular}




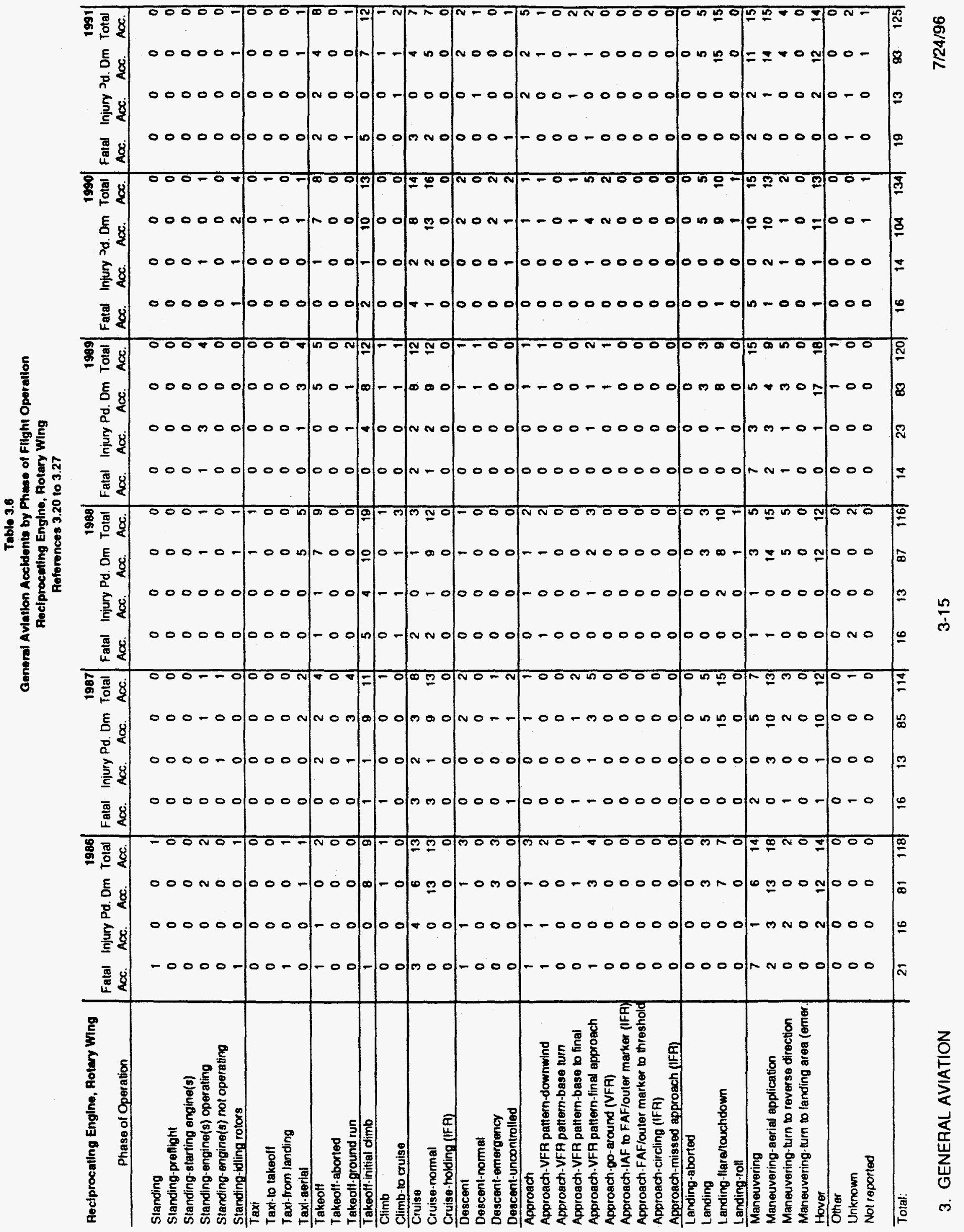


Teblo 3.8 (Continued)

General Aviatlon Accidents by Pheee of Fllght Operation

Aociprocating Englino, Rotary Wing

Reterences 3.20103 .27

\begin{tabular}{|c|c|c|c|c|c|c|c|c|c|c|c|c|c|c|c|}
\hline $\begin{array}{c}\text { Reclprocating Engino, Aotery Wing } \\
\text { Phase ol Operation } \\
\end{array}$ & $\begin{array}{l}\text { Fatal } \\
\text { Aoc. }\end{array}$ & $\begin{array}{l}\text { Injury } \\
\text { Acx. }\end{array}$ & $\begin{array}{l}\text { Pd. Dm } \\
\text { Aoc. }\end{array}$ & $\begin{array}{l}1992 \\
\text { Total } \\
\text { Acc. } \\
\end{array}$ & $\begin{array}{l}\text { Fatal } \\
\text { ACC. }\end{array}$ & $\begin{array}{l}\text { Injury } \\
\text { Aoc. }\end{array}$ & $\begin{array}{c}\text { Pr. Dm } \\
\text { AcC. }\end{array}$ & $\begin{array}{l}1993 \\
\text { Total } \\
\text { Acc. }\end{array}$ & $\begin{array}{l}\text { Fatal } \\
A \subset x \text {. }\end{array}$ & $\begin{array}{c}\text { Injury } \\
\text { Aoc. }\end{array}$ & $\begin{array}{c}\text { Pr. Dm. } \\
\text { Acc. }\end{array}$ & $\begin{array}{c}\text { Total } \\
\text { Tolal } \\
\text { Aoc. } \\
\end{array}$ & $\begin{array}{l}\text { Flight } \\
\text { Phase } \\
\end{array}$ & No. & Pct. \\
\hline Standing & 0 & 0 & 0 & 0 & $\mathbf{0}$ & 0 & $\mathbf{0}$ & 0 & 1 & 0 & 0 & 1 & & & \\
\hline Standing-preflight & 0 & 0 & 0 & 0 & 0 & 0 & $\mathbf{0}$ & 0 & 0 & 0 & 0 & 0) & & & \\
\hline Standing-starting engine(s) & 0 & 0 & 0 & 0 & 0 & 0 & 0 & o) & 0 & 0 & 0 & of & & & \\
\hline Standing-engine(s) operating & 0 & 1 & 0 & 1 & 0 & 0 & 0 & 0 & 1 & 5 & 4 & 10 & & & \\
\hline Standing-enging(s) not operating & 0 & 0 & $\mathbf{0}$ & of & 0 & 0 & 0 & 0) & o & 1 & 0 & 1 & & & \\
\hline Standing-idling rotors & 0 & 0 & 0 & of & 1 & 0 & 0 & 1 & 3 & 1 & 4 & 日 & Standing & 20 & $2.11 \%$ \\
\hline Taxi & 0 & 0 & 1 & 1 & $\mathbf{0}$ & 0 & 0 & of & o & 0 & 2 & 2 & & & \\
\hline Taxi-to takeoff & 0 & 0 & 0 & 0 & $\mathbf{0}$ & 0 & 0 & 0 & 0 & $\mathbf{0}$ & 1 & 1] & & & \\
\hline Taxi-from landing & $\mathbf{0}$ & 0 & 0 & o) & 0 & 0 & 0 & o) & 1 & 0 & 0 & 1 & & & \\
\hline Taxi-aerial & 0 & 0 & 5 & 5 & 0 & 1 & 2 & 3 & 0 & 2 & 20 & 22 & Taxi & 26 & $2.74 x_{0}$ \\
\hline Takeoff & $\overline{0}$ & 1 & 6 & 7 & 0 & $T$ & 3 & 4 & 4 & 9 & 34 & 47 & & & \\
\hline Takeoll-aborted & 0 & 0 & o & o) & 0 & 0 & 0 & of & 0 & 0 & 0 & o) & & & \\
\hline Takeolf-ground run & 0 & 0 & 2 & 2 & 0 & 0 & 0 & 0 & 1 & 2 & 6 & g) & Takeoff & 56 & $5.80 \%$ \\
\hline Takeoff-initial dimb & 2 & 0 & 5 & 7 & 3 & 2 & 8 & 13 & 19 & 12 & 65 & 96 & Initial Climb & 96 & $10.12 \%$ \\
\hline Climb & 1 & 0 & 0 & 1 & 0 & 0 & 2 & 2 & 2 & 1 & 5 & 8 & & & \\
\hline Climb-to cruise & 0 & 2 & 0 & 2 & 0 & 0 & 0 & o] & 1 & 4 & 3 & B & Climb & 16 & $1.69 \%$ \\
\hline Cruiso & 7 & 1 & 10 & 18 & 4 & $T$ & 6 & 11 & 28 & 12 & 46 & 86 & & & \\
\hline Cruise-normal & 5 & 1 & 5 & 11 & 3 & 0 & 4 & 7 & 17 & 7 & 67 & 91] & & & \\
\hline Cruise-holding (IFR) & 0 & 0 & 0 & of & 0 & 0 & 0 & of & 0 & 0 & 0 & 의 & Cruise & 17 & $18.65 \%$ \\
\hline Descent & $\overline{0}$ & 0 & 5 & 5 & 1 & 0 & 0 & 1 & 2 & 1 & 14 & 17 & & & \\
\hline Descent-normal & 0 & 0 & 0 & of & 0 & 0 & 1 & 1] & 0 & 1 & 2 & 3 & & & \\
\hline Descent-emergency & 0 & 0 & 0 & 0 & 0 & 0 & 0 & 0 & 0 & 0 & 6 & 6 & & & \\
\hline Descent-uncontrolled & 0 & 0 & 1 & 1) & 0 & 0 & 2 & 2) & 2 & 1 & 5 & e. & Descent & 34 & $3.58 \%$ \\
\hline Approach & 0 & $\overline{0}$ & 1 & T] & 1 & $T$ & 2 & 4 & $\mathbf{3}$ & 5 & 10 & 18 & & & \\
\hline Approach-VFR pattern-downwind & 1 & 0 & 1 & 2 & 1 & 0 & $\mathbf{0}$ & $1]$ & 4 & 1 & 5 & 10 & & & \\
\hline Approach-VFR pattern-base turn & 0 & 0 & 0 & of & 0 & 0 & 0 & 0 & $\mathbf{0}$ & 0 & 0 & of & & & \\
\hline Approach-VFR pattern-base to final & 0 & 0 & 1 & 1 & 0 & 0 & 1 & $1]$ & 1 & 1 & 6 & 8 & & & \\
\hline Approach-VFR pattern-final approach & 1 & 0 & 5 & 6) & 0 & D & 2 & 2 & 4 & 4 & 21 & 29) & & & \\
\hline Approach-po-around (VFA) & 0 & 0 & 0 & o) & 1 & 0 & 1 & 2 & 1 & 0 & 4 & 5] & & & \\
\hline Approach-1AF to FAF/outer marker (IFA) & 0 & 0 & 0 & o) & 0 & 0 & 0 & 0 & $\mathbf{0}$ & 0 & 0 & of & & & \\
\hline Approach-FAF/outer marker to threshold & 0 & 0 & $\mathbf{0}$ & of & 0 & 0 & 0 & o] & $\mathbf{0}$ & 0 & 0 & of & & & \\
\hline Approach-circling (IFA) & 0 & 0 & o & 0 & 0 & o & 0 & 0 & $\mathbf{0}$ & $\mathbf{0}$ & 0 & 이 & & & \\
\hline Approach-missed approach $\{[F P\}$ & 0 & a & Q & a) & 0 & 0 & $\mathbf{0}$ & o] & $\mathbf{0}$ & 0 & 0 & of & Approach & 70 & $7.38 x$ \\
\hline Landing-abortad & 0 & o & 0 & 0 & $\overline{0}$ & $\overline{0}$ & $\overline{0}$ & 0 & $\overline{0}$ & $\overline{0}$ & 0 & 0 & & & \\
\hline Landing & 1 & o & 3 & 4 & 0 & $\mathbf{0}$ & 4 & 4 & 1 & 0 & 31 & 32 & & & \\
\hline Landing-flarerouchdown & 0 & o & 15 & 15 & 1 & 1 & 10 & 12 & 2 & 4 & B7 & $\infty$ & & & \\
\hline Landing roll & 0 & 0 & 1 & 1 & 0 & 0 & 0 & 0 & 0 & 0 & 3 & 3 & Landing & 128 & $13.48 \%$ \\
\hline Manauvering & 3 & 2 & $\overline{8}$ & 13 & 1 & 1 & 5 & 7 & 28 & 10 & 53 & 91 & & & \\
\hline Maneuvering-aerial application & 0 & $\mathbf{0}$ & 5 & 5 & 1 & 0 & 11 & 12. & 7 & 12 & B1 & 100| & & & \\
\hline Maneuvering-turn to reverse direction & 0 & 0 & 3 & 3 & 0 & 0 & 2 & 2 & 2 & 4 & 20 & 26 & & & \\
\hline Manouvering-turn to landing area (emer. & 0 & $\mathbf{0}$ & $\mathbf{0}$ & 0 & $\mathbf{0}$ & 0 & $\mathbf{0}$ & o) & $\mathbf{0}$ & 0 & 0 & 이 & & & \\
\hline Hover & 1 & 1 & 5 & 7) & 1 & 0 & $B$ & 9. & 4 & 6 & 87 & 99 & Manouvering & 316 & $30.30 \%$ \\
\hline Other & 0 & 0 & 0 & 0 & 0 & 0 & 1 & 1 & 0 & 0 & 2 & 2 & & & \\
\hline Unknown & 0 & 0 & 0 & o. & 0 & 0 & 0 & o] & 4 & 1 & 0 & 5 & & & \\
\hline Not reported & 0 & 0 & 1 & 1 & 0 & 0 & 0 & 0 & 0 & 0 & 3 & 3 & Other/?/NR & 10 & $1.05 \%$ \\
\hline Total & 22 & 9 & 89 & 120 & 19 & 8 & 75 & 102 & 143 & 109 & 697 & 949 & & 949 & $100.00 \%$ \\
\hline
\end{tabular}


Turbine Englne, Rotary Wing Phase ol Operation

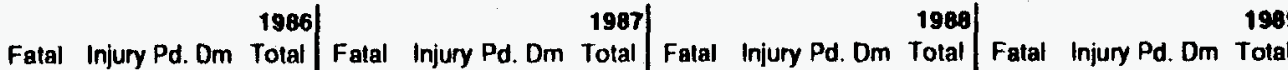

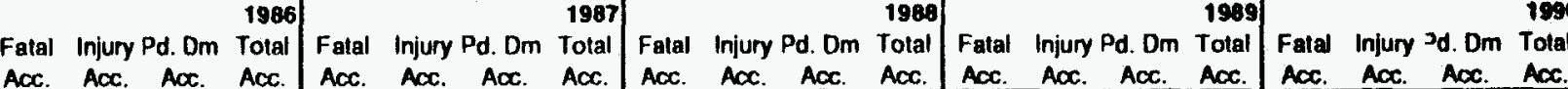

Standing

Standing-prellight

Slanding-starting engine(s)

Standing-engine(s) operating

Standing-engine(s) not operating

Standing-idling rotors

$$
\text { Taxi }
$$

Taxi-to takeoff

Taxi-trom landing

Taxi-aorial

Takeoff

Takeolf-aborted

Takeolf-ground run

Takeoltinitamb

Climb

Climb-to cruise

Cruise

Cruise-normal

Cruise.holding (IFP)

Descent

Descent-normal

Descent-emergency

Descent-uncontrolled

Approach

Approach-VFR pattern-downwind

Approach-VFR pallern-bese turn

Approach-VFR pattern-base to final

Approach-VFA pattern-linal approech

Approach-go-around (VFF)

Approsch-IAF to FAF/outer marker (IFA)

Approach-FAF/outer marker to threshol

Approach-circling (IFR)

Approach-missed approach (IFR)

Landing-aborted

Landing

anding Hare/touchdown

Landing-roll

Manouvering

Manouvering-aerial application

Maneuvering-turn to reverse direction

Manouvering-turn to landing area (emer.

Hover

Unknown

Not reported

Total:

\begin{tabular}{llll|ll}
0 & 0 & 0 & 0 & 0 & 0 \\
0 & 0 & 0 & 0 & 0 & 0 \\
0 & 0 & 0 & 0 & 0 & 0 \\
0 & 0 & 0 & 0 & 0 & 0 \\
0 & 0 & 0 & 0 & 0 & 0 \\
0 & 0 & 1 & 1 & 0 & 0 \\
\hline
\end{tabular}

\begin{tabular}{ll}
0 & 0 \\
0 & 0 \\
0 & 0 \\
0 & 0 \\
0 & 0 \\
0 & 1 \\
\hline
\end{tabular}

\begin{tabular}{ll}
0 & 0 \\
0 & 0 \\
0 & 0 \\
1 & 1 \\
0 & 0 \\
0 & 1 \\
\hline 0 & 0
\end{tabular}

\begin{tabular}{llll|ll}
0 & 0 & 0 & 0 & 0 & 1 \\
0 & 0 & 0 & 0 & 0 & 0 \\
0 & 0 & 0 & 0 & 0 & 0 \\
0 & 0 & 0 & 0 & 0 & 0 \\
0 & 0 & 0 & 0 & 0 & 0 \\
0 & 0 & 2 & 2 & 0 & 1 \\
\hline
\end{tabular}

1990|

Fatal Injury गd. Om Tolal Foc. AOC. AOC. AOC.
Aolal

\begin{tabular}{llll|llll}
0 & 0 & 0 & 0 & 0 & 0 & 0 & 0 \\
0 & 2 & 3 & 5 & 1 & 1 & 1 & 3
\end{tabular}

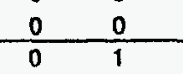

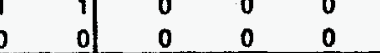

\begin{tabular}{llll|llll|llll}
0 & 0 & 0 & 0 & 0 & 0 & 0 & 0 & 0 & 0 & 0 & 0 \\
1 & 0 & 0 & 1 & 0 & 0 & 1 & 1 & 0 & 0
\end{tabular}

\begin{tabular}{l|lll}
0 & 0 & 0 & 2 \\
\hline 3 & 2 & 1 & 1 \\
0 & 0 & 0 & 0 \\
0 & 0 & 0 & 0 \\
\hline
\end{tabular}

\begin{tabular}{llll|llll|llll|lll}
0 & 4 & 7 & 11 & 1 & 2 & 2 & 5 & 0 & 1 & 2 & 3 & 2 & 4 & 0 \\
\hline 0 & 0 & 0 & 0 & 1 & 0 & 0 & 1 & 0 & 0 & 1 & 1 & 0 & 0 & 2 \\
0 & 0 & 1 & 1 & 0 & 0 & 1 & 1 & 0 & 0 & 0 & 0 & 1 & 0 & 0 \\
\hline 4 & 0 & 4 & 8 & 2 & 1 & 3 & 6 & 1 & 2 & 2 & 5 & 5 & 2 & 4
\end{tabular}

\begin{tabular}{lllr|llll|ll}
0 & 0 & 1 & 1 & 0 & 0 & 1 & 1 & 0 & 0 \\
\hline 4 & 0 & 4 & 8 & 2 & 1 & 3 & 6 & 1 & 2 \\
3 & 2 & 7 & 12 & 2 & 0 & 4 & 6 & 2 & 3 \\
0 & 0 & 0 & 0 & 0 & 0 & 0 & 0 & 0 & 0 \\
\hline
\end{tabular}

\begin{tabular}{|c|c|c|c|c|c|c|c|c|c|c|c|c|c|c|c|c|c|}
\hline 0 & 0 & 0 & o) & 0 & 0 & 0 & 0 & 0 & 0 & 0 & 0 & 0 & 0 & 1 & 1 & 0 & 0 \\
\hline 1 & 0 & $\overline{1}$ & 2 & $\overline{0}$ & 0 & $\overline{0}$ & 0 & 0 & 0 & 3 & 3 & 0 & 0 & 0 & 0 & 0 & 0 \\
\hline 0 & 0 & 2 & 2 & 0 & 0 & 0 & 0 & 0 & 0 & 1 & 1 & 0 & 0 & 1 & 1 & 0 & 0 \\
\hline 0 & 0 & 0 & of & 0 & 0 & 0 & 0 & 0 & 0 & 0 & 0 & 0 & 0 & 0 & of & 0 & 0 \\
\hline 0 & 0 & 0 & 0 & 0 & 0 & 0 & 0 & 0 & 0 & 0 & 0 & 0 & 0 & 0 & 0 & 0 & 0 \\
\hline 0 & 0 & 2 & 2 & 0 & 0 & 0 & 0 & 0 & 0 & 2 & 2 & 1 & 0 & 2 & 3) & 0 & 1 \\
\hline 0 & 0 & 0 & 0 & 0 & 0 & 0 & 0 & 0 & 0 & 0 & 0 & 0 & 0 & 0 & 0 & 0 & 0 \\
\hline 0 & 0 & 0 & 0 & 0 & 0 & 0 & 0 & 1 & 0 & 0 & 1 & 0 & 0 & 0 & 0 & 0 & 0 \\
\hline 0 & 0 & 0 & 0 & 0 & 0 & 0 & 0 & 0 & 0 & 0 & 0 & 0 & 0 & 0 & 0 & 0 & 0 \\
\hline 0 & 0 & 1 & 1 & 0 & 1 & 3 & 4 & 0 & 1 & 1 & 2 & 1 & 0 & 2 & 3 & 0 & 0 \\
\hline 0 & 0 & 0 & 0 & 0 & 0 & 0 & 0 & 0 & 0 & 0 & 0 & 0 & 0 & 0 & 0 & 0 & 0 \\
\hline 0 & 0 & 0 & 0 & 0 & 0 & 0 & 0 & 0 & 0 & 0 & 0 & 0 & 0 & 0 & 0 & 0 & 0 \\
\hline 0 & 0 & 0 & 0 & 0 & 0 & o & 0 & 0 & 0 & 0 & 0 & 0 & 0 & 0 & 0 & 0 & 0 \\
\hline 0 & 0 & 0 & 0 & 0 & 0 & o & 0 & 0 & 0 & 0 & 0 & 0 & 0 & 0 & 0 & 0 & 0 \\
\hline 0 & 0 & 0 & 0 & 0 & 0 & 0 & 0 & 0 & 0 & 0 & 0 & 0 & $\underline{0}$ & 0 & 0 & 0 & 0 \\
\hline 0 & $\overline{0}$ & $\overline{0}$ & 0 & 0 & 0 & 0 & 0 & 0 & 0 & 1 & 1 & 0 & 0 & $\overline{0}$ & 0 & 0 & 0 \\
\hline
\end{tabular}

\begin{tabular}{rrrr|rrrr|rrrrrrrrrrrr}
3 & 2 & 7 & 12 & 2 & 0 & 4 & 6 & 2 & 3 & 5 & 10 & 0 & 1 & 2 & 3 & 0 & 0 \\
0 & 0 & 0 & 0 & 0 & 0 & 0 & 0 & 0 & 0 & 0 & 0 & 0 & 0 & 1 & 1 & 0 & 0 \\
\hline 1 & 0 & 1 & 2 & 0 & 0 & 0 & 0 & 0 & 0 & 3 & 3 & 0 & 0 & 0 & 0 & 0 & 0 \\
0 & 0 & 2 & 2 & 0 & 0 & 0 & 0 & 0 & 0 & 1 & 1 & 0 & 0 & 1 & 1 & 0 & 0 & 0 \\
0 & 0 & 0 & 0 & 0 & 0 & 0 & 0 & 0 & 0 & 0 & 0 & 0 & 0 & 0 & 0 & 0 & 0 & 0 \\
0 & 0 & 0 & 0 & 0 & 0 & 0 & 0 & 0 & 0 & 0 & 0 & 0 & 0 & 0 & 0 & 0 & 0 \\
\hline 0 & 0 & 2 & 2 & 0 & 0 & 0 & 0 & 0 & 0 & 2 & 2 & 1 & 0 & 2 & 3 & 0 & 1 & 0 \\
0 & 0 & 0 & 0 & 0 & 0 & 0 & 0 & 0 & 0 & 0 & 0 & 0 & 0 & 0 & 0 & 0 & 0 & 0 \\
0 & 0 & 0 & 0 & 0 & 0 & 0 & 0 & 1 & 0 & 0 & 1 & 0 & 0 & 0 & 0 & 0 & 0 & 0 \\
0 & 0 & 0 & 0 & 0 & 0 & 0 & 0 & 0 & 0 & 0 & 0 & 0 & 0 & 0 & 0 & 0 & 0 & 0 \\
0 & 0 & 1 & 1 & 0 & 1 & 3 & 4 & 0 & 1 & 1 & 2 & 1 & 0 & 2 & 3 & 0 & 0 & 0 \\
0 & 0 & 0 & 0 & 0 & 0 & 0 & 0 & 0 & 0 & 0 & 0 & 0 & 0 & 0 & 0 & 0 & 0 & 0 \\
0 & 0 & 0 & 0 & 0 & 0 & 0 & 0 & 0 & 0 & 0 & 0 & 0 & 0 & 0 & 0 & 0 & 0 & 0 \\
0 & 0 & 0 & 0 & 0 & 0 & 0 & 0 & 0 & 0 & 0 & 0 & 0 & 0 & 0 & 0 & 0 & 0 & 0 \\
0 & 0 & 0 & 0 & 0 & 0 & 0 & 0 & 0 & 0 & 0 & 0 & 0 & 0 & 0 & 0 & 0 & 0 & 0 \\
0 & 0 & 0 & 0 & 0 & 0 & 0 & 0 & 0 & 0 & 0 & 0 & 0 & 0 & 0 & 0 & 0 & 0 \\
\hline 0 & 0 & 0 & 0 & 0 & 0 & 0 & 0 & 0 & 0 & 1 & 1 & 0 & 0 & 0 & 0 & 0 & 0 & 0
\end{tabular}

3. GENERAL AVIATION 
Table 3.7 (Conlinued)

General Avlation Accidents by Phese of Flighl Operation

Turbine Engine, Rotary Wing

Reterences 3.2010327

\begin{tabular}{|c|c|c|c|c|c|c|c|c|c|c|c|c|c|c|c|}
\hline $\begin{array}{c}\text { Turblne Englne, Rotary Wing } \\
\text { Phase of Operation } \\
\end{array}$ & $\begin{array}{l}\text { Fatal } \\
\text { Acc. }\end{array}$ & $\begin{array}{l}\text { Injury } \mathrm{P} \\
\text { Acc. }\end{array}$ & & $\begin{array}{c}1992 \\
\text { Total } \\
\text { Acc. } \\
\end{array}$ & $\begin{array}{l}\text { Fatal } \\
\text { ACC. }\end{array}$ & $\begin{array}{l}\text { Injury } \\
\text { Acc. }\end{array}$ & $\begin{array}{l}\text { Pr. Dm } \\
\text { Acc. }\end{array}$ & $\begin{array}{l}1993 \\
\text { Total } \\
\text { Acc. }\end{array}$ & $\begin{array}{l}\text { Fatal } \\
\text { Acc. }\end{array}$ & $\begin{array}{l}\text { Injury } \\
\text { Aoc. }\end{array}$ & $\begin{array}{l}\text { Pr. Dm. } \\
\text { Acc. }\end{array}$ & $\begin{array}{l}\text { TOTAL } \\
\text { Total } \\
\text { Acc. }\end{array}$ & $\begin{array}{l}\text { Flight } \\
\text { Phase }\end{array}$ & No. & Pct. \\
\hline Standing & 0 & 0 & 0 & 0 & 0 & 0 & 0 & 0 & 0 & 1 & 0 & 1 & & & \\
\hline Standing-prallight & 0 & $\mathbf{0}$ & 0 & 0 & 0 & 0 & 0 & o & $\mathbf{0}$ & 0 & 0 & 0 & & & \\
\hline Standing-starting engine(s) & 0 & 0 & 0 & 0 & $\mathbf{0}$ & 0 & 0 & 0 & $\mathbf{0}$ & o & 0 & 0 & & & \\
\hline Standing-engine(s) operating & 0 & 0 & 1 & 1 & o & 1 & 0 & 1) & 0 & 2 & 3 & 5 & & & \\
\hline Slanding-engine(s) not operating & 0 & 0 & 0 & 0 & 0 & 0 & 0 & 0 & 0 & 0 & 0 & 0 & & & \\
\hline Standing-ldling rotors & $\underline{0}$ & $\mathbf{0}$ & 0 & 0 & 0 & 0 & 2 & 2 & 0 & 2 & 5 & 7 & Standing & 13 & $2.70 \%$ \\
\hline Taxi & $\overline{0}$ & $\overline{0}$ & 0 & 0 & 0 & 0 & 0 & 0 & 0 & $\overline{0}$ & 0 & 0 & & & \\
\hline Taxi-to takeolf & 0 & 0 & 0 & 0 & 0 & 0 & 0 & 0 & 0 & 0 & 0 & 0 & & & \\
\hline Taxi-from landing & 0 & $\mathbf{0}$ & 0 & 0 & 0 & 0 & 1 & 1] & 0 & 0 & 2 & 2 & & & \\
\hline Taxi-aerial & 1 & $\underline{0}$ & 0 & 1 & $\underline{0}$ & 0 & 2 & 2 & 1 & 0 & 6 & 7 & Taxi & 9 & $1.87 x$ \\
\hline Takeoff & 0 & 0 & 0 & $\overline{0}$ & 0 & $\overline{0}$ & 0 & 0 & 3 & 5 & 13 & 21 & & & \\
\hline Takeoll-aborted & 0 & o & 0 & of & 0 & 0 & 0 & of & 0 & o & 0 & 0 & & & \\
\hline Takeofl-ground run & $\mathbf{0}$ & 0 & 0 & 0 & 0 & 0 & 0 & 0 & 1 & 0 & 1 & 2 & Takeoff & 23 & $4.77 x$ \\
\hline Takeoff-initial climb & $\underline{0}$ & 0 & 2 & 2 & 2 & 0 & 3 & 5 & 10 & 12 & 19 & 41 & Initial Climb & 41 & $8.51 x$ \\
\hline$\overline{\text { Climb }}$ & 0 & 0 & 0 & 0 & 0 & 0 & 0 & 0 & 7 & 0 & 4 & 5 & & & \\
\hline Climb-to cruise & $\underline{0}$ & 0 & 0 & of & 0 & D & 1 & $1]$ & 1 & 0 & 4 & 5 & Climb & 10 & $2.07 x$ \\
\hline Cruise & 6 & 2 & 2 & 10 & 2 & $\overline{2}$ & 3 & 7 & 24 & 11 & 33 & 68 & & & \\
\hline Cruise-nomal & 4 & 1 & $\mathbf{3}$ & 8 & 0 & 1 & 3 & 4) & 12 & B & 28 & 48 & & & \\
\hline Cruise-holding (IFR) & 0 & 0 & 0 & 0 & 0 & 0 & 0 & 0 & 0 & 0 & 1 & 1 & Cruise & 117 & $24.27 x$ \\
\hline Descent & $\mathbf{0}$ & 0 & 1 & 1) & 0 & 0 & 0 & 이 & 7 & 0 & 5 & $\overline{6}$ & & & \\
\hline Descent-normal & 0 & 0 & 0 & 0 & 0 & 0 & 0 & o) & 0 & o & 4 & 4 & & & \\
\hline Descent-emergency & $\mathbf{0}$ & 0 & 0 & o & $\mathbf{0}$ & 0 & o & of & 0 & 0 & 1 & 1 & & & \\
\hline Descent-uncontrolled & 0 & 0 & 0 & 0 & 1 & 0 & 0 & $1]$ & 1 & 0 & 0 & 1 & Descent & 12 & $2.49 \%$ \\
\hline Approach & $T$ & 0 & 1 & 2 & 0 & 1 & 2 & 3 & 4 & 2 & 9 & 15 & & & \\
\hline Approach-VFR pattern-downwind & 0 & 0 & 0 & o) & 0 & 0 & $\mathbf{0}$ & 0 & 0 & 0 & 1 & 1 & & & \\
\hline Approach-VFR pattern-base turn & 0 & 0 & 0 & o] & $\mathbf{0}$ & o & 0 & o & 1 & 0 & o & 1 & & & \\
\hline Approach-VFR pattern-bass to final & $\mathbf{0}$ & 0 & 0 & of & 0 & 0 & 0 & o & 0 & $\mathbf{0}$ & 0 & o & & & \\
\hline Approach-VFA pattern-inal approach & $\mathbf{0}$ & 0 & 2 & 2 & 0 & o & 1 & 1 & 1 & $\mathbf{3}$ & 13 & 17 & & & \\
\hline Approach-go-around (VFA) & 0 & 0 & 0 & o & 0 & o & 0 & 0 & 0 & 0 & 0 & o & & & \\
\hline Approach-1AF to FAFlouter marker (IFR) & $\mathbf{0}$ & 0 & o & of & $\mathbf{0}$ & o & 0 & o & 0 & o & 0 & o & & & \\
\hline Approach-FAFlouter marker to threshold & o & 0 & 0 & 0 & $\mathbf{0}$ & 0 & 0 & o & 0 & o & 0 & o) & & & \\
\hline Approach-circling (IFR) & $\mathbf{0}$ & 0 & 0 & 이 & 0 & 0 & 0 & of & 0 & 0 & 0 & o] & & & \\
\hline Approach-missed approach (IFP) & 0 & 0 & 0 & of & 0 & 0 & o & o. & 0 & 0 & 0 & o & Approach & 34 & $7.05 \%$ \\
\hline Landing-aborted & o & 0 & 0 & of & $\overline{0}$ & $\overline{0}$ & $\overline{0}$ & 0 & o & $\mathbf{0}$ & 1 & 1 & & & \\
\hline Landing & 0 & 1 & 1 & 2 & $\mathbf{0}$ & 0 & 2 & 2 & 0 & 1 & 13 & 14 & & & \\
\hline Landing-flara/louchiown & 0 & 0 & 5 & 5 & 0 & 0 & 1 & 1 & $\mathbf{0}$ & 0 & 34 & 34 & & & \\
\hline Landing-roll & 0 & 0 & 0 & of & $\mathbf{0}$ & $\mathbf{0}$ & $\mathbf{0}$ & of & 0 & 0 & 0 & 0 & Landing & 49 & $10.17 x$ \\
\hline Maneuvering & 5 & 0 & 5 & 10 & 3 & 3 & 9 & 15 & 19 & 9 & 38 & 66 & & & \\
\hline Manouvering-agrial application & 0 & 1 & 0 & 1) & 0 & 1 & 1 & 2 & 2 & $\mathbf{3}$ & 12 & 17 & & & \\
\hline Maneuvering-turn to reverse direction & 0 & $\mathbf{0}$ & 1 & 1 & $\mathbf{0}$ & 0 & 0 & o & 1 & 0 & 2 & 3 & & & \\
\hline Maneuvering-furn to landing area (emer. & 0 & 0 & 0 & o) & o & 0 & o & 0 & 0 & 0 & 0 & 0 & & & \\
\hline Hover & 0 & 1 & 9 & 10 & 5 & 1 & 4 & 10 & 14 & 17 & 48 & 79 & Mancuvering & 165 & $34.23 \%$ \\
\hline Other & 1 & 0 & 0 & 1 & 0 & $\overline{0}$ & $\overline{0}$ & of & 1 & 1 & 2 & 4 & & & \\
\hline Unknown & 0 & 0 & 0 & 0 & 1 & 0 & 0 & 1 & $i$ & 0 & 0 & 1 & & & \\
\hline Not reported & 1 & 0 & 1 & 2 & 0 & 0 & 1 & 1 & 1 & 0 & 3 & 4 & Other/?/NA & 9 & $1.87 \%$ \\
\hline Total & 19 & 6 & 34 & 59 & 14 & $\overline{10}$ & 36 & 60 & 100 & 77 & 305 & 482 & & 482 & $00.00 \%$ \\
\hline
\end{tabular}


Table 3.8

General Awation Aceldents by Phase of Fllght Operation

Total, Rotary Wing

Roterences 3.20103 .27

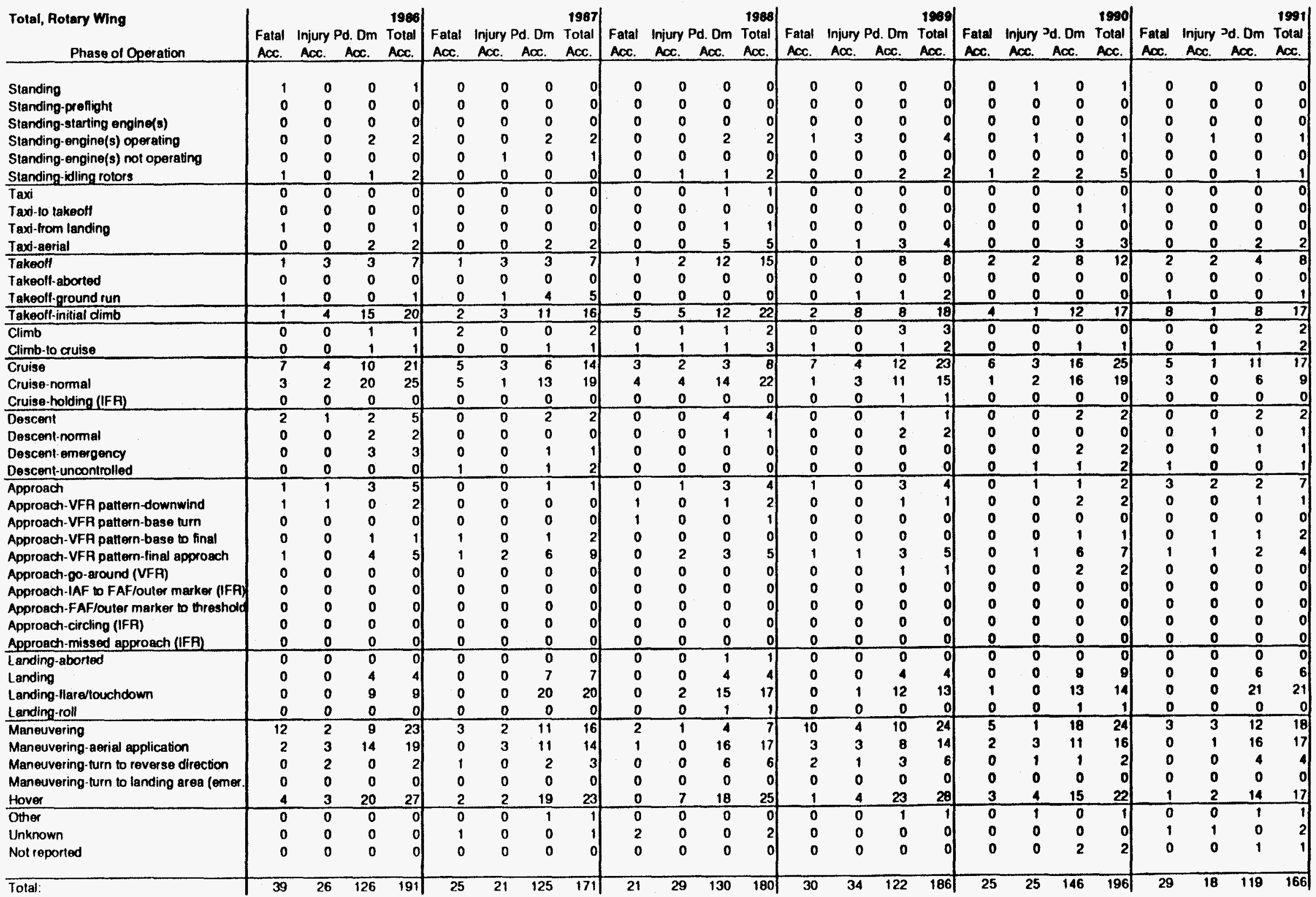

3. GENERAL AVIATION 
Table 3.8 (Continued)

Goneral Aviation Aceidents by Phese of Flight Operallon

Total, Rotary Wing

Peterences 3.20103 .27

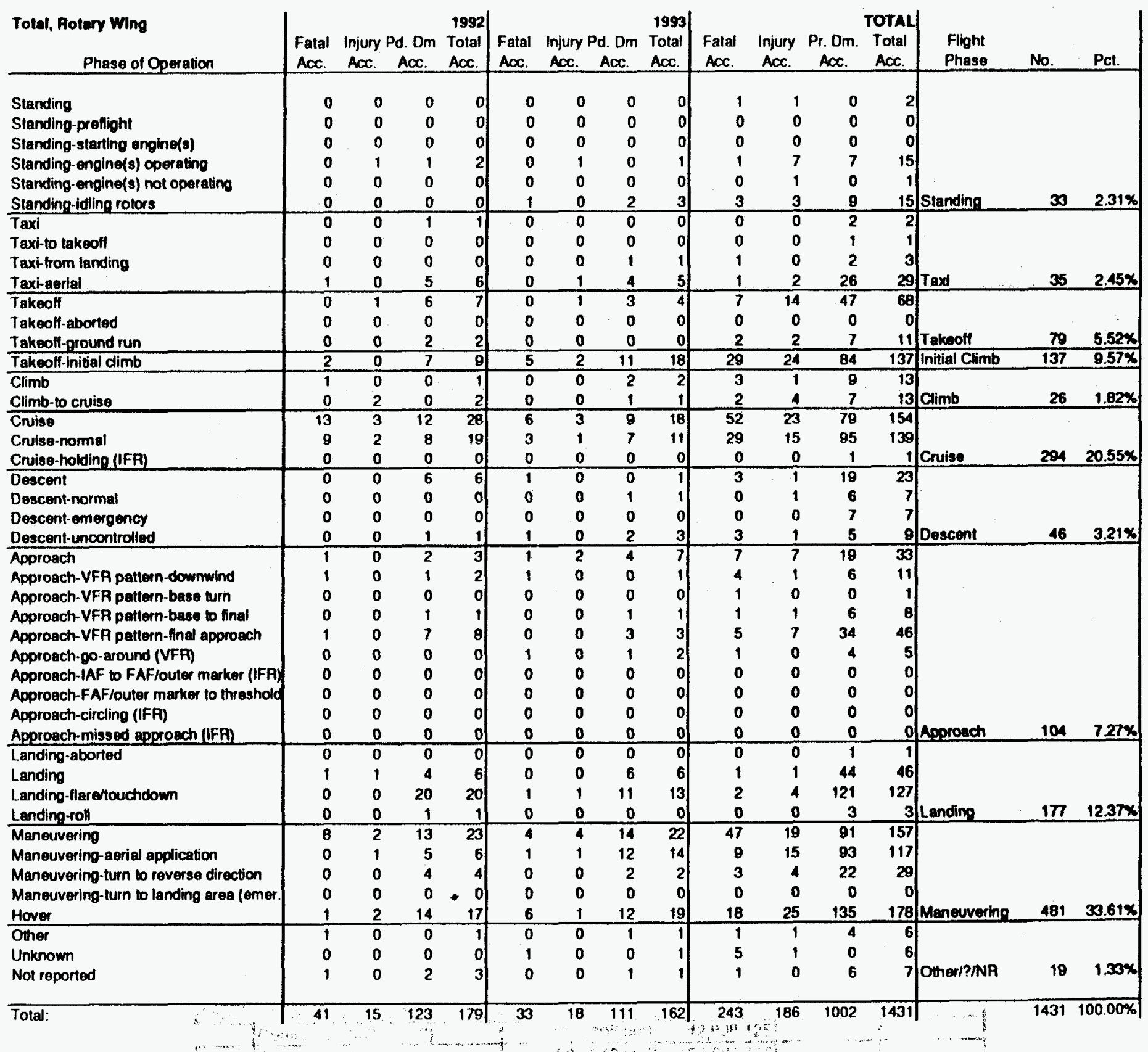


Table 3.9

General Aviation Aecidents by Phese of Filght Operation

Totel Powered Alreraft

Roterences 3.20 to 3.27

\begin{tabular}{|c|c|c|c|c|c|c|c|c|c|c|c|c|c|c|c|c|c|c|c|c|c|c|c|c|}
\hline $\begin{array}{l}\text { Total Powered Alrcrat } \\
\text { Phase of Operation }\end{array}$ & $\begin{array}{l}\text { Fatal } \\
\text { Acc. }\end{array}$ & $\begin{array}{l}\text { Injury } \\
\text { ACE. }\end{array}$ & $\begin{array}{l}\text { Pd. Dm } \\
\text { Aoc. }\end{array}$ & $\begin{array}{l}1906 \\
\text { Total } \\
\text { AOC. } \\
\end{array}$ & $\begin{array}{l}\text { Fatal } \\
\text { ACC. } \\
\end{array}$ & $\begin{array}{l}\text { Injury } \\
\text { Aoc. }\end{array}$ & $\begin{array}{l}\text { Pd. Dm } \\
\text { Acc. }\end{array}$ & $\begin{array}{l}1987 \\
\text { Total } \\
\text { Aoc. } \\
\end{array}$ & $\begin{array}{l}\text { Fatal } \\
\text { ACC. }\end{array}$ & $\begin{array}{l}\text { Injury } \\
\text { Acc. }\end{array}$ & $\begin{array}{l}\text { Pd. Dm } \\
\text { Acc. }\end{array}$ & $\begin{array}{l}1968 \\
\text { Total } \\
\text { Acc. } \\
\end{array}$ & $\begin{array}{l}\text { Fatal } \\
\text { ACx. }\end{array}$ & $\begin{array}{l}\text { Injury } \\
\text { Aoc. }\end{array}$ & $\begin{array}{l}\text { Pd. Dm } \\
\text { Acc. }\end{array}$ & $\begin{array}{l}1989 \\
\text { Total } \\
\text { ACc. }\end{array}$ & $\begin{array}{l}\text { Fatal } \\
\text { AOC. }\end{array}$ & $\begin{array}{l}\text { Injury } \\
\text { ACC. }\end{array}$ & $\begin{array}{l}\text { 2d. Dm } \\
\text { Acc. }\end{array}$ & $\begin{array}{l}1990 \\
\text { Total } \\
\text { Acc. } \\
\end{array}$ & $\begin{array}{l}\text { Fatal } \\
\text { Aoc. }\end{array}$ & $\begin{array}{l}\text { Injury } \\
\text { Acc. }\end{array}$ & $\begin{array}{l}\text { गd. Dm } \\
\text { Aac. }\end{array}$ & $\begin{array}{l}1991 \\
\text { Total } \\
\text { Acc. } \\
\end{array}$ \\
\hline Standing & $t$ & 0 & 1 & 2 & 0 & 0 & 0 & 0 & 0 & 0 & 3 & 3) & 0 & 0 & 1 & 1 & 0 & 2 & 0 & 2 & 0 & o & 2 & 2 \\
\hline Standing-prenight & 1 & 0 & 0 & 1 & 0 & 1 & 0 & 1 & 0 & 0 & 1 & 1) & 0 & 0 & 0 & 0 & 0 & 0 & 0 & 이 & 1 & 1 & 1 & 3 \\
\hline Standing-starting engine(s) & 0 & 5 & 17 & 22 & 1 & 2 & 17 & 20 & 0 & 3 & 15 & 18 & 0 & $\mathbf{0}$ & 13 & 13 & 0 & 1 & 7 & B] & 0 & 2 & 5 & 7 \\
\hline Standing-engine(s) operating & 1 & 0 & 7 & B & 2 & 2 & 11 & 15 & 1 & 1 & 8 & 10 & 2 & 7 & 9 & 18 & 0 & 2 & 6 & 8) & 2 & 2 & 10 & 14 \\
\hline Standing- engine(s) not operating & 0 & 0 & 0 & of & 0 & 2 & 1 & 3 & $\mathbf{0}$ & 0 & 0 & of & 0 & $\mathbf{0}$ & 1 & 1 & 0 & 0 & 0 & o) & $\mathbf{0}$ & o & $\mathbf{0}$ & 0 \\
\hline Slanding-Wding rotors & 1 & 0 & 1 & 2 & 0 & 0 & 0 & of & 0 & 1 & 1 & 2 & 0 & 0 & 2 & 2 & 1 & 2 & 2 & 5 & 0 & 0 & 1 & 1 \\
\hline Taxi & 1 & 0 & 13 & 14 & $\mathbf{0}$ & 0 & 5 & 5 & 1 & $\mathbf{0}$ & 7 & (8) & 0 & 0 & 5 & 5 & 2 & 0 & 9 & 11 & 0 & 1 & 5 & 6 \\
\hline Taxi-to takeolf & 0 & 0 & 21 & 21 & 0 & 0 & 32 & 32 & 0 & 1 & 24 & 25 & 0 & 0 & 22 & 22 & 0 & 0 & 27 & 27 & 0 & o & 16 & 16 \\
\hline Taxi-from landing & 1 & 1 & 18 & 20 & 0 & 0 & 28 & 28 & 1 & 0 & 31 & 32 & 1 & 0 & 26 & 27) & 0 & 0 & 19 & 19 & $\mathbf{0}$ & 2 & 16 & 18 \\
\hline Taxi-aarial & 0 & 0 & 2 & 2 & 0 & 0 & 2 & 2 & 0 & 0 & 5 & 5 & 0 & 1 & 3 & 4 & 0 & 0 & 4 & 4 & 0 & $\mathbf{0}$ & 2 & 2 \\
\hline Takeolf & $\boldsymbol{\theta}$ & 8 & 27 & 44 & $\mathbf{3}$ & 9 & 21 & 33 & 6 & 7 & 32 & 45 & 4 & 3 & 29 & 36 & 10 & 10 & 30 & 50 & 14 & 6 & 29 & 49 \\
\hline Takeoll-aborted & 0 & 0 & 0 & 0 & $\mathbf{0}$ & 0 & 0 & of & 0 & 1 & 15 & 16 & 0 & 0 & 0 & 0 & 0 & 0 & 0 & of & 0 & 0 & 0 & 0 \\
\hline Takooll-ground run & 5 & 6 & 107 & 118 & 2 & 10 & 113 & 125 & 1 & 2 & 77 & 80 & 2 & 2 & 78 & 82 & 5 & 3 & 73 & 81 & 2 & 7 & 81 & 90 \\
\hline Takeofl-initial dimb & 69 & 58 & 232 & 359 & 50 & 70 & 220 & 340 & 71 & 69 & 187 & 327 & 63 & 59 & 202 & 324 & 65 & 61 & 185 & 311 & 57 & 58 & 167 & 302 \\
\hline Climb & 12 & 6 & 20 & 38 & 7 & 4 & 12 & 23 & 5 & 4 & 15 & 24 & 9 & 2 & 10 & 21 & 7 & 5 & 8 & 20 & 15 & 10 & 21 & 46 \\
\hline Climb-to cruise & 21 & 5 & 27 & 53 & 14 & $\boldsymbol{B}$ & 30 & 52 & 26 & 5 & 26 & 57 & 12 & 2 & 15 & 29 & 13 & 7 & 15 & 35 & 13 & $\mathbf{5}$ & 23 & 41 \\
\hline Cruise & 41 & 19 & 115 & 175 & 38 & 11 & 84 & 133 & 44 & 18 & 91 & 153 & 55 & 21 & 107 & 180 & 44 & 19 & 85 & 158 & 55 & 23 & 116 & 194 \\
\hline Cruise-normal & 47 & 37 & 178 & 262 & 64 & 19 & 158 & 241 & 63 & 31 & 164 & 258 & 61 & 20 & 146 & 227 & 35 & 15 & 145 & 195 & 37 & 23 & 116 & 176 \\
\hline Cruise-holding (IFP) & 0 & 0 & 1 & 1 & 0 & 0 & 0 & of & 1 & 0 & $\mathbf{3}$ & 4 & 0 & 0 & 2 & 2 & 0 & 0 & 0 & of & 0 & 0 & 1 & 1 \\
\hline Descent & 14 & 1 & 11 & 26 & 4 & 1 & 6 & 11 & 8 & 1 & 11 & 21 & 9 & 1 & 6 & 16 & 5 & 1 & 10 & 16 & 3 & $\mathbf{5}$ & 8 & 16 \\
\hline Descent-normal & 10 & 7 & 27 & 44 & 8 & 6 & 30 & 44 & 5 & 1 & 39 & 45 & 5 & 2 & 21 & 28 & 9 & 5 & 21 & 35 & 4 & 6 & 23 & 33 \\
\hline Descent-emergency & 2 & 2 & 7 & 11 & 0 & 0 & 6 & 6 & 1 & 0 & 1 & 2 & 0 & o & 2 & 2 & 0 & 0 & $\mathbf{3}$ & 3 & 2 & 1 & 3 & 6 \\
\hline Descent-uncontrolled & 3 & 1 & 1 & 5) & 5 & 0 & 3 & of & 0 & 1 & 0 & 1 & 1 & $\mathbf{0}$ & 2 & 3 & 2 & 1 & 3 & 6 & 4 & $\mathbf{0}$ & 2 & 6 \\
\hline Approach & 12 & 7 & 23 & 42 & 6 & 6 & 23 & 35 & 11 & 7 & 24 & 42 & 16 & 7 & 36 & 50 & 13 & 4 & 16 & 33 & 16 & $\mathbf{3}$ & 23 & 42 \\
\hline Approach-VFR pattern-downwind & 6 & 3 & 16 & 25 & $\mathbf{9}$ & 4 & 11 & 24 & 4 & 4 & 13 & 21) & 6 & 2 & 9 & 17) & 12 & 2 & 18 & 32 & 5 & 4 & 12 & 21 \\
\hline Approach-VFR pattern-base turn & 5 & 0 & $\mathbf{5}$ & 10 & 1 & $\mathbf{3}$ & 3 & $7)$ & 4 & 2 & 5 & 11) & 3 & 1 & 4 & 8) & 5 & 1 & 9 & 15 & 3 & 1 & 3 & 7 \\
\hline Approach-VFA pattern-base to final & 9 & 2 & 8 & 19 & 10 & 1 & 8 & 19 & $\mathbf{5}$ & 2 & 7 & 14 & 10 & 5 & 6 & 21 & 6 & 3 & 13 & 22 & 8 & 3 & 14 & 25 \\
\hline Approach-VFA pattern-final approach & 14 & 30 & 88 & 132 & 12 & 19 & 93 & 124 & $\mathbf{8}$ & 25 & 75 & 100 & 15 & 17 & 82 & 114 & 21 & 10 & 94 & 125 & 16 & 10 & 64 & 90 \\
\hline Approach-go-around (VFR) & 4 & $\mathbf{g}$ & 46 & 59) & 6 & 11 & 32 & 49 & B & 7 & 39 & 54 & 4 & $\mathbf{5}$ & 30 & 39 & 7 & O & 28 & 43 & 7 & $\mathbf{B}$ & 36 & 51 \\
\hline Approsch-IAF to FAF/outer marker (IFA) & 3 & 3 & 1 & 7 & $\mathbf{5}$ & 0 & 0 & 5 & $\boldsymbol{\theta}$ & 1 & 2 & 11. & 8 & 1 & 0 & 9 & 5 & 1 & 2 & 8) & 5 & 1 & 1 & 7 \\
\hline Approach. FAF/ouler marker to threahold & 18 & 6 & a & 32 & 7 & 4 & 5 & 16 & 7 & 5 & 5 & 17 & 4 & o & 4 & 8) & 0 & 1 & 6 & 16] & 6 & 3 & 6 & 15 \\
\hline Approach-circling (IFR) & 2 & 0 & 2 & 4) & 3 & 0 & 0 & 3 & 3 & 1 & 2 & 6) & $\mathbf{0}$ & 0 & 1 & 1 & 1 & 0 & 0 & 1 & 1 & 2 & 0 & 3 \\
\hline Approach-missed approach (IFF) & 2 & 2 & 4 & ge & 4 & 0 & 1 & 5 & 5 & 0 & 2 & 7 & 1 & 0 & 0 & 1 & 4 & 0 & 1 & 5 & $\underline{3}$ & 1 & 1 & 5 \\
\hline Landing-aborted & $\mathbf{0}$ & $\overline{0}$ & $\mathbf{0}$ & 0) & 0 & 0 & 0 & 0 & 3 & 4 & 19 & 26) & $\mathbf{0}$ & $\mathbf{0}$ & $\mathbf{0}$ & 0 & 0 & 0 & 0 & of & $\mathbf{0}$ & 0 & 0 & 0 \\
\hline Landing & 4 & 7 & 44 & 55 & 4 & $\mathbf{3}$ & 41 & 48 & 0 & 1 & 43 & 44. & 2 & 2 & 44 & 4B & 1 & 2 & 31 & 34) & 1 & 3 & 18 & 22 \\
\hline Landing-Hare/touchdown & 3 & 9 & 219 & 231 & 2 & 9 & 243 & 254 & 2 & 7 & 181 & 100 & $\mathbf{3}$ & 9 & 173 & 185 & 4 & 6 & 166 & 176 & 0 & 2 & 180 & 182 \\
\hline Landing-roll & 7 & 6 & $\mathbf{2 9 0}$ & 303 & 0 & 11 & 309 & 320 & 1 & 9 & 263 & 273 & 2 & 7 & 242 & 251 & 2 & 6 & 258 & 266 & 1 & 0 & 233 & 234 \\
\hline Maneuvering & 107 & 32 & 64 & 203 & 122 & 22 & 60 & 204 & 106 & 22 & 60 & 188 & 79 & 27 & 71 & 177 & 86 & 14 & 61 & 171 & 84 & 30 & 68 & 192 \\
\hline Maneuvering-ae & 10 & 11 & 56 & 77 & 9 & 14 & 56 & 79 & 7 & 14 & 58 & 79 & 21 & 10 & 47 & 78 & 13 & 9 & 53 & 75) & 11 & 11 & 62 & 84 \\
\hline Maneuvering-turn to reverse direction & 10 & 4 & 19 & 33 & 6 & 8 & 10 & 24 & 10 & 2 & 15 & 27) & 9 & 3 & 3 & 15 & 11 & $\mathbf{5}$ & 10 & 26 & 9 & 3 & 13 & 25 \\
\hline Maneuvering-turn to landing area (emer. & $\mathbf{0}$ & 0 & 2 & 2 & 1 & 0 & 0 & 1 & 0 & 0 & 7 & 7 & o & 0 & 1 & 1 & 2 & o & 0 & 2 & 1 & $\mathbf{0}$ & 1 & 2 \\
\hline Hover & 4 & 3 & 20 & 27) & 2 & 2 & 18 & 23) & 0 & 7 & 18 & 25 & 1 & 4 & 23 & 20 & 3 & 4 & 15 & 22 & 1 & 2 & 14 & 17 \\
\hline Other & 3 & 0 & 3 & 6 & 4 & 1 & 22 & 27 & 0 & 0 & 1 & 1 & 4 & 6 & 57 & 67 & 6 & 6 & 55 & 67 & 9 & 3 & 42 & 54 \\
\hline Unknown & 13 & 2 & 1 & 16 & 14 & 1 & 3 & 18 & 13 & 1 & 2 & 16 & 17 & 0 & 2 & 19 & 18 & D & 3 & 21 & 13 & 1 & 1 & 15 \\
\hline Not reported & 1 & 0 & 1 & 2 & 7 & 0 & 2 & 9 & 2 & 0 & 4 & 6) & 1 & 0 & 0 & 1 & 6 & 1 & 12 & 19. & 9 & 0 & 10 & 19 \\
\hline Tot: & 476 & 292 & 753 & 521 & 432 & 264 & 1720 & 416 & 442 & 267 & 1601 & 310 & 430 & 226 & 1537 & 2193| & 443 & 217 & 1513 & 2173 & 428 & 243 & 1470 & 214 \\
\hline
\end{tabular}


Table 3.2 (Conitinued)

General Aviation Accldents by Phese of Filght Operation

Total Powered Aircraft

Poferences 3.20 10 3.27

\begin{tabular}{|c|c|c|c|c|c|c|c|c|c|c|c|c|c|c|c|}
\hline $\begin{array}{l}\text { Total Powered Alreratt } \\
\text { Phase of Operation }\end{array}$ & $\begin{array}{l}\text { Fatal } \\
\text { ACC. }\end{array}$ & \multicolumn{2}{|c|}{$\begin{array}{l}\text { Injury Pd. Dm } \\
\text { Aoc. Aoc. }\end{array}$} & $\begin{array}{l}1992 \\
\text { Total } \\
\text { Aoc. }\end{array}$ & $\begin{array}{l}\text { Fatal } \\
\text { Acc. }\end{array}$ & \multicolumn{2}{|c|}{ Injury Pd. Dm } & $\begin{array}{l}1993 \\
\text { Total } \\
\text { Acc. }\end{array}$ & $\begin{array}{l}\text { Fatal } \\
\text { Acc. }\end{array}$ & $\begin{array}{l}\text { Injury } \\
\text { Acc. }\end{array}$ & $\begin{array}{l}\text { Pr. Dm. } \\
\text { Acc. }\end{array}$ & $\begin{array}{l}\text { TOTAL } \\
\text { Total } \\
\text { Acc. } \\
\end{array}$ & $\begin{array}{l}\text { Flight } \\
\text { Phase }\end{array}$ & No. & Pct. \\
\hline Standing & 0 & 0 & 0 & 0 & 0 & 1 & 1 & 2 & 1 & 3 & 8 & 12 & & & \\
\hline Standing-preflight & 0 & 1 & 0 & 1 & 0 & 0 & 0 & 0 & 2 & 3 & 2 & 7 & & & \\
\hline Standing-starting angine(s) & $\mathbf{0}$ & 1 & 4 & 5 & 1 & 0 & 10 & 11 & 2 & 14 & 88 & 104 & & & \\
\hline Slanding-engine(s) operating & 2 & 1 & 3 & 6 & 3 & 4 & 4 & 11 & 13 & 19 & 58 & 90 & & & \\
\hline Standing-engine(s) not operating & 0 & 0 & 0 & o) & o & 0 & 1 & 1 & 0 & 2 & 3 & 5 & & & \\
\hline Standing idling rotors & $\underline{0}$ & $\mathbf{0}$ & 0 & of & 1 & 0 & 2 & 3 & 3 & 3 & $\mathbf{g}$ & 15 & Standing & 233 & $1.31 \%$ \\
\hline Taxi & 2 & 0 & 6 & 8 & 0 & 0 & 8 & 8 & 6 & 1 & 58 & 65 & & & \\
\hline Taxi-10 takeoff & $\mathbf{0}$ & 2 & 15 & 17) & 0 & 1 & 21 & 22 & 0 & 4 & 178 & 182 & & & \\
\hline Taxl-trom landing & 0 & 1 & 15 & 16 & 0 & 1 & 15 & 16 & $\mathbf{3}$ & $\mathbf{5}$ & 168 & 176 & & & \\
\hline Taxt-aerial & 1 & 0 & 5 & 6 & 0 & 1 & 4 & 5 & 1 & 2 & 27 & 30 & Taxi & 453 & $2.55 \%$ \\
\hline Takeoff & 5 & 3 & 19 & 27 & 5 & 4 & 18 & 27 & 56 & 50 & 205 & 311 & & & \\
\hline Takgolf-aborted & 0 & 0 & 0 & 0 & 0 & 0 & 0 & of & 0 & 1 & 15 & 16 & & & \\
\hline Takeolf-pround run & $\mathbf{0}$ & 6 & 75 & 81 & $\mathbf{3}$ & $\mathbf{0}$ & 74 & $\pi$ & 20 & 36 & 678 & 734 & Takooff & 1061 & $5.96 \%$ \\
\hline Takeolf-initial dimb & 57 & 47 & 169 & 273 & 53 & 49 & 178 & 280 & 485 & 471 & 1560 & 2516 & Initial Climb & 2516 & $14.14 x$ \\
\hline$\overline{C l i m b}$ & 9 & 1 & 14 & 24 & 10 & 3 & 20 & 33 & 74 & 35 & 120 & 229 & & & \\
\hline Climb-to cruise & 10 & 5 & 18 & 33 & $\mathbf{9}$ & 5 & 15 & 29 & 118 & 42 & 169 & 329 & Climb & $55 B$ & $3.14 \%_{0}$ \\
\hline Cruise & 57 & 18 & 93 & 168 & 52 & 28 & 117 & 197 & 386 & 157 & 818 & 1361 & & & \\
\hline Cruise-normal & 57 & 18 & 123 & 198 & 35 & 11 & 105 & 151 & 399 & 174 & 1135 & 1708 & & & \\
\hline Cruise-holding (IFP) & 0 & 0 & 0 & o & 0 & 0 & 0 & of & 1 & 0 & 7 & 8 & Cruise & 3077 & $17.28 \%$ \\
\hline Descent & 6 & 2 & 9 & 17 & 11 & 0 & 6 & 17) & 61 & 12 & 67 & 140 & & & \\
\hline Descent-normal & 12 & 2 & 28 & 42 & 4 & 0 & 26 & 30 & 57 & 29 & 215 & 301 & & & \\
\hline -mergency & 1 & 0 & 2 & 3 & 1 & 1 & 3 & 5 & 7 & 4 & 27 & 38 & & & \\
\hline Descent-uncontrolled & 1 & 3 & 3 & 7 & 1 & 1 & 4 & 6 & 17 & 7 & 18 & 42 & Descent & 521 & $2.80 \times$ \\
\hline Approach & 14 & 7 & 13 & 34 & 11 & 4 & 20 & 35 & 99 & 45 & 178 & 322 & & & \\
\hline Approach-VFR pattern-downwind & 4 & 1 & 15 & 20 & 4 & 3 & $\mathbf{B}$ & 15 & 50 & 23 & 102 & 175 & & & \\
\hline Approach-VFA pattern-base turn & 2 & 1 & 6 & g & 4 & 2 & 3 & 9 & 27 & 11 & 38 & 76 & & & \\
\hline Approach-VFF pattern-base to final & 6 & 3 & 7 & 16 & 7 & 0 & 11 & 18 & 61 & 19 & 74 & 154 & & & \\
\hline Approach-VFA pattern-final approach & 14 & 12 & 75 & 101 & 10 & 12 & 71 & 93. & 110 & 135 & 642 & 887 & & & \\
\hline Approach-go-around (VFR) & 7 & 3 & 25 & 35 & 4 & 6 & 38 & 48 & 47 & 57 & 274 & 378 & & & \\
\hline Approach-1AF to FAFlouter marker (IFR) & 4 & 0 & 2 & 6 & 1 & 1 & 1 & 3 & 39 & 8 & $\mathbf{9}$ & 56 & & & \\
\hline Approach-FAF/ouler marker to threshold & 10 & 2 & 5 & 17) & 4 & 2 & 7 & 13 & 65 & 23 & 46 & 134 & & & \\
\hline Approach-circling (IFR) & 1 & 1 & 1 & 3 & 3 & 0 & 1 & 4 & 14 & 4 & 7 & 25 & & & \\
\hline Approach-missed appro & 4 & 1 & 2 & 7 & 1 & 2 & 1 & 4 & 24 & 6 & 12 & 42 & Approach & 2249 & $12.64 x$ \\
\hline Landing-aborted & 0 & 0 & 0 & 0 & 0 & 0 & $\overline{0}$ & 0 & 3 & 4 & 19 & 26 & & & \\
\hline Landing & 4 & 3 & 38 & 45 & 0 & 5 & 39 & 44. & 16 & 26 & 298 & 340 & & & \\
\hline L anding-flare/louchoown & $\mathbf{3}$ & 7 & 178 & 188 & 5 & 4 & 148 & 157 & 22 & 53 & 1488 & 1563 & & & \\
\hline Landing-roll & $\mathbf{3}$ & 4 & 223 & 230 & $\underline{2}$ & 6 & 238 & 246 & 18 & 49 & 2056 & 2123 & Landing & 4052 & $22.77 \%$ \\
\hline Maneuvering & 98 & 20 & 62 & 180 & 109 & 29 & 57 & 195 & 811 & 196 & 503 & 1510 & & & \\
\hline Maner & 6 & 11 & 41 & 58 & 12 & 8 & 43 & 63 & 89 & 88 & 416 & 593 & & & \\
\hline Maneuvering-turn to reverse direction & 10 & 5 & 13 & 28 & 4 & 5 & 9 & 18 & 69 & 35 & 92 & 196 & & & \\
\hline Maneuvering-lurn to landing ares (emer. & 1 & 1 & 1 & 3] & 0 & D & 1 & 1 & 5 & 1 & 13 & 19 & & & \\
\hline Hover & 1 & 2 & 14 & 17 & 6 & 1 & 12 & 19 & 18 & 25 & 135 & 178 & Maneuvering & 2496 & $14.03 \%$ \\
\hline Other & 11 & 5 & 42 & 58 & 3 & 3 & 44 & 50 & 40 & 24 & 266 & 330 & & & \\
\hline Unknown & 14 & 0 & 7 & 21 & 15 & 3 & 1 & 19 & 117 & B & 20 & 145 & & & \\
\hline Not reported & 7 & 0 & 15 & 22 & 9 & 3 & 12 & 24 & 42 & 4 & 56 & 102 & Other/?/NR & 577 & $3.24 \%$ \\
\hline Total: & 444 & 200 & 386 & $2030 \mid$ & 403 & 209 & 1397 & 009 & 3498 & 918 & 12377 & 17793 & & 17793 & $100.00 \%$ \\
\hline
\end{tabular}




\section{Tablo 3.10}

General Aviation Aceldents by Phese of Filght Operation

Gilider:

References 3.20 to 3.27

\begin{tabular}{|c|c|c|c|c|c|c|c|c|c|c|c|c|c|c|c|c|c|c|c|c|c|c|c|c|c|}
\hline Phase of Operation & $\begin{array}{l}\text { Fatal } \\
A \subset C .\end{array}$ & $\begin{array}{l}\text { Injury P } \\
\text { Acc. }\end{array}$ & $\begin{array}{l}\text { Pr. Dm } \\
\text { Aoc. }\end{array}$ & $\begin{array}{l}1968 \\
\text { Total } \\
\text { Acc. } \\
\end{array}$ & $\begin{array}{l}\text { Fatal } \\
\text { Acc. }\end{array}$ & $\begin{array}{l}\text { Injury } \\
\text { Acc. }\end{array}$ & $\begin{array}{l}\text { Pr. Dm. } \\
\text { Acc. }\end{array}$ & $\begin{array}{l}1997 \\
\text { Total } \\
\text { Acc. }\end{array}$ & $\begin{array}{l}\text { Fatal } \\
\text { Acc. }\end{array}$ & $\begin{array}{l}\text { Injury } \\
\text { Aoc. }\end{array}$ & $\begin{array}{c}\text { Pr. Dm } \\
\text { Acc. }\end{array}$ & $\begin{array}{l}1988 \\
\text { Total } \\
\text { Acc. }\end{array}$ & $\begin{array}{l}\text { Fatal } \\
\text { Acc. }\end{array}$ & $\begin{array}{l}\text { Injury } \\
\text { Aoc. }\end{array}$ & $\begin{array}{l}\text { Pr. Dm. } \\
\text { Acc. }\end{array}$ & $\begin{array}{c}1989 \\
\text { Total } \\
A C C .\end{array}$ & $\begin{array}{l}\text { Fatal } \\
\text { Acc. }\end{array}$ & $\begin{array}{l}\text { Injury } \\
\text { Acc. }\end{array}$ & $\begin{array}{l}\text { Pr. Dm } \\
A \propto c .\end{array}$ & & $\begin{array}{l}1990 \\
\text { Total } \\
\text { Acc. }\end{array}$ & $\begin{array}{l}\text { Fatal } \\
\text { Aoc. }\end{array}$ & $\begin{array}{l}\text { Injury } \\
\text { AOC. }\end{array}$ & $\begin{array}{l}\text { Pr. Dm. } \\
\text { Acc. }\end{array}$ & $\begin{array}{l}1991 \\
\text { Total } \\
\text { Aac. }\end{array}$ \\
\hline Standing & $\mathbf{0}$ & 0 & 0 & 0 & 0 & 0 & 0 & o) & 0 & 0 & 0 & 0 & 0 & 0 & o & 0 & 0 & $\mathbf{0}$ & 0 & 0 & 0) & 0 & o & 0 & 0 \\
\hline Standing-preflight & $\mathbf{0}$ & 0 & 0 & 0 & 0 & 0 & o & 0 & 0 & 0 & 0 & of & 0 & 0 & 0 & 0 & 0 & o & o & 0 & of & 0 & 0 & 0 & 0 \\
\hline Standing-starting engine(s) & 0 & 0 & 0 & 0 & 0 & 0 & 0 & 0 & 0 & 0 & 0 & o) & $\mathbf{0}$ & 0 & 0 & of & 0 & $\mathbf{0}$ & 0 & 0 & 이 & 0 & 0 & 0 & 0) \\
\hline Standing-engine(3) operating & o & 0 & 0 & o & 0 & $\mathbf{0}$ & $\mathbf{0}$ & 0 & 0 & 0 & 0 & 0 & 0 & 0 & 0 & of & 0 & $\mathbf{0}$ & 0 & 0 & of & 0 & 0 & $\mathbf{0}$ & o \\
\hline Standing-engine(s) not operating & $\mathbf{0}$ & 0 & 0 & 0 & 0 & 0 & 0 & of & 0 & 0 & 0 & o & 0 & 0 & 0 & o) & 0 & $\mathbf{0}$ & 0 & 0 & of & 0 & 0 & $\mathbf{0}$ & 0 \\
\hline Standing-tolling rotors & 0 & 0 & 0 & of & 0 & $\underline{0}$ & 0 & of & 0 & 0 & 0 & of & 0 & 0 & 0 & of & 0 & 0 & 0 & $\underline{0}$ & 요 & 0 & 0 & 0 & 요 \\
\hline$\overline{T a x i}$ & 0 & 0 & 0 & 0 & 0 & 0 & 0 & 0 & 0 & 0 & 0 & 0 & 0 & 0 & 0 & 0 & 0 & 0 & $\overline{0}$ & 0 & 0 & 0 & $\overline{0}$ & 0 & 0 \\
\hline Taxd-to takeoff & o & 0 & 0 & 0 & $\mathbf{0}$ & 0 & $\mathbf{0}$ & 0 & 0 & 0 & 0 & o & 0 & 0 & $\mathbf{0}$ & of & 0 & 0 & 0 & 0 & of & 0 & 0 & 0 & o) \\
\hline Taxi-trom landing & $\mathbf{0}$ & 0 & 0 & 0 & $\mathbf{0}$ & 0 & 0 & o) & 0 & 0 & 0 & o] & 0 & o & o & o) & 0 & $\mathbf{0}$ & 0 & 0 & of & 0 & o & $\mathbf{0}$ & of \\
\hline Tax-arial & 0 & 0 & 0 & 0 & 0 & 0 & 0 & of & 0 & 0 & 0 & of & 0 & $\underline{0}$ & 0 & of & 0 & 0 & $\underline{0}$ & $\underline{0}$ & of & 0 & o & 0 & 0 \\
\hline Takeolf & 0 & 0 & 1 & 1 & 0 & $\overline{0}$ & 0 & 0 & 0 & 0 & 0 & 0) & 0 & 0 & 0 & 0 & 0 & 0 & 1 & 1 & 1 & 0 & $\overline{0}$ & 1 & 1 \\
\hline Takeolf-aborted & 0 & 0 & 0 & o & 0 & 0 & 0 & 0 & 0 & 0 & 0 & of & 0 & 0 & 0 & of & 0 & 0 & $\mathbf{0}$ & 0 & 이 & 0 & $\mathbf{0}$ & 0 & 이 \\
\hline Takeolf-ground run & 0 & 0 & 1 & 1 & 0 & 0 & 0 & of & 0 & 0 & 1 & 1 & 0 & 0 & 2 & 2 & 0 & 0 & 3 & 3 & 3 & 0 & 1 & 1 & 2 \\
\hline Takeofft-initial dimb & 2 & 1 & 10 & 13 & 0 & 1 & 4 & 5 & 2 & 2 & 4 & 8 & 0 & 1 & 3 & 4 & 0 & 3 & 3 & 3 & 6 & 1 & 1 & 2 & 4 \\
\hline Climb & 0 & 0 & 1 & 1 & 0 & 1 & 0 & 1 & 0 & 0 & 1 & 1 & 0 & 0 & 0 & o) & 0 & 0 & 0 & 0 & 0) & 0 & D & 0 & 이 \\
\hline Climb-to cruise & 0 & 0 & 0 & 0 & 0 & $\underline{0}$ & 0 & of & 0 & 0 & 0 & 의 & 0 & 0 & 0 & of & 0 & 0 & $\underline{0}$ & 0 & of & 0 & $\underline{0}$ & 0 & 0 \\
\hline Cruise & D & 0 & 0 & 0 & 0 & 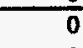 & 0 & 0 & 0 & 0 & 0 & 0 & 0 & 0 & $\overline{0}$ & 0 & 0 & $\overline{0}$ & $\overline{2}$ & 2 & 2 & 0 & $\overline{0}$ & $\overline{0}$ & 0 \\
\hline Cruise-normal & o & 1 & 3 & 4 & 0 & o & 2 & 2 & 2 & 0 & 1 & 3 & 0 & 0 & 2 & 2 & 0 & 0 & 0 & 0 & o & 0 & 0 & 0 & 0 \\
\hline Cruise-holding (IFR) & 0 & 0 & 0 & 0 & 0 & 0 & 0 & of & 0 & 0 & 0 & of & o & 0 & 0 & of & 0 & 0 & $\underline{0}$ & 0 & of & 0 & $\underline{0}$ & 0 & 의 \\
\hline Descent & D & 0 & 0 & 0 & 0 & 0 & 1 & 1 & 1 & 0 & 0 & 1 & 0 & 0 & 0 & 0 & 0 & o & 0 & 0 & 0 & 1 & 0 & 0 & 1 \\
\hline Descent-normal & 0 & 1 & 2 & 3 & 0 & 0 & 0 & of & 0 & 0 & 0 & o) & 0 & 0 & 0 & of & 0 & D & 1 & 1 & 1 & 1 & o & 1 & 2 \\
\hline Descent-emergency & 0 & 0 & 2 & 2 & 0 & 1 & 1 & 2 & 0 & $\mathbf{0}$ & 1 & 1 & 0 & 0 & 1 & 1 & 0 & o & 1 & 1 & 1 & 0 & 1 & 1 & 2 \\
\hline Descent-uncontrolled & $\mathbf{0}$ & 0 & 0 & 0 & $\mathbf{0}$ & $\mathbf{0}$ & 0 & of & 0 & 0 & 0 & of & 0 & 0 & 0 & of & 0 & $\underline{0}$ & $\underline{0}$ & 0 & of $1-1-1-1$ & 0 & $\mathbf{0}$ & $\mathbf{0}$ & 0 \\
\hline Approach & 0 & 1 & 1 & 2 & D & 0 & 1 & 1 & 0 & 0 & 0 & 0 & 0 & 0 & 0 & 0 & 0 & $\overline{0}$ & 7 & 1 & 1 & D & $\overline{0}$ & $\overline{3}$ & 3 \\
\hline Approach-VFR pattern-downwind & 0 & 1 & 0 & 1 & $\mathbf{0}$ & 0 & 0 & 0 & 0 & 0 & 1 & 1 & $\mathbf{0}$ & 1 & 1 & 2 & 0 & 0 & $\mathbf{0}$ & 0 & 0 & 0 & 0 & 0 & 0 \\
\hline Approach-VFA pattern-base turn & o & 1 & 0 & 1) & 0 & 0 & 0 & 0 & 1 & 0 & 0 & 1 & $\mathbf{0}$ & 0 & o & o) & 0 & 1 & o & 0 & 1 & $\mathbf{0}$ & 0 & 0 & 0 \\
\hline Approach-VFR pattern-base to final & 0 & 2 & 0 & $2]$ & 0 & 0 & 2 & 2 & 1 & 0 & 0 & 1 & 0 & 2 & 0 & 2 & 0 & $\mathbf{0}$ & 1 & 1 & 1 & 1 & 1 & 1 & 3 \\
\hline Approach-VFR pattern-final approach & 2 & 3 & 11 & 16 & $\mathbf{0}$ & 0 & 4 & 4 & 0 & 3 & 3 & 6 & 0 & 1 & 3 & 4 & 1 & 1 & 5 & 5 & 7 & 0 & 3 & 7 & 10 \\
\hline Approach-go-around (VFP) & D & 0 & 1 & 1 & $\mathbf{0}$ & 0 & 0 & of & 0 & 0 & 0 & 0 & 0 & 0 & o & of & 0 & 0 & 0 & 0 & of & o & 0 & 0 & 0 \\
\hline Approach-IAF to FAF/outer marker (IFR) & o & 0 & 0 & 0 & 0 & 0 & o & of & $\mathbf{0}$ & $\mathbf{0}$ & 0 & 0 & 0 & 0 & 0 & 0 & 0 & 0 & o & 0 & of & 0 & 0 & 0 & 0 \\
\hline Approach-FAF/outer marker to threshold & 0 & 0 & 0 & 0 & 0 & 0 & 0 & 0 & 0 & $\mathbf{0}$ & 0 & 0 & $\mathbf{0}$ & 0 & 0 & o) & 0 & 0 & 0 & 0 & o) & 0 & 0 & 0 & 0 \\
\hline Approach-circling (IFA) & 0 & 0 & 1 & 1 & $\mathbf{0}$ & 0 & 0 & of & $\mathbf{0}$ & 0 & 0 & 0 & 0 & 0 & 0 & 0 & 0 & 0 & 0 & 0 & of & 0 & o & $\mathbf{0}$ & 0 \\
\hline Approach-missed approach (IFA) & 0 & 0 & 0 & 0 & 0 & 0 & 0 & of & 0 & $\underline{0}$ & 0 & of & $\underline{0}$ & 0 & o & of & 0 & 0 & o & 0 & 의 & 0 & $\underline{0}$ & 0 & 0 \\
\hline Landing-aborted & $\overline{0}$ & 0 & 0 & 0 & 0 & $\overline{0}$ & 0 & of & 0 & 0 & 0 & 0 & 0 & 0 & 0 & 0 & 0 & o & $\overline{0}$ & 0 & 0 & 0 & $\overline{0}$ & 0 & 0 \\
\hline Landing & 0 & 0 & 4 & 4 & 0 & $\mathbf{0}$ & 1 & $1]$ & 0 & 1 & 2 & 3 & 0 & 0 & 0 & of & 0 & 1 & 1 & 1 & 2 & 0 & 1 & 1 & 2 \\
\hline Landing-flare/touchdown & 0 & 1 & 3 & 4 & 0 & 0 & 3 & 3) & 0 & 0 & 4 & 4 & 0 & 1 & 1 & 2 & 0 & 2 & 1 & 1 & 3] & 0 & 1 & 3 & 4 \\
\hline Landing-roll & 1 & 1 & 0 & 2 & 0 & 0 & 3 & 3 & 0 & 0 & 1 & 1 & 0 & 0 & 1 & 1 & 0 & $\mathbf{0}$ & 4 & 4 & 4 & 0 & $\underline{0}$ & 1 & 1 \\
\hline Maneuvering & 3 & 3 & 2 & 8 & 2 & 1 & 2 & 5 & 4 & 1 & 3 & 8 & $T$ & $\overline{0}$ & 1 & 2 & 3 & $\overline{0}$ & 2 & 2 & 5 & 1 & $\overline{1}$ & $\overline{3}$ & 5 \\
\hline Maneuvering-aerial application & 0 & 0 & 0 & 0 & 0 & 0 & 0 & o) & 0 & 0 & 0 & 0 & 0 & 0 & 0 & 0 & 0 & 0 & 0 & 0 & o) & 0 & 0 & 0 & 0 \\
\hline Maneuvering-turn to reverse direction & 1 & 0 & 0 & 1 & 1 & 1 & 1 & 3 & 1 & $\mathbf{0}$ & 3 & 4 & o & 0 & $\mathbf{0}$ & o) & 1 & 0 & 0 & 0 & 1 & 0 & 0 & 0 & 0 \\
\hline Maneuvering-turn to landing area (emer. & 0 & 0 & 0 & 0 & 0 & 1 & 0 & 1 & 0 & 0 & 0 & o & 0 & 0 & 1 & 1) & 0 & $\mathbf{0}$ & 1 & 1 & 1 & $\mathbf{0}$ & $\mathbf{0}$ & 1 & 1 \\
\hline Hover & 0 & 0 & 0 & 0 & $\mathbf{0}$ & 0 & 0 & 0. & 0 & 0 & 0 & 의 & 0 & 0 & 0 & 이 & 0 & 0 & $\underline{0}$ & 0 & D. & 0 & $\underline{0}$ & 0 & 0 \\
\hline Other & 0 & 0 & 0 & 0 & 0 & & 1 & 1 & 0 & 0 & 0 & 0 & 0 & 0 & 0 & of & 0 & $\overline{0}$ & $\overline{0}$ & 0 & 0 & 0 & $\overline{0}$ & 2 & 2 \\
\hline Unknown & 1 & 0 & 0 & 1 & 1 & 0 & $\mathbf{0}$ & 1 & 1 & 0 & 0 & 1 & 0 & 0 & $\mathbf{0}$ & 이 & 0 & 0 & 0 & 0 & o) & 0 & 0 & 0 & 0 \\
\hline Not reported & 0 & 0 & 0 & 0 & 0 & 0 & 0 & of & 0 & 0 & 0 & 0 & 0 & 0 & 0 & 0 & 0 & 0 & 0 & 0 & 0 & 0 & 0 & 0 & 0 \\
\hline Total: & 10 & 16 & 43 & 69 & 4 & 6 & 26 & 36 & 13 & 7 & 25 & 45 & 1 & 6 & 16 & 23 & 5 & $\overline{8}$ & $\overline{27}$ & 7 & 40 & 5 & 10 & 28 & 43 \\
\hline
\end{tabular}


Tablo 3.10 (Continued)

Generel Aviation Accidents by Phase of Filght Operation Gliders

Reterences 3.20 to 3.27

\begin{tabular}{|c|c|c|c|c|c|c|c|c|c|c|c|c|c|c|c|}
\hline Phase ol Operation & $\begin{array}{l}\text { Fatal } \\
\text { Aoc. }\end{array}$ & $\begin{array}{l}\text { Injury } \\
\text { Acc. }\end{array}$ & $\begin{array}{l}\text { Pr. Dm } \\
\text { Aoc. }\end{array}$ & $\begin{array}{l}1992 \\
\text { n. Tolal } \\
\text { Acc. } \\
\end{array}$ & $\begin{array}{l}\text { Fatal } \\
\text { Acc. }\end{array}$ & $\begin{array}{c}\text { Injury } \\
\text { Acc. }\end{array}$ & $\begin{array}{l}\text { Pr. Dm } \\
\text { Acc. }\end{array}$ & $\begin{array}{r}1993 \\
\text { Total } \\
\text { Acc. } \\
\end{array}$ & $\begin{array}{l}\text { Falal } \\
\text { Acc. }\end{array}$ & $\begin{array}{c}\text { Injury } \\
\text { Acc. }\end{array}$ & $\begin{array}{l}\text { Pr. Dm. } \\
\text { Acc. }\end{array}$ & $\begin{array}{c}\text { TotaL } \\
\text { Total } \\
\text { Acc. }\end{array}$ & $\begin{array}{l}\text { Flight } \\
\text { Phase }\end{array}$ & No. & Pct. \\
\hline Standing & 0 & 0 & 0 & 0 & 0 & 0 & 0 & 0 & 0 & 0 & 0 & 0 & & & \\
\hline Standing-preflight & 0 & 0 & 0 & 0 & 0 & 0 & $\mathbf{0}$ & 0 & o & 0 & 0 & 0) & & & \\
\hline Standing-starting enging(s) & 0 & 0 & 0 & 0 & 0 & 0 & $\mathbf{0}$ & 0 & 0 & $\mathbf{0}$ & 0 & 0] & & & \\
\hline Standing-engine(s) operating & 0 & 0 & 0 & 0 & $\mathbf{0}$ & 0 & o & 0 & 0 & 0 & 0 & o) & & & \\
\hline Standing-engine(s) not operating & 0 & 0 & 0 & 0 & 0 & 0 & 0 & 0 & 0 & 0 & 0 & 0 & & & \\
\hline Standing-idling rotors & 0 & 0 & 0 & 0 & o & $\underline{0}$ & 0 & $\underline{0}$ & $\underline{0}$ & $\mathbf{0}$ & 0 & of & Standing & 0 & $0.00 \%$ \\
\hline$\overline{T a x i}$ & $\mathbf{0}$ & 0 & 0 & 0 & $\mathbf{0}$ & $\overline{0}$ & 0 & $\overline{0}$ & $\overline{0}$ & 0 & 0 & 0 & & & \\
\hline Taxi-to takeoff & 0 & 0 & o & 0 & 0 & 0 & 0 & 0 & 0 & 0 & 0 & o) & & & \\
\hline Taxt-hom landing & 0 & 0 & 0 & 0 & 0 & 0 & 0 & 0 & o & 0 & $\mathbf{0}$ & of & & & \\
\hline Text-aerial & 0 & 0 & 0 & 0 & 0 & $\underline{0}$ & 0 & 0 & 0 & $\underline{0}$ & 0 & 이 & Tax & 0 & $0.00 \%$ \\
\hline Takeoff & 0 & 0 & 1 & 1 & 0 & 0 & 1 & 1 & 0 & 0 & 5 & 5 & & & \\
\hline Takeoff-aborted & 0 & 0 & $\mathbf{0}$ & o) & 0 & 0 & 0 & 0 & 0 & 0 & 0 & o & & & \\
\hline Takeoff-ground run & 0 & 0 & 5 & 5 & 0 & 1 & 0 & 1 & 0 & 2 & 13 & 15 & Takoolf & 20 & $6.10 \%_{0}$ \\
\hline Takeoff-initial climb & 2 & 2 & 3 & 7 & 0 & 2 & 3 & 5 & 7 & 13 & 32 & 52 & Initial Climb & 52 & $15.85 \%$ \\
\hline Climb & 0 & $\overline{0}$ & 0 & 0 & 0 & 0 & 0 & 0 & 0 & 1 & 2 & 3) & & & \\
\hline Climb-to cruise & 0 & 0 & 0 & 0 & $\underline{0}$ & $\underline{0}$ & 0 & 0 & 0 & 0 & $\mathbf{0}$ & 의 & Climb & 3 & $0.91 \%$ \\
\hline Cruise & $\overline{0}$ & 2 & 3 & 5 & $\overline{0}$ & $\overline{0}$ & $\overline{2}$ & 2 & $\overline{0}$ & $\overline{2}$ & 7 & 9 & & & \\
\hline Cruise-normal & 1 & 0 & 1 & 2 & 0 & 0 & 1 & 1 & 3 & 1 & 10 & 14 & & & \\
\hline Cruise-holding (IFP) & 0 & 0 & 0 & 0 & o & $\underline{\mathbf{0}}$ & 0 & 0 & 0 & 0 & 0 & of & Cruise & 23 & $7.01 \%$ \\
\hline Descont & 0 & $\overline{0}$ & $\overline{0}$ & $\overline{0}$ & 0 & 0 & 0 & 0 & 2 & 0 & 1 & 3) & & & \\
\hline Descent-normal & $\mathbf{0}$ & 0 & $\mathbf{0}$ & 0 & $\mathbf{0}$ & 0 & 0 & 0 & 1 & 1 & 4 & 6 & & & \\
\hline Descent-emergency & $\mathbf{0}$ & 0 & $\mathbf{0}$ & o & $\mathbf{0}$ & 0 & 0 & 0 & 0 & 2 & 7 & 9 & & & \\
\hline Descent-uncontrolled & $\mathbf{0}$ & 0 & 1 & 1 & 0 & 0 & 0 & 요 & 0 & $\underline{0}$ & 1 & 1 & Descent & 19 & $5.79 \%$ \\
\hline Approach & $\mathbf{0}$ & $\overline{0}$ & 0 & 0 & 0 & 0 & 1 & 1 & 0 & 1 & 7 & 8 & & & \\
\hline Approach-VFR pattern-downwind & $\mathbf{0}$ & 0 & $\mathbf{0}$ & 0 & 0 & 0 & 1 & 1 & $\mathbf{0}$ & 2 & 3 & 5 & & & \\
\hline Approach-VFA pattern-base turn & 0 & 1 & $\mathbf{0}$ & 1 & o & 0 & 0 & 0 & 1 & 3 & $\mathbf{0}$ & 4 & & & \\
\hline Approach-VFR pattern-base to final & $\mathbf{0}$ & 0 & 1 & 1 & 0 & 0 & 1 & 1 & 2 & 5 & 6 & 13 & & & \\
\hline Approach-VFA pattern-final approach & 1 & 3 & 4 & 8 & 0 & 1 & 5 & 6 & 4 & 15 & 42 & 61 & & & \\
\hline Approach-go-around (VFA) & 0 & 0 & 0 & 0 & 0 & 0 & 1 & 1 & 0 & 0 & 2 & 2 & & & \\
\hline Approach-IAF to FAF/outer marker (IFA) & $\mathbf{0}$ & 0 & $\mathbf{0}$ & 0 & $\mathbf{0}$ & 0 & 0 & 0 & 0 & 0 & 0 & 0) & & & \\
\hline Approach.FAF/outer marker to threshold & 0 & 0 & 0 & 0 & 0 & 0 & $\mathbf{0}$ & 0 & 0 & 0 & 0 & 0 & & & \\
\hline Approach-circling (IFA) & $\mathbf{0}$ & 0 & $\mathbf{0}$ & 0 & 0 & 0 & 0 & 0 & 0 & 0 & 1 & 1 & & & \\
\hline Approach-missed approach (IFA) & 0 & 0 & 0 & o & 0 & $\underline{0}$ & 0 & 0 & 0 & 0 & 0 & 요 & Approach & 84 & $28.66 \%$ \\
\hline Landing-abortad & 0 & $\overline{0}$ & $\overline{0}$ & $\overline{0}$ & $\overline{0}$ & $\overline{0}$ & 0 & $\overline{0}$ & 0 & $\overline{0}$ & 0 & 0 & & & \\
\hline Landing & 0 & 1 & $i$ & 2 & 0 & 0 & 0 & 0 & 0 & 4 & 10 & 14 & & & \\
\hline Landing-flarehouchdown & 0 & 0 & 2 & 2 & 0 & 0 & 1 & 1 & 0 & $\mathbf{5}$ & 18 & 23 & & & \\
\hline Landing-roll & 0 & 0 & 2 & 2 & $\underline{0}$ & $\underline{0}$ & 3 & 3 & 1 & 1 & 15 & 17 & Landing & 54 & $16.46 \%$ \\
\hline Maneuvering & 4 & 0 & 3 & 7 & $\overline{0}$ & $\overline{0}$ & 2 & 2 & 18 & $\overline{6}$ & 18 & 42 & & & \\
\hline Maneuvering-aerial application & 0 & 0 & $\mathbf{0}$ & 0 & 0 & 0 & 0 & 0 & 0 & 0 & 0 & o) & & & \\
\hline Maneuvering-turn to reverse direction & 0 & 0 & 0 & 0 & 0 & 0 & o & 0 & 4 & 1 & 4 & 9 & & & \\
\hline Manouvering-furn to landing ares (emer. & 0 & 0 & 0 & 0 & 0 & 1 & 1 & 2 & 0 & 2 & 4 & 6 & & & \\
\hline Hover & $\underline{0}$ & 0 & 0 & o & 0 & $\underline{0}$ & o & 0 & 0 & 0 & 0 & of & Maneuvering & 57 & $17.38 \%$ \\
\hline Other & 0 & 0 & 0 & 0 & 0 & 0 & 0 & $\overline{0}$ & 0 & 0 & 3 & 3 & & & \\
\hline Unknown & 0 & 0 & 0 & 0 & 0 & 0 & 0 & 0 & 3 & 0 & 0 & 3 & & & \\
\hline Not reported & 0 & 0 & 0 & 0 & 0 & 0 & 0 & 0 & 0 & 0 & 0 & o & Other/?NR & 6 & $1.83 \%$ \\
\hline Total & 8 & 9 & 27 & $44 \mid$ & 0 & 5 & $\overline{23}$ & 28 & 46 & 67 & 215 & 328 & & 328 & 0.00 \\
\hline
\end{tabular}


Batloons

Reterences 3.20 to 3.27

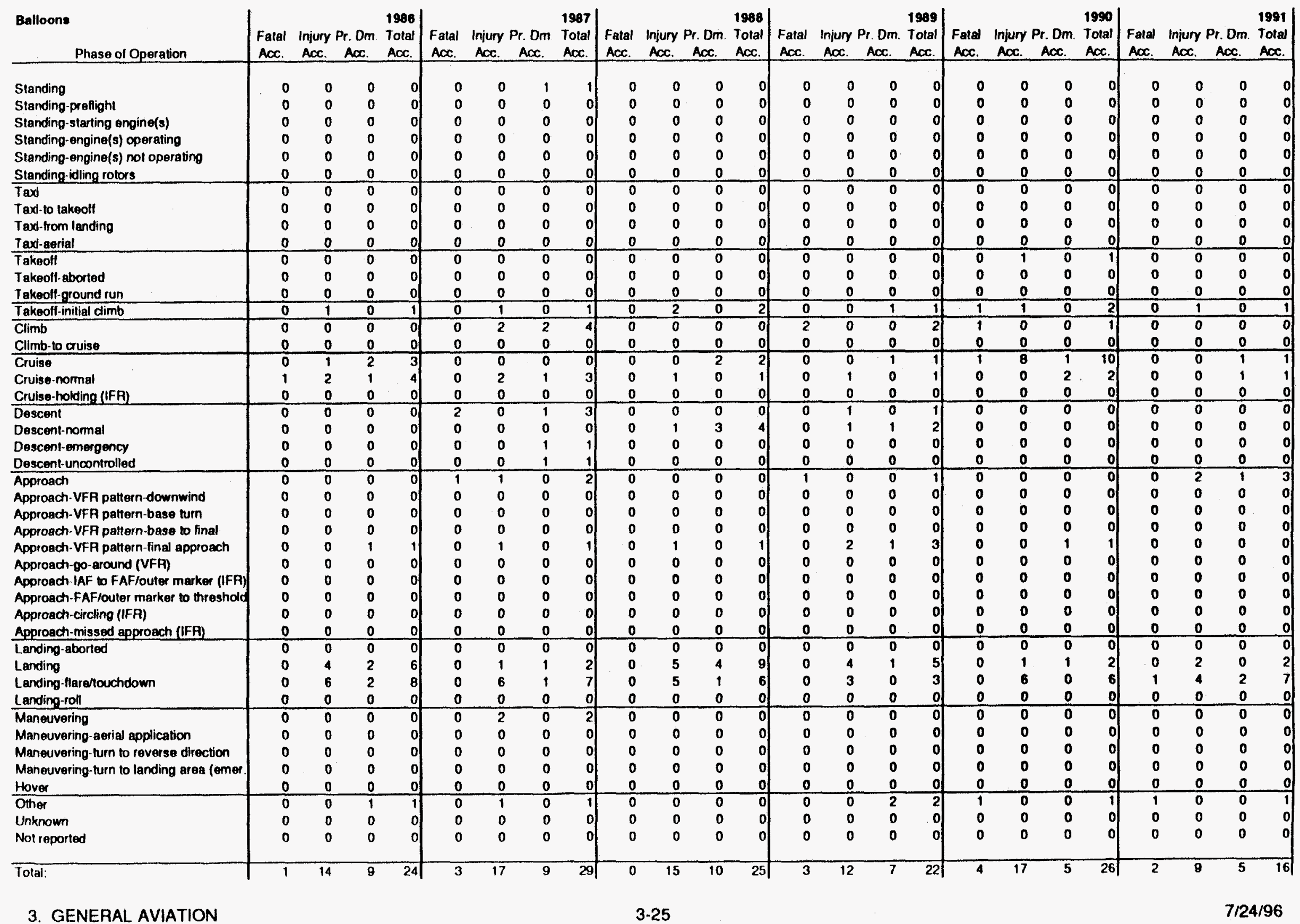




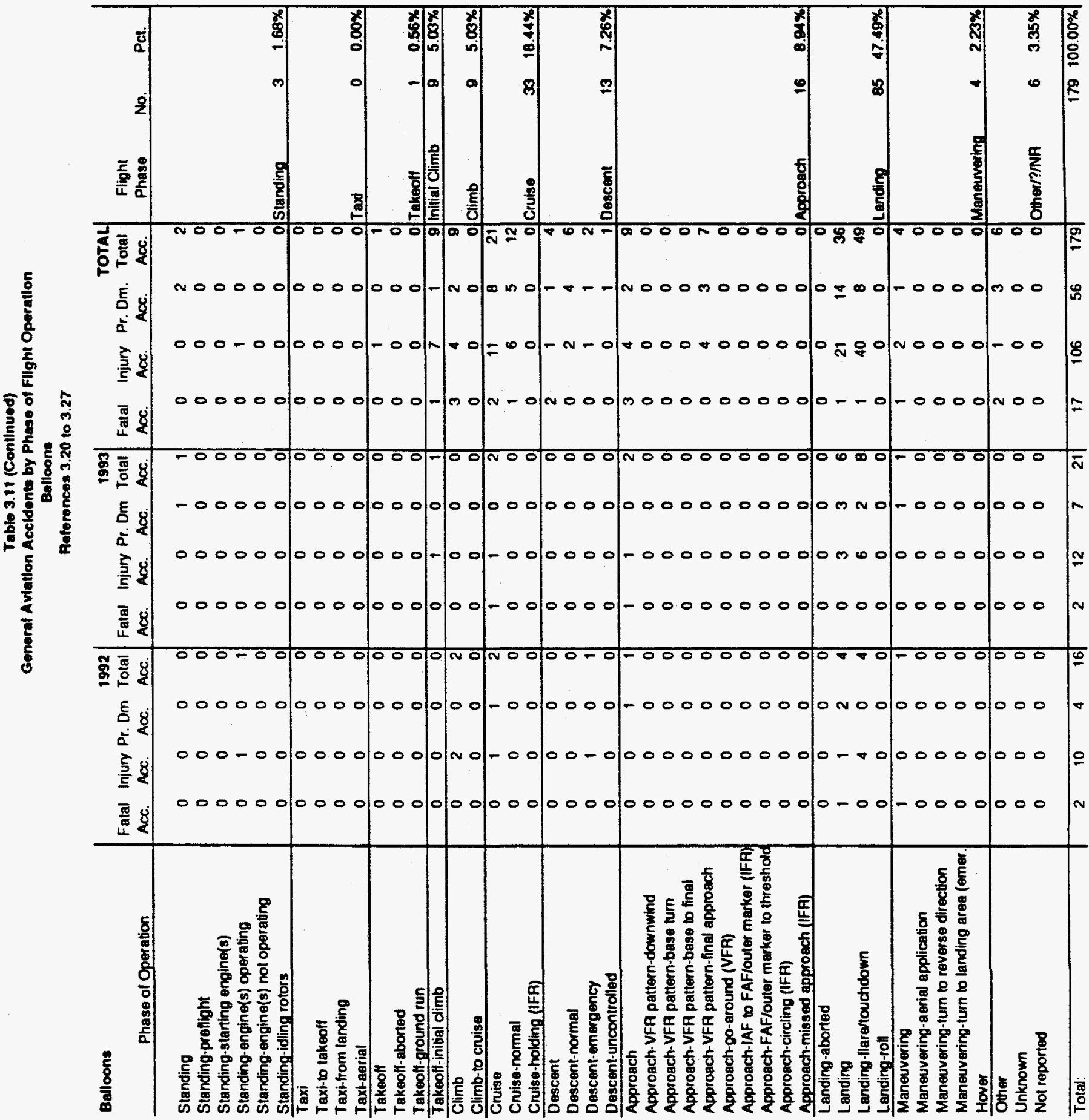


Table 3.12

General Aviation Accidents by Phase of Filght Operation

Other Alrcraft

Rotorences 3.20 to 3.27

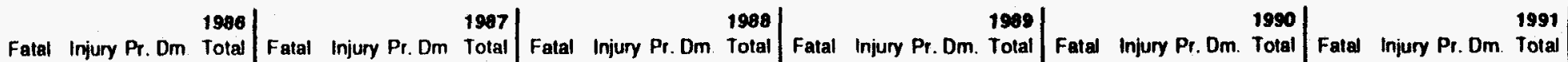

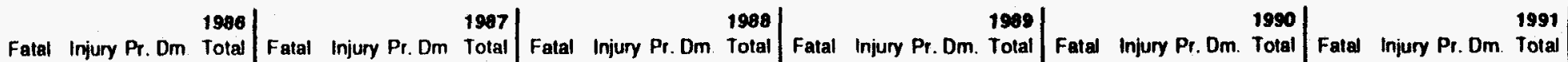

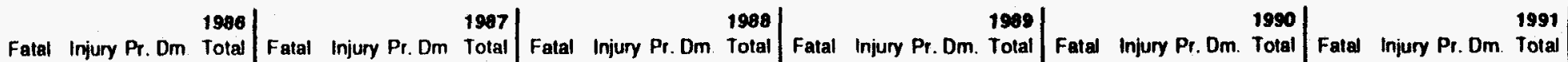
Phase of Operation

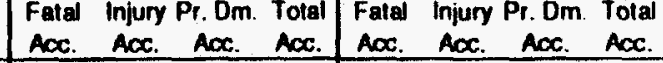
Standing

Standing-preffight

Standing-starting engine(s)

Standing-engine(s) operating

Standing-engine(s) not operating

Standing-idling rotors

Taxi

Taxi-to takeoff

Taxi-hom landing

Taxi-geria!

Takeoff

Takeofl-aborted

Takooll-oround run

Tokeofl-initial climb

Climb

Climb-to cruise

Cruise

Cruise-normal

Cruise-holding (IFA)

Descant

Descent-normal

Descent-emergency

Descent-uncontrolled

Approach

Approact-VFA pattern-downwind

Approach-VFA pattern-base turn

Approach-VFA paftern-base to final

Approach-VFA pattern-linal approach

Approact-go-around (VFP)

Approm-IAF to FAF/outer marker (IFP)

Approach-FAF/outer marker to threshold

Approach-circling (IFP)

Approach-missed approach (IFA)

Landing-aborted

Landing

Landing-Hlare/touchdown

L.anding-roll

Maneuvering

Manouvering-aerial application

Maneuvering-turn to reverse direction

Maneuvering-turn to landing area (emer.

Hover

Unknown

Not reported

Total:

\begin{tabular}{llll|llll|llllllll|}
0 & 0 & 0 & 0 & 0 & 0 & 0 & 0 & 0 & 0 & 0 & 0 & 0 & 0 & 0 & 0 \\
0 & 0 & 0 & 0 & 0 & 0 & 0 & 0 & 0 & 0 & 0 & 0 & 0 & 0 & 0 & 0 \\
0 & 0 & 0 & 0 & 0 & 0 & 0 & 0 & 0 & 0 & 0 & 0 & 0 & 0 & 0 & 0 \\
0 & 0 & 0 & 0 & 0 & 0 & 0 & 0 & 0 & 0 & 0 & 0 & 0 & 0 & 0 & 0 \\
0 & 0 & 0 & 0 & 0 & 0 & 0 & 0 & 0 & 0 & 0 & 0 & 0 & 0 & 0 & 0 \\
0 & 0 & 0 & 0 & 0 & 0 & 0 & 0 & 0 & 0 & 0 & 0 & 0 & 0 & 0 & 0 \\
\hline & 0 & 0 & 0 & 0 & 0 & 0 & 0 & 0 & 0 & 0 & 0 & 0 & 0 & 0 & 0
\end{tabular}

\begin{tabular}{llll|lll}
0 & 0 & 0 & 0 & 0 & 0 & 0 \\
0 & 0 & 0 & 0 & 0 & 0 & 0 \\
0 & 0 & 0 & 0 & 0 & 0 & 0 \\
0 & 0 & 0 & 0 & 0 & 0 & 0 \\
0 & 0 & 0 & 0 & 0 & 0 & 0 \\
0 & 0 & 0 & 0 & 0 & 0 & 0
\end{tabular}

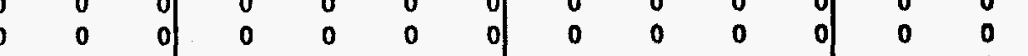

\begin{tabular}{llll|l}
0 & 0 & 0 & 0 & 0 \\
0 & 0 & 0 & 0 & 0
\end{tabular}

\begin{tabular}{ll|ll}
0 & 0 & 0 & 0 \\
0 & 0 & 0 & 0
\end{tabular}

- 1

\begin{tabular}{l|llll|llll|}
0 & 0 & 1 & 0 & 1 & 0 & 0 & 0 & 0 \\
0 & 0 & 0 & 0 & 0 & 0 & 0 & 0 & 0 \\
0 & 0 & 0 & 0 & 0 & 0 & 0 & 0 & 0 \\
0 & 0 & 0 & 0 & 0 & 0 & 0 & 0 & 0 \\
0 & 0 & 0 & 0 & 0 & 0 & 0 & 0 & 0 \\
0 & 0 & 0 & 0 & 0 & 0 & 0 & 0 & 0
\end{tabular}

0
0

\begin{tabular}{|c|c|c|c|c|c|c|c|c|c|}
\hline 0 & 0 & 0 & 0 & 0 & 0 & 0 & 0 & 0 & \\
\hline 0 & 0 & 0 & 0 & $\overline{0}$ & 0 & 0 & 0 & 0 & \\
\hline 0 & 0 & 0 & 0 & 0 & 0 & 0 & 0 & 0 & \\
\hline 0 & 0 & 0 & 0 & 0 & 0 & 0 & of & 0 & 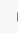 \\
\hline 0 & 0 & 0 & 0 & 0 & 0 & 0 & 0 & 0 & \\
\hline 0 & 1 & 0 & $\overline{1}$ & 0 & 0 & 0 & 0 & 0 & 7 \\
\hline
\end{tabular}

\begin{tabular}{lll}
0 & 0 \\
0 & 0 \\
\hline 0 & 0 & 0 \\
\hline
\end{tabular}

\begin{tabular}{l|llll}
0 & 0 & 1 & 0 & 1 \\
0 & 0 & 0 & 0 & 0 \\
0 & 0 & 0 & 0 & 0 \\
0 & 0 & 0 & 0 & 0 \\
0 & 0 & 0 & 0 & 0 \\
0 & 0 & 0 & 0 \\
0 & 0 & 0 & 0 & 0 \\
0 & 0 & 0 & 0 & 0 \\
0 & 0 & 0 & 0 & 0 \\
0 & 0 & 0 & 0 & 0 \\
\hline 0 & 0 & 0 & 0 & 0
\end{tabular}

\begin{tabular}{ll}
0 & 0 \\
0 & 0 \\
0 & 0 \\
0 & 0 \\
0 & 0 \\
0 & 0 \\
0 & 0 \\
0 & 0 \\
0 & 0 \\
0 & 0 \\
\hline 0 & 0
\end{tabular}

\begin{tabular}{|llll|llll|ll}
0 & 1 & 0 & 1 & 0 & 0 & 0 & 0 & 0 & 0 \\
0 & 0 & 0 & 0 & 0 & 0 & 0 & 0 & 0 & 0 \\
0 & 0 & 0 & 0 & 0 & 0 & 0 & 0 & 0 & 0 \\
\hline & 1 & 0 & 2 & 0 & 1 & 1 & 2 & 1 & 0
\end{tabular}

\begin{tabular}{lll}
0 & 0 \\
0 & 0 \\
\hline 0 & 0 & 0 \\
0 & 0 & 0
\end{tabular}

ner.

\begin{tabular}{l|llll|llll|}
0 & 1 & 1 & 0 & 2 & 0 & 1 & 1 & 2 \\
0 & 0 & 0 & 0 & 0 & 0 & 0 & 0 & 0 \\
0 & 0 & 0 & 0 & 0 & 0 & 0 & 0 & 0 \\
0 & 0 & 0 & 0 & 0 & 0 & 0 & 0 & 0 \\
0 & 0 & 0 & 0 & 0 & 0 & 0 & 0 & 0 \\
\hline 0 & 0 & 0 & 0 & 0 & 0 & 0 & 0 & 0 \\
0 & 0 & 0 & 0 & 0 & 0 & 0 & 0 & 0 \\
0 & 0 & 0 & 0 & 0 & 0 & 0 & 1 & 1 \\
\hline 0 & 1 & 4 & 0 & 5 & 0 & 1 & 2 & 3
\end{tabular}

\begin{tabular}{ll|l}
0 & 0 & 0 \\
\hline 0 & 0 & 0
\end{tabular}

0
0
0

0
0
0
0

0

$\begin{array}{lll}0 & 0 & 0 \\ 0 & 0 & 0\end{array}$

3. GENERAL AVIATION 
Table 3.12 (Continued)

Genoral Avlation Accidents by Phese of Flight Operation

Other Alrcraft

Roterences 3.20 to 3.27

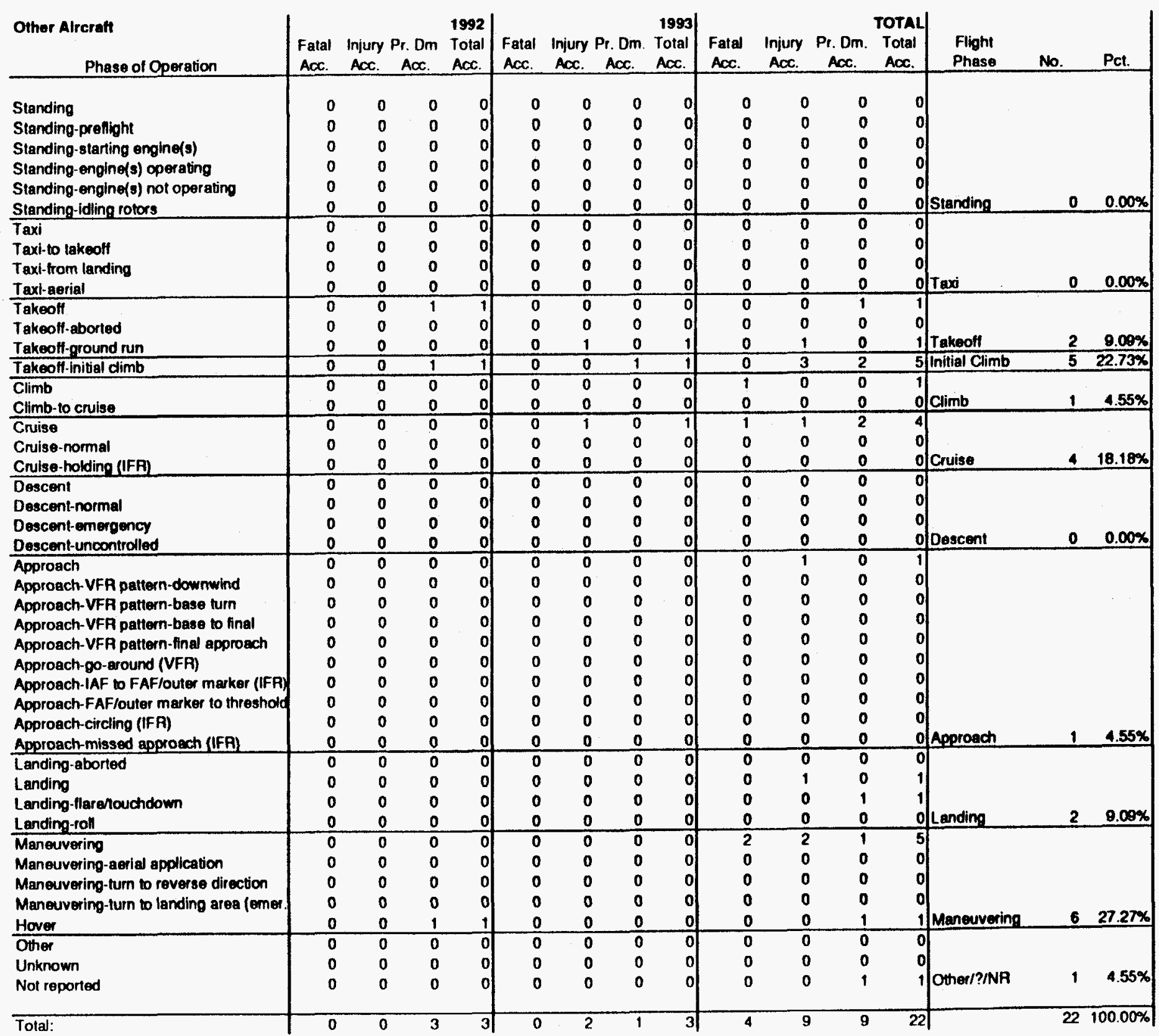


General Avlation Accidents by Phase of Filght Operation Total Alreraft, Aoforences 3.20 to 3.27

Total Alrcraft

Phase of Operation

Standing

Standing-prefight

Standing-starting engine(s)

Standing-engine(s) oper ating

Standing-engine(s) not operating

Standing-idling rotors

Taxi-to takeoff

Taxi-from landing

Taxj-earial

Takeoft

Takeoll-aborted

Takeoll-ground run

Takboff-initial dimb

Climb

Climb-to auise

Cruise

Cruise-normal

Cruise-holding (IFP)

Descent-normal

Descent-emergency

Descent-uncontrolled

Approech

Approach. VFR pattern-downwind

Approach.VFA pattern-base turn

Approach. VFP pattern-base to fing

Approach-VFR pattern-final approach

Approach-go-arcund (VFR)

Approach-IAF to FAFlouter marker (IFR)

Approach. FAF/outer marker to threshol

Approsch-circling (IFR)

Approach-missed approach (IFA)

Landing-aborted

Landing

Landing flarehouchdown

Landing-roll

Maneuvering-aerial application

Maneuvering-turn to reverse direction

Maneuvering-turn to landing erea (emer

Hover

Other

Unknown

Not reported

Total:

Aircralt Damage

1986

1987

1988

1989

Fatal Injury Pr. Dm Total Fatal Injury Pr. Dm Total Fatal Injury Pr. Dm Total Fatal Injury Pr. Dm. Total Fatal Injury Pr. Dm. Total Fatal Injury Pr. Dm Tota ACC. ACC. ACC. ACC. ACC. ACC. ACC. ACC. ACC. ACC. ACC. ACC. ACC. ACC. ACC. ACC. ACC. ACC. ACC. AOC. AOC. ACC. ACC. ACE.

$\begin{array}{llllllllllllllllllllllllllllll}1 & 0 & 1 & 2 & 0 & 0 & 1 & 1 & 0 & 0 & 3 & 3 & 0 & 0 & 1 & 1 & 0 & 2 & 0 & 2 & 0 & 0 & 2 & \end{array}$

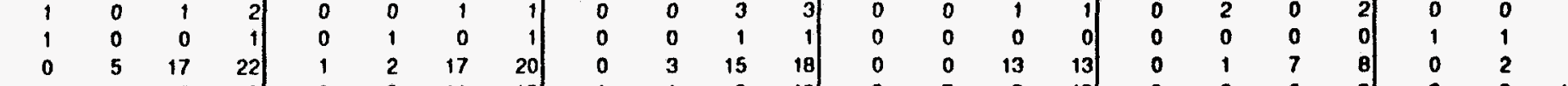

\begin{tabular}{rrrr|rrrr|rrrr}
1 & 0 & 7 & 8 & 2 & 2 & 11 & 15 & 1 & 1 & 8 & 10 \\
0 & 0 & 0 & 0 & 0 & 2 & 1 & 3 & 0 & 0 & 0 & 0 \\
1 & 0 & 1 & 2 & 0 & 0 & 0 & 0 & 0 & 1 & 1 & 2 \\
\hline
\end{tabular}

\begin{tabular}{rrrr|r}
1 & 0 & 1 & 2 & 0 \\
1 & 0 & 13 & 14 & 0 \\
0 & 0 & 21 & 21 \\
1 & 1 & 18 & 20 \\
0 & 0 & 2 & 2 & 0
\end{tabular}

$\begin{array}{rrr}0 & 32 & 32 \\ 0 & 28 & 28 \\ 0 & 2 & 2\end{array}$

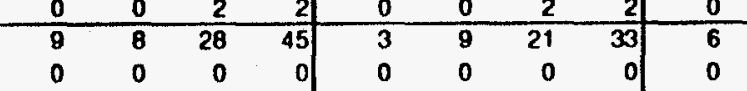

$\begin{array}{lll}1 & 24 & 25 \\ 0 & 31 & 32\end{array}$

$\begin{array}{llll}0 & 0 & 2 & 2 \\ 0 & 0 & 5 & 5\end{array}$

\begin{tabular}{rrrrrrrrrrrrrrr|r|r|r|}
5 & 6 & 108 & 119 & 2 & 10 & 113 & 125 & 1 & 2 & 78 & 81 & 2 & 2 \\
\hline 71 & 60 & 242 & 373 & 50 & 73 & 224 & 347 & 73 & 73 & 191 & 337 & 63 & 60 & 260 \\
21 & 6 & 21 & 39 & 7 & 7 & 14 & 28 & 5 & 4 & 16 & 25 & 12 & 2 \\
21 & 5 & 27 & 53 & 14 & 8 & 30 & 52 & 26 & 5 & 26 & 57 & 12 & 2 \\
\hline
\end{tabular}

\begin{tabular}{|c|c|c|c|c|c|c|c|c|c|c|c|c|c|c|c|}
\hline 21 & 5 & 27 & 53 & 14 & 8 & 30 & 52 & 26 & 5 & 26 & 57 & 12 & 2 & 15 & 29 \\
\hline 41 & 20 & 117 & 178 & 38 & 11 & 84 & 133 & 44 & 18 & 93 & 155 & 55 & 21 & 109 & 185 \\
\hline 48 & 40 & 182 & 270 & 64 & 21 & 161 & 246 & 65 & 32 & 165 & 262 & 61 & 21 & 148 & 230 \\
\hline 0 & 0 & 1 & 1 & 0 & 0 & $\underline{0}$ & & 1 & 0 & 3 & & 0 & 0 & 2 & 2 \\
\hline
\end{tabular}

\begin{tabular}{rrrrrrr|rrrrrrrr}
0 & 0 & 1 & 1 & 0 & 0 & 0 & 0 & 1 & 0 & 3 & 4 & 0 & 0 & 2 \\
\hline 14 & 1 & 11 & 26 & 6 & 1 & 8 & 15 & 10 & 1 & 11 & 22 & 9 & 2 & 6 \\
10 & 8 & 29 & 47 & 8 & 6 & 30 & 44 & 5 & 2 & 42 & 49 & 5 & 3 & 22 \\
2 & 2 & 9 & 13 & 0 & 1 & 8 & 9 & 1 & 0 & 2 & 3 & 0 & 0 & 3 \\
3 & 1 & 1 & 5 & 5 & 0 & 4 & 9 & 0 & 1 & 0 & 1 & 1 & 0 & 2 \\
\hline 12 & 8 & 24 & 44 & 7 & 8 & 24 & 39 & 11 & 7 & 24 & 42 & 17 & 7 & 36
\end{tabular}

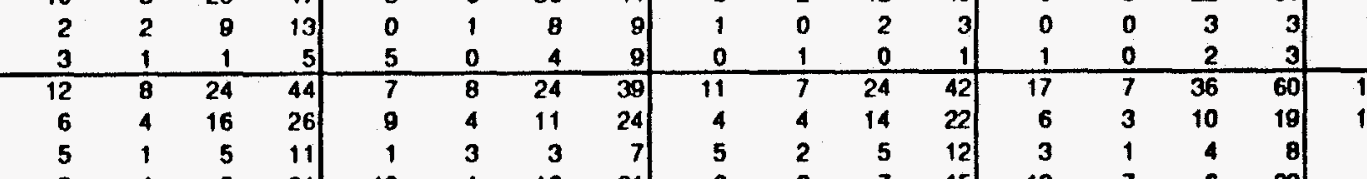

\begin{tabular}{rrrr|rrrr|rrrr|rrr}
6 & 4 & 16 & 26 & 9 & 4 & 11 & 24 & 4 & 4 & 14 & 22 & 6 & 3 & 10 \\
5 & 1 & 5 & 11 & 1 & 3 & 3 & 7 & 5 & 2 & 5 & 12 & 3 & 1 & 4 \\
9 & 4 & 6 & 21 & 10 & 1 & 10 & 21 & 6 & 2 & 7 & 15 & 10 & 7 & 6 \\
16 & 30 & 100 & 149 & 12 & 20 & 97 & 129 & 8 & 29 & 78 & 115 & 15 & 20 & 86 \\
4 & 9 & 47 & 60 & 6 & 11 & 32 & 49 & 8 & 7 & 39 & 54 & 4 & 5 & 30 \\
3 & 3 & 1 & 7 & 5 & 0 & 0 & 5 & 8 & 1 & 2 & 11 & 8 & 1 & 0 \\
18 & 6 & 6 & 32 & 7 & 4 & 5 & 16 & 7 & 5 & 5 & 17 & 4 & 0 & 4 \\
2 & 0 & 3 & 5 & 3 & 0 & 0 & 3 & 3 & 1 & 2 & 6 & 0 & 0 & 1 \\
2 & 2 & 4 & 8 & 4 & 0 & 1 & 5 & 5 & 0 & 2 & 7 & 1 & 0 & 0 \\
\hline 0 & 0 & 0 & 0 & 0 & 0 & 0 & 0 & 3 & 4 & 19 & 26 & 0 & 0 & 0
\end{tabular}

\begin{tabular}{|c|c|c|c|c|}
\hline 2 & 2 & & 8 & \\
\hline 0 & 0 & & 0 & \\
\hline 4 & 11 & 5 & 65 & \\
\hline 3 & 16 & 22 & 243 & \\
\hline 8 & 7 & 29 & 305 & \\
\hline
\end{tabular}

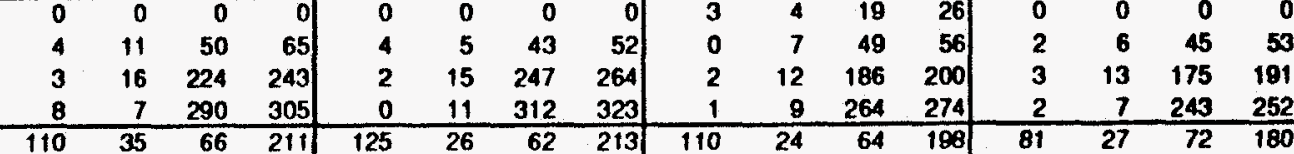

\begin{tabular}{rrrr|rrrr|rrrrrrrrr}
110 & 35 & 66 & 211 & 125 & 26 & 62 & 213 & 110 & 24 & 64 & 198 & 81 & 27 & 72 \\
10 & 11 & 56 & 77 & 9 & 14 & 56 & 79 & 7 & 14 & 58 & 79 & 21 & 10 & 47 \\
11 & 4 & 19 & 34 & 7 & 9 & 11 & 27 & 11 & 2 & 18 & 31 & 9 & 3 & 3 \\
0 & 0 & 2 & 2 & 1 & 1 & 0 & 2 & 0 & 0 & 7 & 7 & 0 & 0 & 2 \\
4 & 3 & 20 & 27 & 2 & 2 & 19 & 23 & 0 & 7 & 18 & 25 & 1 & 4 & 23 \\
\hline
\end{tabular}

1

\begin{tabular}{l|lll}
1 & 1 & 0 & 0 \\
1 & 4 & 0 & 1 \\
\hline 0 & 0 & 0 & 0
\end{tabular}

\begin{tabular}{rrrr}
0 & 0 & 0 & 0 \\
1 & 4 & 30 & 38 \\
4 & 14 & 167 & 185 \\
2 & 6 & 262 & 270 \\
\hline 8 & 14 & 63 & 176
\end{tabular}

3. GENERAL AVIATION

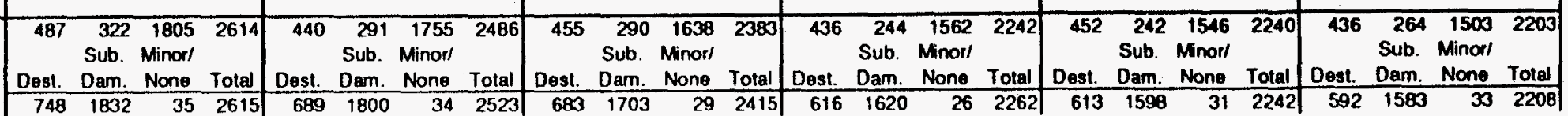

\begin{tabular}{rrrr|lllll|r}
748 & 1832 & 35 & 2615 & 689 & 1800 & 34 & 2523 & 683 \\
& & & & & & & & $3-29$
\end{tabular}

$7 / 24 / 96$ 
Tabb 3.13 (Conilnued)

General Aviation Aceldents by Phass of Fllght Operation

Totel Aircraft, Reterences 3.20 to 3.27

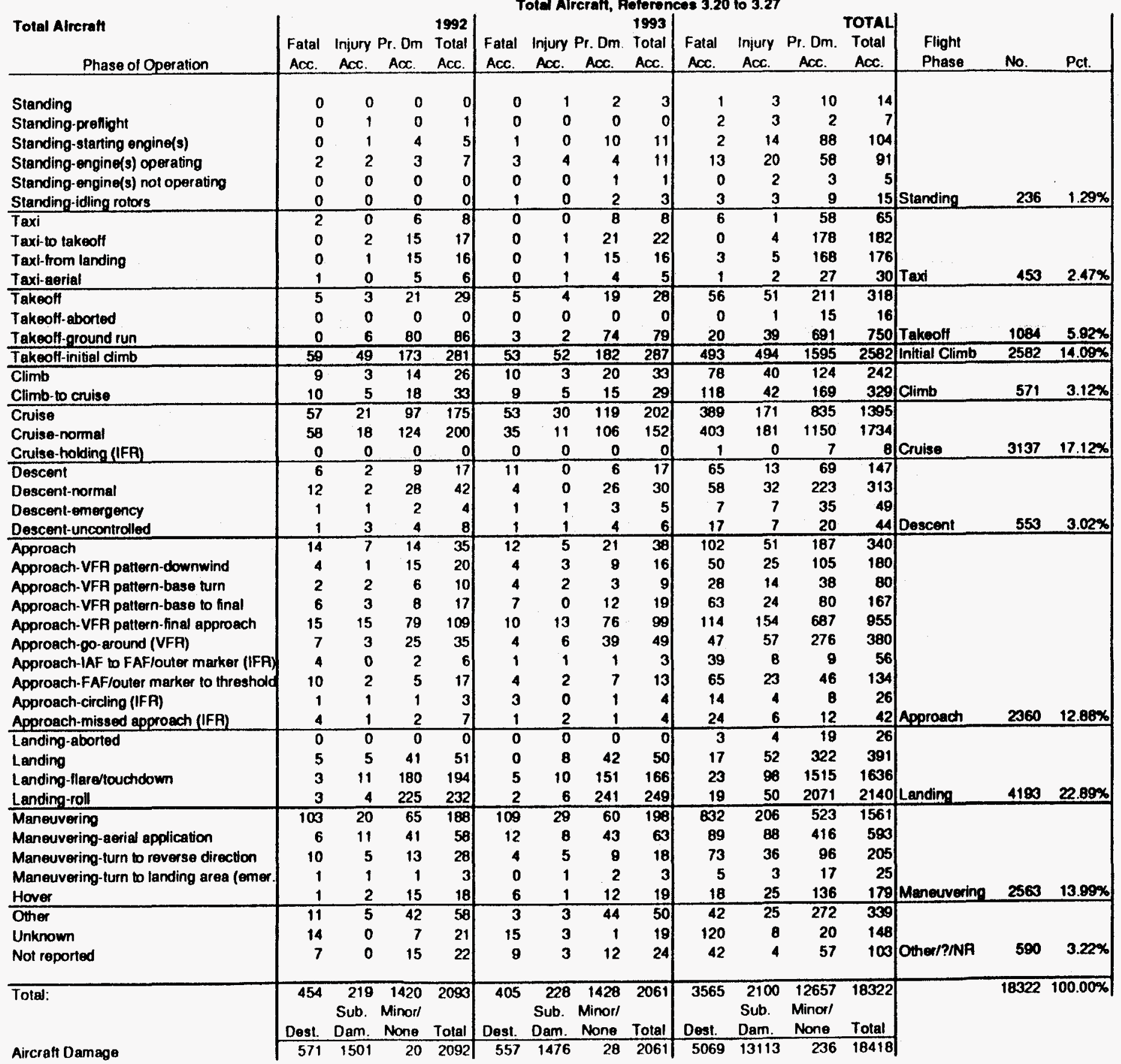

Aircraft Damage 
Table 3.14

General Aviation Accidents, 1986-1993

by Flight Phases and by Aircraft Subcategories Summary

References 3.20 - 3.27

\begin{tabular}{|c|c|c|c|c|c|c|c|c|c|c|c|c|c|c|c|c|c|c|c|c|c|c|c|c|}
\hline Aircraft Subcategories & $\begin{array}{c}\text { Sandin } \\
\text { No. }\end{array}$ & PCL & $\begin{array}{r}\text { Taxi } \\
\text { No. } \\
\end{array}$ & Pct & $\begin{array}{c}\text { Takeoff } \\
\text { No. }\end{array}$ & Pct. & $\begin{array}{c}\text { Initial C } \\
\text { No. }\end{array}$ & $\begin{array}{c}\text { Climb } \\
\mathrm{Pcl}\end{array}$ & \begin{tabular}{|c} 
Climb \\
No.
\end{tabular} & Pct. & $\begin{array}{c}\text { Cruise } \\
\text { No. }\end{array}$ & Pct & $\begin{array}{c}\text { Descent } \\
\text { No. }\end{array}$ & $\mathrm{Pct}$ & $\begin{array}{c}\text { Approe } \\
\text { No. }\end{array}$ & $\begin{array}{l}\text { Pct } \\
\end{array}$ & $\begin{array}{l}\text { Landing } \\
\text { No. }\end{array}$ & $8 \mathrm{Pal}$ & $\begin{array}{c}\text { Manue } \\
\text { No. }\end{array}$ & $\begin{array}{r}\text { vering } \\
\text { Pct } \\
\end{array}$ & $\begin{array}{l}\text { Other? } \\
\text { No. }\end{array}$ & $\begin{array}{c}\mathrm{NA} \\
\mathrm{PcL} \\
\end{array}$ & \begin{tabular}{|c} 
Total \\
No.
\end{tabular} & PcL \\
\hline Single Engine Reciprocating Fixed Wing & 180 & $1.22 \%$ & 364 & $2.48 \%$ & 885 & $6.02 \%$ & 2185 & $14.86 \%$ & 457 & $3.11 \%$ & 2500 & $17.00 \%$ & 429 & $2.82 \%$ & 1840 & $12.51 \%$ & 3500 & $23.60 \%$ & 1885 & $12.82 \times$ & 482 & $3.28 \%$ & 14707 & $100.00 \%$ \\
\hline Mulb Engina Reciprocating Fixed Wing & 13 & $1.03 \%$ & 38 & $3.00 \%$ & 71 & $5.60 \%$ & 161 & $12.70 \%$ & 56 & $4.42 \%$ & 239 & $18.85 \%$ & 31 & $2.44 \%$ & 236 & $18.61 \%$ & 300 & $23.66 \%$ & 64 & $5.05 \%$ & 59 & $4.65 \%$ & 1268 & $100.00 \%$ \\
\hline Turtoprop Fixed Wing & 7 & $2.34 \%$ & 14 & $4.68 \%$ & 17 & $5.69 \%$ & 29 & $9.70 \%$ & 13 & $4.35 \%$ & 38 & $12.71 \%$ & 14 & $4.68 \%$ & 52 & $17.39 \%$ & 51 & $17.06 \%$ & 54 & $16.06 \%$ & 10 & $3.34 \%$ & 299 & $100.00 \%$ \\
\hline Turtojol Fixed Wing & 0 & $0.00 \%$ & 2 & $2.27 \%$ & 9 & $10.23 \%$ & 4 & $4.55 \%$ & 6 & $6.82 \%$ & 6 & $6.82 \%$ & 1 & $1.14 \%$ & 17 & $19.32 \%$ & 24 & $27.27 \times$ & 12 & $13.64 \%$ & 7 & $7.85 \%$ & 68 & $100.00 \%$ \\
\hline Total Fixed Wing & 200 & $1.22 \%$ & 418 & $2.55 \%$ & 982 & $6.00 \%$ & 2379 & $14.54 \%$ & 532 & $3.25 \%$ & 2783 & 17.01x. & 475 & $2.90 \%$ & 2145 & $13.11 \%$ & 3875 & $23.60 \%$ & 2015 & $12.32 \%$ & 550 & $3.41 \times$ & 16362 & $100.00 \times$ \\
\hline Peciprocating Engine Rotary Wing & 20 & $2.11 \%$ & 26 & $2.74 \%$ & 56 & $5.90 \%$ & 86 & $10.12 \%$ & 16 & $1.69 \%$ & 177 & $18.65 \%$ & 34 & $3.58 \%$ & 70 & $7.38 \%$ & 128 & $13.49 \%$ & 316 & $33.30 \%$ & 10 & $1.05 \%$ & 949 & $100.00 \times$ \\
\hline Turbine Engine Rotary Wing & 13 & $2.70 \%$ & 9 & $1.87 \%$ & 23 & $4.77 \%$ & 41 & $8.51 \%$ & 10 & $2.07 \%$ & 117 & $24.27 \%$ & 12 & $2.49 \%$ & 34 & $7.05 \%$ & 49 & $10.17 \times$ & 165 & $34.23 \%$ & 9 & $1.87 x$ & 482 & $100.00 \%$ \\
\hline Total Rotary Wing & 33 & $2.31 \%$ & 35 & $2.45 \%$ & 79 & $5.52 \%$ & 137 & 9.57\% & 26 & $1.82 \%$ & 294 & $20.55 \%$ & 46 & $3.21 \%$ & 104 & $7.27 \times$ & 177 & $12.37 \%$ & 481. & $33.61 x$ & 19 & $1.33 \%$ & 1431 & $100.00 \%$ \\
\hline Total Powered Aircraft & 233 & $1.31 \%$ & 453 & $2.55 \%$ & 1061 & $5.96 \%$ & 2516 & $14.14 \%$ & 550 & $3.14 \%$ & 3077 & $17.29 \%$ & 521 & $2.93 \%$ & 2249 & $12.64 \%$ & 4052 & $22 . \pi x$ & 2486 & $14.03 x$ & 577 & $3.24 x$ & 17783 & $100.00 x$ \\
\hline Gliders & 0 & $0.00 \%$ & 0 & $0.00 \%$ & 20 & $6.10 \%$ & 52 & $15.85 \%$ & 3 & $0.91 \%$ & 23 & $7.01 \%$ & 19 & $5.79 \%$ & 94 & $28.66 \%$ & 54 & $16.46 \%$ & 57 & $17.38 x$ & 6 & $1.83 \%$ & 328 & $100.00 \%$ \\
\hline Balloons & 3 & $1.68 \%$ & 0 & $0.00 \%$ & 1 & $0.56 \%$ & $\theta$ & $5.03 \%$ & 9 & $5.03 \%$ & 33 & $18.44 \%$ & 13 & $7.26 \%$ & 16 & $8.94 \%_{0}$ & 85 & $47.49 \%$ & 4 & $2.23 \times$ & 6 & $3.35 \%$ & 179 & $100.00 \%$ \\
\hline Other & 0 & $0.00 \%$ & 0 & $0.00 \%$ & 2 & $9.09 \%$ & 5 & $22.73 \%$ & 1 & $4.55 \%$ & 4 & $18.18 \%$ & 0 & $0.00 \%$ & 1 & $4.55 \%$ & 2 & $9.09 \%$ & 6 & $27.27 \%$ & 1 & $4.55 x$ & 22 & $100.00 x$ \\
\hline Total Aircraft & 236 & $1.28 \%$ & 453 & $2.47 \%$ & 1084 & $5.92 \%$ & 2582 & $14.09 \%$ & 571 & $3.12 \%$ & 3137 & $17.12 \%$ & 553 & $3.02 \%$ & 2360 & $12.88 \%$ & 4193 & $22.89 \%$ & 2563 & $13.99 \%$ & 590 & $3.22 \%$ & $\mid 18322$ & $100.00 \%$ \\
\hline
\end{tabular}


Table 3.15

General Aviation Accidents, 1986-1993 by Flight Phases and by Aircraft Subcategorlos Summary Manuevering Accidents and Accidents During Standing, Taxi, and Other Flight Phases Deleted

References $3.20-3.27$

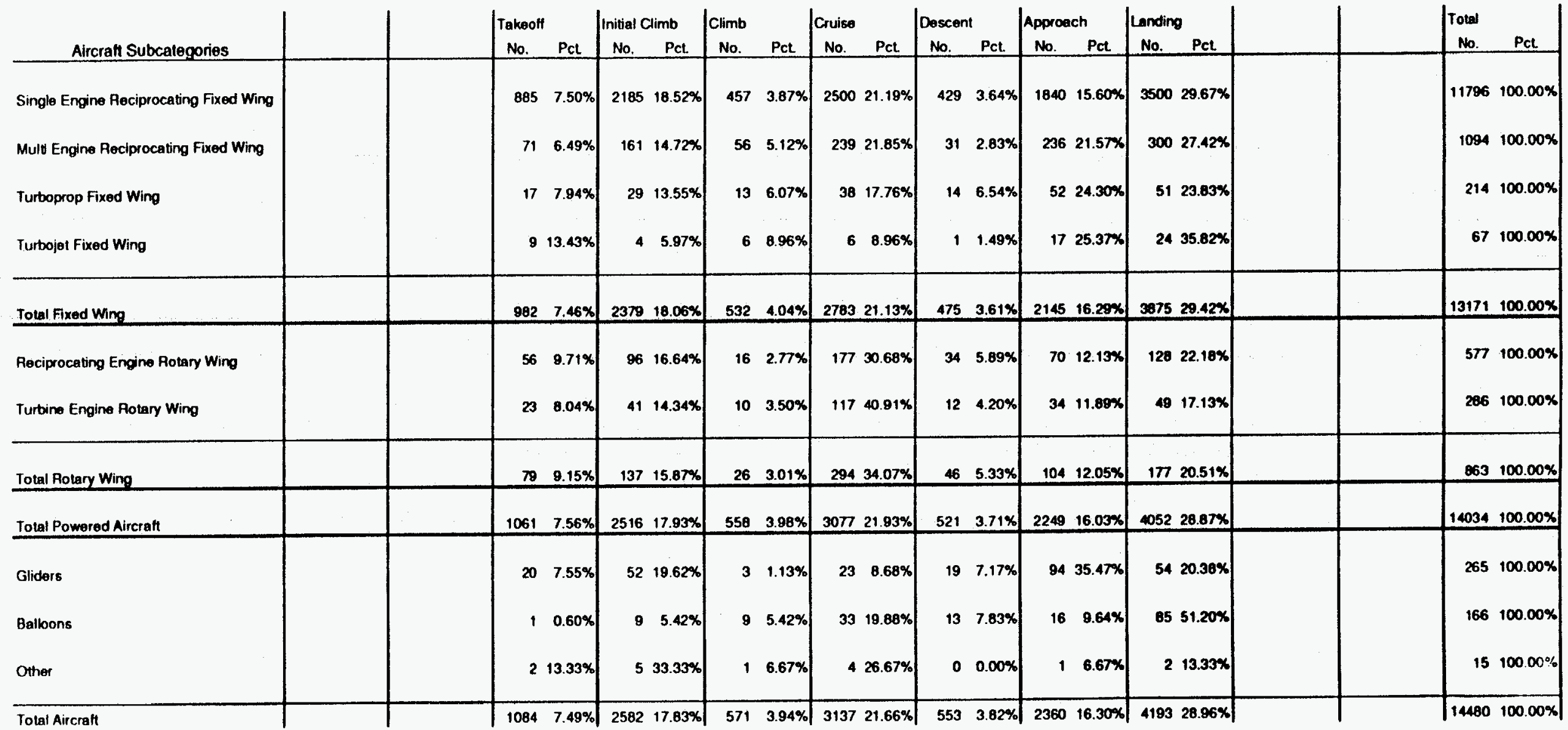




\subsection{GENERAL AVIATION OPERATIONS}

General aviation operations are tabulated by the FAA Office of Aviation Policy, Plans, and Management Analysis in their publication General Aviation Activity and Avionics Survey published annually by calendar year. According to the introduction of this survey, all facets of civil (nonmilitary) aviation are included in this survey except for flights conducted by air carriers under 14 CFR 121 [Ret. 3.9] and 14 CFR 127 [Ref. 3.10]. However, in comparing the operations data for commuter carrier with the data published by the NTSB for scheduled 135 air carriers and in conversations with the FAA staff which prepares the General Aviation Activity and Avionics Survey, it was discovered that no 14 CFR 125 operators and those operators that were known to be scheduled 14 CFR 135 operators were not included in the survey. Despite a conscious effort to exclude these operators, some scheduled 14 CFR 135 operators (commuter air carriers) were included in the survey along with all nonscheduled 14 CFR 135 operators (air taxis).

To determine the general aviation operations consistent with the NTSB definition for general aviation, it is necessary to subtract the scheduled 14 CFR 135 operations (commuter carriers) and the nonscheduled 14 CFR 135 operations (air taxis) from the total operations given by the General Aviation Activity and Avionics Survey. This can be accomplished very readily for hours flown and nautical miles flown since the General Aviation Activity and Avionics Survey lists these numbers separately under total, commuter and air taxi. Determining the number of flights (or landings) for strictly general aviation is another matter. The General Aviation Activity and Avionics Survey gives numbers only on the total number of landings. No breakdown by commuter or air taxi or any other primany use is provided. An estimate of the number of commuter and air taxi flights must be made in order to continue the development of the general aviation accident rate by flight phase.

The number of commuter and air taxi flights can be estimated by two methods. One is to base the estimated number of flights on the number of primary use hours flown divided by the average trip time for that particular subcategory of aircratt. The other method is to base the estimated number of flights on the number of primary use nautical miles flown divided by the average trip length for that particular subcategory of aircraft. Both methods were used and two estimates in the number of commuter and air taxi flights were calculated. The reason for the difference in two estimates is due to rounding in the various factors used to estimate the number of flights.

Tables 3.16 to 3.26 taken from the FAA General Aviation Activity and Avionics Survey [Ref. 3.1 to 3.8] presents the following information by years for the years 1986 to 1993:

\footnotetext{
1. Population (from Ref. Table 2.1),

2. Number active (from Ref. Table 2.1),

3. Total hours flown (from Ref. Table 2.1),

3a. Commuter hours flown (scheduled 14 CFR 135) hours flown (from Ref. Table 3.2),

3b. Air taxi hours flown (nonscheduled 14 CFR 135) hours flown (from Ref. Table 3.2),

3c. Total day hours flown (from Ret. Table 4.1),

3d. Total night hours flown (from Ref. Table 4.1),

3e. Visual Meteorological Conditions (VMC) day hours flown (from Ref. Table 4.2),

3t. Visual Meteorological Conditions (VMC) night hours flown (from Ref. Table 4.2),

3g. Total Visual Meteorological Conditions (VMC) hours flown (from Ret. Table 4.2),

3h. Instrument Meteorological Conditions (IMC) day hours flown (from Ref. Table 4.3),

3i. Instrument Meteorological Conditions (IMC) night hours flown (from Ref. Table 4.3),

3i. Total instrument Meteorological Conditions (IMC) hours flown (from Ref. Table 4.3),

4. General aviation hours flown $=3$. total hours-3a. commuter hours-3b. air taxi hours

5. Total landings (from Ref. Table 2.5)

5a. Local landings (from Ref. Table 2.6),

5b. Cross country landings (from Ref. Table 2.7),

6. Estimated flights (based on primary use hours/average trip time),

6a. Commuter flights (determined from 3a. commuter hours flown/13. average trip time),

6b. Air taxi flights (determined from 3b. air taxi hours flown/13. average trip time),

7. Estimated flights (based on primary use nautical miles/average trip length)

7a. Commuter flights (determined from 9a. commuter nautical miles flown/12. average trip length),
} 

7b. Air taxi flights (determined from 9b. air taxi nautical miles flown/12. average trip length),
8. General aviation flights,
8a. Based on hours/average trip time (determined from 5. total landings-6a. commuter flights-6b. air taxi flights),
8b. Based on nautical miles/average trip length (determined from 5. total landings $-7 a$ commuter flights-7b. air taxi flights),
9. Total nautical miles flown (from Ref. Table 3.3),
9a. $\quad$ Commuter nautical miles flown (from Ref. Table 3.3),
9b. $\quad$ Air taxi nautical miles flown (from Ref. Table 3.3),
10. General aviation nautical miles flown $=9$. total nmiles-9a. commuter nmiles-9b. air taxi nmiles,
11. Average speed (nmiles/hour) (determined from 9. total nmiles flown/5. total landings),
12. Average trip length (nmiles/hour) (determined from 9. total nmiles flown/3. total hours flown),
13. Average trip time (hours/landing) (determined from 3. total hours flown/5. total landings),
14. Average commuter speed (nmiles/hour) (determined from 9a. commuter nautical miles flown/3a. commuter hours flown),
15. Average air taxi speed (nmiles/hour) (determined from 9b. air taxi nautical miles flown/3b. air taxi hours flown),
16. General aviation average speed (nmiles/hour) (determined from 10. general aviation nautical miles flown/4. general aviation hours flown).

The tables listed below gives the general aviation subcategories for which the general aviation operations table were created:

Table 3.16
Table 3.17
Table 3.18
Table 3.19
Table 3.20
Table 3.21
Table 3.22
Table 3.23
Table 3.24
Table 3.25
Table 3.26

Single-engine reciprocating (piston), fixed wing,

Multiple-engine reciprocating (piston), fixed wing,

Turboprop, fixed wing.

Turbojet, fixed wing,

Total fixed wing (summation of Tables 3.16 to 3.19 ),

Reciprocating engine (piston), rotary wing,

Turbine engine, rotary wing,

Total rotary wing (summation of Tables 3.21 and 3.22 ),

Total powered aircratt (summation of Tables 3.16 to 3.19 , and 3.21 to 3.22 ),

Other aircraft (includes gliders and balloons),

Total aircraft (summation of Tables 3.16 to $3.19,3.21$ to 3.22 , and 3.25),

For consistency with the accident data of Section 3.2, general aviation operations data were added and summarized for the 1986 to 1993 time period. General aviation operations data tabulated by the FAA prior to 1986 are not as complete as operations data tabulated from 1986 onwards. Important information such as the estimated number of total landings were not tabulated prior to 1985 . Prior to 1983 , the estimated number of general aviation nautical miles flown were not tabulated. General aviation operations data were found only as far back as 1977 and could be added to Tables 3.16 to 3.26 if it becomes necessary to extend these tables backwards in time further. However, the utility of this exercise would be limited because of the incompleteness of the data. Therefore the decision was made to consider only the 1986 to 1993 time period for general aviation operations.

Table 3.27 summarizes the general aviation operations by aircraft subcategories for 1986-1993 and is based on the information developed in Tables 3.16 through 3.26 . 
Table 3.16

General Aviation Operations

Single-Engine Reciprocating, Fixed Wing References 3.1 to 3.8

Paramoter (Rof. Tablo No.)

1986

1987

1988

1990

1991

1992

1993

1994

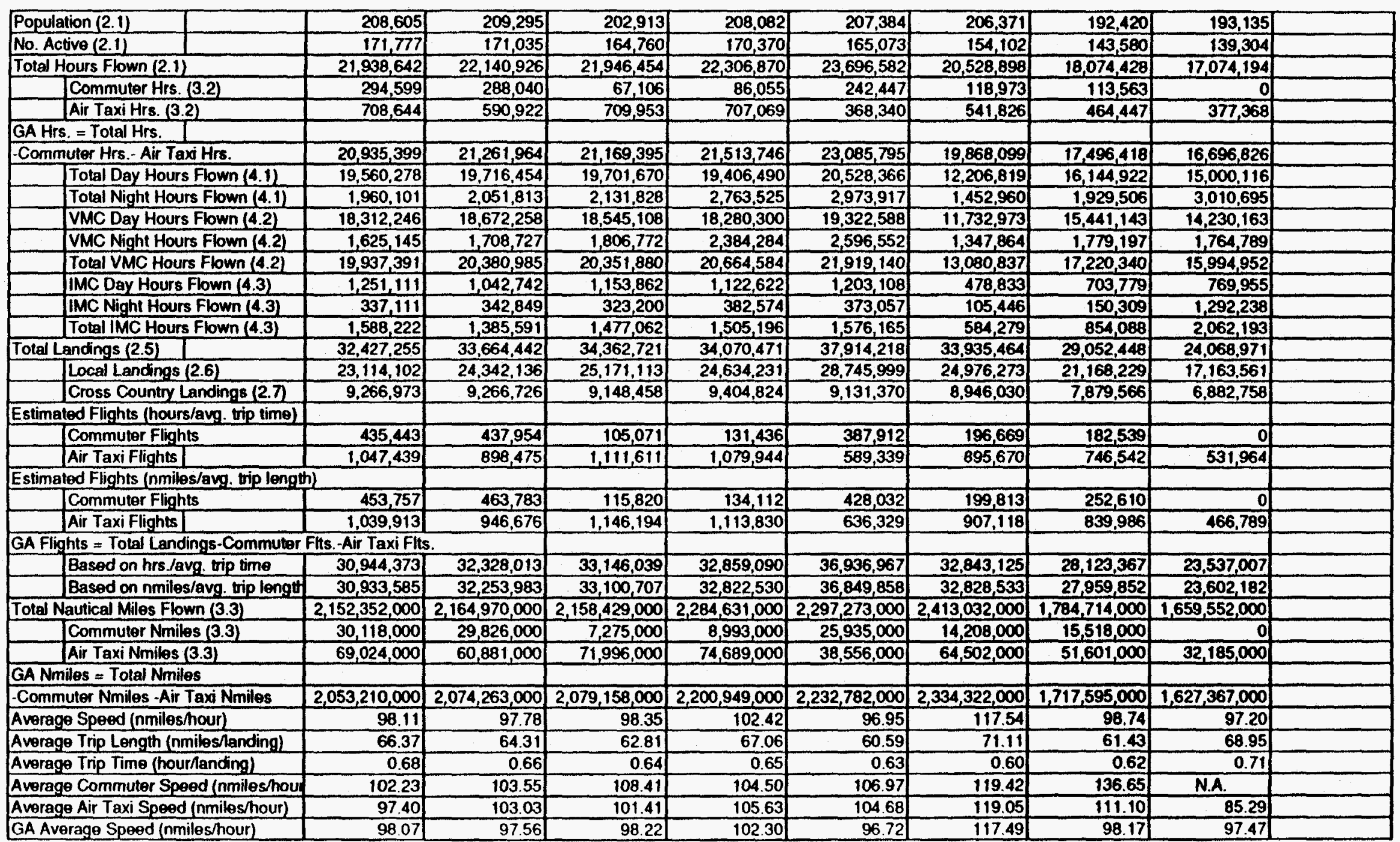

NA. = Not Applicable 
Trble 3.16 (Continued)

General Aviation Operations

Single-Engine Reciprocaling. Fixed Wing

References 3.1 to 3.8

Paramoter (Rof. Tablo No.)

Tolal

Average

Pct. of

Pet. of Tol. Fixed Wing Alrcraft

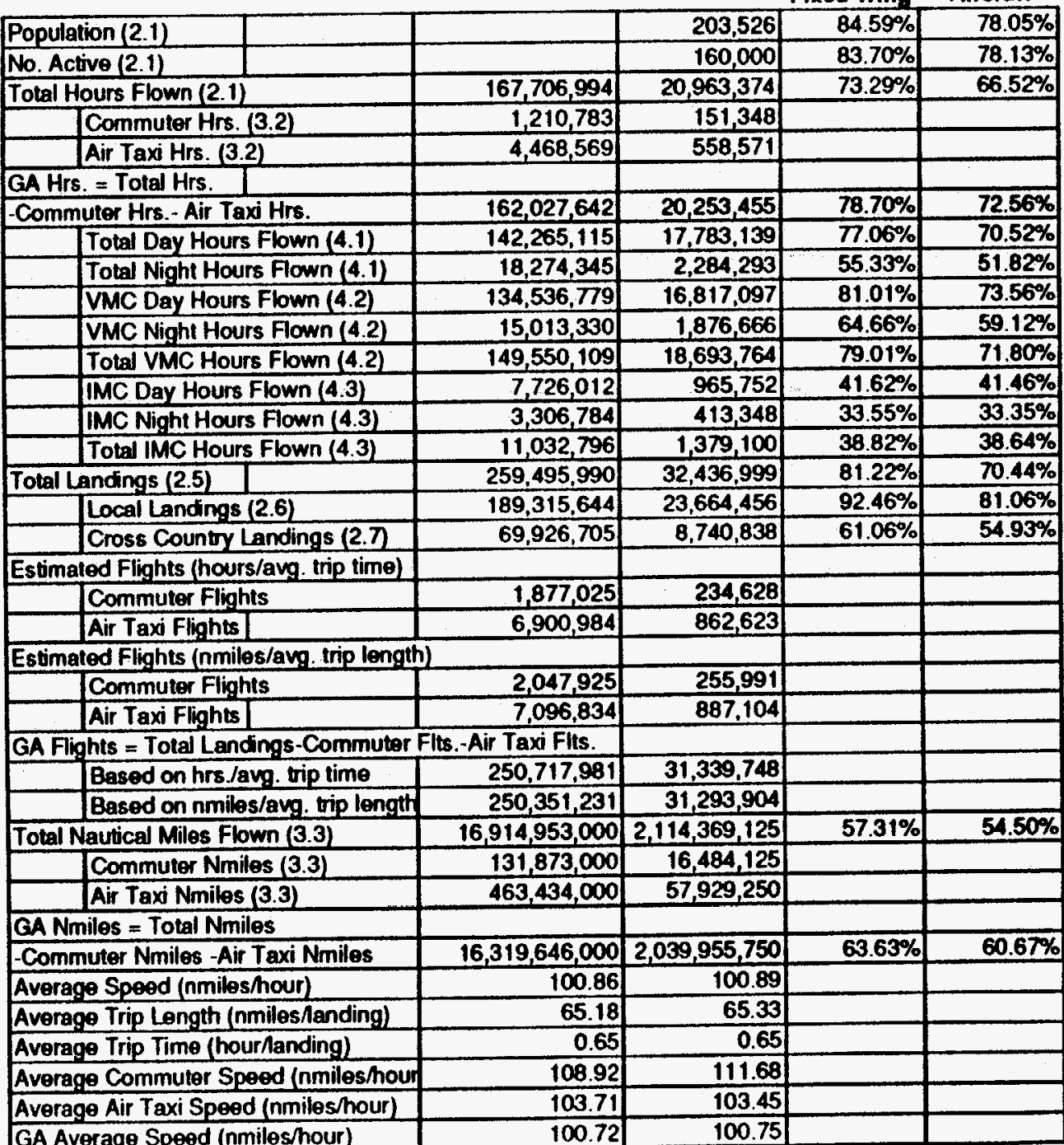

GA Average Speed (nmiles/hour)

100.72

100.75 
Table 3.17

General Aviation Operations

Multi-Engine Reciprocelting, Fixed Wing

Polerences 3.1 to 3.8

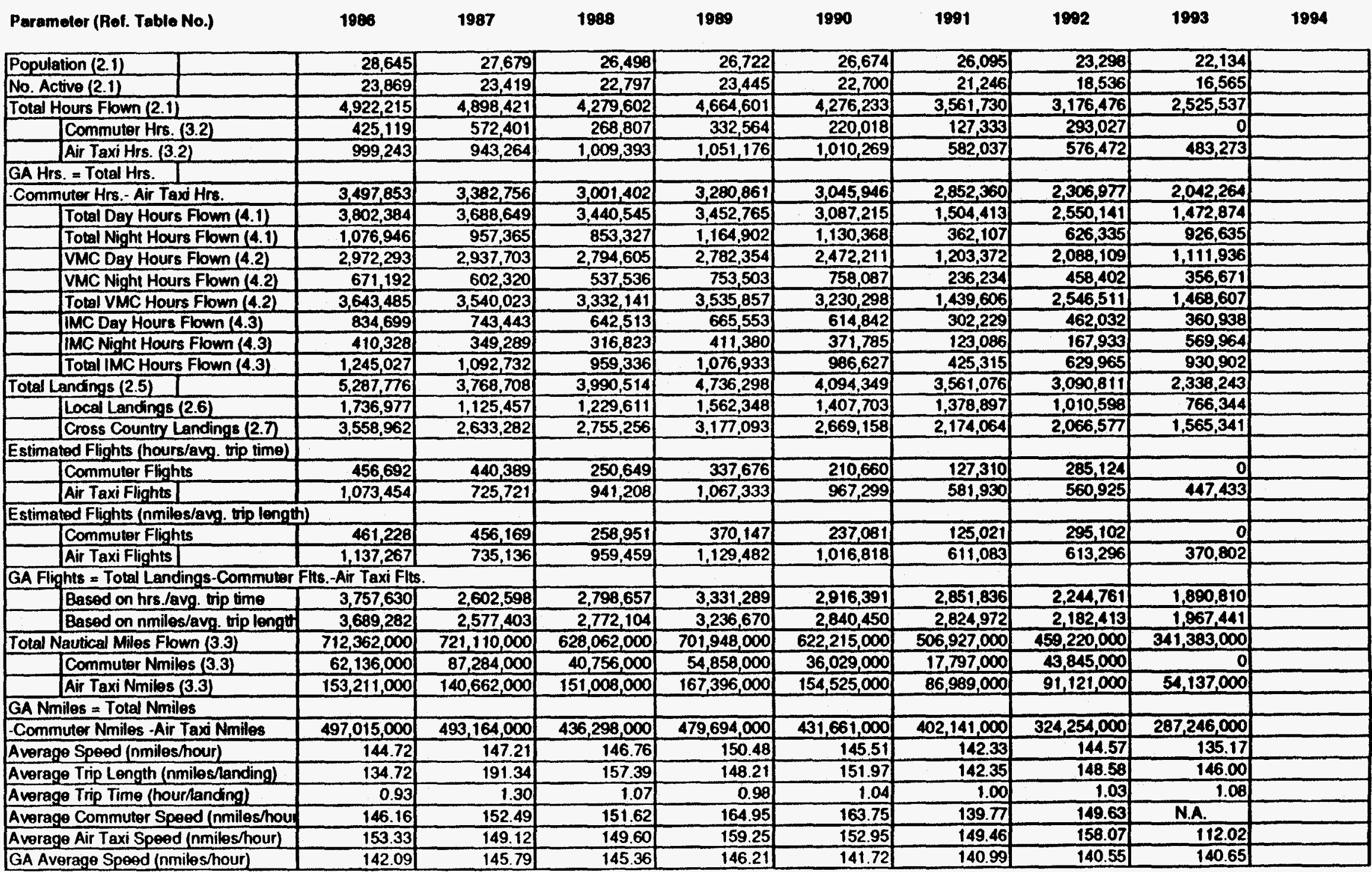

N.A. = Not Applicable 
Table 3.17 (Continued)

General Aviation Operations

Multi-Engine Reciproceting, Fixed Wing

Rolerences 3.1 to 3.8

Paramotor (Rof. Tablo No.)

Tolal

Averago

Pet. of

Pct. of Tol.

\begin{tabular}{|c|c|c|c|c|c|}
\hline \multirow{2}{*}{\multicolumn{2}{|c|}{ Population (2.1) }} & & \multicolumn{2}{|r|}{ Fixed Wing } & \multirow{2}{*}{$\begin{array}{r}\text { Alrcraft } \\
9.96 \%\end{array}$} \\
\hline & & & 25,968 & $10.79 \%$ & \\
\hline No. Active (2.1) & & & 21,572 & $11.28 \%$ & $10.53 \%$ \\
\hline \multicolumn{2}{|l|}{ Total Hours Flown (2.1) } & $32,304,815$ & $4,038,102$ & $14.12 \%$ & $12.81 \%$ \\
\hline \multicolumn{2}{|c|}{ Commuler Hrs. (3.2) } & $2,239,269$ & 279,909 & & \\
\hline \multicolumn{2}{|c|}{ Air Taxi Hrs. (3.2) } & $6,655,127$ & 831,891 & & \\
\hline \multirow{2}{*}{\multicolumn{2}{|c|}{\begin{tabular}{|l|} 
GA Hrs. = Total Hrs. \\
-Commuter Hrs. - Air Taxi
\end{tabular}}} & & & & \\
\hline & & $23,410,419$ & $2,926,302$ & $11.37 \%$ & $10.48 \%$ \\
\hline \multicolumn{2}{|c|}{ Total Day Hours Flown (4.1) } & $22,998,986$ & $2,874,873$ & $12.46 \%$ & $11.40 \%$ \\
\hline \multicolumn{2}{|c|}{ Total Night Hours Flown (4.1) } & 7.097 .985 & 887,248 & $21.49 \%$ & $20.13 \%$ \\
\hline \multicolumn{2}{|c|}{ VMC Day Hours Flown (4.2) } & $18,362,583$ & $2,295,323$ & $11.06 \%$ & $10.04 \%$ \\
\hline \multicolumn{2}{|c|}{ VMC Night Hours Flown (4.2) } & $4,373,945$ & 546,743 & $18.84 \%$ & $17.23 \%$ \\
\hline \multicolumn{2}{|c|}{ Total VMC Hours Flown (4.2) } & $22,736,528$ & $2,842,066$ & $12.01 \%$ & $10.92 \%$ \\
\hline \multicolumn{2}{|c|}{ IMC Day Hours Flown (4.3) } & $4,626,249$ & 578,281 & $24.92 \%$ & $24.83 \%$ \\
\hline \multicolumn{2}{|c|}{ IMC Night Hours Flown (4.3) } & $2,720,588$ & 340,074 & $27.60 \%$ & $27.44 \%$ \\
\hline \multicolumn{2}{|c|}{ Total IMC Hours Flown (4.3) } & $7,346,837$ & 918,355 & $25.85 \%$ & $25.73 \%$ \\
\hline \multicolumn{2}{|l|}{ Total Landings (2.5) } & $30,867,775$ & $3,858,472$ & $9.66 \%$ & $8.38 \%$ \\
\hline \multicolumn{2}{|c|}{ Local Landings (2.6) } & $10,217,935$ & $1,277,242$ & $4.99 \%$ & $4.38 \%$ \\
\hline \multicolumn{2}{|c|}{ Cross County Landings (2.7) } & $20,599,733$ & $2,574,967$ & $17.99 \%$ & $16.18 \%$ \\
\hline \multicolumn{6}{|c|}{ Estimated Flights (hours/avg trip time) } \\
\hline \multicolumn{2}{|c|}{ Commuter Flights } & $2,108,499$ & 263,562 & & \\
\hline Air Taxi Flights & & $6,365,304$ & 795,663 & & \\
\hline \multicolumn{6}{|c|}{ Estimated Flights (nmilos/avg. trip length) } \\
\hline \multicolumn{2}{|c|}{ Commuter Flights } & $2,203,698$ & 275,462 & & \\
\hline Air Taxi Flights & & $6,573,342$ & 821,668 & & \\
\hline \multicolumn{6}{|c|}{ GA Flights = Total Landings-Commuter Flts. - Air Taxi Flts. } \\
\hline \multicolumn{2}{|c|}{ Based on hrs.lavg. trip time } & $22,393,971$ & $2,799,246$ & & \\
\hline \multirow{2}{*}{\multicolumn{2}{|c|}{ Based on nmiles/avg. tip length }} & $22,090,735$ & $2,761,342$ & & \\
\hline Total Nautical Miles Flown (3.3) & & $4,693,227,000$ & $586,653,375$ & $15.90 \%$ & $15.12 \%$ \\
\hline \multicolumn{2}{|c|}{ Commurar Nmiles (3.3) } & $342,705,000$ & $42,838,125$ & & \\
\hline Air Taxi Nmiles & (3.3) & $999,049,000$ & $124,881,125$ & & \\
\hline GA Nmiles $=$ Total Nm & & & & & \\
\hline -Commuter Nmiles -Ai & Taxi Nmiles & $3,351,473,000$ & $418,934,125$ & $13.07 \%$ & $12.46 \%$ \\
\hline Average Speod (nmile & (hour) & 145.28 & 144.59 & & \\
\hline Average Trip Length (n & miles/anding) & 152.04 & 152.57 & & \\
\hline Average Trip Time (ho & Ir/anding) & 1.05 & 1.06 & & \\
\hline Average Commuter Sp & eed (nmiles/hour & 153.04 & 152.62 & & \\
\hline Average Air Taxi Spee & (nmiles/hour) & 150.12 & 147.97 & & \\
\hline GA Average Speed (n) & niles/hour) & 143.16 & 142.92 & & \\
\hline
\end{tabular}


Table 3.18

Genoral Aviation Operations

Turboprop, Flxed Wing

Reforences 3.1 to 3.8

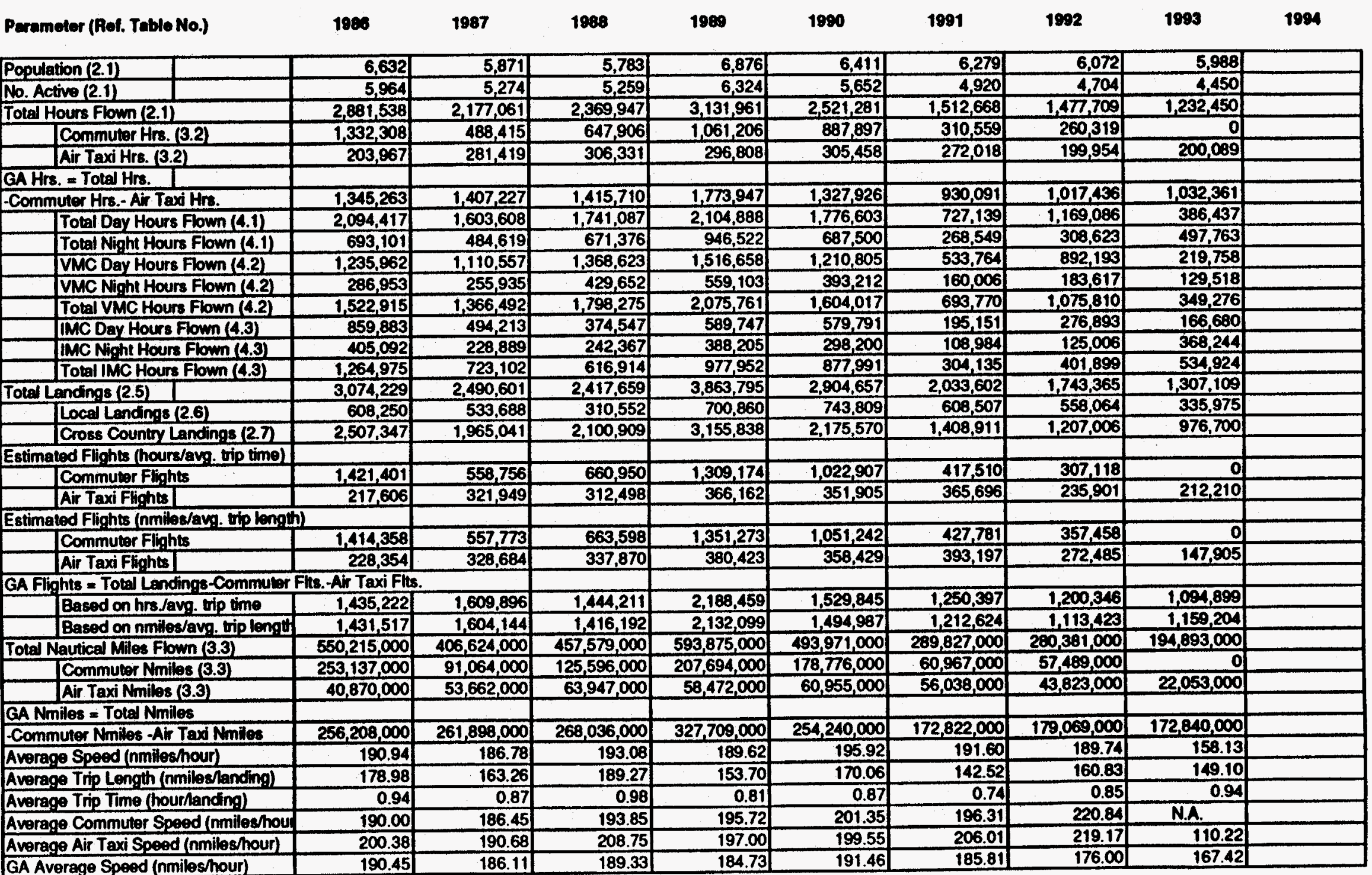

N.A. = Not Applicable 
Table 3.18 (Continued)

General Aviation Operations

Turboprop, Fixed Wing

Peforences 3.1 to 3.8

Paramoler (Rof. Tablo No.)

Tolal

Average

Pet. of

Pol. of Tol.

Fixed Wing Airctent

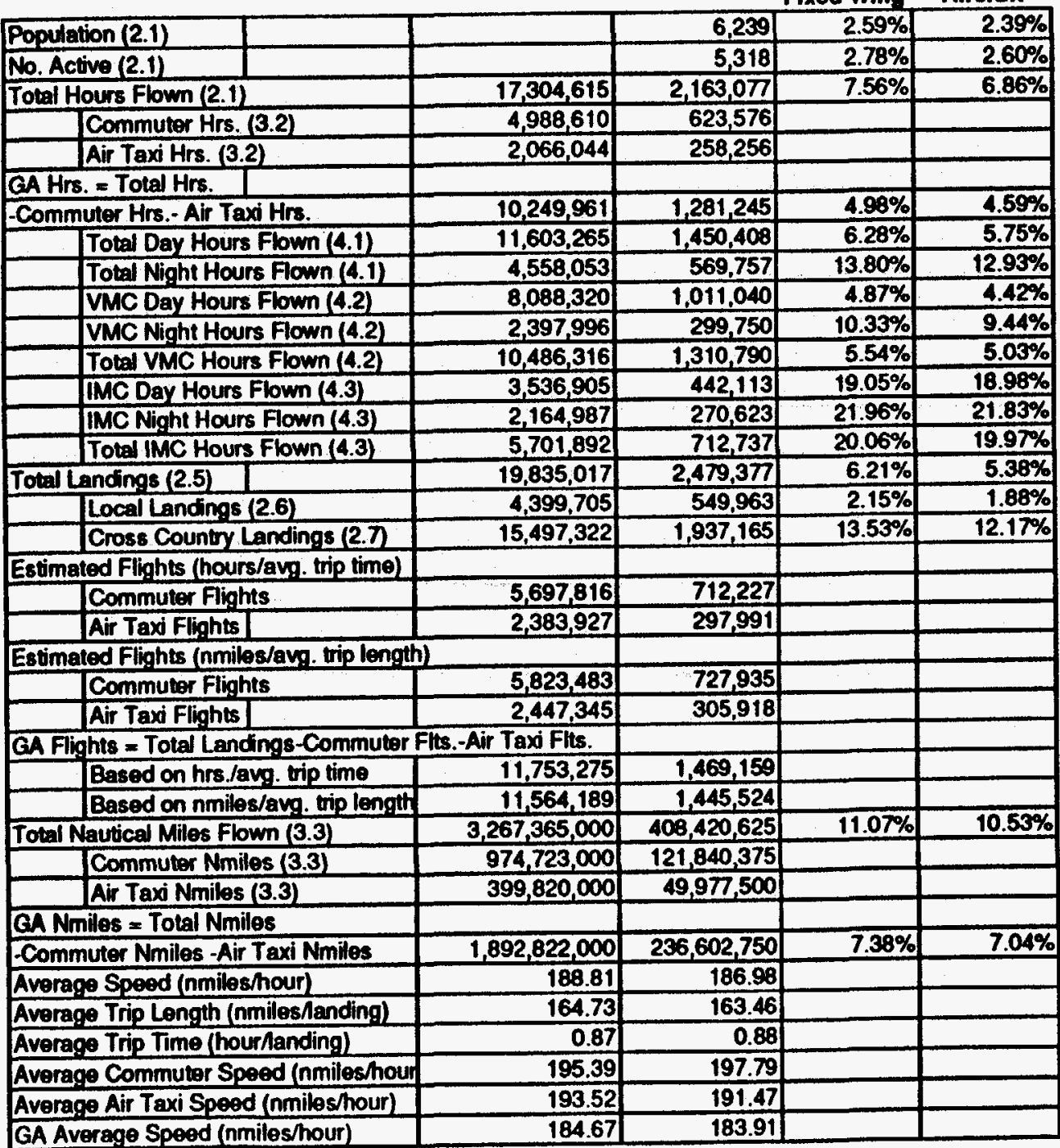


Table 3.19

Goneral Aviation Operations

Turbojet, Fixed Wing

Reforences 3.1 10 3.8

\begin{tabular}{|c|c|c|c|c|c|c|c|c|c|}
\hline Paramoler (Rol. Table No.) & 1986 & 1987 & 1888 & 1989 & 1990 & 1991 & 1992 & 1993 & 1994 \\
\hline Population (2.1) & 4,961 & 4,789 & 4,555 & 4,736 & 4,891 & 5,041 & 4,866 & 5,062 & \\
\hline No. Active (2.1) & 4,480 & 4,338 & 4,187 & 4,402 & 4,374 & 4,353 & 4,022 & 4,068 & \\
\hline Total Hours Flown (2.1) & $1,654,308$ & $1,527,760$ & $1,677,752$ & $1,654,096$ & $1,511,560$ & $1,236,283$ & $1,072,292$ & $1,178,873$ & \\
\hline Commuler Hrs. (3.2) & 381 & 4,625 & 2,805 & 8.593 & 0 & 4,282 & 9,511 & 0 & \\
\hline Air Taxi Hrs. (3.2) & 198,634 & 171,266 & 194,228 & 216,227 & 147,365 & 185,657 & 74,787 & 107,491 & \\
\hline \multicolumn{10}{|l|}{ GA Hrs. = Total Hrs. } \\
\hline - Commuter Hrs. - Air Taxi Hrs. & $1,455,293$ & $1,351,869$ & $1,480,719$ & $1,429,276$ & $1,364,195$ & $1,046,344$ & 987,994 & $1,071,382$ & \\
\hline Total Day Hours Flown (4.1) & $1,276,996$ & $1,138,852$ & $1,227,229$ & $1,168,399$ & $1,085,973$ & $726,5 \pi$ & 838,941 & 297,320 & \\
\hline Total Night Hours Flown (4.1) & 368,424 & 365,923 & 399,843 & 476,069 & 441,692 & 205,652 & 233,351 & 607,918 & \\
\hline VMC Day Hours Flown (4.2) & 782,825 & 708,106 & 814,273 & 831,548 & 752.716 & 499.085 & 576,120 & 119,150 & \\
\hline VMC Night Hours Flown (4.2) & 185,746 & 181,436 & 190,991 & 279,966 & 246,844 & 97,029 & 131,061 & 120,158 & \\
\hline Total VMC Hours Flown (4.2) & 968,571 & 899,542 & $1,005,264$ & $1,111,514$ & 999,560 & 596,114 & 707,181 & 239,308 & \\
\hline IMC Day Hours Flown (4.3) & 488,584 & 431,007 & 411,888 & 338,644 & 334,679 & 228,608 & 262,821 & 178,169 & \\
\hline IMC Night Hours Flown (4.3) & 181,363 & 184,718 & 207,292 & 197,360 & 195,253 & 108,714 & 102,290 & 487,759 & \\
\hline Total IMC Hours Flown (4.3) & 669,947 & 615,725 & 619,180 & 536,004 & 529,932 & 337,322 & 365,111 & 665,928 & \\
\hline Total Landings (2.5) & $1,284,427$ & $1,234,563$ & $1,298,001$ & $1,455,010$ & $1,256,966$ & 971,172 & 929,511 & 884,744 & \\
\hline Local Landings (2.6) & 151,676 & 116,368 & 71,502 & 190.286 & 76,793 & 84,485 & 56,986 & 72,330 & \\
\hline Cross Country Landings (2.7) & $1,130,769$ & $1,115,781$ & $1,225,081$ & $1,269,027$ & $1,182,918$ & 685,844 & 874,324 & 814.790 & \\
\hline \multicolumn{10}{|l|}{ Estimated Flights (hours/avg. trip time) } \\
\hline Commuter Flights & 296 & 3,737 & 2,170 & 7,559 & 0 & 3,364 & 8,245 & 0 & \\
\hline Air Taxi Flights & 154,222 & 138,398 & 150,265 & 190,202 & 122,544 & 145,844 & 64,829 & 80,672 & \\
\hline \multicolumn{10}{|l|}{ Estimated Flights (nmiles/aug. trip length) } \\
\hline Commuter Flights & 305 & 3,883 & 1,964 & 7,422 & 0 & 3,602 & 9,223 & $\mathbf{0}$ & \\
\hline Air Taxi Flights & 156,949 & 143,155 & 154,223 & 186,815 & 126,670 & 151,771 & 70,285 & 94,185 & \\
\hline \multicolumn{10}{|c|}{ GA Flights = Total Landings-Commuler Fis. -Air Taxi Fits. } \\
\hline Besed on hrs./avg. tip time & $1,129,909$ & $1,092,428$ & $1,145,565$ & $1,257,249$ & $1,134,422$ & 821,964 & 856,438 & 804,072 & \\
\hline Based on nmiles/ang trip lengt & $1,127,173$ & $1,087,525$ & $1,141,814$ & $1,260,772$ & $1,130,296$ & 815,799 & 850,000 & 790,559 & \\
\hline Totad Nautical Miles Flown (3.3) & $673,709,000$ & $618,648,000$ & $678,235,000$ & $692,000,000$ & $605,880,000$ & $485,634,000$ & $421,382,000$ & $463,383,000$ & \\
\hline Commuter Nmiles (3.3) & 160,000 & $1,946,000$ & $1,026,000$ & $3,530,000$ & 0 & $1,801,000$ & $4,181,000$ & 0 & \\
\hline Air Taxi Nmiles (3.3) & $82,323,000$ & $71,736,000$ & $80,585,000$ & $88,849,000$ & $61,057,000$ & $75,893,000$ & $31,863,000$ & $49,329,000$ & \\
\hline \multicolumn{10}{|l|}{ GA Nmiles = Total Nmiles } \\
\hline - Commuter Nmiles - Air Taxi Nmiles & $591,226,000$ & $544,966,000$ & $596,624,000$ & $599,621,000$ & $544,823,000$ & $407,940,000$ & $385,338,000$ & $414,054,000$ & \\
\hline Average Speed (nmileshour) & 407.25 & 404.94 & 404.25 & 418.36 & 400.83 & .392 .82 & 392.97 & 393.07 & \\
\hline Average Trip Length (nmileslanding) & 524.52 & 501.11 & 522.52 & 475.60 & 482.02 & 500.05 & 453.34 & 523.75 & \\
\hline Average Trip Time (hourlanding) & 1.29 & 1.24 & 1.29 & 1.14 & 1.20 & 1.27 & 1.15 & 1.33 & \\
\hline Average Commuter Speed (nmiles/hou & 419.95 & 420.76 & 365.78 & 410.80 & 0.00 & 420.60 & 439.60 & N.A. & \\
\hline Averago Air Taxi Speod (nmiles/hour) & 414.45 & 418.86 & 414.90 & 410.91 & 414.32 & 408.78 & 426.05 & 458.91 & \\
\hline GA Average Speed (nmiles/hour) & 406.26 & 403.12 & 402.93 & 419.53 & 399.37 & 389.87 & 390.02 & 386.47 & \\
\hline
\end{tabular}

N.A. = Not Applicable 
Table 3.19 (Continued)

General Aviation Operations

Turbojet, Fixed Wing

Roterences 3.1 to 3.8

Paramoler (Rot. Tablo No.)

Toled

Averago

Pct. of Pat. of Tol.

Fixed Wing Aircratt

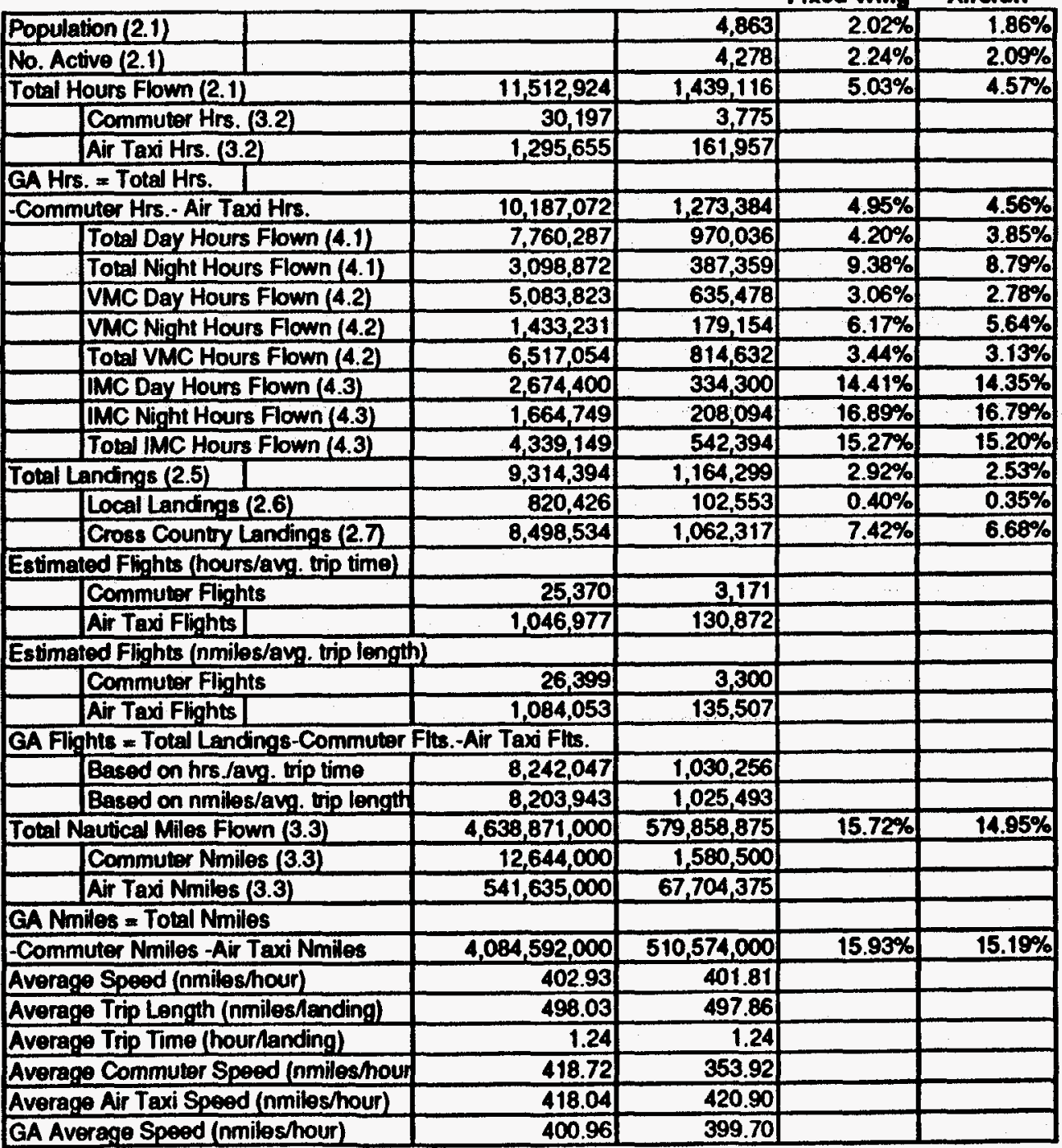


Table 3.20

Gonoral Aviation Operations

Tolal, Fixed Wing

References 3.1 to 3.8

\begin{tabular}{|c|c|c|c|c|c|c|c|c|c|}
\hline Perameler (Rel. Table No.) & 1986 & 1987 & 1988 & 1989 & 1990 & 1991 & 1902 & 1993 & 1994 \\
\hline Population (2.1) & 248,843 & 247,634 & 239,749 & 246,416 & 245,360 & 243,786 & 226.656 & 226,319 & \\
\hline No. Active (2.1) & 206,090 & 204,066 & 197,003 & 204,541 & 197,799 & 184,621 & 170,842 & 164,387 & \\
\hline Total Hours Flown (2.1) & $31,396,703$ & $30,744,168$ & $30,273,755$ & $31,757,528$ & $32,005,656$ & $26,839,579$ & $23,800,905$ & $22,011,054$ & \\
\hline Commuter Hrs. (3.2) & $2,052,407$ & $1,353,481$ & 986,624 & $1,488,418$ & $1,350,362$ & 561,147 & 676,420 & 의 & \\
\hline Air Taxi Hrs. (3.2) & $2,110,488$ & $1,986,871$ & $2,219,905$ & $2,271,280$ & $1,831,432$ & $1,581,538$ & $1,315,660$ & $1,169,221$ & \\
\hline \multicolumn{10}{|l|}{ GA Hrs. = Total Hrs. } \\
\hline - Commuter Hrs. - Ar Texi Hrs. & $27,233,808$ & $27,403,816$ & $27,067,226$ & $27,997,830$ & $28,823,862$ & $24,696,894$ & $21,800,825$ & $20,842,833$ & \\
\hline (Total Day Hours Flown (4.1) & $26,734,075$ & $26,147,563$ & $26,110,531$ & $26,132,542$ & $26,478,157$ & $15,164,948$ & $20,703,090$ & $17,156,747$ & \\
\hline Total Night Hours Flown (4.1) & $4,098,572$ & $3,859,720$ & $4,056,374$ & $5,351,018$ & $5,233,477$ & $2,289,268$ & $3,097,815$ & $5,043,011$ & \\
\hline VMC Day Hours Flown (4.2) & $23,303,326$ & $23,428,624$ & 23.522 .609 & $23,410,860$ & $23,758,320$ & $13,969,194$ & $18,997,565$ & $15,681,007$ & \\
\hline VMC Night Hours Flown (4.2) & $2,769,036$ & $2,748,418$ & $2,964,951$ & $3,976,856$ & $3,994,695$ & $1,841,133$ & $2,552,277$ & $2,371,136$ & \\
\hline Total VMC Hours Flown (4.2) & $26,072,362$ & $26,177,042$ & $26,487,560$ & $27,387,716$ & $27,753,015$ & $15,810,327$ & $21,549,842$ & $18,052,143$ & \\
\hline IMC Day Hours Flown (4.3) & $3,434,277$ & $2,711,405$ & $2,582,810$ & $2,716,566$ & $2,732,420$ & $1,204,821$ & $1,705,525$ & $1,475,742$ & \\
\hline imc Night Hours Flown (4.3) & $1,333,894$ & $1,105,745$ & $1,089,682$ & $1,379,519$ & $1,238,295$ & 446,230 & 545,538 & $2,718,205$ & \\
\hline Total IMC Hours Flown (4.3) & $4,768,171$ & $3,817,150$ & $3,672,492$ & $4,096,085$ & $3,970,715$ & $1,651,051$ & $2,251,063$ & $4,193,947$ & \\
\hline \begin{tabular}{|l|l|} 
Total Landings (2.5) \\
\end{tabular} & $42,073,687$ & $41,158,314$ & $42,068,895$ & $44,125,574$ & $46,170,190$ & $40,501,314$ & $34,816,135$ & $28,599,067$ & \\
\hline Locel Landinge (2.6) & $25,611,005$ & $26,117,649$ & $26,782,778$ & $27,087,725$ & $30,974,304$ & $27,048,162$ & $22,793,8 \pi$ & $18,338,210$ & \\
\hline Cross Country Landings (2.7) & $16,464,051$ & $14,980,830$ & $15,229,704$ & $17,006,782$ & $15,159,016$ & $13,414,849$ & $12,027,473$ & $10,239,589$ & \\
\hline Estimaved Flights (hours/avg. tip time) & & & & & & & & & \\
\hline Commuter Flights & $2,750,363$ & $1,811,953$ & $1,371,029$ & $2,068,086$ & $1,947,883$ & 846,779 & 889,472 & 요 & \\
\hline Air Taxi Flights & $2,828,195$ & $2,659,895$ & $3,084,816$ & $3,155,835$ & $2,641,957$ & $2,386,564$ & $1,924,557$ & $1,517,875$ & \\
\hline Estimated Flights (nmilos/avg. tip leng & & & & & & & & & \\
\hline Commuter Flights & $3,555,855$ & $2,211,047$ & $1,873,250$ & $2,840,953$ & $2,765,383$ & $1,038,700$ & $1,430,528$ & 0 & \\
\hline Air Taxi Flights & $3,554,589$ & $3,440,330$ & $3,942,027$ & $4,021,755$ & $3,619,477$ & $3,106.268$ & $2,581,434$ & $1,696,062$ & \\
\hline GA Flights = Total Landings-Commu & Flts.-Air Taxi Fits & & & & & & & & \\
\hline Based on hrs Javg trip time & $36,495,129$ & $36,686,466$ & $37,613,051$ & $38,901,653$ & $41,580,250$ & $37,267,971$ & $31,902,106$ & $27,091,192$ & \\
\hline Based on nmiles/avg trip lengt & $34,963,242$ & $35,506,937$ & $36,253,617$ & $37,262,866$ & $39,785,330$ & $36,356,347$ & $30,804,173$ & $26,903,005$ & \\
\hline Total Nautical Miles Flown (3.3) & $4,088,638,000$ & $3,911,352,000$ & $3,922,305,000$ & $4,272,454,000$ & $4,019,339,000$ & $3,695,420,000$ & $2,945,697,000$ & $2,659,211,000$ & \\
\hline Commuler Nmiles (3.3) & $345,551,000$ & $210,120,000$ & $174,653,000$ & $275,075,000$ & $240,740,000$ & $94,773,000$ & $121,033,000$ & 0 & \\
\hline Air Taxi Nmilos (3.3) & $345,428,000$ & $326,941,000$ & $367,536,000$ & $389,406,000$ & $315,093,000$ & $283,422,000$ & $218,408,000$ & $157,704,000$ & \\
\hline GA Nmiles = Total Nmiles & & & & & & & & & \\
\hline - Commuter Nmiles - Air Taxi Nmiles & $3,397,659,000$ & $3,374,291,000$ & $3,390,116,000$ & $3,607,973,000$ & $3,463,506,000$ & $3,317,225,000$ & $2,606,256,000$ & $2,501,507,000$ & \\
\hline Average Speed (nmilos/hour) & 130.23 & 127.22 & 129.56 & 134.53 & 125.58 & 137.69 & 123.76 & 120.81 & \\
\hline Average Trip Length (nmileslanding) & 97.18 & 95.03 & 93.24 & 96.82 & 87.05 & 91.24 & 84.61 & 92.98 & \\
\hline Averege Trip Time (hourManding) & 0.75 & 0.75 & 0.72 & 0.72 & 0.69 & 0.66 & 0.68 & 0.77 & \\
\hline Average Commuter Speed (nmiles/hou & 168.36 & 155.24 & 177.02 & 184.81 & 178.28 & 168.89 & 178.93 & N.A. & \\
\hline Average Air Taxi Speed (nmiles/hour) & 163.67 & 164.55 & 165.56 & 171.45 & 172.05 & 179.21 & 166.01 & 135.00 & \\
\hline GA Average Speed (nmiles/hour) & 124.76 & 123.13 & 124.88 & 128.87 & 120.16 & 134.32 & 119.50 & 120.02 & \\
\hline
\end{tabular}

N.A. $=$ Not Applicable

3. GENERAL AVIATION 
Table 3.20 (Continued)

General Aviation Operations

Total, Fixed Wing

Roforonces 3.1 to 3.8

\begin{tabular}{|c|c|c|c|c|}
\hline Parameler (Rof. Table No.) & Totel & Averego & $\begin{array}{l}\text { Pct. of } \\
\text { Fixed Wing }\end{array}$ & $\begin{array}{c}\text { Pet. of Tol. } \\
\text { Airctaf }\end{array}$ \\
\hline Population (2.1) & & 240,595 & $100.00 \%$ & $92.27 \%$ \\
\hline No. Acive (2.1) & & 191,169 & $100.00 \%$ & $93.35 \%$ \\
\hline Total Hours Flown (2.1) & $228,829,348$ & $28,603,669$ & $100.00 \%$ & $90.76 \%$ \\
\hline Commuter Hrs. (3.2) & $8,468,859$ & $1,058,607$ & & \\
\hline Air Taxi Hrs. (3.2) & $14,485,395$ & $1,810,674$ & & \\
\hline GA Hrs. = Total Hrs. & & & & \\
\hline - Commuter Hrs. - Air Taxi Hrs. & $205,875,094$ & $25,734,387$ & $100.00 \%$ & $92.20 \%$ \\
\hline Total Day Hours Flown (4.1) & $184,627,653$ & $23,078,457$ & $100.00 \%$ & $91.52 \%$ \\
\hline Total Night Hours Flown (4.1) & $33,029,255$ & $4,128,657$ & $100.00 \%$ & $93.66 \%$ \\
\hline VMC Day Hours Flown (4.2) & $166,071,505$ & $20,758,938$ & $100.00 \%$ & $90.80 \%$ \\
\hline VMC Night Hours Flown (4.2) & $23,218,502$ & $2,902,313$ & $100.00 \%$ & $91.44 \%$ \\
\hline Total VMC Hours Flown (4.2) & $189,290,007$ & $23,661,251$ & $100.00 \%$ & $90.87 \%$ \\
\hline IMC Day Hours Flown (4.3) & $18,563,566$ & $2,320,446$ & $100.00 \%$ & $99.62 \%$ \\
\hline IMC Night Hours Flown (4.3) & $9,857,108$ & $1,232,139$ & $100.00 \%$ & $99.41 \%$ \\
\hline Total IMC Hours Flown (4.3) & $28,420,674$ & $3,552,584$ & $100.00 \%$ & $99.55 \%$ \\
\hline Total Landings (2.5) & $319,513,176$ & $39,939,147$ & $100.00 \%$ & $86.74 \%$ \\
\hline Local Landings (2.6) & $204,753,710$ & $25,594,214$ & $100.00 \%$ & $87.67 \%$ \\
\hline Cross County Landings (2.7) & $114,522,294$ & $14,315,287$ & $100.00 \%$ & $89.96 \%$ \\
\hline Estimated Flights (hours/avg. trip timo) & & & & \\
\hline Commuter Flights & $11,785,665$ & $1,473,208$ & & \\
\hline Air Tax Flights & $20,199,694$ & $2,524,962$ & & \\
\hline ted Flights (nmiles/t & & & & \\
\hline Commuter Flights & $15,715,716$ & $1,964,465$ & & \\
\hline Air Taxi Flights & $25,961,942$ & $3,245,243$ & & \\
\hline GA Flights = Total Landings-Comme & Air Taxi Flts. & & & \\
\hline Based on hrs./avg. trip time & $287,527,817$ & $35,940,977$ & & \\
\hline Besed on nmiles/avg. trip length & $277,835,518$ & $34,729,440$ & & \\
\hline Total Nautical Miles Flown (3.3) & $29,514,416,000$ & $3,689,302,000$ & $100.00 \%$ & $95.09 \%$ \\
\hline Commuter Nmiles (3.3) & $1,461,945,000$ & $182,743,125$ & & \\
\hline Air Taxi Nmiles (3.3) & $2,403,938,000$ & $300,492,250$ & & \\
\hline GA Nmiles = Total Nmiles & & & & \\
\hline -Commuter Nmiles - Air Taxi Nmiles & $25,648,533,000$ & $3,206,066,625$ & $100.00 \%$ & $95.35 \%$ \\
\hline Average Speed (nmiles/hour) & 128.98 & 128.67 & & \\
\hline Average Trip Length (nmilesfanding) & 92.37 & 92.27 & & \\
\hline Average Trip Time (hournanding) & 0.72 & 0.72 & & \\
\hline Average Commuter Speed (nmiles/hour & 172.63 & 173.08 & & \\
\hline Average Air Taxi Speed (nmiles/hour) & 165.96 & 164.69 & & \\
\hline GA Average Speed (nmiles/hour) & 124.58 & 124.45 & & \\
\hline
\end{tabular}


Table 3.21

Gonoral Aviation Operationa Reciproceting Engine, Rotery Wing

References 3.1 to 3.8

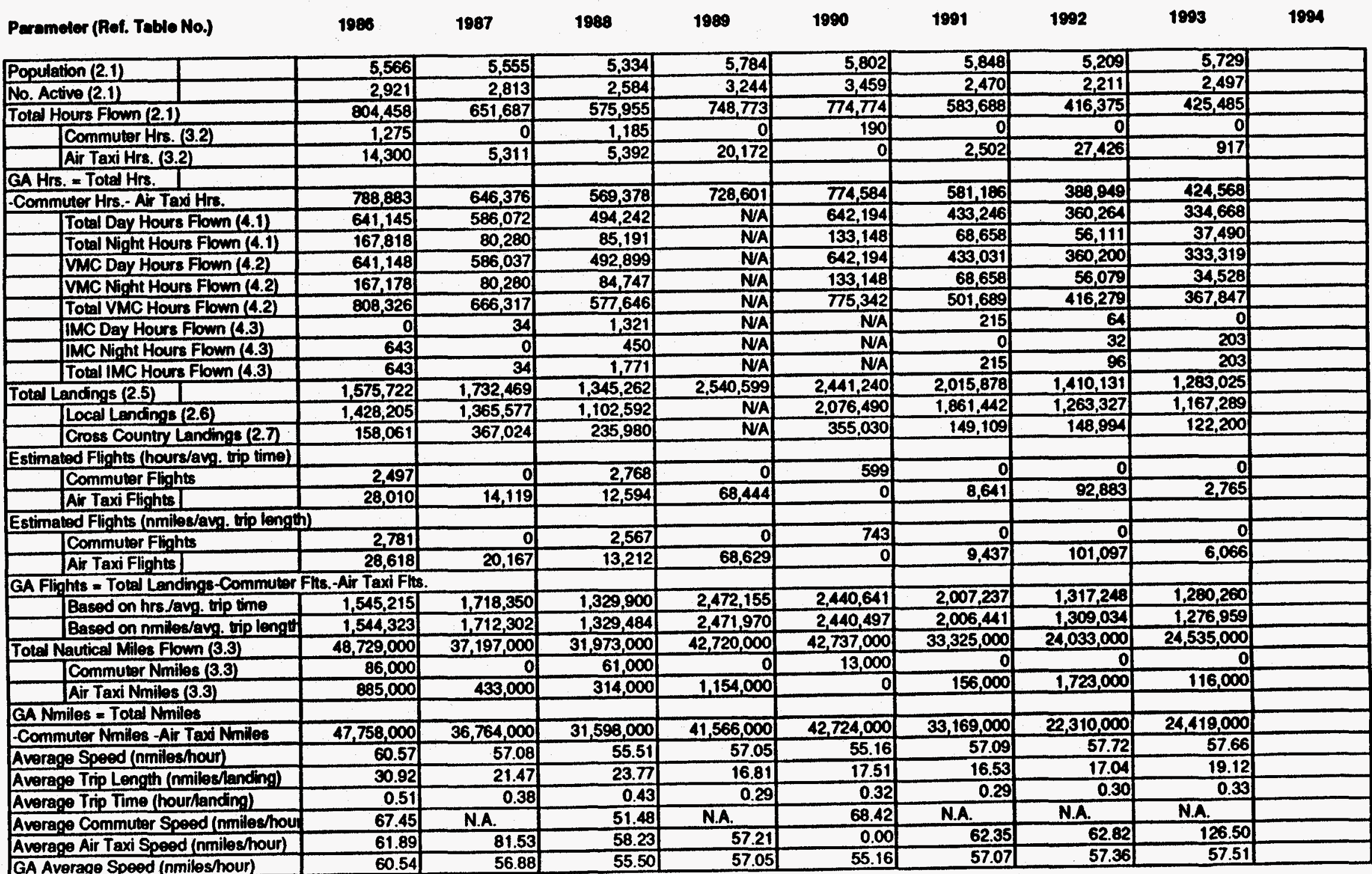

$N A=$ Information not available

N.A. = Not Applicable

3. GENERAL AVIATION 
Table 3.21 (Continued)

General Aviation Operations

Reciprocating Engino, Rotary Wing

Roforences 3.1 to 3.8

\begin{tabular}{|c|c|c|c|}
\hline Parameler (Rol. Table No.) & Tolel & Average & $\begin{array}{l}\text { Pet. of Tol. } \\
\text { Aircraft }\end{array}$ \\
\hline Population (2.1) & & 5,603 & $2.15 \%$ \\
\hline No. Active (2.1) & & 2,775 & $1.35 \%$ \\
\hline Total Hours Flown (2.1) & $4,981,195$ & 622,649 & $1.98 \%$ \\
\hline Commuter Hrs. (3.2) & 2,650 & 331 & \\
\hline Air Taxi Hrs. (3.2) & 76,020 & 9,503 & \\
\hline \multirow{2}{*}{$\begin{array}{l}\text { GA Hrs. = Total Hrs. } \\
\text {-Commuter Hrs. - Air Taxi Hrs. }\end{array}$} & & & \\
\hline & $4,902,525$ & 612,816 & $2.20 \%$ \\
\hline Total Day Hours Flown (4.1) & $3,491,831$ & 498,833 & $1.98 \%$ \\
\hline Total Night Hours Flown (4.1) & 628,696 & 89,814 & $2.04 \%$ \\
\hline VMC Day Hours Flown (4.2) & $3,488,828$ & 498,404 & $2.18 \%$ \\
\hline VMC Night Hours Flown (4.2) & 624,618 & 89,231 & $2.81 \%$ \\
\hline Total VMC Hours Flown (4.2) & $4,113,446$ & 587,635 & $2.26 \%$ \\
\hline IMC Day Hours Flown (4.3) & 1,634 & 272 & $0.01 \%$ \\
\hline IMC Night Hours Fown (4.3) & 1,328 & 221 & $0.02 \%$ \\
\hline Total IMC Hours Flown (4.3) & 2,962 & 494 & $0.01 \%$ \\
\hline \multirow{2}{*}{$\begin{array}{l}\text { Total Landings (2.5) } \\
\text { Local Landings (2.6) }\end{array}$} & $14,344,326$ & $1,793,041$ & $3.89 \%$ \\
\hline & $10,264,922$ & $1,466,417$ & $5.02 \%$ \\
\hline Cross Country Landings (2.7) & $1,536,398$ & 219,485 & $1.36 \%$ \\
\hline \multicolumn{4}{|l|}{ Estimated Flights (hours/ayg. trip time) } \\
\hline Commuter Flights & 5,864 & 733 & \\
\hline Air Taxi Flights & 227,456 & 28,432 & \\
\hline \multicolumn{4}{|l|}{ Estimaled Flights (nmiles/avg. trip length) } \\
\hline Commuter Flights & 6,090 & 761 & \\
\hline Air Taxi Flights & 247,225 & 30,903 & \\
\hline \multicolumn{4}{|c|}{ GA Flights = Total Landings-Commuter Flts.-Air Taxi Flts. } \\
\hline Based on hrs Javg. tip time & $14,111,006$ & $1,763,876$ & \\
\hline \multirow{2}{*}{ Based on nmiles/avg. trip length } & $14,091,011$ & $1,761,376$ & \\
\hline & $285,249,000$ & $35,656,125$ & $0.92 \%$ \\
\hline Commutor Nmiles (3.3) & 160,000 & 20,000 & \\
\hline Air Taxi Nmiles (3.3) & $4,781,000$ & 597,625 & \\
\hline \multicolumn{4}{|l|}{ GA Nmiles = Total Nmiles } \\
\hline -Commuter Nmiles - Air Taxi Nmiles & $280,308,000$ & $35,038,500$ & $1.04 \%$ \\
\hline Average Speed (nmiles/hour) & 57.27 & 57.23 & \\
\hline Averagg Trip Length (nmiles/anding) & 19.89 & 20.40 & \\
\hline Average Trip Time (hour/anding) & 0.35 & 0.36 & \\
\hline Average Commuter Speed (nmileshour & 60.38 & 62.45 & \\
\hline Average Air Taxi Speed (nmiles/hour) & 62.89 & 63.82 & \\
\hline GA Average Speed (nmiles/hour) & 57.18 & 57.13 & \\
\hline
\end{tabular}


Table 3.22

General Aviation Operations

Turbine Engine, Rotery Wing

Roforences 3.1 io 3.8

Parameter (Rof. Table No.)

1988

1987

1988

1989

1990

1991

1992

1993

1994

\begin{tabular}{|c|c|c|c|c|c|c|c|c|c|}
\hline Population (2.1) & 4,899 & 4,479 & 4,434 & 4,685 & 4,620 & 4,626 & 4,390 & 4.090 & \\
\hline No. Active (2.1) & 4,022 & 3,520 & 3,822 & 4,232 & 3,938 & 3,822 & 3,541 & 2,948 & \\
\hline Total Hours Flown (2.1) & $1,820,936$ & $1,631,439$ & $2,130,764$ & $2,079,847$ & $1,617,292$ & $2,171,911$ & $1,866,326$ & $1,467,670$ & \\
\hline Commuter Hrs. (3.2) & 131,669 & 5,198 & 130,227 & 14,378 & 93,508 & 3,224 & 16,135 & 0 & \\
\hline Air Taxi Hrs. (3.2) & 788,841 & 804,820 & 616,419 & 978,686 & 593,753 & 657,035 & 664,835 & 281,595 & \\
\hline GA Hrs. = Total Hrs. & & & & & & & & & \\
\hline - Commuler Hrs - Air Taxi Hrs. & 900,426 & 821,421 & $1,384,118$ & $1,086,783$ & 930,031 & $1,511,652$ & $1,185,356$ & $1,186,075$ & \\
\hline Total Day Hours Flown (4.1) & $1,577,446$ & $1,462,698$ & $1,887,467$ & NA & $1,401,958$ & $1,228,993$ & $1,609,221$ & 989,474 & \\
\hline Total Night Hours Flown (4.1) & 209,023 & 169,794 & 257,096 & NA & 211,881 & 220,469 & 257,105 & 210,530 & \\
\hline VMC Day Hours Flown (4.2) & $1,563,634$ & $1,451,160$ & $1,874,178$ & NA & $1,392,627$ & $1,226,337$ & $1,601,491$ & 985,496 & \\
\hline VMC Night Hours Flown (4.2) & 194,745 & 166,437 & 247,131 & NA & 198,648 & 219,805 & 255,424 & 203,188 & \\
\hline Total VMC Hours Flown (4.2) & $1,758,379$ & $1,617,597$ & $2,121,309$ & NA & $1,591,275$ & $1,446,142$ & $1,856,915$ & $1,188,684$ & \\
\hline IMC Day Hours Flown (4.3) & 13,832 & 11,436 & 13,481 & NA & 9,297 & 2,670 & 7,730 & 3,978 & \\
\hline IMC Night Hours Flown (4.3) & 14,244 & 3,372 & 9,882 & NA & 13,128 & 666 & 1,681 & 7,342 & \\
\hline Total IMC Hours Flown (4.3) & 28,076 & 14,808 & 23,363 & NA & 22,425 & 3,336 & 9,411 & 11,320 & \\
\hline Total Landings (2.5) & $3,450,604$ & $3,182,811$ & $4,003,396$ & $4,715,450$ & $3,763,986$ & $4,725,551$ & $2,783,251$ & $2,639,668$ & \\
\hline Local Landings (2.6) & $2,271,982$ & $1,306,672$ & $2,497,360$ & NA & $1,860,388$ & $3,594,838$ & 521,775 & $1,583,167$ & \\
\hline Cross Country Landinge (2.7) & $1,202,266$ & $1,842,805$ & $1,504,849$ & NA & $1,853,186$ & $1,131,195$ & $2,309,526$ & $1,058,663$ & \\
\hline Estimated Flights (hours/avg. trip time) & & & & & & & & & \\
\hline Commuter Flights & 249,508 & 10,141 & 244,678 & 32,598 & 217,625 & 7,015 & 24,062 & 0 & \\
\hline \begin{tabular}{|l|} 
Ar Taxi Flights \\
\end{tabular} & $1,494,823$ & $1,570,141$ & $1,158,162$ & $2,218,887$ & $1,381,864$ & $1,429,549$ & 991,468 & 506,461 & \\
\hline ated Flights (nmiles/s & & & & & & & & & \\
\hline Commuter Flights & 161,787 & 7,020 & 137,443 & 19,108 & 132,544 & 4,031 & 14,460 & 0 & \\
\hline Air Taxi Flights & 948,372 & $1,094,081$ & 650,226 & $1,289,680$ & 781,728 & 754,589 & 578,210 & 7,374 & \\
\hline A Flights = Total Landings-Commuler & 8. Air Taxi Fin & & & & & & & & \\
\hline Based on hre /avg. tip time & $1,706,273$ & $1,602,529$ & $2,600,557$ & $2,463,965$ & $2,164,497$ & $3,288,988$ & $1,767,721$ & $2,133,207$ & \\
\hline Based on nmiles/avg. tip lengt & $2,340,444$ & $2,081,710$ & $3,215,727$ & $3,406,663$ & $2,849,714$ & $3,966,930$ & $2,190,581$ & $2,182,294$ & \\
\hline Total Nautical Miles Flown (3.3) & $127,051,000$ & $113,348,000$ & $168,940,000$ & $150,292,000$ & $126,513,000$ & $179,343,000$ & $148,975,000$ & $130,981,000$ & \\
\hline Commuler Nmiles (3.3) & $5,957,000$ & 250,000 & $5,800,000$ & 609,000 & $4,455,000$ & 153,000 & 774,000 & 0 & \\
\hline Air Taxi Nmiles (3.3) & $34,919,000$ & $38,963,000$ & $27,439,000$ & $41,105,000$ & $26,275,000$ & $28,638,000$ & $30,949,000$ & $22,695,000$ & \\
\hline GA Nmiles = Total Nmiles & & & & & & & & & \\
\hline - Commuter Nmiles - Air Tax Nmiles & $86,175,000$ & $74,135,000$ & $135,701,000$ & $108,578,000$ & $95,783,000$ & $150,552,000$ & $117,252,000$ & $108,286,000$ & \\
\hline Average Speed (nmileshour) & 69.77 & 69.48 & 79.29 & 72.26 & 78.23 & 82.57 & 79.82 & 89.24 & \\
\hline Average Trip Length (nmiles/anding) & 36.82 & 35.61 & 42.20 & 31.87 & 33.61 & 37.95 & 53.53 & 49.62 & \\
\hline Average Trip Time (hourlanding) & 0.53 & 0.51 & 0.53 & 0.44 & 0.43 & 0.46 & 0.67 & 0.56 & \\
\hline Average Commuter Speod (nmiles/hou & 45.24 & 48.10 & 44.54 & 42.36 & 47.64 & 47.46 & $\mathbf{4 7 . 9 7}$ & N.A. & \\
\hline Average Air Taxi Speod (nmiles/hour) & 44.27 & 48.41 & 44.51 & 42.00 & 44.25 & 43.59 & 46.55 & 80.59 & \\
\hline GA Average Speod (nmiles/hour) & 95.70 & 90.25 & 98.04 & 99.91 & 102.99 & 99.59 & 98.92 & 91.30 & \\
\hline
\end{tabular}

$N A=$ Information not available

N.A. $=$ Not Applicable 
Table 3.22 (Continued)

General Aviation Oporatione

Turbine Engine, Rotery Wing

Rotorences 3.1 to 3.8

Paramoter (Rof. Tabla No.)

Toled

Average

Pet. of Tol.

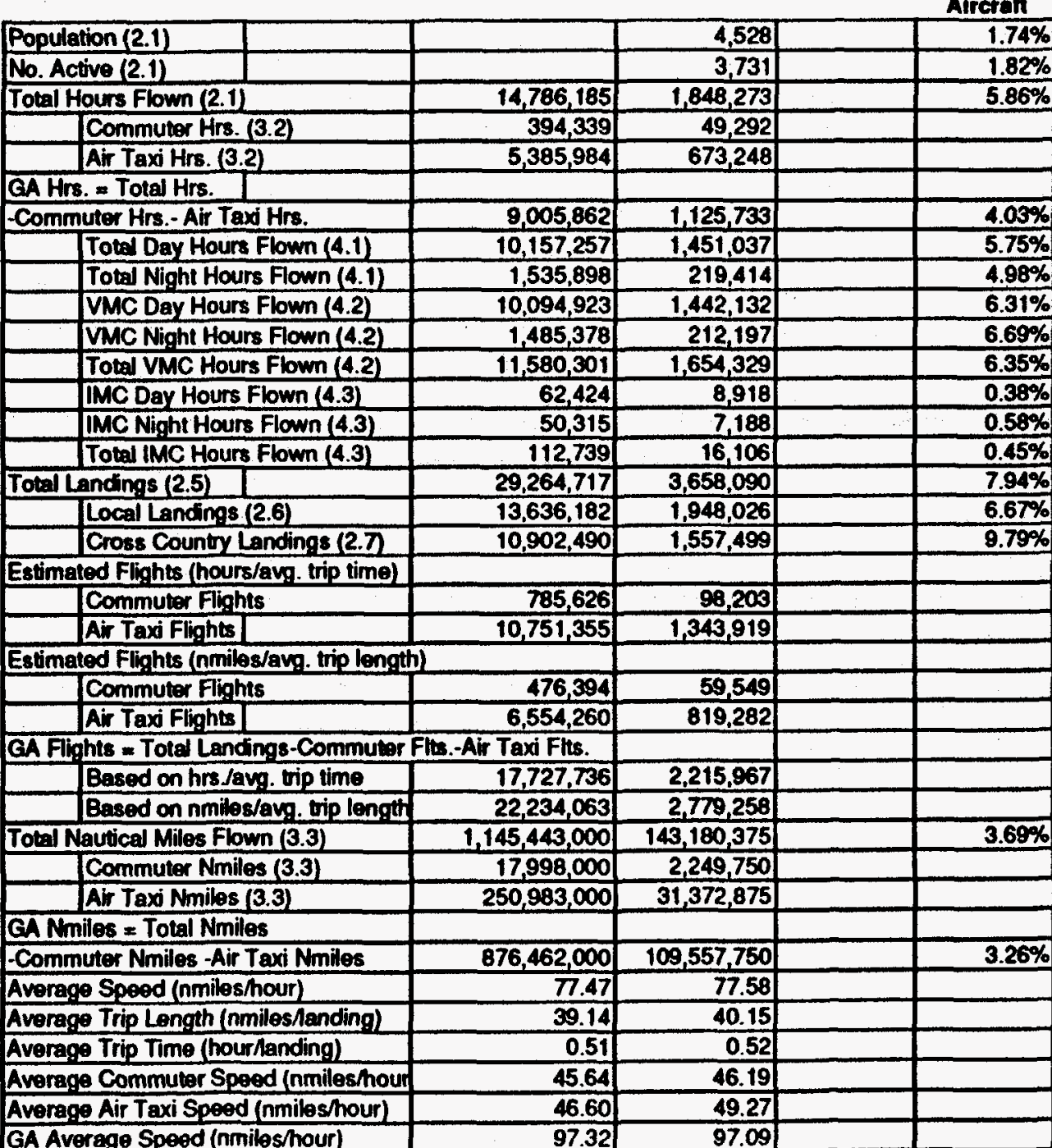

GA Average Speod (nmiles/hour)

97.32

97.09 
Toble 3.23

General Aviation Operatione

Total, Rotary Wing

Roforences 3.1 to 3.8

1988

1969

1990

1991

1892

1998

1994

\begin{tabular}{|c|c|c|c|c|c|c|c|c|c|}
\hline Population (2.1) & 10,465 & 10,034 & 9,768 & 10,469 & 10,422 & 10,474 & 9,599 & 9,819 & \\
\hline No. Active (2.1) & 6.943 & 6,333 & 6,406 & 7,476 & 7,397 & 6,292 & 5,752 & 5,445 & \\
\hline Total Hours Flown (2.1) & $2,625,394$ & $2,283,126$ & $2,706,719$ & $2,828,620$ & $2,392,066$ & $2,755,599$ & $2,282,701$ & $1,893,155$ & \\
\hline Commuter Hrs. (3.2) & 132,944 & 5,198 & 131,412 & 14,378 & 93,698 & 3,224 & 16,135 & 0 & \\
\hline Air Taxi Hrs. (3.2) & 803,141 & 810,131 & 621,811 & 998,858 & 593,753 & 659,537 & 692,261 & 282,512 & \\
\hline \multicolumn{10}{|l|}{ GA Hirs. = Total Hrs. } \\
\hline - Commuter Hrs. - Air Taxi Hrs. & $1,689,309$ & $1,467,797$ & $1,953,496$ & $1,815,384$ & $1,704,615$ & $2,092,838$ & $1,574,305$ & $1,610,643$ & \\
\hline Total Day Hours Flown (4.1) & $2,218,591$ & $2,048,770$ & $2,381,709$ & NA & $2,044,152$ & $1,662,239$ & $1,969,485$ & $1.324,142$ & \\
\hline Total Night Hours Flown (4.1) & 376,841 & 250,074 & 342,287 & NA & 345,029 & 289,127 & 313,216 & 248,020 & \\
\hline VMC Day Hours Fown (4.2) & $2,204,782$ & $2,037,197$ & $2,367,077$ & NA & $2,034,821$ & $1,659,368$ & $1,961,691$ & $1,318,815$ & \\
\hline VMC Night Hours Flown (4.2) & 361,923 & 246,717 & 331,878 & NA & 331,796 & 288,463 & 311,503 & 237,716 & \\
\hline Total VMC Hours Flown (4.2) & $2,566,705$ & $2,283,914$ & $2,698,955$ & NA & $2,366,617$ & $1,947,831$ & $2,273,194$ & $1,556,531$ & \\
\hline IMC Day Hours Flown (4.3) & 13,832 & 11,470 & 14,802 & NA & 9,297 & 2,885 & 7,794 & 3,978 & \\
\hline IMC Night Hours Flown (4.3) & 14,887 & 3,372 & 10,332 & NA & 13,128 & 666 & 1,713 & 7,545 & \\
\hline Total IMC Hours Flown (4.3) & 28,719 & 14,842 & 25,134 & NA & 22,425 & 3.551 & 9,507 & 11,523 & \\
\hline 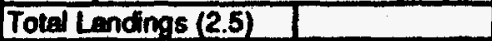 & $5,026,326$ & $4,915,280$ & $5.348,658$ & $7.256,049$ & $6,205,226$ & $6.741,429$ & $4,193,382$ & $3,922,693$ & \\
\hline Locel Landings (2.6) & $3,700,187$ & $2,672,249$ & $3,599,952$ & NA & $3.936,878$ & $5,456,280$ & $1,785,102$ & $2.750,456$ & \\
\hline Cross County Landings (2.7) & $1,360,327$ & $2,209,829$ & $1,740,829$ & NA & $2,208,216$ & 1.280 .304 & $2,458,520$ & $1,180,863$ & \\
\hline \multicolumn{10}{|l|}{ Estimated Flights (hours/avg. trip time) } \\
\hline Commuter Flights & 254,522 & 11,191 & 259,679 & 36,883 & 243,061 & 7,887 & 29,640 & 0] & \\
\hline Air Taxi Flights & $1,537,616$ & $1,744,109$ & $1,228,740$ & $2,562,296$ & $1,540,247$ & $1,613,523$ & $1,271,702$ & 585,376 & \\
\hline \multicolumn{10}{|l|}{ Estimated Flights (nmiles/avg vip length) } \\
\hline Commuter Flights & 172,796 & 8,162 & 156,030 & 22,895 & 163,811 & 4,850 & 18,760 & of & \\
\hline Air Taxi Flights & $1,023,794$ & $1,286,276$ & 738,834 & $1,588,675$ & 963,322 & 912,750 & 791,907 & 575,378 & \\
\hline \multicolumn{10}{|c|}{ GA Flights = Total Landings-Commuter Fits.-Air Taxi Fits. } \\
\hline Based on hrs./avg trip time & $3,234,188$ & $3,159,980$ & $3,860,239$ & $4,656,870$ & $4,421,919$ & $5,120,019$ & $2,892,040$ & $3,337,317$ & \\
\hline Based on nmiles/avg. trip lengt & $3,829,736$ & $3,620,842$ & $4,453,794$ & $5,644,479$ & $5,078,093$ & $5,823,829$ & $3,382,715$ & $3,347,315$ & \\
\hline Total Nautical Miles Flown (3.3) & $175,780,000$ & $150,545,000$ & $200,913,000$ & $193,012,000$ & $169,250,000$ & $212,668,000$ & $173,008,000$ & $155,516,000$ & \\
\hline Commuter Nmiles (3.3) & $6,043,000$ & 250,000 & $5,861,000$ & 609,000 & $4,468,000$ & 153,000 & 774,000 & of & \\
\hline Air Taxi Nmilos (3.3) & $35,804,000$ & $39,396,000$ & $27,753,000$ & $42,259,000$ & $26,275,000$ & $28,794,000$ & $32,672,000$ & $22,811,000$ & \\
\hline \multicolumn{10}{|l|}{ GA Nmiles = Total Nmiles } \\
\hline -Commuter Nmiles -Air Taxi Nmiles & $133,933,000$ & $110,899,000$ & $167,299,000$ & $150,144,000$ & $138,507,000$ & $183,721,000$ & $139,562,000$ & $132,705,000$ & \\
\hline Average Speed (nmileshour) & 66.95 & 65.94 & 74.23 & 68.24 & 70.75 & 77.18 & 75.79 & 82.15 & \\
\hline Average Trip Length (nmiles/anding) & 34.97 & 30.63 & 37.56 & 26.60 & 27.28 & 31.55 & 41.26 & 39.65 & \\
\hline Average Trip Time (hour/anding) & 0.52 & 0.46 & 0.51 & 0.39 & 0.39 & 0.41 & 0.54 & 0.48 & \\
\hline Average Commuter Speed (nmileshou & 45.46 & 48.10 & 44.60 & 42.36 & 47.69 & 47.46 & 47.97 & N.A. & \\
\hline Average Air Taxi Speed (nmiles/hour) & 44.58 & 48.63 & 44.63 & 42.31 & 44.25 & 43.66 & 47.20 & 80.74 & \\
\hline GA Average Speed (nmiles/hour) & 79.28 & 75.55 & 85.64 & 82.71 & 81.25 & 87.79 & 88.65 & 82.39 & \\
\hline
\end{tabular}

NA = Information not available

N.A. $=$ Not Applicable 
Trble 3.23 (Continued)

General Aviation Operations

Total, Rotary Wing

Roforences 3.1 10 3.8

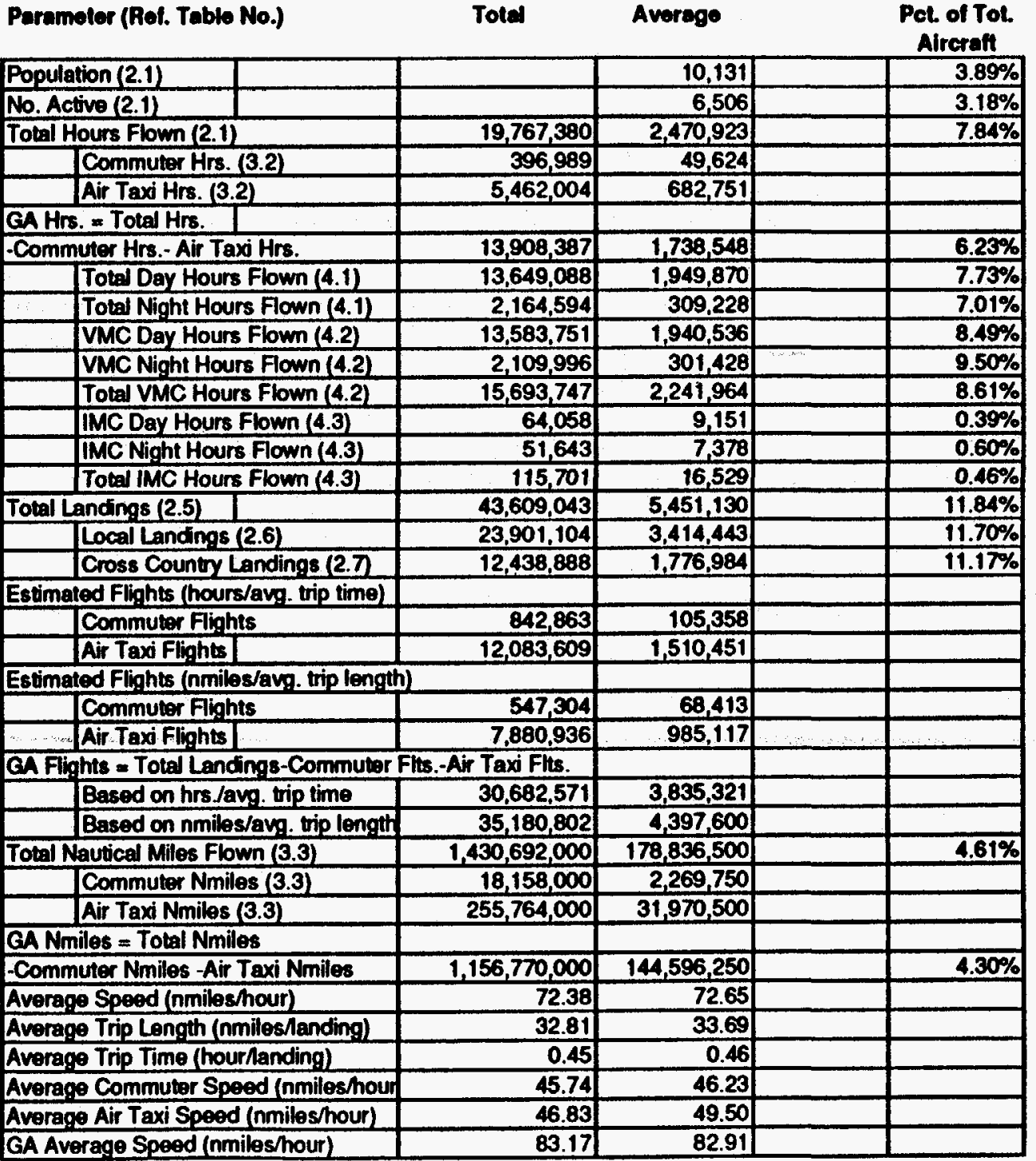


Table 3.24

Genoral Aviation Operations

Tolal Powered Aircraft

Reforences 3.1 to 3.8

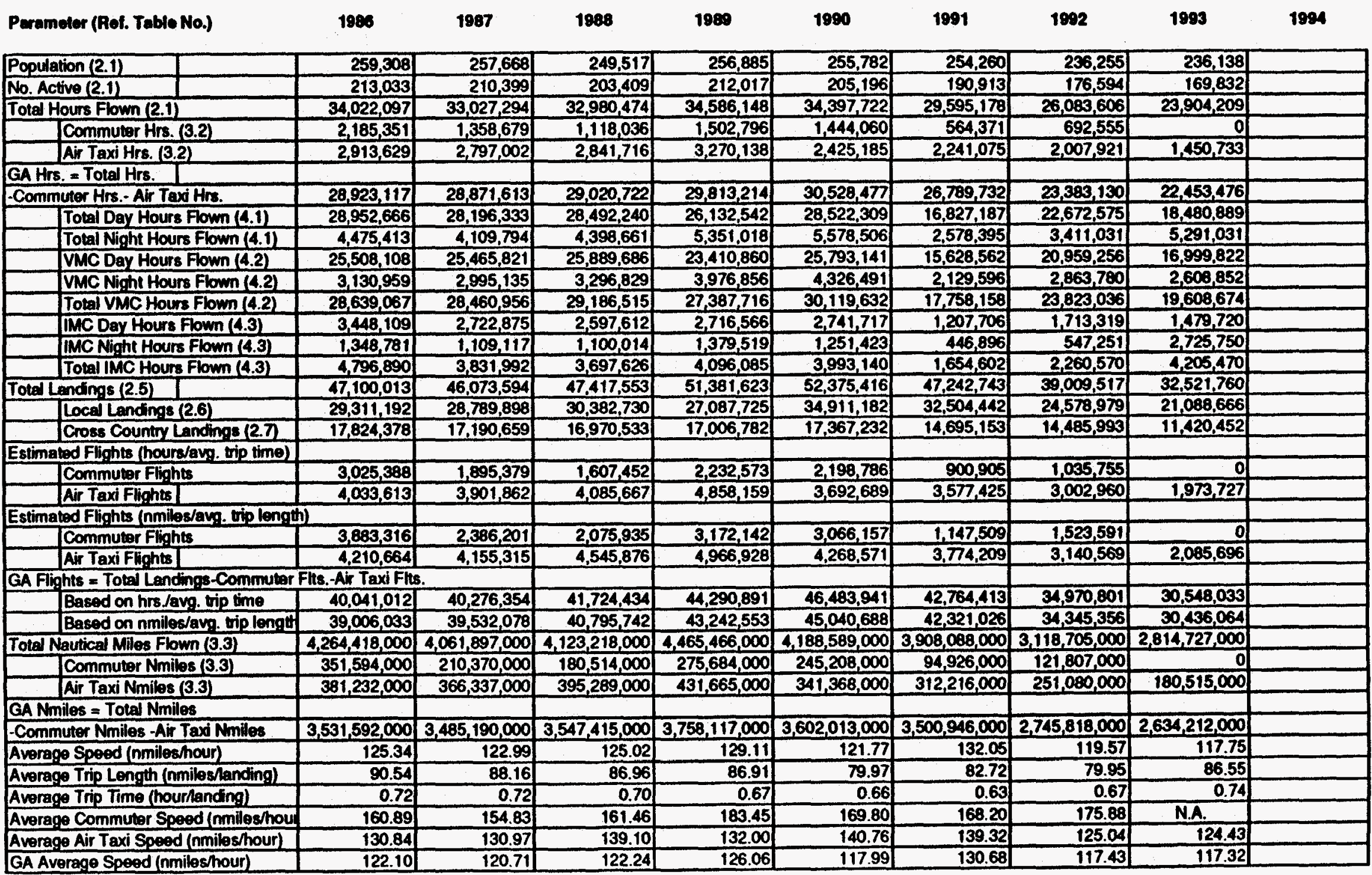

N.A. $=$ Not Applicable 
Table 3.24 (Continued)

General Aviation Operstions

Total Powered Aircraft

Roferences 3.1 to 3.8

Paramoler (Rof. Table No.)

Totel

Averege

Pet. of Tot.

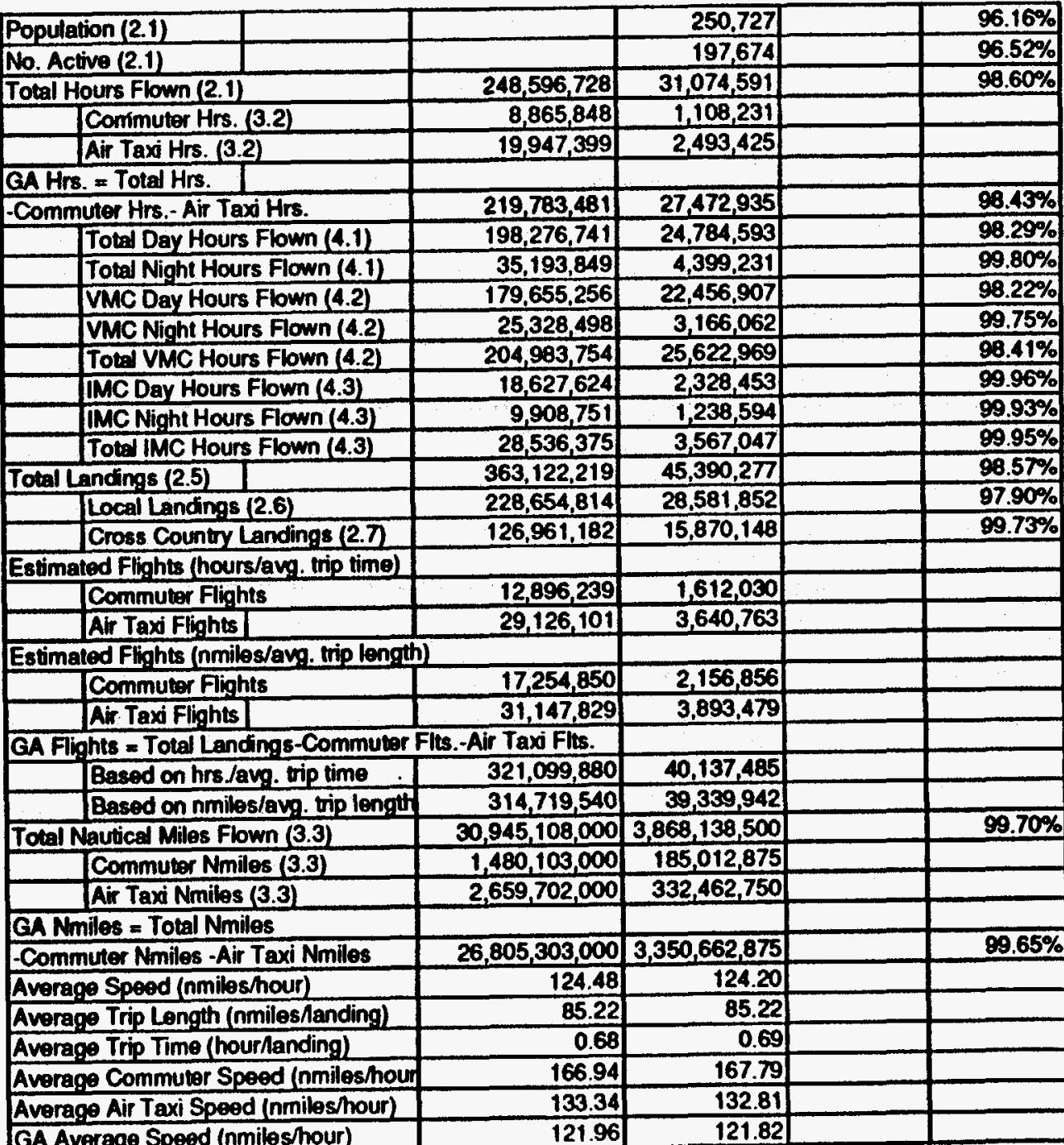


Tablo 3.25

General Aviation Operatione

Other Alrcraft

References 3.1 to 3.8

1987

1988

1989

1990

1991

1992

1993

1994

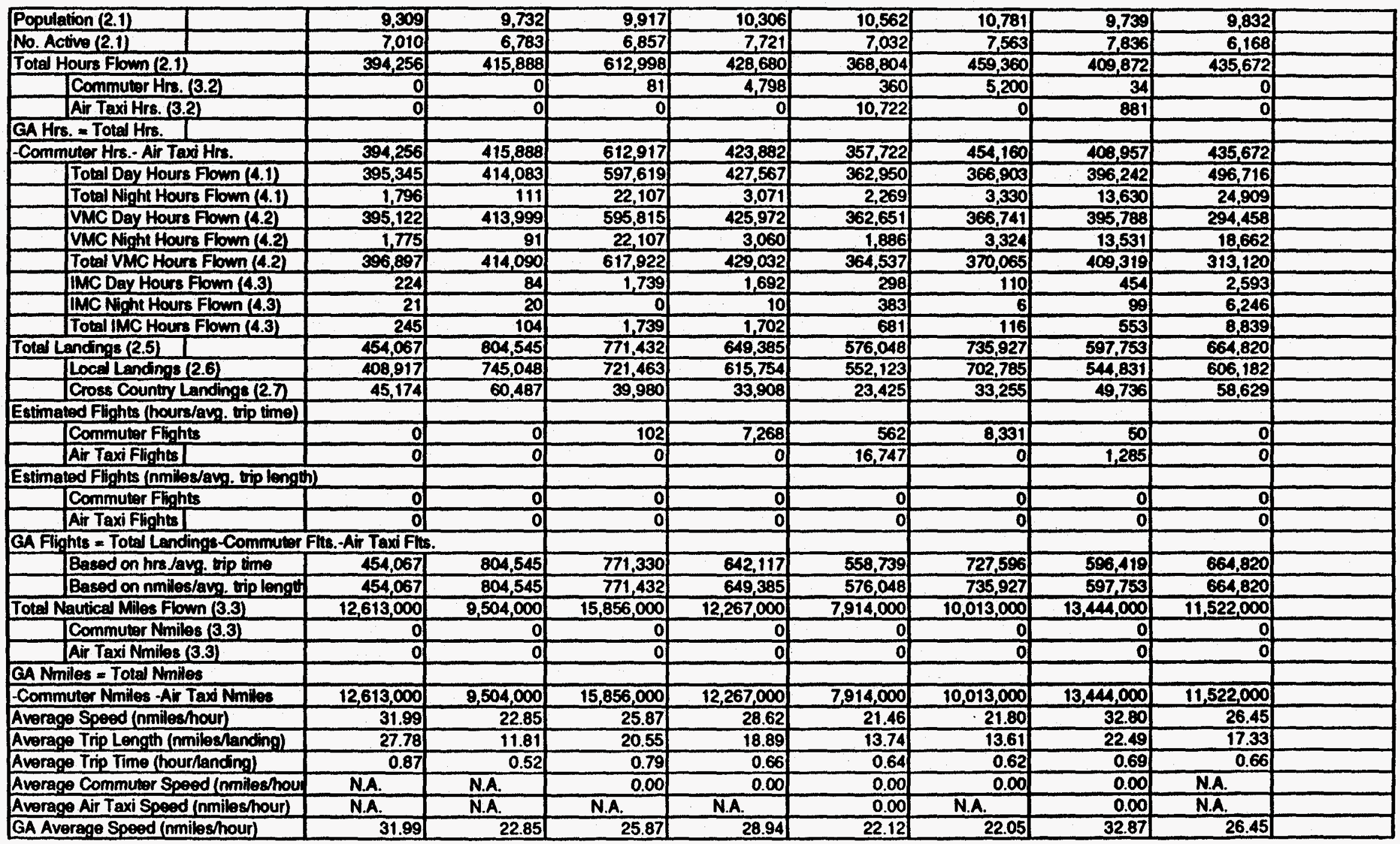

N.A. = Not Applicable 
Table 3.25 (Continued)

General Aviation Operations

Other Aircraft

Reforences 3.1 to 3.8

\begin{tabular}{|c|c|c|c|}
\hline Paramoter (Rof. Table No.) & Total & Averege & $\begin{array}{c}\text { Pot. of Tol. } \\
\text { Alrctaft }\end{array}$ \\
\hline Population (2.1) & & 10,022 & $3.84 \%$ \\
\hline No. Active (2.1) & & 7,121 & $3.48 \%$ \\
\hline Total Hours Flown (2.1) & $3,525,530$ & 440,691 & $1.40 \%$ \\
\hline Commuter Hrs. (3.2) & 10,473 & 1,309 & \\
\hline Air Taxi Hrs. (3.2) & 11,603 & 1,450 & \\
\hline \multicolumn{4}{|l|}{ GA Hrs. = Total Hrs. } \\
\hline - Commuter Hrs. - Air Taxi Hrs. & $3,503,454$ & 437,932 & $1.57 \%$ \\
\hline Total Day Hours Flown (4.1) & $3,457,425$ & 432,178 & $1.7 .1 \%$ \\
\hline Total Night Hours Flown (4.1) & 71.223 & 8,903 & $0.20 \%$ \\
\hline VMC Day Hours Flown (4.2) & $3,250,546$ & 406,318 & $1.78 \%$ \\
\hline VMC Night Hours Flown (4.2) & 64,436 & 8,055 & $0.25 \%$ \\
\hline Total VMC Hours Flown (4.2) & $3,314,982$ & 414,373 & $1.59 \%$ \\
\hline IMC Day Hours Flown (4.3) & 7,194 & 899 & $0.04 \%$ \\
\hline IMC Night Hours Flown (4.3) & 6,785 & 848 & $0.07 \%$ \\
\hline Total IMC Hours Flown (4.3) & 13,979 & 1,747 & $0.05 \%$ \\
\hline Total Landings (2.5) & $5,253,977$ & 656,747 & $1.43 \%$ \\
\hline Local Landings (2.6) & $4,897,103$ & 612,138 & $2.10 \%$ \\
\hline Cross County Landings (2.7) & 344,594 & 43,074 & $0.27 \%$ \\
\hline \multicolumn{4}{|l|}{ Estimated Flights (hours/avg. trip time) } \\
\hline Commuter Flights & 16,313 & 2,039 & \\
\hline Air Taxi Flights & 18,032 & 2,254 & \\
\hline \multicolumn{4}{|l|}{ Estimated Flights (nmiles/avg. trip length) } \\
\hline Commuter Flights & 이 & of & \\
\hline Air Taxi Flights & of & 0 & \\
\hline \multicolumn{4}{|c|}{ GA Flights = Total Landings-Commuter Flts. -Air Taxi Flos. } \\
\hline Based on hrs./avg. trip time & $5,219,632$ & 652,454 & \\
\hline Based on nmilos/avg. trip length & $5,253,977$ & 656,747 & \\
\hline Total Nauticel Miles Flown (3.3) & $93,133,000$ & $11,641,625$ & $0.30 \%$ \\
\hline Commuter Nmiles (3.3) & 0 & 0 & \\
\hline Air Tax Nmiles (3.3) & o & 0 & \\
\hline \multicolumn{4}{|l|}{ GA Nmiles = Total Nmiles } \\
\hline - Commuter Nmiles - Air Taxi Nmiles & $93,133,000$ & $11,641,625$ & $0.35 \%$ \\
\hline Average Speed (nmiles/hour) & 26.42 & 26.48 & \\
\hline Average Trip Length (nmiles/anding) & 17.73 & 18.28 & \\
\hline Average Trip Time (hour/anding) & 0.67 & 0.68 & \\
\hline Average Commuter Speed (nmiles/hour & 0.00 & 0.00 & \\
\hline Average Air Taxi Speed (nmilesshour) & 0.00 & 0.00 & \\
\hline GA Average Speed (nmiles/hour) & 26.58 & 26.64 & \\
\hline
\end{tabular}


Table 3.26

Ceneral Aviation Operations

Totel Aircraft

Roferences 3.1 to 3.8

Perameter (Rof. Table No.)

1988

1887

1888

1890

1991

1992

1993

1894

\begin{tabular}{|c|c|c|c|c|c|c|c|c|c|}
\hline Population (2.1) & 268,617 & 267,400 & 259,434 & 267,191 & 266,344 & 265,041 & 245,994 & 245,970 & \\
\hline No. Active (2.1) & 220,043 & 217,182 & 210,266 & 219,738 & 212,228 & 198,476 & 184,430 & 176,000 & \\
\hline Total Hours Flown (2.1) & $34,416,353$ & $33,443,182$ & $33,593,472$ & $35,014,828$ & $34,766,526$ & $30,054,538$ & $26,493,478$ & $24,339,881$ & \\
\hline Commuter Hrs. (3.2) & $2,185,351$ & $1,358,679$ & $1,118,117$ & $1,507,594$ & $1,444,420$ & 569,571 & 692,589 & 0 & \\
\hline Air TaxiHrs. (3.2) & $2,913,629$ & $2,797,002$ & $2,841,716$ & $3,270,138$ & $2,435,907$ & $2,241,075$ & $2,008,802$ & $1,450,733$ & \\
\hline GA Hrs. = Total Hrs. & & & & & & & & & \\
\hline -Commuter Hrs. - Air Taxi Hrs. & $29,317,373$ & $29,287,501$ & $29,633,639$ & $30,237,096$ & $30,886,199$ & $27,243,892$ & $23,792,087$ & $22,689,148$ & \\
\hline Total Day Hours Flown (4.1) & $29,348,011$ & $28,610,416$ & $29,089,859$ & $26,560,109$ & $28,885,259$ & $17,194,090$ & $23,068,817$ & $18,977,605$ & \\
\hline Total Night Hours Flown (4.1) & $4,477,209$ & $4,109,905$ & $4,420,768$ & $5,354,089$ & $5,580,775$ & $2,581,725$ & $3,424,661$ & $5,315,940$ & \\
\hline VMC Day Hours Flown (4.2) & $25,903,230$ & $25,879,820$ & $26,485,501$ & $23,836,832$ & $26,155,792$ & $15,995,303$ & $21,355,044$ & $17,294,280$ & \\
\hline VMC Night Hours Flown (4.2) & $3,132,734$ & $2,995,226$ & $3,318,936$ & $3,979,916$ & $4,328,377$ & $2,132,920$ & $2,877,311$ & $2,627,514$ & \\
\hline Total VMC Hours Flown (4.2) & $29,035,964$ & $28,875,046$ & $29,804,437$ & $27,816,748$ & $30,484,169$ & $18,128,223$ & $24,232,355$ & $19,921,794$ & \\
\hline IMC Day Hours Flown (4.3) & $3,448,333$ & $2,722,959$ & $2,599,351$ & $2,718,258$ & $2,742,015$ & $1,207,816$ & $1,713,773$ & $1,482,313$ & \\
\hline IMC Night Hours Flown (4.3) & $1,348,802$ & $1,109,137$ & $1,100,014$ & $1,379,529$ & $1,251,806$ & 446,902 & 547,350 & $2,731,996$ & \\
\hline Toual imC Hours Flown (4.3) & $4,797,135$ & $3,832,096$ & $3,699,365$ & $4,097,787$ & $3,993,821$ & $1,654,718$ & $2,261,123$ & $4,214,309$ & \\
\hline Total Landings (2.5) & $47,554,080$ & $46,878,139$ & $48,188,985$ & $52,031,008$ & $52,951,464$ & $47,978,670$ & $39,607,270$ & $33,186,580$ & \\
\hline Local Landings (2.6) & $29,720,109$ & $29,534,946$ & $31,104,193$ & $27,703,479$ & $35,463,305$ & $33,207,227$ & $25,123,810$ & $21,694,848$ & \\
\hline Cross Country Landings (2.7) & $17,869,552$ & $17,251,146$ & $17,010,513$ & $17,040,690$ & $17,390,657$ & $14,728,408$ & $14,535,729$ & $11,479,081$ & \\
\hline Estimated Flights (hours/avg. vip time) & & & & & & & & & \\
\hline Commuter Flights & $3,019,563$ & $1,904,494$ & $1,603,911$ & $2,240,240$ & $2,199,937$ & 909.256 & $1,035,408$ & 0 & \\
\hline Air Taxi Flights & $4,025,846$ & $3,920,627$ & $4,076,370$ & $4.859,329$ & $3.710,030$ & 3.577 .623 & $3,003,123$ & $1,978,024$ & \\
\hline ted Flights /nmies/ & & & & & & & & & \\
\hline Commuter Flights & $3,909,191$ & $2,422,202$ & $2,101,626$ & $3,203,433$ & $3,094,034$ & $1,162,406$ & $1,540,298$ & $\overline{0}$ & \\
\hline Air Taxi Flights & $4,238,720$ & $4,218,007$ & $4,602,135$ & $5,015,923$ & $4,307,381$ & $3,823,206$ & $3,175,006$ & $2,119,656$ & \\
\hline Fights = Total Landings $-C_{c}$ & -Air Taxi Flts & & & & & & & & \\
\hline Based on hrs./avg trip time & $40,508,670$ & $41,063,018$ & $42,508,705$ & $44,931,438$ & $47,041,498$ & $43,491,792$ & $35,568,739$ & $31,208,556$ & \\
\hline Based on nmiles/avg. trip lengt & $39,406,169$ & $40,237,931$ & $41,485,224$ & $43,811,652$ & $45,550,050$ & $42,993,058$ & $34,891,966$ & $31,066,924$ & \\
\hline Total Nautical Miles Flown (3.3) & $4,277,031,000$ & $4,071,401,000$ & $4,139,074,000$ & $4,477,733,000$ & $4,196,503,000$ & $3,918,101,000$ & $3,132,149,000$ & $2,826,249,000$ & \\
\hline Commuter Nmiles (3.3) & $351,594,000$ & $210,370,000$ & $180,514,000$ & $275,684,000$ & $245,208,000$ & $94,926,000$ & $121,807,000$ & 0 & \\
\hline Air Taxi Nmiles (3.3) & $381,232,000$ & $366,337,000$ & $395,289,000$ & $431,665,000$ & $341,368,000$ & $312,216,000$ & $251.080,000$ & $180,515,000$ & \\
\hline GA Nmiles = Total Nmiles & & & & & & & & & \\
\hline -Commuter Nmiles - Air Taxi Nmiles & $3,544,205,000$ & $3,494,694,000$ & $3,563,271,000$ & $3,770,384,000$ & $3,609,927,000$ & $3,510,959,000$ & $2,759,262,000$ & $2,645,734,000$ & \\
\hline Average Speed (nmileshour) & 124.27 & 121.74 & 123.21 & 127.88 & 120.71 & 130.37 & 118.22 & 116.12 & \\
\hline Average Trip Length (nmiles/anding) & 89.94 & 86.85 & 85.89 & 86.06 & 79.25 & 81.66 & 79.08 & 85.16 & \\
\hline Average Trip Time (hour/landing) & 0.72 & 0.71 & 0.70 & 0.67 & 0.66 & 0.63 & 0.67 & 0.73 & \\
\hline Average Commuter Speed (nmiles/hou & 160.89 & 154.83 & 161.44 & 182.86 & 169.76 & 166.66 & 175.87 & N.A. & \\
\hline Average Air Taxi Speed (nmiles/hour) & 130.84 & 130.97 & 139.10 & 132.00 & 140.14 & 139.32 & 124.99 & 124.43 & \\
\hline GA Average Speod (nmiles/hour) & 120.89 & 119.32 & 120.24 & 124.69 & 116.88 & 128.87 & 115.97 & 115.59 & \\
\hline
\end{tabular}

N.A. = Not Applicable 
Table 3.26 (Continued)

General Aviation Operatione

Total Aircraft

References 3.1 to 3.8

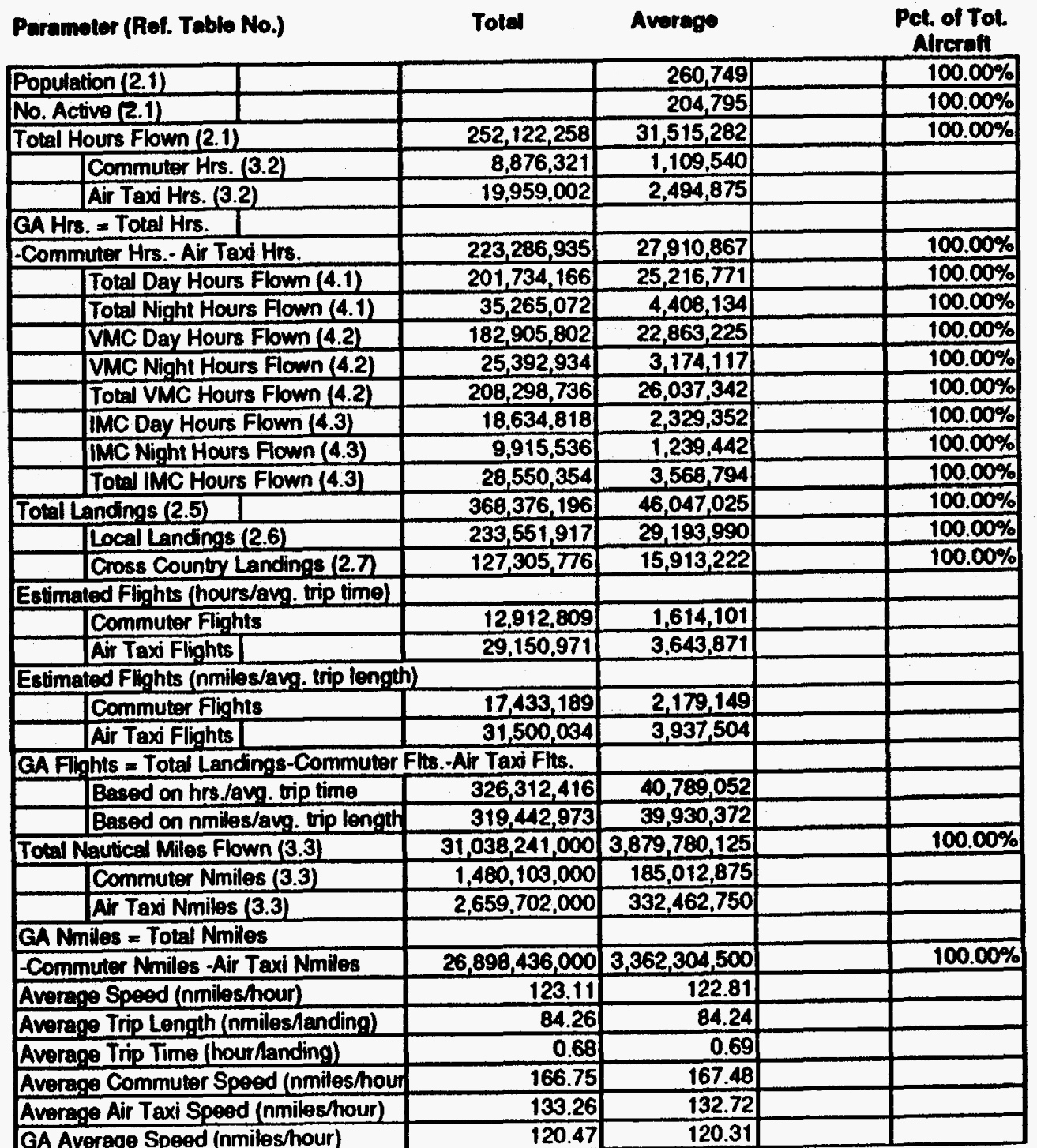


Table 3.27

General Aviation Operations Summery, 1986-1903

Roforences 3.1 to 3.8

\begin{tabular}{|c|c|c|c|c|c|c|c|c|c|c|c|c|c|}
\hline Aircrail Subcategonies & $\begin{array}{c}\text { Averago } \\
\text { No. } \\
\text { Actuo } \\
\end{array}$ & $\begin{array}{l}\text { Total } \\
\text { Hrs. } \\
\text { Flown }\end{array}$ & $\begin{array}{l}\text { Totel } \\
\text { GA Hrs. } \\
\text { Flown }\end{array}$ & $\begin{array}{l}\text { Total Nmi. } \\
\text { Flown } \\
(000)\end{array}$ & $\begin{array}{c}\text { Total GA Nmi. } \\
\text { Flown } \\
000\end{array}$ & $\begin{array}{c}\text { Total } \\
\text { Landings } \\
\text { (itis.) }\end{array}$ & $\begin{array}{c}\text { Est. GA } \\
\text { Flights } \\
\text { (based hrs./ } \\
\text { avg. trip tme) }\end{array}$ & $\begin{array}{c}\text { Est GA } \\
\text { Flights } \\
\text { (based } \mathrm{nmt} \text { / } \\
\text { avg. tip dis.) }\end{array}$ & $\begin{array}{c}\text { Averago } \\
\text { GA } \\
\text { Fliphis }\end{array}$ & $\begin{array}{l}\text { Averago } \\
\text { Spoed } \\
\text { (nml.Mr.) }\end{array}$ & $\begin{array}{c}\text { GA } \\
\text { Average } \\
\text { Speed } \\
\text { (nmi.mi) }\end{array}$ & $\begin{array}{l}\text { Average } \\
\text { Trip } \\
\text { Length } \\
\text { (nmt.m.) }\end{array}$ & $\begin{array}{c}\text { Average } \\
\text { Trip } \\
\text { Time } \\
\text { (hrs.m.) }\end{array}$ \\
\hline $\begin{array}{l}\text { Single-Engine Reciprocating, } \\
\text { Flxed Wing }\end{array}$ & 160,000 & $167,706,994$ & $162,027,642$ & $16,914,953$ & $16,319,646$ & $259,495,990$ & $250,717,981$ & $250,351,231$ & $250,534,606$ & 100.86 & 100.72 & 65.18 & 0.65 \\
\hline $\begin{array}{l}\text { Mult-Engine Reciprocating, } \\
\text { Flxed Wing }\end{array}$ & 21,572 & $32,304,815$ & $23,410,419$ & $4,693,227$ & $3,351,473$ & $30,867,775$ & $22,393,971$ & $22,090,735$ & $22,242,353$ & 145.28 & 143.16 & 152.04 & 1.05 \\
\hline Turboprop Fixed Wing & 5,318 & $17,304,615$ & $10,249,961$ & $3,267,365$ & $1,892,822$ & $19,835,017$ & $11,753,275$ & $11,564,189$ & $11,658,732$ & 188.81 & 184.67 & 164.73 & 0.87 \\
\hline Turbojet fixed Wing & 4,278 & $11,512,924$ & $10,187,072$ & $4,638,871$ & $4,084,592$ & $9,314,394$ & $8,242,047$ & $8,203,943$ & $8,222,895$ & 402.93 & 400.98 & 498.03 & 1.24 \\
\hline Total Fixed Wing & 191,168 & $228,829,348$ & $205,875,094$ & $29,514,416$ & $25,648,533$ & $319,513,176$ & $293,107,274$ & $292,210,098$ & $292,658,686$ & 128.98 & 124.58 & 92.37 & 0.72 \\
\hline $\begin{array}{l}\text { Reciprocaling Engine, } \\
\text { Rotary Wing }\end{array}$ & 2,775 & $4,981,195$ & $4,902,525$ & 285,249 & 280,306 & $14,344,326$ & $14,111,006$ & $14,091,011$ & $14,101,009$ & 57.27 & 57.18 & 19.89 & 0.35 \\
\hline Turbine Engine Rotary Wing & 3,731 & $14,786,185$ & $9,005,862$ & $1,145,443$ & 876,462 & $29,264,717$ & $17,727,736$ & $22,234,063$ & $19,980,900$ & 77.47 & 97.32 & 39.14 & 0.51 \\
\hline Total Rotary Wing & 6.506 & $19,767,380$ & $13,908,387$ & $1,430,692$ & $1,156,770$ & $43,609,043$ & $31,838,742$ & $36,325,074$ & $34,081,008$ & 72.38 & 83.17 & 32.81 & 0.45 \\
\hline Total Powered Aircraft & 197,674 & $248,596,728$ & $219,783,481$ & $30,945,108$ & $26,805,303$ & $363,122,219$ & $324,946,016$ & $328,535,172$ & $326,740,594$ & 124.48 & 121.96 & 85.22 & 0.68 \\
\hline Other Alrcraft & 7,121 & $3,525,530$ & $3,503,454$ & 93,133 & 93,133 & $5,253,977$ & $5,219,632$ & $5,253,977$ & $5,236,805$ & 26.42 & 26.58 & 17.73 & 0.67 \\
\hline Total Aircraft & 204,795 & $252,122,258$ & $223,286,935$ & $31,038,241$ & $26,898,436$ & $368,376,196$ & $330,165,648$ & $333,789,149$ & $331,977,399$ & 123.11 & 120.47 & 84.26 & 0 \\
\hline
\end{tabular}




\subsection{GENERAL AVIATION CRASH RATES}

Table 3.28 presents the general aviation accident (or crash) rates by aircraft subcategories. All accident data are taken from Table 3.15 of Section 3.2. All operations data are taken from Table 3.27 of Section 3.3.

Tables 3.29 to 3.37 presents the following information by flight phases for each subcategory of general aviation:

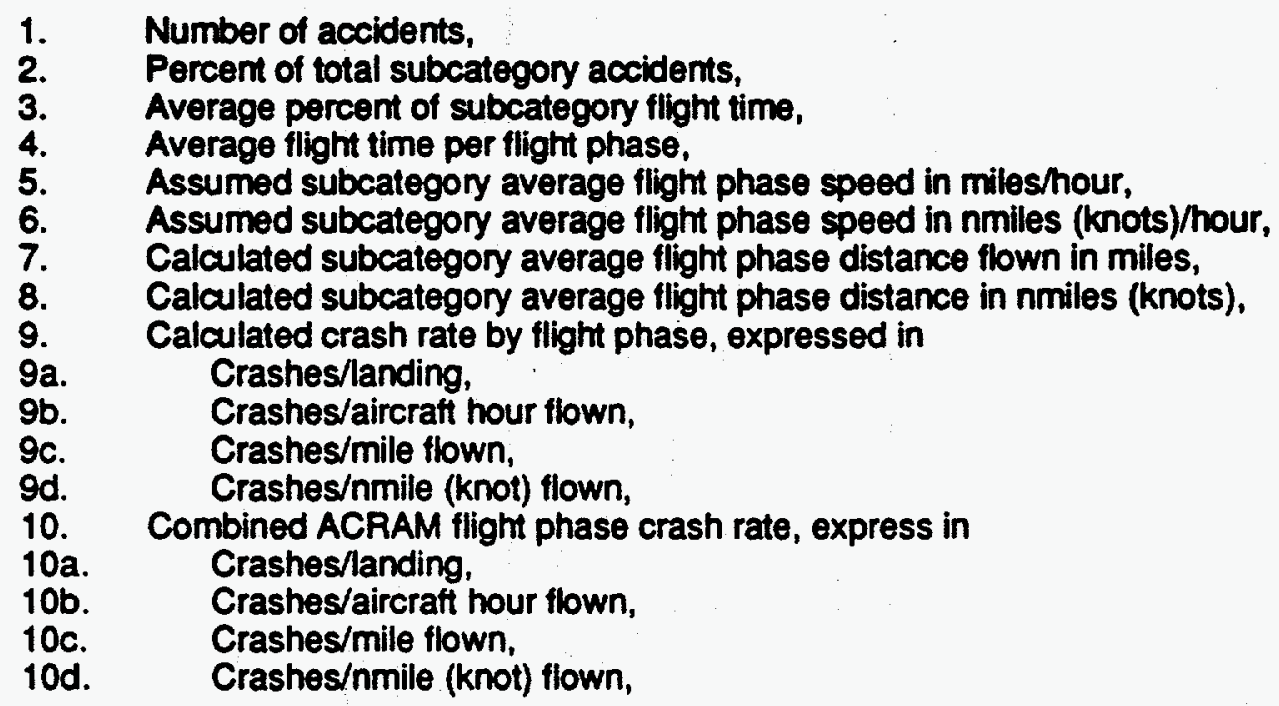

The tables listed below gives the general aviation subcategories for which the general aviation crash rate by flight phase table were created:

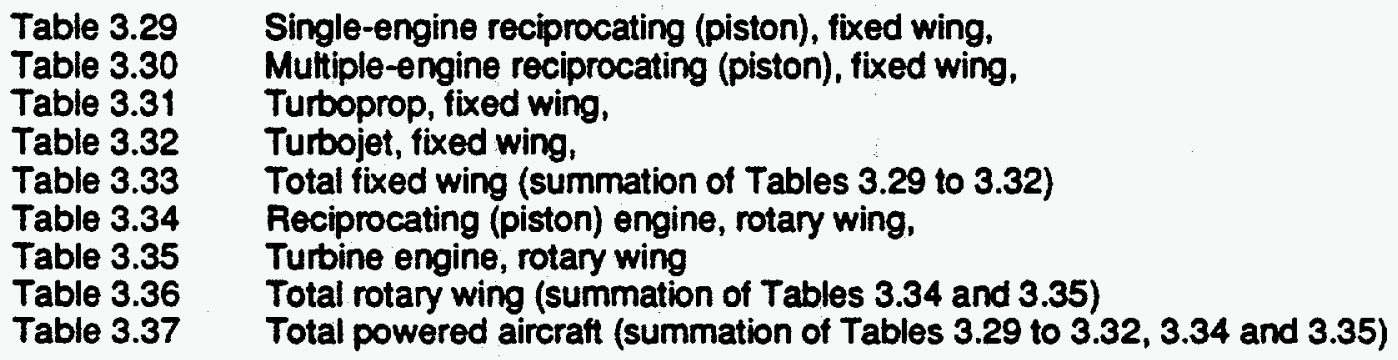

The number of accidents for each flight phase by general aviation subcategory were taken from Table 3.15 of Section 3.2. The hours average flight duration, nmiles average flight distance, and the number of landings were taken from Table 3.27 of Section 3.3. The number of estimated flights were taken to be equal to the estimated number of general aviation landings for that subcategory and is the average of the two methods used to estimate this number. The first method is to base the estimated number of flights on the number of primary use hours flown divided by the average trip time for that particular subcategory of general aviation. The other method is to base the estimated number of flights on the number of primary use nautical miles flown divided by the average trip length for that particular subcategory of general aviation. Flight times for each flight phase were assigned based on conversations with licensed pilots and instructors and engineering judgment and experience. A more formalized process for assigning flight times to the flight phases would be to solicit opinions from qualified pilots and instructors and to adjust the flight times for the flight phases appropriately. A process similar to the NRC expert opinion solicitation process could be followed if a more formalized, accepted procedure was desired. This was not done because of the time and resource constraints. Average flight phase velocities for the subcategory were then adjusted until the average flight distance was approximately equal to the average flight distance of Table 3.27. Again, as with commercial aviation, the seven flight phases used in Tables 3.29 to 3.37 were 
combined to the three defined ACRAM flight phases, takeof, landing, and in-flight or enroute. Again, as with commercial aviation, takeoft is defined as encompassing the actual takeoff roll, and the initial climb flight phase. Landing is defined as including the landing approach, and the actual landing roll. The enroute or in-flight phase is defined as including the climb to cruise, cruise or enroute, and the descent from cruise flight phases.

In the ACRAM Standard's [Ref. 3.19] estimation of the frequency of an impact with a facility, the near aiport contribution is based on the assumption that the facility is located far enough from any runway that only impacts caused by off rumway crashes need be considered, i.e., the contribution from on rumway accidents (considered to be numway overmuns and rumway nunoffs) is negligible. The fixed wing crash rates of Tables 3.29 to 3.33 include both off numway and on numay accidents, and thus they must be adjusted for use in the ACRAM Standard. Rotary wing aircraft are not considered to be capable of numway overnuns and rumway runoffs as their takeoff and landing runs are considered to be small (tens of feet versus hundreds or even thousands of feet for fixed wing aircraft). Analysis of the General Aviation NTSB database by SAIC has determined that approximately $89 \%$ of the general aviation takeoff crashes are off rumway accidents, and $94 \%$ of the general aviation landing crashes are off runway accidents. Therefore, the appropriate crash rates that should be used in the ACRAM Standard are obtained by multiplying the relevant values of Tables 3.29 to 3.33 by 0.89 for takeoffs and 0.94 for landings. These adjusted crash rates are given in Table 3.38 . 
Table 3.28

General Aviation Accident Rates by Aircraft Subcategorles

Based on FAA General Avlation Activity Survey, Calendar Years 1986-1993 [Rof. 3.1 - 3.8]

and NTSB Annual Revlew of Alrcraft Accident Data, U.S. General Avlatlon, Calendar Years 1986-1993 [Ref. 3.20 - 3.27]

\begin{tabular}{|c|c|c|c|c|c|c|c|c|c|}
\hline Aircraft Subcategories & $\begin{array}{c}\text { Total } \\
\text { GA } \\
\text { Accidents }\end{array}$ & $\begin{array}{c}\text { GA } \\
\text { Hrs. } \\
\text { Flown } \\
\end{array}$ & $\begin{array}{c}\text { GA } \\
\text { Nmi. } \\
\text { Flown }\end{array}$ & $\begin{array}{c}\text { GA } \\
\text { Miles } \\
\text { Flown } \\
\end{array}$ & $\begin{array}{c}\text { Avg. GA } \\
\text { Landings } \\
\text { (ilts.) }\end{array}$ & $\begin{array}{l}\text { AccJ } \\
\text { Hrs. }\end{array}$ & $\begin{array}{l}\text { Accd } \\
\text { Nmi. }\end{array}$ & $\begin{array}{l}\text { AccJ } \\
\text { Mille } \\
\end{array}$ & $\begin{array}{c}\text { Acc J } \\
\text { Landing } \\
\end{array}$ \\
\hline Single-Engine Reciprocating, Fixed Wing & 14,707 & $162,027,642$ & $16,319,646,000$ & $18,780,273,265$ & $250,534,606$ & $9.08 E-05$ & 9.01E-07 & 7.83E-07 & 5.87E.05 \\
\hline Multi-Engine Reciprocating, Fixed Wing & 1,268 & $23,410,419$ & $3,351,473,000$ & $3,856,798,045$ & $22,242,353$ & 5.42E-05 & 3.78E-07 & 3.29E-07 & $5.70 \mathrm{E}-05$ \\
\hline Turboprop, Fixed Wing & 299 & $10,249,961$ & $1,892,822,000$ & $2,178,216,023$ & $11,658,732$ & $2.92 E-05$ & $1.58 E-07$ & 1.37E-07 & 2.56E-05 \\
\hline Turbojet, Fixed Wing & 88 & $10,187,072$ & $4,084,592,000$ & $4,700,454,528$ & $8,222,995$ & 8.64E-06 & 2.15E-08 & $1.87 E-08$ & 1.07E-05 \\
\hline Total, Fixed Wing & 16,362 & $205,875,094$ & $25,648,533,000$ & $29,515,741,860$ & $292,658,686$ & 7.95E-05 & 6.38E-07 & 5.54E-07 & 5.59E-05 \\
\hline Reciprocating Engine, Rotary Wing & 949 & $4,902,525$ & $280,308,000$ & $322,571,999$ & $14,101,009$ & $1.94 E-04$ & $3.39 E-06$ & 2.94E-06 & 6.73E-05 \\
\hline Turbine Engine, Rotary Wing & 482 & $9,005,862$ & $876,462,000$ & $1,008,612,311$ & $19,980,900$ & 5.35E-05 & $5.50 E-07$ & 4.78E-07 & 2.41E-05 \\
\hline Total, Rotany Wing & 1,431 & $13,908,387$ & $1,156,770,000$ & $1,331,184,310$ & $34,081,909$ & 1.03E-04 & 1.24E-06 & 1.07E-06 & 4.20E-05 \\
\hline Total Powered Aircraft & 17.793 & $219,783,481$ & $26,805,303,000$ & $30,846,926,170$ & $326,740,595$ & $8.10 E-05$ & 6.64E-07 & 5.T7E-07 & 5.45E-05 \\
\hline Other Aircraft & 529 & $3,503,454$ & $93,133,000$ & $107,175,314$ & $5,236,805$ & 1.51E-04 & 5.68E-06 & 4.94E-06 & 1.01E-04 \\
\hline Total Aircraft & 18,322 & $223,286,935$ & $26,898,436,000$ & $30,954,101,485$ & $331,977,400$ & 8.21E-05 & 6.81E-07 & 5.92E-07 & 5.52E-05 \\
\hline
\end{tabular}


Table 3.29

General Aviation Single-Engine Reciprocating, Fixed Wing Crash Rates by Flight Phase

\begin{tabular}{|c|c|c|c|c|c|c|c|c|c|c|c|c|c|c|c|c|}
\hline Flight Phases & $\begin{array}{l}\text { No. of } \\
\text { Acos. }\end{array}$ & $\begin{array}{l}\text { Accs. } \\
\text { Pet. }\end{array}$ & $\begin{array}{l}\text { Average } \\
\text { Pct. } \\
\text { Fh. Tine }\end{array}$ & $\begin{array}{l}\text { Average } \\
\text { Flt. Time } \\
\text { (hours) }\end{array}$ & \multicolumn{2}{|c|}{$\begin{array}{l}\text { Assumed } \\
\text { Average Velocity }\end{array}$} & \multicolumn{2}{|c|}{$\begin{array}{l}\text { Calculated } \\
\text { Average Distance }\end{array}$} & $\begin{array}{l}\text { Crash Rate by } \\
\text { Crashes } \\
\text { Randing }\end{array}$ & $\begin{array}{l}\text { Flight Phas } \\
\text { Crashes } \\
\text { IAC Hour }\end{array}$ & $\begin{array}{l}\text { ses } \\
\text { Crashes } \\
\text { Mile }\end{array}$ & $\begin{array}{l}\text { Crashes } \\
\text { Knof }\end{array}$ & $\begin{array}{l}\text { Combined A } \\
\text { Crashes } \\
\text { Landing }\end{array}$ & $\begin{array}{l}\text { ACRAM Fligh } \\
\text { Crashes } \\
\text { IAC Hour }\end{array}$ & $\begin{array}{l}\text { ht Phases Cra } \\
\text { Crashes } \\
\text { Mile }\end{array}$ & $\begin{array}{l}\text { ash Rates } \\
\text { Crashes } \\
\text { Knot }\end{array}$ \\
\hline Takeoff & 885 & $7.50 \%$ & $1.28 \%$ & 0.008 & 69.05 & 60.00 & 0.58 & 0.50 & 3.532E-06 & 4.239E-04 & $6.139 E \cdot 06$ & 7.065E-06 & 1.225E-05 & $7.352 E-04$ & $9.127 E-06$ & $1.050 E-05$ \\
\hline Initial Climb & 2,185 & $18.52 \%$ & $1.28 \%$ & 0.008 & 92.06 & 80.00 & 0.77 & 0.67 & 8.721E-06 & 1.047E-03 & 1.137E-05 & $1.308 E-05$ & & & & \\
\hline $\begin{array}{l}\text { Climb to } \\
\text { Cruise }\end{array}$ & 457 & $3.87 \%$ & $20.51 \%$ & 0.133 & 120.83 & 105.00 & 16.11 & 14.00 & 1.824E-06 & $1.368 E-05$ & $1.132 E-07$ & $1.303 E-07$ & & & & \\
\hline $\begin{array}{l}\text { Cruiser } \\
\text { Enroute }\end{array}$ & 2,500 & $21.19 \%$ & $29.49 \%$ & 0.192 & 126.59 & 110.00 & 24.28 & 21.08 & 9.979E-06 & 5.206E-05 & 4.113E-07 & 4.733E-07 & $1.352 E-05$ & 2.749E-05 & 2.233E-07 & 2.570E-07 \\
\hline $\begin{array}{l}\text { Descent } \\
\text { from Cruise }\end{array}$ & 129 & $3.64 \%$ & $25.64 \%$ & 0.167 & 120.83 & 105.00 & 20.14 & 17.50 & $1.712 E-06$ & $1.027 E-05$ & 8.503E-08 & $9.785 E-08$ & & . & & \\
\hline $\begin{array}{l}\text { Landing } \\
\text { Approach }\end{array}$ & 1,840 & $15.60 \%$ & $20.51 \%$ & 0.133 & 97.82 & 85.00 & 13.04 & 11.33 & 7.344E-06 & $5.508 E-05$ & 5.631E-07 & 6.480E-07] & & & & \\
\hline Landing & 3,500 & $29.67 \%$ & $1.28 \%$ & 0.008 & 57.54 & 50.00 & 0.48 & 0.42 & 1.397E-05 & $1.676 E-03$ & 2.914E-05 & $3.353 E-05$ & 2.131E-05 & $1.505 E-04$ & $1.576 \mathrm{E}-06$ & $1.814 E-06$ \\
\hline OTA & .796 & $100.00 \%$ & $100.00 \%$ & 0.650 & & & 75.38 & 65.50 & 4.708E-05 & 7.244E-05 & $6.246 E-07$ & $7.188 E-07$ & 4.708E-05 & 7.244E-05 & 6.246E-07 & $7.180 E-07$ \\
\hline
\end{tabular}

Bases for Crash Rates by Fiight Phase, 1986-1993 Totals:

0.65 Hours Average Flight Duration

65.2 Nmiles Average Flight Distance 250,534,606 Landings 
Table 3.30

General Aviation Multi-Engine Reciprocating, Fixed Wing Crash Rates by Flight Phase

\begin{tabular}{|c|c|c|c|c|c|c|c|c|c|c|c|c|c|c|c|c|}
\hline Flight Phases & $\begin{array}{l}\text { No. of } \\
\text { Acas. }\end{array}$ & $\begin{array}{l}\text { Acos. } \\
\text { Pat. }\end{array}$ & $\begin{array}{l}\text { Average } \\
\text { Pct. } \\
\text { Flt. Time }\end{array}$ & $\begin{array}{l}\text { Average } \\
\text { Flt. Timo } \\
\text { (hours) }\end{array}$ & \multicolumn{2}{|c|}{$\begin{array}{l}\text { Assumed } \\
\text { Average Velocity }\end{array}$} & \multicolumn{2}{|c|}{$\begin{array}{l}\text { Calculated } \\
\text { Average Distance }\end{array}$} & $\begin{array}{l}\text { Crash Rate by } \\
\text { Crashos } \\
\text { Landing }\end{array}$ & $\begin{array}{l}\text { Flight Phase } \\
\text { Crashes } \\
\text { IAC Hour }\end{array}$ & $\begin{array}{l}\text { Crashes } \\
\text { Mile }\end{array}$ & $\begin{array}{l}\text { Crashes } \\
\text { Knot }\end{array}$ & $\begin{array}{l}\text { Combined A } \\
\text { Crashes } \\
\text { Landing }\end{array}$ & $\begin{array}{l}\text { CRAM Fligh } \\
\text { Crashes } \\
\text { IAC Hour }\end{array}$ & $\begin{array}{l}\text { Phases Cra } \\
\text { Crashes } \\
\text { Mile }\end{array}$ & $\begin{array}{l}\text { ash Rates } \\
\text { Crashes } \\
\text { Knot }\end{array}$ \\
\hline Takeoff & 71 & $6.49 \%$ & $0.79 \%$ & 0.008 & 86.31 & 75.00 & 0.72 & 0.63 & 3.192E-06 & $3.831 E-04$ & 4.438E-06 & 5.107E-06 & $1.043 E-05$ & $6.258 E-04$ & $6.043 E-06$ & $6.954 E-06$ \\
\hline Initial Climb & 161 & $14.72 \%$ & $0.79 \%$ & 0.008 & 120.83 & 105.00 & 1.01 & 0.88 & 7.238E-06 & $8.686 E-04$ & 7.189E-06 & $8.273 E-06$ & & & & \\
\hline $\begin{array}{l}\text { Climb to } \\
\text { Cruise }\end{array}$ & 56 & $5.12 \%$ & $11.11 \%$ & 0.117 & 149.60 & 130.00 & 17.45 & 15.17 & 2.518E-06 & $2.158 E-05$ & 1.443E-07 & $1.660 \mathrm{E}-07$ & & & & \\
\hline $\begin{array}{l}\text { Cruised } \\
\text { Enroute }\end{array}$ & 239 & $21.85 \%$ & $59.52 \%$ & 0.625 & 178.37 & 155.00 & 111.48 & 96.88 & $1.075 E-05$ & 1.719E-05 & 9.639E-08 & $1.109 E-07$ & $1.466 E-05$ & 1.614E-05 & $9.238 E-08$ & $1.063 E-07$ \\
\hline $\begin{array}{l}\text { Descent } \\
\text { from Cruise }\end{array}$ & 31 & $2.83 \%$ & $15.87 \%$ & 0.167 & 178.37 & 155.00 & 29.73 & 25.83 & $1.394 E-06$ & $8.362 E-06$ & 4.688E-08 & $5.395 E-08$ & & & & \\
\hline $\begin{array}{l}\text { Landing } \\
\text { Approach }\end{array}$ & & $21.57 \%$ & $11.11 \%$ & 0.117 & 120.83 & 105.00 & 14.10 & 12.25 & $1.061 E-05$ & $9.095 E-05$ & 7.527E-07 & 8.662E-07 & & & & \\
\hline Landing & 300 & $27.42 \%$ & $0.79 \%$ & 0.008 & 74.80 & 65.00 & 0.62 & 0.54 & $1.349 E-05$ & $1.619 \mathrm{E}-03$ & 2.164E-05 & $2.490 E-05$ & 2.410 E-05 & $1.928 E-04$ & 1.637E-06 & $1.884 E-06$ \\
\hline TOTAL: & 1,094 & $100.00 \%$ & $100.00 \%$ & 1.050 & & & 175.11 & 152.17 & 4.919E-05 & 4.684E-05 & $2.809 E-07$ & $3.232 E-07$ & 4.919E-0S & 4.684E-05 & $2.809 E-07$ & $3.232 E-07$ \\
\hline Bases for Cra. & sh Pates b & y Flight P & hase, 1986 & 3-1993 Tota & tals: & & & & $\begin{array}{r}1.05 \\
152.04 \\
22,242,353\end{array}$ & $\begin{array}{l}\text { Hours Avera } \\
\text { Nmiles Avere } \\
\text { Landings }\end{array}$ & $\begin{array}{l}\text { age Flight Du } \\
\text { rage Flight D }\end{array}$ & $\begin{array}{l}\text { uration } \\
\text { istance }\end{array}$ & & & & \\
\hline
\end{tabular}


Table 3.31

General Aviation Turboprop, Fixed Wing Crash Rates by Flight Phase

\begin{tabular}{|c|c|c|c|c|c|c|c|c|c|c|c|c|c|c|c|c|}
\hline Flight Phases & $\begin{array}{l}\text { No. of } \\
\text { Aocs. }\end{array}$ & $\begin{array}{l}\text { Accs. } \\
\text { Pct. }\end{array}$ & $\begin{array}{l}\text { Average } \\
\text { Pct. } \\
\text { Fit Time }\end{array}$ & $\begin{array}{l}\text { Average } \\
\text { Fit. Time } \\
\text { (hours) }\end{array}$ & \multicolumn{2}{|c|}{$\begin{array}{l}\text { Assumed } \\
\text { Average Velocity }\end{array}$} & \multicolumn{2}{|c|}{$\begin{array}{l}\text { Calculated } \\
\text { Average Distance }\end{array}$} & $\begin{array}{l}\text { Crash Rate by } \\
\text { Crashes } \\
\text { Landing }\end{array}$ & $\begin{array}{l}\text { Flight Phas } \\
\text { Crashes } \\
\text { IAC Hour }\end{array}$ & $\begin{array}{l}\text { Crashes } \\
\text { Mile } \\
\end{array}$ & $\begin{array}{l}\text { Crashes } \\
\text { Knot }\end{array}$ & $\begin{array}{l}\text { Combined A } \\
\text { Creshes } \\
\text { Landing }\end{array}$ & $\begin{array}{l}\text { CPAM Flight } \\
\text { Crashes } \\
\text { IAC Hour }\end{array}$ & $\begin{array}{l}\text { Phases Cra } \\
\text { Crashes } \\
\text { Mile }\end{array}$ & $\begin{array}{l}\text { ash Pates } \\
\text { Crashes } \\
\text { Knot }\end{array}$ \\
\hline Takeoff & 17 & $7.84 \%$ & $0.96 \%$ & 0.008 & 100.00 & 85.00 & 0.83 & 0.71 & $1.458 \mathrm{E}-06$ & $1.750 E-04$ & $1.750 E-06$ & $2.059 E-06$ & 3.946E-06 & 2.367E-04 & $1.774 E-06$ & $2.059 E-06$ \\
\hline Initial Climb & 29 & $13.55 \%$ & $0.96 \%$ & 0.008 & 166.86 & 145.00 & 1.39 & 1.21 & 2.487E-06 & $2.985 E-04$ & $1.789 \mathrm{E}-06$ & $2.059 E-06$ & & & & \\
\hline $\begin{array}{l}\text { Climb to } \\
\text { Cruise }\end{array}$ & 13 & $6.07 \%$ & $21.07 \%$ & 0.183 & 224.40 & 195.00 & 41.14 & 35.75 & 1.115E-06 & 6.082E-06 & 2.710E-08 & $3.119 E-08$ & & & & \\
\hline $\begin{array}{l}\text { Cruised } \\
\text { Enroute }\end{array}$ & 38 & $17.76 \%$ & $26.25 \%$ & 0.228 & 241.66 & 210.00 & 55.18 & 47.95 & $3.259 E-06$ & 1.427E-05 & 5.907E-08 & 6.797E-08 & 5.575E-06 & B.426E-06 & 3.557E-08 & $4.093 E-08$ \\
\hline $\begin{array}{l}\text { Descent } \\
\text { from Cruise }\end{array}$ & 14 & $6.54 \%$ & $28.74 \%$ & 0.250 & 241.66 & 210.00 & 60.42 & 52.50 & $1.201 E-06$ & 4.803E-06 & 1.988E-08 & 2.287E-08 & & & & \\
\hline $\begin{array}{l}\text { Landing } \\
\text { Approach }\end{array}$ & 52 & $24.30 \%$ & $21.07 \%$ & 0.183 & 161.11 & 140.00 & 29.54 & 25.67 & $4.460 \mathrm{E}-06$ & $2.433 E-05$ & 1.510E-07 & 1.738E-07 & & & & \\
\hline Landing & 51 & $23.83 \%$ & $0.96 \%$ & 0.008 & 100.00 & 75.00 & 0.83 & 0.63 & 4.374E-06 & 5.249E-04 & 5.249E-06 & $6.999 E-06$ & 8.835E-06 & 4.609E-05 & 2.909E-07 & $3.360 \mathrm{E}-07$ \\
\hline TAL: & 14 & & $\%$ & 0.870 & & & 99.33 & 8.41 & 1.836E-05 & $2.110 E-05$ & $9.695 E-08$ & $1.116 E-07$ & $1.836 E-05$ & 2.110E-05 & 9.695E-08 & $1.116 E-07$ \\
\hline
\end{tabular}

Bases for Crash Raves by Flight Phase, 1986-1993 Totals:

0.87 Hours Average Flight Duration

164.73 Nmiles Average Flight Distance $11,658,732$ Landings 
Table 3.32

General Aviation Turbojet, Fixed Wing Crash Rates by Flight Phase

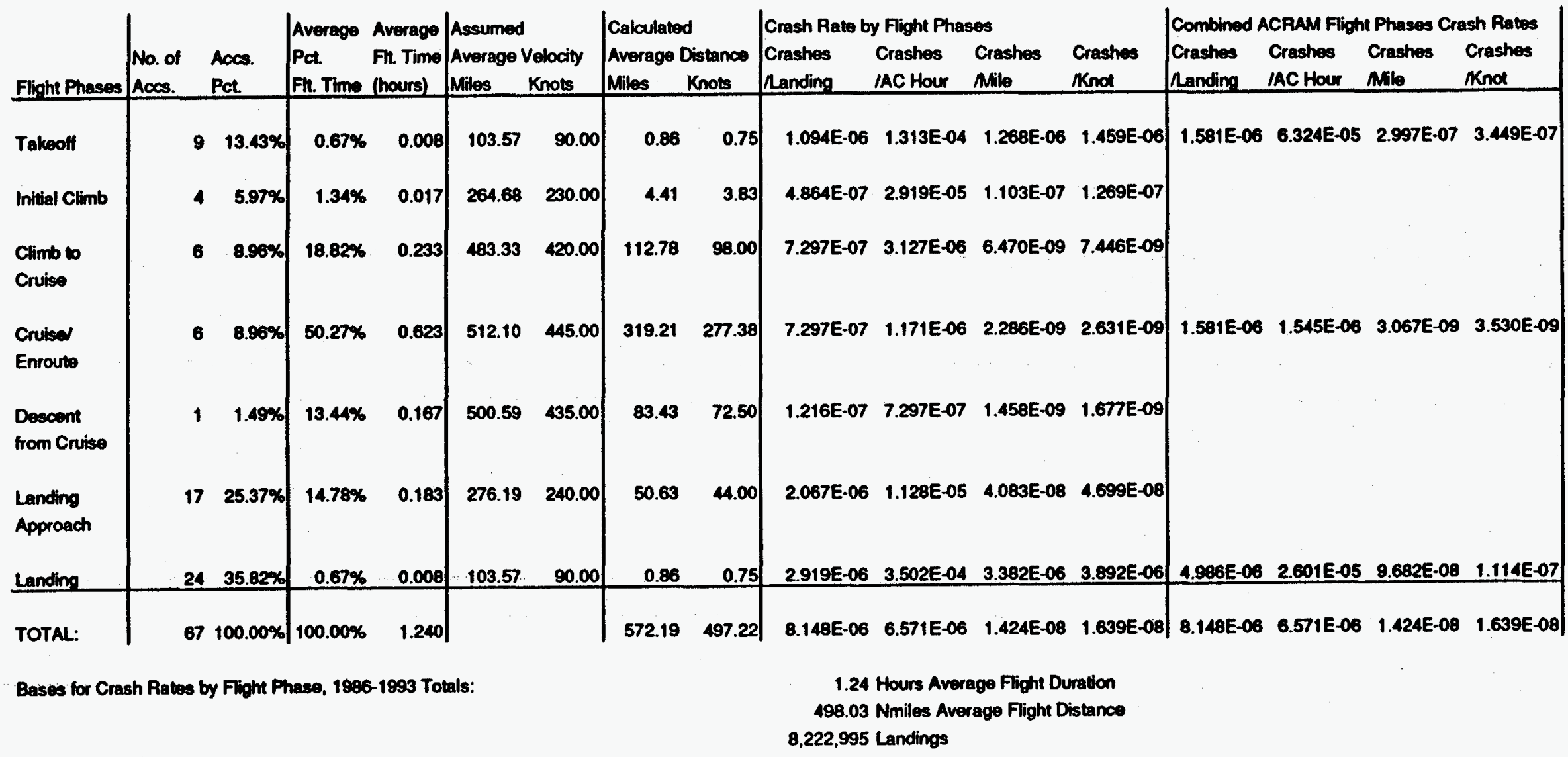


Table 3.33

General Aviation Total Fixed Wing Crash Rates by Fight Phase

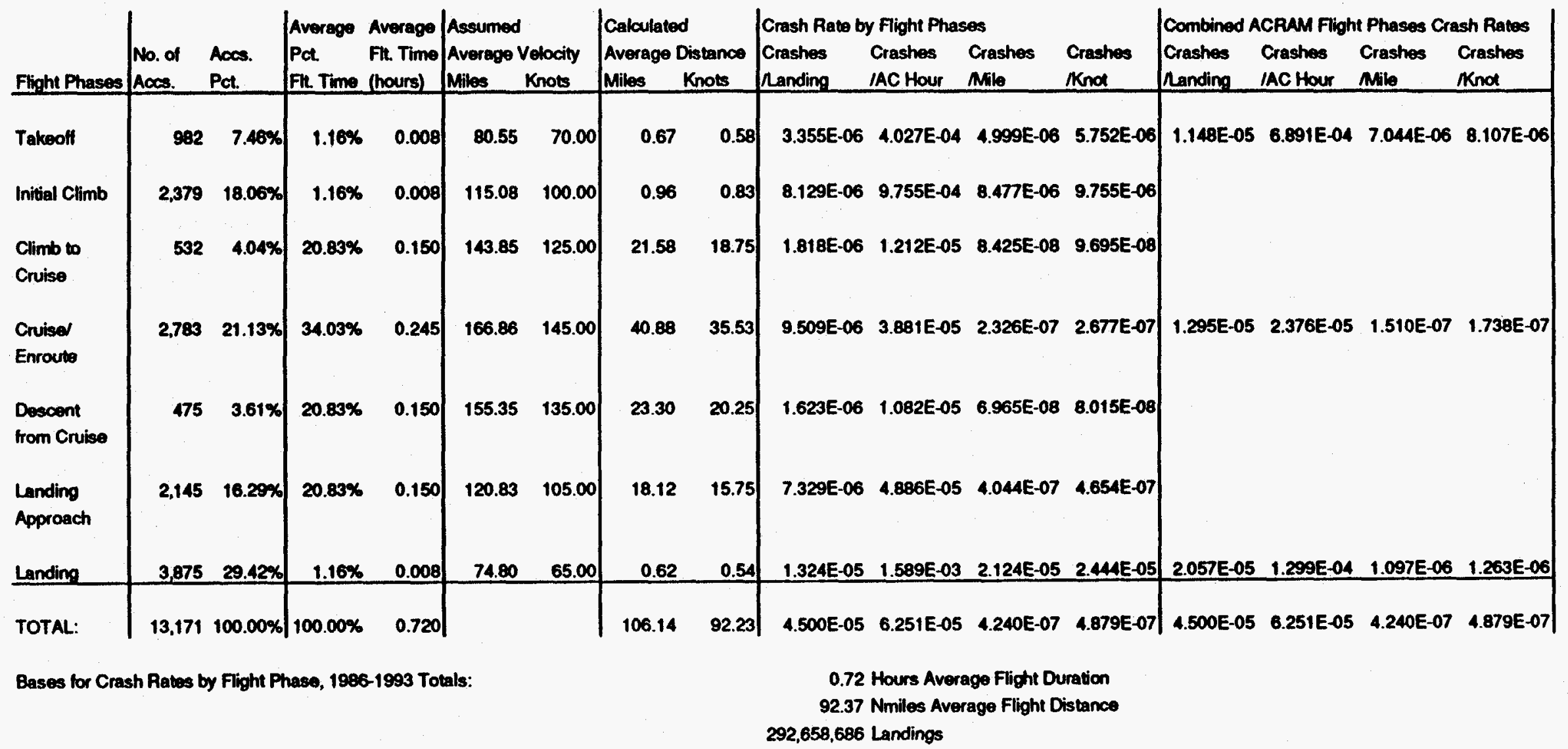


Table 3.34

General Aviation Reciprocating Engine, Rotery Wing Crash Rates by Flight Phase

\begin{tabular}{|c|c|c|c|c|c|c|c|c|c|c|c|c|c|c|c|c|}
\hline Flight Phases & $\begin{array}{l}\text { No. of } \\
\text { Aocs. }\end{array}$ & $\begin{array}{l}\text { Accs. } \\
\text { Pct. }\end{array}$ & \begin{tabular}{|l} 
Average \\
Pct. \\
Fit. Time
\end{tabular} & $\begin{array}{l}\text { Average } \\
\text { Fh. Time } \\
\text { (hours) }\end{array}$ & \multicolumn{2}{|c|}{$\begin{array}{l}\text { Assumed } \\
\text { Average Velocity }\end{array}$} & \multicolumn{2}{|c|}{$\begin{array}{l}\text { Cakculated } \\
\text { Average Distance }\end{array}$} & $\begin{array}{l}\text { Crash Rale by } \\
\text { Crashes } \\
\text { Landing }\end{array}$ & $\begin{array}{l}\text { Flight Phas } \\
\text { Crashos } \\
\text { IAC Hour }\end{array}$ & $\begin{array}{l}\text { ses } \\
\text { Crashes } \\
\text { Mile }\end{array}$ & $\begin{array}{l}\text { Crashes } \\
\text { Knot } \\
\end{array}$ & $\begin{array}{l}\text { Combined A } \\
\text { Crashes } \\
\text { nanding } \\
\end{array}$ & $\begin{array}{l}\text { ACRAM Flight } \\
\text { Crashes } \\
\text { IAC Hour }\end{array}$ & $\begin{array}{l}\text { Phases Cra } \\
\text { Crashes } \\
\text { Mile }\end{array}$ & $\begin{array}{l}\text { ash Rates } \\
\text { Crashes } \\
\text { Knot }\end{array}$ \\
\hline Takeoff & 56 & $9.71 \%$ & $2.38 \%$ & 0.008 & 57.54 & 50.00 & 0.48 & 0.42 & 3.971E-06 & 4.766E- 04 & $8.282 E-06$ & 9.531E-06 & $1.078 E-05$ & $6.468 E-04$ & 1.071E-05 & $1.232 E-05$ \\
\hline Initial Climb & 96 & $16.64 \%$ & $2.38 \%$ & 0.008 & 63.29 & 55.00 & 0.53 & 0.46 & $6.808 E-06$ & 8.170E-04 & 1.291E-05 & 1.485E-05 & & & & \\
\hline $\begin{array}{l}\text { Climb to } \\
\text { Cruise }\end{array}$ & 16 & $2.77 \%$ & $23.81 \%$ & 0.083 & 65.59 & 57.00 & 5.47 & 4.75 & 1.135E-06 & 1.362E-05 & 2.076E-07 & 2.389E-07 & & & & \\
\hline $\begin{array}{l}\text { Cruised } \\
\text { Enroute }\end{array}$ & 177 & $30.68 \%$ & $11.90 \%$ & 0.042 & 69.05 & 60.00 & 2.88 & 2.50 & 1.255E-05 & $3.013 E-04$ & 4.363E-06 & 5.021E-06 & $1.610 E-05$ & $7.727 E-05$ & $1.142 E-06$ & 1.314E-06 \\
\hline $\begin{array}{l}\text { Descent } \\
\text { from Cruise }\end{array}$ & 34 & $5.89 \%$ & $23.81 \%$ & 0.083 & 69.05 & 60.00 & 5.75 & 5.00 & 2.411E-06 & 2.893E-05 & 4.191E-07 & 4.822E-07 & & . & & \\
\hline $\begin{array}{l}\text { Landing } \\
\text { Approach }\end{array}$ & 70 & $12.13 \%$ & $33.33 \%$ & 0.117 & 63.29 & 55.00 & 7.38 & 6.42 & 4.964E-06 & 4.255E-05 & 6.723E-07 & $7.736 E-07$ & & & & \\
\hline Landing & 128 & $22.18 \%$ & $2.38 \%$ & 0.008 & 46.03 & 40.00 & 0.38 & 0.33 & $9.077 E-06$ & 1.089E-03 & $2.366 \mathrm{E}-05$ & 2.723E-05 & 1.404E-05 & $1.123 E-04$ & $1.800 E-06$ & 2.080 E-06 \\
\hline TOTAL: & 577 & $100.00 \%$ & $100.00 \%$ & 0.350 & & & 22.87 & 19.88 & 4.092E-05 & 1.169E-04 & $1.789 E-06$ & $2.059 E-06$ & 4.092E-05 & $1.169 E-04$ & $1.789 E-06$ & 2.059E-06 \\
\hline Bases for Cras & Ih Rates by & Flight Ph & Lase, 1986 & 6-1993 Tou & vals: & & & & $\begin{array}{r}0.35 \\
19.89 \\
14,101.009\end{array}$ & $\begin{array}{l}\text { Hours Avere } \\
\text { Nmiles Aver } \\
\text { Landings }\end{array}$ & $\begin{array}{l}\text { age Flight Du } \\
\text { rage Flight D }\end{array}$ & $\begin{array}{l}\text { mation } \\
\text { istance }\end{array}$ & & & & \\
\hline
\end{tabular}


Teble 3.35

General Aviation Turbine Engino, Rotary Wing Crash Rates by Flight Phase

\begin{tabular}{|c|c|c|c|c|c|c|c|c|c|c|c|c|c|c|c|c|}
\hline Flight Phases & $\begin{array}{l}\text { No. of } \\
\text { Acoss. }\end{array}$ & $\begin{array}{l}\text { Acas. } \\
\text { Pat. }\end{array}$ & $\begin{array}{l}\text { Average } \\
\text { Pct. } \\
\text { Fh. Time }\end{array}$ & $\begin{array}{l}\text { Average } \\
\text { Fh. Time } \\
\text { (hours) }\end{array}$ & \multicolumn{2}{|c|}{$\begin{array}{l}\text { Assumed } \\
\text { Average Velocity }\end{array}$} & \multicolumn{2}{|c|}{$\begin{array}{l}\text { Calculated } \\
\text { Average Distance }\end{array}$} & $\begin{array}{l}\text { Crash Rate by } \\
\text { Crashes } \\
\text { Landing } \\
\end{array}$ & $\begin{array}{l}\text { Flight Phas } \\
\text { Crashes } \\
\text { /AC Hour }\end{array}$ & $\begin{array}{l}\text { Crashes } \\
\text { Mile } \\
\end{array}$ & $\begin{array}{l}\text { Crashes } \\
\text { Annok }\end{array}$ & $\begin{array}{l}\text { Combined A } \\
\text { Crashes } \\
\text { Landing }\end{array}$ & $\begin{array}{l}\text { ACRAM Flight } \\
\text { Crashes } \\
\text { IACHour }\end{array}$ & $\begin{array}{l}\text { It Phases Cro } \\
\text { Crashes } \\
\text { Mile }\end{array}$ & $\begin{array}{l}\text { ash Ratos } \\
\text { Crashos } \\
\text { Knnol }\end{array}$ \\
\hline Takeolt & 23 & $8.04 \%$ & $1.63 \%$ & 0.008 & 63.29 & 55.00 & 0.53 & 0.46 & $1.151 E-06$ & $1.381 E-04$ & $2.182 E-06$ & $2.511 E-06$ & 3.203E-06 & $1.922 E-04$ & $2.904 E-06$ & $3.342 E-06$ \\
\hline Initial Climb & 41 & $14.34 \%$ & $1.63 \%$ & $6 \quad 0.008$ & 69.05 & 60.00 & 0.58 & 0.50 & $2.052 E-06$ & $2.462 E-04$ & $3.566 \mathrm{E}-06$ & $4.104 E-06$ & & & & \\
\hline $\begin{array}{l}\text { Climb to } \\
\text { Cruise }\end{array}$ & 10 & $3.50 \%$ & $22.88 \%$ & 0.117 & 9206 & 80.00 & 10.74 & 9.33 & 5.005E-07 & $4.290 E-06$ & 4.660E-08 & $5.362 E-08$ & & & & \\
\hline $\begin{array}{l}\text { Cruisel } \\
\text { Enroute }\end{array}$ & 117 & $40.91 \%$ & $33.01 \%$ & 0.168 & 103.57 & 90.00 & 17.43 & 15.15 & $5.856 \mathrm{E}-06$ & $3.479 E-05$ & 3.359E-07 & $3.865 E-07$ & 6.957E-06 & $1.732 E-05$ & $1.788 E-07$ & $2.057 E-07$ \\
\hline $\begin{array}{l}\text { Descent } \\
\text { from Cruise }\end{array}$ & 12 & $4.20 \%$ & $22.88 \%$ & 0.117 & 92.06 & 80.00 & 10.74 & 9.33 & 6.006E-07 & $5.148 E-06$ & 5.592E-08 & $6.435 E-08$ & & & & \\
\hline $\begin{array}{l}\text { Landing } \\
\text { Approach }\end{array}$ & 34 & $11.89 \%$ & $16.34 \%$ & 0.083 & 63.29 & 55.00 & 5.27 & 4.58 & $1.702 E-06$ & $2.042 E-05$ & $3.226 \mathrm{E}-07$ & 3.713E-07 & & & & \\
\hline Landing & 49 & $17.13 x$ & $1.63 \%$ & $\quad 0.008$ & 46.03 & 40.00 & 0.38 & 0.33 & 2.452E-06 & 2.943E-04 & 6.393E-06 & 7.357E-06 & 4.15AE-06 & $4.532 E-05$ & 7.342E-07 & 8.499E-07 \\
\hline TOTAL: & 286 & $100.00 \%$ & $100.00 \%$ & 0.510 & & & 45.68 & 39.69| & $1.431 E-05$ & 2.807E-05 & 3.134E-07 & $3.606 E-07$ & 1.431E-05 & 2.807E-05 & 3.134E-07 & $3.606 E-07$ \\
\hline Bases for Cras & sh Raves by & y Flight & hase, 1986 & 8-1993 T & & & & & $\begin{array}{r}0.51 \\
39.14 \\
19,980,900\end{array}$ & $\begin{array}{l}\text { Hours Avera } \\
\text { Nmiles Aver: } \\
\text { Landings }\end{array}$ & $\begin{array}{l}\text { ge Flight Dur } \\
\text { age Flight Di }\end{array}$ & Uration & & & & \\
\hline
\end{tabular}


Table 3.36

General Aviation Total Rotery Wing Crash Rates by Flight Phase

\begin{tabular}{|c|c|c|c|c|c|c|c|c|c|c|c|c|c|c|c|c|}
\hline Flight Phases & $\begin{array}{l}\text { No. of } \\
\text { Aocs. }\end{array}$ & $\begin{array}{l}\text { Acos. } \\
\text { Pct. }\end{array}$ & $\begin{array}{l}\text { Average } \\
\text { Pct. } \\
\text { Fit. Time }\end{array}$ & $\begin{array}{l}\text { Average } \\
\text { Fh. Time } \\
\text { (hours) }\end{array}$ & \multicolumn{2}{|c|}{$\begin{array}{l}\text { Assumed } \\
\text { Average Velocity }\end{array}$} & \multicolumn{2}{|c|}{$\begin{array}{l}\text { Calculated } \\
\text { Average Distance }\end{array}$} & $\begin{array}{l}\text { Crash Rate by } \\
\text { Crashes } \\
\text { Landing }\end{array}$ & $\begin{array}{l}\text { Flight Phas } \\
\text { Crashes } \\
\text { IAC Hour }\end{array}$ & $\begin{array}{l}\text { ses } \\
\text { Crashes } \\
\text { Mile }\end{array}$ & $\begin{array}{l}\text { Crashes } \\
\text { Knol }\end{array}$ & $\begin{array}{l}\text { Combined A } \\
\text { Crashes } \\
\text { Landing } \\
\end{array}$ & $\begin{array}{l}\text { CRAM Fligh } \\
\text { Crashes } \\
\text { IAC Hour }\end{array}$ & $\begin{array}{l}\text { Phases Cra: } \\
\text { Crashes } \\
\text { Mile }\end{array}$ & $\begin{array}{l}\text { ash Rales } \\
\text { Crashes } \\
\text { Knot } \\
\end{array}$ \\
\hline Takeoff & 79 & $9.15 \%$ & $1.85 \%$ & 0.008 & 60.99 & 53.00 & 0.51 & 0.44 & $2.318 E-06$ & 2.782E-04 & 4.561E-06 & $5.248 E-06$ & $6.338 E-06$ & 3.803E-04 & 6.00BE-06 & $6.914 E-06$ \\
\hline Initial Climb & 137 & $15.87 \%$ & $1.85 \%$ & 0.008 & 65.59 & 57.00 & 0.55 & 0.48 & $4.020 \mathrm{E}-06$ & 4.824E-04 & 7.354E-06 & $8.463 E-06$ & & & & \\
\hline $\begin{array}{l}\text { Climb to } \\
\text { Cruise }\end{array}$ & 26 & $3.01 \%$ & $22.22 \%$ & 0.100 & 84.01 & 73.00 & 8.40 & 7.30 & $7.629 E-07$ & 7.629E-06 & $9.081 E-08$ & 1.045E-07 & & & & \\
\hline $\begin{array}{l}\text { Cruisel } \\
\text { Enroute }\end{array}$ & 294 & $34.07 \%$ & $27.78 \%$ & 0.125 & 100.12 & 87.00 & 12.51 & 10.88 & $8.626 E-06$ & $6.901 E-05$ & 6.893E-07 & 7.932E-07 & 1.074E-05 & $3.143 E-05$ & $3.543 E-07$ & 4.077E-07 \\
\hline $\begin{array}{l}\text { Descent } \\
\text { from Cruise }\end{array}$ & 46 & $5.33 \%$ & $25.93 \%$ & 0.117 & 80.55 & 70.00 & 9.40 & 8.17 & $1.350 E-06$ & 1.157E-05 & $1.436 E-07$ & 1.653E-07 & & & & \\
\hline $\begin{array}{l}\text { Landing } \\
\text { Approach }\end{array}$ & 104 & $12.05 \%$ & $18.52 \%$ & 0.083 & 63.29 & 55.00 & 5.27 & 4.58 & 3.051E-06 & $3.662 E-05$ & 5.785E-07 & $6.658 E-07$ & & & & \\
\hline Londing & 177 & $20.51 \%$ & $1.85 \%$ & 0.008 & 46.03 & 40.00 & 0.38 & 0.33 & 5.193E-06 & $6.232 E-04$ & 1.354E-05 & 1.558E-05 & 8.245E-06 & 8.994E-05 & $1.457 E-06$ & 1.677E-06 \\
\hline TOTAL: & 863 & $100.00 \%$ & $100.00 \%$ & 0.450 & & & 37.03 & 32.18 & 2.532E-05 & 5.627E-05 & 6.839E-07 & $7.870 E-07$ & 2.532E-05 & 5.627E-05 & 6.839E-07 & $7.870 \mathrm{E}-07$ \\
\hline Bases for Cras & sh Rates by & y Flight Ph & hase, 1986 & 6-1993 Tot & tals: & & & & $\begin{array}{r}0.45 \\
32.81 \\
34,081,908\end{array}$ & $\begin{array}{l}\text { Hours Avera } \\
\text { Nmiles Aver } \\
\text { Landings }\end{array}$ & $\begin{array}{l}\text { age Flight Du } \\
\text { rage Flight D }\end{array}$ & $\begin{array}{l}\text { mation } \\
\text { istance }\end{array}$ & & & & \\
\hline
\end{tabular}


Table 3.37

General Aviation Total Powered Aircraft Crash Rates by Flight Phase

\begin{tabular}{|c|c|c|c|c|c|c|c|c|c|c|c|c|c|c|c|c|}
\hline Flight Phases & $\begin{array}{l}\text { No. of } \\
\text { Acos. }\end{array}$ & $\begin{array}{l}\text { Acas. } \\
\text { Pct. }\end{array}$ & $\begin{array}{l}\text { Average } \\
\text { Pct. } \\
\text { Fit. Time }\end{array}$ & $\begin{array}{l}\text { Average } \\
\text { Flt. Time } \\
\text { (hours) }\end{array}$ & \multicolumn{2}{|c|}{$\begin{array}{l}\text { Assumed } \\
\text { Average Velocity }\end{array}$} & \multicolumn{2}{|c|}{$\begin{array}{l}\text { Calculated } \\
\text { Average Distance }\end{array}$} & $\begin{array}{l}\text { Crash Rate by } \\
\text { Crashes } \\
\text { nanding }\end{array}$ & $\begin{array}{l}\text { Fight Phas } \\
\text { Crashes } \\
\text { IAC Hour }\end{array}$ & $\begin{array}{l}\text { Crashes } \\
\text { Mile } \\
\end{array}$ & $\begin{array}{l}\text { Crashes } \\
\mathrm{Knnot}\end{array}$ & $\begin{array}{l}\text { Combined A } \\
\text { Crashes } \\
\text { Landing }\end{array}$ & $\begin{array}{l}\text { CRAM Fligh } \\
\text { Crashes } \\
\text { IAC Hour }\end{array}$ & $\begin{array}{l}\text { Phases Cre } \\
\text { Crashes } \\
\text { Mile }\end{array}$ & $\begin{array}{l}\text { ash Rates } \\
\text { Crashes } \\
\text { Knol }\end{array}$ \\
\hline Takeoff & 1,061 & $7.56 \%$ & $1.23 \%$ & 0.008 & 69.05 & 60.00 & 0.58 & 0.50 & $3.247 E-06$ & $3.897 E-04$ & 5.644E-06 & $6,494 E-06$ & 1.095E-05 & $4.379 E-04$ & 4.963E-06 & $5.712 E-06$ \\
\hline Initial Climb & 2,516 & $17.93 \%$ & $2.45 \%$ & 0.017 & 97.82 & 85.00 & 1.63 & 1.42 & $7.700 E-06$ & 4.620E-04 & $4.723 E-06$ & $5.436 \mathrm{E}-06$ & & & & \\
\hline $\begin{array}{l}\text { Climb to } \\
\text { Cruise }\end{array}$ & 558 & $3.98 \%$ & $19.61 \%$ & 0.133 & 143.85 & 125.00 & 19.18 & 16.67 & $1.708 E-06$ & 1.281E.05 & $8.904 E-08$ & $1.025 E-07$ & & & & \\
\hline $\begin{array}{l}\text { Cruised } \\
\text { Enroute }\end{array}$ & 3,077 & $21.93 \%$ & $32.60 \%$ & 0.222 & 166.86 & 145.00 & 36.99 & 32.14 & $9.417 E-06$ & 4.248E.05 & $2.546 E-07$ & $2.930 E-07$ & 1.272E-05 & 2.438E-05 & I.550E-07 & $1.784 E-07$ \\
\hline $\begin{array}{l}\text { Descent } \\
\text { from Cruise }\end{array}$ & 521 & $3.71 \%$ & $24.51 \%$ & 0.167 & 155.35 & 135.00 & 25.89 & 22.50 & $1.595 E-06$ & 9.567E-06 & 6.158E-08 & 7.087E-08 & & & & \\
\hline $\begin{array}{l}\text { Landing } \\
\text { Approach }\end{array}$ & 2,249 & $16.03 \%$ & $17.16 \%$ & 0.117 & 109.32 & 95.00 & 12.75 & 11.08 & 6.883E-06 & $5.900 E-05$ & 5.397E-07 & 6.210E-07 & & & & \\
\hline Landing & 4,052 & $28.87 \%$ & $2.45 \%$ & 0.017 & 63.29 & 55.00 & 1.05 & 0.92 & 1.240 E- 05 & 7.441E-04 & 1.176E-05 & $1.353 E-05$ & 1.928E-05 & $1.446 \mathrm{E}-04$ & $1.396 \mathrm{E}-06$ & $1.607 \mathrm{E}-06$ \\
\hline OTA & 4,034 & $100.00 \%$ & $100.00 \%$ & 0.680 & & & 98.07 & 85.23 & 4.295E-05 & 6.316E-05 & 4.379E-07 & $5.040 E-07$ & 4.295E-05 & 6.316E-05 & 4.379E-07 & $5.040 E-07$ \\
\hline
\end{tabular}

Bases for Crash Rales by Flight Phase, 1986-1993 Totals:

0.68 Hours Average Flight Duration

85.22 Nmiles Average Flight Distance 326,740,594 Landings 
Table 3.38

General Aviation Fixed Wing Crash Rates by Alrcraft Subcategories and Filght Phase, Adjusted for Off Runway Crashes

Based on FAA General Aviation Activity Survey, Calendar Years 1986-1993 [Ref. 3.1 - 3.8], NTSB Annual Revlew of Alrcraft Accident Data, U.S. General Aviation, Calendar Years 1986-1993 [Ref. 3.20 - 3.27], and SAIC Revlew of the NTSB General Aviation Database

\begin{tabular}{l|r|r|}
\multicolumn{1}{c|}{ Alrcraft Subcategories } & $\begin{array}{c}\text { Off Rumway } \\
\text { Crash } \\
\text { Rate } \\
\text { Takeoffs }\end{array}$ & $\begin{array}{c}\text { Off Runway } \\
\text { Crash } \\
\text { Rate } \\
\text { Randings }\end{array}$ \\
\hline Single-Engine Reciprocating, Fixed Wing & $1.09 E-05$ & $2.00 E-05$ \\
Mult-Engine Reciprocating, Fixed Wing & $9.28 E-06$ & $2.27 E-05$ \\
Turboprop, Fixed Wing & $3.51 E-06$ & $8.30 E-06$ \\
Turbojet, Fixed Wing & $1.41 E-06$ & $4.69 E-06$ \\
\hline Total, Fixed Wing & $1.02 E-05$ & $1.93 E-05$ \\
\hline
\end{tabular}




\subsection{GENERAL AVIATION IN FLIGHT NONAIRWAY CRASH PARAMETERS}

Previous efforts to estimate nonairport related aircraft crash frequencies are based on the assumption that aircraft fly within fixed air corridors; such an assumption is not realistic for GA aircraft. Based on the product-kemel method for estimating multidimensional probability density functions, the Standard uses a data driven approach for estimating the GA nonairport crash frequency at a given DOE site. This approach is data driven since it directly uses the coordinates (latitudes and longitudes) of previous GA nonairport crsahes to make its estimates; no assumptions have been made about 1) an aircratt's position (either its coordinates or altitude) prior to a mishap that results in a crash, or 2) an aircraft's trajectory (flight path) from the point where a mishap occurs until ground impact (which would require an assessment of the aircratt's flight characteristics, such as glide ratio and the pilot's ability to maneuver the aircraft following the mishap).

Table A3.1 provides a listing of the 1250 crash locations as longitudes and latitudes with their NTSB number. 


\subsection{GENERAL AVIATION CRASH PARAMETER DISTRIBUTIONS}

\subsubsection{GENERAL AVIATION CRASH LOCATION}

A stepwise process was used to compile data which could be used to evaluate the probability of a damaging general aviation crash for facility sites in the vicinity of airports. The National Transportation Safety Board electronic database for the period between 1983 and 1989 was reviewed. In order to segregate the crashes that provided a potential hazard to either a nuclear or chemical facility, only those accidents were included that were general aviation, that involved substantial damage or destruction of the aircraft, that were off the airstrip/off the airport by at least one mile, and that were helicopters or airplanes (aircraft such as ultralights, gliders, dirigibles, balloons, etc., were exchuded). The narratives for crashes with suspect information were reviewed and some were added and others were deleted. 680 applicable crashes were chosen for evaluation.

The crash locations records obtained from the database allowed for an empirical evaluation of the crash location density $f(x, y)$ for the general aviation category and phase operations. Due to the limited number of records in several GA subcategories, the database for crash location is insufficient for an effective statistical analysis of all subcategories and phases. Specifically, the fixed wing turbojet subcategory has seven observations and the combined rotating wing subcategories have four. Also, the subcategory fixed wing turboprop and takeoff phase has only four observations. It has been decided to combine all the subcategory crash location data for the takeoft phase, and likewise for the landing phase, to determine two GA category crash location density functions, one for takeoffs and one for landings.

The crash location $f(x, y)$, based on a nonparametric bivariate estimation technique [Refs. 3.28 and 3.34] and the crash location data from the GA category, is given in Equation 3.1. Techniques based on parametric models are not as well suited to this bcation data.

$$
\left.f(x, y)=\frac{1}{n} w_{o n} \sum_{i=1}^{n}\left\{\left[\Phi\left(\frac{x_{y}-x_{1}}{h_{x}}\right)-\Phi\left(\frac{x_{1}-x_{1}}{h_{x}}\right)\right]\left[\Phi\left(\frac{y_{y}-y_{1}}{h_{y}}\right)-\Phi\left(\frac{y_{1}-y_{1}}{h_{y}}\right)\right]\right\}+w_{o n}\left[\Phi\left(\frac{x_{y}}{\sigma}\right)-\Phi\left(\frac{x_{y}}{\sigma}\right)\right] \Phi\left(\frac{y_{y}}{\sigma}\right)-\Phi\left(\frac{y_{1}}{\sigma}\right)\right] .
$$

$\Phi$ represents the standard nomal cumulative distribution function. The quantities $h_{\mathbf{x}}, h_{\mathbf{y}}$, and $\sigma$ are "bandwidths" for the estimator. They control the smoothness and diffuseness of the density $f$. Also, $x_{v}=x+0.5, x_{1}=x-0.5, y_{v}=y+0.5$, and $y_{1}=y-0.5$, where the units are miles, and $w_{\text {of }}$ and $w_{o n}$ are the relative frequencies of crash locations in the database off and on the airport, respectively. Values for the quantities $\left(n, h_{x}, h_{y}, \sigma, w_{o n}, w_{o n}\right)$ are included as Table 3.39 .

TABLE 3.39 Constants Required to Formulate Location Estimate Using Equation 3.1

\begin{tabular}{|c|c|c|c|c|c|c|}
\hline PHASE & $n$ & $h_{\gamma}$ & $h_{\gamma}$ & $\sigma$ & $w_{\text {oll }}$ & $w_{m}$ \\
\hline GA Landing & 574 & 0.95 & 0.41 & 0.46 & 0.4915 & 0.5085 \\
\hline GA Takeoff & 106 & 0.69 & 0.45 & 0.46 & 0.4546 & 0.5454 \\
\hline
\end{tabular}

The tables listed below give the GA crash locations and probabilities:

Table 3.40

Table 3.41

Table 3.42

Table 3.43
General Aviation Takeoff Crash Locations

General Aviation Landing Crash Locations

General Aviation Takeoff Crash Location Probabilities

General Aviation Landing Crash Location Probabilities. 


\begin{tabular}{|c|c|c|}
\hline & $x$ coordinate & y coordinate \\
\hline 1 & -1.000 & 0.000 \\
\hline 2 & -1.000 & 0.000 \\
\hline 3 & -0.857 & -0.515 \\
\hline 4 & -0.342 & -0.940 \\
\hline 5 & 0.000 & -1.000 \\
\hline 6) & 0.000 & 1.000 \\
\hline 7 & 0.174 & -0.985 \\
\hline 8 & 0.174 & -0.985 \\
\hline 9 & 0.174 & 0.985 \\
\hline 10 & 0.174 & 0.985 \\
\hline 11 & 0.342 & -0.940 \\
\hline 12 & 0.342 & 0.940 \\
\hline 13 & 0.500 & -0.866 \\
\hline 14 & 0.500 & 0.866 \\
\hline 15 & 0.695 & -0.719 \\
\hline 16 & 0.707 & 0.707 \\
\hline 17 & 0.707 & 0.707 \\
\hline 18 & 0.766 & -0.643 \\
\hline 19 & 0.766 & 0.643 \\
\hline 20 & 0.819 & 0.574 \\
\hline 21 & 0.866 & -0.500 \\
\hline 22 & 0.866 & -0.500 \\
\hline 23 & 0.866 & 0.500 \\
\hline 24 & 0.866 & 0.500 \\
\hline 25 & 0.940 & -0.342 \\
\hline 26 & 0.940 & -0.342 \\
\hline 27 & 0.940 & 0.342 \\
\hline 28 & 0.940 & 0.342 \\
\hline 29 & 0.940 & 0.342 \\
\hline 30 & 0.940 & 0.342 \\
\hline 31 & 0.966 & 0.259 \\
\hline 32 & 0.966 & 0.259 \\
\hline 33 & 0.985 & -0.174 \\
\hline 34 & 0.985 & -0.174 \\
\hline 35 & 0.985 & 0.174 \\
\hline
\end{tabular}

\begin{tabular}{|c|c|c|}
\hline & $x$ coordinate & y coordinate \\
\hline 36 & 0.985 & 0.174 \\
\hline 37 & 0.993 & 0.122 \\
\hline 38 & 0.996 & -0.087 \\
\hline 39 & 0.996 & -0.087 \\
\hline 40 & 0.996 & 0.087 \\
\hline 41 & 0.999 & 0.035 \\
\hline 42 & 0.999 & 0.035 \\
\hline 43 & 1.000 & -0.017 \\
\hline 44 & 1.000 & 0.000 \\
\hline 45 & 1.000 & 0.000 \\
\hline 46 & 1.000 & 0.000 \\
\hline 47 & 1.000 & 0.000 \\
\hline 48 & 1.000 & 0.000 \\
\hline 49 & 1.000 & 0.000 \\
\hline 50 & 1.000 & 0.000 \\
\hline 51 & 1.000 & 0.000 \\
\hline 52 & 1.000 & 0.000 \\
\hline 53 & 1.000 & 0.000 \\
\hline 54 & 1.000 & 0.000 \\
\hline 55 & 1.000 & 0.000 \\
\hline 56 & 1.000 & 0.000 \\
\hline 57 & 1.000 & 0.000 \\
\hline 58 & 1.000 & 0.000 \\
\hline 59 & 1.000 & 0.000 \\
\hline 60 & 1.000 & 0.000 \\
\hline 61 & 1.000 & 0.000 \\
\hline 62 & 1.000 & 0.000 \\
\hline 63 & 1.000 & 0.000 \\
\hline 64 & 1.000 & 0.000 \\
\hline 65 & 1.000 & 0.000 \\
\hline 66 & 1.000 & 0.000 \\
\hline 67 & 1.000 & 0.000 \\
\hline 68 & 1.000 & 0.000 \\
\hline 69 & 1.000 & 0.000 \\
\hline 70 & 1.000 & 0.000 \\
\hline
\end{tabular}

\begin{tabular}{|c|c|c|}
\hline & $x$ coordinate & $y$ coordinate \\
\hline 71 & 1.000 & 0.000 \\
\hline 72 & 1.000 & 0.000 \\
\hline 73 & 1.000 & 0.000 \\
\hline 74 & 1.000 & 0.000 \\
\hline 75 & 1.000 & 0.000 \\
\hline 76 & 1.000 & 0.000 \\
\hline 77 & 1.000 & 0.000 \\
\hline 78 & 1.286 & 1.532 \\
\hline 79 & 1.414 & 1.414 \\
\hline 80 & 1.500 & 2.598 \\
\hline 81 & 1.638 & -1.147 \\
\hline 82 & 1.813 & 0.845 \\
\hline 83 & 1.879 & 0.684 \\
\hline 84 & 1.913 & -0.585 \\
\hline 85 & 1.970 & -0.347 \\
\hline 86 & 1.970 & 0.347 \\
\hline 87 & 2.000 & 0.000 \\
\hline 88 & 2.000 & 0.000 \\
\hline 89 & 2.000 & 0.000 \\
\hline 90 & 2.000 & 0.000 \\
\hline 91 & 2.000 & 0.000 \\
\hline 92 & 2.000 & 0.000 \\
\hline 93 & 2.571 & -3.064 \\
\hline 94 & 2.819 & 1.026 \\
\hline 95 & 2.954 & -0.521 \\
\hline 96 & 3.000 & 0.000 \\
\hline 97 & 3.000 & 0.000 \\
\hline 98 & 3.000 & 0.000 \\
\hline 99 & 4.698 & 1.710 \\
\hline 100 & 4.698 & 1.710 \\
\hline 101 & 5.000 & 0.000 \\
\hline 102 & 5.000 & 0.000 \\
\hline 103 & 5.000 & 0.000 \\
\hline 104 & 5.000 & 0.000 \\
\hline 105 & 5.438 & -2.536 \\
\hline 106 & 6.000 & 0.000 \\
\hline
\end{tabular}

Table 3.40

General Aviation Takeoff Crash Locations 


\begin{tabular}{|c|c|c|}
\hline & $x$ coordinate & y coordinate \\
\hline 1 & -13.000 & 0.000 \\
\hline 2 & -12.951 & 1.133 \\
\hline 3 & -11.998 & -0.209 \\
\hline 4 & .11 .984 & -0.628 \\
\hline 5 &.-11.954 & 1.046 \\
\hline 6 & -11.818 & -2.084 \\
\hline 7 & -11.818 & 2.084 \\
\hline 8 & -10.833 & 1.910 \\
\hline 9 & .10 .000 & 0.000 \\
\hline 10 & -9.996 & 0.523 \\
\hline 11 & -9.962 & -0.872 \\
\hline 12 & -9.848 & -1.736 \\
\hline 13 & -9.848 & 1.736 \\
\hline 14 & -9.000 & 0.000 \\
\hline 15 & 8.000 & 0.000 \\
\hline 16 & -7.995 & 0.279 \\
\hline 17 & -7.878 & 1.389 \\
\hline 18 & -7.000 & 0.000 \\
\hline 19 & -6.983 & 0.488 \\
\hline 20 & -6.928 & -4.000 \\
\hline 21 & -6.914 & -1.095 \\
\hline 22 & 6.894 & 1.216 \\
\hline 23 & -6.578 & -2394 \\
\hline 21 & -6.000 & 0.000 \\
\hline 25 & -6.000 & 0.000 \\
\hline 28 & -6.000 & 0.000 \\
\hline 27 & -6.000 & 0.000 \\
\hline 28 & -6.000 & 0.000 \\
\hline 29 & 6.000 & 0.000 \\
\hline 30 & -6.000 & 0.000 \\
\hline 31 & -5.985 & 0.419 \\
\hline 32 & .5 .909 & 1.062 \\
\hline 33 & .5 .909 & 1.042 \\
\hline 34 & 5.638 & 2.052 \\
\hline 35 & -5.438 & 2.536 \\
\hline 36 & -5.000 & 0.000 \\
\hline 37 & -5.000 & 0.000 \\
\hline 38 & .5 .000 & 0.000 \\
\hline 39 & -5.000 & 0.000 \\
\hline 40 & .5 .000 & 0.000 \\
\hline 41 & -5.000 & 0.000 \\
\hline 42 & -5.000 & 0.000 \\
\hline 43 & -5.000 & 0.000 \\
\hline 4 & -5.000 & 0.000 \\
\hline 45 & -5.000 & 0.000 \\
\hline 46 & -4.981 & 0.436 \\
\hline 47 & -4.973 & -0.523 \\
\hline 48 & 4.924 & 0.668 \\
\hline 49 & 4.698 & -1.710 \\
\hline 50 & -4.532 & -2.113 \\
\hline
\end{tabular}

\begin{tabular}{|c|c|c|}
\hline & $x$ coordinate & yooordingto \\
\hline 51 & -4.243 & -4.243 \\
\hline 52 & -3.995 & 0.209 \\
\hline 53 & -3.985 & -0.349 \\
\hline 54 & -3.939 & 0.695 \\
\hline 55 & -3.830 & -3.214 \\
\hline 56 & -3.830 & 3.214 \\
\hline 57 & -3.830 & 3.214 \\
\hline 58 & 3.825 & -1.169 \\
\hline 59 & 3.214 & -3.830 \\
\hline 60 & -3.000 & 0.000 \\
\hline 61 & -3.000 & 0.000 \\
\hline 62 & -3.000 & 0.000 \\
\hline 63 & -3.000 & 0.000 \\
\hline 64 & 3.000 & 0.000 \\
\hline 65 & 3.000 & 0.000 \\
\hline 66 & 3.000 & 0.000 \\
\hline 67 & 3.000 & 0.000 \\
\hline 68 & 3.000 & 0.000 \\
\hline 69 & -3.000 & 0.000 \\
\hline 70 & -3.000 & 0.000 \\
\hline 71 & -3.000 & 0.000 \\
\hline 72 & -3.000 & 0.052 \\
\hline 73 & -2.989 & 0.261 \\
\hline 74 & -2.954 & 0.521 \\
\hline 75 & -2.954 & -0.521 \\
\hline 76 & -2.954 & 0.521 \\
\hline$\pi$ & -2.723 & -4.193 \\
\hline 78 & -2.677 & 2.973 \\
\hline 79 & -2.516 & 1.634 \\
\hline 80 & -2.500 & 4.330 \\
\hline 81 & -2.298 & -1.928 \\
\hline 82 & -2.298 & 1.928 \\
\hline 83 & -2.298 & 1.928 \\
\hline 84 & -2.298 & 1.928 \\
\hline 85 & -2.298 & 1.928 \\
\hline 86 & -2.121 & -2.121 \\
\hline 87 & -2.000 & 0.000 \\
\hline 88 & -2.000 & 0.000 \\
\hline 89 & -2.000 & 0.000 \\
\hline 90 & -2.000 & 0.000 \\
\hline 91 & -2.000 & 0.000 \\
\hline 92 & -2.000 & 0.000 \\
\hline 93 & -2.000 & 0.000 \\
\hline 94 & -2.000 & 0.000 \\
\hline 95 & -2.000 & 0.000 \\
\hline 96 & -2.000 & 0.000 \\
\hline 97 & -2.000 & 0.000 \\
\hline 98 & -2.000 & 0.000 \\
\hline 99 & -2.000 & 0.000 \\
\hline 100 & -2.000 & 0.000 \\
\hline
\end{tabular}

\begin{tabular}{|c|c|c|}
\hline & $x$ coordinate & y coordinate \\
\hline 101 & -2.000 & 0.000 \\
\hline 102 & -2.000 & 0.000 \\
\hline 103 & -2.000 & 0.000 \\
\hline 104 & -2.000 & 0.000 \\
\hline 105 & -2.000 & 0.000 \\
\hline 106 & -2.000 & 0.000 \\
\hline 107 & -2.000 & 0.000 \\
\hline 108 & -2.000 & 0.000 \\
\hline 109 & -2.000 & 0.000 \\
\hline 110 & -2.000 & 3.464 \\
\hline 111 & -1.999 & 0.070 \\
\hline 112 & -1.995 & -0.140 \\
\hline 113 & -1.992 & 0.174 \\
\hline 114 & -1.992 & -0.174 \\
\hline 115 & -1.992 & 0.174 \\
\hline 116 & -1.981 & 0.278 \\
\hline 117 & -1.975 & 0.313 \\
\hline 118 & -1.970 & -0.347 \\
\hline 119 & -1.970 & -0.347 \\
\hline 120 & -1.970 & 0.347 \\
\hline 121 & -1.970 & 0.347 \\
\hline 122 & -1.970 & 0.347 \\
\hline 123 & -1.970 & 0.347 \\
\hline 124 & -1.970 & 0.347 \\
\hline 125 & -1.970 & 0.347 \\
\hline 126 & -1.932 & 0.518 \\
\hline 127 & -1.928 & -2.298 \\
\hline 128 & -1.902 & -0.618 \\
\hline 129 & -1.879 & 0.684 \\
\hline 130 & -1.879 & 0.684 \\
\hline 131 & -1.879 & 0.684 \\
\hline 132 & -1.813 & 0.845 \\
\hline 133 & -1.732 & -1.000 \\
\hline 134 & -1.732 & 1.000 \\
\hline 135 & -1.732 & -1.000 \\
\hline 136 & -1.721 & 2.457 \\
\hline 137 & -1.634 & -2.516 \\
\hline 138 & -1.532 & -1.286 \\
\hline 139 & -1.532 & .1 .286 \\
\hline 140 & -1.500 & -2.598 \\
\hline 141 & -1.500 & 2.598 \\
\hline 142 & -1.414 & 1.414 \\
\hline 143 & -1.414 & 1.414 \\
\hline 144 & -1.368 & 3.759 \\
\hline 145 & -1.362 & 2.673 \\
\hline 146 & -1.286 & -1.532 \\
\hline 147 & -1.286 & -1.532 \\
\hline 148 & -1.286 & 1.532 \\
\hline 149 & -1.286 & 1.532 \\
\hline 150 & -1.231 & 1.576 \\
\hline
\end{tabular}

\begin{tabular}{|c|c|c|}
\hline & $x$ coordinate & y coordinate \\
\hline 151 & -1.000 & 0.000 \\
\hline 152 & -1.000 & 0.000 \\
\hline 153 & -1.000 & 0.000 \\
\hline 154 & -1.000 & 0.000 \\
\hline 155 & -1.000 & 0.000 \\
\hline 156 & -1.000 & 0.000 \\
\hline 157 & -1.000 & 0.000 \\
\hline 158 & -1.000 & 0.000 \\
\hline 159 & -1.000 & 0.000 \\
\hline 160 & .1 .000 & 0.000 \\
\hline 161 & -1.000 & 0.000 \\
\hline 162 & -1.000 & 0.000 \\
\hline 163 & -1.000 & 0.000 \\
\hline 164 & -1.000 & 0.000 \\
\hline 165 & .1 .000 & 0.000 \\
\hline 166 & -1.000 & 0.000 \\
\hline 167 & -1.000 & 0.000 \\
\hline 168 & -1.000 & 0.000 \\
\hline 169 & -1.000 & 0.000 \\
\hline 170 & -1.000 & 0.000 \\
\hline 171 & -1.000 & 0.000 \\
\hline 172 & .1 .000 & 0.000 \\
\hline 173 & -1.000 & 0.000 \\
\hline 174 & -1.000 & 0.000 \\
\hline 175 & .1 .000 & 0.000 \\
\hline 176 & -1.000 & 0.000 \\
\hline 177 & -1.000 & 0.000 \\
\hline 178 & -1.000 & 0.000 \\
\hline 179 & -1.000 & 0.000 \\
\hline 180 & -1.000 & 0.000 \\
\hline 181 & -1.000 & 0.000 \\
\hline 182 & -1.000 & 0.000 \\
\hline 183 & -1.000 & 0.000 \\
\hline 184 & -1.000 & 0.000 \\
\hline 185 & -1.000 & 0.000 \\
\hline 186 & -1.000 & 0.000 \\
\hline 187 & .1 .000 & 0.000 \\
\hline 188 & -1.000 & 0.000 \\
\hline 189 & -1.000 & 0.000 \\
\hline 190 & -1.000 & 0.000 \\
\hline 191 & -1.000 & 0.000 \\
\hline 192 & -1.000 & 0.000 \\
\hline 193 & -1.000 & 0.000 \\
\hline 194 & -1.000 & 0.000 \\
\hline 195 & -1.000 & 0.000 \\
\hline 196 & -1.000 & 0.000 \\
\hline 197 & -1.000 & 0.000 \\
\hline 198 & .1 .000 & 0.000 \\
\hline 199 & -1.000 & 0.000 \\
\hline 200 & -1.000 & 0.000 \\
\hline
\end{tabular}

\begin{tabular}{|c|c|c|}
\hline & $x$ coondind & ycoondinate \\
\hline 201 & -1.000 & 0.000 \\
\hline 202 & -1.000 & 0.000 \\
\hline 203 & -1.000 & 0.000 \\
\hline 204 & -1.000 & 0.000 \\
\hline 205 & -1.000 & 0.000 \\
\hline 206 & -1.000 & 0.000 \\
\hline 207 & -1.000 & 0.000 \\
\hline 208 & -1.000 & 0.000 \\
\hline 209 & -1.000 & 0.000 \\
\hline 210 & -1.000 & 0.000 \\
\hline 211 & -1.000 & 0.000 \\
\hline 212 & -1.000 & 0.000 \\
\hline 213 & -1.000 & 0.000 \\
\hline 214 & -1.000 & 0.000 \\
\hline 215 & -1.000 & 0.000 \\
\hline 216 & -1.000 & 0.000 \\
\hline 217 & -1.000 & 0.000 \\
\hline 218 & -1.000 & 0.000 \\
\hline 219 & -1.000 & 0.000 \\
\hline 220 & -1.000 & 0.000 \\
\hline 221 & -1.000 & 0.000 \\
\hline 222 & -1.000 & 0.000 \\
\hline 223 & -1.000 & 0.000 \\
\hline 224 & -1.000 & 0.000 \\
\hline 225 & -1.000 & 0.000 \\
\hline 226 & -1.000 & 0.000 \\
\hline 227 & -1.000 & 0.000 \\
\hline 220 & -1.000 & 0.000 \\
\hline 229 & -1.000 & 0.000 \\
\hline 230 & -1.000 & 0.000 \\
\hline 231 & -1.000 & 0.000 \\
\hline 232 & -1.000 & 0.000 \\
\hline 233 & -1.000 & 0.000 \\
\hline 234 & -1.000 & 0.000 \\
\hline 236 & -1.000 & 0.000 \\
\hline 236 & -1.000 & 0.000 \\
\hline 237 & -1.000 & 0.000 \\
\hline 238 & -1.000 & 0.000 \\
\hline 239 & -1.000 & 0.000 \\
\hline 240 & -1.000 & 0.000 \\
\hline 241 & -1.000 & 0.000 \\
\hline 212 & -1.000 & 0.000 \\
\hline 213 & -1.000 & 0.000 \\
\hline 244 & -1.000 & 0.000 \\
\hline 245 & -1.000 & 0.000 \\
\hline 246 & -1.000 & 1.732 \\
\hline 247 & -0.999 & 0.035 \\
\hline 248 & -0.999 & 0.035 \\
\hline 249 & -0.999 & -0.052 \\
\hline 250 & -0.996 & -0.087 \\
\hline & & \\
\hline
\end{tabular}

Table 3.41

General Aviation Landing Crash Locations

3. GENERAL AVIATION 


\begin{tabular}{|c|c|c|}
\hline & $x$ coondingte & Yocordinate \\
\hline 251 & -0.996 & -0.087 \\
\hline 252 & -0.996 & 0.087 \\
\hline 253 & -0.985 & -0.174 \\
\hline 254 & -0.965 & -0.174 \\
\hline 255 & -0.985 & -0.174 \\
\hline 256 & -0.985 & -0.174 \\
\hline 257 & -0.985 & -0.174 \\
\hline 258 & -0.995 & 0.174 \\
\hline 259 & -0.985 & 0.174 \\
\hline 260 & -0.985 & 0.174 \\
\hline 261 & -0.985 & 0.174 \\
\hline 262 & -0.935 & 0.174 \\
\hline 263 & -0.995 & 0.174 \\
\hline 264 & -0.985 & 0.174 \\
\hline 265 & -0.985 & 0.174 \\
\hline 266 & -0.995 & 0.174 \\
\hline 267 & -0.965 & 0.174 \\
\hline 268 & -0.992 & 0.191 \\
\hline 269 & -0.966 & 0.259 \\
\hline 270 & -0.966 & 0.259 \\
\hline 271 & -0.966 & 0.259 \\
\hline 272 & -0.966 & 0.259 \\
\hline 273 & -0.940 & -0.342 \\
\hline 274 & 0.940 & 0.342 \\
\hline 275 & -0.940 & 0.342 \\
\hline 276 & -0.940 & 0.342 \\
\hline $2 \pi$ & -0.940 & 0.342 \\
\hline 278 & -0.940 & -0.342 \\
\hline 279 & -0.940 & -0.342 \\
\hline 280 & -0.940 & -0.342 \\
\hline 281 & -0.940 & 0.342 \\
\hline 282 & -0.940 & 0.342 \\
\hline 283 & -0.940 & 0.342 \\
\hline 284 & -0.940 & 0.342 \\
\hline 285 & -0.906 & -0.423 \\
\hline 286 & -0.875 & -0.495 \\
\hline 287 & -0.868 & 4.924 \\
\hline 288 & -0.666 & -0.500 \\
\hline 289 & -0.866 & 0.500 \\
\hline 290 & -0.866 & -0.500 \\
\hline 291 & -0.866 & 0.500 \\
\hline 292 & -0.866 & 0.500 \\
\hline 293 & -0.866 & 0.500 \\
\hline 294 & 0.819 & -0.574 \\
\hline 295 & -0.819 & -0.574 \\
\hline 296 & -0.788 & 0.616 \\
\hline 297 & -0.766 & -0.643 \\
\hline 298 & -0.766 & 0.643 \\
\hline 299 & -0.766 & -0.643 \\
\hline 300 & -0.766 & -0.643 \\
\hline
\end{tabular}

\begin{tabular}{|c|c|c|}
\hline & $x$ coordinate & ycoondind \\
\hline 301 & -0.766 & -0.643 \\
\hline 302 & -0.766 & -0.643 \\
\hline 303 & -0.707 & -0.707 \\
\hline 304 & -0.695 & 0.719 \\
\hline 305 & -0.695 & 3.939 \\
\hline 306 & -0.643 & -0.766 \\
\hline 307 & -0.643 & -0.766 \\
\hline 308 & -0.643 & -0.766 \\
\hline 309 & -0.643 & 0.766 \\
\hline 310 & -0.643 & -0.766 \\
\hline 311 & -0.643 & 0.766 \\
\hline 312 & -0.574 & 0.819 \\
\hline 313 & -0.515 & -0.857 \\
\hline 314 & -0.515 & -0.857 \\
\hline 315 & -0.500 & 0.866 \\
\hline 316 & -0.500 & 0.066 \\
\hline 317 & -0.500 & 0.866 \\
\hline 318 & -0.500 & 0.866 \\
\hline 319 & -0.342 & -0.940 \\
\hline 320 & -0.342 & -0.940 \\
\hline 321 & -0.342 & -0.940 \\
\hline 322 & -0.309 & 0.951 \\
\hline 323 & -0.259 & 0.966 \\
\hline 324 & -0.174 & -0.985 \\
\hline 325 & -0.174 & -0.985 \\
\hline 326 & -0.174 & 0.985 \\
\hline 327 & -0.174 & 0.985 \\
\hline 328 & -0.174 & 0.985 \\
\hline 329 & -0.174 & 0.985 \\
\hline 330 & -0.156 & 0.988 \\
\hline 331 & -0.067 & -0.996 \\
\hline 332 & -0.087 & -0.996 \\
\hline 333 & -0.070 & 0.998 \\
\hline 334 & -0.017 & -1.000 \\
\hline 335 & 0.000 & -5.000 \\
\hline 336 & 0.000 & 5.000 \\
\hline 337 & 0.000 & -1.000 \\
\hline 338 & 0.000 & -1.000 \\
\hline 339 & 0.000 & -1.000 \\
\hline 340 & 0.000 & -1.000 \\
\hline 341 & 0.000 & -1.000 \\
\hline 342 & 0.000 & -1.000 \\
\hline 343 & 0.000 & -1.000 \\
\hline 344 & 0.000 & -1.000 \\
\hline 345 & 0.000 & 1.000 \\
\hline 346 & 0.000 & 1.000 \\
\hline 347 & 0.000 & 1.000 \\
\hline 348 & 0.000 & 1.000 \\
\hline 349 & 0.000 & 1.000 \\
\hline 350 & 0.000 & 1.000 \\
\hline & & \\
\hline
\end{tabular}

\begin{tabular}{|c|c|c|}
\hline & $x$ coondinale & $y$ coondinate \\
\hline 351 & 0.000 & -2.000 \\
\hline 352 & 0.000 & 3.000 \\
\hline 353 & 0.000 & 3.000 \\
\hline 354 & 0.000 & 3.000 \\
\hline 355 & 0.000 & -5.000 \\
\hline 356 & 0.174 & -0.985 \\
\hline 357 & 0.174 & -0.985 \\
\hline 358 & 0.174 & 0.985 \\
\hline 359 & 0.174 & 0.985 \\
\hline 360 & 0.174 & -0.985 \\
\hline 361 & 0.174 & 0.985 \\
\hline 362 & 0.174 & -0.985 \\
\hline 363 & 0.174 & 0.985 \\
\hline 364 & 0.174 & 0.985 \\
\hline 365 & 0.174 & 0.985 \\
\hline 366 & 0.174 & 0.985 \\
\hline 367 & 0.309 & 0.951 \\
\hline 368 & 0.342 & -0.940 \\
\hline 369 & 0.342 & 0.940 \\
\hline 370 & 0.342 & 0.940 \\
\hline 371 & 0.342 & 0.940 \\
\hline 372 & 0.342 & 0.940 \\
\hline 373 & 0.342 & 0.940 \\
\hline 374 & 0.342 & 0.940 \\
\hline 375 & 0.438 & -0.899 \\
\hline 376 & 0.485 & -0.875 \\
\hline 37 & 0.500 & 0.066 \\
\hline 378 & 0.500 & -0.866 \\
\hline 379 & 0.500 & -0.866 \\
\hline 380 & 0.500 & -0.866 \\
\hline 381 & 0.500 & 0.866 \\
\hline 382 & 0.500 & 0.866 \\
\hline 383 & 0.500 & 0.866 \\
\hline 384 & 0.500 & 0.866 \\
\hline 385 & 0.643 & 0.766 \\
\hline 386 & 0.643 & 0.766 \\
\hline 387 & 0.643 & 0.766 \\
\hline 388 & 0.684 & -1.879 \\
\hline 389 & 0.707 & 0.707 \\
\hline 390 & 0.766 & -0.643 \\
\hline 391 & 0.766 & 0.643 \\
\hline 392 & 0.766 & -0.643 \\
\hline 393 & 0.766 & 0.643 \\
\hline 394 & 0.766 & 0.643 \\
\hline 395 & 0.766 & 0.643 \\
\hline 396 & 0.766 & 0.643 \\
\hline 397 & 0.766 & 0.643 \\
\hline 398 & 0.766 & 0.643 \\
\hline 399 & 0.766 & 0.643 \\
\hline 400 & 0.819 & -0.574 \\
\hline
\end{tabular}

\begin{tabular}{|c|c|c|}
\hline & $x$ coordinalo & ycoordinate \\
\hline 401 & 0.819 & 0.574 \\
\hline 402 & 0.857 & -0.515 \\
\hline 403 & 0.866 & -0.500 \\
\hline 404 & 0.866 & 0.500 \\
\hline 405 & 0.866 & -0.500 \\
\hline 406 & 0.866 & -0.500 \\
\hline 407 & 0.866 & -0.500 \\
\hline 408 & 0.866 & -0.500 \\
\hline 409 & 0.656 & 0.500 \\
\hline 410 & 0.866 & 0.500 \\
\hline 411 & 0.866 & 0.500 \\
\hline 412 & 0.866 & 0.500 \\
\hline 413 & 0.866 & 0.500 \\
\hline 414 & 0.866 & 0.500 \\
\hline 415 & 0.866 & 0.500 \\
\hline 416 & 0.868 & 4.924 \\
\hline 117 & 0.906 & -0.423 \\
\hline 418 & 0.940 & -0.342 \\
\hline 419 & 0.940 & -0.342 \\
\hline 420 & 0.940 & -0.342 \\
\hline 421 & 0.940 & -0.342 \\
\hline 422 & 0.940 & -0.342 \\
\hline 423 & 0.940 & -0.342 \\
\hline 124 & 0.940 & 0.342 \\
\hline 425 & 0.940 & 0.342 \\
\hline 426 & 0.940 & 0.342 \\
\hline 427 & 0.940 & 0.342 \\
\hline 428 & 0.985 & -0.174 \\
\hline 429 & 0.985 & -0.174 \\
\hline 430 & 0.985 & 0.174 \\
\hline 431 & 0.985 & -0.174 \\
\hline 432 & 0.985 & 0.174 \\
\hline 433 & 0.985 & -0.174 \\
\hline 434 & 0.985 & -0.174 \\
\hline 435 & 0.985 & -0.174 \\
\hline 436 & 0.985 & 0.174 \\
\hline 437 & 0.985 & 0.174 \\
\hline 438 & 0.985 & 0.174 \\
\hline 439 & 0.985 & 0.174 \\
\hline 440 & 0.965 & 0.174 \\
\hline 41 & 0.985 & 0.174 \\
\hline 442 & 0.985 & 0.174 \\
\hline 443 & 0.985 & 0.174 \\
\hline 444 & 0.985 & 0.174 \\
\hline 445 & 0.985 & 0.174 \\
\hline 446 & 0.996 & -0.087 \\
\hline 447 & 0.996 & -0.087 \\
\hline 448 & 0.999 & 0.035 \\
\hline 449 & 0.999 & 0.035 \\
\hline 450 & 1.000 & -1.732 \\
\hline
\end{tabular}

\begin{tabular}{|c|c|c|}
\hline & $\times$ coondinate & ycoordinate \\
\hline 451 & 1.000 & 0.000 \\
\hline 452 & 1.000 & 0.000 \\
\hline 453 & 1.000 & 0.000 \\
\hline 454 & 1.000 & 0.000 \\
\hline 455 & 1.000 & 0.000 \\
\hline 456 & 1.000 & 0.000 \\
\hline 457 & 1.000 & 0.000 \\
\hline 458 & 1.000 & 0.000 \\
\hline 459 & 1.000 & 0.000 \\
\hline 460 & 1.000 & 0.000 \\
\hline 461 & 1.000 & 0.000 \\
\hline 462 & 1.000 & 0.000 \\
\hline 463 & 1.000 & 0.000 \\
\hline 461 & 1.000 & 0.000 \\
\hline 465 & 1.000 & 0.000 \\
\hline 466 & 1.000 & 0.000 \\
\hline 467 & 1.000 & 0.000 \\
\hline 468 & 1.000 & 0.000 \\
\hline 469 & 1.000 & 0.000 \\
\hline 470 & 1.000 & 0.000 \\
\hline 471 & 1.000 & 0.000 \\
\hline 472 & 1.000 & 0.000 \\
\hline 473 & 1.000 & 0.000 \\
\hline 474 & 1.000 & 0.000 \\
\hline 475 & 1.000 & 0.000 \\
\hline 476 & 1.000 & 0.000 \\
\hline 477 & 1.000 & 0.000 \\
\hline 478 & 1.000 & 0.000 \\
\hline 479 & 1.000 & 0.000 \\
\hline 460 & 1.000 & 0.000 \\
\hline 491 & 1.000 & 0.000 \\
\hline 482 & 1.000 & 0.000 \\
\hline 463 & 1.000 & 0.000 \\
\hline 484 & 1.000 & 0.000 \\
\hline 485 & 1.000 & 0.000 \\
\hline 466 & 1.000 & 0.000 \\
\hline 487 & 1.000 & 0.000 \\
\hline 488 & 1.000 & 0.000 \\
\hline 469 & 1.000 & 0.000 \\
\hline 490 & 1.000 & 0.000 \\
\hline 491 & 1.000 & 0.000 \\
\hline 492 & 1.000 & 0.000 \\
\hline 493 & 1.000 & 0.000 \\
\hline 494 & 1.000 & 0.000 \\
\hline 495 & 1.000 & 0.000 \\
\hline 496 & 1.000 & 0.000 \\
\hline 497 & 1.000 & 0.000 \\
\hline 498 & 1.000 & 0.000 \\
\hline 499 & 1.000 & 0.000 \\
\hline 500 & 1.000 & 0.000 \\
\hline & & \\
\hline
\end{tabular}

Table 3.41 (continued)

General Aviation Landing Crash Locations 


\begin{tabular}{|c|c|c|}
\hline & $\times$ coondinalo & ycoondinato \\
\hline 501 & 1.000 & 0.000 \\
\hline 502 & 1.000 & 0.000 \\
\hline 503 & 1.000 & 0.000 \\
\hline 504 & 1.000 & 0.000 \\
\hline 505 & 1.000 & 0.000 \\
\hline 506 & 1.000 & 0.000 \\
\hline 507 & 1.000 & 0.000 \\
\hline 508 & 1.000 & 0.000 \\
\hline 509 & 1.000 & 0.000 \\
\hline 510 & 1.000 & 0.000 \\
\hline 511 & 1.000 & 0.000 \\
\hline 512 & 1.000 & 0.000 \\
\hline 513 & 1.000 & 0.000 \\
\hline 514 & 1.000 & 0.000 \\
\hline 515 & 1.000 & 0.000 \\
\hline 516 & 1.000 & 0.000 \\
\hline 517 & 1.000 & 1.732 \\
\hline 518 & 1.000 & 1.732 \\
\hline 519 & 1.286 & -1.532 \\
\hline 520 & 1.206 & 1.532 \\
\hline 521 & 1.368 & 3.759 \\
\hline 522 & 1.782 & 0.008 \\
\hline 523 & 1.879 & 0.684 \\
\hline 524 & 1.928 & -2.298 \\
\hline 525 & 1.932 & -0.518 \\
\hline 526 & 1.970 & -0.347 \\
\hline 527 & 1.970 & -0.347 \\
\hline 528 & 1.970 & -0.347 \\
\hline 529 & 1.970 & 0.347 \\
\hline 530 & 2.000 & 0.000 \\
\hline 531 & 2.000 & 0.000 \\
\hline 532 & 2.000 & 0.000 \\
\hline 533 & 2.000 & 0.000 \\
\hline 534 & 2.000 & 0.000 \\
\hline 535 & 2.000 & 0.000 \\
\hline 536 & 2.000 & 0.000 \\
\hline 537 & 2.500 & 4.330 \\
\hline 538 & 2.598 & -1.500 \\
\hline 539 & 2.598 & -1.500 \\
\hline 540 & 2.819 & 1.026 \\
\hline 541 & 2.898 & 0.776 \\
\hline 542 & 2.954 & -0.521 \\
\hline 543 & 2.954 & -0.521 \\
\hline 544 & 2.954 & 0.521 \\
\hline 545 & 2.954 & 0.521 \\
\hline 546 & 3.000 & 0.000 \\
\hline 547 & 3.000 & 0.000 \\
\hline 548 & 3.000 & 0.000 \\
\hline 549 & 3.000 & 0.000 \\
\hline 550 & 3.064 & 2.571 \\
\hline & & \\
\hline
\end{tabular}

\begin{tabular}{|c|c|c|}
\hline & $x$ coordinne & ycoordinate \\
\hline 551 & 3.830 & -3.214 \\
\hline 552 & 3.830 & 3.214 \\
\hline 553 & 3.857 & -4.596 \\
\hline 554 & 3.939 & -0.695 \\
\hline 555 & 4.000 & 0.000 \\
\hline 556 & 4.000 & 0.000 \\
\hline 557 & 4.000 & 0.000 \\
\hline 558 & 4.330 & -2.500 \\
\hline 559 & 4.330 & 2.500 \\
\hline 560 & 4.455 & 2.270 \\
\hline 561 & 4.596 & 3.857 \\
\hline 562 & 4.830 & 1.294 \\
\hline 563 & 4.924 & 0.868 \\
\hline 564 & 4.924 & 0.868 \\
\hline 565 & 4.924 & 0.868 \\
\hline 566 & 4.981 & 0.436 \\
\hline 567 & 5.000 & 0.000 \\
\hline 568 & 5.000 & 0.000 \\
\hline 569 & 5.000 & 0.000 \\
\hline 570 & 5.000 & 0.000 \\
\hline 571 & 5.000 & 0.000 \\
\hline 572 & 5.000 & 0.000 \\
\hline 573 & 5.000 & 0.000 \\
\hline 574 & 5.000 & 0.000 \\
\hline
\end{tabular}

Teblo 3.41 (continued)

Ceneral Aviation Lending Crash Locations 


\begin{tabular}{|c|c|c|c|c|c|c|c|c|c|c|c|c|}
\hline$x$ & $(-4,-3)$ & $(-3,-2)$ & $(-2,-1)$ & $(-1,0)$ & $(0,1)$ & $(1,2)$ & $(2,3)$ & $(3,4)$ & $(4,5)$ & $(5,6)$ & $(6,7)$ & $(7,8)$ \\
\hline$(3,4)$ & & & & 1.2E-05 & 1.7E-04 & 4.2E-04 & 1.7E-04 & $1.4 E-05$ & & & & \\
\hline$(2,3)$ & & & $1.1 E-05$ & $1.5 \mathrm{E}-04$ & $1.1 E-03$ & $2.2 \mathrm{E}-03$ & $8.9 E-04$ & 4.0E-04 & $1.1 E-03$ & $6.5 \mathrm{E}-04$ & $6.3 \mathrm{E}-05$ & \\
\hline$(1,2)$ & & $1.6 \mathrm{E}-05$ & 5.2E-04 & $6.4 E-03$ & $1.3 \mathrm{E}-02$ & $9.8 \mathrm{E}-03$ & $4.0 E-03$ & $1.9 \mathrm{E}-03$ & $3.2 E-03$ & $1.9 \mathrm{E}-03$ & $2.1 E-04$ & \\
\hline$(0,1)$ & & $3.4 E-04$ & 5.1E-03 & $1.5 \mathrm{E}-01$ & 2.0E-01 & $6.9 E-02$ & $2.2 E-02$ & 5.8E-03 & $4.5 \mathrm{E}-03$ & 4.5E-03 & $1.5 \mathrm{E}-03$ & 4.6E-04 \\
\hline$(-1,0)$ & 1.1E- 05 & $4.8 \mathrm{E}-04$ & $6.4 E-03$ & $1.5 E-01$ & $2.0 \mathrm{E}-01$ & $6.3 E-02$ & $2.1 E-02$ & $6.1 E-03$ & $4.3 E-03$ & $4.4 \mathrm{E}-03$ & $1.5 E-03$ & $1.6 \mathrm{E}-04$ \\
\hline$(-2,-1)$ & & $5.9 \mathrm{E}-05$ & $1.0 E-03$ & $7.2 E-03$ & 1.1E-02 & 5.7E-03 & $2.0 E-03$ & $5.1 E-04$ & $2.7 E-04$ & $3.8 E-04$ & $1.4 \mathrm{E}-04$ & $1.0 \mathrm{E}-05$ \\
\hline$(-3,-2)$ & & & 1.7E-05 & $1.0 \mathrm{E}-04$ & 1.7E-04 & 4.5E-04 & $1.0 E-03$ & 5.1E-04 & 2.7E-04 & $3.8 \mathrm{E}-04$ & 1.4E-04 & $1.0 \mathrm{E}-05$ \\
\hline$(-4,-3)$ & & & & & 2.6E-05 & 4.4E-04 & $1.2 E-03$ & 5.7E-04 & $2.0 E-04$ & $3.4 \mathrm{E}-04$ & $1.2 E-04$ & \\
\hline$(-5,-4)$ & & & & & & 1.5E-05 & 4.2E-05 & $2.0 E-05$ & & & & \\
\hline
\end{tabular}

Table 3.42

Crash Location Probability $f(x, y)$ for

General Aviation Takeofis 


\begin{tabular}{|c|c|c|c|c|c|c|c|c|c|c|c|c|c|c|c|c|c|c|c|c|c|c|c|c|}
\hline$x$ & $(-16,-15)$ & $(-15,-14)$ & $(-14,-13)$ & $(-13,-12)$ & $(-12,-91)$ & $(-11,-10)$ & $(-10,-9)$ & $(-9,8)$ & $(-8,-7)$ & $(-7,-6)$ & $(-6,-5)$ & $(-5,-4)$ & $(-4,-3)$ & $(-3,-2)$ & $(-2,-1)$ & $(-1,0)$ & $(0,1)$ & $(1,2)$ & $(2,3)$ & $(3,4)$ & $(4,8)$ & $5,6)$ & $(6, n)$ & $(7.8)$ \\
\hline$(5,6)$ & & & & & & & & & & & & & 1.5E-0.5 & 6.3E-0.5 & $1.9 E-04$ & $3.5 E-09$ & 3.5E-04 & $1.0 E-04$ & 6.2E-05 & $1.5 E-05$ & & & & \\
\hline$(4,5)$ & & & & & & & & & & & & 4.3E-0S & $1.9 E-04$ & 4.3E-04 & 8.1E-04 & $8.8 \mathrm{E}-04$ & $6.0 \mathrm{E}-04$ & $4.8 E-04$ & $3.8 E-04$ & $2.4 E-04$ & 1.7E-04 & $8.7 E-06$ & $2.0 E-05$ & \\
\hline$(3,4)$ & & & & & & & & & & $3.3 E-05$ & $1.1 E-04$ & $2.7 E-04$ & $5.2 E-04$ & B.3E-04 & $9.7 E-04$ & 7.BE-OA & 5.0E-0a & $3.8 E-04$ & 3.TE-OA & $4.8 E-04$ & $4.8 E-04$ & 2.3E-0 & 4.4E-05 & \\
\hline$(2,3)$ & & & 5.6E-05 & $2.05-04$ & 3.3E-04 & 2.8E-04 & 1.0E-04 & $7.1 E-05$ & 9.9E-05 & 3.1E-04 & 5.0E-04 & 4.5E-04 & $7.5 E-04$ & $1.5 E-03$ & $1.7 E-09$ & 1.1E-03 & $6.0 E-08$ & 4.0E-04 & 4.5E-O4 & $7.1 \mathrm{E}-04$ & $7.1 E-04$ & 3.3E-04 & 6.0E-05 & \\
\hline$(1,2)$ & & $7.2 E-05$ & $2.8 \mathrm{E}-0 \mathrm{~A}$ & 5.2E-01 & 6.1E-04 & 5.6E-04 & 4.5E-04 & 4.5E-04 & $6.5 E-04$ & $8.8 E-04$ & 6.7E-04 & $6.6 E-09$ & 1.1E-03 & 3.0E-03 & $5.8 E-09$ & $1.2 E-02$ & 1.1E-02 & 4.4E-03 & $1.5 E-00$ & $7.0 \mathrm{E}-04$ & 5.3E-04 & 3.3E-O4 & Q.0E-05 & \\
\hline$(0,1)$ & $1.2 E-05$ & $1.0 E-04$ & 3.5E-04 & 3.3E-04 & $5.0 \mathrm{E}-04$ & $3.8 \mathrm{E}-04$ & 7.4E-OA & 9.5E-O4 & $1.0 E-\infty$ & $2.9 E-03$ & $4.0 E-\infty$ & 4.3E- -0 & $7.2 E-\infty$ & $18 \mathrm{~B}-02$ & $3.9 E-02$ & 1.6E-01 & $1.6 E-01$ & $2.0 E-02$ & 1.1E-02 & $3.0 E-03$ & $2.8 E-03$ & $1.7 E-03$ & 5.6E-04 & 6.8E-05 \\
\hline$(-1,0)$ & & 7.3E-05 & $3.1 \mathrm{E}-04$ & 6.0E-04 & 6.3E-04 & $0.0 E-04$ & 6.5E-04 & 6.7E-04 & 1.1E-03 & $2.2 E-03$ & $3.3 E-03$ & $3.8 E-03$ & $6.8 E-03$ & 1.7E-02 & 3.7E-02 & $1.6 E-01$ & 1.6E-01 & 2.8E-02 & $1.0 E-02$ & $4.0 E-03$ & $3.0 E-\infty$ & 2.1E-00 & B.5E-08 & 7.7E-05 \\
\hline$(-2,-1)$ & & & $5.8 E-05$ & $1.8 E-04$ & $3.0 \mathrm{E}-04$ & $3.9 E-04$ & $3.7 E-04$ & 2.1E-04 & $2.5 E-04$ & $3.5 E-04$ & 5.1E-04 & 7.4E-04 & $1.0 E-09$ & 2.3E-03 & $4.9 E-09$ & 1.1E-02 & 1.0E-02 & $3.8 E-\infty$ & $1.6 E-\infty$ & 8.2E-04 & $6.0 E-04$ & $4.0 E-04$ & 1.2E-04 & 1.4E-05 \\
\hline$(-3,-2)$ & & & 4.6E-05 & $1.6 E-04$ & 2.1E-04 & $1.5 E-04$ & $1.0 \mathrm{E}-04$ & 7.0E-05 & $1.8 E-04$ & $3.2 E-04$ & $3.8 E-04$ & $5.3 \mathrm{E}-04$ & $6.8 E-04$ & $1.1 \mathrm{E}-03$ & 1.4E-03 & $1.2 E-03$ & 9.4E-O4 & 6.8E-04 & 4.7E-04 & $4.2 \mathrm{E}-04$ & $3.7 E-04$ & 1.0E-04 & $2.7 E .05$ & \\
\hline$(-1,-3)$ & & & & & & & & $5.2 E-05$ & $1.6 E-04$ & 2.0E-04 & $2.5 E-04$ & $6.0 E-04$ & 8.3E-04 & $5.8 E-04$ & $3.6 E-04$ & $3.8 E-04$ & $3.3 E-04$ & 1.4E-OA & 1.4E-OA & $2.8 E-04$ & $2.4 E-04$ & $0.1 E 05$ & & \\
\hline$(-5,-1)$ & & & & & & & & $4.8 E-05$ & 1.5E-04 & $1.7 \mathrm{E}-\mathrm{OA}$ & $1.0 E-04$ & 3.5E-OA & $\triangle 0 E-04$ & $3.8 \mathrm{E}-04$ & 2.5E-OA & $3.3 E-04$ & 3.1E-OA & $1.2 E-04$ & 1.2E-04 & $2.0 E-04$ & $2.2 E-04$ & 6.8E-05 & & \\
\hline$(-6,-5)$ & & & & & & & & & & & & $1.3 \mathrm{E}-0.5$ & $1.6 \mathrm{E}-05$ & 2.5E-05 & $1.1 E-04$ & $3.0 E-04$ & 3.0E-04 & 3.5E-05 & 1.1E-04 & 3.3E-05 & $45 E-05$ & $1.4 E-05$ & & \\
\hline
\end{tabular}

Table 3.43

Crash Location Probability $f(x, y)$ for

General Avlation Landings 


\subsubsection{IMPACT VELOCITY DISTRIBUTION}

An important input to the detailed calculation of aircraft crash likelihood is the distribution of aircraft velocity at the time of impact. The NTSB database records airspeeds at impact using 11 ordinal categories; this information was regrouped into five segments to ensure adequate cell counts for further statistical analysis, as shown in Table 3.44.

\begin{tabular}{|c|c|}
\hline \multicolumn{2}{|c|}{ Table 3.44} \\
DISTRIBUTION OF IMPACT VELOCITIES \\
\hline $\begin{array}{c}\text { Impact Velocity } \\
\text { (knots) }\end{array}$ & Percentage \\
\hline $0-30$ & 6.3 \\
\hline $30-60$ & 29.7 \\
\hline $60-90$ & 38.4 \\
\hline $90-120$ & 14.2 \\
\hline$>120$ & 11.4 \\
\hline
\end{tabular}

An investigation was conducted to see if impact velocities were correlated to the angle of impact or to aircraft weight; results of an analysis of variance (ANOVA) indicated no significant interactions at the 0.05 significance level. 


\subsubsection{IMPACT ANGLE DISTRIBUTION}

An important input to the detailed caiculation of aircraft crash likelihood is the distribution of aircraft impact angle. In this study, the term impact angle is defined as the aircraft's flight path angle as measured with respect to horizontal (e.9., 0 degrees implies level flight while 90 degrees implies that the aircraft is traveling straight down); impact angle is not related to an aircraft's orientation, as measured by its roll, pitch, and yaw angles. The NTSB database records impact angles using nine ordinal categories; this information was regrouped into five segments to ensure adequate cell counts for further statistical analysis, as shown in Table 3.45.

\begin{tabular}{|c|c|}
\hline $\begin{array}{l}\text { Table } \\
\text { DISTRIBUTION OF }\end{array}$ & $\begin{array}{l}3.45 \\
\text { IMPACT ANGLES }\end{array}$ \\
\hline $\begin{array}{c}\text { Impact Angle } \\
\text { (dearees) }\end{array}$ & Percentage \\
\hline $0-10$ & 42.6 \\
\hline $10-20$ & 19.0 \\
\hline $20-30$ & 11.2 \\
\hline $30-60$ & 14.9 \\
\hline$>60$ & 12.3 \\
\hline
\end{tabular}

As noted in Section 3.6.2, results of an analysis of variance (ANOVA) indicated no significant interactions among impact velocity, impact angle, and aircraft weight at the 0.05 significance level. 


\subsubsection{SKID DISTANCE DISTRIBUTION}

In order to support the screening analysis, an empirical distribution of skid distances was developed from the NTSB database (Figure 3.1) by combining the data across all aircratt subcategories, impact velocities, and impact angles. It should be noted that about $32.1 \%$ of all aircratt crashes do not result in a measurable skid distance. Approximately $90 \%$ of all skids are less than 135 feet, and this value is suggested for use in the screening analysis.

In order to support the detailed analysis, multiple regression analysis was used to correlate skid distances to aircraft weight, impact velocity, and impact angle since these parameters dictate an aircraft's momentum at the time of impact. Regression analysis is well-suited since skid distance and aircraft weight are continuous quantities, and the influence of the ordinal impact velocity and impact angle groups can be readily accommodated using indicator variables. The basic form of the regression equation is:

$$
\ln L=b^{*} \operatorname{mln} W=\sum_{i=1_{\alpha_{i}}}^{5} S_{i}+\sum_{j=1_{\beta_{j}}}^{5} A_{j}+\varepsilon
$$

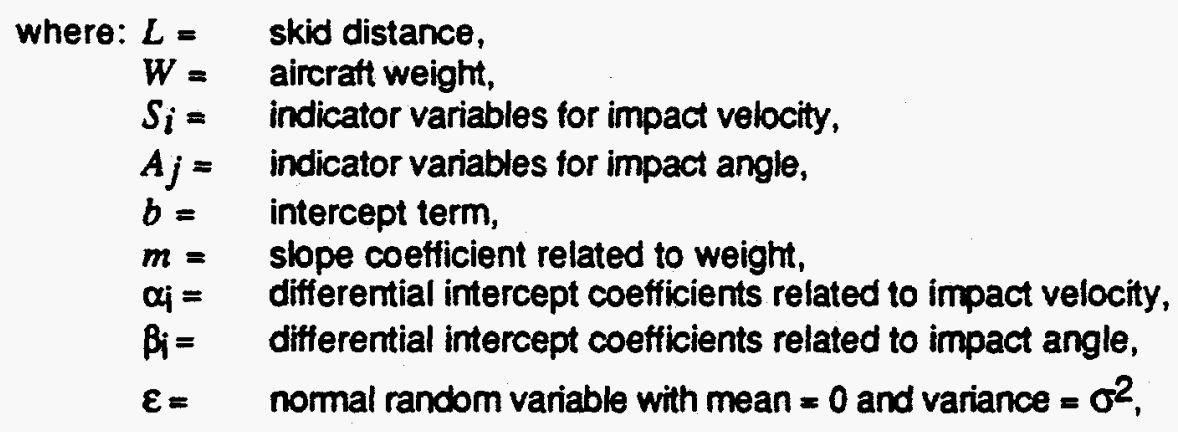

It should be noted that the indicators variables are set as ordered quintuples when using the regression equation. For example, if the impact velocity range is 30-60 knots and the impact angle range is 0-10 degrees, then $\left\{S_{1}=0, S_{2}=1, S_{3}=0, S_{4}=0, S_{5}=0\right\}$ and $\left\{A_{1}=1, A_{2}=0, A_{3}=0, A_{4}=0, A_{5}=0\right\}$.

Results of the multiple regression are shown in Table 3.46. As expected, the signs and magnitudes of the regression parameters coincide with common sense: skid distance increases as the aircraft weight and impact velocity increase, and decreases as the impact angle increases. A plot of the residuals (Figure 3.2) shows a linear trend (distorted at the tails), tending to confirm the normality of the error term.

The results of the regression analysis can be used to predict the skid distance distribution given aircraft weight, impact velocity, and impact angle. For example, consider the situation in which the aircraft weight is 1500 pounds, the impact velocity range is 60-90 knots, and the impact angle range is 20-30 degrees. Then,

$$
\begin{aligned}
\operatorname{In} L= & 0.184+0.349 \ln 1500+\left(-0.485^{*} 0-0.310^{*} 0+0.000989 * 1+0.206^{*} 0+0.626^{*} 0\right) \\
& +\left(0.471^{*} 0+0.116^{*} 0-0.236^{*} 1-0.405^{*} 0-0.559^{*} 0\right) \\
= & 2.50
\end{aligned}
$$


Recalling that in $L$ is normally distributed, then $L$ is $\log$-normally distributed, l.e.,

$$
\operatorname{Pr}\{L \leq x\}=\Phi\left[\frac{\ln x-2.50}{1.32}\right]
$$

where:

$$
\Phi[y]=\text { standard normal distribution function }
$$

Thus, the 90th percentile of the skid distance for this example (i.e., the value of $x$ such that $\operatorname{Pr}\{L \leq x\}=0.90\})$ is given by:

$$
\begin{aligned}
x_{0.9} & =\exp [2.50+1.2816 \cdot 1.32] \\
& =66.1 \text { feet }
\end{aligned}
$$

\begin{tabular}{|c|c|}
\hline \multicolumn{2}{|c|}{ Table 3.46 } \\
\hline MULTIPLE REGRESSION ANALYSIS & FOR SKID DISTANCE \\
\hline Parameter & Value \\
\hline b (intercept term) & 0.184 \\
\hline m (aircraft weight-related slope) & 0.349 \\
\hline differential intercept coefficients & \\
for impact velocity & -0.485 \\
0-30 knots: $\alpha_{1}$ & -0.310 \\
$30-60$ knots: $\alpha_{2}$ & 0.000989 \\
$60-90$ knots: $\alpha_{3}$ & 0.206 \\
90-120 knots: $\alpha_{4}$ & 0.626 \\
$>120$ knots: $\alpha_{5}$ & \\
\hline differential intercept coefficients & \\
for impact angle & 0.471 \\
$0-10$ degrees: $\beta_{1}$ & 0.116 \\
10-20 degrees: $\beta_{2}$ & -0.236 \\
$20-30$ degrees: $\beta_{3}$ & -0.405 \\
$30-60$ degrees: $\beta_{4}$ & -0.559 \\
$>60$ degrees: $\beta_{5}$ & \\
\hline coefficient of multiple regression $(R)$ & 0.44 \\
\hline sigma & 1.32 \\
\hline
\end{tabular}




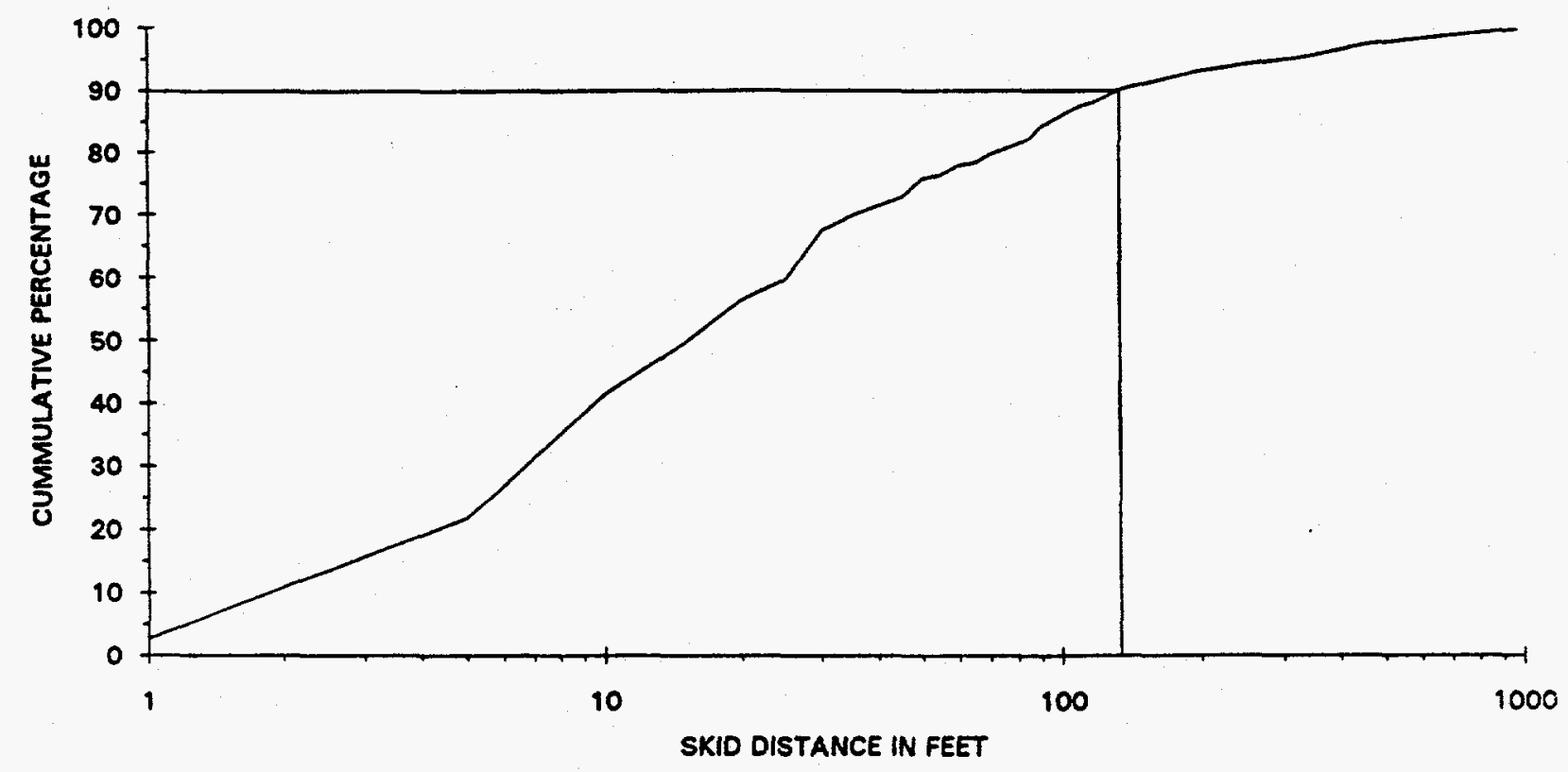

Flgure 3.1

General Aviation Empirical Skid Distance Distribution

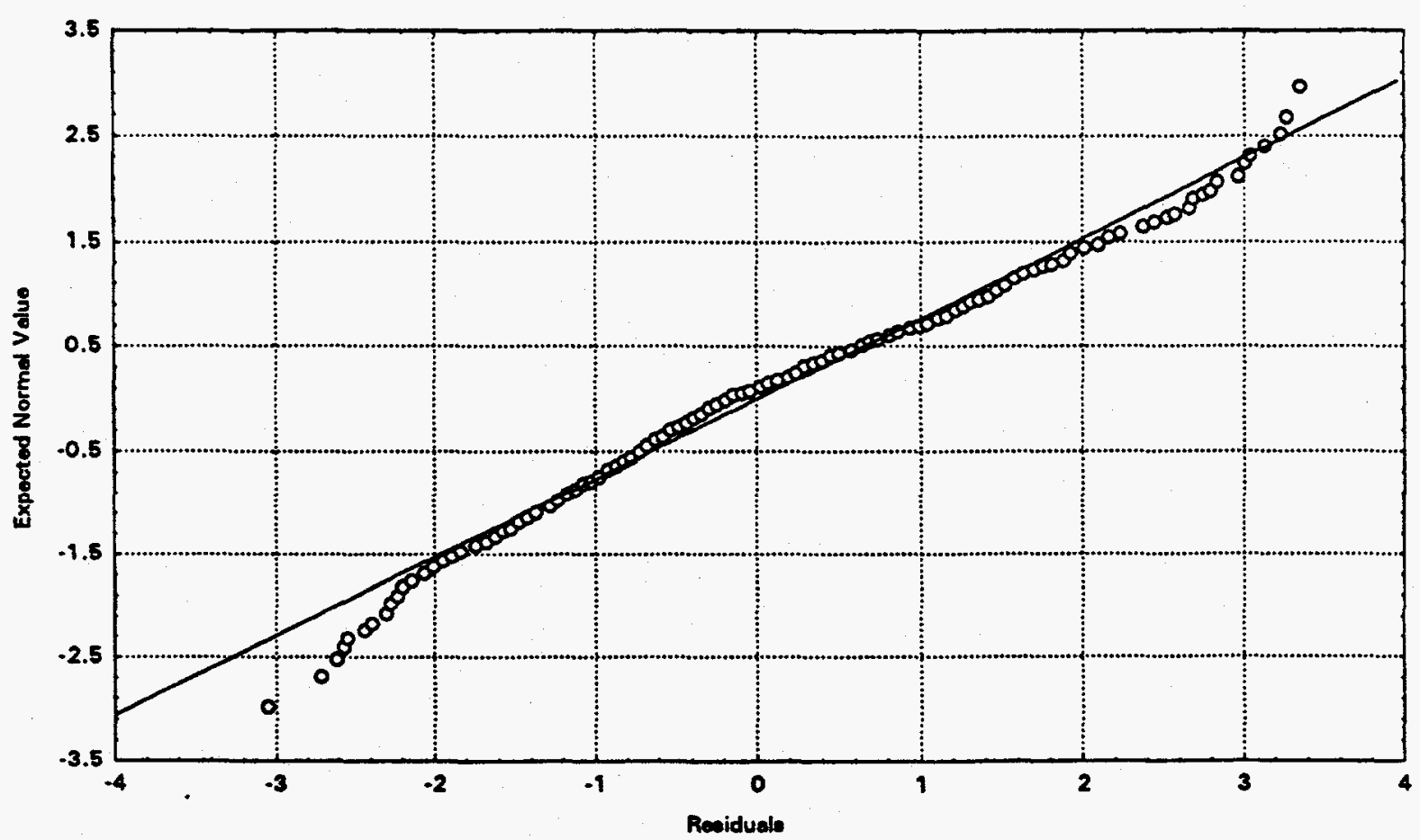

Figure 3.2

Normal Probablilty Plots of Reslduals 


\subsection{GENERAL AVIATION AIRCRAFT CHARACTERISTICS}

This section was prepared to provide to the analyst those characteristics of aircraft that could be important to determine the effect of the aircraft on the facility. Such aircratt characteristics which could be crucial in determine whether a facility could withstand an impact from an aircraft could be the weight of the aircraft, the amount of fuel aboard the aircraft, the speed of the aircraft, the dimensions of the aircratt, and potential missiles generated by the aircratt as its structure failed. Many of these parameters would vary greatly during the course of the flight, but the values of the characteristics given in this section could be considered the upper bound of these parameters. To determine the characteristics of an aircraft at the moment of impact in a particular accident, the analyst should refer to the specific accident report.

Tables 3.47 to 3.52 taken from References 3.29 to 3.35 presents the following information on general aviation aircraft characteristics:
1. Aircratt type,
2. Powerplant(s), number and type,
3. Nominal power rating of each powerplant,
4. Operating empty weight expressed in pounds, and kilograms,
5. Maximum allowed (or certificated) takeoff weight expressed in pounds, and kilograms,
6. Maximum allowed (or certificated) landing weight expressed in pounds, and kilograms,
7. Maximum zero-fuel weight expressed in pounds, and kilograms,
8. Maximum fuel-capacity expressed in imperial gallons, and liters,
9. Overall wing span expressed in feet and inches, and in meters,
10. Overall length expressed in feet and inches, and in meters,
11. Overall height expressed in feet and inches, and in meters,
12. Wing area expressed in $\mathrm{tt}^{2}$ and meters ${ }^{2}$,
13. Takeoff rumway length according to Federal Air Regulations (FAR) requirements, expressed in feet,
14. Landing rumway length according to Federal Air Regulations (FAR) requirements, expressed in feet,
15. Stall speed with gear flaps deployed, expressed in miles/hour.

Powerplant(s) were included as potential missile generators. Weights were given for mass during various points of the flight. Fuel capacity was included in order to provide the maximum fuel available for a fire following the crash. Aircraft dimensions will be needed when the facility effective area is determined. The takeoff runway length and landing numway length were provided so that an analyst could screen certain aircraft from certain airports due to their inability to operate from the airport's rumway(s). Stall speed was provided so that an estimate of the impact speed could be made as a large percentage of general aviation aircraft crashes occur during the landing approach flight phase, and most general aviation aircraft fly the approach phase at about 1.3 times the stall speed.

The tables listed below gives the general aviation subcategories for which the general aviation aircraft characteristics table were created:

Table 3.47

Table 3.48

Table 3.49

Table 3.50

Table 3.51

Table 3.52
Single-engine reciprocating (piston), fixed wing,

Single-engine agricultural, fixed wing,

Multi-engine reciprocating (piston), fixed wing,

Turboprop, fixed wing,

Turbojet, fixed wing,

Rotary wing,

Aircraft are listed in descending order of maximum takeoff weight within each subcategory.

A separate table for single-engine agricultural, fixed wing aircraft was created because the size, weight, engine power, and flight profiles of these aircraft differed so greatly from the standard single-engine piston, fixed wing aircraft population. We have not attempted to characterize the agricultural aircraft population in terms of number of active aircraft, distribution of active aircraft, number of opertions, etc. 
This could be an area in which additional research would be justified, particularty if a facility has a large number of agricultural aircraft operations in the nearby vicinity.

Finally, additional research is needed to characterize the missiles (projectiles) generated during the course of an aircraft's crash. Obvious examples of such missiles are the aircraft's engines, the auxiliary power units (APUs), and the landing gear. Much of the information on these aircraft components is not readily available and may have to be obtained directly from the aircraft or engine manufacturer or from the company that produces the component. 
Table 3.47

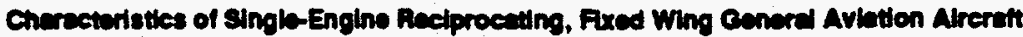
Roturences 329 to 325

\begin{tabular}{|c|c|c|c|c|c|c|c|c|c|c|c|}
\hline \multirow[b]{2}{*}{ Arenth Type } & \multicolumn{2}{|c|}{ Pomerientis) } & \multirow{2}{*}{$\begin{array}{l}\text { Nomina } \\
\text { Power } \\
\text { Pating (oni) }\end{array}$} & \multicolumn{2}{|c|}{$\begin{array}{l}\text { Operating } \\
\text { Empir Woinht }\end{array}$} & \multicolumn{2}{|c|}{$\begin{array}{l}\text { Maximum } \\
\text { Takeor Weight }\end{array}$} & \multicolumn{2}{|c|}{$\begin{array}{l}\text { Maximum } \\
\text { Lending Weight }\end{array}$} & \multicolumn{2}{|c|}{$\begin{array}{l}\text { Maximum } \\
\text { Zero-Fuel Weight }\end{array}$} \\
\hline & No. & Type & & Pound is & Kloorent & Pounds & Klogram & Pound & Kilogren & Pound: & Kilogran \\
\hline \multicolumn{12}{|c|}{ SINGLE-ENGINE RECIPROCATWG (PISTON), FIXED WING } \\
\hline Cersna Prossurized Conturion & 1 & Continoma TSIO-520-P PP & $310 \mathrm{hp}$ & 2,340 & 1,081 & 4,018 & 1.022 & N.A. & N.A. & N.A. & N.A. \\
\hline Cecsene Tutoo Conturion & 1 & Continental TS10-520-APP & 310hg & 2221 & 1,007 & 4,018 & 1,822 & N.A. & N.A. & N.A. & N.A. \\
\hline Cesena Tutoo Stationai 8 & 1 & Continom I TS10-520-4 PP & 310 ho & 2,183 & $\infty$ & 3,816 & 1.731 & N.A. & N.A. & N.A. & N.A. \\
\hline Cenesna Stationair 8 & 1 & Continomiat 10-520-F PP & 300 hp & 2105 & ess & 3,812 & 1,720 & N.A. & N.A. & N.A. & N.A. \\
\hline Cesene Centurion & 1 & Continemil 10-520-L PP & soohp & 2,130 & e日) & 3.812 & 1.729 & N.A. & N.A. & N.A. & N.A. \\
\hline Eeochcren Eonanza ABBTC & 1 & Continend TS10-520-up PP & $300 \mathrm{hg}$ & 2.200 & 1,00 & 3,060 & 1,068 & N.A. & N.A. & N.A. & N.A. \\
\hline Cossna Turbo Stationair 6 & 1 & Continenal TSIO-520-M PP & 310 ho & 2000 & $\infty$ & 3.618 & 1.840 & N.A. & N.A. & N.A. & N.A. \\
\hline Piper Serenga & 1 & Lroomin $10540-K_{1650}$ PP & soongl & 1,200 & 671 & 3,615 & 1.040 & N.A. & N.A. & N.A. & N.A. \\
\hline Cessna Sudonair 6 & 1 & Continemal 10-520-F PP & $300 \mathrm{np}$ & 1,027 & 874 & 3.612 & $1,0 \times 8$ & N.A. & N.A. & N.A. & N.A. \\
\hline Piper Turbo Sarabas SP & 1 & LLcomin T10-540-51AD PP & soong & 2,073 & 240 & 3.600 & 1.033 & N.A. & N.A. & N.A. & N.A. \\
\hline Piper Turoo Sarabog & 1 & Lreoming $10-540-K_{1}$ G5D PP & soong & 1,083 & e.1 & 3,000 & $1,0 \times 3$ & N.A. & N.A. & N.A. & N.A. \\
\hline Beochereft Bonenza A33 & 1 & Continontal 10-520-88 PP & $205 \mathrm{hp}$ & 2,181 & e94 & 3.600 & 1.053 & N.A. & N.A. & N.A. & N.A. \\
\hline Beochcran Bonenza F33A & 1 & Continental 10-520-83 PP & $295 \mathrm{ng}$ & 2,132 & 067 & 3,400 & 1,542 & N.A. & N.A. & N.A. & N.A. \\
\hline Beechcran Bonenza V353 & 1 & Continental 10-520-B8 PP & $295 \mathrm{hp}$ & 2,117 & 80 & 3,400 & 1,542 & N.A. & N.A. & N.A. & N.A. \\
\hline Cesena 185 Skywagon & 1 & Continental 10-520-D PP & $300 \mathrm{np}$ & 1,688 & 786 & 3,382 & 1,525 & N.A. & N.A. & N.A. & N.A. \\
\hline Cessna Turbo Skyiane RG & 1 & Lycoming O-540-L3C50 PP & $235 \mathrm{hp}$ & 1,791 & 812 & 3,112 & 1,412 & N.A. & N.A. & N.A. & N.A. \\
\hline Cessna Skyane AC & 1 & Lyeoming 0-540-13C50 PP & $235 \mathrm{hp}$ & 1,750 & 794 & 3,112 & 1,412 & N.A. & N.A. & N.A. & N.A. \\
\hline Cessna Turbo Sirylane RG II & 1 & Lycomin $0-540-43 c 50$ PP & $235 \mathrm{no}$ & 1781 & 800 & 3,100 & 1.408 & N.A. & N.A. & N.A. & N.A. \\
\hline Piper Turbo Sarabge & 1 & ILeomin TL-540-SIAD PP & soong & 1.617 & 733 & 3,000 & 1,381 & N.A. & N.A. & N.A. & N.A. \\
\hline Pipsor Dakoth & 1 & Lreoming $0-540$ J345D PP & $235 \mathrm{hp}$ & 1,834 & 741 & 3,000 & 1,381 & N.A. & N.A. & N.A. & N.A. \\
\hline Cossna 182R Shylene II & 1 & Continental O-470-U PP & $230 \mathrm{np}$ & 1,730 & 788 & 3,100 & 1,408 & N.A. & N.A. & N.A. & N.A. \\
\hline Piper Turbo Arrow IV & 1 & Continonal TSIO-380-F PP & $200 \mathrm{hp}$ & 1,680 & 787 & 2,800 & 1,315 & N.A. & N.A. & N.A. & N.A. \\
\hline Piper Turto Dakota & 1 & Continonal TSIO-330-FB PP & $200 \mathrm{ng}$ & 1.579 & 716 & 2800 & 1,315 & N.A. & N.A. & N.A. & N.A. \\
\hline Mooney Turbo 231 & 1 & Continental TS10-sso-GB PP & $210 \mathrm{hp}$ & 1,800 & 818 & 2,800 & 1,315 & N.A. & N.A. & N.A. & N.A. \\
\hline Maula M-5-180C & 1 & Lyeoming 0-360-CIF PP & $180 \mathrm{hp}$ & 1,300 & 500 & 2,800 & 1,315 & N.A. & N.A. & N.A. & N.A. \\
\hline Cossne 180 Shywagon & 1 & Continental O-470-U PP & $230 \mathrm{ng}$ & 1,843 & 745 & 2,810 & 1,275 & N.A. & N.A. & N.A. & N.A. \\
\hline Piper Arrow IV & 1 & Lyeoming $10-300-C_{1}$ C6 PP & $200 \mathrm{ng}$ & 1,641 & 744 & 2,750 & 1,247 & N.A. & N.A. & N.A. & N.A \\
\hline Beecheratt Sierta 200 & 1 & Lycomin $10-360-A 1 B 6$ PP & $200 \mathrm{hg}$ & 1.713 & $\pi 7$ & 2,750 & 1,247 & N.A. & N.A. & N.A. & N.A. \\
\hline Mooney 201 & 1 & Lycomin $10-360-1386$ PP & $200 \mathrm{hp}$ & 1,640 & 74 & 2,740 & 1,243 & N.A. & N.A. & N.A. & N.A. \\
\hline Leke Aircrat Lake Buccanneer & 1 & Lyeoming PP & $200 \mathrm{hp}$ & 1.555 & 705 & 2,090 & 1,220 & N.A. & N.A. & N.A. & N.A. \\
\hline Cutlass RG & 1 & Lreomin O-360-FiA6 PP & teo hp & 1.558 & 707 & 2,658 & 1,208 & N.A. & N.A. & N.A. & N.A. \\
\hline Cessna Hawk XP & 1 & Continental 10-360-KB PP & $195 \mathrm{hp}$ & 1,538 & 698 & 2,558 & 1,160 & N.A. & N.A. & N.A. & N.A. \\
\hline Piper Archer II & 1 & Lycominn 0-360-AM & $180 \mathrm{hp}$ & 1,418 & 643 & 2,550 & 1,157 & N.A. & N.A. & N.A. & N.A. \\
\hline Maule M-6 & 1 & N.A. & N.A. & 1,450 & 658 & 2,500 & 1,134 & N.A. & N.A. & N.A. & N.A \\
\hline Maule M5-235C & 4 & Lrcoming 0-540-J1A50 PP & $235 \mathrm{hg}$ & 1,400 & 635 & 2,500 & 1,134 & N.A. & N.A. & N.A. & N.A \\
\hline Meule M5-210TC & 1. & Lycoming TO-360-F1 A6D PP & $210 \mathrm{np}$ & 1,400 & 635 & 2,500 & 1,134 & N.A. & N.A. & N.A. & N.A. \\
\hline Maub M5-210C & 1. & Continental 10-360-D PP & $210 \mathrm{hg}$ & 1,350 & 612 & 2,500 & 1,134 & N.A. & N.A. & N.A. & N.A. \\
\hline Beochcraft Sundowner & 1. & ILreoming 0-360-A4K PP & 180 ng & 1.502 & 681. & 2,450 & 1,111 & N.A. & N.A. & N.A. & N.A. \\
\hline Piper Warior II & 1 & Lrcomina 0-320-D3G PP & $160 \mathrm{hp}$ & 1,340 & 608 & 2,325 & 1,055 & N.A. & N.A. & N.A. & N.A. \\
\hline Ceasna Shnnaw & 1 & Lyeoming O-320-H2AD PP & $160 \mathrm{ng}$ & 1,403 & 636 & 2,307 & 1,048 & N.A. & N.A. & N.A. & N.A. \\
\hline Verga 2180 & 1 & Lreomin 0-320A & N.A. & 1,175 & 533 & 1,870 & 848 & N.A. & N.A. & N.A. & N.A. \\
\hline Vanga Kachina 2150A & 1 & Lycoming 0-320-A2C PP & $150 \mathrm{hp}$ & 1,125 & 510 & 1,817 & 824 & N.A. & N.A. & N.A. & N.A. \\
\hline Piper PA-10-150 Super Cub & 1 & Lrcoming 0-320 PP & 150 hg & 946 & 420 & 1.750 & 794 & N.A. & N.A. & N.A. & N.A. \\
\hline Beechcrati 77 Skjpger & 1 & Lrooming O-235L2C PP & $115 \mathrm{hp}$ & 1.103 & 500 & 1.675 & 780 & N.A. & NA. & N.A. & N.A. \\
\hline Pioer PA-38-112 Tomahawk & 1 & I rooming 0-235-L2C PP & $112 \mathrm{hp}$ & 1,128 & 512 & 1.670 & 757 & N.A. & N.A. & N.A. & N.A. \\
\hline Cossna 150 Commuter II & 1 & Continental Q-200-A PP & $100 \mathrm{ng}$ & 1,035 & 492 & 1,000 & 720 & N.A. & N.A. & N.A. & N.A. \\
\hline Coesna 150 & 1 & Continental 0-200-A PP & $100 \mathrm{hg}$ & es5 & 447 & 1,500 & 880 & N.A. & N.A. & N.A. & N.A. \\
\hline Totorchen F-21 & 1 & Lreomin 0-235-L2C PP & $11 \mathrm{ghp}$ & 890 & 49 & 1,500 & 60 & N.A. & N.A. & N.A. & N.A. \\
\hline Plper J-3C-65 Cub & 1 & Continental A-85-i PP & .6 hp & 840 & 200 & 1,100 & 490 & N.A. & N.A. & N.A. & N.A. \\
\hline
\end{tabular}

N.A. = intormation not avilable when wbib prepered.

$P P=$ Pistonprop

hp a horepower

the o breice horeperwor 
Table 347 (Contunued)

Chemetertatice of 8ingle-Engin Acelprocatting, Fxed Wing Ceneral Avtation Areraft

Pelerences 3.20 b 3.35

\begin{tabular}{|c|c|c|c|c|c|c|c|c|c|c|c|c|c|}
\hline \multirow[b]{2}{*}{ Alrenan Type } & \multicolumn{2}{|c|}{$\begin{array}{l}\text { Meximum } \\
\text { Ful Capoin }\end{array}$} & \multicolumn{2}{|c|}{$\begin{array}{l}\text { Overall } \\
\text { Wing Span }\end{array}$} & \multicolumn{2}{|c|}{ loverll Lenen } & \multicolumn{2}{|c|}{ loverel Height } & \multicolumn{2}{|c|}{ Wing Area } & \multirow{2}{*}{$\begin{array}{c}\text { Trkeofit } \\
\text { Diat. (ti) } \\
\text { (FAR) }\end{array}$} & \multirow{2}{*}{$\begin{array}{c}\text { Landing } \\
\text { Dist. (th) } \\
\text { (FAP) }\end{array}$} & \multirow{2}{*}{$\begin{array}{l}\text { Stell Spoed } \\
\text { (ooentinps } \\
\text { dom, mph) }\end{array}$} \\
\hline & $\operatorname{lmp} \mathrm{Ce}$ & Lines & Fi. In. & Whiters & Fi. In. & nowers & Ft. in. & Moten: & Fi. ${ }^{\infty} 2$ & $M^{\prime * 2}$ & & & \\
\hline \multicolumn{14}{|c|}{ SWGLE-ENGINE RECIPAOCATING (PISTON), FIXED WING } \\
\hline Cocent Pressurized Centurion & Q8) & 408 & NA. & N.A. & N.A. & N.A. & N.A. & NA: & N.A. & N.A. & 2.100 & 1500 & 68 \\
\hline Covena Tutoo Centurion & 89 & 405 & N.A. & N.A. & N.A. & N.A. & N.A. & N.A. & N.A. & N.A. & 2,160 & 1,500 & 68 \\
\hline Cesena Tuto Sutioneit 8 & 73 & 332 & N.A. & N.A. & N.A & N.A. & N.A. & N.A. & M.A. & N.A. & 1.660 & 1500 & 68 \\
\hline Cesene Sudoner 8 & 70 & 382 & N.A. & N.A. & N.A. & N.A. & N.A. & N.A. & NA. & N.A. & 1970 & 1,500 & 66 \\
\hline Ceesna Centurion & 8 & 405 & N.A. & N.A. & N.A. & N.A. & N.A. & N.A. & N.A. & N.A. & 2.030 & 1,500 & 64) \\
\hline Becheron Borenta A36TC & 74 & $3 \times 3$ & N.A. & N.A. & NA. & N.A. & NA. & N.A. & NA. & NA. & 2.012 & 149 & 65 \\
\hline Cerene Turbo Stationair 8 & 88 & 400 & N.A. & N.A. & N.A. & N.A. & N.A. & N.A. & NA. & NA. & 1.840 & 1,395 & 62 \\
\hline Piper Serever & 107 & 48 & N.A. & N.A. & N.A. & N.A. & N.A. & N.A. & NA. & N.A. & 1,573 & 1,550 & 6 \\
\hline Cesen Stavionir 6 & 8 & 400 & N.A. & N.A. & N.A. & N.A. & N.A. & NA. & N.A. & N.A. & 1.780 & 1,395 & 62 \\
\hline Piser Tuto Serapos SP & 107 & 48 & N.A. & N.A. & N.A. & N.A. & N.A. & N.A. & N.A. & N.A. & 1420 & 1,840 & 69 \\
\hline Piper Turbo Saratoge & 107 & 4as & N.A. & N.A. & N.A. & N.A. & N.A. & N.A. & NA. & NA. & 1573 & 1,612 & 68 \\
\hline Beochernth Bomenza $A 36$ & 74 & $3<8$ & N.A. & N.A. & $\mathbf{N A}$ & NA. & N.A. & NA. & N.A. & N.A. & 2,040 & $\$ 450$ & 60 \\
\hline Beochcraft Bonanza F33A & 74 & 303 & N.A. & N.A. & N.R. & N.A. & N.A. & N.A. & N.A. & NA. & 1769 & 1,324 & 58 \\
\hline Beecheraft Bonanza V358 & 74 & 368 & N.A. & N.A. & N.A. & N.A. & N.A. & N.A. & N.A. & N.A. & 1,760 & 1,324 & 58 \\
\hline Cesene 785 Sbrwagon & 84 & 382 & N.A. & N.A. & $\mathbf{N A}$ & N.A. & NA. & N.A. & N.A. & N.A. & 1,430 & 1,400 & 56 \\
\hline Cessna Turto Swylane RG & 88 & 400 & NA & N.A. & N.A. & N.A. & N.A. & N.A. & N.A. & NA. & 1,570 & 1,320 & 57 \\
\hline Cosena Skylane RG & 88 & 400 & N.A. & N.A. & N.A. & N.A. & N.A. & N.A. & N.A. & N.A. & 1,570 & 1,320 & 57 \\
\hline Coseng Turbo Shylane RG II & 92 & 418 & N.A. & N.A. & NA. & N.A. & N.A. & N.A. & N.A. & N.A. & N.A. & N.A. & 56 \\
\hline Piper Tutoo Sarabga & 107 & 488 & N.A. & N.A. & N.A. & N.A. & NA. & N.A. & N.A. & N.A. & 1420 & 1,700 & 69 \\
\hline Piper Dakote & 77 & 350 & NA. & N.A. & N.A. & N.A. & N.A. & N.A. & N.A. & N.A. & 1,216 & 1410 & 64 \\
\hline Cosena 182R Shylane II & 88 & 400 & $35^{\prime} 10^{\circ}$ & $10.82 \mathrm{~m}$ & $28^{\prime} 0^{\circ}$ & $8.53 \mathrm{~m}$ & $0^{\circ} 3^{\circ}$ & $2.02 \mathrm{~m}$ & 174.0 & 18.18 & 1,350 & 1,350 & 57 \\
\hline Piper Turbo Arow IV & 77 & 350 & N.A & N.A. & N.A. & N.A. & N.A. & N.A. & N.A. & N.A. & 1,820 & 1,555 & 66 \\
\hline Piper Turoo Dakota & 77 & 350 & N.A. & N.A. & N.A. & N.A. & N.A. & N.A. & N.A. & N.A. & 1402 & 1,697 & 66 \\
\hline Mooney Turbo 231 & 72 & 327 & N.A. & N.A. & N.A. & NA. & N.A. & NA. & NA. & NA. & 2,080 & 2,280 & 65 \\
\hline Mule M-5-180C & 6 & 203 & N.A. & N.A. & N.A. & N.A. & N.A. & N.A. & NA. & N.A. & $\infty$ & $\infty$ & 39 \\
\hline Cossne 180 Shymagon & 84 & 382 & N.A. & N.A. & N.A. & N.A. & N.A. & NA. & N.A. & N.A. & 1.205 & 1,365 & 55 \\
\hline Piper Arrow IV & $\pi$ & 350 & N.A. & N.A. & N.A. & N.A. & N.A. & N.A. & NA. & NA. & 1,600 & 1,525 & 63 \\
\hline Eeochcraft Sierra 200 & 57 & 250 & 329 & $8.88 \mathrm{~m}$ & $25^{\circ} 8^{\prime}$ & $7.85 \mathrm{~m}$ & $8 \cdot 10$ & $2.46 \mathrm{~m}$ & N.A. & N.A. & 1,880 & 1,462 & 69 \\
\hline Mooney 201 & 64 & 291 & N.A. & N.A. & N.A. & N.A. & N.A. & N.A. & N.A. & N.A. & 1,517 & 1,610 & 61 \\
\hline Lake Aircraft Lake Buccanneer & 54) & 245 & N.A. & N.A. & N.A. & N.A. & N.A. & NA. & N.A. & N.A. & $1100(w)$ & $600(w)$ & 45 \\
\hline Cutlass RG & 62 & 282 & N.A. & N.A. & N.A. & N.A. & N.A. & N.A. & N.A. & N.A. & 1,775 & 1,340 & 57 \\
\hline Cossng Hawk XP & 8 & 300 & N.A. & N.A. & N.A. & N.A. & N.A. & N.A. & N.A. & N.A & 1,360 & 1.270 & 50 \\
\hline Piper Avcher If & so & 227 & N.A. & N.A. & N.A. & N.A. & N.A. & N.A. & NA. & N.A. & 1,625 & 1,400 & 61 \\
\hline Meuls M6 & 6 & 314 & N.A. & N.A. & N.A. & N.A. & N.A. & N.A. & N.A. & N.A. & N.A. & N.A. & 26 \\
\hline Maule M5-235C & 63 & 288 & N.A. & N.A. & N.A. & N.A. & N.A. & N.A. & N.A. & N.A. & e⿻o & 600 & 38 \\
\hline Maule M5-210TC & 63 & 208 & N.A. & N.A. & N.A. & N.A. & N.A. & N.A. & N.A. & N.A. & $6 \infty$ & 600 & 38 \\
\hline Maule MS-210C & 83 & 283 & N.A. & N.A. & N.A. & NA. & N.A. & N.A. & NA. & N.A. & 600 & 600 & 39 \\
\hline Beschcraft Sundownor & 57 & 230 & N.A. & N.A. & N.A. & N.A. & N.A. & N.A. & N.A. & N.A. & 1,955 & 1,484 & 58 \\
\hline Piper Wartior II & 50 & 227 & N.A. & N.A. & N.A. & N.A. & N.A. & NA. & N.A. & N.A. & 1480 & 1,115 & 57 \\
\hline Cossne Sinnewk & 50 & 227 & N.A. & N.A. & N.A. & N.A. & N.A. & NA. & N.A. & NA. & 1,390 & 1,250 & 50 \\
\hline Varpa 2180 & 35 & 159 & N.A. & N.A. & N.A. & NA. & NA. & N.A. & N.A. & N.A. & 1,310 & 1425 & 52 \\
\hline Varpa Kaching 2150A & 35 & 159 & N.A. & N.A. & N.A. & N.A. & N.A. & N.A. & N.A. & N.A. & 40 & 450 & 52 \\
\hline Plper PA-18-150 Super Cub & 38 & 164 & $35: 3$ & $10.75 \mathrm{~m}$ & 227 & $6.88 \mathrm{~m}$ & 6.8 & $2.02 \mathrm{~m}$ & 178.5 & 16.58 & 500 & 885 & 42 \\
\hline Beschoreft 77 Skiger & 29 & 132 & 300 & $8.14 \mathrm{~m}$ & $24^{\prime} 0$ & $7.32 \mathrm{~m}$. & 7119 & $2.41 \mathrm{~m}$. & 129.8 & 12.08 & 1280 & 1313 & 54 \\
\hline Piger PA-38-112 Tomahawk & 32 & 145 & 340 & $10.36 \mathrm{~m}$ & $231^{\circ}$ & $7.04 \mathrm{~m}$ & $8+19$ & $2.77 \mathrm{~m}$ & 124.7 & 11.59 & 1460 & 1,544 & 56 \\
\hline Cossna 150 Commuter II & 37 & 108 & 332 & $10.11 \mathrm{~m}$ & 23119 & $7.29 \mathrm{~m}$ & $8^{\prime} 8^{\prime}$ & $2.59 \mathrm{~m}$ & 157.0 & 14.58 & 1.340 & 1,200 & 50 \\
\hline Cesena 150 & 37 & 188 & 3549 & $10.17 \mathrm{~m}$. & $21 \cdot 8$ & $8.56 \mathrm{~m}$. & $8^{\prime} 11^{\circ}$ & $2.11 \mathrm{~m}$. & 150.5 & 14.80 & 1340 & 1,200 & 50 \\
\hline Texperenti F-21 & 24 & 10 & 380 & $10.88 \mathrm{~m}$ & 223 & $6.78 \mathrm{~m}$ & $6^{\prime} \mathrm{B}^{\prime}$ & $1.08 \mathrm{~m}$ & 183.5 & 17.10 & 350 & 350 & 43 \\
\hline Piper J-sc-es Cub & N.A. & N.A. & $35+3$ & $10.75 \mathrm{~m}$ & 223 & $8.78 \mathrm{~m}$ & $6^{2} 8^{\circ}$ & $2.00 \mathrm{~m}$ & 178.5 & 18.00 & N.A. & N.A. & N.A. \\
\hline
\end{tabular}

N.A. = Intormation not evilable whon able prepered.

wowater 
Table 3.40

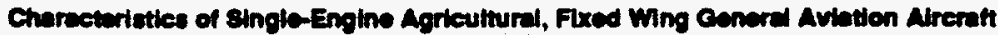
Poderences 329 to 3.35

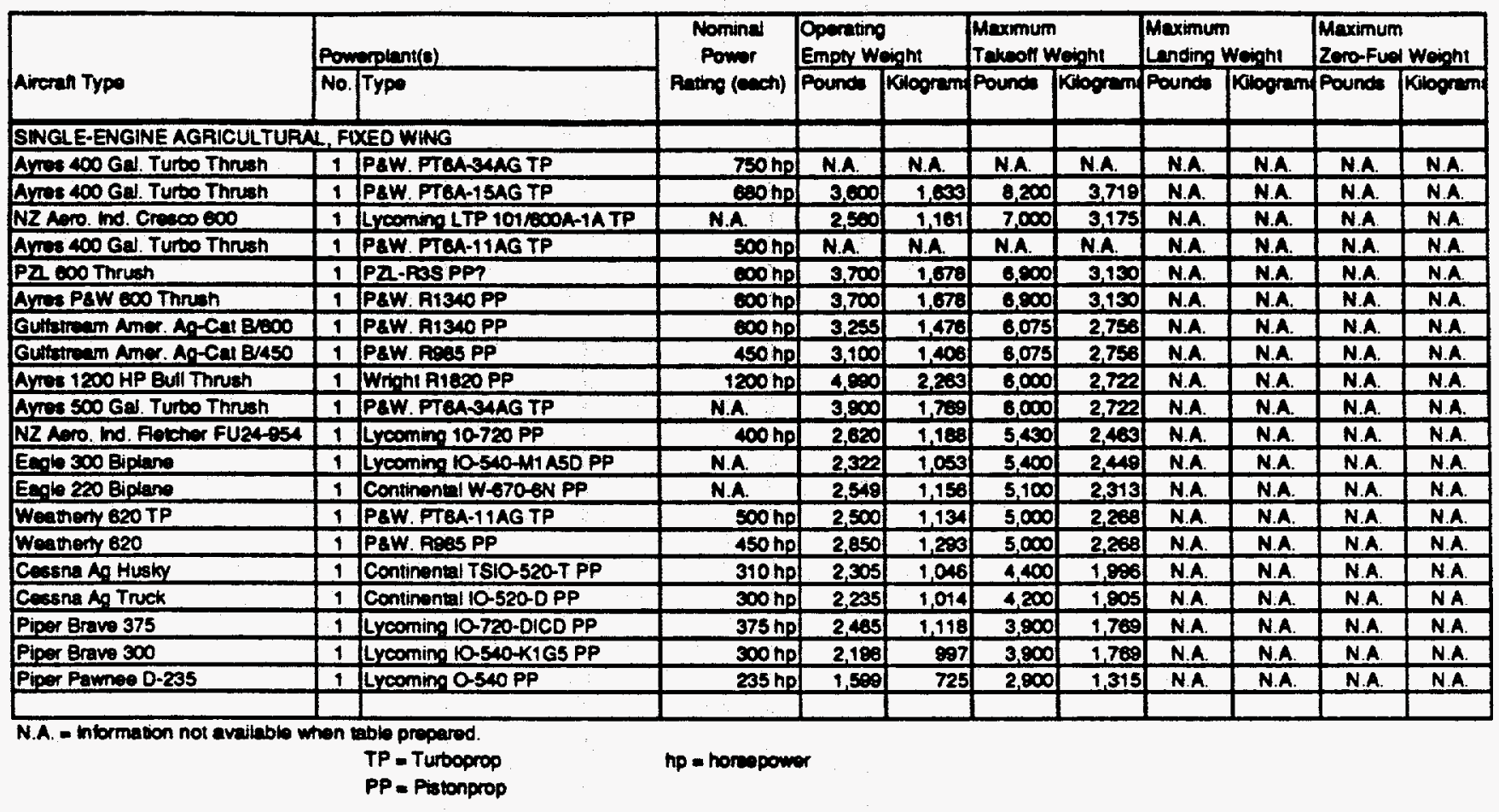


Tablo 3.48 (Contrued)

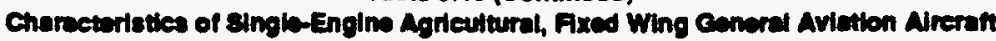

Rubrencese 3.29 to 3.35

\begin{tabular}{|c|c|c|c|c|c|c|c|c|c|c|c|c|c|}
\hline \multirow[b]{2}{*}{ Aircrah Typo } & \multicolumn{2}{|c|}{$\begin{array}{l}\text { Merimum } \\
\text { Fuel Capaciv }\end{array}$} & \multicolumn{2}{|c|}{$\begin{array}{l}\text { Overell } \\
\text { Wing Span }\end{array}$} & \multicolumn{2}{|c|}{ Ooverell Lengen } & \multicolumn{2}{|c|}{ loverell Hoight } & \multicolumn{2}{|l|}{ Wing Area } & \multirow{2}{*}{$\begin{array}{c}\text { Takeoth } \\
\text { Dist. (n.) } \\
\text { (FAR) }\end{array}$} & \multirow{2}{*}{$\begin{array}{l}\text { Lending } \\
\text { Dist. (t). } \\
\text { (FAR) }\end{array}$} & \multirow{2}{*}{$\begin{array}{l}\text { Srall Speed } \\
\text { (gooumaps } \\
\text { oown, mph) }\end{array}$} \\
\hline & Imp. Gal. & Lhere & Fi. in. & Motern & Ft. In. & Motere & Ft. In. & Maver & Ft:“2 & $M=2$ & & & \\
\hline \multicolumn{14}{|c|}{ SNGLE-ENGINE AGRICULTURAL, FIXED WING } \\
\hline Aroes 400 Gel. Turbo Thrush & N.A. & N.A. & N.A. & N.A. & N.A. & N.A. & N.A. & NA. & N.A. & N.A. & NA. & N.A. & N.A. \\
\hline Arees 400 Gel. Turto Thrush & 100 & 482 & N.A. & N.A. & N.A. & N.A. & N.A. & N.A. & N.A. & N.A. & 815 & 500 & 57 \\
\hline WZ Aero. ho. Grose0 600 & N.A. & N.A. & N.A. & N.A. & N.A. & N.A. & N.A. & N.A. & NA. & N.A. & NA. & NA & 52 \\
\hline Aves 400 Gal. Turbo Thresh & N.A. & N.A. & N.A. & N.A. & N.A. & N.A. & N.A. & N.A. & NA & N.A. & N. & N.A. & N.A. \\
\hline PA eoo Thrugh & 108 & 482 & N.A. & N.A. & N.A. & N.A. & N.A. & N.A. & NA & NA. & 775 & 500 & 64 \\
\hline Ayes PaW eoo Thruh & 100 & 482 & N.A. & N.A. & N.A. & N.A. & N.A. & NA. & N.A. & N.A. & 775 & 500 & 64 \\
\hline 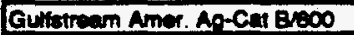 & 8 & 3en & N.A. & N.A. & N.A. & N.A. & N.A. & NA. & N.A. & N.A. & 850 & 1.054 & 54 \\
\hline Gulletream Amer. Ap-Cat B/450 & $\infty$ & 394 & N.A. & N.A. & N.A. & N.A. & N.A. & NA. & NA. & N.A. & 1,090 & 2833 & 53 \\
\hline Area 1200 HP Bull Thrush & 100 & Q⿻日木 & N.A. & N.A. & N.A. & N.A. & NA. & N.A. & NA. & N.A. & 350 & 550 & 57 \\
\hline Arree 500 Gal. Turto Thrueh & 100 & 455) & N.A. & NA & N.A. & N.A. & N.A. & N.A. & N.A. & N.A. & $\infty$ & 500 & 57 \\
\hline N2 Aero. ind. Fercher FU24-054 & M.A. & N.A. & N.A. & N.A. & N.A. & N.A. & N.A. & N.A. & N.A. & NA. & 1,220 & 1,280 & 56 \\
\hline Eeple 300 Biplene & 0 & 314 & N.A. & N.A. & N.A. & N.A. & N.A. & N.A. & N.A. & N.A. & N.A. & N.A. & 51 \\
\hline Eeque 220 Biplene & 69 & 314 & N.A & N.A. & N.A. & N.A. & N.A. & $\mathbf{N A}$ & N.A. & N.A. & N.A. & N.A. & 51 \\
\hline Weathery $620 \mathrm{TP}$ & 65 & 295 & N.A. & N.A. & N.A. & N.A. & N.A. & NA & N.A. & N.A. & N.A. & 800 & 66 \\
\hline Weatherty 620 & 65 & 295 & N.A & N.A. & N.A. & N.A. & N.A. & N.A. & N.A. & N.A. & N.A. & 800 & 68 \\
\hline Cesene Aq Husky & 54 & 245 & $41^{\circ} 8^{\circ}$ & $12.70 \mathrm{~m}$ & $26^{\circ} 6^{\circ}$ & $8.03 \mathrm{~m}$ & $6 \cdot 2$ & $2.49 \mathrm{~m}$ & 205.0 & 19.05 & 1,975 & 1,265 & 61 \\
\hline Cessing Ag Truck & 54 & 245 & $41^{\prime} 8^{\prime}$ & $12.70 \mathrm{~m}$ & $25^{\prime} 11^{\prime}$ & $7.80 \mathrm{~m}$ & $\theta^{\prime 2}$ & $2.49 \mathrm{~m}$ & 205.0 & 18.05 & 2,140 & 1,265 & 65 \\
\hline Piper Brave 375 & $\theta$ & 391 & N.A. & N.A. & N.A. & NA. & N.A. & N.A. & N.A. & N.A. & 1,200 & 1850 & 76 \\
\hline Piper Brave 300 & 86 & 391 & N.A. & N.A. & N.A. & N.A. & N.A. & N.A. & N.A. & N.A. & 1,525 & 1,650 & 71 \\
\hline Piper Pennee D-235 & 38 & 173 & N.A. & N.A. & N.A. & N.A. & N.A. & N.A. & N.A. & N.A. & 1,350 & N.A. & 70 \\
\hline & & & & & & & & & & & & & \\
\hline
\end{tabular}

N.A. - Intormation not evailebie whon able prepered. 
Table 3.49

Cherecterlettes of Mult-Engine Pedproeating, Flxed Wing Canerel Avlation Airenft Riderences 329 to 3.35

\begin{tabular}{|c|c|c|c|c|c|c|c|c|c|c|c|}
\hline \multirow[b]{2}{*}{ Atreran Typo } & \multicolumn{2}{|c|}{ Pomerplan $(8)$} & \multirow{2}{*}{$\begin{array}{c}\text { Nomina } \\
\text { Powor } \\
\text { Rating (cech) }\end{array}$} & \multicolumn{2}{|c|}{$\begin{array}{l}\text { Operating } \\
\text { Emper Weight }\end{array}$} & \multicolumn{2}{|c|}{$\begin{array}{l}\text { Maximum } \\
\text { Takeoff Weight }\end{array}$} & \multicolumn{2}{|c|}{$\begin{array}{l}\text { Meximum } \\
\text { Landing Weinh! }\end{array}$} & \multicolumn{2}{|c|}{$\begin{array}{l}\text { Maximum } \\
\text { Zero-Fuel Waint }\end{array}$} \\
\hline & No. & Type & & Pound: & Klogram f & Pound & Kilogram & Founds & Kilogram & Pounds & Kilogram \\
\hline \multicolumn{12}{|c|}{ MULTI-ENGINE RECIPROCATING (PISTON), FIXED WING } \\
\hline Pilatus BN.2A MK III-2 Trietander & 3 & Lroomin 0-540-E4CS PP & $200 \mathrm{hpl}$ & 5,000 & 2540 & 10,000 & 4,536 & N.A. & N.A. & N.A. & N.A. \\
\hline Cesene Timn Courier & 2 & Continental GTS10-520-M PP & $375 \mathrm{np}$ & 4,84 & 2.197 & 8,450 & 3,833 & N.A. & N.A. & N.A. & N.A. \\
\hline Cesena Timan Ambasesedor & 2 & Continentil GTS10-520-M PP & $375 \mathrm{hp}$ & 4.818 & 2,185 & 8,450 & 3.803 & N.A. & N.A. & N.A. & N.A. \\
\hline Cosene Titen Freighter & 2 & Cominental GTS10-520-M PP & $375 \mathrm{hp}$ & 4,085 & 2.125 & 8.450 & 3.803 & N.A. & N.A. & N.A. & N.A. \\
\hline Ceseng 421 Colden Egab & 2 & Continunial TSIO-520-L PP & $375 \mathrm{hg}$ & 4,023 & 2,097 & 7,500 & 3,402 & N.A. & N.A. & N.A. & N.A. \\
\hline Piper Chiptrain & 2 & Lyeoming T10-540-J28D PP & ssong & 4,221 & 1.815 & 7,000 & 3,175 & N.A. & N.A. & N.A. & N.A. \\
\hline Cesenn 402 C Bupinessliner & 2 & Continentel TS10-520-VB PP & $325 \mathrm{hpl}$ & 4,074 & 1,84 & 6,885 & 3,123 & N.A. & N.A. & N.A. & N.A. \\
\hline Cesene $402 \mathrm{C}$ voliner & 2 & Continental TSIO-520-VB PP & $325 \mathrm{hp}$ & 4,128 & 1,872 & 6,850 & 3,107 & 6,850 & 3.107 & 6,515 & 2,855 \\
\hline Cecena 402 & 2 & Continental TSIO-520-VB PP & $325 \mathrm{hp}$ & N.A. & N.A. & 6,895 & 3,123 & N.A. & N.A. & N.A. & N.A. \\
\hline Cosena Chancellor & 2 & Cominont ISIO-520-NB PP & $310 \mathrm{hp}$ & 4,350 & 1,978 & 6.785 & 3,078 & N.A. & N.A. & NA. & N.A. \\
\hline Beechant Dule B60 & 2 & Lycoming T10-541-E1C4 PP & seonp & 4,408 & 1,000 & 6,775 & 3,073 & N.A. & N.A. & N.A. & N.A. \\
\hline Pilatus BN.28-26 lsiander II & 2 & Lyecomino 0-540-E4C5 PP & $280 \mathrm{hp}$ & 3,612 & 1.638 & 6.600 & 2,894 & N.A. & N.A. & N.A. & N.A. \\
\hline Pilalus BN.2A-21 (lg) & 2 & Lycoming $10-540-K 185$ PP & $300 \mathrm{np}$ & 3.702 & 1,708 & 6,000 & 2.994 & N.A. & N.A. & N.A. & N.A. \\
\hline Pitalus BN.2A-20 (la) & 2 & Lycoming lo-540-K1B5 PP. & $300 \mathrm{no}$ & 3.722 & 168 & 6.500 & 2,876 & N.A. & N.A. & N.A. & N.A. \\
\hline Piper Navajo CAR & 2 & Lycoming T10-540-F2BD PP & $325 \mathrm{ho}$ & 4,000 & 1859 & 8,500 & 2,848 & N.A. & N.A. & N.A. & N.A. \\
\hline Piper Navaio & 2 & ILycoming T10-540-A2C PP & $310 \mathrm{hg}$ & 4,000 & 1,816 & 6,500 & 2.948 & N.A. & N.A. & N.A. & NA. \\
\hline Beechcratt Baron 58P & 2 & Continental TSIO-520-WB PP & $325 \mathrm{ng}$ & 4.020 & 1,823 & 6,200 & 2,812 & N.A. & N.A. & N.A. & N.A. \\
\hline Beechcratt Beron 58TC & 2 & Continental TSIO-520-WB PP & $325 \mathrm{ho}$ & 3793 & 1,720 & 6,200 & 2,B12 & N.A. & N.A. & N.A. & N.A. \\
\hline Cessna 340 & 2 & Continental TS10-520NB PP & $310 \mathrm{ho}$ & 3.911 & 1774 & 6,025 & 2.733 & N.A. & N.A. & N.A. & N.A. \\
\hline Plper Aerostar 601 P & 2 & Lreoming 10-540-S1A5 PP & $290 \mathrm{ng}$ & 4,056 & 1,840 & 6,000 & 2,722 & NA. & N.A. & N.A. & N.A. \\
\hline Piper Aorostar 601B & 2 & Lycoming 10-540-S1A5 PP & $200 \mathrm{hg}$ & 3,985 & 1,800 & 6,000 & 2.722 & N.A. & N.A. & N.A. & N.A. \\
\hline Beochcratt Baron 58 & 2 & Continental 10-520-CQ PP & $285 \mathrm{np}$ & 3,383 & 1,525 & 5,400 & 2,449 & N.A. & N.A. & N.A. & N.A. \\
\hline Beecherat Baron E55 & 2 & Continenta! $10-520-C B$ PP & $235 \mathrm{hp}$ & 3,208 & 1,491 & 5,300 & 2,404 & N.A. & N.A. & N.A. & N.A. \\
\hline Cessne 303 Crusader & 2 & Continontal TS10-520-AE PP & $250 \mathrm{hp}$ & 3,305 & 1,400 & 5,175 & 2,347 & N.A. & N.A. & N.A. & N.A. \\
\hline Beecherath Baron 855 & 2 & Continental $10-470-\mathrm{LPP}$ & $280 \mathrm{np}$ & 3,233 & 1,468 & 5,100 & 2,313 & NA. & N.A. & N.A. & N.A. \\
\hline Piper Sencea II & 2 & Continental TSIO-360-E & $200 \mathrm{hp}$ & 2,841 & 1,209 & 4.570 & 2,073 & N.A. & N.A. & N.A. & N.A. \\
\hline Piper Turbo Seminde & 2 & Lrcoming LO-380-E1A6D PP & $180 \mathrm{hp}$ & 2,450 & 1,402 & 3,925 & 1,780 & N.A. & N.A. & N.A. & N.A. \\
\hline Beechcraft Duchess & 2 & Lreoming 0-3e0-A1 G6D PP & $180 \mathrm{np}$ & 2,460 & 1.118 & 3,800 & 1,700 & N.A. & N.A. & N.A. & N.A. \\
\hline Piper Seminole & 2 & Lycoming O-360-E1 $A 60 \mathrm{PP}$ & $180 \mathrm{hp}$ & 2,354 & 1,008 & 3,800 & 1,724 & N.A. & N.A. & N.A. & N.A. \\
\hline Wing D-1 Derringer & 2 & Lreoming 10-320-81C PP & $160 \mathrm{ho}$ & 2,100 & 953 & 3,050 & 1,383 & N.A. & N.A. & N.A. & N.A. \\
\hline
\end{tabular}


Table 3.19 (Continuad)

Charectoristles of Mutt-Engine Acelprecating, Fxed Wing Coneral Avition Areraft

Aeterences 329 w0 3.35

\begin{tabular}{|c|c|c|c|c|c|c|c|c|c|c|c|c|c|}
\hline \multirow[b]{2}{*}{ Areran Type } & \multicolumn{2}{|c|}{$\begin{array}{l}\text { Moximum } \\
\text { Fuel Capecioy }\end{array}$} & \multicolumn{2}{|c|}{$\begin{array}{l}\text { Overall } \\
\text { Wing Spen }\end{array}$} & \multicolumn{2}{|c|}{ overdil Lengn } & \multicolumn{2}{|c|}{ Overall Hoight } & \multicolumn{2}{|c|}{ Wing Area } & \multirow{2}{*}{$\begin{array}{c}\text { Takeoff } \\
\text { Dist. (f..) } \\
\text { (FAR) }\end{array}$} & \multirow{2}{*}{$\begin{array}{c}\text { Landing } \\
\text { Oist. (t.) } \\
\text { (FAP) }\end{array}$} & \multirow{2}{*}{$\begin{array}{l}\text { Stall Spoed } \\
\text { (ocarmape } \\
\text { com, mph) }\end{array}$} \\
\hline & $\operatorname{limp.~Gell~}$ & Where & Ft. In. & Moteres & FI. In. & Molers & FI. In. & Nomess & $F I^{-\infty} 2$ & $M=2$ & & & \\
\hline \multicolumn{14}{|c|}{ MULTI-ENGINE RECIPROCATNG (PISTON), FIXED WING } \\
\hline Pliatue BN.2A MK III-2 Trislander & 108 & 691 & IN.A. & N.A. & N.A. & N.A. & N.A. & N.A. & N.A. & N.A. & 1,019 & 1430 & 58 \\
\hline Cessina Titan Courier & 34) & 1.58 & N.A. & N.A. & N.A. & N.A. & N.A. & N.A. & N.A. & N.A. & 2,387 & 2,130 & 8 \\
\hline Cesesma Titan Amberesedor & 34 & 1,564 & N.A. & N.A. & N.A. & N.A. & N.A. & N.A. & N.A. & N.A. & 2,387 & 2,130 & 80 \\
\hline Cesene Titen Freighter & 34 & 1.584 & N.A. & N.A. & N.A. & N.A. & NA. & NA. & N.A. & N.A. & 2,367 & 2.130 & $\infty$ \\
\hline Cecene 421 Golden Eagle & 232 & 1,191 & N.A. & N.A. & N.A. & NA. & N.A. & N.A. & NA. & N.A. & 2,323 & 2,293 & 85 \\
\hline Piper Chiofrain & 102 & 673 & N.A. & N.A. & N.A. & N.A. & N.A. & NA. & $\overline{\text { N.A. }}$ & N.A. & 2450 & 1,880 & N.A. \\
\hline Cocens $402 C$ Busin maliner & 200 & $\infty 6$ & N.A. & N.A. & N.A. & N.A. & N.A. & N.A. & NA. & N.A. & 2,105 & 2,485 & 78 \\
\hline Conene 402C Utiliner & 170 & $\infty$ & $44^{\prime} \cdot 9.5$ & $13.45 \mathrm{~m}$ & $364.5^{\circ}$ & $111.00 \mathrm{~m}$ & $11 \cdot 5.5^{\circ}$ & $3.40 \mathrm{~m}$ & 225.8 & 21.0 & 2,105 & 2,485 & 78 \\
\hline Cosena 402 & 213 & 68 & N.A. & N.A. & N.A. & N.A. & NA. & Na. & N.A. & N.A. & 2,185 & 2,485 & 78 \\
\hline Cosens Chencellor & 200 & 856 & N.A. & N.A. & N.A. & N.A. & N.A. & NA. & N.A. & NA. & 2595 & 2,383 & 82 \\
\hline Beechcreft Duke BeO & 232 & 1,055 & N.A & N.A. & N.A. & N.A. & N.A. & NA. & N.A. & N.A. & 2,628 & 3,065 & 8 \\
\hline Pilatus BN.29-26 lalander II & 137 & 823 & N.A. & N.A. & N.A. & N.A. & N.A. & N.A. & N.A: & N.A. & 1,000 & 880 & 48 \\
\hline Pitatus BN.2A-21 (th) & 189 & 691 & N.A. & N.A. & N.A. & N.A. & N.A. & NA. & N.A. & N.A. & 1,100 & 860 & 46 \\
\hline Pltariue BN.2A-20 (19) & 135 & 614 & N.A. & N.A. & N.A. & N.A. & N.A. & N.A. & N.A. & N.A. & 1,100 & 860 & 46 \\
\hline Piper Naveo C/A & 192 & 873 & N.A. & N.A. & N.A. & NA. & N.A. & NA. & N.A. & NA. & 2,080 & 1818 & 80 \\
\hline Piper Nevajo & 192 & 873 & N.A. & N.A. & N.A. & N.A. & N.A. & NA. & N.A. & NA. & 2,095 & 1818 & 80 \\
\hline Beecherat Baron 58P & 180 & 864 & N.A. & N.A. & N.A. & N.A. & N.A. & N.A. & N.A. & N.A. & 2,643 & 2,427 & 80 \\
\hline Beecheratt Baron 58TC & 180 & 804 & N.A. & N.A. & N.A. & N.A. & N.A. & N.A. & N.A. & N.A. & 2,643 & 2,427 & $\infty$ \\
\hline Cossna 340 & 207 & 941 & N.A. & N.A. & N.A. & N.A. & N.A. & N.A. & N.A. & N.A. & 2,175 & 1,850 & 81 \\
\hline Piper Merostar 601P & 174 & 791 & N.A. & N.A. & N.A. & N.A. & N.A. & N.A. & N.A. & N.A. & 2,490 & 2,030 & 87 \\
\hline Piper Aorosuar 6018 & 174 & 791 & N.A. & N.A. & N.A. & N.A. & N.A. & N.A. & N.A. & N.A. & 2,480 & 2,030 & 87 \\
\hline Beochcrath Baron 58 & 189 & 882 & N.A. & N.A. & N.A. & N.A. & N.A. & N.A. & N.A. & N.A. & 2,101 & 2,498 & 65 \\
\hline Beecheran Baron E55 & 168 & 755 & N.A. & N.A. & N.A. & N.A. & N.A. & N.A. & N.A. & N.A & 2,050 & 2,202 & 84 \\
\hline Cossni 303 Cnuseder & 155 & 705 & N.A. & N.A. & N.A. & N.A. & N.A. & N.A. & N.A. & N.A. & 2,100 & 1,535 & 72 \\
\hline Beectheraft Baron 855 & 138 & 618 & N.A. & N.A. & N.A. & N.A. & N.A. & N.A. & N.A. & N.A. & 2,154 & 2,148 & 84 \\
\hline Piper Senece II & 1201 & 582 & N.A. & N.A. & N.A. & N.A. & N.A. & NA. & N.A. & N.A. & 1,240 & 2,090 & 70 \\
\hline Piper Turbo Seminole & 110 & 500 & N.A. & N.A. & N.A. & N.A. & N.A. & N.A. & NA. & N.A. & 1,500 & 1,180 & 70 \\
\hline Beecharath Duchess & 100 & 455 & N.A. & N.A. & N.A. & N.A. & N.A. & N.A. & N.A. & N.A. & 2,119 & 1,181 & 69 \\
\hline Piper Seminole & 110 & 500 & N.A. & N.A. & N.A. & N.A. & N.A & N.A. & N.A. & N.A. & 1,400 & 1,190 & 68 \\
\hline Wing D-1 Demnger & B7) & 396 & N.A. & N.A. & N.A. & N.A. & N.A. & NA. & N.A. & N.A. & 1,240 & N.A. & 72 \\
\hline
\end{tabular}

N.A. = Informaton not available when mole prepered. 
Tablo 3.50

Charectortatles of Turboprop, Fixed Wing Caneral Aviation Alreratt

Rotorences 32.29 to 3.35

\begin{tabular}{|c|c|c|c|c|c|c|c|c|c|c|c|}
\hline \multirow[b]{2}{*}{ Arcrat Type } & \multicolumn{2}{|c|}{ Pownotant(s) } & \multirow{2}{*}{$\begin{array}{c}\text { Nomina } \\
\text { Powor } \\
\text { Alung (cach) }\end{array}$} & \multicolumn{2}{|c|}{$\begin{array}{l}\text { Operating } \\
\text { Empty Weight }\end{array}$} & \multicolumn{2}{|c|}{$\begin{array}{l}\text { Maximum } \\
\text { Tekeof Weight }\end{array}$} & \multicolumn{2}{|c|}{$\begin{array}{l}\text { Meximum } \\
\text { Lending Weint }\end{array}$} & \multicolumn{2}{|c|}{$\begin{array}{l}\text { Maximum } \\
\text { Zoro-Fuol Woight }\end{array}$} \\
\hline & No. & Type & & Pouncs & Klooram & Pound: & Koogram & Pounds & Klogrem & Pounds & Klogrum \\
\hline \multicolumn{12}{|l|}{ TURBOPROP FIXED WING } \\
\hline Grummen 159 Gulferean I & 2 & AR. Den 529 QX or BE TP & $1000 \sin 0$ & 21,800 & e.ess & 35,100 & 15,920 & 33,000 & 15,240 & 28,170 & 11,870 \\
\hline Beechorift 350 Super King Air & 2 & P8WC. PTQAEOATP & 1,050 eng & $0,0<0$ & 4,008 & 15.000 & 8,804 & N.A. & N.A. & N.A. & N.A. \\
\hline Feirchild Swoavingen Morlin NC & 2 & GUTA TPE-331-110-001GTP & N.A. & 0.100 & 4.128 & 14.200 & 6,441 & N.A. & N.A. & N.A. & N.A. \\
\hline Eeecheran soo Super King Air & 2 & PQWC. PTEA-OATP & 1,000 ong & 8,400 & 3.851 & 14,000 & 0.350 & N.A. & N.A. & N.A. & N.A. \\
\hline Feirenild Swoaringen Mertin IIC & 2 & Gerven TPE-331-KU-503G TP & $\infty \infty \operatorname{chp}$ & 8,150 & 3.697 & 12,500 & 5.670 & N.A. & N.A. & N.A. & N.A. \\
\hline Becherafl zoo Super Kon? Air & 2 & PAW. PTOA-41TP & $850 \operatorname{chp}$ & 7,543: & 3,421 & 12.500 & 5.070 & N.A. & N.A. & N.A. & N.A. \\
\hline Beechereth B100 King Air & 2 & GeTOI TPE-331- -2528 TP & 715 thp & 7,112 & 3,228 & 11,800 & 5,352 & N.A. & N.A. & N.A. & N.A. \\
\hline Mrautiani MU.2 Marquise & 2 & Genot TPE-331-10-501M TP & 778 cho & 7,650 & 3.470 & 11,575 & 5,250 & N.A. & N.A. & N.A. & N.A. \\
\hline Gullstream Am. Commendor 100 & 2 & Geron TPE-331.10 TP & N.A. & N.A. & N.A. & 11,200 & 5,103 & N.A. & N.A. & N.A. & N.A. \\
\hline Ploes Chereme III & 2 & PaW. PTBA-11 TP & 720 sho & 6.210 & 2,800 & 11,000 & 4000 & N.A. & N.A. & N.A. & N.A. \\
\hline Beecheratt Feo Kin A Ar & 2 & PEW. PTEA-135 TP & 750 sho & 6,840 & 3,012 & 10.050 & 4.837 & N.A. & N.A. & N.A. & N.A. \\
\hline Guthtroan An. Commander 800 & 2 & N.A. & N.A. & N.A. & N.A. & 10,775 & 4,887 & N.A. & N.A. & N.A. & N.A. \\
\hline Missubishi MU.2 Soliuare & 2 & Gerren TPE-331-10-501M TP & 727 and & 7,010 & 3,180 & 10,470 & 4,749 & N.A. & N.A. & N.A. & N.A. \\
\hline Gultstream An. Commander 880 & 2 & Garmet TPE-331-10-Solk TP & 717 chp & 6.271 & 2.84 & 10,325 & 4.693 & N.A. & N.A. & N.A. & N.A. \\
\hline Gulfstrean Am. Commander 840 & 2 & Garron TPE-331-5-524K TP & 717 ang & 8,120 & 2,776 & 10,325 & 4.898 & N.A. & N.A. & N.A. & N.A. \\
\hline Cosena 441 Conquest & 2 & Gamett TPE-331-6-401S TP & 635 tho & 5,706 & 2,588 & 8,850 & 4,468 & N.A. & N.A. & N.A. & N.A. \\
\hline Beecheraft E8O King Air & 2 & PQW.PTBA-21TP & 550 sho & 5,778 & 2,621 & 9.850 & 4,377 & N.A. & N.A. & N.A. & NA. \\
\hline Beechcraf Cso King Air & 2. & PAW. PTBA-21 TP & 550 anp & 5,785 & 2,615 & 9650 & 4,377 & 8,188 & 4,159 & NA. & N.A. \\
\hline Beechareft 80 King Air & 2 & PAWC. PTEA- TP & 500 chp & 5,318 & 2,615 & 8,300 & 4,377 & N.A. & NA. & N.A. & N.A. \\
\hline Piper PA-31T Cheyenne II & 2 & PAW. PTGA-28 TP & 620 thp & 4,883 & 2,097 & 8,000 & 4,082 & 0.000 & 4,082 & 7,600 & 3,447 \\
\hline Pinatue PC-12 & 1 & PQWC. PT6A-67B.TP & $1,200 \operatorname{shp}$ & 5,260 & 2,388 & 8,818 & 4,000 & N.A. & N.A. & N.A. & N.A. \\
\hline Piper Chovenne I & 2 & PAW. PT6A-11 TP & $500 \mathrm{shp}$ & 4,904 & 2.224 & 8,700 & 3.846 & N.A. & N.A. & N.A. & N.A. \\
\hline Cessna 425 Corsair & 2 & PEW. PTEA-112 TP & $450 \mathrm{shp}$ & 4,800 & 2,250 & 8,200 & 3,719 & N.A. & N.A. & N.A. & N.A. \\
\hline Cesena 200 Caravan 1 & 1 & PQW. PT6A-114TP & $600 \operatorname{shp}$ & 3.800 & 1.724 & 8,750 & 3,909 & 7,300 & 3.311 & N.A. & N.A. \\
\hline Lear Fen 2100 & 2 & PEW. PTe8-35F TP & N.A. & 4,000 & 1.814 & 7,250 & 3,200 & N.A. & N.A. & N.A. & N.A. \\
\hline Pilate PC-6/B2-144 Turbo Porer & 1 & P\&WC. PTBA-27 TP & 550 thp & 1411 & 20 & 2,028 & 920 & N.A. & N.A. & N.A. & N.A. \\
\hline
\end{tabular}


Table 3.50 (Conthued)

Cherectudaties of Turtoprep, Fxed Wing Comerel Aviedion Aireraft

Anterencees 3.29 to 3.35

\begin{tabular}{|c|c|c|c|c|c|c|c|c|c|c|c|c|c|}
\hline \multirow[b]{2}{*}{ Airenen TYPo } & \multicolumn{2}{|c|}{$\begin{array}{l}\text { Meximum } \\
\text { Fuel Capecios }\end{array}$} & \multicolumn{2}{|c|}{$\begin{array}{l}\text { Overall } \\
\text { Wine Span }\end{array}$} & \multicolumn{2}{|c|}{ loweril Length } & \multicolumn{2}{|c|}{ Overel Height } & \multicolumn{2}{|c|}{ Wing Area } & \multirow{2}{*}{$\begin{array}{c}\text { Telceoff } \\
\text { Diet. (th.) } \\
\text { (FAA) }\end{array}$} & \multirow{2}{*}{$\begin{array}{c}\text { Lending } \\
\text { Dist. (ft.) } \\
\text { (FAA) }\end{array}$} & \multirow{2}{*}{$\begin{array}{l}\text { Stall Spoed } \\
\text { (ooernaps } \\
\text { down, mph) }\end{array}$} \\
\hline & inp. en. & Litane & Fi. in. & Nowers & Ft. in. & Mowrs & Ft. In. & Maver & $F_{t} \cdot{ }^{\infty}$ & $M^{* * 2}$ & & & \\
\hline \multicolumn{14}{|l|}{ TUREOPROP, FIXED WING } \\
\hline Grummen ise Gultstoem I & 1200 & 5,005 & 760 & $23.82 \mathrm{~m}$ & 658 & $19.43 \mathrm{~m}$ & 228 & $8.84 \mathrm{~m}$. & 610.3 & 88.70 & 2.875 & 2,125 & N.A. \\
\hline Bancherit 350 super $\mathrm{Kin}$ & N.A. & N.A. & $5711^{\circ}$ & $17.65 \mathrm{~m}$ & 468 & $14.2 \mathrm{~m}$ & $14^{4} 40$ & $4.37 \mathrm{~m}$ & 310.0 & 28.80 & NA & NA. & N.A. \\
\hline Feirchild Swerineen Mertin NC & 48 & 2.48 & N.A. & N.A. & N.A. & N.A. & N.A. & NA. & N.A. & NA. & 2,850 & 2,532 & 90 \\
\hline Bechereft soo Super Kno Air & N.A. & N.A. & 546 & $18.61 \mathrm{~m}$ & $4310^{\circ}$ & $13.38 \mathrm{~m}$ & $14^{4} 4$ & $4.37 \mathrm{~m}$ & 300.0 & 20.15 & N.A. & N.A. & N.A. \\
\hline Furchild Sweringen Mortin HIC & 298 & 2846 & N.A. & N.A. & N.A. & N.A. & NA. & N.A. & NA. & N.A. & 3850 & 3,150 & 102 \\
\hline Becenaren 200 Super King Air & Sa4 & 2,473 & $54^{\prime} 6^{\circ}$ & $16.61 \mathrm{~m}$ & 480 & $13.34 \mathrm{~m}$. & $15^{\prime} 0$ & $4.57 \mathrm{~m}$. & 300.0 & 28.15 & 2579 & 2.074 & 88 \\
\hline Bewencrin Bico Kin Air & 470 & 2,137 & $45: 111$ & $14.00 \mathrm{~m}$ & $39^{\circ} 11^{\circ}$ & $12.17 \mathrm{~m}$. & $15^{\prime} 5^{\prime \prime}$ & $4.70 \mathrm{~m}$ & 270.7 & 25.80 & 2,004 & 2,670 & 86 \\
\hline Minsubish MU.2 Marouies & 405 & 1.832 & N.A. & N.A. & N.A. & N.A. & N.A. & N.A. & N.A. & N.A. & 2,170 & 2,200 & 87 \\
\hline Guffetren Am. Commancer 100 & 474 & 2,155 & N.A. & N.A. & N.A. & N.A. & N.A. & N.A. & N.A. & N.A. & N.A. & N.A. & N.A. \\
\hline Pioer Cheyenne ill & 400 & 1,818 & N.A. & N.A. & N.A. & N.A. & N.A. & N.A. & NA. & N.A. & 2,390 & 2,820 & 85 \\
\hline Beechenth Feo King Air & 470 & 2,137 & $45^{3} 110$ & $14.00 \mathrm{~m}$ & $30 \cdot 10$ & $12.13 \mathrm{~m}$ & $15^{\prime} 1{ }^{\circ}$ & $4.60 \mathrm{~m}$. & 270.7 & 25.83 & 2,856 & 2,224 & 87 \\
\hline Guthetrean Am. Commander 800 & N.A. & N.A. & N.A. & N.A. & N.A. & N.A. & N.A. & N.A. & N.A. & N.A. & 1,937 & 2,698 & N.A. \\
\hline Mitubishi MU.2 Solizaire & 403 & 1,832 & N.A. & N.A. & N.A. & N.A. & N.A. & N.A. & N.A. & N.A. & 1,800 & 1,850 & 84 \\
\hline Gulfstream Am. Commander sao & 450 & 1.855 & N.A. & N.A. & N.A. & N.A. & NA. & N.A. & N.A. & N.A. & 1,830 & 2,150 & 86 \\
\hline Gulfstream Am. Commander 840 & 450 & 1.855 & N.A. & N.A. & N.A. & N.A. & N.A. & N.A. & N.A. & N.A. & 1.833 & 2,030 & 86 \\
\hline Ceseng 441 Conquest & 475 & 2,159 & 494 & $15.04 \mathrm{~m}$. & $39^{\circ} 0^{\circ}$ & $11.8 \mathrm{~m}$ & 1311 & $4.01 \mathrm{~m}$ & 253.6 & 23.56 & 2,465 & 1,875 & 87 \\
\hline Beechcrath Eso King Air & 384 & 1,746 & N.A. & N.A. & N.A. & NA. & N.A. & N.A. & N.A. & N.A. & 2,261 & 1,672 & 87 \\
\hline Beechcrath C90 King Air & 320 & 1454 & 503 & $15.32 \mathrm{~m}$ & $35^{\prime} 8^{\prime}$ & $10.82 \mathrm{~m}$ & $143^{\prime}$ & $4.34 \mathrm{~m}$ & 293.9 & 27.31 & 2,261 & 2,010 & 87 \\
\hline Beechcratt so King Air & N.A. & N.A. & $45 ! 117$ & $13.88 \mathrm{~m}$ & 356 & $10.82 \mathrm{~m}$ & $14^{\prime} 8^{\circ}$ & $4.47 \mathrm{~m}$ & 279.7 & 25.80 & N.A. & N.A. & N.A. \\
\hline Pioer PA-31T Cheyenne & 405 & 1,385 & 428 & $13.01 \mathrm{~m}$ & $34^{\prime} 8$ & $10.57 \mathrm{~m}$ & 129 & $3.89 \mathrm{~m}$ & 220.0 & 21.30 & 2,650 & 2,100 & N.A. \\
\hline Pitans PC-12 & N.A. & N.A. & $520^{\circ}$ & $16.08 \mathrm{~m}$ & 472 & $14.39 \mathrm{~m}$ & $14^{\prime} 0^{\circ}$ & $4.28 \mathrm{~m}$ & 277.8 & 25.81 & N.A. & N.A. & N.A. \\
\hline Piper Chevenne I & 308 & 1,400 & NA & NA & N.A & N.A. & N.A. & N.A. & N.A. & N.A. & 2444 & 2,223 & 83 \\
\hline Cesesna 125 Corserr & 387 & 1.068 & $44^{\prime} 1.5^{\circ}$ & $13.45 \mathrm{~m}$ & $35 \cdot 10^{\circ}$ & $10.83 \mathrm{~m}$ & 127 & $3.84 \mathrm{~m}$ & 225.0 & 20.20 & N.A. & N.A. & N.A. \\
\hline Comens 208 Caraven 1 & 335 & 1,288 & 5211 & $15.88 \mathrm{~m}$ & 377 & $11.46 \mathrm{~m}$ & $14^{\prime} 2$ & $4.32 \mathrm{~m}$ & 279.4 & 25.86 & 1.865 & 1,550 & N.A. \\
\hline Lear Fan 2100 & 250 & 1,137 & N.A. & N.A. & N.A. & N.A. & N.A. & N.A. & N.A. & N.A. & N.A. & N.A. & N.A. \\
\hline Pllatus PC-6B2-H4 Turbo Porbr & N.A. & N.A. & 5211 & $15.87 \mathrm{~m}$ & $30^{\circ} 11$ & $11.00 \mathrm{~m}$ & $100^{\circ}$ & $3.20 \mathrm{~m}$ & 324.5 & 30.15 & 3,525 & 3270 & 50 \\
\hline
\end{tabular}

N.A. - hiormation not evailable when table prepared. 
Toble 3.51

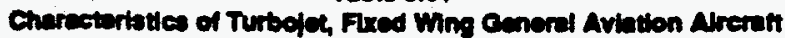

Redurnese 329 to 3.36

\begin{tabular}{|c|c|c|c|c|c|c|c|c|c|c|c|}
\hline \multirow[b]{2}{*}{ Arean Type } & \multicolumn{2}{|c|}{ Pomplent(o) } & \multirow{2}{*}{$\begin{array}{l}\text { Noring } \\
\text { Power } \\
\text { Pating (cach) }\end{array}$} & \multicolumn{2}{|c|}{$\begin{array}{l}\text { Operating } \\
\text { Empry Weight }\end{array}$} & \multicolumn{2}{|c|}{$\begin{array}{l}\text { Maximum } \\
\text { Takeoff Waigh! }\end{array}$} & \multicolumn{2}{|c|}{$\begin{array}{l}\text { Maximum } \\
\text { Lending Weight }\end{array}$} & \multicolumn{2}{|c|}{$\begin{array}{l}\text { Meximum } \\
\text { Zorofud Weight }\end{array}$} \\
\hline & No. & Trpe & & Pounde & Klogrem & Pound & Klogren & Pound & Klogram & Founds & Klogram \\
\hline \multicolumn{12}{|l|}{ TUABONET, FIXED WING } \\
\hline Gultzmean III & 2 & AR. Sog Mlks11 8 TF & 10,342 tos. & 32,500 & 14,742 & 60,700 & 31,615 & N.A. & N.A. & N.A. & N.A \\
\hline Cenadair cos Challenger & 2 & GE. CF35-39 TF & 8.729 thes. & N.A. & N.A. & 47,700 & 21.838 & N.A. & N.A. & N.A. & N.A. \\
\hline Derapult Fucon $800 \mathrm{~B}$ & 3 & Germin TFE 731-58ATF & $4,750 \mathrm{Bb}$ & 22,575 & 10.240 & 45,500 & 20,020 & N.A. & N.A. & N.A. & NA. \\
\hline Cenedair cos Chellenger & 2 & GE. CF34OA TF & $9.140 \mathrm{bs}$ & 25.500 & 11.567 & 45,100 & 20,457 & N.A. & N.A. & N.A. & N.A. \\
\hline Lochtoed vetetar II & 4 & Gerrot TFE 731.3 TF & 3,7000 & 24,178 & 10,987 & 43,750 & 18,845 & N.A. & N.A. & N.A. & N.A. \\
\hline Dasenuti Faloon 50 & 3 & Gerren TFE 731.3 TF & $3,700 \mathrm{Ds}$ & 20,170 & 8,140 & 40,780 & 18.497 & N.A. & N.A. & N.A. & N.A. \\
\hline Cenedar eoo Chellenocer & 2 & Avoo Lycoming ALF so2 TF & 7,500 thes. & 22,075 & 10,285 & 40,125 & 18,200 & N.A. & N.A. & N.A. & N.A. \\
\hline Deseant Facon 2000 & 2 & CFE CFE732-1-1日TF & 5.725 thes. & 10980 & 9,083 & 35,000 & 15,878 & N.A. & N.A. & N.A. & N.A. \\
\hline Desiesth Foloon 200 & 2 & Gemin ATF 3-6A-4C TF & 5,200 bes & 18,200 & 0.209 & $32,0,0$ & 14,515 & N.A. & N.A. & N.A. & N.A. \\
\hline Desesun Fuloon 20F & 2 & GE. CF700-2D-2 TF & 4.50010. & 16,000 & $7,5 \times 0$ & 28,000 & 13,000 & N.A. & N.A. & N.A. & N.A. \\
\hline IA 112SSP Astre & 2 & GeTOH TFE-731-3C-2006 TF & 3,700 tos. & 10,225 & 5,800 & 24,650 & 11,181 & N.A. & N.A. & N.A. & N.A. \\
\hline IAl 1124A Wostwind II & 2 & Gemen TFE-731-1-100G TF & $3,700 \mathrm{lbs}$ & 13,250 & 0,010 & 23,050 & 10.727 & N.A. & N.A. & N.A. & N.A. \\
\hline Leaript 6 & 2 & PEWC PWEOSA TF & 4.00010. & 13,750 & 6,237 & 23,100 & 10,478 & N.A. & N.A. & N.A. & N.A. \\
\hline Ceosena 850 Citation VII & 2 & GOMOH TFE $731-4 R-25$ TF & 4,0000165 & 11,686 & 5,301 & 22,450 & 10,183 & N.A. & N.A. & N.A. & N.A. \\
\hline Cessna 650 Citation IIVVI & 2 & Gamon TFE 731-33-100S TF & 3,650 tbs, 81 & 12,200 & 5.534 & 22,000 & 8,879 & 17,000 & 7.711 & N.A. & N.A. \\
\hline Leajet 55 & 2 & Gamen TFE-731-34-2B TF & 3,700 tos. & 13,258 & 6,014 & $21, \infty 00$ & 8,525 & N.A. & N.A. & N.A. & NA. \\
\hline |A| 1123 Westwind I & 2. & GE. CJ-610-9 TJ & $3,100 \mathrm{tb}$ & 11,750 & 5,350 & 20,700 & 0,389 & N.A. & N.A. & N.A. & N.A. \\
\hline Dessauth Falcon 100 & 2 & Gamen TFE 731-2C TF & 3,230166 & 11,145 & 5,055 & 18,300 & 8.754 & N.A. & N.A. & N.A. & N.A. \\
\hline Desseutit Falcon 10 & 2 & Gemott TFE 731-2C TF & $3,230 \mathrm{lbs}$ & 10,780 & 4,081 & 18,740 & 8,500 & N.A. & N.A. & N.A & N.A. \\
\hline Leariat 38A & 2 & Gerrot TFE-731-28 TF & $3,500 \mathrm{lbs}$ & 10,120 & 4,500 & 18,300 & B,301 & N.A. & N.A. & N.A. & N.A. \\
\hline Loana1 35A & 2 & Garrent TFE-731-28 TF & 3.500 thes. & 10,120 & 4,590 & 18,300 & 8,301 & N.A. & N.A. & N.A. & N.A. \\
\hline Cessne 560 Cimion V & 2 & PEW. JT150-5A TF & $2,900106.81$ & 0,828 & 4,004 & $15, \infty 00$ & 7,212 & N.A. & N.A. & N.A. & N.A. \\
\hline Cessna S550/551 Citation SN1, SI & 2 & PaW. J150-4B TF & $2,50010 \mathrm{~s} . \mathrm{st}$ & 8,080 & 3,656 & 15,100 & 6,849 & N.A. & N.A. & N.A. & N.A. \\
\hline Leariel 29 & 2 & GE. CJB10-8A TF & 2,050100 & 8,224 & 3,730 & 15,000 & 6,604 & N.A. & N.A. & N.A & N.A. \\
\hline Leeriel 28 & 2 & GE. C.S10-8ATF & 2.950105. & 8,268 & 3,750 & 15,000 & 6,804 & N.A. & N.A. & N.A. & N.A. \\
\hline Leariel 250 & 2. & GE. CNB10-8A TF & 2,950106 & 7,840 & 3,465 & 15,000 & 6,804 & N.A. & N.A. & N.A. & N.A. \\
\hline Cecenn 550/551 Citation II & 2 & P\&W. JT150- TF & $2,500100.11$ & 7,181 & 3,257 & 13,300 & 6,030 & N.A. & N.A. & N.A. & N.A. \\
\hline Leariel 24D & 2 & N.A. & N.A. & N.A. & N.A. & N.A. & N.A. & N.A. & N.A. & N.A. & N.A. \\
\hline Mitsubishi Diemond VBeechiet & 2 & P8W. JT15D-5 TF & $2,900 \mathrm{lbs} .81$ & 8,050 & 3,651 & 15,780 & 7,150 & N.A. & N.A. & N.A. & N.A. \\
\hline Learat 23 & 2 & GE. C.8310-4 TJ & $2,850 \mathrm{lbs}$ & 6,550 & 2,871 & 12,500 & 5,670 & N.A. & N.A. & N.A. & N.A. \\
\hline Cessna 500/501 Citation USP & 2 & PEW. JI5D-1A1BTF & $2,200165.81$ & 8,631 & 3,008 & 11,850 & 5,375 & 11,350 & 5,148 & N.A. & N.A. \\
\hline Cessina 500 Citation & 2 & P\&W. Л150-1 TF & 2,200 lbs. 11 & 5,400 & 2,453 & 10,850 & 4,921 & N.A. & N.A. & N.A. & N.A \\
\hline
\end{tabular}

N.A. = thtormation not evailable when bble prepared

be, at. a pounds thrust static

$T d=$ Tutboipl 
Teble 9.51 (Continued)

Charecturistics of Turtojpt, Fxed Wing Ceneral Aviation Alreran

Reterences 3.29 to 3.35

\begin{tabular}{|c|c|c|c|c|c|c|c|c|c|c|c|c|c|}
\hline \multirow[b]{2}{*}{ Areren Type } & \multicolumn{2}{|c|}{$\begin{array}{l}\text { Marimum } \\
\text { Fud Capeciry }\end{array}$} & \multicolumn{2}{|c|}{$\begin{array}{l}\text { Orenall } \\
\text { Wing Span }\end{array}$} & \multicolumn{2}{|c|}{ Orendll Length } & \multicolumn{2}{|c|}{ loverell Hoight } & \multicolumn{2}{|l|}{ Wing Ane } & \multirow{2}{*}{$\begin{array}{c}\text { Takeoff } \\
\text { Orst. (fi.) } \\
\text { (FAF) }\end{array}$} & \multirow{2}{*}{$\begin{array}{l}\text { Landing } \\
\text { Oist. (tI.) } \\
\text { (FAR) }\end{array}$} & \multirow{2}{*}{$\begin{array}{l}\text { Stat Spoed } \\
\text { (oparmaps } \\
\text { dom, mph) }\end{array}$} \\
\hline & inp. Cal. & Litere & Ft. In. & Movers & Ft. In. & Molers & Ft. In. & Mons & Fi."-2 & $M \cdot 2$ & & & \\
\hline \multicolumn{14}{|c|}{ GENERAL AVIATION TURBONET, FIXED WING } \\
\hline Gulfotrean III & 4.133 & 18,789 & $7710^{\circ}$ & $23.72 \mathrm{~m}$ & Q5:10 & $25.32 \mathrm{~m}$ & $245^{n}$ & $7.43 \mathrm{~m}$. & es4.6) & 88.83 & 5,850 & 3,400 & 139 \\
\hline Cenndair eos Challenper & N.A. & N.A. & 65' 10 & $18.85 \mathrm{~m}$ & 885 & $20.85 \mathrm{~m}$ & $20^{\circ} 8^{\circ}$ & $6.30 \mathrm{~m}$ & 520.0 & 48.31 & N.A. & N.A. & NA. \\
\hline Deasenti Faloon 9003 & N.A. & N.A. & 655 & $18.33 \mathrm{~m}$ & 084 & $20.21 \mathrm{~m}$ & $24^{\prime} 8^{\prime \prime}$ & $7.55 \mathrm{~m}$ & 527.4 & 40.00 & N.A. & N.A. & N.A. \\
\hline Cenedeir eos Chetlenear & N.A. & N.A. & $61^{\prime} 10$ & $18.85 \mathrm{~m}$ & 685 & $20.85 \mathrm{~m}$ & 200 & $8.30 \mathrm{~m}$ & 80.0 & 48.31 & N.A. & N.A. & N.A. \\
\hline Lockleed betar II & N.A. & N.A. & 54:57 & $16.60 \mathrm{~m}$ & 605 & $18.42 \mathrm{~m}$ & 205 & $6.23 \mathrm{~m}$ & S12.5 & 50.40 & N.A. & N.A. & N.A. \\
\hline Deserenil Feloon 50 & 2.316 & 10,539 & $61^{\prime} 119$ & $18.68 \mathrm{~m}$ & $61 \cdot 119$ & $18.52 \mathrm{~m}$. & 22119 & $8.97 \mathrm{~m}$ & 504.1 & 46.83 & 4,000 & 3.600 & 87 \\
\hline Cenadar epo Challenger & N.A. & N.A. & $61+10$ & $18.85 \mathrm{~m}$ & 685 & $20.85 \mathrm{~m}$ & 208 & $6.30 \mathrm{~m}$ & 450.0 & 41.82 & N.A. & N.A. & N.A. \\
\hline Desseull Falcon 2000 & NA. & N.A. & 255 & $18.34 \mathrm{~m}$ & 604 & $20.23 \mathrm{~m}$. & 232 & $7.08 \mathrm{~m}$ & 527.7 & 40.02 & N.A. & NA. & N.A. \\
\hline Desenut Faloon 200 & 1,568 & 7,210 & 587 & $16.32 \mathrm{~m}$. & $503^{2}$ & $17.15 \mathrm{~m}$. & 176 & $5.32 \mathrm{~m}$ & 441.3 & $41 . \infty$ & 5,700 & 2,610 & NA. \\
\hline Desseun Felcon zof & 1,360 & 0.224 & 557 & $16.32 \mathrm{~m}$ & $50 \times 3$ & $17.15 \mathrm{~m}$ & $176^{\circ}$ & $5.32 \mathrm{~m}$ & 41.3 & 41.00 & 4.000 & 1,975 & 86 \\
\hline IAl 11255 PAstr: & N.A. & N.A. & 528 & $10.05 \mathrm{~m}$ & $55^{\prime} 7$ & $16.84 \mathrm{~m}$ & $10^{\prime} 2$ & $5.53 \mathrm{~m}$ & 316.8 & 29.41 & N.A. & N.A. & N.A. \\
\hline 1A) 1124A Wesnwind II & 1424 & 6474 & $44^{\prime} 3$ & $13.85 \mathrm{~m}$ & 523 & $15.03 \mathrm{~m}$ & $15^{\prime} 10^{\circ}$ & $4.81 \mathrm{~m}$ & 308.3 & 28.64 & 5,250 & 2,450 & 114 \\
\hline Leajel eo & 1,212 & 5,510 & $43^{\circ}$ & $13.34 \mathrm{~m}$ & $588^{4}$ & $17.88 \mathrm{~m}$ & $14^{\prime} 8^{\prime}$ & $4.47 \mathrm{~m}$ & 204.5 & 24.57 & 5,130 & 2,950 & 113 \\
\hline Cosena 650 Citation VIf & N.A. & N.A. & 556 & $16.31 \mathrm{~m}$. & $55^{\prime} 6^{\circ}$ & $16.80 \mathrm{~m}$ & $16^{\prime} 10^{\circ}$ & $5.12 \mathrm{~m}$ & 312.0 & 29.00 & NA. & NA. & N.A. \\
\hline Cossna 650 Citation IIVWI & 921 & 4,505 & $53^{\circ} 6^{\circ}$ & $16.31 \mathrm{~m}$ & $55^{\prime} 6$ & $16.00 \mathrm{~m}$ & $16^{\prime} 10^{\circ}$ & $5.12 \mathrm{~m}$ & 312.0 & 29.00 & 3.800 & 2,690 & 101 \\
\hline Learat 55 & 1015 & 4.614 & $48^{\prime \prime}$ & $13.34 \mathrm{~m}$ & $55^{\prime} 1^{\prime \prime}$ & $16.79 \mathrm{~m}$ & $14^{\prime} 8^{\circ}$ & $4.47 \mathrm{~m}$ & 284.5 & 24.57 & 4,550 & 2,950 & 113 \\
\hline IAl 1123 Westwind I & 1,300 & 5.010 & $44^{\prime} 5^{\circ}$ & $13.65 \mathrm{~m}$ & $523^{\prime}$ & $15.83 \mathrm{~m}$ & $15^{\prime} 10^{\circ}$ & $4.81 \mathrm{~m}$ & 300.3 & 28.64 & 4,950 & 2,450 & 114 \\
\hline Dessaul Falcon 100 & 882 & 4,010 & 42111 & $13.08 \mathrm{~m}$ & $45^{\prime} 8^{\circ}$ & $13.88 \mathrm{~m}$ & $15^{\prime} 2^{\prime}$ & $4.61 \mathrm{~m}$ & 259.0 & 24.10 & 4,500 & 2,750 & 90 \\
\hline Desseunt Faicon 10 & 882 & 4,010 & 42114 & $13.08 \mathrm{~m}$ & $45^{\prime} 6^{\circ}$ & $13.83 \mathrm{~m}$ & $15^{\prime} 2$ & $4.61 \mathrm{~m}$ & 259.0 & 24.10 & 4,500 & 2,750 & 80 \\
\hline Leariat 33A & 1,110 & 5,048 & $39^{\prime} 6$ & $12.04 \mathrm{~m}$ & $48^{\prime} 8^{\circ}$ & $14.63 \mathrm{~m}$. & 123 & $3.73 \mathrm{~m}$ & 253.3 & 23.53 & 4.784 & 2,884 & 110 \\
\hline Leeriat 35A & 831 & 4,232 & $39^{\prime} 6^{\circ}$ & $12.04 \mathrm{~m}$ & $488^{1}$ & $14.83 \mathrm{~m}$. & $123^{\circ}$ & $3.73 \mathrm{~m}$ & 253.3 & 23.53 & 4,224 & 2,884 & 110 \\
\hline Cessina 560 Ciration V & N.A. & N.A. & 523 & $15.00 \mathrm{~m}$ & 48.119 & $1480 \mathrm{~m}$ & $15^{\circ} 0^{\prime \prime}$ & $4.57 \mathrm{~m}$ & 342.6 & 31.83 & NA. & NA. & N.A. \\
\hline Cocena 5550/551 Citation S/1, SA & N.A. & N.A. & $523^{1}$ & $15.90 \mathrm{~m}$ & $473^{0}$ & $14.39 \mathrm{~m}$ & $15^{\prime} 0^{\circ}$ & $4.57 \mathrm{~m}$ & 342.6 & 31.83 & N.A. & N.A. & N.A. \\
\hline Leariat 29 & 602 & 3,846 & $4310^{\circ}$ & $13.35 \mathrm{~m}$ & 477 & $1450 \mathrm{~m}$ & 123 & $3.73 \mathrm{~m}$ & N.A. & N.A. & 3,040 & 2.734 & 104 \\
\hline Leeriet 28 & $\infty$ & 3.178 & 357 & $10.85 \mathrm{~m}$ & 477 & $14.50 \mathrm{~m}$ & N.A. & N.A. & N.A. & N.A. & 3.040 & 2,734 & 10 \\
\hline Leariat 25D & 810 & 4,137 & $35^{\prime} 8^{\circ}$ & $10.84 \mathrm{~m}$ & 477 & $14.50 \mathrm{~m}$ & 123 & $3.73 \mathrm{~m}$ & 231.8 & 21.53 & 3,937 & 2,744 & 112 \\
\hline Coesna 5sors5t Citution II & 714 & 3.246 & $57^{\prime} 8^{*}$ & $15.75 \mathrm{~m}$ & $456^{\circ}$ & $13.26 \mathrm{~m}$ & N.A. & N.A. & N.A. & NA. & 2,000 & 2,270 & 83 \\
\hline Leariet 240 & N.A. & N.A. & $35 \cdot 7$ & $10.84 \mathrm{~m}$ & $43^{\circ}$ & $12.5 \mathrm{~m}$. & N.A. & N.A. & N.A. & N.A. & N.A. & N.A. & N.A. \\
\hline Mitsubishi Diamond VBeachiat & 732 & 3,320 & $43^{\prime} 6^{\prime \prime}$ & $13.28 \mathrm{~m}$ & $48^{\prime} 5^{\circ}$ & $14.78 \mathrm{~m}$. & $13^{\prime} 9.5^{\circ}$ & $4.2 \mathrm{~m}$ & N.A. & N.A. & 4,100 & 2,700 & 87 \\
\hline Learigt 23 & N.A. & N.A. & $35^{\prime} 8^{\circ}$ & $10.84 \mathrm{~m}$ & $433^{\circ}$ & $13.18 \mathrm{~m}$. & 1237 & $3.73 \mathrm{~m}$ & 231.8 & 21.53 & N.A. & N.A. & N.A. \\
\hline Cossna 500/501 Citation USP & sen & 2,564 & 4719 & $14.35 \mathrm{~m}$ & $430^{\circ}$ & $13.28 \mathrm{~m}$ & $14 \cdot 30$ & $4.36 \mathrm{~m}$ & 278.5 & 25.80 & 2,830 & 2,270 & 8 \\
\hline Cessna 500 Citation & se4 & 2504 & $43^{\prime} 9^{\prime}$ & $13.32 \mathrm{~m}$ & $43^{\circ} 0^{\circ}$ & $13.28 \mathrm{~m}$ & $14^{\prime} 3$ & $4.36 \mathrm{~m}$ & 200.0 & 24.15 & N.A. & N.A. & N.A. \\
\hline
\end{tabular}

N.A. - Iniormatoon not avaiable when bable propered. 
Toble 3.52

Characteristes of Botery Wing Cenerd Avtetion Airereft Poterencese 3.29 ip 3.35

\begin{tabular}{|c|c|c|c|c|c|c|c|c|c|c|c|}
\hline \multirow[b]{2}{*}{ Aneen Typo } & \multicolumn{2}{|c|}{ Powerplant(8) } & \multirow{2}{*}{$\begin{array}{c}\text { Nouning } \\
\text { Powner } \\
\text { Pating (cach) }\end{array}$} & \multicolumn{2}{|c|}{$\begin{array}{l}\text { Oponting } \\
\text { Empry Woight }\end{array}$} & \multicolumn{2}{|c|}{$\begin{array}{l}\text { Maximum } \\
\text { Tekoofl Weight }\end{array}$} & \multicolumn{2}{|c|}{$\begin{array}{l}\text { Maximum } \\
\text { Lendino Wenght }\end{array}$} & \multicolumn{2}{|c|}{$\begin{array}{l}\text { Maximum } \\
\text { Zero-Fuel WoighI }\end{array}$} \\
\hline & No. & Typo & & Pound & Kloonen & Pounde 1 & Kiogram & Pound & Klooran & Pound: & Kiogram \\
\hline \multicolumn{12}{|l|}{ ROTARY WING } \\
\hline Booin Verol zoulA Chinook & 2 & ILcomin AL S512TS & $4,075 \sin 0$ & 25000 & 11.748 & 31,000 & 23,133 & N.A. & N.A. & N.A. & N.A. \\
\hline Slkondy S-84N MK II & 2 & GE. CTSe-140-2 TS & $1.500 \sin$ & 12,510 & 5,674 & 22,000 & 0.070 & N.A. & N.A. & N.A. & N.A. \\
\hline Eurocopler As332L Supor Puma & 2 & Turbomect Metila & $1,755 \mathrm{sh}$ & 2.005 & 4370 & 19,840 & 0.00 & N.A. & N.A. & N.A. & N.A. \\
\hline Boeing Vertol BV 107 & 2 & GE. CTS8-110-1 TS & $1,250 \operatorname{shp}$ & 10.723 & 4,804 & 18,000 & 8.618 & N.A. & N.A. & N.A. & N.A. \\
\hline Bell 214ST & 2 & GE. CT 7-2ATS & $1,025 \operatorname{sh} 2$ & 0.401 & 4301 & 17,500 & 7,030 & N.A. & N.A. & N.A. & N.A. \\
\hline Acocopatiale SA3sol Puing & 2 & Tutomece Tumo NC & $1,575 \mathrm{shd}$ & 8,305 & 3,787 & 10,505 & 7,500 & N.A. & N.A. & N.A. & NA. \\
\hline Befl 2148 Bin Liner & 1 & Lroomino TS50-80 TS & $2,800 \operatorname{chp}$ & 7,827 & 3,550 & 18.000 & 7,257 & N.A. & N.A. & N.A. & N.A. \\
\hline Sliporisy S-58T & 1 & PAWC PTET- TS & $1,075 \operatorname{shn}$ & 7,400 & 3,357 & 13,000 & 5,007 & N.A. & N.A. & N.A. & N.A. \\
\hline Bell 412 & 2 & PAW. PTET-39TS & $1,308 \mathrm{shp}$ & 8,070 & 2,750 & 11,500 & 5,218 & N.A. & N.A. & N.A. & N.A. \\
\hline Bell 212 & 2 & PEW. FTOT-3 TS & $1200 \operatorname{shp}$ & 6,007 & 2786 & 11,200 & 5,000 & N.A. & N.A. & N.A. & N.A. \\
\hline Bent 205A-1 & 1 & Lyeomin TS31-138 TS & 1,250 shp & 5,323 & 2,414 & 10,500 & 4,783 & N.A. & N.A. & N.A. & N.A. \\
\hline Sthoraky 5.78 Mk II & 2 & Allieon 250C-30 TS & $250 \mathrm{chp}$ & 5,000 & 2,540 & 10,300 & 4.672 & N.A. & N.A. & N.A. & N.A. \\
\hline Aerospatiale SA365N2 Deyphin 2 & 2 & Tutbomect Ariel $1 \mathrm{C}_{2}$ & 739 chp & 4.90 & 2241 & 9,370 & 4,250 & N.A. & N.A. & N.A. & N.A. \\
\hline Bell 2223 & 2 & Lrecoming LTS-101 650C TS & $880 \operatorname{shp}$ & 4,577 & 2,078 & 8,250 & 3,742 & N.A. & N.A. & N.A. & N.A. \\
\hline Siloonthy S-62C & 1 & GE. CTSB-110-1 TS & $1,250 \mathrm{chp}$ & 4,800 & 2,204 & 7,800 & 3,583 & N.A. & N.A. & N.A. & N.A. \\
\hline Eurocoplor Kawrasakj BK.1178-2 & 2 & Ireoming LTS-101-750-8-1 TS & 592 thp & 3,607 & 1,727 & 7,385 & 3,350 & N.A. & N.A. & N.A. & N.A. \\
\hline Sikoraky S.55T & 1 & JAlPeeearch TSE $331-3 U-3003$ TS & 650 shp & 4,700 & 2,132 & 7,200 & 3,268 & N.A. & N.A. & N.A. & N.A. \\
\hline Aorospatiale SAseOC Dauphin & 1 & Turoomeca Astezou XVIIIA TS & 1,050 shp & 3,428 & 1.555 & 6,613 & $3,0,0$ & N.A. & N.A. & N.A. & N.A. \\
\hline Aousta 109A Mk II & 2 & Allieon 250C-20B TS & 420 chp & 3,128 & 1418 & 5.732 & 2,000 & N.A. & N.A. & N.A. & N.A. \\
\hline Eurocopter AS355F Eareuil 2 & 2 & Allieon 250C-20F TS & 420 ahp & 2.877 & 1,305 & 5,732 & 2,000 & N.A. & N.A. & N.A. & N.A. \\
\hline Eurocopter BO. 105 CBS & 2 & Allison 250C-208 TS & 420 enp & 2,808 & 1,301 & 5,511 & 2,500 & N.A. & N.A. & N.A. & N.A. \\
\hline Aerospatiale SA3t 58 Lama & 1 & Turbomea Arousto llfB & S50 ethe & 2,250 & 1,021 & 5,070 & 2,300 & N.A. & N.A. & N.A & NA. \\
\hline Aerospatiele SA319B Alounth III & 1 & Turboneca Astazou XIVB & $600 \operatorname{sing}$ & 2.513 & 1,140 & 4,860 & 2.250 & N.A. & N.A. & N.A. & N.A. \\
\hline Bell zobLT Twin Panger & 2 & Allieon 250C-20R TS & 450 sho & 2,748 & 1,246 & 4,550 & 2,084 & N.A. & N.A. & N.A. & N.A. \\
\hline Euroeopter AS35032 Ecureuil & 1 & Turbonece Ariel 101 & $732 \sin$ & 2.541 & 1,153 & 4,860 & 2,250 & N.A. & N.A. & N.A. & N.A. \\
\hline Acrospatiale SA342 Gezelle & 1 & Turbomeca Asiazou XIV & 858 cho & 2,149 & 975 & 4,180 & 1.801 & N.A. & N.A. & N.A. & N.A. \\
\hline Bell 20aL-1 Long Ranger if & 1 & Alleon 250C-28B TS & 500 shp & 2,160 & 880 & 4,150 & 1,882 & N.A. & N.A. & NA & N.A. \\
\hline Bell 206B Jel Ranoer III & 1 & Allison 250C-201 TS & 420 ehp & 1.635 & 742 & 3,350 & 1,520 & N.A. & N.A. & N.A. & N.A. \\
\hline Hitier UH12E4T & 1 & Allison 250C-20B TS & Sol ho & 1,650 & 748 & 3,100 & 1405 & N.A. & N.A. & N.A. & N.A. \\
\hline Hiller UH12ET & 1 & Allison $250 \mathrm{C}-20 \mathrm{~B}$ TS & $301 \mathrm{hg}$ & 1,650 & 748 & 3,100 & 1,408 & N.A. & N.A. & N.A. & N.A. \\
\hline Hillor UH12E4 & 1 & Lreomin: Vo-540-C2A PS & $305 \mathrm{hp}$ & 1.858 & 833 & 3,100 & 1.406 & N.A. & N.A. & N.A. & N.A. \\
\hline Hiller UH12E & 1 & Lreoming Vo-540-C2A PS & $\operatorname{coshp}$ & 1,759 & 798 & 3,100 & 1.408 & N.A. & N.A. & N.A. & N.A. \\
\hline Huphes 500D & 1 & Allison 250C-203 TS & 420 cho & 1,300 & 617 & 3,000 & 1,331 & N.A. & N.A. & N.A. & N.A. \\
\hline Eoll 47G-3B-2A & 1 & Lrooming TVO-435-FIA & $280 \mathrm{hp}$ & 1,893 & 850 & 2950 & 1,338 & N.A. & N.A. & N.A. & N.A. \\
\hline Hillar UH FH1100 & 1 & Allieon 250C-11B TS & N.A. & 1,305 & 633 & 2,750 & 1,247 & N.A. & N.A. & N.A. & N.A. \\
\hline Erstrom 260FX Shark & 1. & Lrcoming HO-s60-FIAD PS & $225 \mathrm{hp}$ & 1,505 & 719 & 2,600 & 1,179 & N.A. & N.A. & N.A. & N.A. \\
\hline Enstrom F-28F & 1 & Lrcoming HO-360-F1AD PS & $225 \mathrm{np}$ & 1,500 & 690 & 2,350 & 1,086 & N.A. & N.A. & N.A. & N.A. \\
\hline Enstrom 280C Shark & 1 & Lycoming HO-SBO-EIAD PS & $205 \mathrm{hp}$ & 1,500 & 680 & 2,350 & 1,086 & N.A. & N.A. & N.A. & N.A. \\
\hline Enstrom F-28A & 1 & Lrcoming HL-3so-C1B PS & $205 \mathrm{hpl}$ & 1,450 & 658 & 2,150 & 975 & N.A. & N.A. & N.A. & N.A. \\
\hline Hughes $300 \mathrm{C}$ & 1 & Lreoming HO-380-DIAPS & $225 \mathrm{hp}$ & 1,046 & 474 & $2,0 \leq 0$ & 800 & N.A. & N.A. & N.A. & N.A. \\
\hline Branty-thnes B28 & 1 & Lreoming IVO-360-AIA PS & $180 \mathrm{ho}$ & 1,020 & 463 & 1,670 & 757 & N.A. & N.A. & N.A. & N.A. \\
\hline Robinson P-22 & 1 & Lycoming 0-320 PS & $124 \mathrm{hp}$ & 798 & 381 & 1,300 & 500 & N.A. & N.A. & N.A. & N.A. \\
\hline Rotonway Exec & 1 & AW-145 PS & $145 \mathrm{hp}$ & 780 & 354 & 1,283 & 573 & N.A. & N.A. & N.A & N.A. \\
\hline Scorpion 133 & 1 & N.A. & N.A. & N.A. & N.A. & N.A. & N.A. & N.A. & N.A. & NA. & N.A. \\
\hline
\end{tabular}

N.A. - Information not evereble when thele prepared.

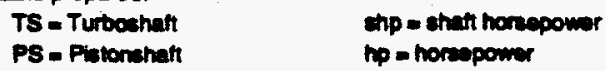


Toble 3.52 (Continued)

Charecteriatles of Potan Wing Coneral Aviation Areraft

Alterences 3.20 to 3.35

\begin{tabular}{|c|c|c|c|c|c|c|c|c|c|c|c|c|}
\hline \multirow[b]{2}{*}{ Aretall Typo } & \multicolumn{2}{|c|}{$\begin{array}{l}\text { Maximum } \\
\text { Fud Capecive }\end{array}$} & \multicolumn{2}{|c|}{ Porox Diamoter } & \multicolumn{2}{|c|}{ Fucedeco Lemph } & \multicolumn{2}{|c|}{ loversll Height } & \multicolumn{2}{|c|}{$\begin{array}{l}\text { Mein Aotox } \\
\text { Diec Area }\end{array}$} & \multirow{2}{*}{$\begin{array}{l}\text { Number } \\
\text { Pootr(s) }\end{array}$} & \multirow{2}{*}{$\begin{array}{l}\text { Number } \\
\text { Folor } \\
\text { Bladese }\end{array}$} \\
\hline & Imp. G & Liten & Ft. In. & Moters & Fi. In. & Mares & Ft. In. & Moters & Ft." & $M+\infty 2$ & & \\
\hline \multicolumn{13}{|l|}{ POTARY WING } \\
\hline Booing Verol 234LA Chinook & 2,000 & e.501 & $\cos$ & $18.29 \mathrm{~m}$. & 5210 & $15.87 \mathrm{~m}$ & $188^{-1}$ & $5.08 \mathrm{~m}$ & 305.0 & 525.34 & 2 & 3 \\
\hline Sileordy S-61N Mk II & N.A. & N.A. & 820 & $18.00 \mathrm{~m}$ & $58 \cdot 19$ & $17.83 \mathrm{~m}$ & 176 & $5.32 \mathrm{~m}$. & 30180 & 280.47 & 1 & 5 \\
\hline Eurecopter AS332L Super Puma & 400 & 1,053 & 408 & $15.08 \mathrm{~m}$ & $50^{\prime} 119$ & $15.52 \mathrm{~m}$ & 1619 & $4.02 \mathrm{~m}$. & 1022.5 & 178.00 & 1 & 4 \\
\hline Boein Verol BV 107 & N.A. & N.A. & 500 & $15.24 \mathrm{~m}$ & 47 & $13.50 \mathrm{~m}$. & $16 \%$ & $5.00 \mathrm{~m}$. & 3005.0 & 324.00 & 2 & 3 \\
\hline Bef 2145T & 410 & 1,084 & 520 & $15.85 \mathrm{~m}$. & soof & $15.24 \mathrm{~m}$ & $15^{\prime} 11^{9}$ & $4.8 \mathrm{~m}$. & 2124.0 & 197.30 & 1 & 2 \\
\hline Acrospatiale SA3sal Puma & N.A. & N.A. & 498 & $15.06 \mathrm{~m}$ & 482 & $14.00 \mathrm{~m}$ & $10^{\circ} 11^{\circ}$ & $5.14 \mathrm{~m}$ & 1905.0 & 177.00 & 1 & 4 \\
\hline Bell 2148 Bin Liner & 204 & 927 & 5000 & $15.24 \mathrm{~m}$ & N.A. & N.A. & N.A. & N.A & 1803.5 & 182.40 & 1 & 2 \\
\hline Stroraty S-59T & N.A. & N.A. & 500 & $17.07 \mathrm{~m}$ & 473 & $14.41 \mathrm{~m}$. & 144 & $4.38 \mathrm{~m}$ & 2460.0 & 228.50 & 1 & 4 \\
\hline Bell 412 & 215 & 97 & 4800 & $14.02 \mathrm{~m}$ & $41^{\circ} \theta^{-1}$ & $12.70 \mathrm{~m}$ & $150^{\prime}$ & $4.57 \mathrm{~m}$. & 1032.0 & 154.40 & 1 & 4 \\
\hline Bell 212 & 215 & on & 482 & $14.69 \mathrm{~m}$ & 4250 & $12.22 \mathrm{~m}$ & $14^{\prime} 10^{\circ}$ & $4.53 \mathrm{~m}$. & 1800.6 & 188.10 & 1 & 2 \\
\hline Bell 205A-1 & 215 & on & 4800 & $14.63 \mathrm{~m}$. & 41.8 & $12.65 \mathrm{~m}$ & 147 & $4.45 \mathrm{~m}$. & 1800.0 & 168.00 & 1 & 2 \\
\hline Sikorsky S-78 Mk II & 200 & 1,273 & 400 & $13.41 \mathrm{~m}$. & 434 & $13.22 \mathrm{~m}$ & $14^{\prime} 6^{\circ}$ & $4.41 \mathrm{~m}$ & 1520.5 & 141.30 & 1 & 4 \\
\hline Acrospatiale SA365N2 Dauphin 2 & $\mathbf{2 9 1}$ & 1,323 & 39.2 & $11.94 \mathrm{~m}$ & 377 & $10.80 \mathrm{~m}$. & 1310 & $3.88 \mathrm{~m}$. & 1205.0 & 119.80 & 1 & 4 \\
\hline Bell 2228 & 184 & 836) & 420 & $12.80 \mathrm{~m}$ & 422 & $12.85 \mathrm{~m}$ & $11^{\prime} 6^{\prime}$ & $3.51 \mathrm{~m}$. & 1385.4 & 128.70 & 1 & 2 \\
\hline Sikorsky $S-62 C$ & N.A. & N.A. & 5300 & $16.17 \mathrm{~m}$ & $4: 7$ & $13.00 \mathrm{~m}$ & $14^{\prime} 2$ & $4.32 \mathrm{~m}$. & 2207.7 & 205.10 & 1 & 3 \\
\hline Eurocopoter Kawesadk BK.117B-2 & 1764 tos. & N.A. & 3619 & $11.00 \mathrm{~m}$ & 326 & $0.81 \mathrm{~m}$. & 128 & $3.85 \mathrm{~m}$ & 1023.0 & 95.03 & 1 & 4 \\
\hline Sikorany S.55T & N.A. & N.A. & 5300 & $18.16 \mathrm{~m}$. & 423 & $12.87 \mathrm{~m}$. & 1344 & $4.08 \mathrm{~m}$. & 2207.7 & 200.10 & & \\
\hline Aerospatiale SA3eOC Dauphin & 109 & 788 & 378 & $11.50 \mathrm{~m}$ & 380 & $10.89 \mathrm{~m}$ & 196 & $3.50 \mathrm{~m}$ & 1118.0 & 103.87 & 1 & 4 \\
\hline ANLSB 109A Mk II & 140 & Q64) & $36,1^{\circ}$ & $11.00 \mathrm{~m}$ & 35,2 & $10.71 \mathrm{~m}$ & $10^{\circ} 10^{\circ}$ & $3.30 \mathrm{~m}$ & 1022.8 & 85.03 & 1 & 4 \\
\hline Eurocopter AS355F Equreuil 2 & 100 & 864 & $35: 17$ & $10.69 \mathrm{~m}$ & $35^{\circ} 10^{\prime \prime}$ & $10.91 \mathrm{~m}$ & 104 & $3.14 \mathrm{~m}$ & 808.1 & 89.75 & 1 & 3 \\
\hline Eurocopier BO.105 CBS & 17200 & N.A. & 324 & $8.84 \mathrm{~m}$ & $29^{\prime}+11$ & $8.81 \mathrm{~m}$. & 9111 & $3.02 \mathrm{~m}$ & 618.8 & 76.05 & 1 & 4 \\
\hline Aoroepatiajo SA315B Lama & 152 & $\infty 1$ & 352 & $11.02 \mathrm{~m}$ & $358^{-1}$ & $10.28 \mathrm{~m}$ & 102 & $3.00 \mathrm{~m}$ & 1026.7 & 95.38 & 1 & 2 \\
\hline Aerospatiale Abuete SA319B & 152 & 091 & $36^{2}$ & $11.02 \mathrm{~m}$ & 32,119 & $10.03 \mathrm{~m}$ & $8 \cdot 10^{\circ}$ & $3.00 \mathrm{~m}$ & 1026.7 & 25.38 & 1 & 3 \\
\hline Bell zosLT T win Ranger & N.A. & N.A. & 370 & $11.28 \mathrm{~m}$ & 325 & $8.81 \mathrm{~m}$ & 104 & $3.14 \mathrm{~m}$ & 1075.2 & 99.89 & 1 & 2 \\
\hline Eurocopter A535032 Eeurouil & 140 & 68 & 35.19 & $10.69 \mathrm{~m}$ & $35,10^{\circ}$ & $10.91 \mathrm{~m}$ & 104 & $3.14 \mathrm{~m}$ & 806.1 & 89.75 & 1 & 3 \\
\hline Acrospatiale SA342 Gezelle & N.A. & N.A. & $34^{\prime} 6^{\prime}$ & $10.5 \mathrm{~m}$ & $31 \cdot 3$ & $9.53 \mathrm{~m}$ & $105^{\circ}$ & $3.18 \mathrm{~m}$ & 201.0 & 86.50 & 1 & 3 \\
\hline Bell zo6L-1 Lon Rangor II & 8 & 40 & 370 & $11.28 \mathrm{~m}$. & $333^{\circ}$ & $10.13 \mathrm{~m}$ & 1049 & $3.14 \mathrm{~m}$ & 1075.2 & 89.69 & 1 & 2 \\
\hline Bell 2068 Jel Fianger III & 76 & 346 & 3340 & $40.16 \mathrm{~m}$ & $31^{\circ} 2$ & $8.50 \mathrm{~m}$ & $\theta^{\prime} 7^{\circ}$ & $2.91 \mathrm{~m}$ & 872.7 & B1.07 & 1 & 2 \\
\hline Hiller UH12E4T & 46 & 200 & N.A. & N.A. & N.A. & N.A. & N.A. & N.A. & N.A. & N.A. & N.A. & N.A. \\
\hline Hillar UH12ET & 46) & 200 & N.A. & N.A. & N.A. & N.A. & N.A. & N.A. & N.A. & N.A. & N.A. & N.A. \\
\hline Hiller UH12E4 & 46. & 2000 & N.A. & N.A. & N.A. & N.A. & N.A. & N.A. & N.A. & N.A. & N.A. & N.A. \\
\hline Hiller UH12E & (16) & 200 & N.A. & N.A. & N.A. & N.A. & N.A. & NA. & N.A. & N.A. & N.A. & N.A. \\
\hline Hunthes 5000 & 64 & 291 & N.A. & N.A. & N.A. & N.A. & N.A. & N. & N.A. & N.A. & N.A. & N.A. \\
\hline Bell 47G-3B-2A & N.A. & N.A. & 372 & $11.32 \mathrm{~m}$ & $31 \cdot 7$ & $8.63 \mathrm{~m}$ & $9^{\prime} 4^{\circ}$ & $2.83 \mathrm{~m}$ & 1005.0 & 100.80 & 1 & 2 \\
\hline Hiller UH FH1100 & 138 & 618 & NA. & N.A. & N.A. & N.A. & NA. & N.A. & NA. & N.A. & N.A. & N.A. \\
\hline Enctrom 280FX Shark & 42 & 191 & $320^{\circ}$ & $9.75 \mathrm{~m}$ & N.A. & N.A. & N.A. & N.A. & 8010 & 74.60 & 1 & 3 \\
\hline Ens500m F-28F & 42 & 181 & NA. & N.A. & N.A. & N.A. & N.A. & N.A. & N.A. & N.A. & N.A. & N.A. \\
\hline Enstrom $200 \mathrm{C}$ Shark & 40 & 182 & N.A. & N.A. & N.A. & NA & N.A. & N.A. & N.A. & N.A. & N.A. & N.A. \\
\hline Enstrom F-28A & 40 & 182 & $320^{\circ}$ & $9.75 \mathrm{~m}$ & N.A. & N.A. & N.A. & N.A. & 8040 & 74.69 & 1 & 3 \\
\hline Hughes $3000 \mathrm{C}$ & $\mathbf{3 0}$ & 136 & N.A. & N.A. & N.A. & N.A. & N.A. & N.A. & N.A. & N.A. & N.A. & N.A. \\
\hline Branity-Hynes BzB & 3i) & 149 & $239^{\circ}$ & $7.24 \mathrm{~m}$ & $21.9^{\circ}$ & $6.62 \mathrm{~m}$ & 6'8 & $2.08 \mathrm{~m}$ & 43.0 & 41.18 & 1 & 3 \\
\hline Pobinson R-22 & 20 & 91 & N.A. & N.A. & N.A. & N.A. & NA. & N.A. & N.A: & N.A. & N.A. & N.A. \\
\hline Rolonway Exec & 19 & 86 & N.A. & N.A. & N.A. & NA. & N.A. & NA & N.A. & N.A. & N.A. & N.A. \\
\hline Scorpion 133 & N.A. & N.A. & N.A. & N.A. & N.A. & N.A. & N.A. & N.A. & N.A. & N.A. & N.A. & N.A. \\
\hline
\end{tabular}

N.A. = information not avellabie when wbie pregered. 
[3.1] General Aviation Activity and Avionics Survey Annual Summaxy Beport. 1986 Data. U.S. Department of Transportation, Federal Aviation Administration, Office of Management Systems, Washington, DC, December 1987.

[3.2] General Aviation Activity and Avionics Sunyey Annual Summany Repoort 1987 Data U.S. Department of Transportation, Federal Aviation Administration, Ottice of Management Systems, Washington, DC, November 1988.

[3.3] General Aviation Activity and Avionics Survey, Calendar Year 1988. U.S. Department of Transportation, Federal Aviation Administration, Office of Management Systems, Washington, DC, (No Date).

[3.4] General Aviation Activity and Avionics Sunvey, Calendar Year 1989 U.S. Department of Transportation, Federal Aviation Administration, Office of Management Systems, Washington, DC, (No Date).

[3.5] General Aviation Activity and Avionics Survey, Calendar Year 1990. U.S. Department of Transportation, Federal Aviation Aưministration, Office of Management Systems, Washington, DC, (No Date).

[3.6] General Aviation Activity and Avionics Survey, Calendar Year 1991 U.S. Department of Transportation, Federal Aviation Administration, Office of Aviation Policy, Plans and Management Analysis, Washington, DC, (No Date).

[3.7] General Aviation Activity and Avionics Survev. Calendar Year 1992. U.S. Department of Transportation, Federal Aviation Administration, Office of Aviation Policy, Plans and Management Analysis, Washington, DC, (No Date).

[3.8] General Aviation Activity and Avionics Survey, Calendar Year 1993. U.S. Department of Transportation, Federal Aviation Administration, Ottice of Aviation Policy, Plans and Management Analysis, Washington, DC, (No Date).

[3.9] "Certification and Operations: Domestic, Flag, and Supplemental Air Carriers and Commercial Operators of Large Aircraft", Iitle 14 Aeronautics and Space. Code of Federal Requlations. Chapter 1-Federal Aviation Administration. Department of Transportation. Subchapter G-Air Carriers, Air Travel Clubs, and Operators for Compensation or Hire: Certification and Operations. Pant 121 (14CFB 1211, U.S. National Archives and Record Administration, Washington, DC, January 1, 1994.

[3.10] "Certification and Operations of Scheduled Air Carriers with Helicopters", Intle 14 Aeronautics and Space, Code of Federal Requlations. Chapter L-Federal Aviation Administration. Department of Transportation Subchapter G-Air Carriers. Air Travel Clubs, and Operators for Compensation or Hire: Certification and Operations. Part 122114 CFB 127), U.S. National Archives and Record Administration, Washington, DC, January 1,1994.

[3.11] "Air Traffic and General Operating and Flight Rules", Irte 14 Aemanutics and Space. Code of Federal Reoulations, Chapter L-Federal Aviation Administration. Department of Transpontation. Subchapter F. Air Traffic and General Oeeratino Rules. Part 91 (14 CFR 911, U.S. National Archives and Record Administration, Washington, DC, January 1, 1994.

[3.12] "Uttralight Vehicles", Intle 14 Aeronautics and Space, Code of Federal Requlations, Chapter 上Federal Aviation Administration. Department of Transportation. Subchapter $F$. Air Traftic and General Operating Bules. Part 103 (14 CER 103) U.S. National Archives and Record Administration, Washington, DC, January 1, 1994. 
[3.13] "Parachute Jumping", Iitle 14 Aeronautics and Space, Code of Federal Bequlations, Chapter L. Eederal Aviation Administration. Department of Transportation. Subchapter F. Air Traffic and General Ooeratino Rules. Part 105 (14 CFR 105) U.S. National Archives and Record Administration, Washington, DC, January 1, 1994.

[3.14] "Certification and Operations: Airplanes Having a Seating Capacity of 20 or More Passengers or a Maximum Payload of 6,000 Pounds or More". Iitle 14 Aeronautics and Space. Code of Federal Requlations. Chapter l-Federal Aviation Administration. Department of Transportation. Subchapter G-Air Camiers, Air Iravel Clubs, and Operators tor Compensation or Hire: Certification and Operations. Part 125 (14 CER 125), U.S. National Archives and Record Administration, Washington, DC, January 1, 1994.

[3.15] "Rotorcraft Extemat-Load Operations", Title 14 Aeronautics and Space, Code of Federal Bequlations. Chapter L-Federal Aviation Administration. Department of Transportation. Subchapter G-Air Carriers. Air Travel Clubs, and Operators for Comoensation or Hire: Certification and Operations. Part 133 (14 CFB 133), U.S. National Archives and Record Administration, Washington, DC, January 1, 1994.

[3.16] "Air Taxi Operators and Commercial Operators", Iitle 14 Aeronautics and Soace Code of Eederal Requlations. Chapter LFederal Aviation Administration. Department of Transportation. Subchapter G-Air Carriers, Air Travel Clubs, and Operators for Compensation or Hire: Certification and Operations. Par 135 (14 CFA 135), U.S. National Archives and Record Administration, Washington, DC, January 1, 1994.

[3.17] "Agricultural Aircraft Operations", Iitle 14 Aeronautics and Space. Code of Federal Bequlations. Chapter L-Federal Aviation Administration. Department of Transportation. Subchapter G-Air Carriers Air Iravel Clubs, and Operators for Compensation or Hire: Certification and Operations. Part 137 (14 CER 137) U.S. National Archives and Record Administration, Washington, DC, January 1, 1994.

[3.18] "Mperations: Foreign Air Carriers and Foreign Operators of U.S.-Registered Aircratt Engaged in Common Carriage", Iitle 14 Aeronautics and Soace, Code of Federal Bequlations. Chapter 1Eederal Aviation Administration. Department of Transoortation. Subchaoter G-Air Carriers. Air Iravel Clubs, and Operators for Compensation or Hire: Centification and Operations. Part 129 (14 CFR 129), U.S. National Archives and Record Administration, Washington, DC, January 1, 1994.

[3.19] DOE Standard Accident Analysis for Aircratt Crash into Hazardous Facilities, DOE-STD-3014-96, U.S. Department of Energy, Washington, DC, July 1996.

[3.20] Annual Review of Aircraft Accident Data. U.S. General Aviation Calendar Year 1986. NTSB/ABG. 88/01 U.S. National Transportation Safety Board, Bureau of Safety Programs, Washington, DC, October 25, 1988.

[3.21] Annual Review of Aircratt Accident Data, U.S. General Aviation Calendar Year 1987. NTSB/ARG89/01 U.S. National Transportation Safety Board, Bureau of Safety Programs, Washington, DC, December 12, 1989.

[3.22] Annual Review of Aircratt Accident Data, U.S. General Aviation Calendar Year 1988, NTSB/ABG. 91/01. U.S. National Transportation Safety Board, Office of Research \& Engineering, Washington, DC, March 27, 1991.

[3.23] Annual Review of Aircratt Accident Data. U.S. General Aviation Calendar Year 1989, NTSB/ARG93/01 U.S. National Transportation Safety Board, Office of Research \& Engineering, Washington, DC, (No Date). 

[3.24] Annual Beview of Aircratt Accident Data, U.S. General Aviation Calendar Year 1990. NTSB/ARG. 93/02. U. S. National Transportation Satety Board, Office of Research \& Engineering, Washington, DC, December 17, 1993.

[3.25] Annual Beview of Aircraft Accident Data, U.S. General Aviation Calendar Year 1991.NTSB/ARG94/01. U.S. National Transportation Safefy Board, Office of Research \& Engineering, Washington, DC, April 4, 1994.

[3.26] Annual Beview of Aircraft Accident Data, U.S. General Aviation Calendar Year 1992, NTSB/ARG$94 / 02$, U.S. National Transportation Safety Board, Office of Research \& Engineering, Washington, DC, June 15, 1994.

[3.27] Annual Beview of Aircratt Accident Data. U.S. General Aviation Calendar Year 1993. NTSB/ARG. 95/01 U.S. National Transportation Safety Board, Office of Research \& Engineering, Washington, DC, May 12, 1995.

[3.28] ACRAM Modeling Technical Support Document. LA-UR-96-2460/TSA-11-95-R12 C. Bolig, D. Sanzo, D. Stack, R. Mensing, and R. Glasser, Los Alamos National Laboratory, Los Alamos, NM, July 1996.

[3.29] Modem Commercial Aircratt William Green, Gordon Swanborough, and John Mowinski, Portland House, New York, NY, 1987.

[3.30] The Aviator's Source Book, Barbara B. Buchholz, editor, St. Martin's Press, New York, NY, 1982.

[3.31] AField Guide te Airplanes of North America. 2nd Edition M.R. Montgomery, Gerald Foster, Houghton Mifflin Co., New York, NY, 1984.

[3.32] Winos of Cessna, Model 120 to the Citation Ill Eoward H. Phillips, Flying Books, Eagan, MN, 1986.

[3.33] Beecheratt. Stagoenwing to Starship Edward H. Phillips, Flying Books, Eagan, MN, 1987.

[3.34] Multivariate Density Estimation. Theory. Practice and Visualization, David W. Scott, John Wiley \& Sons, Inc., New York, NY, 1992.

[3.35] The Intemational Directory of Civil Aircraft. 1995/96, Gerard Frawley and Jim Thom, Aerospace Publications Pty Ltd, Weston Creek, Australia, 1995. 
APPENDIX A3.1 GENERAL AVIATION IN FLIGHT CRASH LOCATIONS

\begin{tabular}{|c|c|c|c|}
\hline & NTSB number & $\begin{array}{l}\text { Crash } \\
\text { Latitude }\end{array}$ & $\begin{array}{c}\text { Crash } \\
\text { Longitude }\end{array}$ \\
\hline & ATL83AA184 & 28.6333 & 80.9167 \\
\hline 2 & ATL83AA235 & 31.0333 & 87.5167 \\
\hline & ATL83AA305 & 36.2000 & 80.7833 \\
\hline 4 & ATL83FA128 & 36.6667 & 87.3333 \\
\hline 5 & ATL83FA140 & 35.7667 & 84.9667 \\
\hline$\underline{6}$ & ATL83FA144 & 35.8333 & 87.4333 \\
\hline 7 & ATL83FA155 & 34.8333 & 85.0833 \\
\hline 8 & ATL83FA198 & 32.3167 & 85.8833 \\
\hline 9 & ATL83FA262 & 31.1833 & 90.4667 \\
\hline 10 & ATLBSFA263 & 33.5000 & 85.1500 \\
\hline 11 & ATL $83 F 1 G 06$ & 38.9000 & 79.2167 \\
\hline 12 & ATLQAFAO28 & 35.9833 & 86.7333 \\
\hline 13 & ATL BAFA033 & 31.2667 & 81.4667 \\
\hline 14 & ATL $84 F A 054$ & 35.6667 & 88.3000 \\
\hline 15 & ATLQAFA058 & 35.4667 & 83.1333 \\
\hline 16 & ATLEAFA075 & 34.7167 & 82.8333 \\
\hline 17 & ATLBAFA090 & 34.0000 & 78.9333 \\
\hline 18 & ATL84FAO92 & 37.9000 & 78.4833 \\
\hline 19 & ATL84FA111 & 31.3333 & 87.4333 \\
\hline 20 & ATLBAFA116 & 38.7667 & 77.5333 \\
\hline 21 & ATL8AFA129 & 34.1000 & 84.4333 \\
\hline 22 & ATL84FA133 & 33.8333 & 84.3000 \\
\hline$\overline{23}$ & ATL84FA158 & 37.0333 & 82.3000 \\
\hline 24 & ATLQAFA168 & 33.8333 & 84.8333 \\
\hline 25 & ATL8AFA169 & 37.5000 & 87.2167 \\
\hline 26 & ATL84FA184 & 33.5833 & 87.8000 \\
\hline 27 & ATL84FA187 & 35.2333 & 80.9500 \\
\hline 28 & ATL84FA190 & 34.0833 & 84.6667 \\
\hline 29 & ATL84FA193 & 34.5500 & 88.6000 \\
\hline 30 & ATL84FA203 & 34.1167 & 84.2000 \\
\hline 31 & ATLOAFA216 & 32.3000 & 82.5667 \\
\hline 32 & ATL84FA225 & 33.5000 & 90.2500 \\
\hline 33 & ATL84FA241 & 30.4167 & 88.7500 \\
\hline 34 & ATL QAFA248 & 34.0167 & 84.6167 \\
\hline 35 & ATL84FA268 & 33.9000 & 80.6500 \\
\hline 36 & ATL84FA292 & 32.8167 & 85.2500 \\
\hline 37 & ATLBAFA298 & 33.9333 & 82.3833 \\
\hline 38 & ATL84FLT10 & 35.7667 & 84.3500 \\
\hline 39 & ATL84MAOG3 & 35.3833 & 87.3000 \\
\hline 40 & ATL8AMA101 & 38.0167 & 78.5500 \\
\hline 41 & ATLBAMA208 & 34.3000 & 84.3833 \\
\hline 42 & ATL85FA011 & 36.6500 & 80.7000 \\
\hline 43 & ATLBSFA020 & 32.2333 & 90.9333 \\
\hline 44 & ATL85FA039 & 36.0833 & 84.4500 \\
\hline 45 & ATL85FA041 & 33.4500 & 85.8167 \\
\hline 46 & ATL85FA043 & 34.4167 & 83.6667 \\
\hline 47 & ATL $85 F A 050$ & 35.9667 & 78.3667 \\
\hline
\end{tabular}

APPENDIX 3. GENERAL AVIATION

\begin{tabular}{|c|c|c|c|}
\hline & nber & $\begin{array}{c}\text { Crash } \\
\text { Latitude }\end{array}$ & $\begin{array}{c}\text { Crash } \\
\text { Longitude }\end{array}$ \\
\hline 48 & ATL85FA070 & 39.8667 & \\
\hline 49 & ATL85FA143 & 32.4833 & 88.8000 \\
\hline 50 & ATLB5FA146 & 34.5167 & 90.4167 \\
\hline 51 & ATL85FA147 & 35.0167 & 80.6167 \\
\hline 52 & ATL85FA165 & 37.0000 & 83.0000 \\
\hline 53 & ATL85FA191 & 34.5833 & 83.7667 \\
\hline 54 & ATL85FA198 & 31.2167 & 85.4833 \\
\hline 55 & ATL85FA221 & 33.9667 & 84.6500 \\
\hline 56 & ATLB5FA237 & 38.7333 & 84.6667 \\
\hline 57 & ATLB5FA242 & 34.1500 & 82.3500 \\
\hline 58 & ATL85FA252 & 32.6333 & 84.9833 \\
\hline 59 & ATL85FA256 & 35.2167 & 82.5667 \\
\hline 60 & ATL85FA261 & 35.7000 & 81.2167 \\
\hline 61 & ATL85FA266 & 34.2167 & 81.0833 \\
\hline 62 & ATL85FA272 & 33.1667 & \\
\hline 63 & $5 F A 283$ & 38.3667 & 82.5500 \\
\hline 64 & 5 FA285 & 34.6833 & 85.2833 \\
\hline 65 & $\mathrm{MGO3}$ & 36.0667 & 78.8000 \\
\hline 66 & A275 & 34.3667 & 86.7667 \\
\hline 67 & ATI & 35.9333 & 79.1000 \\
\hline 68 & 4002 & 38.4667 & 82.0333 \\
\hline 69 & $\overline{A T}$ & 34.1333 & 87.6667 \\
\hline 70 & A034 & 33.9833 & 83.9667 \\
\hline 71 & $\overline{\mathrm{AT}}$ & 33.5667 & 86.0500 \\
\hline 72 & AT & 33.8333 & 86.1667 \\
\hline 73 & $A 062$ & 34.7833 & 83.9500 \\
\hline 74 & & 33. & 85.8000 \\
\hline 75 & FA064 & 36.5667 & 77.1833 \\
\hline 76 & FA066 & 35.7 & 80.3000 \\
\hline 77 & A074 & 40.0500 & 77.4667 \\
\hline 78 & A079 & 36.1500 & 81.1167 \\
\hline 79 & & 36 & 167 \\
\hline 80 & GFA097 & 35.1167 & 90.1333 \\
\hline 81 & F102 & 33.1667 & 84.7167 \\
\hline 82 & ATI & 36.4667 & 76.9167 \\
\hline 83 & ATL & 30.7833 & 87.7000 \\
\hline 84 & & 33.9333 & 83.3167 \\
\hline 85 & A156 & 33.4333 & 87.3667 \\
\hline 86 & AT & 35.8 & 667 \\
\hline 87 & FA220 & 34.6000 & 83.2833 \\
\hline 88 & A222 & 36.7833 & \\
\hline 89 & A239 & 37.7833 & 80.3333 \\
\hline 90 & A242 & 34.4500 & \\
\hline 91 & A259 & 31.5333 & 82.0167 \\
\hline 92 & $\bar{A}$ & 36.5667 & 8081 \\
\hline 93 & 2265 & 32.2500 & 88.2500 \\
\hline 94 & ATL86FA266 & 35.7333 & 78.9167 \\
\hline
\end{tabular}

\begin{tabular}{|c|c|c|c|}
\hline & $b$ & $\begin{array}{l}\text { Crash } \\
\text { Atitudo }\end{array}$ & $\begin{array}{c}\text { Crash } \\
\text { ngitude }\end{array}$ \\
\hline 95 & ATL86FE106 & 41.0333 & 81.4667 \\
\hline 96 & ATL86FEl10 & 41.2500 & 81.9167 \\
\hline 97 & ATL86FEK02 & 40.3667 & 82.5000 \\
\hline 98 & ATL86FKG01 & 32.5000 & 83.7500 \\
\hline 99 & ATL 86FKG07 & 32.5000 & 81.7500 \\
\hline 100 & ATL86FKG08 & 34.3000 & 84.4167 \\
\hline 101 & ATL $86 F L Q 02$ & 37.1667 & 82.6333 \\
\hline 102 & ATL86FLQ05 & 38.1667 & \\
\hline 103 & ATL86LA189 & 41.6167 & 81.3667 \\
\hline 104 & ATL86MA011 & 33.0000 & 83.7667 \\
\hline 105 & ATLB6MA080 & 35.0500 & 84.3000 \\
\hline 106 & ATLB6MA087 & 31.4167 & 86.4500 \\
\hline 107 & ATLB6MA114A & 42.2167 & 71.4167 \\
\hline 108 & ATL 86 MA1148 & 42.2167 & 71.4187 \\
\hline 109 & ATL $87 D E G 03$ & 39.3333 & 82.4333 \\
\hline 110 & ATL87DEG04 & 39.1833 & 83.53 \\
\hline 111 & ATLBTDEIO1 & 41.0000 & 81.7667 \\
\hline 112 & ATLO7DE10B & 41.3000 & 81.7500 \\
\hline 113 & ATL87DMG02 & 36.4667 & 77.6667 \\
\hline 114 & ATLOTFAOO1A & 35.4500 & 7.9667 \\
\hline 115 & ATLETFAO01B & 35.4500 & 77.9667 \\
\hline 116 & IFAOOA & 32.1667 & 81.3667 \\
\hline 117 & FA010 & 36.7833 & 87.4333 \\
\hline 118 & ATLBTFA016 & 32.8333 & 83.5667 \\
\hline 119 & $A 030$ & 34.9667 & 78.3500 \\
\hline 120 & ATL87FAO45 & 35.4333 & 82.5333 \\
\hline 121 & ATL87FA052 & 31.6000 & 85.233 \\
\hline 122 & $\mathrm{A071}$ & & 84.0167 \\
\hline 123 & 7FA074 & 35.9500 & 85.0833 \\
\hline 124 & FA122 & 32.7500 & 84.4167 \\
\hline 125 & ATL87FA123 & 34.2667 & 77.9000 \\
\hline 126 & ATL87FA136 & 35.0500 & 89.6833 \\
\hline 127 & 12 & & 84.7 \\
\hline 128 & FA147 & 35.0000 & 82.0000 \\
\hline 129 & A160 & 36.3833 & 87.0167 \\
\hline 130 & ATL87FA173 & 26.2833 & 80.6333 \\
\hline 131 & FA174 & 35.4500 & 82.5167 \\
\hline 132 & ATL87FA194 & 33.8167 & 78.7167 \\
\hline 133 & A230 & 31.4167 & 82.1000 \\
\hline 134 & FA243 & & 81.0833 \\
\hline 135 & A257 & 32.0833 & 80.4333 \\
\hline 136 & FA262 & 32.4500 & 87.9500 \\
\hline 137 & FA268 & 35.7500 & 75.5000 \\
\hline 138 & ATI & & 84.58 \\
\hline 139 & A035 & 36.4000 & 85.0000 \\
\hline 140 & 1057 & 35.0167 & 77.4000 \\
\hline 14 & ATL88DLT01 & 36.1667 & 87.133 \\
\hline
\end{tabular}

A3-1 


\section{APPENDIX A3.1 GENERAL AVIATION IN FLIGHT CRASH LOCATIONS}

\begin{tabular}{|c|c|c|c|}
\hline & NTSB number & $\begin{array}{l}\text { Crash } \\
\text { Letitude }\end{array}$ & $\begin{array}{c}\text { Crash } \\
\text { Longitude }\end{array}$ \\
\hline 142 & ATL $880 \mathrm{MGO3}$ & 35.0000 & 80.0000 \\
\hline 143 & ATL $88 F A 007$ & 35.0167 & 90.0000 \\
\hline 144 & ATLB8FA010 & 34.8833 & 82.3333 \\
\hline 145 & ATL88FA025 & 39.6333 & 84.5833 \\
\hline 146 & ATLB8FAO40 & 36.9833 & 86.9500 \\
\hline 147 & ATLE8FAO44 & 39.3333 & 83.0833 \\
\hline 148 & ATL8BFAO46 & 36.0000 & 84.3167 \\
\hline 149 & ATLB8FAOS9 & 32.2333 & 80.7167 \\
\hline 150 & ATLB8FAOCOO & 31.0000 & 83.0000 \\
\hline 151 & ATLBQFAO66 & 35.1833 & 80.5333 \\
\hline 152 & ATLBBFA067 & 35.7667 & 84.9667 \\
\hline 153 & ATLE8FAOB1 & 35.0000 & 82.4333 \\
\hline 154 & ATLBBFACB2 & 33.3667 & 81.9667 \\
\hline 155 & ATLBBFA085 & 35.9667 & 87.8667 \\
\hline 156 & ATLBBFA088 & 41.1167 & 80.6167 \\
\hline 157 & ATLBBFA104 & 36.7000 & 85.3333 \\
\hline 158 & ATLBBFA109 & 35.0000 & 83.0000 \\
\hline 159 & ATL B8FA123 & 35.8500 & 83.5167 \\
\hline 160 & ATLBBFA136 & 36.6167 & 83.6167 \\
\hline 161 & ATLBBFA137 & 34.2500 & 78.9167 \\
\hline 162 & ATL88FA155 & 35.8500 & 84.0667 \\
\hline 163 & ATLBBFA191 & 35.0000 & 84.0000 \\
\hline 164 & ATLBBFA200 & 32.2667 & 87.9500 \\
\hline 165 & ATL8BFA211 & 34.1333 & 81.9667 \\
\hline 166 & ATL8BFA220 & 33.0333 & 85.1667 \\
\hline 167 & ATLBBFA230 & 34.7333 & 80.3500 \\
\hline 168 & ATLBBFA233 & 35.0000 & 79.0000 \\
\hline 169 & ATL8BFA254 & 36.1667 & 84.4000 \\
\hline 170 & ATLBBFA256 & 33.4167 & 87.2167 \\
\hline 171 & ATL88MA089 & 39.8333 & 83.8333 \\
\hline 172 & ATL88MA184 & 40.8833 & 74.2000 \\
\hline 173 & ATL89DMGO1 & 35.4000 & 80.1500 \\
\hline 174 & ATLBOFAOOA & 36.4000 & 84.1333 \\
\hline 175 & ATLBOFAODB & 32.9167 & 85.9500 \\
\hline 176 & ATLEOFA015 & 36.4333 & 84.0667 \\
\hline 177 & ATLBOFA019 & 33.8000 & 84.6333 \\
\hline 178 & ATLB9FA035 & 36.1667 & 83.1667 \\
\hline 179 & ATLEOFA047 & 36.0000 & 82.0000 \\
\hline 180 & ATLO9MAO23 & 36.0000 & 86.0000 \\
\hline 181 & ATL89MA070. & 39.5500 & 83.9167 \\
\hline 182 & BFO84FA001 & 38.4667 & 75.1833 \\
\hline 183 & BF085FA004 & 39.0000 & 79.0000 \\
\hline 184 & BF085FA006 & 39.1667 & 77.5667 \\
\hline 185 & BF085FA009 & 38.7167 & 77.5167 \\
\hline 186 & BF085FA023 & 39.3833 & 77.4333 \\
\hline 187 & BFO85FA048 & 38.6833 & 77.3167 \\
\hline 188 & BFO $55 F A 049$ & 38.0333 & 77.3667 \\
\hline
\end{tabular}

\begin{tabular}{|c|c|c|c|}
\hline & NTSB number & $\begin{array}{c}\text { Crash } \\
\text { Latitude }\end{array}$ & $\begin{array}{c}\text { Crash } \\
\text { Longitude }\end{array}$ \\
\hline 189 & BFO $85 F A 051$ & 38.9667 & 76.9167 \\
\hline 190 & BF085FA061 & 38.4500 & 76.7333 \\
\hline 191 & BFOB5FAOS2 & 39.6333 & 79.0500 \\
\hline 192 & BF085FA068 & 38.5167 & 77.9667 \\
\hline 193 & BFO $85 F A 070$ & 38.0333 & 78.8667 \\
\hline 194 & BF085FA077 & 38.7333 & 76.9167 \\
\hline 195 & BF086FA011 & 39.4667 & 77.0167 \\
\hline 196 & BF086FA027 & 38.9667 & 76.9167 \\
\hline 197 & BFOB6FA036 & 38.9333 & 76.7667 \\
\hline 198 & BFOBGFA037 & 38.9500 & 76.5667 \\
\hline 199 & BFOBGFAO42 & 39.3167 & 76.3833 \\
\hline 200 & BFO $86 F A 047$ & 39.4167 & 77.1167 \\
\hline 201 & BFOB6FIA02 & 38.5833 & 78.4000 \\
\hline 202 & BFO 66 FIDO1 & 38.1500 & 78.1667 \\
\hline 203 & BFOBGFID03 & 38.0833 & 78.9500 \\
\hline 204 & BFOB7FAO22 & 39.5500 & 77.5833 \\
\hline 205 & BFOB7FA037 & 39.7167 & 75.8000 \\
\hline 206 & BFOB7FAO46 & 36.8167 & 82.0667 \\
\hline 207 & BF087FA057 & 38.8667 & 77.0333 \\
\hline 208 & BFOBQDIAOI & 36.6333 & 80.0167 \\
\hline 200 & BFO88DIDOA & 36.9833 & 77.0000 \\
\hline 210 & BF088FA007 & 38.7167 & 77.7000 \\
\hline 211 & BFOB8FA008 & 37.3333 & 79.0000 \\
\hline 212 & BFOB8FA009 & 36.8000 & 78.1500 \\
\hline 213 & BFOBBFA011 & 39.6500 & 77.2500 \\
\hline 214 & BFOB8FA024 & 38.9000 & 80.0667 \\
\hline 215 & BFO88FA025 & 38.9333 & 76.7667 \\
\hline 216 & BFOB8FA026 & 38.5000 & 77.3167 \\
\hline 217 & BFO $88 F A 068$ & 38.9833 & 76.3500 \\
\hline 218 & BFOB8FIDO3 & 36.7833 & 76.4500 \\
\hline 219 & CHIBSFA076 & 41.8000 & 88.0667 \\
\hline 220 & CHI83FA090 & 45.8167 & 92.3667 \\
\hline 221 & CHI83FA135 & 40.5500 & 89.2500 \\
\hline 222 & CHI83FA161 & 39.8333 & 88.3333 \\
\hline 223 & CHIO3FA213 & 40.4333 & 88.9667 \\
\hline 224 & CHI83FA334 & 41.0833 & 88.7000 \\
\hline 225 & CHIB3FA336 & 41.4667 & 87.0167 \\
\hline 226 & CHI83FA346 & 41.9500 & 88.4833 \\
\hline 227 & CHI83FA350 & 44.0500 & 88.7167 \\
\hline 228 & CHI83FA366 & 40.4167 & 80.8333 \\
\hline 229 & CHI83FA402 & 43.9500 & 86.0000 \\
\hline 230 & CHI83FA407 & 45.2500 & 93.3333 \\
\hline 231 & CHI83FA437 & 45.6833 & 92.9500 \\
\hline 232 & CHIB4FA028 & 40.8333 & 85.1667 \\
\hline 233 & CHI84FA037 & 42.1333 & 86.1167 \\
\hline 234 & CHIB4FA054 & 40.2333 & 84.2000 \\
\hline 235 & CHI84FA063 & 40.3000 & 84.7500 \\
\hline
\end{tabular}

\begin{tabular}{|c|c|c|c|}
\hline & NTSB number & $\begin{array}{l}\text { Crash } \\
\text { Lotiuds }\end{array}$ & $\begin{array}{c}\text { Crash } \\
\text { Longitude }\end{array}$ \\
\hline 236 & CHI84FA121 & 42.1167 & 87.8833 \\
\hline 237 & CHI84FA250A & 48.2000 & 96.6667 \\
\hline 238 & CHIB4FA289 & 42.4833 & 86.0333 \\
\hline 239 & CHIBAFA313 & 48.3333 & 96.5833 \\
\hline 240 & CHIBAFA 343 & 45.2500 & 83.4333 \\
\hline 241 & CHIB4FA352 & 41.6333 & 82.8167 \\
\hline 242 & CHIB4FA402 & 42.1167 & 87.9000 \\
\hline 243 & CHIB4MA249 & 39.1000 & 84.4167 \\
\hline 244 & CHI85FAO20 & 45.5000 & 91.4167 \\
\hline 245 & CHIB5FA036 & 43.8000 & 89.0333 \\
\hline 246 & CHIlo5FA053 & 41.0000 & 87.0000 \\
\hline 247 & CHIBSFA070 & 42.6667 & 03.5000 \\
\hline 248 & CHIB5FA104 & 38.7833 & 83.0167 \\
\hline 249 & CHI85FA156 & 43.6833 & 85.6500 \\
\hline 250 & CHIB5FA158 & 41.2167 & 81.3000 \\
\hline 251 & CHIBSFA211 & 38.4667 & 89.6667 \\
\hline 252 & CHIB5FA213 & 39.7500 & 86.1167 \\
\hline 253 & CHIB5FA292 & 38.7167 & 88.1833 \\
\hline 254 & CHIBSFA301 & 41.4167 & 87.3833 \\
\hline 255 & CHI85FA316 & 40.2000 & 83.2333 \\
\hline 256 & CHI85FA351 & 42.4500 & 83.6833 \\
\hline 257 & CHIBSFA370 & 41.6000 & 88.0833 \\
\hline 258 & CHIBSFEC01 & 41.3500 & 83.4167 \\
\hline 259 & CHI85FEl02 & 41.1000 & 81.2000 \\
\hline 260 & CHI85FEK01 & 41.0000 & 84.0000 \\
\hline 261 & CHI85FEKO2 & 40.8000 & 84.7000 \\
\hline 262 & CHIQ5FEP02 & 42.0000 & 89.0000 \\
\hline 263 & CHI85FEPO4 & 42.9667 & 88.8000 \\
\hline 264 & CHIBSFERO1 & 44.8333 & 93.0033 \\
\hline 265 & CHI85FER02 & 45.7500 & 93.8333 \\
\hline 266 & CHIBSFEV03 & 40.0500 & 89.6500 \\
\hline 267 & CHI86FA007 & 44.2833 & 85.4167 \\
\hline 268 & CHIB6FA031 & 40.9833 & 85.2000 \\
\hline 269 & CHIBGFAO46 & 41.9500 & 84.2000 \\
\hline 270 & CHIB6FAO68 & 41.9167 & 88.3000 \\
\hline 271 & CHIB6FAO77 & 38.6833 & 87.1167 \\
\hline 272 & CHIBGFAOSB & 41.7667 & 88.2667 \\
\hline 273 & CHIB6FA100A & 41.9000 & 88.2333 \\
\hline 274 & CH186FA102 & 44.6333 & 86.2167 \\
\hline 275 & CHIB6FA120 & 41.8667 & 84.4833 \\
\hline 276 & CHIB6FA137 & 30.0000 & 83.0000 \\
\hline 277 & CHI86FA141 & 41.6333 & 85.0833 \\
\hline 278 & CHIB6FA146 & 47.9333 & 97.0000 \\
\hline 279 & CHI86FA170 & 42.1333 & 86.4333 \\
\hline 280 & CHIB6FA171 & 45.2167 & 88.0667 \\
\hline 281 & CHIB6FA174 & 43.5500 & 87.9667 \\
\hline 282 & CHIBGFA183 & 41.6833 & 88.1167 \\
\hline
\end{tabular}


APPENDIX A3.1 GENERAL AVIATION IN FLIGHT CRASH LOCATIONS

\begin{tabular}{|c|c|c|c|}
\hline & NTSB number & $\begin{array}{l}\text { Crash } \\
\text { Lotitude }\end{array}$ & $\begin{array}{c}\text { Crash } \\
\text { Longitude }\end{array}$ \\
\hline 283 & CHI86FA194 & 43.9833 & 88.5500 \\
\hline 284 & CHI86FA198 & 43.1667 & 84.6667 \\
\hline 285 & CH186FA233 & 41.7833 & 87.7333 \\
\hline 286 & CHI86FEE05 & 41.7500 & 88.2000 \\
\hline 287 & CHI86FEM10 & 42.2500 & 85.0667 \\
\hline 288 & CHIB6FEM12 & 44.5333 & 84.7000 \\
\hline 289 & CHIB6FEM14 & 41.8000 & 85.5833 \\
\hline 290 & $\mathrm{CH} 186 \mathrm{FEPO2}$ & 44.3500 & 89.8333 \\
\hline 291 & CHI86FER03 & 44.6167 & 93.2167 \\
\hline 292 & CHIB6FER06 & 46.5000 & 94.4833 \\
\hline 293 & CHIBSFETO1 & 41.6333 & 86.5833 \\
\hline 294 & CHIB6FEX02 & 38.0500 & 87.6167 \\
\hline 295 & CHI86FEX07 & 38.7667 & 87.5333 \\
\hline 296 & CHI86FEX09 & 40.6333 & 85.6167 \\
\hline 297 & CHI86FEX10 & 40.5000 & 86.0833 \\
\hline 298 & CHIB6MA071 & 43.3333 & 93.2500 \\
\hline 299 & CHIB7DCAOI & 38.5667 & 80.1500 \\
\hline 300 & $\mathrm{CH} 187 \mathrm{DECO1}$ & 42.5500 & 83.1833 \\
\hline 301 & CHI87DECO4 & 42.6500 & 83.4167 \\
\hline 302 & CHI870EE03 & 41.7167 & 88.2667 \\
\hline 303 & CHIB70EE08 & 41.4000 & 87.8500 \\
\hline 304 & CHIB7DEE09 & 41.0500 & 87.8000 \\
\hline 305 & CHI87DEE10 & 41.6167 & 88.2667 \\
\hline 306 & CHI87DEM01 & 43.0333 & 86.2000 \\
\hline 307 & CHI87DEMO5 & 42.1500 & 85.8333 \\
\hline 308 & CHI87DEP06 & 44.0500 & 89.0833 \\
\hline 309 & CH1870EP10 & 43.9667 & 90.4833 \\
\hline 310 & CHI87DER05 & 46.3000 & 95.5000 \\
\hline 311 & CHIB7DERO9 & 45.5000 & 93.2000 \\
\hline 312 & CHI87DETO7 & 41.7000 & 86.6667 \\
\hline 313 & CHI87DEXO4 & 39.8333 & 85.1667 \\
\hline 314 & CHI87DEX07 & 39.9000 & 85.9167 \\
\hline 315 & CHI87FA010 & 41.7167 & 87.7500 \\
\hline 316 & CHIB7FA012 & 48.5667 & 93.4000 \\
\hline 317 & CHIB7FAO4O & 40.6687 & 89.6833 \\
\hline 318 & CHIB7FA058 & 41.4167 & 83.1667 \\
\hline 319 & CHIB7FAOG9 & 41.7833 & 87.7500 \\
\hline 320 & CHI87FA085 & 39.9500 & 91.2000 \\
\hline 321 & CHI87FA104 & 41.4833 & 87.8500 \\
\hline 322 & CHI87FA129 & 42.2500 & 85.5333 \\
\hline 323 & CHI87FA140 & 43.1167 & 89.5333 \\
\hline 324 & CHI87FA149 & 43.8667 & 90.4833 \\
\hline 325 & CHI88DCAO1 & 38.5667 & 90.1667 \\
\hline 326 & CHI8BDEE06 & 41.0000 & 87.9000 \\
\hline 327 & CHI88DEMO7 & 42.2667 & 85.2333 \\
\hline 328 & CHI88DEP02 & 43.4167 & 88.2833 \\
\hline 329 & CHI88DEP11 & 44.3333 & 89.3667 \\
\hline
\end{tabular}

\begin{tabular}{|c|c|c|c|}
\hline & NTSB number & $\begin{array}{l}\text { Crash } \\
\text { Latitude }\end{array}$ & $\begin{array}{c}\text { Crash } \\
\text { Longitude }\end{array}$ \\
\hline 330 & CHI88DERO2 & 48.0000 & 95.3333 \\
\hline 331 & CHI8BDERO4 & 45.1667 & 96.0833 \\
\hline 332 & CHI8BDERO7 & 48.5333 & 93.1500 \\
\hline 333 & CHI88DET01 & 41.1333 & 500 \\
\hline 334 & CHI88DEX01 & 38.0000 & 86.0000 \\
\hline 335 & CHI8BOEX02 & 39.5333 & 86.8333 \\
\hline 336 & CHI88DEX07 & 39.1333 & 86.6333 \\
\hline 337 & CHI88DEX08 & 30.3333 & 333 \\
\hline 338 & CHI88FAOOB & 44.7000 & 500 \\
\hline 339 & CHI88FA075 & & 667 \\
\hline 340 & FA079 & & 000 \\
\hline 341 & DCA83AAO17 & & 3000 \\
\hline 342 & DCA83AAD25 & & 67 \\
\hline 343 & DCAB7MAO20B & & \\
\hline 344 & DCA & & 33 \\
\hline 345 & $\overline{\mathrm{DC}}$ & & \\
\hline 346 & & & \\
\hline 347 & & & 33 \\
\hline 348 & 056 & & 67 \\
\hline 349 & 062 & & 500 \\
\hline 350 & 4070 & 39 & 3167 \\
\hline 351 & 4087 & 33 & 3833 \\
\hline 352 & A108 & 67 & 3833 \\
\hline 353 & $\mathbf{A 1 2 3}$ & 67 & 2167 \\
\hline 354 & 131 & 00 & 667 \\
\hline 355 & FA150 & 44. & 5167 \\
\hline 356 & FA203 & 00 & 4500 \\
\hline 357 & 214 & & 3333 \\
\hline 358 & A221 & & 667 \\
\hline 359 & A222 & & 8000 \\
\hline 360 & & & 1500 \\
\hline 361 & 3FTGO1 & & 96.9667 \\
\hline 362 & 3FTIO3 & 667 & 112.7500 \\
\hline 363 & $A 039$ & 000 & 110.7333 \\
\hline 364 & & & 3833 \\
\hline 365 & FA08B & & 500 \\
\hline 366 & AFA137 & & 100.8500 \\
\hline 367 & 4FA144 & & 105.6667 \\
\hline 368 & 4FA155 & 333 & 105.4167 \\
\hline 369 & 4FA188 & 39.3667 & 104.6667 \\
\hline 370 & 4FA199 & 39.9000 & 105.1167 \\
\hline 371 & DEN84FA202 & 35.2000 & 106.6667 \\
\hline 372 & DEN84FA211 & 40.0167 & 104.9000 \\
\hline 373 & DEN84FA219 & 40.9667 & 103.1167 \\
\hline 374 & DEN84FA223 & 36.8000 & 105.4833 \\
\hline 375 & DEN84FA242 & 41.5000 & 113.1833 \\
\hline 376 & DEN84FA274 & 48.3000 & 114.2500 \\
\hline
\end{tabular}

\begin{tabular}{|c|c|c|c|}
\hline & NTSB number & $\begin{array}{c}\text { Crash } \\
\text { Lotitude }\end{array}$ & $\begin{array}{l}\text { Crash } \\
\text { Longitude }\end{array}$ \\
\hline 377 & DENBAFA275 & 41.6000 & 109.0667 \\
\hline 378 & DEN84FA278 & 34.7833 & 106.6167 \\
\hline 379 & DEN84FA295 & 47.4500 & 111.3667 \\
\hline 380 & DEN84FA298 & 37.0833 & 110.9000 \\
\hline 381 & DENB4MA247 & 40.1167 & 104.8167 \\
\hline 382 & DEN84MA266 & 41.5000 & 110.0167 \\
\hline 383 & DENBSFAOO2 & 45.4500 & 99.4167 \\
\hline 384 & DENASFAOOA & 35.1833 & 105.8167 \\
\hline 385 & DEN85FA010 & 39.5667 & 333 \\
\hline 386 & 5FAO15 & 41.0000 & 104 \\
\hline 387 & $5 \overline{F A 017}$ & 500 & 104.8000 \\
\hline 388 & $5 F A 027$ & 000 & 112 \\
\hline 389 & DENBSFAO28 & 867 & 333 \\
\hline 390 & FA034 & 100 & 104.7333 \\
\hline 391 & FA035 & 500 & 111.716 \\
\hline 392 & FA037 & $\infty$ & 101.0167 \\
\hline 393 & FA043 & 34.9833 & 06.8500 \\
\hline 394 & DEN85FA048 & 45.8333 & 08.5500 \\
\hline 395 & DEN85FA055 & 35.1500 & 1667 \\
\hline 396 & DENBSFAOG2 & 000 & 9667 \\
\hline 397 & DEN85FA069 & 333 & 333 \\
\hline 398 & FA084 & 48.4833 & 667 \\
\hline 399 & DEN85FAO96 & 41.7833 & 500 \\
\hline 400 & FA097 & 833 & 833 \\
\hline 401 & FA103 & 500 & \\
\hline 402 & A167 & 33 & \\
\hline 403 & DEN & & 67 \\
\hline 404 & $\overline{D E}$ & & 500 \\
\hline 405 & 202 & 00 & 1333 \\
\hline 406 & $\overline{D I}$ & 500 & 104.6000 \\
\hline 407 & $\bar{D}$ & 667 & 104.8667 \\
\hline 408 & $\overline{D E}$ & 37.5167 & 112.9667 \\
\hline 409 & $\overline{D E}$ & 167 & 104.4667 \\
\hline 410 & $\overline{D E}$ & 667 & 667 \\
\hline 411 & 840 & 40.5333 & 105.1833 \\
\hline 412 & 241 & 40.7500 & 107.3167 \\
\hline 413 & 46 & 34.9333 & 104.6333 \\
\hline 414 & 204 & 35.0000 & 108.0000 \\
\hline 415 & & 45.7333 & 111.0000 \\
\hline 416 & & 500 & 101.9667 \\
\hline 417 & & 48.2167 & 101.3500 \\
\hline 418 & & 43.8333 & 9.3333 \\
\hline 419 & & 667 & 103.1500 \\
\hline 420 & & 39.7333 & 107.3167 \\
\hline 421 & & 35.1833 & 106.6500 \\
\hline 422 & & 38.7500 & 102.3167 \\
\hline 42 & DEN86FA054 & 39.0167 & 110 \\
\hline
\end{tabular}




\begin{tabular}{|c|c|c|c|}
\hline & NTSB number & $\begin{array}{l}\text { Crash } \\
\text { Latitude }\end{array}$ & $\begin{array}{c}\text { Crash } \\
\text { Longitude }\end{array}$ \\
\hline 424 & DENBGFA056 & 40.7833 & 111.9667 \\
\hline 425 & DENBGFA060 & 40.7833 & 111.9667 \\
\hline 426 & DENBGFA071 & 48.2000 & 114.6000 \\
\hline 427 & DENB6FA075 & 46.7667 & 100.7500 \\
\hline 428 & DENB6FA078 & 35.0333 & 106.6000 \\
\hline 429 & DENBGFA092 & 46.0500 & 113.3167 \\
\hline 430 & DEN86FA142 & 46.4167 & 101.8333 \\
\hline 431 & DENBGFA146 & 41.1500 & 104.7667 \\
\hline 432 & DEN 86FA147 & 38.8167 & 106.1167 \\
\hline 433 & DENB6FA150 & 33.9667 & 97.0167 \\
\hline 434 & DEN86FA160 & 38.0667 & 102.6833 \\
\hline 435 & DEN86FA163 & 43.8000 & 110.1333 \\
\hline 436 & DEN86FA165 & 35.8000 & 104.8333 \\
\hline 437 & DEN86FA170 & 33.4167 & 108.1333 \\
\hline 438 & DEN86FA175 & 44.3833 & 108.7167 \\
\hline 439 & DENB6FA1B1 & 46.3833 & 111.0500 \\
\hline 440 & DEN86FA207 & 36.4500 & 105.6667 \\
\hline 441 & DENB6FA211 & 48.4500 & 1113833 \\
\hline 442 & DEN86FA213 & 41.8167 & 107.2500 \\
\hline 443 & DEN86FA232 & 38.9500 & 111.7000 \\
\hline$\frac{774}{444}$ & DEN 8 GFA252 & 39.8667 & 104.5167 \\
\hline 445 & DENB6FTE02 & 39.1833 & 104.7667 \\
\hline 446 & DENB6FTC02 & 47.0833 & 97.4500 \\
\hline 447 & DENB6FTKO1 & 44.5000 & 98.1000 \\
\hline 448 & DENBGFTMO2 & 41.1833 & 112.0000 \\
\hline 449 & DENE6MA129 & 38.5500 & 110.2000 \\
\hline 450 & DEN87DTIO1 & 44.4167 & 108.4167 \\
\hline 451 & DEN87DTKO1 & 45.5000 & 98.3333 \\
\hline 452 & DENBTDTMO1 & 40.3667 & 112.0333 \\
\hline 453 & DENB7FACOB & 44.2500 & 107.0500 \\
\hline 454 & DENB7FAO11 & 35.3667 & 108.5833 \\
\hline 455 & DENB7FAO14 & 45.5000 & 111.2500 \\
\hline 456 & DEN87FA018 & 45.7833 & 108.5000 \\
\hline 457 & DENB7FA024 & 44.3167 & 103.5333 \\
\hline 458 & DENB7FA037 & 17.2000 & 108.9000 \\
\hline 459 & DENB7FA046 & 35.1833 & 105.4500 \\
\hline 460 & DENB7FAO48 & 37.6333 & 102.2833 \\
\hline 461 & DENB7FA050 & 44.2167 & 110.4000 \\
\hline 462 & DENB7FA061 & 38.4333 & 108.4833 \\
\hline 463 & DENB7FA064 & 32.4833 & 104.7167 \\
\hline 464 & DENBTFAOB1 & 41.1333 & 111.7500 \\
\hline 465 & DENB7FAOSO & 48.3500 & 101.8500 \\
\hline 466 & DENB7FA110 & 40.4833 & 107.5167 \\
\hline 467 & DEN87FA111 & 40.0333 & 105.2167 \\
\hline 468 & DENB7FA130 & 44.5167 & 109.0167 \\
\hline 469 & DENB7FA134 & 41.2167 & 111.9667 \\
\hline 470 & DENQ7FA147 & 47.7167 & 112.7333 \\
\hline
\end{tabular}

APPENDIX 3. GENERAL AVIATION

\begin{tabular}{|c|c|c|c|}
\hline & NTSB number & $\begin{array}{l}\text { Crash } \\
\text { Latitude }\end{array}$ & $\begin{array}{c}\text { Crash } \\
\text { Longitude }\end{array}$ \\
\hline 471 & DENB7FA159 & 33.7000 & \begin{tabular}{|l|}
108.8500 \\
\end{tabular} \\
\hline 472 & DENB7FA177 & 39.5667 & \begin{tabular}{|c|}
104.8333 \\
\end{tabular} \\
\hline 473 & DENB7FA178 & 40.7167 & 107.2833 \\
\hline 474 & DENB7FA186 & 39.6167 & 106.1667 \\
\hline 475 & DENB7FA188 & 38.3667 & 105.7500 \\
\hline 476 & DENB7FA203 & 48.3000 & 114.2500 \\
\hline 477 & DENB7FA216 & 45.7000 & 111.0500 \\
\hline 478 & DENB7FA219 & 40.4500 & 107.1000 \\
\hline 479 & DENB7FA220 & 39.5667 & 104.8500 \\
\hline 480 & DEN87FA224 & 48.1333 & 99.1500 \\
\hline 481 & DENB7FA226 & 34.7500 & \begin{tabular}{|c|}
107.8000 \\
\end{tabular} \\
\hline 482 & DEN87FA230 & 37.7000 & 113.0000 \\
\hline 483 & DENB7LA170 & 39.3000 & \begin{tabular}{|c|}
107.1500 \\
\end{tabular} \\
\hline 484 & DENB8DQAO1 & 33.1333 & 104.5833 \\
\hline 485 & DENB8DTEO2 & 40.0833 & 104.4667 \\
\hline 486 & DENB8DTKO1 & 45.3333 & 98.1000 \\
\hline 487 & DENB8DTMO1 & 40.5333 & 111.9667 \\
\hline 488 & DENBSDTM03 & 40.0000 & 113.0000 \\
\hline 489 & DENB8FA001 & 46.2833 & 105.2500 \\
\hline 490 & DENB8FA014 & 39.3000 & 105.4333 \\
\hline 491 & DEN88FA021 & 37.4000 & 107.7500 \\
\hline 492 & DENB8FA023 & 37.3833 & 113.0833 \\
\hline 493 & DEN88FA030 & 40.8000 & 113.0333 \\
\hline 494 & DENB8FA050 & 39.3000 & 104.1667 \\
\hline 495 & DENB8FA071 & 45.1667 & 109.7833 \\
\hline 496 & DENB8FA096 & 38.3333 & 112.9667 \\
\hline 497 & DENB8FA098 & 37.4333 & 113.1667 \\
\hline 498 & DENBBFA100 & 44.0333 & 103.0500 \\
\hline 499 & DENBBFA110 & 40.5167 & 111.5500 \\
\hline 500 & DENB8FA112 & 35.0000 & 106.0000 \\
\hline 501 & DENB8FA119 & 32.8500 & 103.7500 \\
\hline 502 & DENB8FA128 & 42.1833 & 110.6333 \\
\hline 503 & DENB8FA138 & 36.6333 & 105.1833 \\
\hline 504 & DENB8FA141 & 33.6000 & 105.3667 \\
\hline 505 & DENB8FA186 & 43.4167 & 97.8333 \\
\hline 506 & DENBBFA202 & 43.0000 & 110.0000 \\
\hline 507 & DENB8FA212 & 38.0000 & 112.0000 \\
\hline 508 & DEN8BGA068 & 35.2833 & 106.0500 \\
\hline 509 & DENB8GA185 & 40.0167 & 105.7333 \\
\hline 510 & DENB9FA017 & 38.5000 & 106.3167 \\
\hline 511 & DENB9FA019 & 39.1167 & 105.3333 \\
\hline 512 & DENB9FA020 & 41.0000 & 111.0000 \\
\hline 513 & DENB9FA034 & 40.0000 & 112.0000 \\
\hline 514 & DENB9FA03B & 39.2833 & 105.8667 \\
\hline 515 & DENB9FA041 & 44.0000 & 106.0000 \\
\hline 516 & DENB9FA101 & 40.0000 & 111.0000 \\
\hline 517 & FTWB3FA117 & 32.3333 & 102.5 \\
\hline
\end{tabular}

\begin{tabular}{|c|c|c|c|}
\hline & NTSB number & $\begin{array}{l}\text { Crash } \\
\text { Latitudo }\end{array}$ & $\begin{array}{c}\text { Crash } \\
\text { Longitude }\end{array}$ \\
\hline 518 & FTWO3FA142 & 27.8833 & 98.0333 \\
\hline 519 & FTMO3FA144 & 34.9333 & 92.8667 \\
\hline 520 & FTWB3FA149 & 34.0000 & 96.6500 \\
\hline 521 & FTMO3FA226 & 29.6667 & 98.2000 \\
\hline 522 & FTMO3FA245 & 36.1000 & 95.7833 \\
\hline 523 & FTMB3FA252 & 30.0333 & 90.0333 \\
\hline 524 & FTMB3FA254 & 33.2500 & 99.7667 \\
\hline 525 & FTMB3FA259 & 29.9167 & 90.2500 \\
\hline 526 & FTUB3FA276 & 29.9000 & 98.9000 \\
\hline 527 & FTMO3FA299 & 35.2500 & 97.4833 \\
\hline 528 & FTWB3FA387 & 35.3500 & 104.1667 \\
\hline 529 & FTWB3FA415 & 30.0667 & 95.1667 \\
\hline 530 & FTWO3FA416 & 34.7000 & 99.3333 \\
\hline 531 & FTWB3FA430 & 29.5167 & 92.9833 \\
\hline 532 & FTWO3FA441 & 31.1500 & 93.3333 \\
\hline 533 & FTWB4FA023 & 34.4833 & 92.1000 \\
\hline 534 & FTWBAFA032 & 31.9167 & 107.1000 \\
\hline 535 & FTWOAFA060 & 36.7333 & 92.5500 \\
\hline 536 & FTUBAFA083 & 27.4333 & 98.6833 \\
\hline 537 & FTWOAFA104 & 35.4833 & 107.4500 \\
\hline 538 & FTMOAFA107 & 29.0000 & 91.0000 \\
\hline 539 & FTMOAFA125 & 30.6000 & 96.4167 \\
\hline 540 & FTWBAFA171 & 35.9000 & 98.1167 \\
\hline 541 & FTWBAFA180 & 30.1333 & 94.1000 \\
\hline 542 & FTWOAFA206 & 30.0333 & 95.7500 \\
\hline 543 & FTMBAFA214 & 33.1833 & 96.6000 \\
\hline 544 & FTUBAFA218 & 29.2667 & 98.4500 \\
\hline 545 & FTMB4FA220 & 29.3833 & 95.0333 \\
\hline 546 & FTWO4FA224 & 31.6667 & 98.1000 \\
\hline 547 & FTWBAFA242 & 35.1667 & 96.5333 \\
\hline 548 & FTMB4FA244 & 29.3000 & 98.3333 \\
\hline 549 & FTMOAFA272 & 34.9833 & 99.1667 \\
\hline 550 & FTWBAFA297 & 31.0833 & 97.6833 \\
\hline 551 & FTMOAFA300 & 30.6667 & 97.6667 \\
\hline 552 & FTWBAFA331 & 29.9000 & 101.0833 \\
\hline 553 & FTMOAFA354 & 29.5000 & 90.0833 \\
\hline 554 & FTWBAFA404 & 32.8833 & 97.0667 \\
\hline 555 & FTMOAFA 408 & 35.3833 & 95.1333 \\
\hline 556 & FTWBALAA16 & 30.3000 & 91.3667 \\
\hline 557 & FTWB5FA006 & 32.1667 & 91.9167 \\
\hline 558 & FTWBSFA010 & 35.3833 & 95.6333 \\
\hline 559 & FTWO5FA014 & 29.6000 & 97.9833 \\
\hline 560 & FTMBSFA019 & 32.9667 & 96.8333 \\
\hline 561 & FTWBSFA032 & 29.2333 & 96.0167 \\
\hline 562 & FTWO5FA034 & 35.5000 & 98.0333 \\
\hline 563 & FTWB5FA040 & 32.3000 & 92.2333 \\
\hline 564 & FTMO5FA050 & 31.5333 & 106.1500 \\
\hline
\end{tabular}

A3-4 
APPENDIX A3.1 GENERAL AVIATION IN FLIGHT CRASH LOCATIONS

\begin{tabular}{|c|c|c|c|}
\hline & NTSB mumber & $\begin{array}{l}\text { Crash } \\
\text { Latitude }\end{array}$ & $\begin{array}{c}\text { Crash } \\
\text { Longitude }\end{array}$ \\
\hline 565 & FTW05FAOS5 & 32.3833 & 96.7833 \\
\hline 566 & FTW05FA065 & 25.7500 & 97.4167 \\
\hline 567 & FTMO5FA088 & 29.5333 & 98.4667 \\
\hline 568 & FTM85FA090 & 29.2667 & 97.2000 \\
\hline 569 & FTW05FA130 & 29.3333 & 98.4667 \\
\hline 570 & FTMO5FA159 & 35.9500 & 95.9667 \\
\hline 571 & FTM05FA171 & 31.4500 & 97.0167 \\
\hline 572 & FTWB5FA181 & 32.6667 & 93.0500 \\
\hline 573 & FTW85FA188 & 29.8667 & 90.8333 \\
\hline 574 & FTMB5FA204 & 35.5000 & 98.0000 \\
\hline 575 & FTWOSFA224 & 30,3667 & 104.0167 \\
\hline 576 & FTWB5FA245 & 33.4167 & 96.8667 \\
\hline 577 & FTWB5FA247 & 31.3333 & 92.4333 \\
\hline 578 & FTWB5FA257 & 32.7667 & 98.0500 \\
\hline 579 & FTWBSFA259 & 29.7667 & 100.3500 \\
\hline 580 & FTMB5FA265 & 32.6667 & 96.7833 \\
\hline 581 & FTWB5FA287 & 32.9000 & 97.4333 \\
\hline 582 & FTMB5FA300 & 29.6000 & 90.6833 \\
\hline 583 & FTW $55 F A 306$ & 33.5500 & 102.3667 \\
\hline 584 & FTWB5FA310 & 29.9167 & 90.8833 \\
\hline 585 & FTWB5FA335 & 34.8000 & 86.6667 \\
\hline 586 & FTWB5FA346 & 35.2500 & 100.6667 \\
\hline 587 & FTVS5FQGO3 & 33.7167 & 102.6500 \\
\hline 588 & FTWB5FRAO1 & 29.6667 & 95.0000 \\
\hline 589 & FTUS5FRDO2 & 30.2000 & 94.8667 \\
\hline 590 & FTWB5FRD03 & 30.0000 & 92.8333 \\
\hline 591 & FTWB5MA297 & 35.4333 & 98.0000 \\
\hline 592 & FTMBGFAOOS & 31.4167 & 103.4833 \\
\hline 593 & FTMB6FA019 & 30.5167 & 101.0667 \\
\hline 594 & FTWB 6 FA023 & 32.4667 & 92.8000 \\
\hline 595 & FTW 6 6FA025 & 30.5833 & 96.3500 \\
\hline 596 & FMB $6 F A 026$ & 35.3500 & 96.9500 \\
\hline 597 & FTWB6FA027A & 33.5333 & 96.2333 \\
\hline 598 & FTWO6FA027B & 33.5333 & 96.2333 \\
\hline 599 & FTWB6FA033 & 28.4667 & 96.4833 \\
\hline 600 & FTMB6FA035 & 33.5167 & 97.0500 \\
\hline 601 & FTMBGFAO46 & 35.2667 & 95.2833 \\
\hline 602 & FTWB6FA050 & 32.6000 & 96.5167 \\
\hline 603 & FTW06FA054 & 36.3167 & 95.1667 \\
\hline 604 & FTWB6FA059 & 33.7167 & 97.6333 \\
\hline 605 & FTM86FA063 & 33.0000 & 96.8000 \\
\hline 606 & FTUB6FA086B & 36.7833 & 97.3000 \\
\hline 607 & FTWB6FA092 & 35.5833 & 98.1833 \\
\hline 603 & FTWB6FA098 & 36.3333 & 95.9500 \\
\hline 609 & FTWB6FA127 & 29.2167 & 95.0333 \\
\hline 610 & FTW66FA133 & 32.9500 & 96.8333 \\
\hline 611 & FTWB6FA142 & 34.4333 & 94.5167 \\
\hline
\end{tabular}

\begin{tabular}{|c|c|c|c|}
\hline & NTSB number & $\begin{array}{l}\text { Crash } \\
\text { Latitude }\end{array}$ & $\begin{array}{c}\text { Crash } \\
\text { Longitude }\end{array}$ \\
\hline 612 & FTWB6FA154 & 47.9500 & 109.7167 \\
\hline 613 & FTWB6FA171 & 29.5833 & 95.2667 \\
\hline 614 & FTWB6FPA07 & 31.4167 & 97.7833 \\
\hline 615 & FTWB6FPA13 & 32.6667 & 96.3833 \\
\hline 616 & FTW86FPA18 & 33.5167 & 96.6500 \\
\hline 617 & FTMB6FPA22 & 32.5333 & 96.9000 \\
\hline 618 & FTWB6FPA27 & 31.3333 & 96.8500 \\
\hline 619 & FTW36FPJ05 & 36.2500 & 95.7667 \\
\hline 620 & FTWB6FOGO2 & 31.6000 & 102.8500 \\
\hline 621 & FTWB6FQGO4 & 33.5833 & 8833 \\
\hline 622 & FTMB6FRD25 & 32.8167 & 8167 \\
\hline 623 & FTMB6FRG02 & 30.3833 & 667 \\
\hline 624 & FTYB6FRG19 & 667 & 667 \\
\hline 625 & FTMO6MA001A & 833 & 333 \\
\hline 626 & $3 M A 010$ & 333 & 3000 \\
\hline 627 & MA058 & 167 & 167 \\
\hline 628 & PJ03 & 67 & 67 \\
\hline 629 & 5.08 & & 33 \\
\hline 630 & 5.509 & 567 & 33 \\
\hline 631 & $\mathrm{RDO3}$ & 333 & 667 \\
\hline 632 & G01 & 3500 & 4000 \\
\hline 633 & G04 & 500 & 8333 \\
\hline 634 & 029 & 33.9000 & 101.8500 \\
\hline 635 & 7FA031 & 167 & 97.6833 \\
\hline 636 & FTVB7FAO43 & 31.0000 & 0000 \\
\hline 637 & FTWB7FA047 & 32.2167 & 97.3167 \\
\hline 638 & FTWB7FA064 & 833 & 94.6833 \\
\hline 639 & FTWBTFA088 & 333 & 96.8500 \\
\hline 640 & FTW87FAO96 & 32.4667 & 333 \\
\hline 641 & FTW87FA123 & 35.8500 & 5167 \\
\hline 642 & FTWB7FA168 & 133 & 9833 \\
\hline 643 & FTWB7FA177 & 00 & 0000 \\
\hline 644 & FTWB7FA183 & 40.4333 & 500 \\
\hline 645 & FTVB7FA206A & 29.5000 & 96.2500 \\
\hline 646 & FTWB7FA206B & 29.5000 & 96.2500 \\
\hline 647 & FTWOTFA209 & 30.1000 & 95.9167 \\
\hline 648 & FTWB7FA210 & 167 & 98.4333 \\
\hline 649 & FTWBTFA223 & 32.5333 & 93.9667 \\
\hline 650 & FTMB7MA133 & 36.1167 & 99.8667 \\
\hline 651 & FTWB8DPJ02 & 36.1167 & 95.6167 \\
\hline 652 & FTV88DPNO3 & 35.2500 & 97.4667 \\
\hline 653 & FTW88DPJO7 & 36.0000 & 95.0000 \\
\hline 654 & FTW88DP 08 & 34.7000 & 94.4833 \\
\hline 655 & FTM88DQG02 & 34.0833 & 101.5167 \\
\hline 656 & FTWBBDRAO4 & 29.0000 & 95.0000 \\
\hline 657 & FTVB8DRA11 & 30.0000 & 95.0000 \\
\hline 658 & FTVBBDRDO8 & 32.7167 & 92.7667 \\
\hline
\end{tabular}

\begin{tabular}{|c|c|c|c|}
\hline & NTSB number & $\begin{array}{l}\text { Crash } \\
\text { Latiude }\end{array}$ & $\begin{array}{c}\text { Crash } \\
\text { Longitude }\end{array}$ \\
\hline 659 & FTMB8DRO12 & 32.4833 & 91.8667 \\
\hline 660 & FTWB8DRD14 & 30.1833 & 92.3167 \\
\hline 661 & FTWB8DRD18 & 30.2333 & 92.2500 \\
\hline 662 & FTWB8DRD20 & 30.0000 & 89.0000 \\
\hline 663 & FTMa8DRGO4 & 26.2167 & 97.6500 \\
\hline 664 & FTWB8DRGO6 & 29.0000 & 98.0000 \\
\hline 665 & FTM $88 F A 011$ & 30.9333 & 95.6667 \\
\hline 666 & FTMO8FAOZ3 & 28.0833 & 333 \\
\hline 667 & FTWB8FAO24 & 30.7167 & 167 \\
\hline 668 & FTMB8FA032 & 26.0000 & 000 \\
\hline 669 & BFA035 & 833 & 67 \\
\hline 670 & FAO46 & 33 & 33 \\
\hline 671 & FA051 & & 00 \\
\hline 672 & FTM & & 67 \\
\hline 673 & $\overline{F T V}$ & 67 & 667 \\
\hline 674 & $F T$ & 33 & 5000 \\
\hline 675 & $74 B$ & 67 & 333 \\
\hline 676 & FAOB4 & 167. & 93.4833 \\
\hline 677 & FA103 & 33.8000 & 95.5333 \\
\hline 678 & FTMB8FAT04 & 34.8333 & 95.1000 \\
\hline 679 & FTWB8FA110 & 31.3667 & 100.4667 \\
\hline 680 & FTWB8FA119 & 667 & 97.4167 \\
\hline 681 & BFA162 & 000 & 97.1667 \\
\hline 682 & FTMA90PJO1 & 34 & 95.8333 \\
\hline 683 & FA006 & 833 & 94.7500 \\
\hline 684 & FA028 & & 95.5500 \\
\hline 685 & A090 & 167 & 667 \\
\hline 686 & $\mathrm{LA}$ & 667 & 3000 \\
\hline 687 & LA & 67 & 333 \\
\hline 688 & LA & 67 & 67 \\
\hline 689 & LA & 00 & 33 \\
\hline 690 & LA & 00 & 200 \\
\hline 691 & & 67 & 100 \\
\hline 692 & & & 33 \\
\hline 693 & & & 133 \\
\hline 694 & $\overline{L A}$ & & 667 \\
\hline 695 & LA & & 33 \\
\hline 696 & & & \\
\hline 697 & & & 333 \\
\hline 698 & & & 667 \\
\hline 699 & & & 333 \\
\hline 700 & FA071 & & 667 \\
\hline 701 & FA087 & & 115.2667 \\
\hline 702 & FA107 & 500 & 118.4000 \\
\hline 703 & $\mathbf{L A}$ & & 117.4667 \\
\hline 704 & LAX & & 119.6000 \\
\hline 705 & LAX84FA129 & 32.5167 & 109.4833 \\
\hline
\end{tabular}


APPENDIX A3.1 GENERAL AVIATION IN FLIGHT CRASH LOCATIONS

\begin{tabular}{|c|c|c|c|}
\hline & NTSB number & $\begin{array}{l}\text { Crash } \\
\text { Latitude }\end{array}$ & $\begin{array}{c}\text { Crash } \\
\text { Longitude }\end{array}$ \\
\hline 706 & LAX84FA138 & 37.6833 & 121.4333 \\
\hline 707 & LAX84FA142 & 34.2000 & 117.6667 \\
\hline 708 & LAX84FA152 & 36.3500 & 121.3667 \\
\hline 709 & LAX84FA193 & 34.2333 & 118.9667 \\
\hline 710 & LAX84FA221 & 34.9000 & 117.8000 \\
\hline 711 & LAX84FA227 & 38.1167 & 121.2000 \\
\hline 712 & LAX84FA253 & 40.8833 & 124.1167 \\
\hline 713 & LAX84FA258 & 34.5000 & 112.6667 \\
\hline 714 & LAX84FA280 & 38.8000 & 120.7833 \\
\hline 715 & LAX84FA300 & 33.8833 & 117.6000 \\
\hline 716 & LAX84FA319A & 37.5167 & 122.5000 \\
\hline 717 & LAX84FA337 & 34.7667 & 116.4000 \\
\hline 718 & LAXBAFA367 & 40.7833 & 122.9667 \\
\hline 719 & LAXBAFA378A & 37.9000 & 122.1667 \\
\hline 720 & LAXBAFA390 & 33.0000 & 115.3333 \\
\hline 721 & LAX84FA396 & 35.8000 & 120.7000 \\
\hline 722 & LAX84FA429 & 33.0667 & 116.6000 \\
\hline 723 & LAX84FA439 & 34.9833 & 113.0500 \\
\hline 724 & LAX84FA460 & 35.3667 & 118.7500 \\
\hline 725 & LAX84FA467 & 33.9833 & 117.4167 \\
\hline 726 & LAX84FA473 & 37.0000 & 118.0667 \\
\hline 727 & LAX84FA481 & 34.3167 & 119.1000 \\
\hline 728 & LAXBAFA498 & 34.4333 & 112.3833 \\
\hline 729 & LAX85FA036 & 33.9667 & 117.0333 \\
\hline 730 & LAX85FAO48 & 35.7333 & 118.5500 \\
\hline 731 & LAX85FAOG8 & 36.2000 & 119.3333 \\
\hline 732 & LAXB5FA071 & 34.9000 & 118.6500 \\
\hline 733 & LAX85FA024 & 33.5167 & 117.2000 \\
\hline 734 & LAX85FA086 & 37.7167 & 121.6667 \\
\hline 735 & LAX85FA08B & 34.1000 & 117.6833 \\
\hline 736 & LAX85FA093 & 34.0000 & 116.0000 \\
\hline 737 & LAX85FA100 & 36.8667 & 117.0500 \\
\hline 738 & LAX85FA102 & 34.5667 & 117.2000 \\
\hline 739 & LAX85FA123A & 34.1167 & 117.8833 \\
\hline 740 & LAX85FA123B & 34.1167 & 117.8833 \\
\hline 741 & LAX85FA137 & 33.0000 & 117.0000 \\
\hline 742 & LAX85FA159 & 33.2167 & 117.3500 \\
\hline 743 & LAXB5FA163 & 37.6167 & 121.0000 \\
\hline 744 & LAX85FA192 & 33.8333 & 117.5033 \\
\hline 745 & LAX85FA213 & 34.4833 & 120.0667 \\
\hline 746 & LAX85FA228 & 33.6833 & 117.7333 \\
\hline 747 & LAX85FA232 & 37.2333 & 121.9667 \\
\hline 748 & LAX85FA241 & 36.3500 & 119.3000 \\
\hline 749 & LAX85FA251 & 37.6500 & 122.1167 \\
\hline 750 & LAX85FA253 & 34.5000 & 119.2167 \\
\hline 751 & LAX85FA259 & 34.0000 & 118.0000 \\
\hline 752 & LAX85FA280 & 33.9000 & 118.4167 \\
\hline
\end{tabular}

APPENDIX 3. GENERAL AVIATION

\begin{tabular}{|c|c|c|c|}
\hline & NTSB number & $\begin{array}{c}\text { Crash } \\
\text { Labitude }\end{array}$ & $\begin{array}{c}\text { Crash } \\
\text { Longitude }\end{array}$ \\
\hline 753 & LAX85FA283 & 38.6833 & 120.9833 \\
\hline 754 & LAX85FA286 & 39.2167 & 120.1167 \\
\hline 755 & LAX85FA301 & 33.8333 & 117.5167 \\
\hline 756 & LAX85FA311 & 31.3500 & 111.1667 \\
\hline 757 & LAX85FA385 & 32.1333 & 111.1667 \\
\hline 758 & LAX85MAOOB & 37.6167 & 122.3667 \\
\hline 759 & LAX86DVA04 & 37.8667 & 120.4167 \\
\hline 760 & LAX860VA07 & 37.2833 & 120.5000 \\
\hline 761 & LAX86DVAO9 & 35.7500 & 119.2333 \\
\hline 762 & LAX86DVA17 & 36.2500 & 119.4000 \\
\hline 763 & LAX86FA003 & 37.8667 & 122.2167 \\
\hline 764 & LAX86FAO07 & 34.3000 & 110.9167 \\
\hline 765 & LAX86FA014 & 38.2167 & 122.4500 \\
\hline 766 & LAX86FA024 & 33.7000 & 118.1167 \\
\hline 767 & LAX86FA038 & 39.1833 & 120.6500 \\
\hline 768 & LAXBGFAO63 & 36.7000 & 118.3500 \\
\hline 769 & LAX86FAOT7 & 33.0500 & 113.3667 \\
\hline 770 & LAXB6FAOB9 & 35.9833 & 112.1500 \\
\hline 771 & LAXB6FAO92 & 33.8000 & 116.1167 \\
\hline 772 & LAX86FA106 & 34.1000 & 110.9333 \\
\hline 773 & LAX86FA107 & 39.2500 & 123.7500 \\
\hline 774 & LAX86FA114 & 38.6833 & 122.6333 \\
\hline 775 & LAX86FA130 & 34.0500 & 117.6000 \\
\hline 776 & LAXB6FA178 & 33.9500 & 116.9500 \\
\hline 77 & LAX86FA183 & 33.7667 & 116.2333 \\
\hline 778 & LAX86FA220 & 33.9667 & 117.6333 \\
\hline 779 & LAX86FA240 & 33.9333 & 117.9500 \\
\hline 780 & LAXB6FA247 & 37.5000 & 118.6333 \\
\hline 781 & LAX86FA314 & 41.3333 & 122.9167 \\
\hline 782 & LAX86FA326 & 33.7667 & 118.2667 \\
\hline 783 & LAX86FA328 & 35.9167 & 112.1667 \\
\hline 784 & LAX86FUM02 & 32.9667 & 116.9000 \\
\hline 785 & LAX86FUMO4 & 32.6333 & 116.9500 \\
\hline 786 & LAX86FVA10 & 37.2333 & 120.2500 \\
\hline 787 & LAX86FVA12 & 36.6167 & 120.2500 \\
\hline 788 & LAX86FVD07 & 37.8500 & 121.2667 \\
\hline 789 & LAX86MA311 & 37.8667 & 121.0167 \\
\hline 790 & LAX870UG07 & 34.0833 & 117.1333 \\
\hline 791 & LAX870U 109 & 32.2333 & 109.1333 \\
\hline 792 & LAX87DUM01 & 33.1167 & 115.5167 \\
\hline 793 & LAX87DUM05 & 33.0500 & 117.0833 \\
\hline 794 & LAX87DVA02 & 36.7167 & 119.8833 \\
\hline 795 & LAX870VA04 & 35.8500 & 119.1667 \\
\hline 796 & LAX87DVM04 & 36.9333 & 121.7833 \\
\hline 797 & LAX87FA017 & 34.4500 & 120.6167 \\
\hline 798 & LAX87FA084 & 34.2500 & 118.4167 \\
\hline 799 & LAX87FA087 & 35.5333 & 115.6333 \\
\hline
\end{tabular}

\begin{tabular}{|c|c|c|c|}
\hline & NTSB number & $\begin{array}{l}\text { Crash } \\
\text { Latiude }\end{array}$ & $\begin{array}{c}\text { Crash } \\
\text { Longitude }\end{array}$ \\
\hline 800 & LAX87FA102 & 34.9333 & 119.4667 \\
\hline 801 & LAX87FA117 & 34.3500 & 118.5167 \\
\hline 802 & LAX87FA136 & 34.8833 & 118.9000 \\
\hline 803 & LAX87FA149 & 37.9167 & 120.3667 \\
\hline 804 & LAX87FA151 & 34.6500 & 1124167 \\
\hline 805 & LAX87FA196 & 34.3500 & 118.4500 \\
\hline 806 & LAX87FA207 & 34.0000 & 118.4500 \\
\hline 807 & LAX87FA212 & 37.1667 & 121.9167 \\
\hline 808 & LAX87FA239 & 39.2667 & 133 \\
\hline 809 & LAX87FA264 & 36. & \\
\hline 810 & LAX87FA267 & 33. & 1 \\
\hline 811 & LAX87FA298 & 33 & \\
\hline 812 & LAX & & 12 \\
\hline 813 & LAX & 34 & \\
\hline 814 & $\operatorname{LAx}$ & 35 & 1 \\
\hline 815 & $\operatorname{LAX}$ & 33 & 11 \\
\hline 816 & $A 03$ & 37.1667 & 121.0167 \\
\hline 817 & VAO4 & 37.2833 & 120.5167 \\
\hline 818 & DVDO3 & 37.9333 & 121.7833 \\
\hline 819 & LAX88FA052 & 34.5667 & 111.8000 \\
\hline 820 & LAX88FA148 & 333 & 1667 \\
\hline 821 & LAX8BFA149 & 33 & 000 \\
\hline 822 & LAX88FA196 & 33.4167 & 112 \\
\hline 823 & LAXB8FAZ30 & 34.2500 & 115.9167 \\
\hline 824 & LAX6BFA258 & 33.7167 & 5500 \\
\hline 825 & LAX88FA264 & 37.0000 & 000 \\
\hline 826 & LAX8BFA278 & 33.9500 & 333 \\
\hline 827 & LAX890VD03 & 333 & \\
\hline 828 & FAO32 & 00 & \\
\hline 829 & A065 & 30 & 0 \\
\hline 830 & $A 073$ & 33 & 67 \\
\hline 831 & $\mathrm{MI}$ & & 0 \\
\hline 832 & 3 & Xo & 33 \\
\hline 833 & 0 & 0 & 33 \\
\hline 834 & $\overline{M I}$ & $\mathbf{0 0}$ & 00 \\
\hline 835 & 5 & 833 & 33 \\
\hline 836 & $\bar{M}$ & 27.6 & 333 \\
\hline 837 & & & 33 \\
\hline 838 & & & 333 \\
\hline 839 & & & 233 \\
\hline 840 & & & 3.2500 \\
\hline 841 & & & 3.0500 \\
\hline 842 & & & 0.4167 \\
\hline 843 & & 27.7667 & 82.6000 \\
\hline 844 & & & 82.5500 \\
\hline 845 & MIAB4FA101 & & 81.5167 \\
\hline$\overline{846}$ & MIAB4FA149 & 29.9833 & 84.3833 \\
\hline
\end{tabular}


APPENDIX A3.1 GENERAL AVIATION IN FLIGHT CRASH LOCATIONS

\begin{tabular}{|c|c|c|c|}
\hline & NTSB number & $\begin{array}{l}\text { Crash } \\
\text { Latitude }\end{array}$ & $\begin{array}{c}\text { Crash } \\
\text { Longitude }\end{array}$ \\
\hline 847 & MIA84FA161 & 28.7667 & 81.2333 \\
\hline 848 & MIAB4FA170 & 27.6333 & 82.7500 \\
\hline 849 & MIA84FA190 & 30.7333 & 84.8333 \\
\hline 850 & MIABAFA200 & 29.9500 & 81.3333 \\
\hline 851 & MIABAFA206 & 27.4167 & 81.8667 \\
\hline 852 & MIABAFA225 & 30.4000 & 86.8167 \\
\hline 853 & MIA84FA234 & 27.6500 & 80.4167 \\
\hline 854 & MLABAFA240 & 26.0000 & 80.2333 \\
\hline 855 & MIAB4FA265 & 30.3833 & 81.9000 \\
\hline 856 & MIAB5FA009 & 26.9167 & 81.9833 \\
\hline 857 & MIAB5FA037 & 27.5000 & 80.2333 \\
\hline 858 & MIAB5FA038 & 27.8167 & 80.6667 \\
\hline 859 & MIAB5FA041 & 28.3500 & 81.5500 \\
\hline 860 & MIA65FA065 & 26.0000 & 81.0000 \\
\hline 861 & MIAB5FA067 & 26.9167 & 81.9833 \\
\hline 862 & MIA85FA098 & 29.3667 & 82.4500 \\
\hline 863 & MIAB5FA101 & 26.0000 & 81.0000 \\
\hline 864 & MIAB5FA105 & 30.4833 & 81.6833 \\
\hline 865 & M1AB5FA106 & 29.3667 & 83.2833 \\
\hline 866 & MIA85FA128 & 28.8167 & 81.8167 \\
\hline 867 & MIAB5FA146 & 30.0000 & 85.0000 \\
\hline 868 & M1A85FA150 & 28.0000 & 82.0000 \\
\hline 869 & MIAB5FA227 & 29.1500 & 83.0667 \\
\hline 870 & MIA85FA234 & 26.4167 & 81.1667 \\
\hline 871 & MIA85FA239 & 29.2833 & 82.1167 \\
\hline 872 & MIAB5FA248 & 28.9167 & 80.8667 \\
\hline 873 & MIABSFA251 & 27.6167 & 80.9500 \\
\hline 874 & MIAB6FA012 & 30.4667 & 87.3500 \\
\hline 875 & MIAB6FA028 & 28.7833 & 81.5500 \\
\hline 876 & MIAB6FA033 & 26.5833 & 80.0333 \\
\hline 877 & MIAB6FAOB4 & 27.3000 & 82.2667 \\
\hline 878 & MIAB6FAO44 & 30.4667 & 87.1833 \\
\hline 879 & MIA86FA055 & 30.4167 & 82.5833 \\
\hline 880 & MIAB6FA059 & 29.0333 & 82.0167 \\
\hline 881 & MIAB6FA085 & 28.2167 & 81.0500 \\
\hline 882 & MIAB6FAO91 & 29.0667 & 81.2833 \\
\hline 883 & MLAB6FA093 & 28.2833 & 81.4333 \\
\hline 884 & MIABGFA097 & 27.9167 & 82.0333 \\
\hline 885 & MLAB6FA101 & 27.9833 & 83.5000 \\
\hline 886 & MIABGFA110 & 29.8500 & 81.9333 \\
\hline 887 & MIAB6FA113 & 27.4833 & 80.3667 \\
\hline 888 & MIAB6FA119 & 29.0833 & 82.1667 \\
\hline 889 & MIAB6FA126 & 28.8500 & 81.6000 \\
\hline 890 & MIAB6FA135 & 27.7500 & 82.6167 \\
\hline 891 & MIAB6FA146 & 25.9333 & 80.4167 \\
\hline 892 & MIAB6FA184 & 26.5333 & 80.2000 \\
\hline 893 & MIAB6FA202 & 33.3833 & 90.2167 \\
\hline
\end{tabular}

\begin{tabular}{|c|c|c|c|}
\hline & NTSB number & $\begin{array}{c}\text { Crash } \\
\text { Latitude }\end{array}$ & $\begin{array}{c}\text { Crash } \\
\text { Longitude }\end{array}$ \\
\hline 894 & M1A86MAO32 & 26.3667 & 80.1333 \\
\hline 895 & MIA86MA182 & 30.3167 & 81.7667 \\
\hline 896 & MIA87FA025 & 30.3000 & 84.2500 \\
\hline 897 & MIAB7FA031 & 33.4500 & 88.5833 \\
\hline 898 & MIA87FA056 & 30.0500 & 81.8833 \\
\hline 899 & MIA87FA084 & 25.7500 & 80.7167 \\
\hline 900 & MIA87FA091 & 28.5333 & 81.3167 \\
\hline 901 & MIAB7FA135 & 25.3833 & 80.6167 \\
\hline 902 & MIAB7FA150 & 33.1667 & 3167 \\
\hline 903 & MIA87FA186 & 30.2167 & 85.6833 \\
\hline 904 & MIA87FA189 & 26.0000 & 2333 \\
\hline 905 & M1A87FA203 & 27.6333 & 333 \\
\hline 906 & MIA87FA205 & 500 & 833 \\
\hline 920 & MIA88FA094 & 667 & 333 \\
\hline 921 & 8 FA114 & & 000 \\
\hline 922 & FA127 & & 300 \\
\hline 923 & A134 & & \\
\hline 924 & MI & & \\
\hline 925 & $\bar{M}$ & & 67 \\
\hline 926 & 34 & 33 & 500 \\
\hline 927 & 39 & 000 & 000 \\
\hline 928 & A254 & 167 & 3833 \\
\hline 929 & FA257 & 833 & 80.1000 \\
\hline 930 & FA044 & 30.7500 & 3167 \\
\hline 931 & $\mathrm{AO48}$ & 29.0000 & 000 \\
\hline 932 & MIA69FA054 & 29.2000 & 667 \\
\hline 933 & MKCB3FA065 & 000 & 92.6000 \\
\hline 934 & FA069 & 38.5667 & 1833 \\
\hline 935 & FAO9O & 37.8000 & 97.2633 \\
\hline 936 & FAO98 & 667 & 92.9833 \\
\hline 937 & 136 & 333 & 333 \\
\hline 938 & FA177 & 37.8500 & 6667 \\
\hline 939 & FA033 & 39.1500 & 6000 \\
\hline 940 & 4FA147 & 40.6500 & 0833 \\
\hline 941 & AFA157 & & 1333 \\
\hline 942 & MKC84FA164 & 37.4167 & 9833 \\
\hline 943 & AFA174 & 41.2833 & 99.5667 \\
\hline 944 & MKCBAFA197 & 34.4333 & 92.0833 \\
\hline 945 & MKCBAFA225 & 33.9833 & 93.8333 \\
\hline 946 & MKCB4FA256 & 41.2167 & 92.4833 \\
\hline 947 & MKC84FA271 & 41.1000 & 92.4667 \\
\hline 948 & MKCBAFA285 & 33.0667 & 91.5833 \\
\hline 949 & MKC85FA007 & 38.4000 & 100.2000 \\
\hline 950 & MKC85FA030 & 39.3000 & 100.9333 \\
\hline 951 & MKCB5FA036 & 38.0500 & 92.5667 \\
\hline 952 & MKCB5FAO42 & 40.0000 & 96.0000 \\
\hline 953 & MKC85FA059 & 34.3167 & 93.3333 \\
\hline
\end{tabular}

\begin{tabular}{|c|c|c|c|}
\hline & NTSB numb & $\begin{array}{l}\text { Crash } \\
\text { Lotitude }\end{array}$ & $\begin{array}{c}\text { Crash } \\
\text { Longitude }\end{array}$ \\
\hline 954 & MKC85FA078 & 34.8667 & 93.4167 \\
\hline 955 & MKC85FA080 & 33.0000 & 93.0000 \\
\hline$\overline{956}$ & MKC85FA084 & 38.4833 & 90.8667 \\
\hline 957 & MKC85FA087 & 34.5500 & 91.8833 \\
\hline 958 & MKC85FA088 & 34.7000 & 94.3667 \\
\hline 959 & MKC85FA105 & 34.0000 & 91.0000 \\
\hline 960 & MKCB5FA111 & 38.8333 & 94.5500 \\
\hline 961 & MKC85FA124 & 41.4833 & 93.5000 \\
\hline$\overline{962}$ & MKC85FA180 & 38.0000 & 92.0000 \\
\hline 963 & MKC85FA199 & 38.0000 & 90.0000 \\
\hline 964 & MKC85FPG01 & 35.1333 & 90.2333 \\
\hline 965 & MKC85FPG02 & 35.0000 & 91.0000 \\
\hline 966 & MKC85FPG03 & 35.9000 & 93.8667 \\
\hline 967 & MKC86FA008 & 38.8167 & 97.7333 \\
\hline 968 & MKC86FA011 & 38.2167 & 91.1667 \\
\hline 969 & MKC $86 F A 023$ & 39.1833 & $\mathbf{9 4 . 1 3 3 3}$ \\
\hline 970 & MKC86FA030 & 36.2000 & 94.2833 \\
\hline 971 & MKC86FA048 & 333 & 333 \\
\hline 972 & $6 F A 053$ & 500 & \\
\hline 973 & $A 077$ & 38.7333 & 167 \\
\hline 974 & FA080 & 39.7167 & 91.4500 \\
\hline 975 & FA082 & 833 & \\
\hline 976 & & & 33 \\
\hline 977 & $\mathbf{N}$ & & 67 \\
\hline 978 & 01 & 67. & 67 \\
\hline 979 & 9 & 367 & 000 \\
\hline 980 & FA151 & 500 & 500 \\
\hline 981 & $\overline{F A 166}$ & 667 & 833 \\
\hline 982 & FA181 & 000 & 000 \\
\hline 983 & FA189 & 38.7167 & 833 \\
\hline 984 & FA195 & 333 & 167 \\
\hline 985 & MKC86FCAD1 & 333 & 667 \\
\hline 986 & $6 F C \sqrt{03}$ & 833 & 667 \\
\hline 987 & $\overline{\mathrm{CQ001}}$ & 667 & 333 \\
\hline 988 & $\longdiv { \mathrm { COO2 } }$ & 833 & 99.1333 \\
\hline 989 & 0003 & 167 & 98.0333 \\
\hline 990 & $\overline{\mathrm{DPGO2}}$ & 34.7667 & 91.6333 \\
\hline 991 & MKC87DPG03 & 33.7167 & 91.4500 \\
\hline 992 & $\overline{37 F A 002}$ & 37.8333 & 94.7000 \\
\hline 993 & MKC87FA012 & 36.2000 & 94.4833 \\
\hline 894 & MKC87FA015 & 37.0333 & 100.9500 \\
\hline 995 & MKC67FA017 & 33.4500 & 94.0000 \\
\hline 996 & MKC87FAO20 & 37.0333 & 97.9167 \\
\hline 997 & MKC87FA022 & 37.2167 & 91.5000 \\
\hline 998 & MKC87FA027 & 39.3500 & 101.0500 \\
\hline 999 & & 39.0167 & 94.2333 \\
\hline 000 & FA036 & 37.9500 & 91.0333 \\
\hline
\end{tabular}




\section{APPENDIX A3.1 GENERAL AVIATION IN FLIGHT CRASH LOCATIONS}

\begin{tabular}{|c|c|c|c|}
\hline & NTSB number & $\begin{array}{l}\text { Crash } \\
\text { Lattitudo }\end{array}$ & $\begin{array}{c}\text { Crash } \\
\text { Longitude }\end{array}$ \\
\hline 1001 & MKC87FA051 & 40.7667 & 102.0500 \\
\hline 1002 & MKCB7FA077 & 41.2500 & 95.7500 \\
\hline 1003 & MKC87FA078 & 37.1000 & 93.2833 \\
\hline 1004 & MKC87FA083 & 38.8000 & 94.6333 \\
\hline 1005 & MKC87FA097 & 42.0333 & 94.0500 \\
\hline 1006 & MKC87FA094 & 36.5667 & 90.5000 \\
\hline 1007 & MKC87FA103 & 39.2500 & 95.8833 \\
\hline 1008 & MKC87FA121 & 33.7167 & 93.6500 \\
\hline 1009 & MKC87FA131 & 40.4333 & 99.3333 \\
\hline 1010 & MKC87FA165 & 35.7333 & 91.6500 \\
\hline 1011 & MKCB7FA169 & 39.3167 & 102.0500 \\
\hline 1012 & MKC8BDCDO1 & 41.7000 & 94.9167 \\
\hline 1013 & MKC8BDCDO2 & 41.5167 & 93.2167 \\
\hline 1014 & MKC88DCG01 & 36.0000 & 94.0000 \\
\hline 1015 & MKCB8DCQOS & 39.1500 & 97.8667 \\
\hline 1016 & MKC88DCOO4 & 37.0000 & 98.0000 \\
\hline 1017 & MKC8BDPG01 & 34.7333 & 92.2333 \\
\hline 1018 & MKCBBDPGO2A & 34.4000 & 91.4500 \\
\hline 1019 & MKCBBFAOO7 & 39.0167 & 92.2833 \\
\hline 1020 & MKCBBFAO18 & 37.6667 & 97.0833 \\
\hline 1021 & MKCBBFAO21 & 34.7000 & 94.2333 \\
\hline 1022 & MKCBBFAO23 & 41.0833 & 98.2333 \\
\hline 1023 & MKCBBFAO26 & 30.3500 & 95.4167 \\
\hline 1024 & MKC88FA039 & 33.6187 & 92.7833 \\
\hline 1025 & MKC8BFA012 & 37.8833 & 100.7167 \\
\hline 1026 & MKC88FA059 & 38.0833 & 91.9500 \\
\hline 1027 & MKC88FA070 & 37.0000 & 93.0000 \\
\hline 1028 & MKC88FA119 & 39.7500 & 100.7500 \\
\hline 1029 & MKCBBFA147 & 39.0000 & 94.0000 \\
\hline 1030 & MKC8BFA166 & 36.7000 & 93.5000 \\
\hline 1031 & MKC89FAO14 & 38.9167 & 94.7500 \\
\hline 1032 & NYCB3AA208 & 39.5167 & 74.5333 \\
\hline 1033 & NYCB3FA060 & 40.6500 & 76.7333 \\
\hline 1034 & NYC83FAO91 & 40.7500 & 74.5000 \\
\hline 1035 & NYCB3FA105 & 40.7333 & 73.4167 \\
\hline 1036 & NYC83FA121 & 41.3000 & 72.3167 \\
\hline 1037 & NYC83FA125 & 42.2167 & 74.0667 \\
\hline 1038 & NYC83FA141 & 41.3500 & 73.5333 \\
\hline 1039 & NYC83FA144 & 43.7667 & 71.3833 \\
\hline 1040 & NYC83FA187 & 39.3667 & 75.0667 \\
\hline 1041 & NYC83FA192 & 42.2833 & 74.1000 \\
\hline 1042 & NYC83FA207 & 41.3333 & 75.7167 \\
\hline 1043 & NYCB3FA253 & 42.0167 & 74.5167 \\
\hline 1044 & NYC83FA256 & 44.4667 & 72.8833 \\
\hline 1045 & NYCB3FFJ02 & 43.2000 & 77.9833 \\
\hline 1046 & NYC84FA024 & 42.8500 & 78.7167 \\
\hline 1047 & NYCBAFA032A & 41.0833 & 74.5833 \\
\hline
\end{tabular}

APPENDIX 3. GENERAL AVIATION

\begin{tabular}{|c|c|c|c|}
\hline & & $\begin{array}{l}\text { Crash } \\
\text { Latitude }\end{array}$ & $\begin{array}{l}\text { Crash } \\
\text { Longitude }\end{array}$ \\
\hline 1048 & NYC84FA032B & 41.0833 & 74.5833 \\
\hline 1049 & NYC84FA033 & 41.4500 & 79.8500 \\
\hline 1050 & NYC84FA039 & 40.0333 & 76.9833 \\
\hline 1051 & NYC84FA053 & 41.6167 & 73.6500 \\
\hline 1052 & NYC84FA074 & 41.3500 & 72.0333 \\
\hline 1053 & NYC84FA090 & 42.5833 & 70.9167 \\
\hline 1054 & NYC84FA107 & 43.3500 & 76.3833 \\
\hline 1055 & NYC84FA129 & 41.7667 & 72.5333 \\
\hline 1056 & NYCBAFA137 & 42.3667 & 74.2500 \\
\hline 1057 & NYC84FA143 & 42.4667 & 71.3333 \\
\hline 1058 & NYC84FA148 & & 79.6833 \\
\hline 1059 & NYC84FA157 & & \\
\hline 1060 & NYC84FA158 & 40.8167 & 72.8500 \\
\hline 1061 & NYCBAFA189 & & \\
\hline 1062 & NYCBAFA218 & & 000 \\
\hline 1063 & NYCBAFA224 & & \\
\hline 1064 & NYCB4FA247 & & 74.1667 \\
\hline 1065 & NYC84FA295 & 41.0667 & 73.2167 \\
\hline 1066 & NYCBAFA306 & & 71.0000 \\
\hline 1067 & NYCBAFA307 & & 300 \\
\hline 1068 & NYCB4F & & \\
\hline $10 \div 9$ & NYC84FA326 & & 76.7500 \\
\hline 1070 & NYCBAFFAOS & & 75.0167 \\
\hline 1071 & NYCBAFGMO1 & & \\
\hline 1072 & NYC84FHMOS & 367 & 77.8167 \\
\hline 1073 & & & 167 \\
\hline 1074 & NYC84MA270 & 000 & 74.0000 \\
\hline 1075 & NYCB5FAO03 & 41.4667 & 74.5000 \\
\hline 1076 & & & \\
\hline 1077 & NYC & 367 & 79.8833 \\
\hline 1078 & & & 74 \\
\hline 1079 & NYCB5FA047 & & 77.6667 \\
\hline 1080 & NYC & & 75.1833 \\
\hline 1081 & NYC & 200 & 73.0500 \\
\hline 1082 & NYC & & 73. \\
\hline 1083 & NYC85 & & \\
\hline 1084 & NYC85 & & 75.5500 \\
\hline 1085 & $\overline{N Y C}$ & & 73.9500 \\
\hline 1086 & NYC & & 79.0333 \\
\hline 1087 & NYC85FA222 & & 77.1667 \\
\hline 1088 & NYC & & \\
\hline 1089 & 5 FA241 & & \\
\hline 1090 & $\mathrm{NY}$ & & 79. \\
\hline 1091 & NYC85FFA01 & 44.2833 & 73.3667 \\
\hline 109 & NYC & & 76.1000 \\
\hline 109 & NYC85FGMO4 & & \\
\hline 1094 & YCR5ECON5 & & \\
\hline
\end{tabular}

\begin{tabular}{|c|c|c|c|}
\hline & NTSB number & $\begin{array}{c}\text { Crash } \\
\text { Lotiude }\end{array}$ & $\begin{array}{l}\text { Crash } \\
\text { Longitude }\end{array}$ \\
\hline 1095 & NYC85FGTO2 & 34.8833 & 77.0667 \\
\hline 1096 & NYCBSFHA03 & 41.7000 & 76.0500 \\
\hline 1097 & NYC85FHDO2 & 0.5667 & 74.7333 \\
\hline 1098 & NYC85FNA02 & 42.4667 & 71.5167 \\
\hline 1099 & NYCB6FAOO1 & 40.2000 & 74.6000 \\
\hline 1100 & NYCBGFAOO2 & 42.5333 & 74.4167 \\
\hline 1101 & NYCB6FA006 & 40.5167 & 79.1500 \\
\hline 1102 & NYCB6FA030 & 44.6667 & 68.8000 \\
\hline 1103 & NYCB6FA037 & 41.5000 & 72.8167 \\
\hline 1104 & NYCB6FA068 & 42.4667 & 72.5667 \\
\hline 1105 & NYCB6FAOT7 & 41.0000 & 74.7333 \\
\hline 1106 & NYCB6FA112 & 41.9167 & \\
\hline 1107 & NYC86FA127 & 41.5500 & 73.8333 \\
\hline 1108 & NYC86FA135 & 39.8000 & 75.3500 \\
\hline 1109 & NYCB6FA138 & 42.3 & 833 \\
\hline 1110 & NYCB6FA176 & 39.8667 & 78.8333 \\
\hline 1111 & NYC86FA201 & & \\
\hline 1112 & NYCB6FA214 & 40.0000 & 000 \\
\hline 1113 & NYCB6FA216 & & 71.1167 \\
\hline 1114 & NYCB6FA218 & & 74.4500 \\
\hline 1115 & NrCB6FA219 & 667 & 76.6000 \\
\hline 1116 & NYC86FGMOS & & \\
\hline 1117 & NYCBGFNCO2 & & 367 \\
\hline 1118 & NYC86FNC07 & 33 & 167 \\
\hline 1119 & NYC86FNCOB & & 71.33333 \\
\hline 1120 & NYC86MA148 & 42.1167 & 71.7167 \\
\hline 1121 & NYCB7L & & 333 \\
\hline 1122 & NYC87DNC05 & 367 & 69.3833 \\
\hline 1123 & NYC87DNC12 & & 73.0000 \\
\hline 1124 & NYCB7FAOOB & & 72.6833 \\
\hline 1125 & NYCB7FAO21 & & 79.5000 \\
\hline 1126 & NYCB7FA023 & & \\
\hline 1127 & NYCB7FAO45 & 667 & 500 \\
\hline 1128 & NYC87FA095 & & 78.0000 \\
\hline 1129 & NYCBTFAT19 & 667 & 77.8667 \\
\hline 1130 & NYCB7FA127 & & 76.8667 \\
\hline 1131 & NYC87MAO24 & & $\underline{00}$ \\
\hline 1132 & NYC88DGTO1 & 40.6667 & 77.8333 \\
\hline 1133 & NYCB8FA051 & & \\
\hline 1134 & SEAB3FA037 & 45.5333 & 122.5667 \\
\hline 1135 & SEAB3FA038 & & 121.5833 \\
\hline 1136 & & & 116.7500 \\
\hline 1137 & SEAQ3FA072 & 48.2 & 122.4333 \\
\hline 1138 & SEAQ3FA087 & & 122.9500 \\
\hline 1139 & SEAB3FA093 & & 122.2167 \\
\hline 1140 & SEAB3FA118 & 45.2333 & 121.7333 \\
\hline 1141 & SEAB3FA168 & 43.2 & 123.1667 \\
\hline
\end{tabular}


APPENDIX A3.1 GENERAL AVIATION IN FLIGHT CRASH LOCATIONS

\begin{tabular}{|c|c|c|c|}
\hline & NTSB number & $\begin{array}{l}\text { Crash } \\
\text { Latthude }\end{array}$ & $\begin{array}{c}\text { Crash } \\
\text { Longitude }\end{array}$ \\
\hline 1142 & SEA83FA178 & 48.0667 & 122.0167 \\
\hline 1143 & SEA83FA2O4 & 48.7167 & 122.8333 \\
\hline 1144 & SEAB4FA058 & 45.5333 & 123.0500 \\
\hline 1145 & SEABAFA063 & 43.7167 & 116.6667 \\
\hline 1146 & SEAB4FA068 & 45.6667 & 121.6667 \\
\hline 1147 & SEA84FA078 & 44.9500 & 117.8000 \\
\hline 1148 & SEABAFAOBB & 43.0833 & 122.1167 \\
\hline 1149 & SEABAFAO9O & 39.0167 & 119.7167 \\
\hline 1150 & SEABAFA101 & 48.6830 & 122.8167 \\
\hline 1151 & SEABAFA102 & 40.1667 & 118.4500 \\
\hline 1152 & SEABAFA111 & 45.5167 & 122.7500 \\
\hline 1153 & SEABAFA135 & 46.1833 & 123.9833 \\
\hline 1154 & SEABAFA15B & 35.9667 & 115.1167 \\
\hline 1155 & SEA84FA164 & 47.4833 & 122.7500 \\
\hline 1156 & SEABAFA173 & 46.4667 & 122.9167 \\
\hline 1157 & SEABAFA175 & 40.8167 & 115.7833 \\
\hline 1158 & SEABAFA178 & 35.9333 & 115.1500 \\
\hline 1159 & SEAB4FA196 & 48.7167 & 116.2833 \\
\hline 1160 & SEAB4FA215B & 45.6333 & 121.2167 \\
\hline 1161 & SEABAFA217 & 44.7667 & 115.0833 \\
\hline 1162 & SEAB4FA223 & 45.6333 & 122.0000 \\
\hline 1163 & SEA84FYKO1 & 43.9167 & 123.0000 \\
\hline 1164 & SEABSFAOO1 & 39.3667 & 117.1167 \\
\hline 1165 & SEA85FA016 & 47.0500 & 123.7500 \\
\hline 1166 & SEA05FA035 & 39.0500 & 119.6667 \\
\hline 1167 & SEABSFAO36 & 40.0000 & 117.0000 \\
\hline 1168 & SEA85FA043 & 47.3333 & 122.1000 \\
\hline 1169 & SEAB5FAO50 & 42.5333 & 122.9000 \\
\hline 1170 & SEABSFA051 & 39.0000 & 119.0000 \\
\hline 1171 & SEAB5FA056 & 42.3833 & 122.8667 \\
\hline 1172 & SEABSFAD65 & 39.7833 & 114.3667 \\
\hline 1173 & SEA $85 F A 084$ & 45.3667 & 123.3500 \\
\hline 1174 & SEABSFA100 & 42.8833 & 114.9167 \\
\hline 1175 & SEA1 5 FA103 & 47.5333 & 122.3000 \\
\hline 1176 & SEAB5FA113 & 43.1167 & 116.8833 \\
\hline 117 & SEAB5FA121 & 48.0500 & 122.9500 \\
\hline 1178 & SEAB5FA122 & 47.0000 & 117.0000 \\
\hline 1179 & SEAB5FA156 & 48.3000 & 122.5000 \\
\hline 1180 & SEA85FA162 & 43.9333 & 115.7500 \\
\hline 1181 & SEAB5FA181 & 43.8667 & 114.7167 \\
\hline 1182 & SEABSFA203 & 43.3167 & 114.3167 \\
\hline 1183 & SEAB5FA203 & 47.3667 & 120.2333 \\
\hline 1184 & SEAB5FA211 & 44.0167 & 116.4833 \\
\hline 1185 & SEA $85 F A 225$ & 46.5167 & 114.6333 \\
\hline 1186 & SEABSLAOSA & 42.4833 & 122.8167 \\
\hline 1187 & SEABSLAOSS & 40.4833 & 115.5000 \\
\hline 1188 & SEABGFAOO4 & 42.2500 & 113.8667 \\
\hline
\end{tabular}

\begin{tabular}{|c|c|c|c|}
\hline & NTSB number & $\begin{array}{l}\text { Crash } \\
\text { Latitude }\end{array}$ & $\begin{array}{c}\text { Crash } \\
\text { Longitude }\end{array}$ \\
\hline 1189 & SEA86FA034 & 46.3000 & 123.9333 \\
\hline 1190 & SEAB6FA039 & 44.2000 & 123.4000 \\
\hline 1191 & SEAB6FAO46 & 38.9167 & 120.0000 \\
\hline 1192 & SEAB6FA047 & 39.4833 & 119.7667 \\
\hline 1193 & SEAB6FA052 & 47.3167 & 122.0500 \\
\hline 1194 & SEA86FA054 & 47.4833 & 122.2167 \\
\hline 1195 & SEAB6FA066 & 44.7500 & 120.6000 \\
\hline 1196 & SEABGFA071 & 46.8667 & 122.9500 \\
\hline 1197 & SEAB6FA073 & 44.9667 & 123.9167 \\
\hline 1198 & SEAB6FA075 & 833 & 122.5000 \\
\hline 1199 & SEA86FA092A & 47.7333 & 122.2667 \\
\hline 1200 & SEA86FA092B & 333 & 122.2667 \\
\hline 1201 & SEABGFAC96 & 1000 & 120.1000 \\
\hline 1202 & SEAB6FA098 & 000 & 112.2833 \\
\hline 1203 & SEA86FA121A & 47.7833 & 118.3000 \\
\hline 1204 & SEAB6FA121B & 47.7833 & 118.3000 \\
\hline 1205 & SEAB6FA126 & 44.2167 & 122.3167 \\
\hline 1206 & SEAB6FA137 & 47.3333 & 667 \\
\hline 1207 & SEA86FA144 & 000 & \\
\hline 1208 & SEA86FA145 & 33 & 000 \\
\hline 1209 & SEABGFA155 & 167 & 333 \\
\hline 1210 & SEA86FA165 & 000 & \\
\hline 1211 & SEA86FA215 & & \\
\hline 1212 & SEA86FA216 & & \\
\hline 1213 & SEAB6FA225 & & \\
\hline 1214 & SEA86FA232 & & \\
\hline 1215 & $S E$ & 00 & \\
\hline 1216 & SEA & & 00 \\
\hline 217 & SE & & 67 \\
\hline 1218 & SE & & 67 \\
\hline 1219 & SE & & 00 \\
\hline 20 & $\overline{S E}$ & & 300 \\
\hline 21 & $\overline{S E}$ & & 500 \\
\hline 1222 & $\mathrm{SE}$ & 67 & 11 \\
\hline 23 & & 00 & 117.4333 \\
\hline 1224 & & 500 & 833 \\
\hline 1225 & & & 5000 \\
\hline 1226 & & & 000 \\
\hline 1227 & & & 115.5833 \\
\hline & & 00 & 1000 \\
\hline 1229 & & 1667 & 333 \\
\hline 1230 & & & 2667 \\
\hline 1231 & & 167 & 118.7667 \\
\hline 1232 & & 667 & 122.6000 \\
\hline 1233 & & 0667 & 115.0667 \\
\hline 1234 & SEAB7MA035 & 36.5000 & 115.2500 \\
\hline 1235 & 17 MA192 & 42.4833 & 114.4667 \\
\hline
\end{tabular}

\begin{tabular}{|c|c|c|c|}
\hline & NTSB mumber & $\begin{array}{c}\text { Crash } \\
\text { Lotitude }\end{array}$ & $\begin{array}{c}\text { Crash } \\
\text { Longitude }\end{array}$ \\
\hline 1236 & SEAB8FA012 & 48.5500 & 117.2167 \\
\hline 1237 & SEAB8FA021 & 48.0167 & 122.9167 \\
\hline 1238 & SEAB8FA023 & 45.5333 & 122.9500 \\
\hline 1239 & SEAB8FA024 & 44.6167 & 116.8167 \\
\hline 1240 & SEAB8FA031 & 39.1167 & 123.0167 \\
\hline 1241 & SEAB8FA034 & 44.9167 & 122.4833 \\
\hline 1242 & SEAB8FA039 & 44.1333 & 112.0833 \\
\hline 1243 & SEAB8FA069 & 42.6833 & 111.3167 \\
\hline 1244 & SEAB8FA083 & 38.0000 & 115.0000 \\
\hline 1245 & SEAB8FA095 & 47.0500 & 124.1333 \\
\hline 1246 & SEAB8FA120 & 47.7500 & 121.1000 \\
\hline 1247 & SEAB8FA122 & 45.3167 & 115.0333 \\
\hline 1248 & SEAB8FA176 & 47.6167 & 120.3167 \\
\hline 1249 & SEAB8LA094 & 39.8833 & 119.5167 \\
\hline 1250 & SEAB9FAO01 & 48.6500 & 122.5833 \\
\hline
\end{tabular}




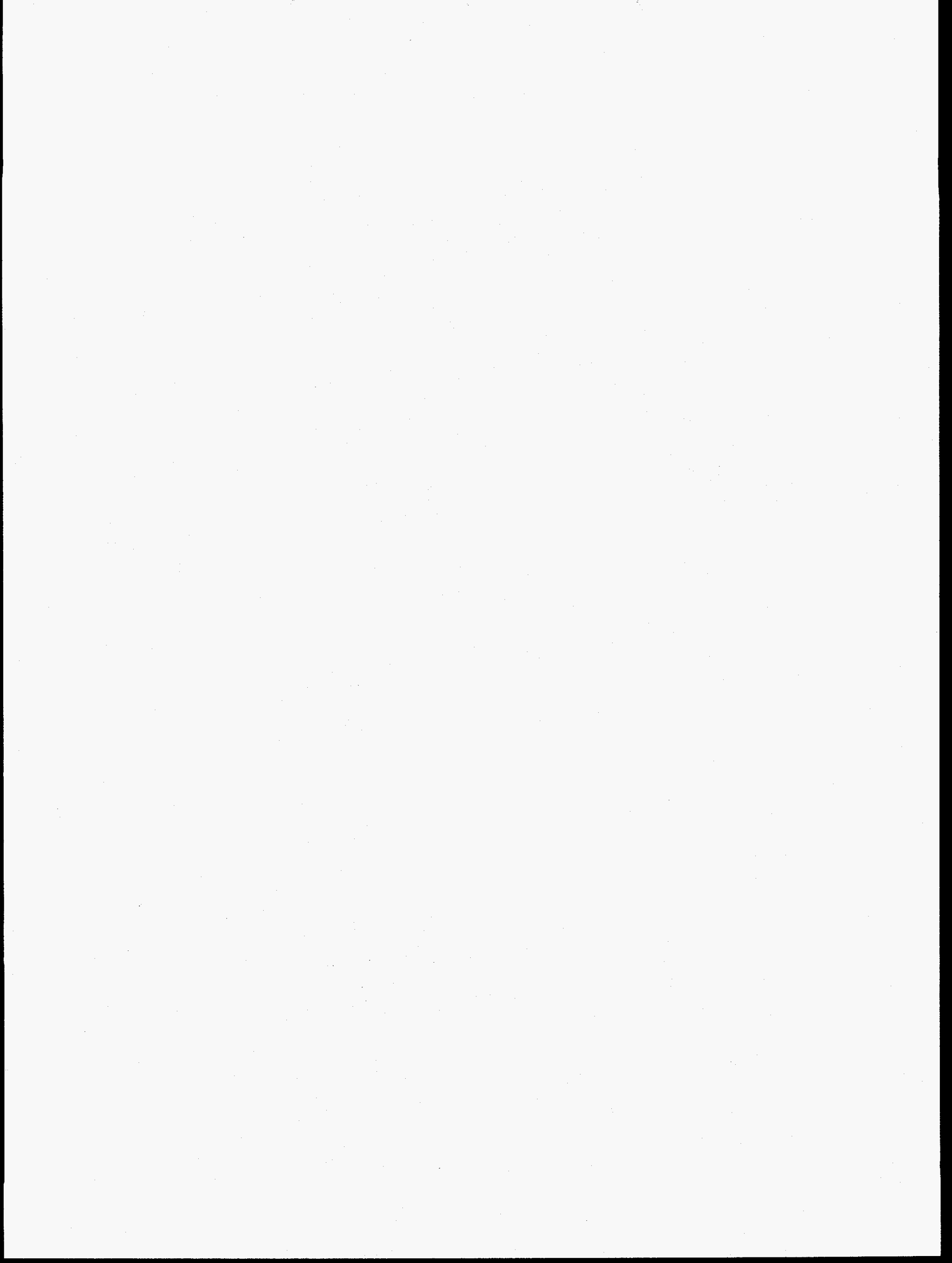




\section{MILITARY AVIATION}

\subsection{INTRODUCTION}

For the ACRAM Standard [Ret. 4.1], military aviation is defined as the broad categorization of aviation activities performed by military personnel in fulfillment of their official duties. Such activities include passenger and cargo transport, in-flight refueling, flight training, etc. Activities associated with military operations areas (MOA) and training ranges, such as air combat training, low level navigation, personnel and stores drops, are not included. However, cruise phase of flight between an originating airfield and the MOAs, as well as training associated with takeoff and landing at an airfield, including touch and go's; simulated emergency landings, e.g., no flap, flameout; and missed approach/go-arounds, are included.

To quantify the risk of a military aircraft crashing into a facility it is necessary to estimate the number of military flights in the vicinity of the facility, the frequency of military aircraft crashes and the probability that the aircraft crashes into the facility. The latter requires knowledge about the location of aircraft crashes, given an incident leading to a crash, as well as some crash kinematics, e.g., glide or impact angle, heading angle, and skid distance.

To estimate military aircraft crash frequencies, relevant crash and flight information was solicited from the U.S. Air Force, Army and Navy safety agencies. Useable information for fixed wing and rotary wing (helicopter) aircraft were received from the Air Force. The Army provided data on Army helicopters. Data received from the Navy was judged to be of limited value for this application, and is not included in the estimates presented here. Analysis of available crash data and the resulting estimates of military aircraft crash frequencies are presented in Section 4.2.

Crash kinematic and crash location data were derived by reviewing Air Force aircraft mishap reports from 1976 to 1993. A database of aircratt crash data was developed as part of the DNA supported W78M87 Minuteman III Weapon System Safety Assessment (WSSA) [Ref. 4.2]. That formed the basis for developing crash kinematics and crash location probability distributions. Data analysis and distributions are summarized in Section 4.3.

Section 4.4 includes a summary of some of the appropriate characteristics of military aircraft which are necessary for the structural analyses. 


\subsection{MILITARY AVIATION CRASH FREQUENCIES}

Development of estimates of aircraft crash frequencies is based on an analysis of aircraft crash and flight data supplied by Air Force and Army safety agencies [Ref. 4.3-4.6] and the database of Air Force mishaps developed for the DNA supported Minuteman III WSSA [Ref. 4.2].

For the ACRAM Standard, the flight phases of interest for military aviation are

Takeoffs at airfields in the vicinity of the facility of interest

Landings at airfields in the vicinity of the facility of interest

Overlights, during the cruise phase of flight, in the vicinity of the facility of interest

Analysis of the miltary crash data required the identification of the phase of flight in which the crash occurred as well as screening of crashes to delete those not applicable to crashes into structures, e.g., crashes involving taxiing and/or parked aircraft. This required a review of the individual accident reports/summaries to assign each accident to the proper phase and, occasionally, involved judgments in the applicability of an accident. Thus, there is some uncertainty in the estimates of crash frequencies in addition to inherent "statistical" variation due to the limited amount of historical data. Athough recognized, this uncertainty is neither quantified nor included in the provided estimates of crash frequencies. Rather, the estimates provided are considered plausible point estimates of the appropriate frequencies.

Two analyses were considered in developing estimates for crash frequencies. One analysis is based on a review of the brief summaries of mishaps as provided by the Air Force/Army safety agencies. This approach is discussed and the resulting estimates of crash frequencies are presented in Section 4.2.2. The second analysis is based on the Air Force mishap database developed for the Minuteman III WSSA. This analysis and the resulting estimates are discussed in Section 4.2.3.

This Standard is expected to be applicable to facilities off an airfield and not in the immediate vicinity of a nunway. Therefore, military takeoff and landing crashes were identified as "on runway" (i.e., crashes in which the initial impact occurred on the runway and the rolling/skidding aircraft departed the rumway or remained on the numway) or "off nunway" (i.e., the initial impact occurred off the runway). Although the off runway crashes include some that occurred on an airtield, the off rumway crash frequency estimated from the historical data is considered a reasonable conservative estimate applicable for this Standard.

For military aviation the cruise phase of flight involved a number of different types of operations in addition to "normal" flight from one base to another. A significant part of the cruise portion of flight, particularly for military attack, fighter and trainer aircraft, involved maneuvers. Since this type of activity is not expected to affect facilities covered by this Standard, accidents occurring during maneuvers, air shows, and other special operations were not included in developing the estimated inflight crash frequencies. Deleting those accidents from consideration required that the mileage flown, which is the denominator of crash frequencies, be adjusted. Since the available military flight information is in flight hours, considerable judgment was involved in developing estimates of the number of miles flown during "normal" flight. Again, this needs to be recognized in using the provided estimates of crash frequencies.

Ideally, estimates of crash frequencies can be developed for each type of military aircraft. This was the goal in the second analysis (Section 4.2.3). Due to limited data and the reasonable expectation that actual frequencies are comparable for some subsets of aircraft, estimated crash frequencies are provided per aircraft type or group of types. The philosophy of this Standard is to use three subcategories of military aircraft:

Large aircraft: bomber and cargo aircraft such as the B-1, B-2, B-52, C-5, C-9, KC-10, C-21, C$130, \mathrm{KC}-135$ and $\mathrm{C}-141$

Small aircraft: attack, fighter and trainer aircratt such as the A-7, A-10, A-37, F-4, F-5, F-15, F-16, F-106, F-111, F-117, T-33, T-37, T-38, T-39 and T-41

Helicopters: $H-1, H-3, H-53$ and $H-60$ 
Estimates of crash frequencies are provided for each subcategory as well as for all military aircratt. These combined estimates are based on a weighted average of the individual frequencies, weighted by the respective normalized number of takeotts/landings and number of miles flown during "nomal" flight. Application of the combined frequencies is appropriate only when (1) the distribution, among the different types of aircratt (e.g., T-38, F-16, B-52, ...), of the number of takeotts/landings at the airfield of interest and (2) the distribution, among aircratt types, of the enroute overfights in the vicinity of the facility of interest are comparable to the distributions in the historical data. If operations at a given location are much different than the overall distribution in the historical data, alternative estimates of crash frequencies, based on the appropriate mix of aircraft at the location, should be considered. 


\subsubsection{ESTIMATES OF CRASH FREQUENCIES BASED ON AN ANALYSIS OF IMPACT ACCIDENTS}

Air Force mishap data solicited from the Air Force Safety Agency [Ref. 4.3-4.5] provides one basis for estimating crash frequencies for military aviation. Air Force classification of mishaps is based on the economic costs of damage to the aircraft and personnel costs and are defined as follows:

Flight Mishap - A mishap involving an Air Force aircraft when intent for flight exists.

Class A - $\quad$ A mishap in which the resulting total cost of property damage, injury and iness is $\$ 1,000,000$ or greater; or an Air Force aircraft is destroyed; or a fatality occurs.

Class B - A mishap in which the resulting total cost of property damage, injury and iliness is $\$ 200,000$ or more, but less than $\$ 1,000,000$.

Class C - $\quad$ A mishap in which the resulting total cost of property damage is $\$ 10,000$ or more, but less than $\$ 200,000$ or injury or occupational illness resulted in a lost workday case involving days away from work.

Destroyed - Destroyed means uneconomical to repair, defined by the number of man-hours estimated as needed to repair the aircraft. Repair time varies depending on the type of aircraft.

Mishap Rates - Rates are computed on basis of the number of mishaps per 100,000 flying hours.

Flight Related - When there is a mishap with little or no damage to the aircratt, the incident does not affect the mishap rate.

One set of data provided by the AFSA is based on the lifetime mishap history, up to the early 1994 time frame, of a large number of Air Force aircraft. A total of 5171 Class A, 2450 Class B, and 3598 Destroyed mishaps are included in this data set. A summary of these mishaps, by aircratt type, is given in Table 4.1. Related flight information included in the table is the total flight hours for each type of aircraft. Also included in the table are estimates of mishap rates, given as rates per 100,000 flight hours.

The various aircraft types were grouped by the three subcategories of military aviation, small aircratt, large aircraft, and helicopter. The mishap data for the three subcategories are summarized in Tables 4.2 to 4.4. Again, estimates of mishap rates are per 100,000 flight hours. Since T-33 aircraft have been out of the Air Force inventory for some time, mishap rates for small aircraft are estimated excluding the T-33 data as well.

The mishaps recorded in Tables 4.1 to 4.4 include a large number of mishaps not applicable to crashes into off airfield facilities, e.g., nonimpact mishaps, on airfield crashes, etc. Therefore, the mishap rates provided in these tables may be over estimates of crash rates into off airfield structures. In addition, takeoff and landing incidents are likely to be significant contributors to crashes into structures; thus, it is appropriate to develop crash rates per takeoff and landing. To do this it is necessary to have a more detailed description of the mishap.

A second set of data provided by the AFSA included summary information for mishaps in the 1979-1993 time period. Useable information was derived from 1426 mishaps. To develop crash frequency estimates, the 1426 mishaps were classified either as impact or non-impact mishaps. Impact mishaps included accidents involving ground, numay, water, midair, terrain, vehicle, and building impact. Non-impact mishaps included incidents involving foreign objects, birds, etc., and parked aircraft fires and other such incidents. Non-impact mishaps are considered not applicable. There were 1093 impact mishaps. Those mishaps included all classes of damage to the crashing aircraft. The mishaps were partitioned by aircraft "size" (large, small and helicopter) and by flight phase (takeoff, landing and inflight). Takeoff and landing were further partitioned into "on nunway" and "oft runway" mishaps. In-flight mishaps were partitioned into "normal" and "special", i.e., low altitude and maneuvering operation mishaps. A graphical description of the classification of the mishaps is shown in Figure 4.1. For this analysis, takeoff includes takeoff roll, abort/discontinue, and initial climb portions of a flight; landing includes the pattern, final approach, flare 
and rollout portions; normal in-flight includes climb to cruise, cruise between an originating airfield and an operations area, if applicable, and cruise descent portions; and special in-flight includes low level and maneuvering operations in restricted airspace.

The number of impacting mishaps for each subcategory of aircraft and flight phase, appropriate flight information and the resulting estimates of crash frequencies are summarized in Table 4.5.

Crash trequencies for takeofts and landings are per takeotf/anding. Crash frequencies for "normal" inflight operation, are "per mile." The estimated mileage derived to estimate inflight rates is based on an analysis of the expected number of miles flown during "normal" flight. This analysis attempted to account for the time in the takeoff and landing phases of flight as well as the time in maneuvers and other special operations. This required a considerable amount of judgment by the analyst.

For some facilities, particularly hardened structures, a more appropriate estimate of a crash frequency may be one based on only considering impact mishaps in which the crashing aircraft was destroyed. Of the 1093 impact mishaps, a crashing aircratt was classified as "destroyed" in 819 mishaps. These impact destroyed mishaps were partitioned in the same way as impact mishaps as shown in Figure 4.1. A summary of this data and the resulting estimates of crash frequencies are given in Table 4.6.

Basic mishap data, flight information and partitioning of mishaps by aircraft subcategory and flight phase were developed by T. Lin at Sandia National Laboratory [Ref. 4.6]. Development of estimated miles flown during "normal" inflight operations is based on an analysis of aircratt operations by Logicon RDA [Ref. 4.7]. 


\subsubsection{ESTIMATES OF CRASH FREQUENCIES BASED ON AN ANALYSIS OF THE MISHAP DATABASE DEVELOPED FOR THE MINUTEMAN III WSSA}

Another source of mishap information is the mishap database developed for the DNA-supported Minuteman III WSSA [Ref. 4.2]. This database was developed from data extracted from individual mishap reports, also available trom the AFSA. Its primary use was as a resource for crash location and crash kinematic information, but it is also useful as a basis for estimating crash frequencies. Development of crash frequency estimates using this database is based on identifying mishaps involving a "crash" which is defined as "An aircraft mishap associated with flight that prevents the aircraft from coming to a full stop landing on its gear." In addition, for this analysis, flight phases are defined as:

Takeoff: The phase of flight from the application of takeoff power on the rumway to the point where the aircraft altitude is not affected by its proximity from the departure rumway.

Landing: The phase of flight from the point where the aircraft attitude is affected by its proximity from the approach rumway to its departure from the rumway under a controlled taxi.

In-flight: The phase of fight where the aircraft altitude is not affected by its proximity to the numway.

A summary of the crash data, estimated crash frequencies and applicable flight information for individual aircraft types and groups of aircraft types, as well as for the three subcategories of aircraft, is presented in Tables 4.7 and 4.8 .

The basic crash data and flight information were developed by $M$. Fuentes at Sandia National Laboratories [Ref. 4.6]. The estimated miles used for estimating crash frequencies during the inflight phase of flight is based on analysis of military aircraft operations by Logicon RDA. 


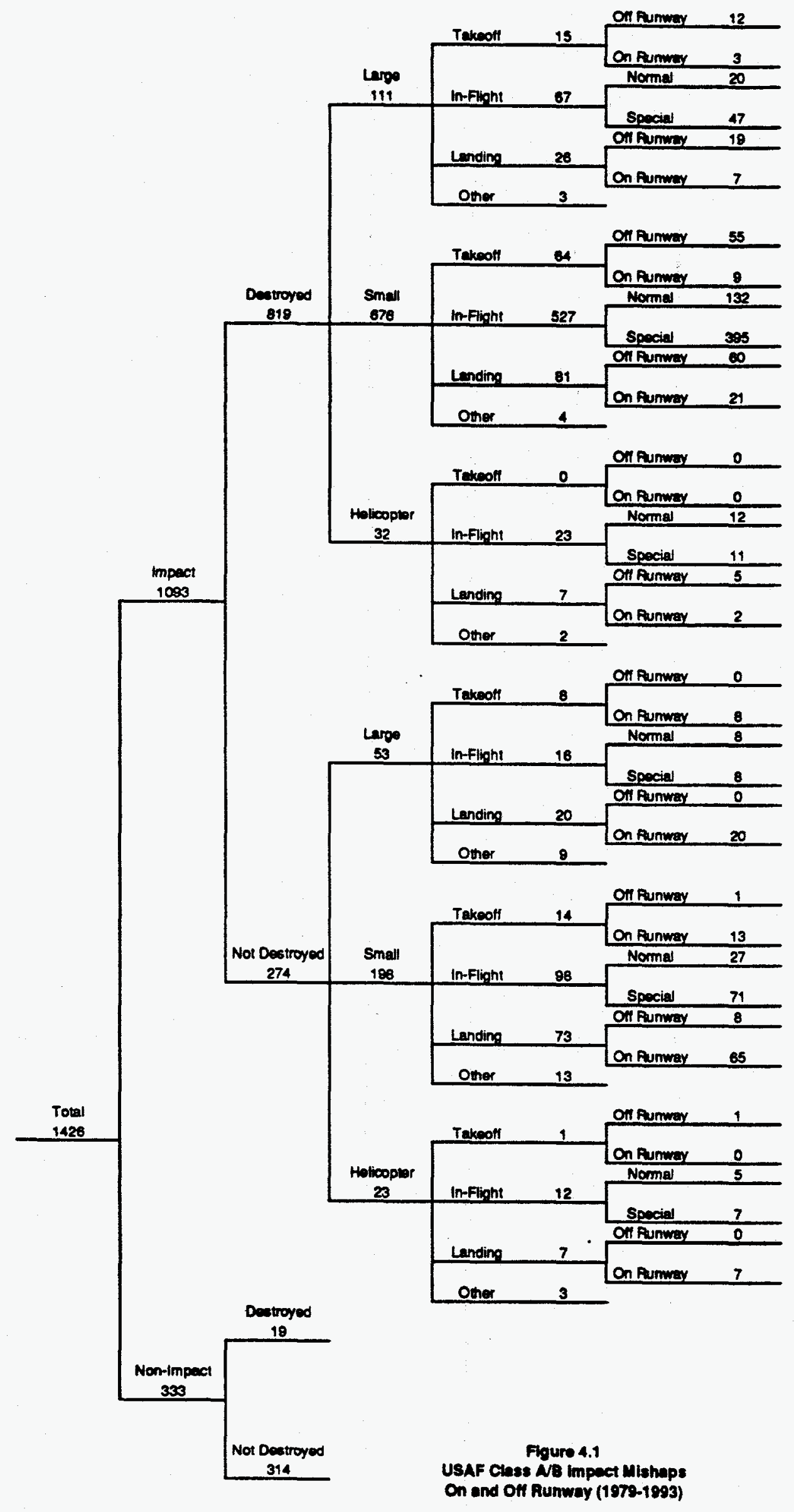


Table 4.1

Summary of U.S. Alr Force Crash Mishap History (Mishap Rates, per 100000 Flight Hours)

\begin{tabular}{|c|c|c|c|c|c|c|c|}
\hline Aircraft & Class A & Class A & Class B & Class B & Destroyed & Destroyed & Flight \\
\hline Model & Number & Rate & Number & Rate & Number & Rate & Hours \\
\hline A-7 & 101 & 5.71 & 24 & 1.36 & 102 & 5.77 & $1,768,958$ \\
\hline$A-10$ & 79 & 2.62 & 44 & 1.46 & 78 & 2.58 & $3,020,198$ \\
\hline A-37 & 36 & 4.92 & 6 & 0.82 & 32 & 4.38 & 730,977 \\
\hline B-1 & 10 & 5.25 & 8 & 4.20 & 4 & 2.10 & 190,551 \\
\hline $\mathrm{B}-2$ & o & 0.00 & 0] & 0.00 & 0 & 0.00 & 663 \\
\hline B-52 & 94 & 1.28 & 162 & 2.20 & 74 & 1.00 & $7,363,422$ \\
\hline C.5 & 15 & 1.04 & 34 & 2.37 & 4 & 0.28 & $1,436,120$ \\
\hline C.9 & 2 & 0.30 & 1 & 0.15 & 1 & 0.15 & 657,344 \\
\hline C-10 & 2 & 0.42 & 5 & 1.06 & 이 & 0.00 & 473,334 \\
\hline C.12 & 2 & 0.58 & 0 & 0.00 & 1 & 0.29 & 346,438 \\
\hline C-21 & 1 & 0.20 & 0 & 0.00 & 1 & 0.20 & 500,859 \\
\hline C. 130 & 138 & 1.03 & 139 & 1.03 & 79 & 0.59 & $13,449,405$ \\
\hline C.135 & 77 & 0.73 & 115 & 1.09 & 63. & 0.59 & $10,596,663$ \\
\hline C-141 & 32 & 0.33 & 28 & 0.29 & 14 & 0.14 & $9,715,118$ \\
\hline$F-4 / R F-4$ & 593 & 5.75 & 387 & 3.76 & 516 & 5.01 & $10,304,926$ \\
\hline$F-4$ & 468 & 5.80 & 308 & 3.82 & 411 & 5.09 & $8,066,975$ \\
\hline$F-5$ & 39 & 8.82 & 16 & 3.62 & 40 & 9.05 & 442,176 \\
\hline RF $-4 C$ & 125 & 5.59 & 79 & 3.53 & 105 & 4.69 & $2,237,767$ \\
\hline$F-15$ & 83 & 2.74 & 127 & 4.19 & 80 & 2.64 & $3,031,730$ \\
\hline$F-16$ & 201 & 5.06 & 24 & 0.60 & 191 & 4.81 & $3,971,946$ \\
\hline$F-106$ & 151 & 9.35 & 61 & 3.78 & 118 & 7.31 & $1,614,846$ \\
\hline F-111 & 113 & 6.23 & 94 & 5.18 & 94 & 5.18 & $1,814,333$ \\
\hline F-117 & 1 & 2.01 & 2 & 4.03 & 1) & 2.01 & 49,640 \\
\hline$T-33$ & 2,351 & 13.67 & 589 & 3.43 & 1,189 & 6.92 & $17,193,534$ \\
\hline$T-37$ & 131 & 1.15 & 31 & 0.27 & 129 & 1.13 & $11,439,050$ \\
\hline T-38 & 183 & 1.57 & 90 & 0.77 & 180 & 1.54 & $11,664,996$ \\
\hline T-39 & 29 & 1.10 & 21 & 0.80 & 12 & 0.45 & $2,641,197$ \\
\hline$T-41$ & 7 & 1.16 & 5 & 0.83 & 3 & 0.50 & 602,788 \\
\hline $\mathrm{H}-1$ & 48 & 3.35 & 14 & 0.98 & 34 & 2.37 & $1,434,316$ \\
\hline $\mathrm{H}-3$ & 31 & 4.29 & 20 & 2.77 & 21 & 2.91 & 722,328 \\
\hline $\mathrm{H}-53$ & 24 & 6.69 & 15 & 4.18 & 18 & 5.01 & 359,002 \\
\hline $\mathrm{H}-60$ & 4 & 3.74 & 1 & 0.93 & 3 & 2.80 & 107,083 \\
\hline Totals: & 5,171 & 4.04 & 2.450 & 1.91 & 3,598 & 2.81 & $127,948,683$ \\
\hline
\end{tabular}


Table 4.2

Summary of Small (High-Performance) Aircraft Mlshap History

(Mlshap Rates, per 100000 fllght Hours)

\begin{tabular}{|c|c|c|c|c|c|c|c|}
\hline Aircraft & Class A & Class A & Class B & Class B & Destroyed & Destroyed & Flight \\
\hline Model & Number & Rate & Number & Rate & Number & Rate & Hours \\
\hline A.7 & 101 & 5.71 & 24 & 1.36 & 102 & 5.77 & $1,768,958$ \\
\hline$A-10$ & 79 & 2.62 & 44 & 1.46 & 78 & 2.58 & $3,020,198$ \\
\hline A-37 & 36 & 4.92 & 6 & 0.82 & 32 & 4.38 & 730,977 \\
\hline F-4/RF-4 & 593 & 5.75 & 387 & 3.76 & 516 & 5.01 & $10,304,926$ \\
\hline$F-4$ & 468 & 5.80 & 308 & 3.82 & 411 & 5.09 & $8,066,975$ \\
\hline F.5 & 39 & 8.82 & 16 & 3.62 & 40 & 9.05 & 442,176 \\
\hline RF-4C & 125 & 5.59 & 79 & 3.53 & 105 & 4.69 & $2,237,767$ \\
\hline F.15 & 83 & 2.74 & 127 & 4.19 & 80 & 2.64 & $3,031,730$ \\
\hline$F-16$ & 201 & 5.06 & 24 & 0.60 & 191 & 4.81 & $3,971,946$ \\
\hline$F-106$ & 151 & 9.35 & 61 & 3.78 & 118 & 7.31 & $1,614,846$ \\
\hline F-111 & 113 & 6.23 & 94 & 5.18 & 94 & 5.18 & $1,814,333$ \\
\hline$F-117$ & 1 & 2.01 & 2 & 4.03 & 1 & 2.01 & 49,640 \\
\hline T-33 & 2,351 & 13.67 & 589 & 3.43 & 1,189 & 6.92 & $17,193,534$ \\
\hline$T-37$ & 131 & 1.15 & 31 & 0.27 & 129 & 1.13 & $11,439,050$ \\
\hline$T-38$ & 183 & 1.57 & 90 & 0.77 & 180 & 1.54 & $11,664,996$ \\
\hline T.39 & 29 & 1.10 & 21 & 0.80 & 12 & 0.45 & $2,641,197$ \\
\hline$T-41$ & 7 & 1.16 & 5 & 0.83 & 3 & 0.50 & 602,788 \\
\hline Totals: & 4,691 & 5.82 & 1,908 & 2.37 & 3,281 & 4.07 & $80,596,037$ \\
\hline & & & & & & & \\
\hline \multicolumn{8}{|c|}{ Calculation of crash rate without T-33. } \\
\hline Totals: & 2,340 & 3.69 & 1,319 & 2.08 & 2,092 & 3.30 & $63,402,503$ \\
\hline
\end{tabular}

-T-33 excluded from crash rate calculation due to its withdrawal from active service. 
Table 4.3

Summary of Large (Bomber and Cargo) Aircraft Mishap History

(Mishap Rates, per 100000 Fllght Hours)

\begin{tabular}{|l|r|r|r|r|r|r|r|}
\hline Aircratt & \multicolumn{1}{|c|}{ Class A } & \multicolumn{1}{c|}{ Class A } & \multicolumn{1}{c|}{ Class B } & \multicolumn{1}{c|}{ Class B } & Destroyed & Destroyed & \multicolumn{1}{c|}{ Flight } \\
\hline Model & Number & \multicolumn{1}{|c|}{ Rate } & Number & Rate & Number & Rate & \multicolumn{1}{c|}{ Hours } \\
\hline B-1 & 10 & 5.25 & 8 & 4.20 & 4 & 2.10 & 190,551 \\
\hline B-2 & 0 & 0.00 & 0 & 0.00 & 0 & 0.00 & 663 \\
\hline B-52 & 94 & 1.28 & 162 & 2.20 & 74 & 1.00 & $7,363,422$ \\
\hline C-5 & 15 & 1.04 & 34 & 2.37 & 4 & 0.28 & $1,436,120$ \\
\hline C-9 & 2 & 0.30 & 1 & 0.15 & 1 & 0.15 & 657,344 \\
\hline C-21 & 1 & 0.20 & 0 & 0.00 & 1 & 0.20 & 500,859 \\
\hline C-130 & 138 & 1.03 & 139 & 1.03 & 79 & 0.59 & $13,449,405$ \\
\hline C-135 & 77 & 0.73 & 115 & 1.09 & 63 & 0.59 & $10,596,663$ \\
\hline C-141 & 32 & 0.33 & 28 & 0.29 & 14 & 0.14 & $9,715,118$ \\
\hline & & & & & & & \\
\hline Totals: & 369 & 0.84 & 487 & 1.11 & 240 & 0.55 & $43,910,145$ \\
\hline
\end{tabular}

Table 4.4

Summary of Helicopter Aircraft Mishap History

(Mishap Rates, per 100000 Flight Hours)

\begin{tabular}{|l|r|r|r|r|r|r|r|}
\hline Aircraft & \multicolumn{1}{|c|}{ Class A } & \multicolumn{1}{c|}{ Class A } & Class B & \multicolumn{1}{c|}{ Class B } & Destroyed & Destroyed & \multicolumn{1}{c|}{ Flight } \\
\hline Model & Number & \multicolumn{1}{|c|}{ Rate } & Number & Rate & Number & Rate & Hours \\
\hline $\mathrm{H}-1$ & 48 & 3.35 & 14 & 0.98 & 34 & 2.37 & $1,434,316$ \\
\hline $\mathrm{H}-3$ & 31 & 4.29 & 20 & 2.77 & 21 & 2.91 & 722,328 \\
\hline $\mathrm{H}-53$ & 24 & 6.69 & 15 & 4.18 & 18 & 5.01 & 359,002 \\
\hline $\mathrm{H}-60$ & 4 & 3.74 & 1 & 0.93 & 3 & 2.80 & 107,083 \\
\hline & & & & & & & \\
\hline Totals: & 107 & 4.08 & 50 & 1.91 & 76 & 2.90 & $2,622,729$ \\
\hline
\end{tabular}


Table 4.5

Mishaps and Estimates of Crash Frequencles Based on Impact Mishaps,

Dlsregarding Damage Condition of the Crashing Alreraft

\begin{tabular}{|c|c|c|c|c|c|c|c|c|c|c|c|c|c|c|c|c|}
\hline \multirow[b]{2}{*}{$\begin{array}{c}\text { Aircraft } \\
\text { Subcategony }\end{array}$} & \multirow[b]{2}{*}{$\begin{array}{c}\text { Number } \\
\text { Operations }\end{array}$} & \multicolumn{4}{|c|}{ TAKEOFF } & \multicolumn{4}{|c|}{ LANDING } & \multirow[b]{2}{*}{$\begin{array}{l}\text { Fllght } \\
\text { Hours }\end{array}$} & \multicolumn{3}{|c|}{ NOPMAL IN-FLIGHT } & \multicolumn{3}{|c|}{ SPECIAL OPERATIONS } \\
\hline & & \multicolumn{2}{|c|}{ Number of Mishaps } & \multicolumn{2}{|c|}{ Crash Rate/Takeoff } & \multicolumn{2}{|c|}{ Number of Mishaps } & \multicolumn{2}{|c|}{ Crash Ratelanding } & & \begin{tabular}{|c|}
$\begin{array}{c}\text { Estlmated } \\
\text { Milles }\end{array}$ \\
\end{tabular} & \begin{tabular}{|c|} 
No. of \\
Mishaps \\
\end{tabular} & $\begin{array}{c}\text { Crashes: } \\
\text { Mile } \\
\end{array}$ & $\begin{array}{l}\text { Estmated } \\
\text { Miles }\end{array}$ & $\begin{array}{c}\text { No. of } \\
\text { Mishaps }\end{array}$ & $\begin{array}{c}\text { Crashes/ } \\
\text { Mille } \\
\end{array}$ \\
\hline Large & $15,337,472$ & 11 & 12 & 7.17E- -07 & $7.82 E-07$ & 27 & 19 & 1.76E-06 & $1.24 E-06$ & $19,435,867$ & $4.50 E+09$ & 28 & 6.22E-09 & $4.50 E+09$ & 55 & $1.22 E-08$ \\
\hline Small & $41,245,806$ & 22 & 56 & 5.33E-07 & $1.36 E-06$ & 86 & 68 & $2.09 E-06$ & 1.65E-06 & $29,591,424$ & $40 E+09$ & 159 & $2.48 E-08$ & $7.10 E+09$ & 466 & 6.56E-08 \\
\hline Hellicopter & $2,933,967$ & 0 & 1 & $<3.4 E-7$ & 3.41E-07 & 9 & 5 & 3.07E- -06 & $1.70 E-06$ & $1,209,057$ & $7.00 E+07$ & 17 & $2.43 E-07$ & $7.70 E+07$ & 18 & $2.34 E-07$ \\
\hline Milltary & $59,517,245$ & 33 & 69 & 5.54E-07 & $1.16 E-06$ & 122 & 92] & $2.05 E-06$ & $1.55 E-06$ & $50,236,348$ & $1.10 E+10$ & 204 & $1.86 E-08$ & $1.17 E+10$ & 5391 & $4.62 E-08$ \\
\hline
\end{tabular}

\section{Table 4.6}

Mlshaps and Estlmates of Crash Frequencles Based on Impact Mlshape In Which the Crashing Aircraft Was Destroyed

\begin{tabular}{|c|c|c|c|c|c|c|c|c|c|c|c|c|c|c|c|c|}
\hline \multirow{3}{*}{$\begin{array}{c}\text { Alrcraft } \\
\text { Subcategory }\end{array}$} & \multirow{3}{*}{$\begin{array}{l}\text { Number } \\
\text { Operations }\end{array}$} & \multicolumn{4}{|c|}{ TAKEOFF } & \multicolumn{4}{|c|}{ LANDING } & & \multicolumn{3}{|c|}{ NORMAL INFUGHT } & \multicolumn{3}{|c|}{ SPECIAL OPEAATIONS } \\
\hline & & \multicolumn{2}{|c|}{ Number of Mishaps } & \multicolumn{2}{|c|}{ Crash Rate/Takeoff } & \multicolumn{2}{|c|}{ Number of Mishaps } & \multicolumn{2}{|c|}{ Crash Ratelanding } & \multirow{2}{*}{$\begin{array}{l}\text { Fllght } \\
\text { Hours }\end{array}$} & \multirow{2}{*}{\begin{tabular}{|c|} 
Estimated \\
Milies \\
\end{tabular}} & \multirow{2}{*}{$\begin{array}{c}\text { No. of } \\
\text { Mishaps }\end{array}$} & \multirow{2}{*}{$\begin{array}{c}\text { Crashes } \\
\text { Mllo } \\
\end{array}$} & \multirow{2}{*}{$\begin{array}{c}\text { Estimated } \\
\text { Mlles }\end{array}$} & \multirow{2}{*}{$\begin{array}{c}\text { No. of } \\
\text { Mishaps }\end{array}$} & \multirow{2}{*}{$\begin{array}{c}\text { Crashes } \\
\text { Mile }\end{array}$} \\
\hline & & On RW & OH RW & On RW & Off RW & On RW & Off RW & On RW & Off RW & & & & & & & \\
\hline Large & $15,337,472$ & 3 & 12 & $1.96 \mathrm{E}-07$ & $7.82 E-07$ & 7 & 19 & 4.56E-07 & 1.24E-06 & $19,435,867$ & $4.50 E+09$ & 20 & 4.44E-09 & $4.50 E+09$ & 47 & $1.04 E-08$ \\
\hline Small & $41,245,806$ & 9 & 55 & 2.18E-07 & $1.33 E-06$ & 21 & 60 & $5.09 E-07$ & $1.45 E-06$ & $29,591,424$ & $6.40 E+09$ & 132 & $2.06 E-08$ & $7.10 E+09$ & 395 & 5.56E-08 \\
\hline Hellcopler & $2,933,967$ & 0 & 0 & $<3.4 E-7$ & $<3.4 E-7$ & 2 & 5 & 6.82E-07 & $1.70 E-06$ & $1,209,057$ & $7.00 E+07$ & 12 & 1.71E-07 & $7.70 E+07$ & 11 & $1.43 E-07$ \\
\hline All Milltary & $59,517,245$ & 12 & 67 & 2.02E-07 & $1.13 E-06$ & 30 & 84 & 5.04E-07 & $1.41 E-06$ & $50,236,348$ & $1.10 E+10$ & 164 & $1.49 E-08$ & $1.17 E+10$ & 453 & 3.8BE-08 \\
\hline
\end{tabular}


Table 4.7

Crash Data and Estimates of Crash Frequencies Based on the Minuteman III Mishap Database

\begin{tabular}{|c|c|c|c|c|c|c|c|c|c|c|c|c|}
\hline & & & \multirow{3}{*}{$\begin{array}{l}\text { Number of } \\
\text { Landings }\end{array}$} & \multicolumn{4}{|c|}{ TAKEOFF } & \multicolumn{4}{|c|}{ LANOING } \\
\hline & & & & & \multicolumn{2}{|c|}{ Number of Crashes } & \multicolumn{2}{|c|}{ Crash Rate/Takeoff } & \multicolumn{2}{|c|}{ Number of Crashes } & \multicolumn{2}{|c|}{ Crash Raten anding } \\
\hline & & & & & On AW & Off RW & On RW & Off AW & On RW & OH RW & On RW & Off RW \\
\hline \multirow{7}{*}{ Lange } & \multicolumn{2}{|l|}{ Bombers (Bs) } & $B 1, B 52, B 57$ & $1,072,000$ & 3] & 2 & $2.80 E-06$ & 1.87E-06 & 2 & 6 & $1.87 E-06$ & $5.60 E-06$ \\
\hline & \multirow{6}{*}{\multicolumn{2}{|c|}{ Cargo (Cs) }} & $C 130$ & $6,341,000$ & 1 & 2 & $1.58 \mathrm{E}-07$ & $3.15 E-07$ & 8 & 8 & $1.26 E-06$ & $1.26 E-06$ \\
\hline & & & KC135 & $3,512,000$ & 3 & 4 & 8.54E-07 & 1.14E-06 & 5 & 8 & $1.42 E-06$ & $2.28 E-06$ \\
\hline & & & Other Large Cs & $4,932,000$ & of & 2 & $<2.0 \mathrm{E}-7$ & $4.06 E-07$ & 11 & 5 & 2.23E-06 & $1.01 E-06$ \\
\hline & & & Small Cs & $1,628,000$ & 0] & 0 & $<6.1 \mathrm{E}-7$ & $<6.1 \mathrm{E}-7$ & 2 & 1 & 1.23E-06 & 6.14E-07 \\
\hline & & & All Cargo & $16,413,000$ & 4 & 8 & 2.44E-07 & 4.87E- -07 & 26 & 22 & $1.58 \mathrm{E}-06$ & 1.34E-06 \\
\hline & & & All Large (Bs, C8) & $17,485,000$ & 7 & 10 & $4.00 E-07$ & 5.72E-07 & 28 & 28 & $1.60 E-06$ & $1.60 E-06$ \\
\hline \multirow{15}{*}{ Small } & \multirow{8}{*}{$\begin{array}{l}\text { Attack (As) } \\
\text { Fighters (Fs) }\end{array}$} & \multirow{3}{*}{$\begin{array}{l}\text { Single } \\
\text { Engine }\end{array}$} & A7 & 940,000 & 2 & 9 & $2.13 E-06$ & 9.57E-06 & 12 & 5 & $1.28 E-05$ & 5.32E-06 \\
\hline & & & F16 & $2,818,000$ & 2 & 16 & $7.10 E-07$ & 5.68E-06 & 24 & 34 & 8.52E-06 & 1.21E-05 \\
\hline & & & All Single-engine & $3,758,000$ & 4) & 25 & $1.06 \mathrm{E}-06$ & $6.65 \mathrm{E}-06$ & 36 & 39 & $9.58 E-06$ & 1.04E-05 \\
\hline & & \multirow{7}{*}{$\begin{array}{l}\text { Multiple } \\
\text { Engine }\end{array}$} & A10 & $1,738,000$ & of & 2 & $<5.8 \mathrm{E}-7$ & 1.15E-06 & 9 & 10 & $5.18 E-06$ & 5.75E-06 \\
\hline & & & $F_{4}$ & $4,462,000$ & 7 & 21 & 1.57E-06 & 4.71E-06 & 41 & 24 & $9.19 E-06$ & 5.38E-06 \\
\hline & & & F15 & $2,235,000$ & 1 & 5 & 4.47E-07 & 2.24E-06 & 17. & 7 & 7.61E-06 & 3.13E-06 \\
\hline & & & F111, FB111 & 891,000 & 1 & 5 & $1.12 E-06$ & 5.61E-06 & 8 & 14 & $8.98 E-06$ & 1.57E-05 \\
\hline & & & All Multiplo-engine & $9,326,000$ & 9 & 33 & $9.65 \mathrm{E}-07$ & 3.54E-06 & 75 & 55 & 8.04E-06 & $5.90 \mathrm{E}-06$ \\
\hline & & & Other As, Fs & $1,677,000$ & 5 & 12 & $2.98 E-06$ & 7.16E-06 & 23 & 26 & $1.37 E-05$ & 1.55E-05 \\
\hline & & & $\mathrm{All}_{\mathrm{As}, \mathrm{F}_{\mathrm{8}}}$ & $14,761,000$ & 18 & 70 & $1.22 E-06$ & 4.74E-06 & 134 & 120 & $9.08 E-06$ & $8.13 E-06$ \\
\hline & \multirow{4}{*}{\multicolumn{2}{|c|}{ Trainers (Ts) }} & Subsonk: T37 & $14,147,000$ & 1 & 4 & 7.07E-.08 & $2.83 E-07$ & 2 & 6 & 1.41E-07 & 4.24E-07 \\
\hline & & & Supersonic: T38 & $15,118,000$ & 6 & 9 & 3.97E-07 & 5.95E-07 & 17 & 20 & $1.12 E-06$ & $1.32 E-06$ \\
\hline & & & Other T8 & $2,896,000$ & 3 & 3 & $1.04 E-06$ & 1.04E--06 & 4. & 8 & $1.38 E-06$ & $2.76 E-06$ \\
\hline & & & All Ts & $32,161,000$ & 10 & 16 & $3.11 E-07$ & 4.97E-07 & 23) & 34 & 7.15E-07 & $1.06 E-06$ \\
\hline & & & All Small $\left(A_{8}, F_{8}, T_{8}\right)$ & $46,922,000$ & 28 & 86 & \begin{tabular}{|l|}
$5.97 E-07$ \\
\end{tabular} & $1.83 E-06$ & 157 & 154 & $3.35 E-06$ & $3.28 E-06$ \\
\hline \multicolumn{3}{|c|}{ Hellcopter } & & $3,507,000$ & 2 & 3 & $5.70 \mathrm{E}-07$ & 8.55E-07 & 2 & 10 & $5.70 E-07$ & 2.85E-06 \\
\hline \multicolumn{3}{|c|}{ All Military Arcreft } & & $67,914,000$ & 37 & 99 & 5.45E-07 & $1.46 E-06$ & 187 & 192 & $2.75 E-06$ & 2.83E-06 \\
\hline
\end{tabular}


Table 4.8

Crash Data and Estimates of Crash Frequencies Based on the Minuteman III Mishap Database

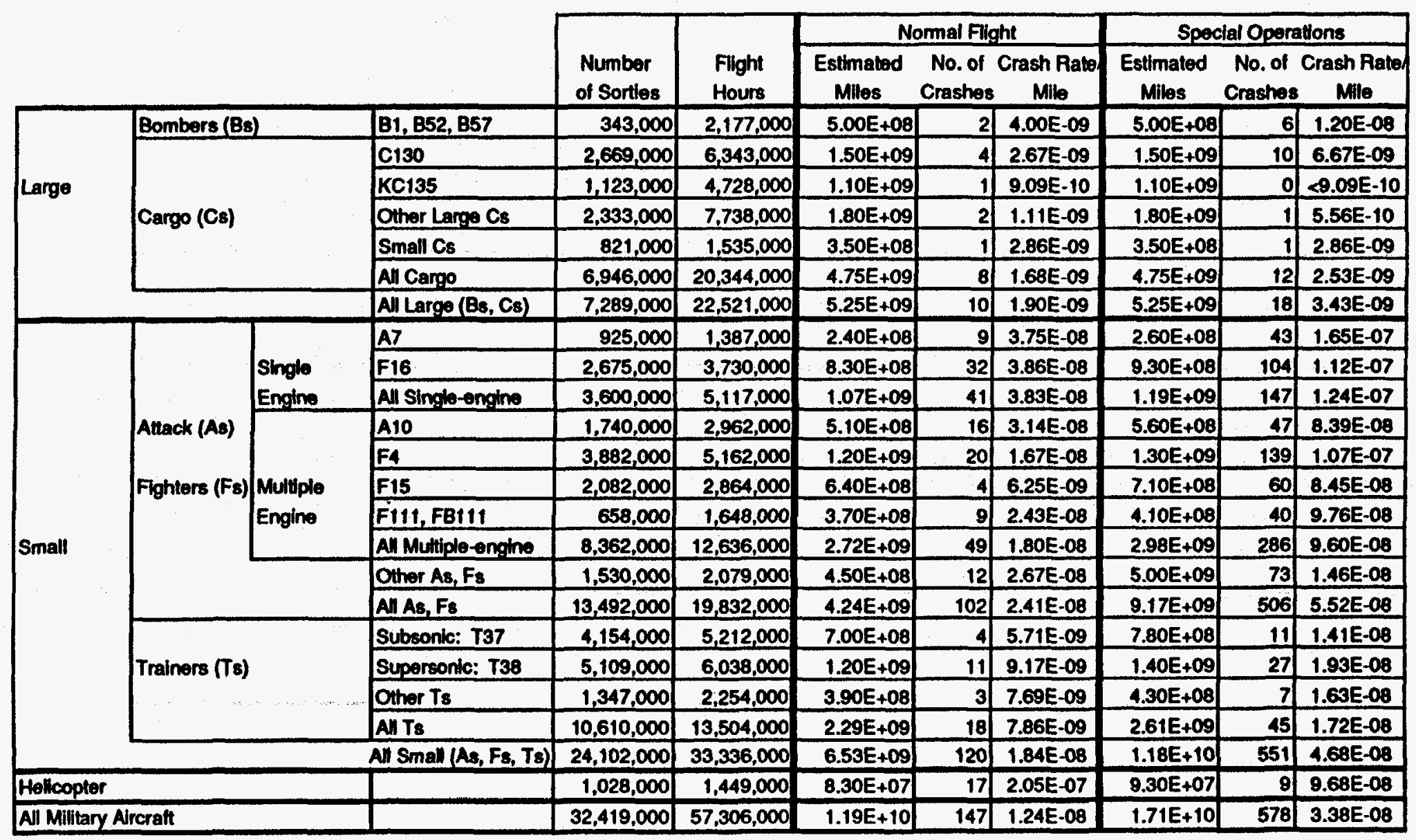




\subsection{MILITARY AVIATION CRASH PARAMETER DISTRIBUTIONS}

In addition to an assessment of crash frequencies and lovel of activity (i.e., numbers of flights), an analysis of the effects of a crashing aircraft on the safety of a facility requires a description of crash conditions, given a crash. Relevant crash parameters include crash locations as well as crash kinematics, ө.g., crash velocity, impact angle, etc. Since crash locations and crash kinematics vary between crashes, these variables are considered stochastic (random) variables. Descriptions of the crash conditions are based on developing probability distributions for each of the crash parameters. The specitic crash parameters considered relevant for this Standard are
1. crash location
2. impact angle of the crashing aircraft
3. air speed and horizontal velocity of the crashing aircraft
4. skid distance of the aircraft, given a crash.

The data used to develop probability distributions for the crash parameters was extracted from the crash information in the database developed for the Minuteman III WSSA [Ref. 4.2]. The data in the database was derived from Air Force mishap reports covering the 1976-1993 time period. Quantification of the crash parameters trom the mishap descriptions in the reports often required considerable judgment. In addition, information about some crash parameters is very limited. Thus, there is considerable uncertainty associated with the probability distributions derived from the available data. Following the philosophy stated earlier, this uncertainty is not buitt into the distributions provided, rather the distributions provided are considered plausible estimates of reality.

A variety of statistical techniques were used to analyze the data and develop the probability distributions. Both parametric and non-parametric methods were used. The choice of the final distribution selected as the estimated distribution was primarily based on analyst judgment although some statistical tests of "goodness of fit" were applied. The 2-dimensional crash locations are correlated. Because of the scarcity of data, a non-parametric bivariate kernel estimation technique [Ret. 4.8] was used to develop the crash location density function. The same technique was used to develop the joint distributions of impact angle and crash velocity, and horizontal velocity and skid distance, which were also considered correlated. For the distribution of heading angle and the marginal distributions of impact angle, crash velocity and skid distance, a parametric fit to the data was attempted. Initially, a computer code, Best Fit [Ret. 4.9], was used to select an appropriate family of probability distributions. Then, either (1) the distributional parameter values provided by the program were used; or (2) if applicable, when truncated distributions are appropriate, maximum likelihood estimates of the distributional parameters, were developed.

Distributions were developed for the following classes of military aircraft types and flight phases:

Large aircraft, takeoff

Large aircraft, landing

Small aircraft, takeoff

Small aircraft, landing

Analysis of helicopter crash parameter data, derived from Air Force and Army mishap reports, is in progress.

Section 4.3.1 provides a summary of crash bcation analysis and probability distributions. The analysis and distributions for impact angle and the cotangent of the impact angle are discussed in Section 4.3.2. The air speed and horizontal velocity of the crashing aircraft is covered in Section 4.3.3. The marginal distribution of skid distance is the topic of Section 4.3.4. 


\subsubsection{CRASH LOCATION DISTRIBUTIONS}

Before describing the probability distributions of crash locations, it is necessary to specity the coordinate convention used to quantity locations. For military aviation, crash locations are quantitied in Cartesian coordinates consistent with the coordinate convention established for the Standard, specifically:

1. The origin of the coordinate system is at the center of the runway.

2. The $x$ axis of the coordinate system coincides with the extended centerline of the rumway such that the positive direction is the direction of tlight during takeoff and landing.

3. The positive y axis coincides with a line defined by a 90 degree counterclockwise rotation of the positive $x$ axis.

For military aviation, landings and operations at an aibase, e.g., touch and go's, involve flight patterns associated with each runway. At each airbase there is a well defined side of the rumway for flight pattems. Since more traffic exists on the pattern side of the rumway, crash locations tend to be biased to that side as well. For descriptive purposes in this document, the pattern side is assumed to be to the right of the direction of flight, i.e., in the negative y direction. As discussed in the Standard, in applying crash location distributions it is appropriate to identify where the facility of interest exists relative to the pattern side of the source numay. At non-military airfields, unless there is a well established pattern side, a conservative analysis would be based on assuming that the facility is on the pattern side.

Crash locations in the Minuteman III WSSA database, derived from mishaps reports about Air Force aircraft crashes between 1976 and 1994, were used to develop conditional, given a crash, distributions of crash location in terms of the joint distribution of the $x, y$ coordinates of the crash location. A nonparametric kernel estimation technique (Ref. 4.8) was used to model the joint distributions. Because of differences in flight patterns and operations, separate crash location distributions were developed for:

- Large aircraft, landing

- Large aircraft, takeoft

- Small aircraft, landing

- Small aircratt, takeotf

Plots of the estimated joint distributions are shown in Figures 4.2 to 4.5.

For application in the crash frequency calculations, the crash location distributions are used to assess the probability, per unit area, of an aircraft crashing into a given location, given the aircraft crashes. Tables of these probabilities, per square mile, are given in Tables 4.9 to 4.12 for the four combinations of aircraft size and phase of flight. The entries in the tables were computed by integrating the appropriate joint probability distributions. 


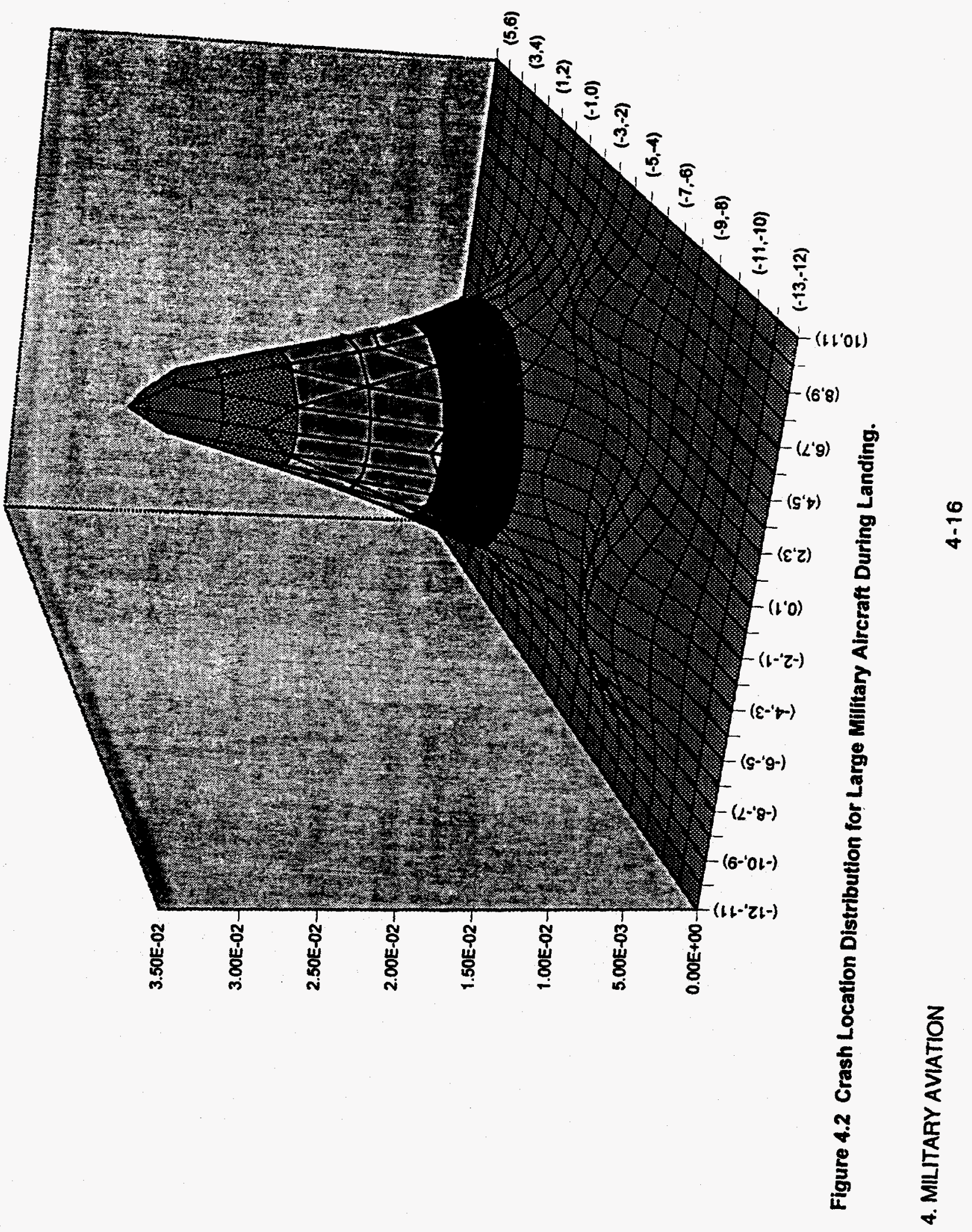




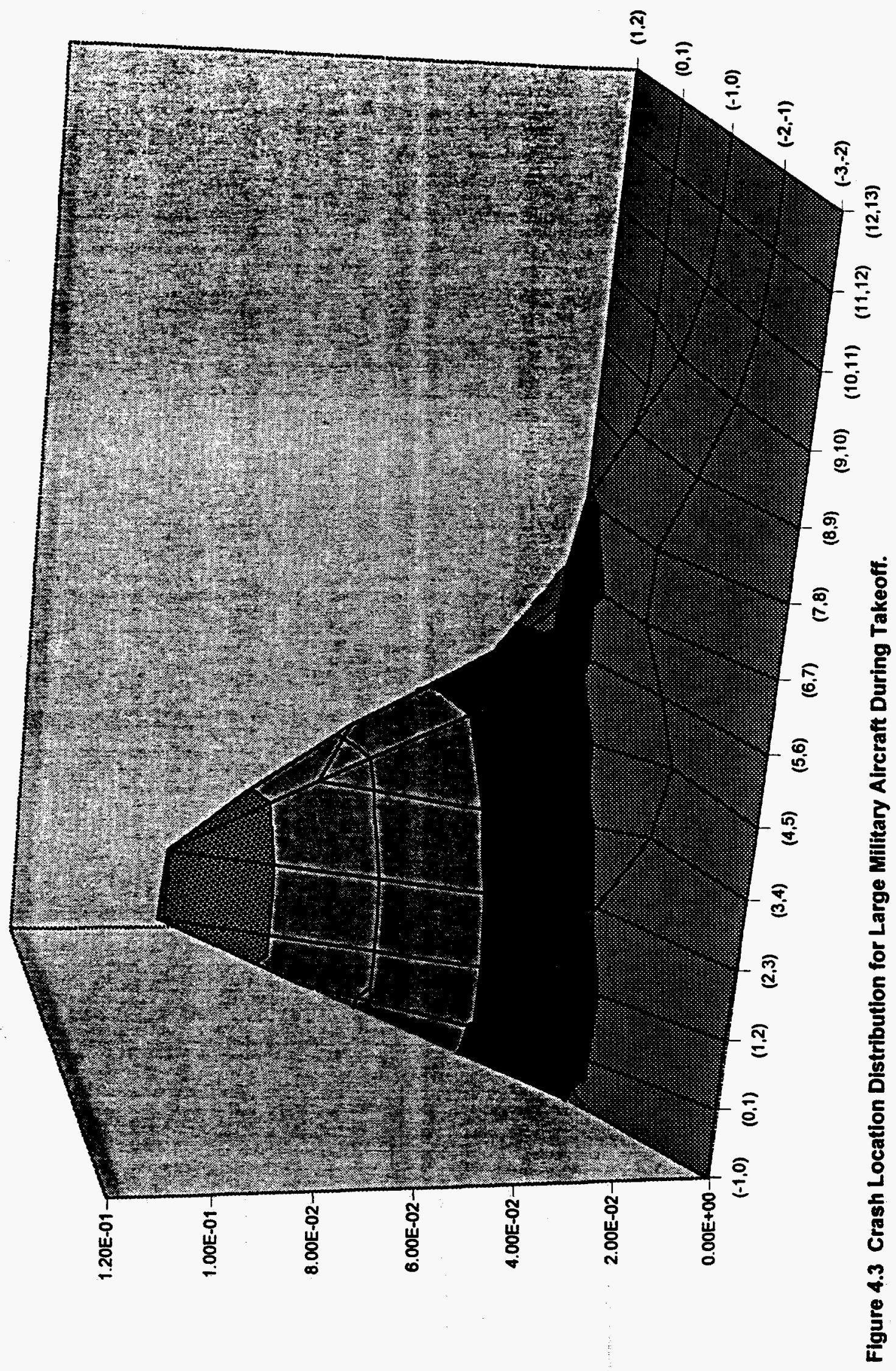

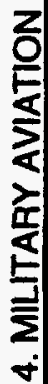




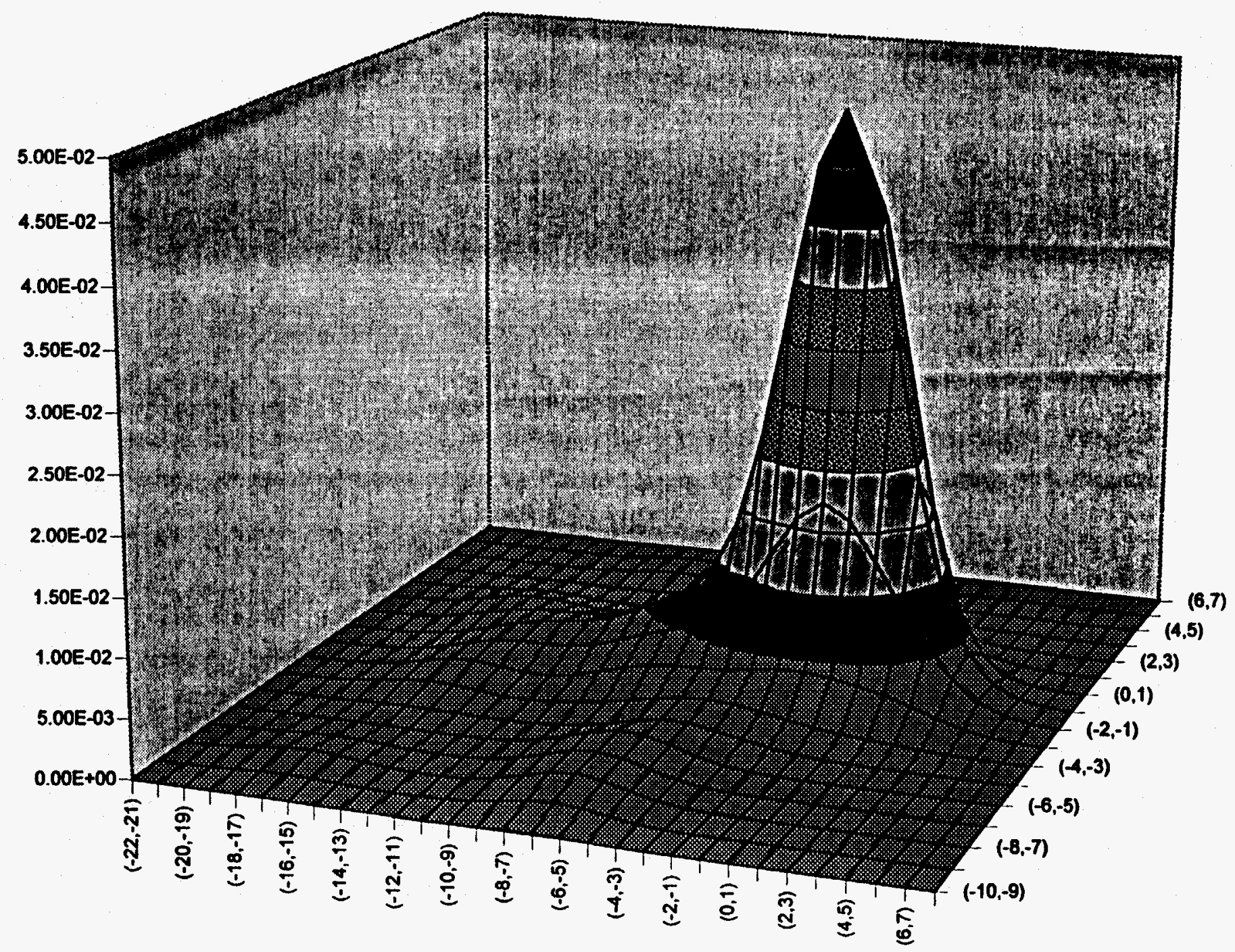

Figure 4.4 Crash Location Distribution for Small Military Aircraft During Landing. 


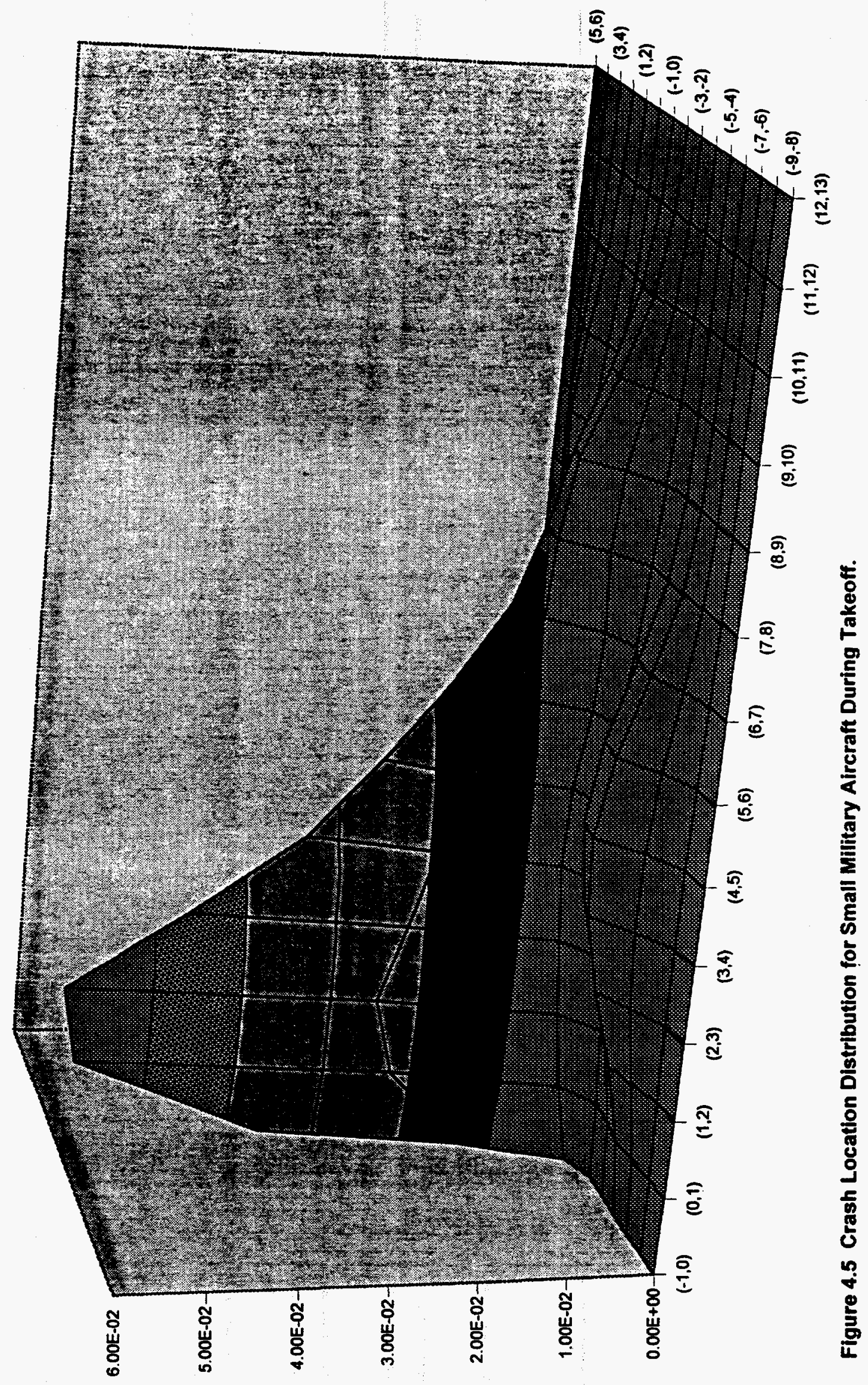




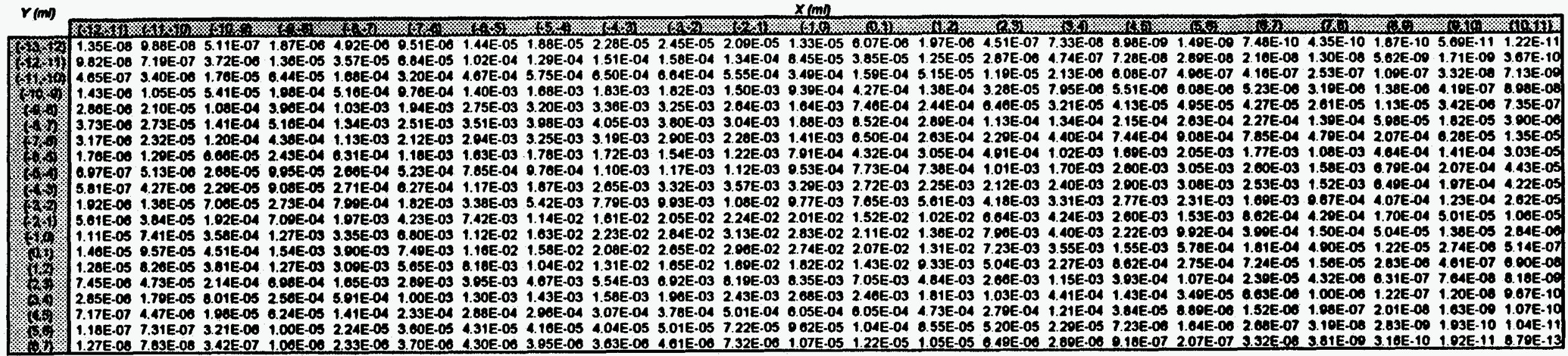

Table 4.9 Per Square Mile Crash Location Probability, Oiven a Crash, for Large Mulitary Alrctaft Ouring Lunding

romis

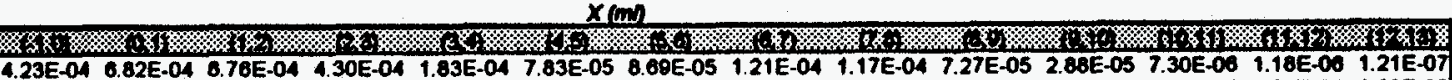

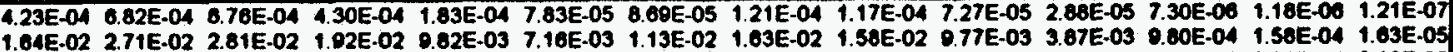

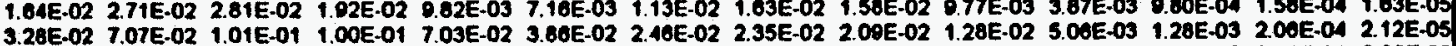

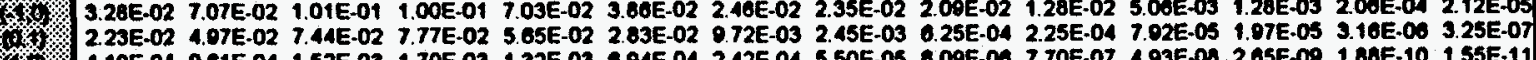

Table 4.10 Per Square Mile Crash Location Probability, Oiven a Crash, for Large Military Aireran During Takeoff 


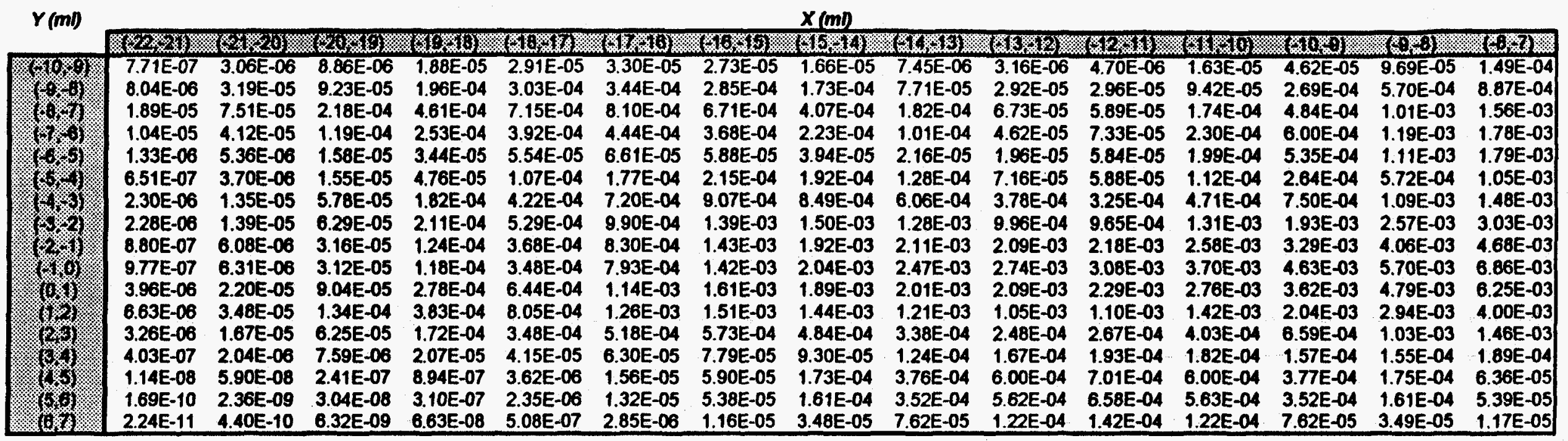

\begin{tabular}{|c|c|c|c|c|c|c|c|c|c|c|c|c|c|c|c|}
\hline \multirow[t]{2}{*}{$Y(m)$} & \multicolumn{15}{|c|}{$(m)$} \\
\hline & 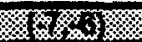 & $8 x^{2}$ & 怒 & rar & \% 6 & 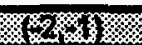 & 繁 & 008 & 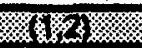 & 26 & 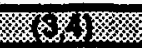 & 6 & 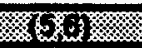 & 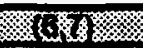 & 8 \\
\hline 663 & 1.67E-04 & $1.38 E-04$ & 8.36E-05 & 3.71E-05 & $1.21 E-05$ & $2.88 E-06$ & $5.03 E-07$ & $6.44 E-08$ & 6.05E-09 & $4.16 E-10$ & $2.09 E-11$ & 7.70E-13 & $2.08 E-14$ & $4.09 E-16$ & $3.92 E-18$ \\
\hline & & $1.52 E-03$ & $8.48 E-04$ & $\begin{array}{l}2.36 E-04 \\
4.37 E-04\end{array}$ & 1.49 & 3.7 & $\begin{array}{l}3.39 E-06 \\
6.89 E-06\end{array}$ & $\begin{array}{l}4.44 E-07 \\
9.39 E-07\end{array}$ & $9.46 t$ & $\begin{array}{l}2.99 E-09 \\
7.11 E-09\end{array}$ & & 1.98 & $\begin{array}{l}E-13 \\
E-13\end{array}$ & 0.8 & \\
\hline & & $1.75 \mathrm{E}$ & $1.15 E-03$ & & 2.1 & 5.9 & 1.31E-05 & -06 & 3.58 & 5.38E-08 & & 1.22 & & & \\
\hline & 03 & 2.17 & $1.62 E-03$ & .04 & 4.4 & 1.7 & 6.2 & -05 & 6.1 & 1.6 & & 1.37 & 08 & 8.5 & \\
\hline & & & $1.86 \mathrm{E}-03$ & & 1.0 & & & -04 & & & & 7.1 & & 6. & \\
\hline & & 2.2 & 2.36 & & 1.8 & & & -04 & 5.8 & & & & & & \\
\hline & & $3.3 t$ & & 3.7 & & & 3.7 & & & & & & & 7.8 & \\
\hline & -03 & 6.43 & 8.79 & -02 & 1.5 & & 1.6 & -02 & & & & & & 1.6 & \\
\hline & .03 & 1.32 & 2.15 & 3.33 & 4.4 & & 4.4 & -02 & & & & & & & \\
\hline & 8 & 1.29 & 2.06 & $3.13 E-02$ & 4.1 & & & & & & & & & & \\
\hline & & 6.10 & 7.50 & 9.4 & 1.1 & 1.1 & 1.0 & 7.3 & & & & & & & \\
\hline & & 1.94 & 1.96 & 2.01E-03 & 2.08 & 2.0 & $1.7 \mathrm{~s}$ & 1.35E-03 & 8.35 & & & & & 2.0 & \\
\hline & 2 & 3.2 & 4.26 & 5.3 & 5.7 & 5.3 & 4.15 & $2.7 \epsilon$ & 1.53 & 6.8 & & & & & \\
\hline & & & & $E-05$ & -05 & & & 2.09E-05 & 1.0 & 4.00 & 1.2 & & & 7. & \\
\hline & & & & & & & & & & & & & & 1 & \\
\hline & $2.85 E-06$ & 5.12E-07 & $7,18 E-08$ & $1.40 E-08$ & 8.66E-09 & 6.55E-09 & $3.92 E-09$ & $1.82 E-09$ & $6.75 E-10$ & $2.08 E-10$ & 5.34E-11 & $1.12 E-11$ & 1.85E-12 & $2.34 E-13$ & $2.22 E^{-}$ \\
\hline
\end{tabular}

Table 4.11 Per Square Mile Crash Location Probabillty, Given a Crash, for Small Military Aircraft During Landing 


\begin{tabular}{|c|c|c|c|c|c|c|c|c|c|c|c|c|c|c|}
\hline \multirow[t]{2}{*}{$Y(m)$} & \multicolumn{14}{|c|}{$x(m)$} \\
\hline & (X) & OXX & 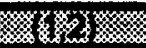 & (6x) & 6 & $(x)$ & (3) & 66 & 36 & (6) & 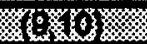 & KTtKX: & 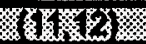 & (26) \\
\hline & $2.18 E-06$ & $9.79 \mathrm{E}-06$ & $2.50 \mathrm{E}-05$ & $3.85 E-05$ & 4.01E-05 & $3.45 E-05$ & $2.60 \mathrm{E}-05$ & $1.41 E-05$ & 4.77E-06 & $9.38 \mathrm{E}-07$ & $1.06 \mathrm{E}-07$ & $6.75 \mathrm{E}-09$ & $2.44 E-10$ & $5.00 \mathrm{E}-12$ \\
\hline 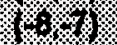 & 3 & 1.4 & 3.7 & 5.90 & 6.47 & 5.9 & 4 & 2.52 & 5 & 1.6 & 1.8 & 1.2 & 99 & \\
\hline & 1.45 & 6.68 & 1.7BE-03 & 2.97E-03 & 3.53 & 3.47 & 2.75 & 1.51E-03 & 5.0 & 1.0 & 1.1 & & & \\
\hline & & $1.03 E-03$ & 2.93E-03 & 5.45 & 7.35 & & 6.34 & $3.46 \mathrm{E}-03$ & & & & & & \\
\hline & & 5.77 & 1.87 & 4.10E-03 & 6.41 & 7.4 & 6.16 & $3.41 E-03$ & 1.2 & & & & & \\
\hline & $1.38 \mathrm{E}$ & $3.98 E-04$ & 9.40E-04 & $1.91 E-03$ & 3.07 & 3.60 & $3.07 E-03$ & $2.12 \mathrm{E}$ & 1.4 & 9.6 & & & & \\
\hline & $1.84 E-03$ & $3.51 E-03$ & $4.68 E-03$ & $5.44 E-03$ & 6.16 & 5.90 & $4.62 t$ & 4.20E-03 & & & & & & \\
\hline & 13 & 2.231 & 2.47 & 2.19 & 1.9 & & 1.2 & 9.78 & & & & & & \\
\hline & 3.61 & $5.82 E-02$ & 5.99E-02 & 4.63E-02 & 3.34 & 2.5 & 1.81 & $1.22 \mathrm{E}-02$ & 9.1 & & & & & \\
\hline & 3.51 & $5.55 E-02$ & 5.51E-02 & $3.98 \mathrm{E}-02$ & 2.57 & & 1.14 & $6.81 \mathrm{E}$ & & & & & & \\
\hline & 1230 & $1.94 E-02$ & 186 & & 7.6 & & & 2.13 & & & & & & \\
\hline & 1 & 2.63E-03 & $2.44 E$ & 1.5 & 8.46 & 5.5 & 6.29 & 1.40 & 2.7 & & & & & \\
\hline & & $1.44 E-04$ & 1.29 & 7.47 & & & & & & & & & & 1.45E-05 \\
\hline & & .06 & 2.58 & $1.42 E-06$ & 1.12 & 6.60 & 4.10 & $1.50 E$ & & & & & -05 & 2.78E-06 \\
\hline & $20 L-00$ & $97 E-08$ & $73 E-08$ & $1.06 E-08$ & $3.59 E-08$ & 3.73E-07 & $2.39 E-06$ & 8.78E-06 & $1.84 E-05$ & 2.19E-05 & & & & $1.63 E-07$ \\
\hline
\end{tabular}

Table 4.12 Per Square Mile Crash Location Probability, Given a Crash, for Small Military Aircraft During Takeoff 


\subsubsection{IMPACT ANGLE DISTRIBUTIONS}

The impact angle associated with a crashing aircraft is one of the crash kinematics involved in the crash frequency calculations. It is used in the effective area calculations in the form of the cotangent of the impact angle. It, combined with the crash velocity, determines the horizontal velocity of the aircraft at impact which, in turm, affects the distance a crashing aircraft skids prior to coming to its final location after a crash and which is an important input into analyses of the response of a structure to the impact of a crashing aircraft or the impact of a signiticant component, e.g., an engine, of the crashing aircraft. For purposes of describing the crash kinematics associated with military aviation in this Standard, the impact angle is defined as the angle between the velocity vector of the crashing aircraft and the horizontal, often referred to as the flight path angle.

Development of the marginal distribution of the impact angle and the cotangent of the impact angle, the variable used in the effective area calculation, are based on data in the Minuteman III WSSA database. Given the data, the computer code Best Fit (Ref 4.9) was used to select candidate families of distributions to describe the distribution of impact angles of future crashes. Statistical goodness of fit tests and analyst judgment were combined to select an appropriate family. Given the selected distributional family, maximum likelihood methodology was used to estimate values of the distributional parameters. For the impact angle, truncated lognomal distributions were chosen for large aircraft and beta distributions were chosen for small aircraft. For the cotangent of the impact angle, Weibull distributions were selected for both large and small aircraft.

Tabulated values of the cumulative probability distributions for the impact angle and the cotangent of the impact angle, for the four combinations of aircraft size and phase of operation, are given in Tables 4.13 and 4.14 respectively. 
Impact Angle

\begin{tabular}{|c|c|c|c|c|}
\hline \multirow[b]{2}{*}{$\begin{array}{l}\text { Cumulative } \\
\text { Probability }\end{array}$} & \multicolumn{2}{|c|}{ Large Aircraft } & \multicolumn{2}{|c|}{ Small Aircrant } \\
\hline & Landing & Takeoff & Landing & Takeoff \\
\hline $5 \%$ & 1.62 & 2.02 & 1.05 & 1.09 \\
\hline $10 \%$ & 2.46 & 2.98 & 1.07 & 1.52 \\
\hline $15 \%$ & 3.26 & 3.86 & 1.16 & 2.48 \\
\hline $20 \%$ & 4.07 & 4.74 & 1.37 & 4.08 \\
\hline $25 \%$ & 4.93 & 5.66 & 1.78 & 6.40 \\
\hline $30 \%$ & 5.84 & 6.63 & 2.49 & 9.53 \\
\hline $35 \%$ & 6.84 & 7.67 & 3.59 & 13.47 \\
\hline $40 \%$ & 7.95 & 8.81 & 5.20 & 18.23 \\
\hline $45 \%$ & 9.18 & 10.07 & 7.44 & 23.78 \\
\hline $50 \%$ & 10.57 & 11.48 & 10.44 & 30.02 \\
\hline $55 \%$ & 12.16 & 13.07 & 14.31 & 36.86 \\
\hline $60 \%$ & 14.01 & 14.91 & 19.17 & 44.14 \\
\hline $65 \%$ & 16.20 & 17.07 & 25.11 & 51.68 \\
\hline $70 \%$ & 18.84 & 19.66 & 32.19 & 59.26 \\
\hline $75 \%$ & 22.13 & 22.85 & 40.42 & 66.63 \\
\hline $80 \%$ & 26.37 & 26.93 & 49.75 & 73.51 \\
\hline $85 \%$ & 32.13 & 32.45 & 60.02 & 79.61 \\
\hline $90 \%$ & 40.63 & 40.56 & 70.87 & 84.61 \\
\hline $95 \%$ & 55.10 & 54.46 & 81.58 & 88.14 \\
\hline $100 \%$ & 80.00 & 90.00 & 90.00 & 90.00 \\
\hline
\end{tabular}

Table 4.13 Estimated Cumulative Distributions for Impact Angle
Cotangent of Impact Angle

\begin{tabular}{|c|c|c|c|c|}
\hline \multirow[b]{2}{*}{$\begin{array}{l}\text { Cumulative } \\
\text { Probability }\end{array}$} & \multicolumn{2}{|c|}{ Large Aircraft } & \multicolumn{2}{|c|}{ Small Aircrait } \\
\hline & Landing & Takeoff & Landing & Takeoff \\
\hline $5 \%$ & 0.24 & 0.45 & 0.09 & 0.02 \\
\hline $10 \%$ & 0.58 & 0.89 & 0.27 & 0.07 \\
\hline $15 \%$ & 0.98 & 1.34 & 0.52 & 0.17 \\
\hline $20 \%$ & 1.43 & 1.82 & 0.84 & 0.30 \\
\hline $25 \%$ & 1.95 & 2.32 & 1.22 & 0.48 \\
\hline $30 \%$ & 2.52 & 2.84 & 1.69 & 0.71 \\
\hline $35 \%$ & 3.17 & 3.40 & 2.23 & 1.01 \\
\hline $40 \%$ & 3.89 & 4.00 & 2.88 & 1.38 \\
\hline $45 \%$ & 4.70 & 4.65 & 3.64 & 1.85 \\
\hline $50 \%$ & 5.62 & 5.35 & 4.54 & 2.43 \\
\hline $55 \%$ & 6.66 & 6.13 & 5.61 & 3.16 \\
\hline $60 \%$ & 7.87 & 6.98 & 6.89 & 4.07 \\
\hline $65 \%$ & 9.27 & 7.95 & 8.44 & 5.24 \\
\hline $70 \%$ & 10.93 & 9.06 & 10.36 & 6.76 \\
\hline $75 \%$ & 12.95 & 10.36 & 12.78 & 8.77 \\
\hline $80 \%$ & 15.51 & 11.94 & 15.97 & 11.56 \\
\hline $85 \%$ & 18.90 & 13.97 & 20.41 & 15.68 \\
\hline $90 \%$ & 23.87 & 16.80 & 27.26 & 22.44 \\
\hline $95 \%$ & 32.78 & 21.58 & 40.37 & 36.54 \\
\hline $00 \%$ & 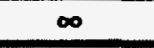 & $\infty$ & $\infty$ & $\infty$ \\
\hline
\end{tabular}

Table 4.14 Estimated Cumulative Distributions for Cotangent of Impact Angle 


\subsubsection{CRASH VELOCITY DISTRIBUTIONS}

Two other crash kinematic parameters are needed for the calculations associated with this Standard are the airspeed and horizontal velocity of the aircraft at the time of impact. Both of these parameters are necessary inputs into the structural response calculations. For purposes of describing the crash kinematics for military aviation in this Standard, the airspeed of the crashing aircraft is referred to as the crash velocity. The horizontal velocity is the crash velocity in the horizontal direction, i.e., the crash velocity adjusted by the cosine of the impact angle.

The Minuteman III WSSA database was the source of the data used in developing the distributions of the crash and horizontal velocities. The same analysis techniques as were used for the impact angle were used in selecting and estimating appropriate distributions for the velocities associated with future military aviation crashes. Lognormal distributions were selected for the crash velocity for both large and small aircraft. The logistic distribution seemed to be the best descriptor for the horizontal velocity.

Tabulated values of the cumulative probability distributions for the crash velocity and the horizontal velocity, for the four combinations of aircraft size and phase of operation, are given in Tables 4.15 and 4.16 respectively. 

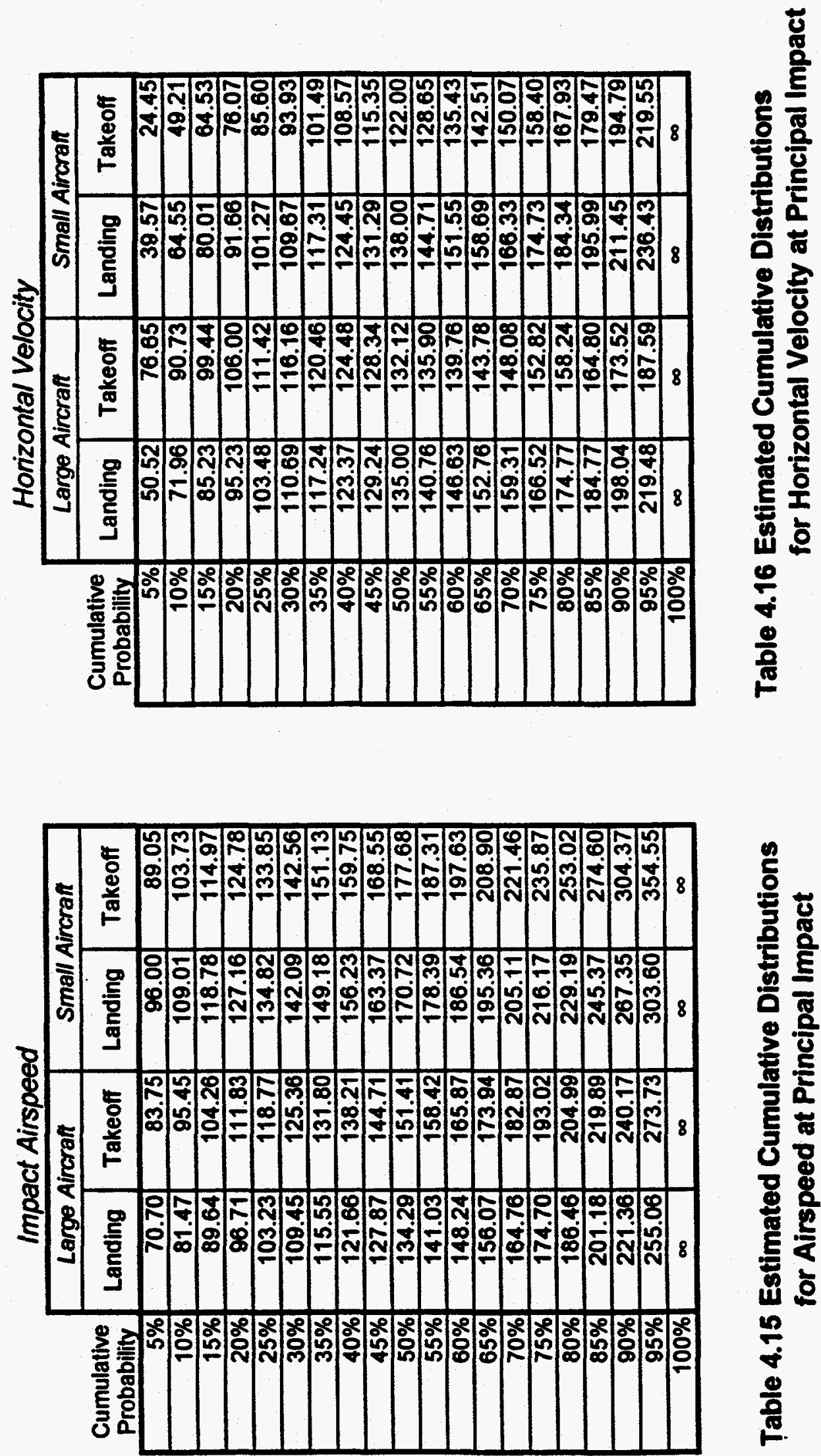


\subsubsection{SKID DISTANCE DISTRIBUTIONS}

The final crash parameter applicable to the aircraft crash risk analysis methodology, as presented in this Standard, is the distance an aircraft travels after its initial principle impact, assuming that the initial principle impact point is the recorded crash location. This parameter is an importam element of the effective area calculation. Use of the distance described above implies that impacts with 'insignificant' objects, such as telephone poles or trees, as the aircratt descends are not considered in establishing crash locations. It also implies that the distance traveled can include the flight distance if the aircratt becomes airbome after its initial principle impact. For purposes of describing the distributions of crash kinematic parameters for military aviation for this Standard, the distance traveled, as described above, is referred to as the skid distance. In most aircraft crashes there is considerable breakup of the aircraft after its initial impact. For purposes of assessing skid distance; judgment must be used regarding when a 'significant' part of the skidding aircraft still exists rather than it being just debris. This is a judgment made by the analysts who derived the information from the available mishap reports.

As with the other crash kinematic parameters, development of the marginal distributions for the skid distance was based on the data in the Minuteman III WSSA database. Since this Standard is applicable only to facilities not on an airfield, the data in the database was screened to delete mishaps for which part of the skid distance involved travel along a nuwway. This is appropriate since skidding along the paved runway is different than what is expected to be experienced by a crashing aircratt at a location away from the airfield. Based on a review of the military mishap data, it was observed that in almost all cases, if the impact angle is greater than 20 degrees, there was no skid. Rather, there was an initial crater and considerable breakup of the aircraft and a scattering of debris. Thus, the appropriate model for describing the distribution of skid distance is a mixture distribution in which a portion of the distribution is at zero, i.e., no skid, and the remainder of the distribution is defined on the positive axis. This is the model selected to describe the marginal distributions of skid distance for military aviation crashes. The proportion of the skid distance distribution at zero is $50 \%, 57 \%, 41 \%$, and $62 \%$, for large aircraft during landing and takeoff and for small aircraft during landing and takeoff, respectively. The distributions of skid distance, conditional on the distance being greater than zero, seemed to be best described by Pearson VI distributions for both large and small aircraft.

Tabulated values of the cumulative probability distributions for skid distance, for the four combinations of aircraft size and phase of operation, are given in Table 4.17. 
Skid Distance

\begin{tabular}{|c|c|c|c|c|}
\hline \multirow[b]{2}{*}{$\begin{array}{l}\text { Cumulative } \\
\text { Probability }\end{array}$} & \multicolumn{2}{|c|}{ Large Aircraft } & \multicolumn{2}{|c|}{ Small Aircraft } \\
\hline & Landing & Takeoff & Landing & Takeoff \\
\hline $40 \%$ & 0 & 이 & 0 & 0 \\
\hline $45 \%$ & 0 & o) & 131 & 0 \\
\hline $50 \%$ & 0 & 0 & 200 & $\overline{0}$ \\
\hline $55 \%$ & 185 & ol & 262 & 0 \\
\hline $60 \%$ & 262 & 1326 & 326 & $\overline{0}$ \\
\hline $65 \%$ & 335 & 1498 & 396 & 125 \\
\hline $70 \%$ & 414 & 1568 & 477 & 242 \\
\hline $75 \%$ & 505 & 1717 & 574 & 330 \\
\hline $80 \%$ & 617 & 1817 & 698 & 431 \\
\hline $85 \%$ & 768 & 1925 & 868 & 560 \\
\hline $90 \%$ & 999 & 2056 & 1142 & $\overline{752}$ \\
\hline $95 \%$ & 1461 & 2245 & 1740 & 1134 \\
\hline $100 \%$ & $\infty$ & $\infty$ & $\infty$ & $\infty$ \\
\hline
\end{tabular}

Table 4.17 Estimated Cumulative Distributions for Skid Distance 


\subsection{MILITARY AVIATION AIRCRAFT CHARACTERISTICS}

This section was prepared to provide to the analyst those characteristics of aircraft that could be important to determine the effect of the aircraft on the facility. Such aircratt characteristics which could be crucial in determining whether a facility could withstand an impact from an aircraft could be the weight of the aircraft, the amount of fuel aboard the aircratt, the speed of the aircraft, the dimensions of the aircraft, and potential missiles generated by the aircratt as its structure failed. Many of these parameters would vary greatty during the course of the flight, but the values of the characteristics given in this section could be considered the upper bound of these parameters. To determine the characteristics of an aircraft at the moment of impact in a particular accident, the analyst should refer to the specific accident report.

Tables 4.18 to 4.20 , taken from References 4.10 to 4.18 , presents the following information on military aviation aircraft characteristics:

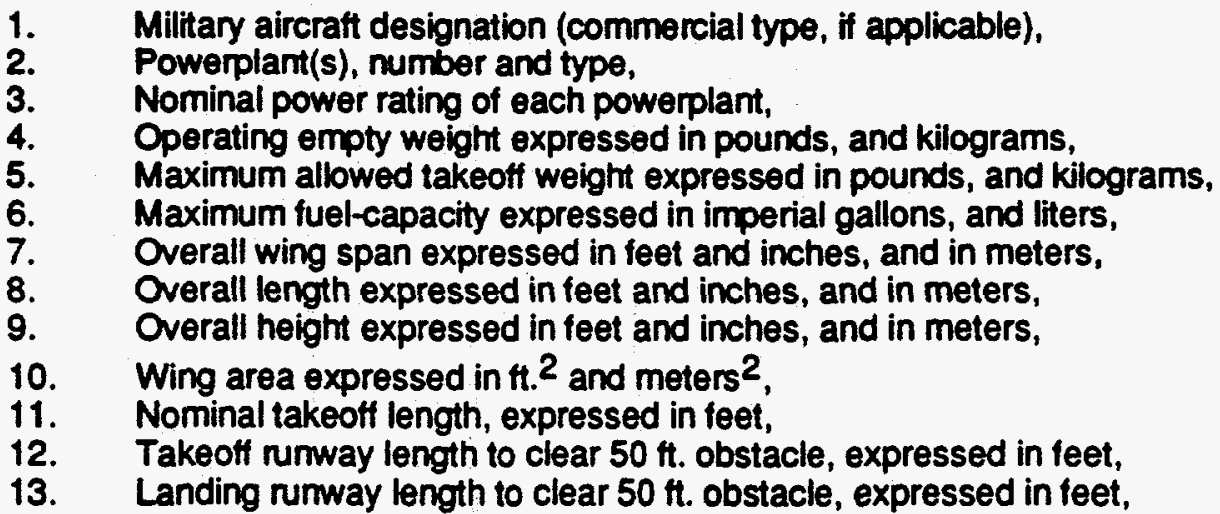

Powerplant(s) were included as potential missile generators. Weights were given for mass during various points of the flight. Fuel capacity was included in order to provide the maximum fuel available for a fire following the crash. Aircraft dimensions will be needed when the facility effective area is determined. The takeotf rumway length and landing numway length were provided so that an analyst could screen certain aircraft from certain airports due to their inability to operate from the airport's rumway(s).

Table 4.21, taken from References 4.10 to 4.18 , presents the following information on military aviation helicopter characteristics:

1. Military aircraft designation (commercial type),

2. Powerplant(s), number and type,

3. Nominal power rating of each powerplant,

4. Operating empty weight expressed in pounds, and kilograms,

5. Maximum allowed takeoff weight expressed in pounds, and kilograms,

6. Maximum fuel-capacity expressed in imperial gallons, and liters,

7. Rotor diameter, expressed in feet and inches, and in meters,

8. Fuselage overall length expressed in feet and inches, and in meters,

9. Overall height expressed in feet and inches, and in meters,

10. Number of rotor(s),

11. Number of rotor blades per rotor.

Powerplant(s) were included as potential missile generators. Weights were given for mass during various points of the flight. Fuel capacity was included in order to provide the maximum fuel available for a fire following the crash. Aircraft dimensions will be needed when the facility effective area is determined. Helicopter rotor diameter should be considered equivalent to aircraft wingspan. The number of rotor(s) and number of blades per rotor were provided as potential missile generators.

The tables listed below gives the general aviation subcategories for which the military aviation aircraft characteristics table were created: 
Table 4.18

Table 4.19

Table 4.20

Table 4.21
Large military aircraft,

Small high performance military aircraft, Small low performance military aircraft, Military helicopters.

Aircraft are listed in descending order of maximum takeoft weight within each subcategory. Aircraft are also listed in ascending order according to military aircraft designation, i.e., A for attack aircraft, B for bomber, $C$ for cargo aircraft, E for electronic warfare aircraft, $F$ for fighter aircraft, $H$ for helicopter, $O$ for observation aircraft, $P$ for maritime patrol aircraft, $P$ for reconnaissance aircraft, $S$ for anti-submarine warfare aircraft, $T$ for training aircratt, $T$ for training aircratt and $U$ for utility aircraft.

The criterion for the determination of large military aircraft from small military aircraft was arbitrarily set at 100,000 lbs. maximum takeotf weight. Also, because of the wide dissimilarity of flight performance, small military aircraft were further divided into high pertormance aircraft and low performance aircraft. All turbofan and turbojet aircraft were classified as the high performance aircraft. All small military aircraft which derived their propulsion power primarily through propellers were classified as low performance aircraft.

Finally, additional research is needed to characterize the missiles (projectiles) generated during the course of an aircraft's crash. Obvious examples of such missiles are the aircraft's engines, the auxiliary power units (APUs), and the landing gear. Much of the information on these aircratt components is not readily available and may have to be obtained directly from the aircraft or engine manutacturer or from the company that produces the component. 
Tablo 4.18

Charactoritalice of Lango Milltary Aviation Airerah

Aoferences 4.10 to 4.18

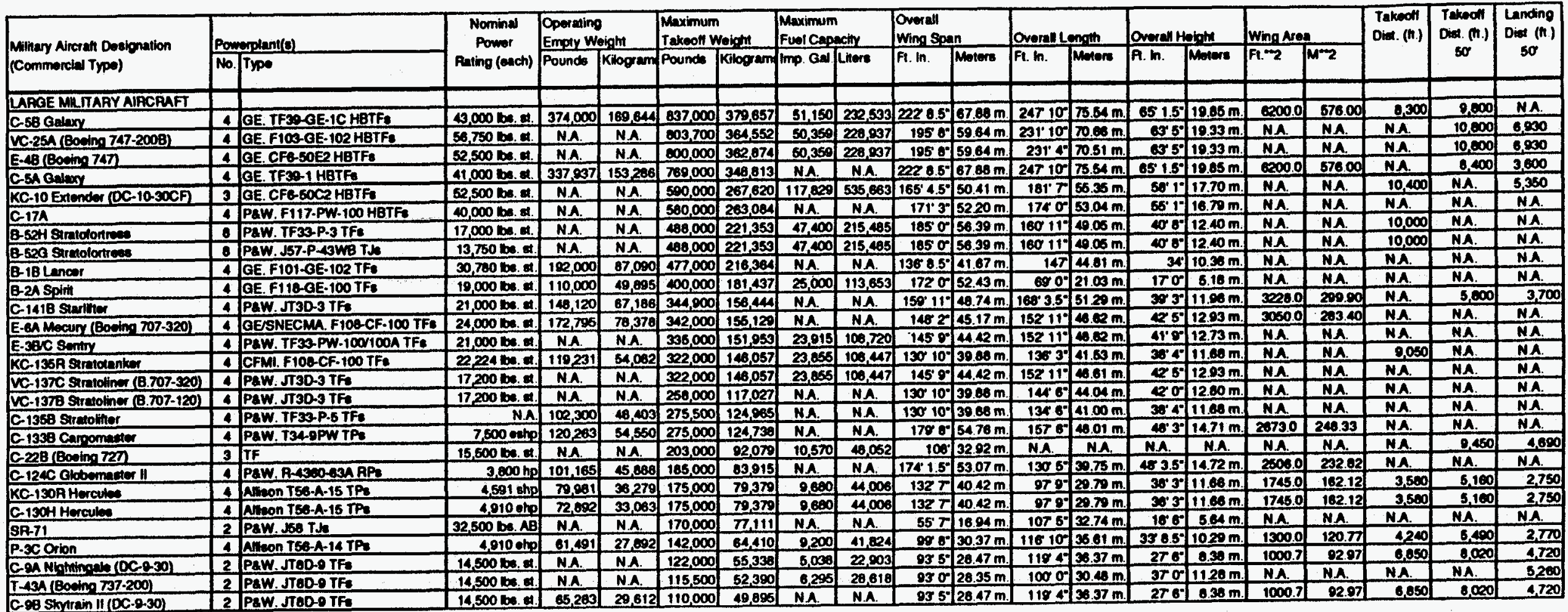
Paw. JTEDOT

N.A. - Intormation not avaluble when table prepared.

\begin{tabular}{|c|c|}
\hline $\begin{array}{l}\text { HBTF - High Bypas Turbolen } \\
\text { Tf - Turbolan } \\
\text { TJ - Turbolet }\end{array}$ & 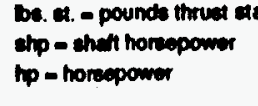 \\
\hline
\end{tabular}

TP - Tutroprop

hip - torecpower

PP - Redial Pitionprop 


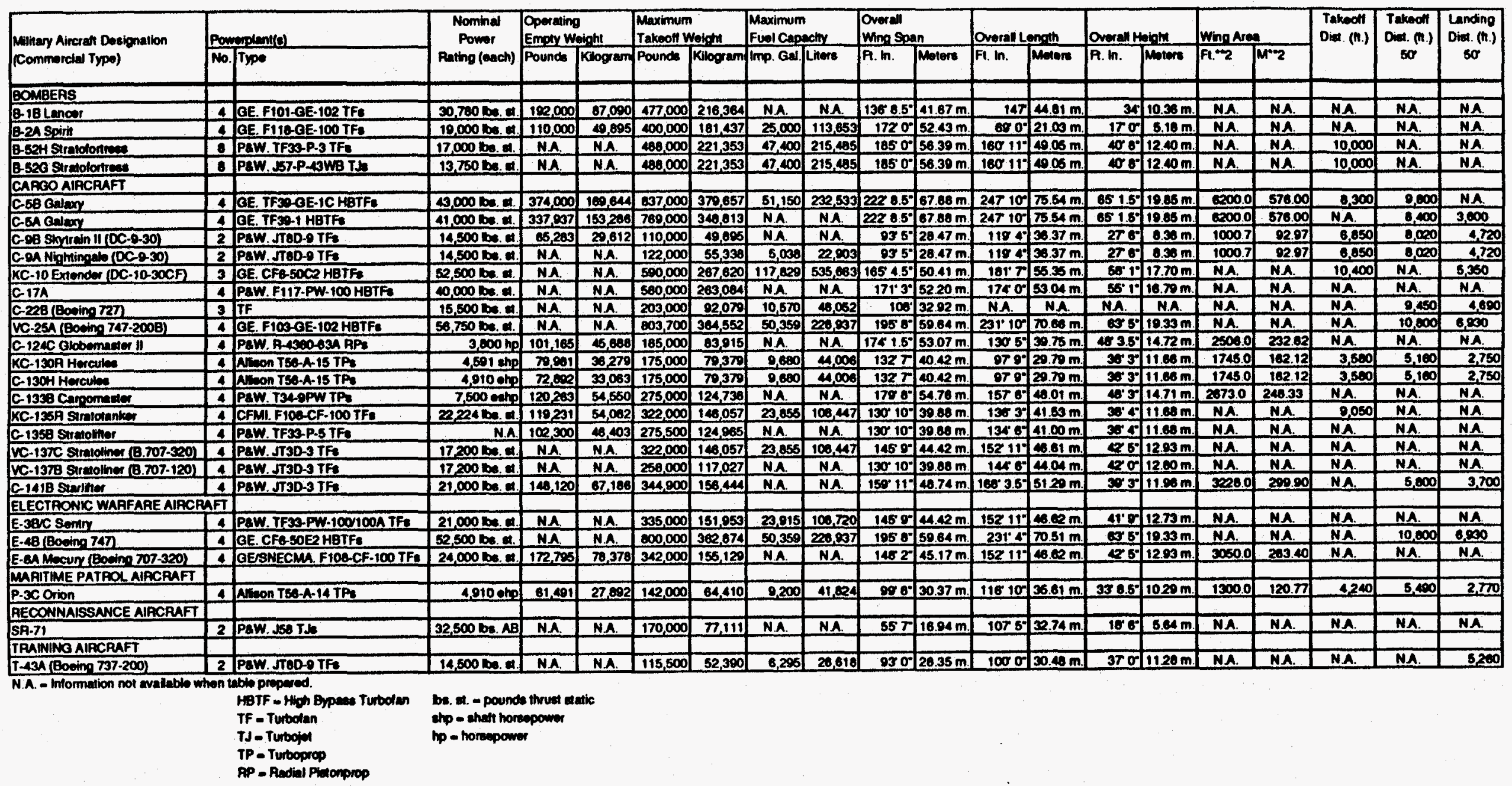




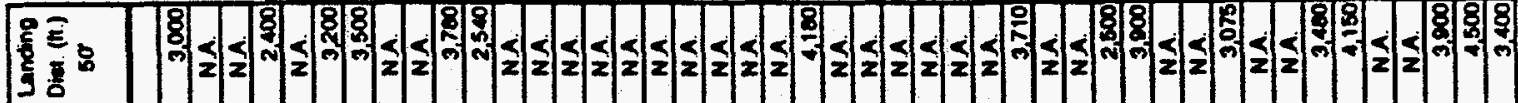

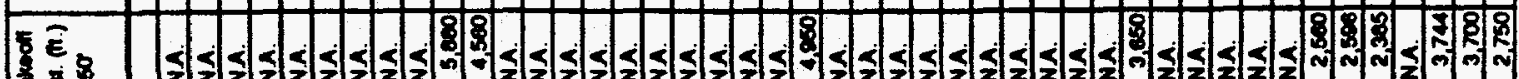

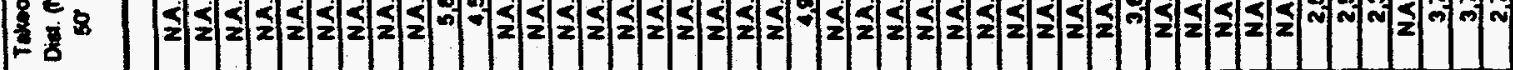

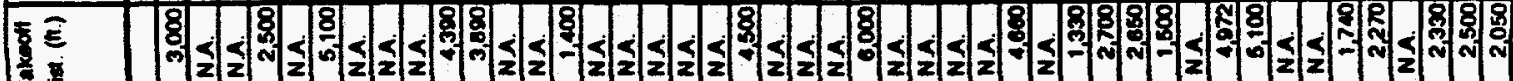
E

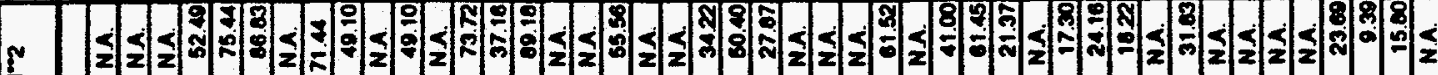

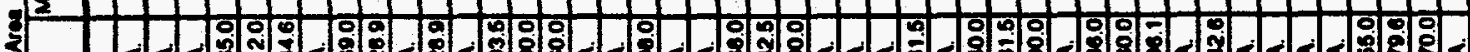

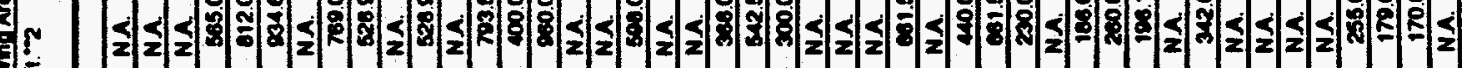

EE

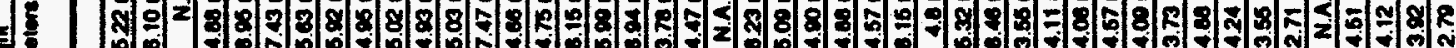

:0

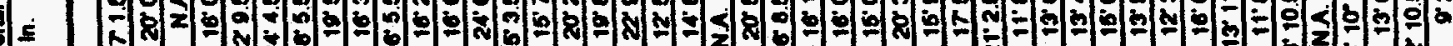

CE

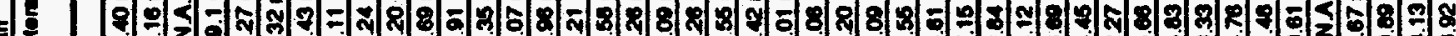

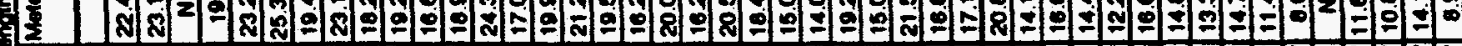

200 bF-

을

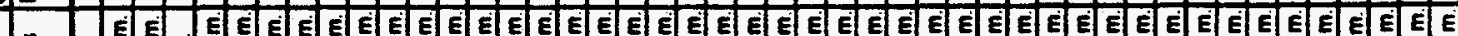

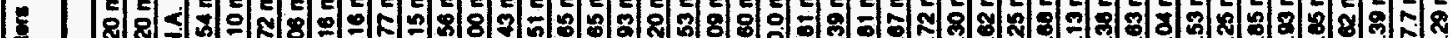

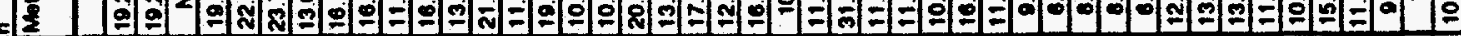

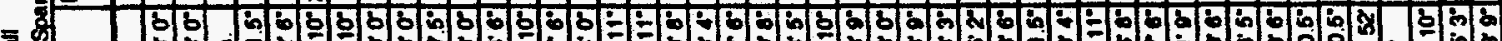

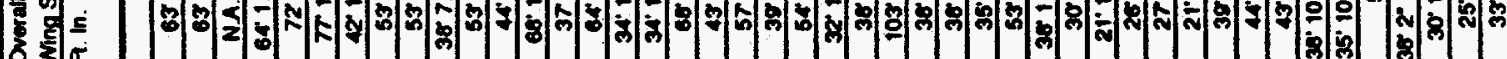

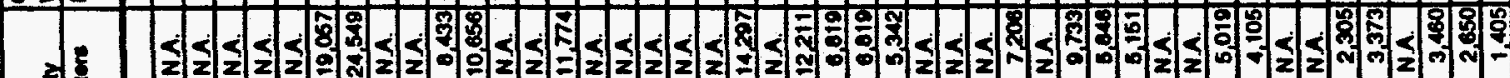
ह 일 空

E / 5 ;

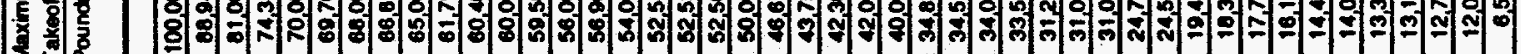

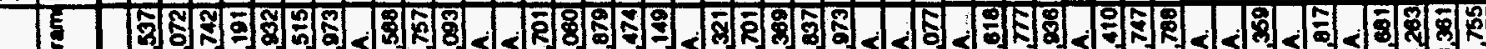

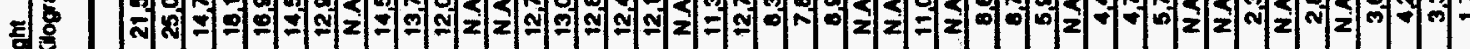

\% 3 -

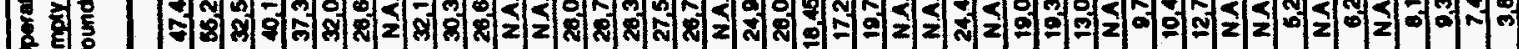

(20.

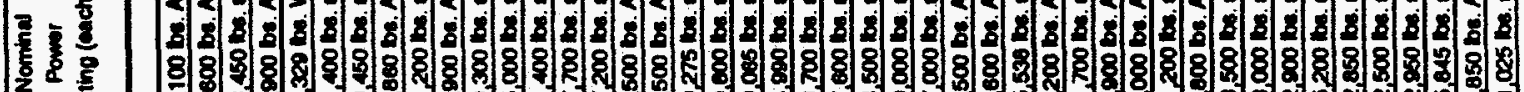

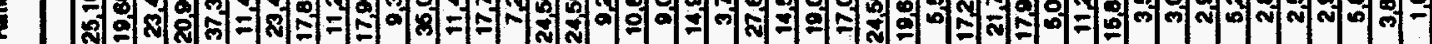

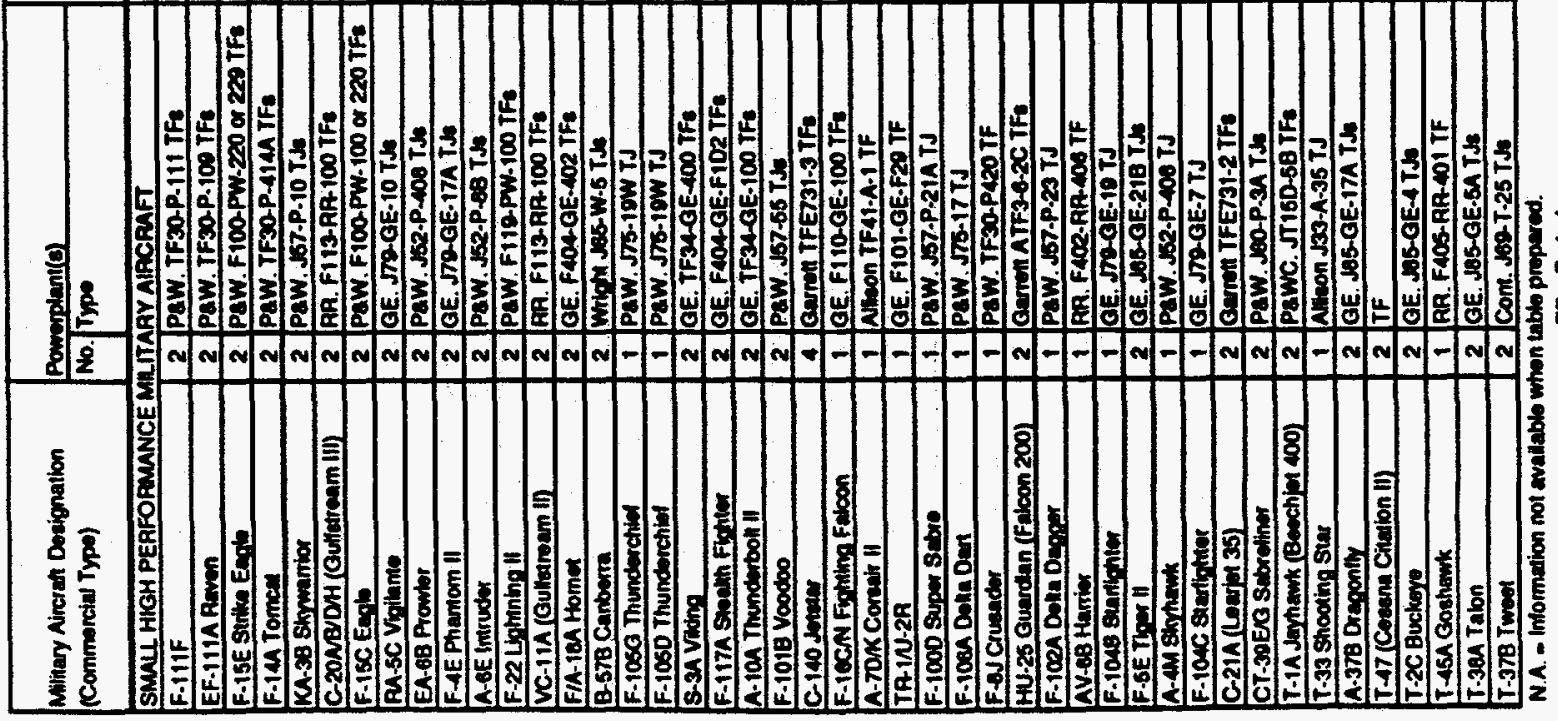

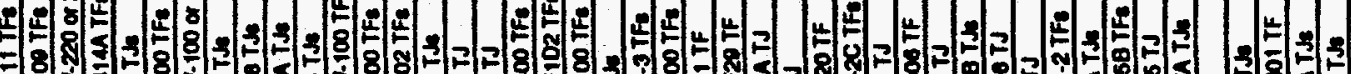

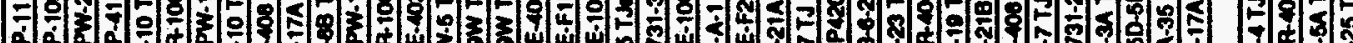

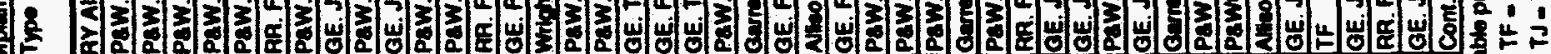


Table 4.19 (Coninued)

Charecteriatice of Small Hoh Performance Mithary Avlation Aireran

Roferences 4.10 to 4.18

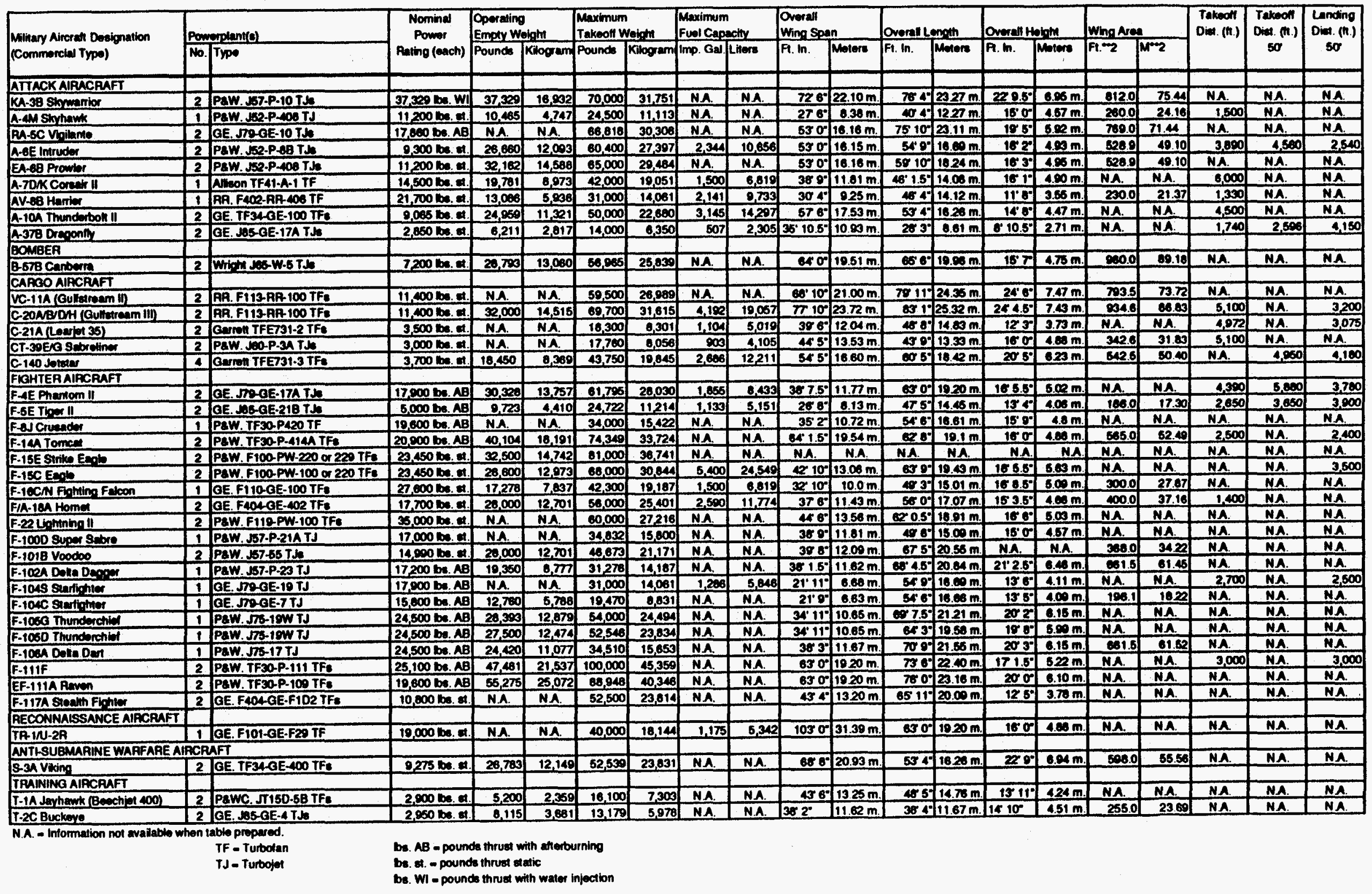


Tablo 4.19 (Comilmued)

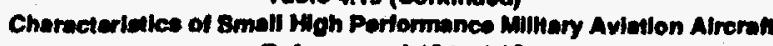

Folerences 4.10 to 4.18

\begin{tabular}{|c|c|c|c|c|c|c|c|c|c|c|c|c|c|c|c|c|c|c|c|c|}
\hline \multirow{2}{*}{$\begin{array}{l}\text { Milnary Aircraft Dessignation } \\
\text { (Commercial Typo) }\end{array}$} & \multicolumn{2}{|c|}{ Pomempint(8) } & \multirow{2}{*}{$\begin{array}{l}\text { Nominal } \\
\text { Power } \\
\text { Pating (each) }\end{array}$} & \multicolumn{2}{|c|}{$\begin{array}{l}\text { Operating } \\
\text { Empry Woight }\end{array}$} & \multicolumn{2}{|c|}{$\begin{array}{l}\text { Maximum } \\
\text { Takeoll Woigth }\end{array}$} & \multicolumn{2}{|c|}{$\begin{array}{l}\text { Maximum } \\
\text { Fuol Capacity }\end{array}$} & \multicolumn{2}{|c|}{$\begin{array}{l}\text { Overall } \\
\text { Wing Soan }\end{array}$} & \multicolumn{2}{|c|}{ Overall Lenoth } & \multicolumn{2}{|c|}{ loveren Heinth } & \multicolumn{2}{|c|}{ Wing Area } & \multirow[t]{2}{*}{$\begin{array}{l}\text { Takeont } \\
\text { Dim. (n.) }\end{array}$} & \multirow{2}{*}{$\begin{array}{c}\text { Takeoft } \\
\text { Died. (m.) } \\
\text { so }\end{array}$} & \multirow{2}{*}{$\begin{array}{c}\text { Lending } \\
\text { Oist (in.) } \\
50\end{array}$} \\
\hline & No. & Typo & & Pounde & Klogram & Poundes & Kilogram & Imp. Gal. & Linore & A. $\ln$. & Moters & Fit.m. & Mators & A.m. & Molen & f."-2 & $M-2$ & & & \\
\hline \multicolumn{21}{|l|}{ TRMINING AIRCARFT Continued } \\
\hline T.33 Shoolinn Star & 1 & Atseon $133-A-35$ TJ & $5,200 \mathrm{be} .1$ & NA. & NA. & 14,42 & 6,551 & N.A. & NA & $30^{\prime} 10.5^{\circ}$ & $11.85 \mathrm{~m}$ & 379 & $11.40 \mathrm{~m}$ & $110^{\circ}$ & $3.56 \mathrm{~m}$ & NA & NA & NA. & 2,560 & 3,400 \\
\hline $7.37 \mathrm{~T}$ Tmeat & 2 & Cont 100 T-25 Tde & 1,025 bet at. & 3,870 & 1,755 & 8,575 & 2,982 & 300 & 1,005 & $339^{\circ}$ & $10.29 \mathrm{~m}$ & 293 & $6.2 \mathrm{~m}$ & 92 & $2.7 \mathrm{~m}$ & NA & NA. & 2,050 & 2,750 & 3,400 \\
\hline T.38n Tabn & 2 & GE. JOS-GE-GA Th & 3,050 b8.AB & 7,410 & 3,361 & 12,093 & 5,405 & 583 & 2,650 & $25 \cdot 3$ & $7.7 \mathrm{~m}$ & $46 \cdot 10^{\circ}$ & $14.13 \mathrm{~m}$ & $1210.5^{\circ}$ & $3.20 \mathrm{~m}$ & 1700.0 & 15.00 & 2,500 & 3,700 & $\$, 500$ \\
\hline T-45n coshent & 1 & RA. FAOS-AR-NOITF & 5,845 bos. A & 9,399 & 4263 & 12,750 & 5,787 & 761 & 3,460 & $30^{\circ} 10^{\circ}$ & $2.39 \mathrm{~m}$ & $35^{\circ} 9^{\circ}$ & $10.00 \mathrm{~m}$ & 136 & $4.12 \mathrm{~m}$ & 170.8 & 0.30 & 2,330 & 3,74 & 3,900 \\
\hline$T-47$ (Coesna Cimion II) & 2 & IF & $2,500 \mathrm{be}$. A & NA. & NA. & 13,300 & 6,033 & 742 & 3,373 & 52 & $15.85 \mathrm{~m}$. & NA. & NA & NA. & N.A. & NA & NA & 2270 & 2,305 & NA. \\
\hline \multicolumn{21}{|l|}{ UTILITY AIRCAAFT } \\
\hline
\end{tabular}

HU-25 Querdian (Fucon 200$)$ L

N.A. - Information not available when lablo propered.

TF - Turbolan

b8. AB - pounds thruet with atherburning

be. - a pounds thruet seatic

be. W1 - pounds thruet with water iniection 
Table 4.20

Charactertatices of Small Low Portormance Mulltary Avietion Aircran

Relerencese 4.10104 .18

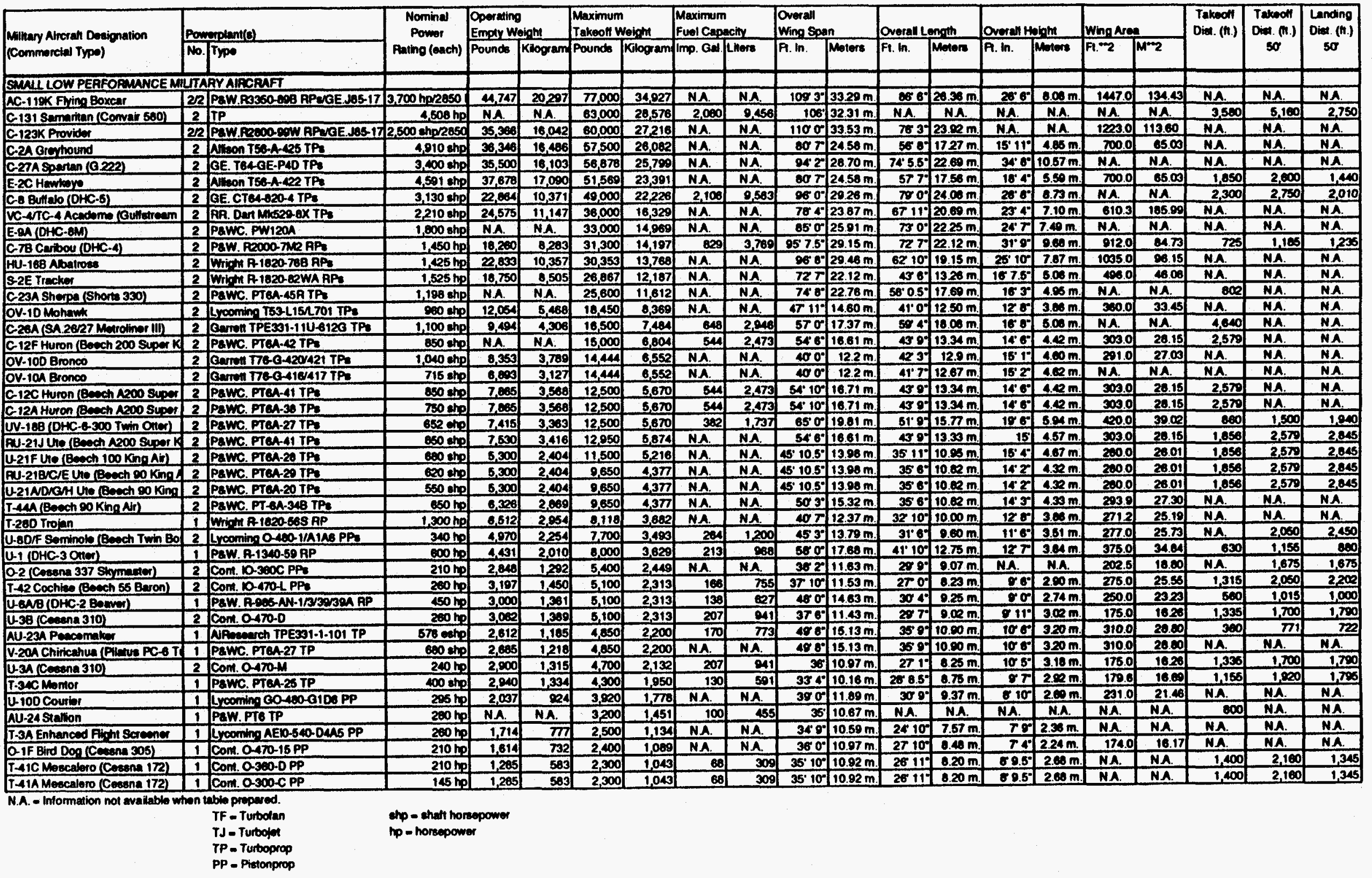


Tablo 4.20 (Conklnued)

Charecterlatice of Small Low Porformanco Milhery Avietion Alrerat Roferences 4.10104 .18

\begin{tabular}{|c|c|c|c|c|c|c|c|c|c|c|c|c|c|c|c|c|c|c|c|}
\hline \multirow{2}{*}{ 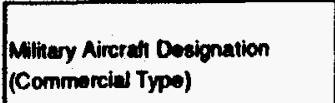 } & Powemplantse) & \multirow{2}{*}{$\mid \begin{array}{c}\text { Nominal } \\
\text { Powor } \\
\text { Rating (oach) }\end{array}$} & \multicolumn{2}{|c|}{$\begin{array}{l}\text { Operaling } \\
\text { Empoy Woight }\end{array}$} & \multicolumn{2}{|c|}{ Maximum } & \multicolumn{2}{|c|}{$\begin{array}{l}\text { Maximum } \\
\text { Fuel Capactir }\end{array}$} & \multicolumn{2}{|l|}{$\begin{array}{l}\text { Overall } \\
\text { Whing Span }\end{array}$} & \multicolumn{2}{|c|}{ overall Length } & \multicolumn{2}{|c|}{ loverell Hainte } & \multirow{2}{*}{\multicolumn{2}{|c|}{ Wing Ave }} & \multirow[t]{2}{*}{$\begin{array}{l}\text { Takeoth } \\
\text { Dirat. (n.) }\end{array}$} & \multirow{2}{*}{ 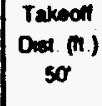 } & \multirow{2}{*}{\begin{tabular}{|c|} 
Landing \\
Dint (n) \\
Sog
\end{tabular}} \\
\hline & \begin{tabular}{|l|l|} 
No. Trpeo \\
\end{tabular} & & Pounds & Klogra & Pounds & Kilogram & $\mid \mathrm{mp.}$ Gal. & Linores & A. In & Mot & F.. $\ln$ & Metors & P. In. & Moters & & 2 & & & \\
\hline RGO AIPCRAFT & & & & & & & & & & & & & & & & & & & \\
\hline 219rohtound & 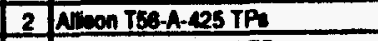 & 4,010 ethp & 33,346 & 16,406 & 57,500 & 26,082 & NA. & $\overline{\mathrm{NA}}$ & 807 & $24.58 \mathrm{~m}$ & 5600 & $1727 \mathrm{~m}$ & $15^{\circ} 11$ & $4.05 \mathrm{~m}$. & $\pi 00$. & 05.00 & NA. & NA. & NA \\
\hline Acadome (Qulloticeam & 2 AR. Oen Muses-8X TP & 2210 ahp & 24,575 & 11,147 & 36,000 & 16,329 & N.A. & NA. & 784 & $23.87 \mathrm{~m}$ & 67110 & $20.00 \mathrm{~m}$ & $23 \times 4$ & $7.10 \mathrm{~m}$ & 810.3 & 105.09 & NA. & NA. & NA. \\
\hline C.7B Carbou (DHC-4) & \begin{tabular}{|l|l|}
2 & $P A W$ P2000.7M2APQ \\
\end{tabular} & $1,450 \mathrm{hp}$ & 18,260 & 8,283 & 31,300 & 14,197 & 829 & 3,761 & $957.5^{-}$ & $29.15 \mathrm{~m}$ & 727 & $22.12 \mathrm{~m}$ & $310^{\circ}$ & $0.60 \mathrm{~m}$ & 9120 & 84.73 & 723 & $\frac{1,106}{1,70}$ & $\frac{1235}{2010}$ \\
\hline C-BBuffol (OHC-5) & 2 QE. CTRA-20-4 TPE & $3,130 \mathrm{ehp}$ & 22,864 & 10,371 & 49,000 & 22228 & 2,100 & 2,58: & $260^{\circ}$ & $29.28 \mathrm{~m}$ & $\pi 00^{\circ}$ & $24.00 \mathrm{~m}$ & $20^{\circ} 0^{\circ}$ & $0.73 \mathrm{~m}$ & NA. & NA. & $\frac{2,300}{3500}$ & & $\frac{2,010}{N A}$ \\
\hline C.12F Huron (Booch 200 Super K & 2 PAWC. PTQA- & $050 \mathrm{ehp}$ & NA. & NA. & 15,000 & 6,804 & 54 & 2,47 & $54^{6} 6^{\circ}$ & $16.61 \mathrm{~m}$ & & $13.34 \mathrm{~m}$ & $14: 6$ & $4.4 \mathrm{~m}$ & 303.0 & 20.15 & $\frac{2,570}{2570}$ & NA. & NA. \\
\hline$C_{-12 C}$ Huron (Beoch 12000 super & \begin{tabular}{|l|l|l|}
2 & PEWC, PTQA-A \\
\end{tabular} & $850 \mathrm{ehp}$ & 7,805 & 3,568 & 12,500 & 5,870 & 54 & 2,47 & $54 \cdot 10^{\circ}$ & $18.71 \mathrm{~m}$ & 438 & $13.34 \mathrm{~m}$ & $14 \cdot 6^{\circ}$ & $4.2 \mathrm{~m}$ & 3030 & $\frac{28.15}{3.15}$ & $\frac{2,579}{3570}$ & $\frac{N A .}{M A}$ & NA \\
\hline C.12A Huron Beoch N200 Super & 2 PQWC. PTEA-30 & $750 \mathrm{ehp}$ & 7,605 & 3,568 & 12,500 & 5,670 & 54 & 2,47 & 54. 10 & $16.71 \mathrm{~m}$ & 450 & $13.34 \mathrm{~m}$ & $14 \cdot 6$ & $4.2 \mathrm{~m}$ & 303. & 28.15 & 2,579 & NA. & NA. \\
\hline C.23A sherpe (Shorte 330) & 2 PQWC. PTEA- $25 \mathrm{APTPS}$ & 1,100 ghp & NA. & NA. & 25,600 & 11,612 & N.A. & N.A. & $7 \times 0$ & $22.76 \mathrm{~m}$ & $50^{0} 0.57$ & $17.00 \mathrm{~m}$ & $16 \cdot 39$ & $4.06 \mathrm{~m}$ & NA. & NA & 002 & NA. & $\frac{\text { NA }}{M A}$ \\
\hline C.28A (SA 2827 Metroliner III) & 2 Gerron TPE331-11U-8120 TPE & $1,100 \mathrm{ghg}$ & 2,04 & 4,306 & 16,500 & 7,480 & 640 & 2,2 & 570 & $17.37 \mathrm{~m}$ & 504 & $10.00 \mathrm{~m}$ & $10 \cdot 0^{\circ}$ & $5.00 \mathrm{~m}$ & NA & NA & 4,040 & A. & NA \\
\hline G-27A Spartan (G.222) & 2 QE. TOLQEE-PAD TPA & $3,400 \mathrm{shp}$ & 35,500 & 16,103 & 58,876 & 25,799 & N.A. & NA & $\operatorname{arc} 2$ & $28.70 \mathrm{~m}$ & 74.5 .57 & $22.00 \mathrm{~m}$ & $34 \cdot 0$ & $10.57 \mathrm{~m}$ & NA. & NA. & NA. & N.A. & NA. \\
\hline AG-119K Fying Boxcar & 222 P\&W.R3360-098 APEGE.J05-17 & 3,700 hp/2850 & 4,747 & 20,297 & 77,000 & 34,927 & N.A. & N.A. & 1003 & $3329 \mathrm{~m}$ & $\infty 0$ & $2.36 \mathrm{~m}$ & 206 & $8.00 \mathrm{~m}$ & 14970 & 134.43 & NA & NA. & NA \\
\hline 5.000 & 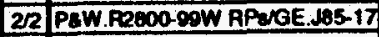 & 2,500 ehp 2850 & 35,306 & 16,042 & 60,000 & 27216 & m n & NA & 1100 & $33.53 \mathrm{~m}$ & & $m$ & & N. & 12230 & 113.80 & NA & MA. & NA \\
\hline 31 semerichen (Convair 560$)$ & \begin{tabular}{|l|l|l|}
2 & $\mathrm{TP}$ \\
\end{tabular} & $4,500 \mathrm{hp}$ & NA. & NA & 63,000 & 28,576 & 2,000 & 0,45 & .106 & $32.31 \mathrm{~m}$ & $\overline{N A}$ & $\mathrm{NA}$ & $\sqrt{1 a}$ & IA. & A. & NA. & 3,500 & 5,100 & 2,75 \\
\hline \multirow{2}{*}{$\begin{array}{l}\text { ELECTAONIC WAAFAAE AIACAAF } \\
\text { E-2C HEWtor" }\end{array}$} & AF & & & & & & & & & & & & & & & & & & \\
\hline & 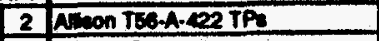 & 4.591 thp & $37,6 \pi$ & 17,000 & 51,560 & 23,399 & N.A. & N.A. & 807 & $24.50 \mathrm{~m}$ & 677 & $7.50 \mathrm{~m}$ & 104 & $5.60 \mathrm{~m}$. & 700.8 & 65.0 & 1,05 & 2,000 & $\sqrt{14}$ \\
\hline 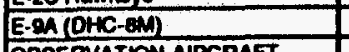 & 2 PSWC. PWizon & $1,000 \mathrm{ehp}$ & NA & NA. & 33,000 & 14,968 & N.A. & NA. & 850 & $25.91 \mathrm{~m}$ & $7300^{\circ}$ & $223 \mathrm{~m}$ & $26^{7} 7$ & $80 \mathrm{~m}$ & NA & NA. & NA. & Na. & NA \\
\hline (ARCART & & & & & & & & & & & & & & & & & & & \\
\hline 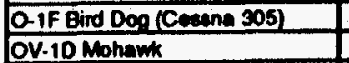 & 2 com O-A70-15PP & $210 \mathrm{mp}$ & 1,614 & 735 & 2,400 & 1,000 & N.A. & NA. & 380 & $10.97 \mathrm{~m}$ & $\frac{2710}{410}$ & $0.4 \mathrm{~m}$ & $\frac{74}{120}$ & $2.24 \mathrm{~m}$ & 174.0) & $\frac{16.17}{33.65}$ & NA & A. & NA. \\
\hline 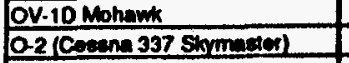 & 2 Lyooming T53L1502701 TPA & 9008ho & 12,054 & 5,4868 & 18,450 & 8,369 & NA. & N.A. & $4 \pi 11^{\circ}$ & $\frac{11.60 \mathrm{~m}}{1.09}$ & $\frac{41009}{2009}$ & $\frac{12.50 \mathrm{~m}}{0.07 \mathrm{~m}}$ & $\frac{128}{N A}$ & \begin{tabular}{|l|}
$\mid \frac{3.03 \mathrm{~m}}{\mathrm{~N} . \mathrm{m}}$ \\
\end{tabular} & $\frac{300.0}{2005}$ & $\frac{33.45}{1600}$ & $\frac{N A}{N A}$ & $\frac{N A}{1.875}$ & $\frac{N A}{1.675}$ \\
\hline 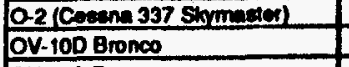 & 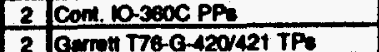 & $210 \mathrm{ho}$ & 2840 & $\frac{1,202}{3200}$ & $\frac{5,400}{14,44}$ & $\frac{2,499}{6.552}$ & NA. & NA. & $\frac{382}{400^{\circ}}$ & $\frac{11.63 \mathrm{~m}}{12.2 \mathrm{~m}}$ & $\frac{297}{293}$ & $9.07 \mathrm{~m}$. & $\frac{n .4}{15 \cdot 1}$ & \begin{tabular}{|l|l|}
$.000 \mathrm{~m}$ \\
\end{tabular} & 2010 & 2700 & NA. & NA. & $\frac{1,675}{N A}$ \\
\hline$\frac{\text { OV-100 Bronce }}{\text { OQV-10A Bronce }}$ & 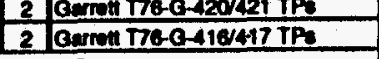 & $\frac{1040 \mathrm{ehp}}{715 \mathrm{enp}}$ & 8,853 & $\frac{3,709}{3,127}$ & $\frac{14,44}{14,44}$ & $\frac{6,552}{6,552}$ & $\frac{N A}{N A}$ & $\frac{N A .}{N A .}$ & 400 & $12.2 \mathrm{~m}$ & A17 & $12.67 \mathrm{~m}$ & $15 \cdot 2$ & $1.02 \mathrm{~m}$. & NA. & NA & NA. & NA. & NA. \\
\hline \multicolumn{2}{|c|}{ 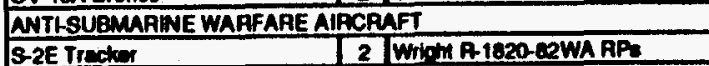 } & & & & & & & & & & & & & & & & & & \\
\hline S-2E Trecker & \begin{tabular}{|l|l|l|}
2 & $A_{1}$ \\
\end{tabular} & $625 \mathrm{hp}$ & 10,750 & $0.50 !$ & 20,867 & 12,107 & NA & NA. & 727 & $22.12 \mathrm{~m}$ & 486 & $328 \mathrm{~m}$ & 0.5 & $6.00 \mathrm{~m}$ & $\infty$ & 480 & NA & NA. & NA. \\
\hline TPQUNINA AIACRAFT & & & & & & & & & & & & & & & & & & & \\
\hline noed Firtit Screener & 1 Lyeomina A E10-640-DASS PP & ong & $\frac{1,714}{0501}$ & $\frac{m}{205}$ & $\frac{2,500}{6.100}$ & $\frac{1,134}{3600}$ & NA & MA. & $\frac{340}{407}$ & $\frac{\mid 10.59 \mathrm{~m}}{12.37 \mathrm{~m}}$ & $\frac{2 \times 109}{3210}$ & $\frac{7.57 \mathrm{~m}}{10.00 \mathrm{~m}}$ & $\frac{18}{120}$ & $\frac{2.006 \mathrm{~m}}{3.006}$ & 2712 & 25.10 & NA & NA. & NA. \\
\hline T-39c Mnnor & 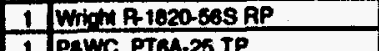 & $\frac{1,300 \mathrm{hp}}{1600 \mathrm{sh}}$ & $\frac{0,512}{2020}$ & $\frac{2.054}{1,334}$ & $\frac{0,1100}{4,300}$ & $\frac{3,662}{1.050}$ & & $\frac{5 .}{50}$ & $334^{\circ}$ & $10.16 \mathrm{~m}$ & $20^{\circ} .0 .5$ & $8.75 \mathrm{~m}$ & 97 & $2.92 \mathrm{~m}$ & 179.6 & 1860 & $.1,186$ & 1,0200 & $\frac{1,200}{1,703}$ \\
\hline 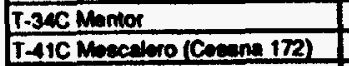 & \begin{tabular}{|l|l}
1 & PaWC. PTEA.25 TP \\
1 & Com 0.360.DPP \\
\end{tabular} & $\frac{200 \mathrm{enp}}{210 \mathrm{hop}}$ & $\frac{2,290}{1,225}$ & 503 & 00 & 1.043 & 6 & & $35^{\prime} 10^{\circ}$ & $10.92 \mathrm{~m}$ & 26110 & $0.20 \mathrm{~m}$ & & $2.00 \mathrm{~m}$ & NA. & MA & 1,400 & 2.100 & $\frac{1.345}{1.345}$ \\
\hline 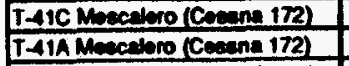 & \begin{tabular}{|l|l|}
1 & Con $0.300-D P p$ \\
1 & con $0.300-C P P$ \\
\end{tabular} & $\frac{210 \mathrm{hp}}{145 \mathrm{hp}}$ & $\frac{1,205}{1,205}$ & 503 & $\frac{2,300}{2,300}$ & $\frac{1,040}{1,043}$ & $\frac{6}{6}$ & 30 & $36^{\circ} 10^{\circ}$ & $10.92 \mathrm{~m}$ & 2610 & $8.20 \mathrm{~m}$ & $80.5^{4}$ & $2.00 \mathrm{~m}$ & NA. & MA. & 1,400 & 100 & $\frac{1,345}{1,345}$ \\
\hline 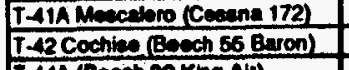 & \begin{tabular}{|l|l|}
1 & $\operatorname{con} 0.300-C P P$ \\
2 & $\operatorname{con} 10.470-1 P p Q$ \\
\end{tabular} & $\frac{105 \mathrm{hp}}{200 \mathrm{hp}}$ & $\frac{1,200 \mid}{3,197}$ & 1,450 & 5,100 & $\frac{1,0,313}{2,313}$ & $\frac{106}{160}$ & $\bar{\pi}$ & $3710^{\circ}$ & $11.53 \mathrm{~m}$ & 2700 & $0.23 \mathrm{~m}$ & 96 & $2.00 \mathrm{~m}$ & 275.0 & 25.56 & 1,315 & 2,050 & 2,200 \\
\hline 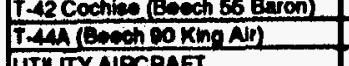 & 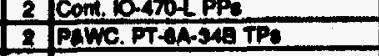 & 60019 & 8,200 & 2000 & 0,060 & 4,377 & NA. & NA. & $603^{\circ}$ & $16.32 \mathrm{~m}$ & 3609 & $10.02 \mathrm{~m}$ & $14 \cdot 3$ & $4.33 \mathrm{~m}$ & 203.9 & 27.30 & NA. & NA. & $\overline{N A}$ \\
\hline CRAFT & & & & & & & & & & & & & & & & & & & \\
\hline 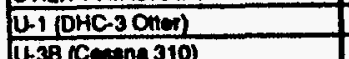 & $R-3360-59 \mathrm{AP}$ & $600 \mathrm{he}$ & 2,433) & 2,010 & (0) & 3,629 & 213 & 3 & 500 & $17.68 \mathrm{~m}$ & 410 & $12.76 \mathrm{~m}$ & 127 & Am. & & & & & \\
\hline & $21 c$ & $200 \mathrm{hp}$ & 3,002 & 1,309 & 100 & 2,313 & 207 & 9 & $376^{\circ}$ & $11.43 \mathrm{~m}$ & 207 & $2.00 \mathrm{~m}$ & 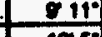 & m. & & & & & 1,700 \\
\hline 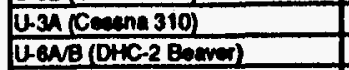 & $2 a$ & $240 \mathrm{hp}$ & 2,900 & 1,315 & 4,700 & 2,132 & 207 & 9u & 36 & $10.97 \mathrm{~m}$ & 2719 & $0.25 \mathrm{~m}$ & $1055^{\circ}$ & $3.10 \mathrm{~m}$ & & 16 & 1,33 & & 1,700 \\
\hline & 1 PaW.R-AOS-AN-1/233 & 450 & 3,000 & 1,361 & 5 & 2,313 & 130 & 62 & 450 & $14.63 \mathrm{~m}$ & 304 & $0.25 \mathrm{~m}$ & $m$ & $2.74 \mathrm{~m}$ & 250.0 & & 56 & & 1,000 \\
\hline 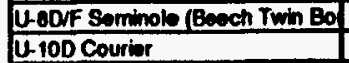 & 2 Lyeoming $0-100-1$ & $340 \mathrm{~h}$ & 4.5 & 2254 & & 3 & 264 & 1,20 & $25^{3}$ & $13.79 \mathrm{~m}$. & $31 \cdot 6$ & $0.00 \mathrm{~m}$. & $11 \%$ & $3.51 \mathrm{~m}$. & 277.0 & 25.73 & - & & $2,4,55$ \\
\hline$\frac{U-100 \text { Courier }}{\text { HU-180 Nomiroes }}$ & 1 LYeoming co-180-G108 PP & $206 \mathrm{kp}$ & 2,037 & 22 & & 1.7 & NA. & NA. & $380^{\circ}$ & $11.09 \mathrm{~m}$ & 309 & $0.37 \mathrm{~m}$ & $810^{\circ}$ & $2.00 \mathrm{~m}$ & 110 & 21.46 & NA. & A. & 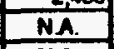 \\
\hline 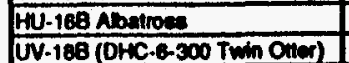 & 2 Whom A-1020-78BAPB & $25 \mathrm{hp}$ & 22,833 & 10,357 & 153 & 13,780 & NA & $\mathrm{NA}$ & $060^{\circ}$ & $29.46 \mathrm{~m}$ & 8210 & $10.15 \mathrm{~m}$ & $25: 10$ & $7.07 \mathrm{~m}$ & 1003.0 & .15 & MA. & 1A. & n \\
\hline 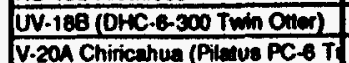 & 2 PaWC. PTGA-27 TPB & $652 \mathrm{chp}$ & 7,415 & 3,363 & 500 & 5,670 & 30 & 1,73 & & & & & $186^{\circ}$ & $5.04 \mathrm{~m}$. & 200 & 39.02 & 86 & 1,500 & 1,940 \\
\hline 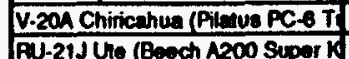 & 1 PAWC. PTGA-27 TP & $600 \mathrm{ehp}$ & 2,625 & 1218 & 4,850 & 2200 & $\mathbf{N A}$ & NA & & & & & 100 & $320 \mathrm{~m}$ & 3100 & 20.00 & NA & NA. & NA. \\
\hline Beoch 1200 Supork & 2 PQWC. PTEA-A1 TPB & $850 \mathrm{shp}$ & 7,530 & 3,416 & 12,950 & 5,874 & 1] N.A. & $N A$ & $\frac{6}{6^{\circ}}$ & $18.61 \mathrm{~m}$ & & $13.30 \mathrm{~m}$ & & & 303.0 & 20.15 & 1,056 & 2,570 & 2,045 \\
\hline \multicolumn{20}{|c|}{ 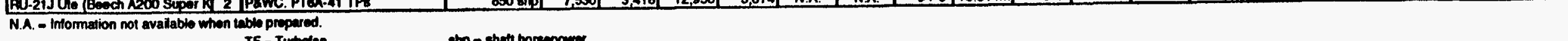 } \\
\hline N.A. - Irtormation & $\begin{array}{l}\text { TJ - Turtolit } \\
T P=\text { Trutoprop } \\
\text { PP = Pistopprop }\end{array}$ & & & & & & & & & & & & & & & & & & \\
\hline
\end{tabular}


Tabto 4.20 (Contimuca)

Charectertatice of Small Low Performence minary Avietion Aireren

Pelerence 4.10 to 4.18

\begin{tabular}{|c|c|c|c|c|c|c|c|c|c|c|c|c|c|c|c|c|c|c|c|}
\hline \multirow{2}{*}{$\begin{array}{l}\text { Milmary Arereh Deaignation } \\
\text { (Commeried Typo) }\end{array}$} & Powarplant(8) & \multirow{2}{*}{\begin{tabular}{c|} 
Nominal \\
Powor \\
Rating (aach)
\end{tabular}} & \multicolumn{2}{|c|}{$\begin{array}{l}\text { Operating } \\
\text { Empay Woight }\end{array}$} & \multicolumn{2}{|c|}{$\begin{array}{l}\text { Maximum } \\
\text { Takeoff Weight }\end{array}$} & \multicolumn{2}{|c|}{$\begin{array}{l}\text { Maximum } \\
\text { Fuel Capancity }\end{array}$} & \multicolumn{2}{|c|}{$\begin{array}{l}\text { Ovmall } \\
\text { Wing Span }\end{array}$} & \multicolumn{2}{|c|}{ Overall Lenoth } & \multicolumn{2}{|c|}{ Overall Heing } & \multicolumn{2}{|c|}{ Who Aven } & \multirow[t]{2}{*}{$\begin{array}{l}\text { Takeof } \\
\text { Din (in.) }\end{array}$} & \multirow{2}{*}{$\begin{array}{c}\text { Takeon } \\
\text { Dias. (m.) } \\
\text { sor }\end{array}$} & \multirow{2}{*}{$\begin{array}{l}\text { Landing } \\
\text { Diat. (n.) } \\
50\end{array}$} \\
\hline & No. Type & & Pounds & Klogram & Pound & Kilogram & $\operatorname{Imp.~Gat|t}$ & Lhere & F. $\ln$ & Motere & Fi. $\ln$. & Moters & R.m. & Mienes & $f_{1}=-2$ & $M=2$ & & & \\
\hline \multicolumn{20}{|l|}{ UTHITY AIRCPAFT Continued } \\
\hline U-21F Uin (Beoch 100 King Ab) & 2 PRMC. PTQA-20 TP & $600 \mathrm{ch}$ & 5,300 & 2,104 & 11,500 & 5216 & NA & NA. & $46^{\circ} \cdot 10.5^{\circ}$ & $13.90 \mathrm{~m}$ & $35^{\circ} 11^{\circ}$ & $10.95 \mathrm{~m}$ & $18 \cdot 4$ & $4.67 \mathrm{~m}$. & 200.0 & 20.01 & 1.056 & 2,570 & 2,045 \\
\hline 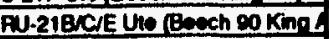 & 2 Pewc. PTOA-ZOTPZ & $600 \mathrm{en}$ & 5,300 & 2,404 & 2,850 & 4,37 & NA & NA & $45 \cdot 10.5^{\circ}$ & $13.90 \mathrm{~m}$ & $35 \cdot 6$ & $10.02 \mathrm{~m}$ & $14 \cdot 2$ & $4.32 \mathrm{~m}$ & 200.0 & 20.01 & 1.056 & 2,570 & 2,845 \\
\hline U-21 Novant Une (Boech 50 King & 2 PaWc. PTRA-zo TP & 550 enf & 5,300 & 2,404 & 9.850 & 4,37 & N.A. & NA. & $45^{\circ} 10.5^{\circ}$ & $13.90 \mathrm{~m}$ & $35^{\circ} \cdot 6^{\circ}$ & $10.82 \mathrm{~m}$ & $14: 2$ & $4.32 \mathrm{~m}$ & 200.0 & 28.01 & 1.056 & 2,570 & 2,045 \\
\hline AU-23A Peacomeles & 1 AiRmeanch TPE331-1-101 TP & 578 ehp & 2,612 & 1,185 & 4,850 & 2,200 & 170 & 73 & $190^{\circ}$ & $15.13 \mathrm{~m}$ & $35 \cdot 9$ & $10.00 \mathrm{~m}$ & $106-1$ & $320 \mathrm{~m}$ & 3100 & 28.80 & 360 & $\pi 1$ & 722 \\
\hline AU-24 Stalion & I PEW.PTE.TP & $280 \mathrm{hpl}$ & N.A. & NA & 3,200 & 1,451 & 100 & 455 & 35 & $10.67 \mathrm{~m}$ & NA. & NA. & NA. & N.A. & NA. & NA. & 800 & NA. & NA \\
\hline \multicolumn{20}{|c|}{ 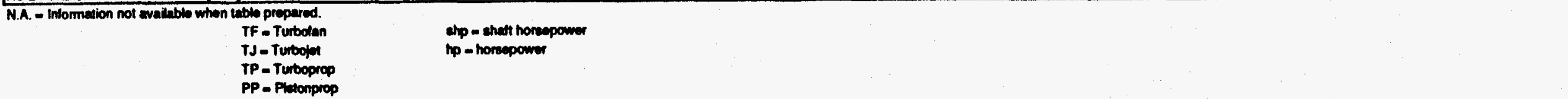 } \\
\hline
\end{tabular}


Tablo 4.21

Charactoriatice of Milinary Holloopters

Roferencese 4.10 to 4.18

\begin{tabular}{|c|c|c|c|c|c|c|c|c|c|c|c|c|c|c|c|c|c|}
\hline \multirow{2}{*}{$\begin{array}{l}\text { Military Aircrat Dosignation } \\
\text { (Commercial Typo) }\end{array}$} & \multicolumn{2}{|c|}{ Poworpliant(o) } & \multirow{2}{*}{$\begin{array}{c}\text { Nominal } \\
\text { Pownot } \\
\text { Rating (each) }\end{array}$} & \multicolumn{2}{|c|}{$\begin{array}{l}\text { Operating } \\
\text { Emply Woight }\end{array}$} & \multicolumn{2}{|c|}{$\begin{array}{l}\text { Maximum } \\
\text { Takooll Woigth }\end{array}$} & \multicolumn{2}{|c|}{$\begin{array}{l}\text { Maximum } \\
\text { Fuel Capacity }\end{array}$} & \multicolumn{2}{|c|}{ Folor Diameter } & \multicolumn{2}{|c|}{$\begin{array}{l}\text { Fuselage } \\
\text { Overal Length }\end{array}$} & \multicolumn{2}{|c|}{ Overalls Heipm } & \multirow{2}{*}{$\begin{array}{l}\text { Number } \\
\text { Rotor(a) }\end{array}$} & \multirow{2}{*}{$\begin{array}{l}\text { Number } \\
\text { Aotor } \\
\text { Blades }\end{array}$} \\
\hline & No. & Type & & \begin{tabular}{|l|l} 
Pounds \\
\end{tabular} & Kllogram & Pounds & Kilogram & $\operatorname{limp.~GN~}$ & Litores & A. $\ln$. & Moters & Ft. In. & Menen & A. $\ln$. & Molore & & \\
\hline \multicolumn{18}{|l|}{ MILITARY HELICOPTEA } \\
\hline CH-S3E SLper Stallion & 3 & GE. TQAOE-416 TS & 4,380 ehp & 33,220 & 15,072 & 73,500 & 33,339 & N.A. & Na. & $790^{\circ}$ & $24.08 \mathrm{~m}$ & $734^{4}$ & $22.39 \mathrm{~m}$ & $20 \cdot 5=$ & $0.07 \mathrm{~m}$ & 1 & 7 \\
\hline MV-22O-prer & 2 & Alieon T $400-A D-400$ TS & 6,150 ehp & NA & N.A. & 59,000 & 26,762 & NA & NA & 360 & $10.90 \mathrm{~m}$ & $560^{\circ}$ & $17.33 \mathrm{~m}$ & 174 & $5.20 \mathrm{~m}$ & 2 & 3 \\
\hline MH.53N Pave Lom & 2 & GE. TGA-GE-7A TSS & $4,325 \mathrm{shp}$ & NA. & N.A. & 50,000 & 22,600 & 1,300 & 5,910 & 723 & $22.02 \mathrm{~m}$ & 672 & $20.47 \mathrm{~m}$ & $24 \cdot 11$ & $7.50 \mathrm{~m}$ & 1 & 7 \\
\hline CH-SAB Turhe (S-6A) & 2 & PEW. T73.700 TSE & $4,600 \mathrm{shp}$ & 10,234 & 8,724 & 47,000 & 21,319 & 630 & 2,864 & 720 & $21.95 \mathrm{~m}$ & NA & NA. & $10 \cdot 7$ & $5.67 \mathrm{~m}$. & 1 & 6 \\
\hline CH- 47 CMMH- 47 Chinook & 2 & Lycoming T55-L-11A TSE & $3.570 \mathrm{chp}$ & 20,616 & 9351 & 46,000 & 20,865 & 1,042 & 4,737 & $800^{\circ}$ & $18.29 \mathrm{~m}$ & $51^{\circ} 0^{\circ}$ & $16.54 \mathrm{~m}$ & $10 \cdot 7$ & $5.67 \mathrm{~m}$ & 2 & 3 \\
\hline CH.53D Sea Stallion & 2 & OE. TO4-GE-413 TSB & $3,225 \mathrm{shp}$ & 23,628 & 10,717 & $\$ 2,000$ & 19,051 & $\mathrm{NA}$ & NA & $723^{\circ}$ & $22.04 \mathrm{~m}$ & 672 & $20.46 \mathrm{~m}$ & 2411 & $7.50 \mathrm{~m}$ & 1 & - \\
\hline H-37A Mojave (S-56) & 2 & PEW. A-2000-50154 APSE & $1,900 \mathrm{hp}$ & 20,831 & 9,40 & 31,000 & 14,061 & NA. & NA & $720^{\circ}$ & $21.05 \mathrm{~m}$ & 64. $3^{\circ}$ & $19.50 \mathrm{~m}$ & 200 & $6.71 \mathrm{~m}$ & 1 & 5 \\
\hline CH- 46 E Soa Knight & 2 & QE. TSO-OE-18 TSB & $1,870 \mathrm{shp}$ & 15,180 & 6,694 & 24,300 & 11,022 & NA & NA. & $256^{\circ}$ & $7.81 \mathrm{~m}$ & $460^{\circ}$ & $13.22 \mathrm{~m}$ & $168^{-1}$ & $5.00 \mathrm{~m}$ & 2 & 3 \\
\hline MHHHH-600 Pave Hemk & 2 & GE.T700-9E-700701C TSQ & 1,580 shp & 10,624 & 4819 & 22,500 & 10.206 & 820 & 3,728 & $530^{\circ}$ & $16.36 \mathrm{~m}$ & $501^{\circ}$ & $15.20 \mathrm{~m}$ & $1610^{-1}$ & $5.13 \mathrm{~m}$ & 1 & 1 \\
\hline CH-3EJHH-3E Joly Green Giant & 2 & GE. T5Q-GE-5 TSE & $1,500 \mathrm{chp}$ & 13,255 & 6,012 & 22,050 & 10,002 & 1,200 & 5,496 & $620^{-}$ & $18.9 \mathrm{~m}$ & $573^{\circ}$ & $17.45 \mathrm{~m}$ & 10.10 & $5.81 \mathrm{~m}$ & 1 & 5 \\
\hline HH-3F Polican & 2 & GE. T58-GE-5 TSB & $1,500 \mathrm{shp}$ & NA. & N.A. & 22.050 & 10,002 & N.A & NA. & $620^{-1}$ & $18.9 \mathrm{~m}$ & $57^{\circ}$ & $17.45 \mathrm{~m}$ & $100^{\circ}$ & $5.51 \mathrm{~m}$. & 1 & 5 \\
\hline SH-608 Sechemenk & 2 & OE. T700-GE-401C TS: & $1,800 \mathrm{shp}$ & 13,640 & 6,191 & 21,000 & 0,525 & $\mathrm{NA}$ & NA. & $538^{-1}$ & $16.36 \mathrm{~m}$ & 5010 & $1520 \mathrm{~m}$ & 172 & $5.23 \mathrm{m.}$ & 1 & 4 \\
\hline AH-Bi Apecho & 2 & GE. T700-GE-700 TSO & $1,000 \mathrm{chp}$ & 10,269 & 4,857 & 21,000 & 0,525 & 376 & 1,709 & $480^{\circ}$ & $1463 \mathrm{~m}$ & 405 & $15.06 \mathrm{~m}$ & $12 T$ & $3.03 \mathrm{~m}$ & 1 & 4 \\
\hline SH-3H San KaO $(\mathrm{S}-61)$ & 2 & GE. T5Q-GE-10 TSA & 1,400 shp & 13,465 & 8,108 & 21,000 & 9,525 & NA. & NA. & $620^{-1}$ & $18.9 \mathrm{~m}$ & 549 & $16.09 \mathrm{~m}$ & $16^{\circ} 10^{-1}$ & $5.13 \mathrm{~m}$. & 1 & 5 \\
\hline WH-OON Saahauk & 2 & GE. T700-GE-700 TSO & $1,560 \mathrm{shp}$ & 11,204 & 5,118 & 16,999 & 7,700 & NA. & NA & 538 & $18.38 \mathrm{~m}$ & 5010 & $15.20 \mathrm{~m}$ & $16 \cdot 10$ & $5.13 \mathrm{~m}$ & 1. & 1 \\
\hline H-21 Shamenes & 1 & Wripth R-1020-103 APS & $1,425 \mathrm{hp}$ & 8,000 & 3,902 & 15,000 & 6,804 & NA. & NA. & $440^{-1}$ & $13.41 \mathrm{~m}$ & 526 & $16.0 \mathrm{~m}$ & $15^{6} \cdot{ }^{\circ}$ & $4.7 \mathrm{~m}$. & 2 & 3 \\
\hline AH-IW Son Cobra & 2 & GE. T700-GE-401 TSE & ochp & 10,200 & 4,627 & 14,750 & 6,690 & NA. & NA. & 100 & $14.63 \mathrm{~m}$ & $45 \cdot 6$ & $13.87 \mathrm{~m}$ & $14^{\prime} 2^{\prime \prime}$ & $4.32 \mathrm{~m}$ & 1 & 2 \\
\hline AH-1T Cobra & 2 & PaWC. T400-WV-402 & $1,025 \mathrm{chp}$ & 8,600 & 3,005 & 14,000 & 0,350 & NA. & NA. & 400 & $14.63 \mathrm{~m}$ & 4020 & $14.60 \mathrm{~m}$ & $13 \cdot 6$ & $4.12 \mathrm{~m}$. & 1 & 2 \\
\hline CH-34C Choctern (S-58) & 1 & PQW. A-1000-04APS & $1,525 \mathrm{hp}$ & 7.750 & 3,515 & 14,000 & 0.350 & NA. & NA & 560 & $17.07 \mathrm{~m}$ & 469 & $14.25 \mathrm{~m}$ & $153.6^{\circ}$ & $4.33 \mathrm{~m}$ & 1 & 4 \\
\hline SH.2F Seenorito & 2 & QE. TS6-GE-AFTSE & $1,350 \mathrm{shp}$ & 6,652 & 3,017 & 13,500 & 6,123 & NA & NA & 40 & $13.42 \mathrm{~m}$ & 364 & $11.60 \mathrm{~m}$ & 15.6 & $4.73 \mathrm{~m}$. & 1 & 4 \\
\hline UH-1N hoquois & 2 & PAWC. T $400-C P-100$ TSA & $1,290 \mathrm{shp}$ & 5,549 & 2,517 & 11,200 & 5,080 & 410 & 1,864 & 402 & $14.7 \mathrm{~m}$ & 425 & $12.03 \mathrm{~m}$ & 14:-6: & $4.39 \mathrm{~m}$ & 1 & 2 \\
\hline AH-18 Cobra & 1 & Lycoming T53-703 TS & 0 shp & 6,479 & 2,039 & 10,000 & 4,536 & NA. & NA. & $440^{\circ}$ & $13.41 \mathrm{~m}$ & 477 & $13.50 \mathrm{~m}$. & 13.6 & $4.12 \mathrm{~m}$ & 1 & 2 \\
\hline AH-1 J Cobra & 2 & PaWC. T400 TSS & $900 \mathrm{shp}$ & 7,281 & 3,294 & 10,000 & 4,536 & NA & NA. & $400^{\circ}$ & $13.41 \mathrm{~m}$ & 47 & $13.59 \mathrm{~m}$ & 13.6 & $4.12 \mathrm{~m}$. & 1 & 2 \\
\hline AH-10 Cobra & 1 & Lycoming T63-13 TS & $1,400 \mathrm{chp}$ & 6,073 & 2,755 & 8,500 & 4,309 & 256 & 1,177 & $440^{\circ}$ & $13.41 \mathrm{~m}$ & 47 & $13.50 \mathrm{~m}$ & $13^{\prime} \cdot 6$ & $4.12 \mathrm{~m}$. & 1 & 2 \\
\hline HH-1H hoouois & 1 & Lyooming TS3-L-139 TS & $1,400 \mathrm{shp}$ & NA. & N.A. & 9,500 & 4,309 & 330 & 1,500 & 404 & $14.73 \mathrm{~m}$ & $220^{\circ}$ & $12.00 \mathrm{~m}$ & $13 \cdot 0-$ & $3.90 \mathrm{~m}$ & 1 & 2 \\
\hline HH. 65A Dohphin & 2 & Niseongancen LHTEC T800-000 & $1200 \mathrm{shp}$ & 6,022 & 2,686 & 8,928 & 4,050 & N.A. & NA. & $392^{2}$ & $11.9 \mathrm{~m}$ & $37 \theta^{-1}$ & $11.43 \mathrm{~m}$ & 120 & $3.00 \mathrm{~m}$ & 1 & 4 \\
\hline HH- 43 F Huakin & 1 & Lyeoming T53-L-11A TS & $825 \mathrm{shp}$ & 4,469 & 2,027 & 8,800 & 3,992 & NA. & N.A. & $470^{\circ}$ & $14.33 \mathrm{~m}$ & 252 & $7.67 \mathrm{~m}$ & $156.5^{\circ}$ & $4.74 \mathrm{~m}$ & 2 & 2 \\
\hline H-100 Chickasew & 1 & Whoth A-1300-3 APS & $700 \mathrm{tp}$ & 5,250 & 2,381 & 8,000 & 3,089 & NA & NA. & $490^{\circ}$ & $14.94 \mathrm{~m}$ & $233^{\circ}$ & $12.00 \mathrm{~m}$ & 134 & $4.00 \mathrm{~m}$ & 1 & 3 \\
\hline H.25 Asmy Mulo & 1 & Com. A-976-10/46A RPS & $350 \mathrm{hp}$ & 3,928 & 1,782 & 0,100 & 2,767 & NA. & NA. & $3500^{\circ}$ & $10.67 \mathrm{~m}$ & $31 \cdot 10^{-1}$ & $9.7 \mathrm{~m}$ & $13^{\prime} 2$ & $4.01 \mathrm{~m}$ & 2 & 3 \\
\hline H-SA (S-51) & 1 & PAW. R-906-4B APS & $450 \mathrm{np}$ & 3,770 & 1,710 & 5,000 & 2,268 & NA & NA. & $450^{\circ}$ & $14.6 \mathrm{~m}$ & 40110 & $12.47 \mathrm{~m}$ & $12 \cdot 11^{\circ}$ & $3.24 \mathrm{~m}$ & 1 & 3 \\
\hline AHenit-6 Dedender & 1 & Whicon T250-C208 TS & $420 \mathrm{shp}$ & NA. & NA & 3.850 & 1,746 & 62 & 202 & 264 & $8.02 \mathrm{~m}$ & $230^{\circ}$ & $7.0 \mathrm{~m}$ & 61.5 & $2.47 \mathrm{~m}$ & 1 & 3 \\
\hline OH.6eVIH-50 Kama & 1 & Aleon TE3.720 (C200) TS & 650 dho & 1,464 & 684 & 3200 & 1,451 & 112 & 509 & 354 & $10 . \pi \mathrm{m}$ & N.A. & NA. & 8.5 & $2.91 \mathrm{~m}$ & 1 & 2 \\
\hline TH-57 Sen Panger & 1 & Nhion T60-A-700 IS & $317 \mathrm{che}$ & 1,164 & 684 & 3,000 & 1,361 & NA. & NA & $354^{4}$ & $10.78 \mathrm{~m}$ & 327 & $0.04 \mathrm{~m}$ & 9 & $2.01 \mathrm{~m}$. & 1 & 2 \\
\hline$H-13$ Shoux (Bell 47) & 1 & Lyooming TVO-435 PS & $270 \mathrm{hp}$ & 1,819 & 825 & 2,850 & 1,336 & NA & NA. & 371.5 & $11.32 \mathrm{~m}$ & NA & NA & $93.5^{\circ}$ & $2.03 \mathrm{mf}$ & 1 & 2 \\
\hline OH-6BCayes & 1 & Alizon TES-A-SA TS & $317 \mathrm{shp}$ & 1,220 & 557 & 2,700 & 1225 & NA & NA. & 264 & $0.02 \mathrm{~m}$ & $230^{\circ}$ & $7.0 \mathrm{~m}$ & $81.5^{\circ}$ & $2.47 \mathrm{mI}$ & 1 & 3 \\
\hline H-230 Raven & 1 & Lreoming 0-540-238 PS & $250 \mathrm{hp}$ & 1,816 & 824 & 2,700 & 1225 & NA. & NA. & 350 & $10.67 \mathrm{~m}$ & 270.5 & $0.40 \mathrm{~m}$ & $80.5^{-1}$ & $2.00 \mathrm{~m}$. & 1 & 2 \\
\hline TH-56A Oagap & 1 & Lycoming HIO-330-BIA PS & $180 \mathrm{hp}$ & 1,000 & 457 & 1.850 & 839 & 49 & 223 & $253.5^{\circ}$ & $7.71 \mathrm{~m}$ & NA. & NA. & 0.3 & $2.50 \mathrm{~m}$. & 1 & 3 \\
\hline
\end{tabular}
Lyooming HIO-360-B1A PS

N.A. - Information not avatiablo whon lable propered.

TS - Turbochunt

RPS - Redial Pictonehnt 
Table 4.21 (Contimued)

Charectertatles of Milinary Helicoptors

Roterencese 4.10 to 4.18

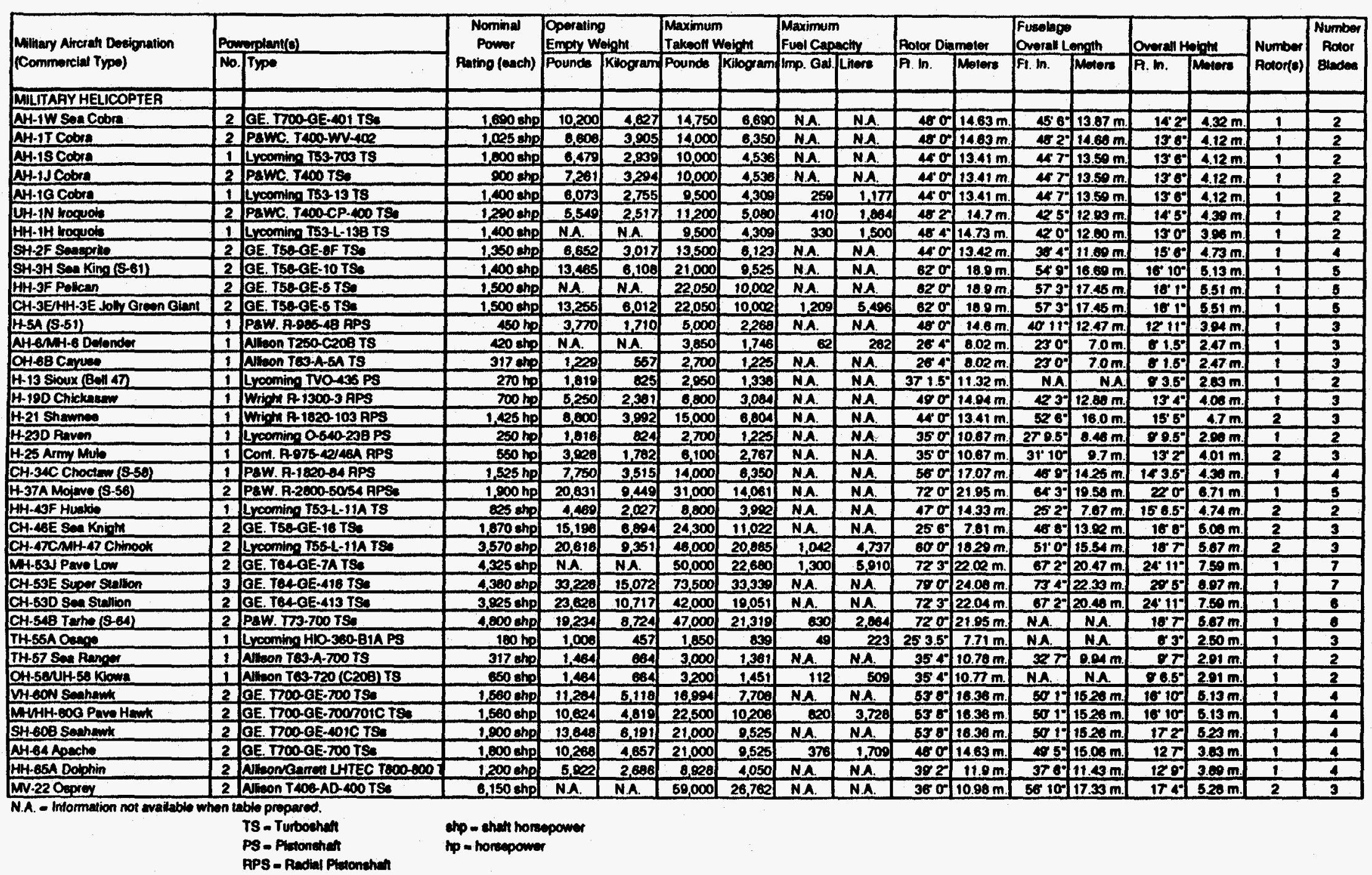




\subsection{MILITARY AVIATION REFERENCES}

[4.1] DOE Standard Accident Analysis for Aircraft Crash into Hazardous Facilities DOE-STD-3014-96, U.S. Department of Energy, Washington, DC, July 1996.

[4.2] Defense Nuclear Aoency. W78 W87 Minuteman lll Weapon System Safety Assessment. Draft Beport, Logicon RDA, Alexandria, VA., July, 1994.

[4.3] Class AB Mishaps, 1979 to Date, Memo from J.J.Clark, Jr. Chiet, Report Division, HQ AFSAIMR, to Y.T.Lin, SNL, August 2, 1994.

[4.4] Bomber/Cargo/F/ATrainer Class AB Mishaps, 1979 to Date, Memo from J.J. Clark, Jr. Chief, Report Division, HQ AFSAIMR, to Y.T. Lin, SNL, August 2, 1994.

[4.5] Flying Hours by FYMDS, 1979 to Date, Memo from J.J. Clark, Jr. Chief, Report Division, HQ AFSAIMR, to Y.T. Lin, SNL, August 2, 1994.

[4.6] Data Development and Analysis Task for Aircraft Crash Risk Analysis Methodology, Memo from Y.T. Lin, SNL12333, MS-0491 to Kamiar Jamali, DOE/HQ/DP-31, March 18, 1995.

[4.7] Memo, First Order Estimate of Times and Airspeeds Associated with Cnuise Phases for Fighter, Attack, Trainer and Bomber Aircratt, Logicon RDA, February, 10, 1995.

[4.8] Multivariate Density Estimation. Theor, Practice, and Visualization, David W. Scott, John Wiley \& Sons, Inc., New York, NY, 1992.

[4.9] Best Fit, Distribution Fitting Software for Windows, Release 1.0, Palisade Corporation, Newtield, N.Y., June, 1993.

[4.10] Jane's All the World's Aircrafts, yarious editions.

[4.11] Federal Aviation Administration Air Trattic Control Manual. Apoendix A. Aircraft Information, $7110.65 \mathrm{H}$, Federal Aviation Administration

[4.12] USAF Almanac. Air Force Magazine, various issues.

[4.13] The Naval Institute Guide to the Shios and Aircratt of the U.S. Fleet various editions, Norman Polmar, Naval Institute Press, Annapolis, MD.

[4.14] An lllustrated Guide to USAE Bill Gunston, Arco Publishing, Inc., New York, NY, 1982.

[4.15] An Illustrated Guide to Militan Helicopters, Bill Gunston, Arco Publishing, Inc., New York, NY, 1981.

[4.16] The lliustrated Encralopedia of Modem Warplanes, Doug Richardson, Crescent Books, New York, NY, 1982.

[4.17] U.S. Amm Aircraft Since 1947, Stephen Harding, Specially Press, Stilwater, MN, 1990.

[4.18] Yietnam, the War in the Air Rene' J. Francillon, Arch Cape Press, Now York, NY, 1987. 



\section{AIRCRAFT OPERATIONS}

\subsection{INTRODUCTION}

The determination of the number of aircraft operations near the facility under consideration in the aircraft crash frequency analysis is usually the foremost piece of information needed to perform this analysis. This determination of aircraft operations may be quite complicated depending on the particular bcation of the facility and the type and scale of aircraft operations that may take place nearby

The term Aircratt Operation, as defined by the Federal Aviation Administration (FAA), is the number of arrivals and departures from the airport at which an airport traffic control tower is located. For the ACRAM Standard [Ref. 5.1], the term Aircraft Operations will be used in a broader sense. Aircratt Operations will refer to the operation of any powered aircraft in any airspace. Aircraft Operations which occur in the Near Airport Environment will be referred to as Airport Operations. Aircraft Operations which occur in the Nonairport Environment, l.e. the Enroute/In-Flight and the TRACON Environments will simply be referred to as Aircraft Operations.

Before describing Aircraft Operations further, it may be useful at this point to describe how an aircratt is guided by the various air trattic facilities to ensure that aircraft are safely separated from each other and are guided safely to their destinations. As an aircraft takes off from an airport, they are initially given directions by the Airport Traffic Control Tower (ATCT). As the aircraft climbs, it is guided by the TRACON or Terminal Radar Control. As the aircraft climbs to its assigned cruising altitude, the Air Route Traffic Control Center or ARTCC guides the aircraft towards its destination. As the aircraft approaches its destination, it begins its descent. As it leaves 11,000 feet Mean Sea Level, and approximately 35 miles from its destination airport, the aircratt is handed over for guidance from the ARTCC to the TRACON. At about 5 to 20 miles, the aircraft is handed over for guidance from the TRACON to the Airport Traffic Control Tower. This narrative has described a typical flight by an Air Carrier or Air Taxi. Not all aircraft go through this process for every flight. Most General Aviation flights never enter the airspace controlled by an ARTCC because they fly below their airspace responsibility. Many Military Aviation flights remain under the control of military air trattic control facilities for most if not all of their flight. However, near busy airports, all forms of aviation, Commercial, General and Military do come under the guidance of a TRACON and/or the Airport Traffic Control Tower

This chapter is an attempt to provide some general guidance on the determination of aircraft operations near a facility. It should be noted that in many parts of the United States, unique situations may have developed in the air traffic flow patterns and thus, what is generally true in one part of the U.S., may not be applicable to other parts of the U.S.

Table 5-1 shows the results of a survey pertormed on 15 of the major Department of Energy (DOE) sites, airports near the site, and air traffic control facilities which provide guidance to air traffic near the site. Below each DOE site name in the first column of Table 5-1 is the name of the sectional map where the aiport information was found. The second through fourth columns of Table 5-1 provides the name of the nearby airport(s), whether it has a control tower, and its longest nunway in hundreds of feet. After each airport name is the three character designation for that airport. If an airport lacks the three character designation, it is considered a private airport. For larger airports, the class of airspace around that airport is given directly below its name, i.e. Class B, Class.C. Further discussion on airspaces around airports is provided in Section 5.2. The fitth column of Table 5-1 gives the air traffic control facility which provides terminal approach and departure control. Note that TRACONs, or Airport Trafilic Control Towers could provide this service. This is discussed further in Section 5.4. Finally, the last column of Table 5.1 gives the air traffic control facility which provides Class A overflight control. This is always an ARTCC. More discussion on this service is given in Section 5.3. Table 5.1 is not intended to be an exhaustive or a conclusive list of Airports near DOE sites which should be considered in an aircraft crash frequency analysis. There may be Airports known locally which are not listed in Table 5.1. Some of the Airports listed in Table 5.1 may be too small or even closed to be considered further. All of these factors should be considered by the analyst in their aircratt crash frequency analysis of their facility.

This chapter discusses aircraft operations in the following Sections: 5.2) Near Airport Environment, 5.3) Enroute/ln-Flight Environment, 5.4) TRACON Environment, and 5.5) Refẹrences. 


\begin{tabular}{|c|c|c|c|c|c|}
\hline DEPT. OF ENERGY SITE & NEARBY AIRPORT(S) & $\begin{array}{l}\text { Towered? } \\
\text { Yeano) }\end{array}$ & $\begin{array}{l}\text { Longest } \\
\text { RW (100) }\end{array}$ & $\begin{array}{l}\text { Terminal Approach and } \\
\text { Departure Control }\end{array}$ & $\begin{array}{l}\text { Clase A Overtlight } \\
\text { Control }\end{array}$ \\
\hline $\begin{array}{l}\text { Haniord/Richland Nationd Engineering } \\
\text { Laboratory, Richland/Pasco, WA } \\
\text { (Seattle Sectional) }\end{array}$ & 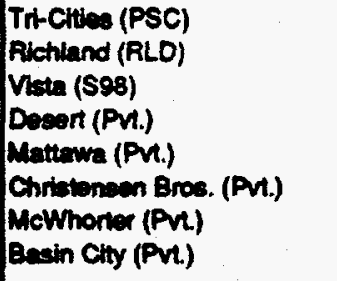 & $\begin{array}{l}\text { Yes } \\
\text { No } \\
\text { No } \\
\text { No } \\
\text { No } \\
\text { No } \\
\text { No } \\
\text { No }\end{array}$ & $\begin{array}{l}77 \\
40 \\
35 \\
36 \\
26 \\
25 \\
23 \\
14\end{array}$ & N.A. & Soatto ARTCC \\
\hline $\begin{array}{l}\text { Lewrence Livermore Nationel Leboraton, } \\
\text { (LLNL), Livermore, CA } \\
\text { (San Francticos Sectional) }\end{array}$ & $\begin{array}{l}\text { Uremorn Municipal (LVK) } \\
\text { Meadowlark (PV.) }\end{array}$ & $\begin{array}{l}\text { Yes } \\
\text { No }\end{array}$ & $\begin{array}{l}52 \\
18\end{array}$ & Bay TRACON $(0 \infty 0)$ & Oakland ARTCC \\
\hline $\begin{array}{l}\text { Nevada Test Sto (NTS), NV } \\
\text { (Las Vepas Sectional) }\end{array}$ & $\begin{array}{l}\text { Indian Springs (INS) } \\
\text { Dosert Rock (Pvt.) } \\
\text { Jeckass (U75) } \\
\text { Pahuib Mese (PVt.) } \\
\text { Beatty (BTM } \\
\text { Frans Star (2L1) }\end{array}$ & $\begin{array}{l}\text { Yes } \\
\text { No } \\
\text { No } \\
\text { No } \\
\text { No } \\
\text { No }\end{array}$ & $\begin{array}{l}20 \\
75 \\
62 \\
58 \\
56 \\
27\end{array}$ & N.A. & Los Angeles ARTCC \\
\hline $\begin{array}{l}\text { Idaho National Engineering Laboratory } \\
\text { (INEL), Idaho Falls, ID } \\
\text { (Salt Lake City Sectional) }\end{array}$ & $\begin{array}{l}\text { Idaho Falls Municipel (IDA) } \\
\text { Arco/Butte Co. (AOC) } \\
\text { Howe (U97) } \\
\text { Midway (U37) } \\
\text { Mud Lake (1U2) } \\
\text { Big Southern Butte (U46) } \\
\text { AB One (PVt.) }\end{array}$ & $\begin{array}{l}\text { Yes } \\
\text { No } \\
\text { No } \\
\text { No } \\
\text { No } \\
\text { No } \\
\text { No }\end{array}$ & $\begin{array}{l}\infty 0 \\
66 \\
38 \\
38 \\
33 \\
26 \\
26\end{array}$ & N.A. & Salt Lake City ARTCC \\
\hline $\begin{array}{l}\text { Rocky Flats Plant, Boulder, co } \\
\text { (Denver Sectiona) }\end{array}$ & $\begin{array}{l}\text { Denver IntI (DEN) } \\
\text { Class B Airspace } \\
\text { Jetterson CO. (BUC) } \\
\text { Boulder (1 V5) }\end{array}$ & $\begin{array}{l}\text { Yes } \\
\text { Yes } \\
\text { No }\end{array}$ & $\begin{array}{l}120 \\
90 \\
41\end{array}$ & Denver TRACON (D84) & Denver ARTCC \\
\hline $\begin{array}{l}\text { Los Alamos National Laboratory (LNL) } \\
\text { Los Alamos, NM } \\
\text { (Abuquerque Sectional) }\end{array}$ & Los Alemos (PM.) & No & 55 & N.A. & Abuquerque ARTCC \\
\hline $\begin{array}{l}\text { Sandia National Leboratory (SNL), } \\
\text { Abuquerque, NM } \\
\text { (Abuquerque Sectional) }\end{array}$ & $\begin{array}{l}\text { Abuquerque intn'l (ABO) } \\
\text { Class C Airspace }\end{array}$ & Yes & 138 & Abuquerque Intn'l (ABO) & Abuquerque ARTCC \\
\hline $\begin{array}{l}\text { Pantex Plant, Amarillo, TX } \\
\text { (Dallas Ft. Worth Sectionai) }\end{array}$ & $\begin{array}{l}\text { Amarillo Intn'l (AMA) } \\
\text { Class C Arspece } \\
\text { Tradewind (TDW) } \\
\text { Panthandle Carson (T45) } \\
\text { Eegles (PVt) } \\
\text { Finley Ranch (PVt) } \\
\text { True (Put.) } \\
\text { Stumpts (Pvt) } \\
\text { Finiey Fam (Put) }\end{array}$ & $\begin{array}{l}\text { Yes } \\
\text { No } \\
\text { No } \\
\text { No } \\
\text { No } \\
\text { No } \\
\text { No } \\
\text { No }\end{array}$ & $\begin{array}{l}135 \\
51 \\
44 \\
48 \\
30 \\
26 \\
23 \\
22\end{array}$ & Amarillo Intn'l (AMA) & Abuquerque ARTCC \\
\hline
\end{tabular}

Table 5-1

Survey of Major DOE Sites and Ait Tratfic Control Facilities

N.A. = not applicable 


\begin{tabular}{|c|c|c|c|c|c|}
\hline DEPT. OF ENEAGY SITE & NEARBY ARPORT(S) & $\begin{array}{l}\text { Towered? } \\
\text { (Yeano) }\end{array}$ & $\begin{array}{l}\text { Longest } \\
\text { RW (100) }\end{array}$ & $\begin{array}{l}\text { Teminal Approach and } \\
\text { Depanture Control }\end{array}$ & $\begin{array}{l}\text { Class A Overtlight } \\
\text { Controf }\end{array}$ \\
\hline $\begin{array}{l}\text { Argonne National Laboratory (ANL). } \\
\text { Argonne, IL } \\
\text { (Chicago Sectiona) }\end{array}$ & $\begin{array}{l}\text { Chicage OHare (ORD) } \\
\text { Clases B Arapace } \\
\text { Chicago Miolway (MDW) } \\
\text { Class C Arapace } \\
\text { Schaumburg (O6C) } \\
\text { Brookeridge (PM.) }\end{array}$ & $\begin{array}{l}\text { Yes } \\
\text { Yes } \\
\text { No } \\
\text { No }\end{array}$ & $\begin{array}{l}130 \\
65 \\
30 \\
28\end{array}$ & Chicago TRACON (CSO) & Chicago ARTCC \\
\hline $\begin{array}{l}\text { Kanses Clty Plant } \\
\text { Kanses Clty, MO } \\
\text { (Kansas Cly Sectional) }\end{array}$ & $\begin{array}{l}\text { Kanses City Intn't (MCl) } \\
\text { Class B } \\
\text { Rlethards Cobaur (GVW) } \\
\text { Kanses City Downtown (RC } \\
\text { Hoart (MOO6) } \\
\text { Lee Summit (K84) } \\
\text { Independence Memorial (3 }\end{array}$ & $\begin{array}{l}\text { Yes } \\
\text { Yes } \\
\text { Yes } \\
\text { No } \\
\text { No } \\
\text { No }\end{array}$ & $\begin{array}{l}108 \\
95 \\
70 \\
50 \\
30 \\
25\end{array}$ & Kanses City lntn1 (MCl) & Kansas City ARTCC \\
\hline $\begin{array}{l}\text { Paducah Gesoous Dittusion Plant, } \\
\text { Paducah, KY } \\
\text { (St. Louis Sectional) }\end{array}$ & $\begin{array}{l}\text { Barkloy Regional (PAH) } \\
\text { Metropolis (M30) } \\
\text { Farringlon (PVt.) } \\
\text { Terry (PVI.) }\end{array}$ & $\begin{array}{l}\text { Yes } \\
\text { No } \\
\text { No } \\
\text { No }\end{array}$ & $\begin{array}{l}65 \\
30 \\
29 \\
25\end{array}$ & N.A. & Memphis ARTCC \\
\hline $\begin{array}{l}\text { Portsmouth Gaseous Diffusion Plant, } \\
\text { Piketon, OH } \\
\text { (Cincinnati Sectional) }\end{array}$ & $\begin{array}{l}\text { Plke Co. (157) } \\
\text { Morkassel (PVI.) } \\
\text { Hidden Quarry (PVt.) } \\
\text { Glade STOL (Pvt.) }\end{array}$ & $\begin{array}{l}\text { No } \\
\text { No } \\
\text { No } \\
\text { No }\end{array}$ & $\begin{array}{l}42 \\
19 \\
11 \\
10\end{array}$ & N.A. & Indianapolis ARTCC \\
\hline $\begin{array}{l}\text { Brookhaven National Leboration (BNL), } \\
\text { Upton, NY } \\
\text { (New York Sectional) }\end{array}$ & $\begin{array}{l}\text { Long island islip (ISP) } \\
\text { Class C Airspace } \\
\text { Nay Caverton (CTO) } \\
\text { Gabresk (FOK) } \\
\text { Brookhaven (HWM } \\
\text { Lutker (4NY7) } \\
\text { Spedard (IN2) }\end{array}$ & $\begin{array}{l}\text { Yes } \\
\text { Yes } \\
\text { Yes } \\
\text { No } \\
\text { No } \\
\text { No }\end{array}$ & $\begin{array}{l}60 \\
100 \\
90 \\
43 \\
23 \\
22\end{array}$ & Now YoKK TRACON (N90) & New York ARTCC \\
\hline $\begin{array}{l}\text { Savannah River Sile, Aken, SC } \\
\text { (Atlanta Sectional) }\end{array}$ & $\begin{array}{l}\text { Augusta Bush Field (AGS) } \\
\text { Class C Airspace? } \\
\text { Bamwell Co. (BNL) } \\
\text { Wade (Pvt) } \\
\text { Windy Acres (Pvt.) } \\
\text { Harman (PVI.) }\end{array}$ & $\begin{array}{l}\text { Yes } \\
\text { No } \\
\text { No } \\
\text { No } \\
\text { No }\end{array}$ & $\begin{array}{l}80 \\
51 \\
45 \\
23 \\
20\end{array}$ & Augusta Bush Field (AGS) & Atlanta ARTCC \\
\hline $\begin{array}{l}\text { Oak Pidge National Leborabry (OANL), } \\
\text { Oak Ridge, TN } \\
\text { (Attanta Sectional) }\end{array}$ & $\begin{array}{l}\text { Otver Springs (Pvt.) } \\
\text { Higdon (Pvt.) }\end{array}$ & $\begin{array}{l}\text { No } \\
\text { No }\end{array}$ & $\begin{array}{l}28 \\
15\end{array}$ & N.A. & Attanta ARTCC \\
\hline
\end{tabular}

N.A. = not applicable 


\subsection{NEAR AIRPORT ENVIRONMENT}

The Near Aiport Environment is defined as that airspace area that extends from the center of the Aiport outwards to a radius of approximately 5 miles to 35 miles depending on the presence or absence of a TRACON and other air traffic control considerations. This airspace near the Airport encompasses the local airport traffic pattem, the airport nunway approach pattem(s), and the airport initial climb departure pattem(s). Generally, this is the airspace near an airport where aircratt are in the Rurway Approach Flight Phase, the Landing Flight Phase, the Takeotf Flight Phase, and the Initial Climb Flight Phase. The airspace near an airport is classified as Class B Airspace, Class C Airspace, or Class D Airspace.

Formerty called Terminal Control Areas (TCAS), Class B Airspace is generally the airspace from the surface to 10,000 feet mean sea level (MSL) surrounding U.S. airports with more than 150,000 annual instrument tlight rule (IFR) operations or 650,000 annual passenger enplanements. The configuration of each Class B Airspace is individually tailored and consists of a surface area, usually 5 nautical miles in radius from the primary airport, and two or more layers extending laterally from the core at typically 3 to 10 nautical mile increments to a radius of approximately 20 nautical miles, with each layer having a prescribed attitude floor. In many respects, Class B Airspace has been likened to upside down wedding cakes. Class B Airspace is designed to contain all published instrument procedures once an aircraft enters the airspace. Operations may be under either IFR or visual flight rules (VFR) with all aircraft subject to air traffic control clearances and instructions. Air traffic control provides aircraft separation and safety advisories.

Class C Airspace, formerty called Airport Radar Service Areas (ARSAs), is generally the airspace from the surface to approximately 4,000 feet MSL surrounding U.S. airports with more than 75,000 annual IFR operations at the primary airpont, or 100,000 annual IFR operations at the primary and secondary aiponts in the terminal hub area, or 250,000 annual passenger enplanements at the primary airport. The configuration of each Class C Airport is individually tailored and consists of a surface area of 5 nautical miles radius from the primary airport, and another layer with a prescribed altitude floor, extending laterally from the core to a radius of 10 nautical miles. Operations may be under either IFR or VFR, with all aircraft subject to air traffic control clearances and instructions. Air traftic control provides aircraft separation between IFR/IFR and IFR/special VFR (SVFR) aircraft. VFR operations are given traftic advisories and, upon request, collision resolution instructions.

Class D Airspace, formerty called Airport Traffic Areas (ATAs) and Control Zones (Czs), is the airspace from the surface to 2,500 feet above ground level (AGL) within a 5 statue mile (4.3 nautical mile) radius of the center of the airport. Operations may be under either IFR or VFR, with all aircraft subject to air traffic control clearances and instructions. Air traffic control separation service is provided to IFR aircraft only, but all aircraft will be given traffic advisories and, upon request, conflict resolution instructions.

For the ACRAM Standard [Ref. 5.1], the term Airport Operation will be used to describe Aircraft Operations that occur in the Near Airport Environment. An Aircraft Operation, as defined by the Federal Aviation Administration (FAA), is the number of arrivals and departures from the airpon at which an Aiport Traffic Control Tower is located. Operations which include aircraft that: 1) operate in the local traffic pattem or within sight of the airpon, 2) are known to be departing for, arriving from flight in the local practice areas located within a 20-mile radius of the airport, or 3) execute simulated instrument approaches or low passes at the aiport, are classified as local operations. All other operations are classified as itinerant operations.

Air traffic controllers at the Aipont Traffic Control Towers classity the Aircraft Operations into the following four categories: 1) Air Carriers (AC), 2) Air Taxis (AT), 3) General Aviation (GA), and 4) Military Aviation (MA). Air carriers are defined as aircraft in general commercial service capable of carrying 70 or more passengers, regardless of the flight rules under which the flight is conducted, e.g. 14 CFR 121 or 14 CFR 91 rules. Foreign air carriers are also included under the category of air carriers as long as the aircraft type is capable of carnying 70 or more passengers. All other aircraft in general commercial service that carry less than 70 passengers are classitied as Air Taxis. However, since many Air Taxis operate small single-engine aircraft, air traftic controllers often count these flights as General Aviation flights unless the flight is identified by a flight plan or a commercial livery is carried by the aircraft. Again, air traffic controllers classity Air Taxi flights by the aircraft type (carries less than 70 passengers), regardless of the 
flight rules under which the flight is conducted, e.g. 14 CFR 135 or 14 CFR 91. (See Section 2 for further discussion on the regulations under which Air Carriers and Air Taxis flights are conducted). Military Aviation operations are classified as all Aircraft Operations operated by the Armed Forces of the United States. Finally, General Aviation operations are classified as all Aircraft Operations which were not included under the previous three categories.

Airports, may be classified as military or civilian. These airports may be further divided as to whether or not they have a control tower. A control tower is very important to the airport in that it not only provides control over the air tratfic near the airport, it is also the location where counts are made on Airport Operations. Amost all civilian airports with a control tower are either staffed by F.A.A. personnel or under contract to the F.A.A. Most military ainports with a control tower are staffed by military personnel. However, there are a few joint-use military aipports where the control tower is staffed by F.A.A. personnel, e.g. Charleston AFB, SC. For the most current information on airport operations and flight patterns, the analyst should contact the Aipon Traffic Control Tower directly. Airport operations and flight patterns do change due to population growth, new development, new businesses, airtines establishing or moving traffic hubs, and changes at other nearby airports. The analyst should obtain sufficient historical information on airport operations to assure the growth patterns are not undergoing radical changes. Most Airport Traffic Control Towers keep only limited historical data on Airport Operations. For historical data on airport operations at airports with F.A.A. control towers, the analyst should obtain the document "F.A.A. Air Traffic Activity" distributed by the F.A.A. Office of Aviation Policy, Plans and Management Analysis each fiscal year. In addition, future airport plans could radically atter current airport operations so the analyst should be aware of future airport developments. For projections of tuture airport operations, the analyst should obtain the document "Terminal Area Forecasts" again distributed by the F.A.A. Office of Aviation Policy, Plans and Management Analysis each fiscal year. For detailed information on future airport developments, the analyst should contact local airport planning offices. 


\subsection{ENROUTE/N-FLIGHT ENVIRONMENT}

The Enroute or In-Flight Environment is defined as the airspace outside of the Near Airport and TRACON Environments. The Near Airport Environment is described in Section 5.2. The TRACON Environment is described in Section 5.4. The Enroute/n-Flight Environment encompasses all airspace above 11,000 feet mean sea level (MSL) and most airspace above 10,000 feet MSL. Generally, this is the airspace where aircratt are in the Climb to Cruise Flight Phase, Enroute or Cnuise Flight Phase, and the Descent from Cruise Flight Phase. Included within the Enroute/n-Flight Environment is Class A Airspace. All airspace over the Continental United States from 18,000 feet MSL to 60,000 feet MSL has been established as Class A Airspace (formerty called Positive Control Area or PCA) by 14 CFR 71.31 [Ref. 5.2]. For General Aviation, the Enroute/n-Flight Environment can include the airspace outside of the Near Airport and TRACON Environments and below 10,000 feet MSL.

In past aircraft crash frequency analyses, aircraft flights outside of the Near Airport Environment were considered as flying on established airways. Airways were established to assist aircratt in their navigation in the United States. A system of air routes were established based on radio navigation facilities called VORTACs (Very high frequency Omnidirectional Radio range and Tactical Navigation). When a VOR is colocated with a TACAN navigation aid (which is basically a military version of a VOR), it is referred to as a VORTAC. Airways are classitied as Low Altitude Airways or Victor Airways, High Altitude Routes or Jet Routes, and Military Training Routes. Low Altitude (Victor) Airways are the designated air routes between VOR or VORTAC stations located below 18,000 feet MSL. Air routes established in Class A Airspace from 18,000 feet MSL to 45,000 feet MSL are called Jet Routes. Jet Routes are actually available to any aircraft capable of operating at 18,000 feet or above, not just jet traffic [Ref. 5.3]. Military Training Routes are the system of designated routes used by the armed forces of the United States.

During the late 1950s and earty 1960s, several serious high altitude midair accidents in the United States spurred the development of an air traffic control system which would control all high altitude air traffic and ensure a minimum separation distance between aircraft. The facilities established to provide air traffic control service to aircraft operating on Instrument Flight Rules (IFR) flight plans within controlled airspace, principally during the en route phase of flight are the Air Route Traffic Control Centers (ARTCCs). ARTCCs are the central authority for issuing IFR clearances, and provide nationwide monitoring of each IFR flight. Within the Continental United States, there are 20 ARTCCs, each responsible for handling en route traffic passing through a specific geographic area. Because of the size of the area covered by each ARTCC, өach ARTCC's area is further divided into smaller blocks of airspace called Sectors. Each sector is monitored by one or more controllers who maintain lateral and/or vertical separation of aircraft within its airspace boundaries. Figure 5-1 presents the ARTCCs and their associated airspace boundaries within the Continental United States. [Rets. 5.3 to 5.6]. This situation has remained unchanged since 1976 when the Great Falls, Montana ARTCC was merged with the Salt Lake City, Utah ARTCC.

All civil aviation operations conducted in Class A Airspace must be conducted under IFR as established by 14 CFR 91.135 [Ref. 5.7]. Therefore, all air trattic in Class A Airspace including traffic in Jet Routes are under the direction and control of the ARTCCs. However, in discussions with the Federal Aviation Administration (FAA), the recent trend for routing of air traffic in Class A Airspace is toward increased point-to-point routing rather than the assignment along specific Jet Routes. Beginning in 1995, all flights above 41,000 feet MSL in Class A Airspace were allowed to be routed point-to-point, if so desired. At approximately two month intervals, the lower limit for point-10-point routing in Class A Airspace was decreased. It is currently at 31,000 feet MSL as of January 1996 and can be expected to be decreased even further in the near future. As a point of explanation, point-to-point routing is not equivalent to the concept of free flight. All separation margins between aircraft in Class A Airspace are maintained by the ARTCCs in point-to-point flights. What has been changed is that the point-to-point flights are not restricted to within a 4 mile air corridor on each side of the Jet Route centerline. This allows more direct flights between points (hence the name point-to-point). In contrast, the free flight concept, in its fullest application, would not be under the control of the ARTCCs and separation margins between aircraft in Class A Airspace would be maintained by onboard aircratt systems, perhaps an advanced version of the Traftic Collision Avoidance System (TCAS. 
Data on the number of IFR aircratt handled by each ARTCC is available from the FAA Office of Aviation Policy, Plans, and Management Analysis document EAA Air Traffic. Activity published annually by Fiscal Year. The FAA detines IFR Aircratt Handled by an ARTCC as including IFR Departures, and IFR Overs, where IFR Departures are defined as IFR Flights originating in the ARTCC's area, accepted by the ARTCC under Sole Enroute clearance procedures, and extended by the ARTCC. IFR Overs are defined as IFR Flights that originates outside the ARTCC area and passes through the area without landing. IFR Aircraft Handled are then the number of IFR Departures multiplied by two plus the number of IFR Overs. This definition assumes that the number of departures (acceptances, extensions, and originations of IFR flight plans) is equal to the number of landings (IFR flight plans closed).

Data on the number of IFR Aircraft Handled for the 20 Continental United States ARTCCs has been tabulated and summarized for the 1975 to 1994 time period in Table 5-2. 


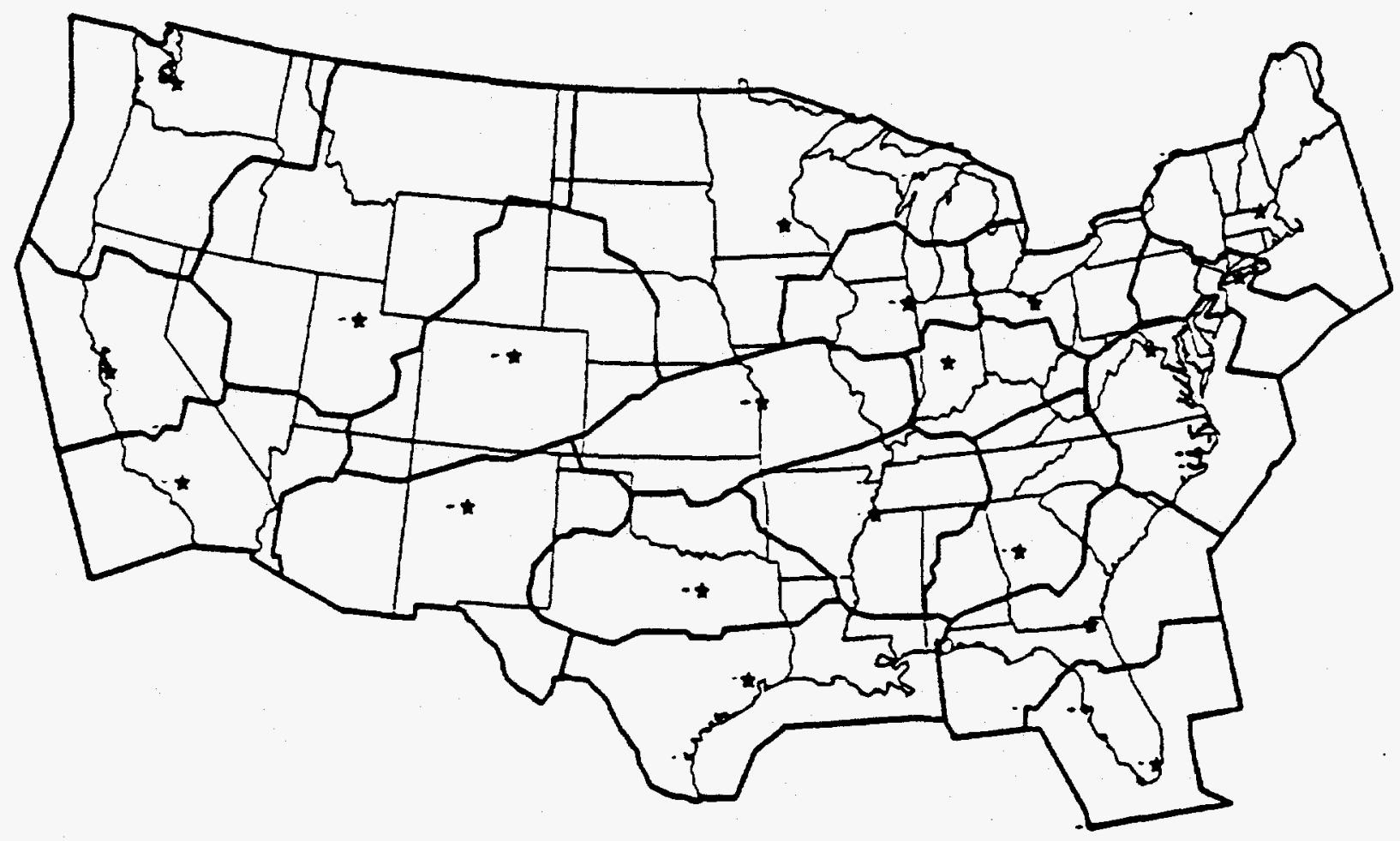

Figure 5-1

ARTCCs and Airspace Boundaries within the Continental United States 


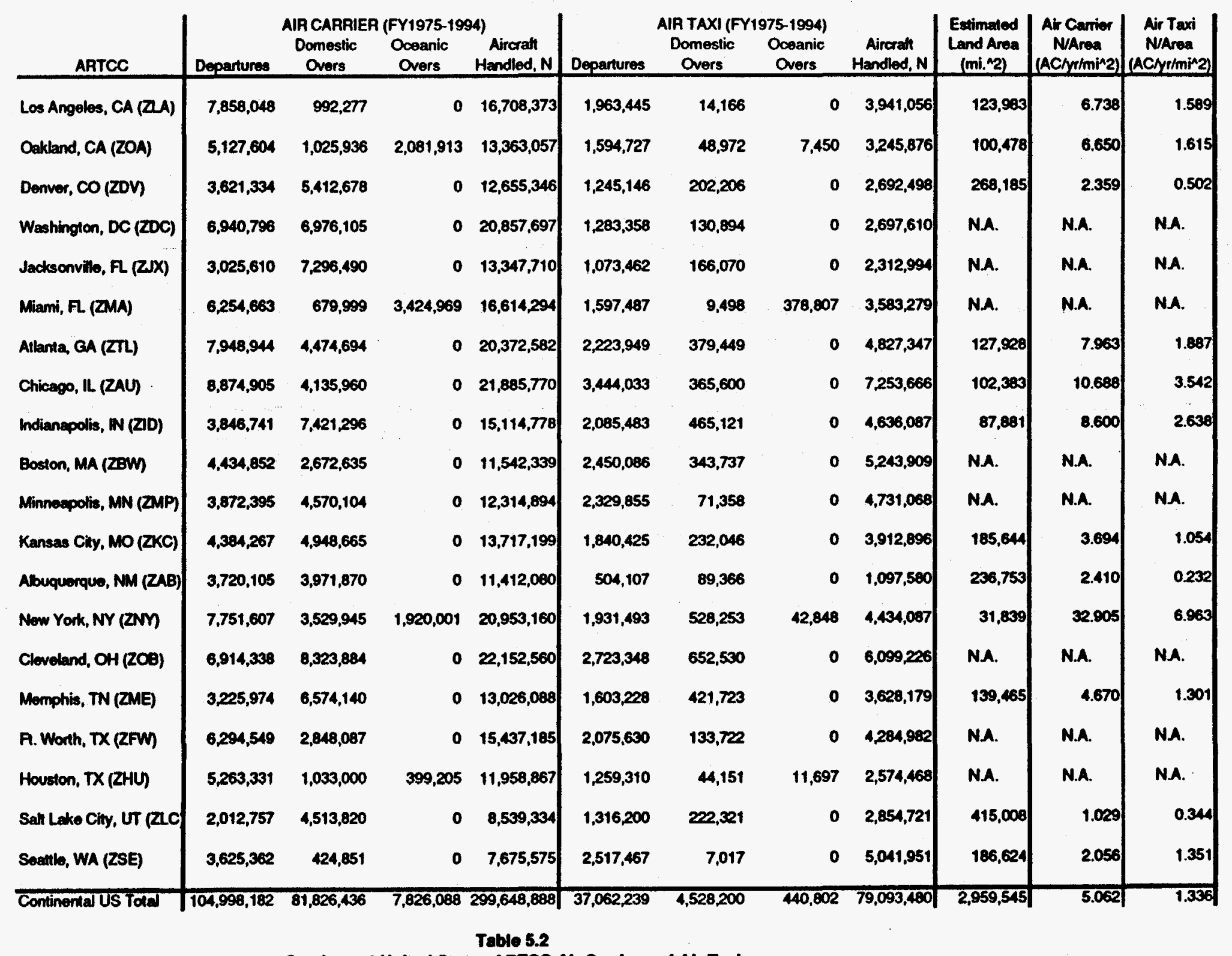

Continental United States ARTCC Air Carrier and Air Taxi

Aircraft Handied for FY1975-1994, AFTCC Land Area and Traffic Density

NA. = information not available at time of table preparation. 


\subsection{TRACON ENVIRONMENT}

TRACON or Terminal Radar Control, refers to the air tratfic control facility which is responsible for providing approach/departure aircraft separation, especially for IFR aircraft operations, for the airspace area from approximately 5 miles radius from the primary airport extending outwards to approximately 35 miles from the primary airport. The TRACON Environment is defined as that airspace outside of the Near Airport Environment (which is usually under the control of the Aiport Traffic Control Tower) and inside and beneath the Enroute/ln-Flight Environment Environment (which is below the minimum control altitude of the ARTCC).

The TRACON Environment may be important for those facilities which are located outside or beneath the Class B, or Class C Airspaces of Aiports but are within the airspace controlled by the TRACON. An example of such a situation is the Lawrence Livermore National Laboratory (LLNL) which is located outside of the Class B Airspace of the San Francisco Imtemational Airport (SFO) and the Class C Airspaces of the Oakland Intemational Airport (OAK) and the San Jose International Airport (SJC). The LLNL is located (barely) within the Class D Airspace of the Livermore Municipal Airport (LVK). Because of the peculiarities of the terrain in the San Francisco Bay area and the air traffic patterns which have developed, much of the air traftic entering the San Francisco Bay area from the Southeast, East, and Northeast is directed by the Bay TRACON $(O 90)$ to the V195 Airway located within 5 miles of LLNL. This air tratfic is then directed to intersect the SUNOL intersection where Bay TRACON directs the air traffic to their respective airport. In the Enroute/in-Flight Environment, air traffic was assumed to be spread more or less uniformly across the airspace of the ARTCC. In the TRACON Environment, the control of the TRACON serves to concentrate the air traffic within TRACON airspace especially in Low Aftitude Airways. If the facility is located outside the controlled airspace of nearby Airports, then the air trattic being directed by the local TRACON will not be counted by the Airport Operations of the local Airports. The number of aircraft handled by the local TRACON could be used as an estimate of air traffic handled by the TRACON near the facility of interest except for two impontant reasons. One is the that the number of aircraft handled by the TRACON refers to only the number of instrument operations handied by the TRACON and does not include visual flight rules (VFR) flights. The second reason is that the TRACON coumt includes the total number of instrument operations coming into the TRACON airspace from all directions. Directionality is not considered in the TRACON counts. So for the case of LLNL, the Bay TRACON undercounts due to the exclusion of VFR flights, then overcounts due to counting of flights coming into the San Francisco Bay area from the South, West and North.

Because of the difficulty of obtaining reasonable estimates of the air traffic handied by a TRACON near a facility, it is fortunate that in only a few situations is it necessary to perform the aircraft crash frequency analysis for the TRACON Environment. In most cases, if the facility is located near a large population center, the facility is located within the Class B, or Class C Airspace associated with the primary airport and thus the Airport Operations of the Airport Traffic Control Tower (ATCT) can be used. The aircraft crash frequency analysis would be done as for the Near Airport Environment Environment except that the radial distance that the airspace would be considered within the Near Aiport Analysis could be extended beyond 5 miles depending upon the class of airspace designated for that Airport and other air traffic considerations. For those cases where the facility is located far from a large population center, there is usually no TRACON to provide terminal approach and departure service since TRACONs exist to provide air traffic guidance and separation because of air traffic congestion. No large population center implies that air traffic is minimal and therefore the need for a TRACON does not exist. It is only for those cases where a facility is located outside the Class B or Class C Airspaces of Airports and where a TRACON exists to provide terminal approach and departure control service, is it necessary to consider the aircraft crash frequency analysis in the TRACON Environment.

The terminal approach and departure control service is not always provided by a TRACON facility. Many of the major U.S. Airports have their teminal approach and departure control service provided by the Airport Traftic Control Tower. Of the 33 major U.S. Airports designated with Class B Airspaces, only the 19 have terminal approach and departure control service provided by a TRACON facility. Three of these airports have their terminal approach and departure control service provided by the same TRACON facility (the New York TRACON). These aiports are: 
Major U.S. Airports

with Class B Airspace

Las Vegas NV

Los Angeles, CA

San Diogo, CA

San Francisco, CA

Denver, CO

Battimore, MD

Chicago O'Hare, IL

Boston, MA

Minneapolis/St. Paul, MN

St. Louis, MO

Phoenix, AZ

Nowark NJ

New York Kennedy, NY

New York LaGuardia, NY

Detroit, MI

Dallas/Ft. Worth, TX

Houston, TX

Salt Lake City, UT

Seattle/Tacoma, WA
TRACON

Eacility (FAA Desionation)

Las Vegas TRACON (N90)

Southem Califomia TRACON (SCT)

San Diego TRACON (NKX)

Bay TRACON (O90)

Derver TRACON (D84)

Baltimore TRACON (B95)

Chicago TRACON (C90)

Boston TRACON (A90)

Minneapolis TRACON (M98)

St. Louis TRACON (T75)

Phoonix TRACON (P50)

New York TRACON (N90)

New York TRACON (N90)

Now York TRACON (N90)

Detroit TRACON (D21)

Dallas/Ft. Worth TRACON (D10)

Houston TRACON (190)

Salt Lake City TRACON (S56)

Seattle/Tacoma TRACON (S46)

Most U.S. Aipports with Class $C$ Airspaces have their terminal approach and departure control provided by the Airport Traffic Control Tower. There are several U.S. Airports designated with Class $\mathrm{C}$ Airspaces that have their terminal approach and departure control service provided by a TRACON facility. These Airports are:

\author{
U.S. Aiports \\ with Class C Airspace \\ Anchorage, AK \\ Ontario, CA \\ Oakland, CA \\ San Jose, CA \\ Sacramento, CA \\ Pensacola, FL \\ Chicago Midway, IL \\ Hartford, CT \\ Omaha, NE \\ Tucson, AZ \\ Portland, OR
}

\author{
TRACON \\ Eacility (FAA Desionation) \\ Anchorage TRACON (A11) \\ Ontario TRACON (O40) \\ Bay TRACON (O90) \\ Bay TRACON (O9O) \\ Sacramento TRACON (MCC) \\ Pensacola TRACON (P31) \\ Chicago TRACON (C90) \\ Yankee TRACON (Y90) \\ Omaha TRACON (R90) \\ Tucson TRACON (U90) \\ Portland TRACON (P80)
}




\subsection{REFERENCES}

[5.1] DOE Standard Accident Analysis for Aircratt Crash into Hazardous Facilities, DOE-STD-3014-96, U.S. Department of Energy, Washington, DC, July 1996.

[5.2] Designation of Class A, Class B, Class C, Class D, and Class E Airspace Areas; Ainways; Routes; and Reporting Points," Ittle 14 Aeronautics and Space. Code of Federal Requlations. Chapter -Federal Aviation Administration. Departmem of Transpontation. Subchapter E-Airspace. Rart 71 114 CFR 711. U.S. National Archives and Record Administration, Washington, DC, January 1, 1995.

[5.3] The Aviator's Guide to Modem Navigation, Donald J. Clausing, TAB Books, Inc., Blue Ridge Summit, PA, 1987.

[5.4] Instnument Ratina Manual, Jeppesen Sanderson, Inc., Englewood, CO, 1990.

[5.5] Why Aimlanes Crash, Clinton V. Oster, John S. Strong, and C. Kurt Zorn, Oxford University Press, New York, NY, 1992.

[5.6] The Pilot's Air Traffic Control Handbook 2nd Edition, Paul E. Illman, TAB Books, Inc., Blue Ridge Summit, PA, 1993.

[5.7] "General Operating and Flight Rules," Itle 14 Aeronautics and Space. Code of Federal Requlations. Chapter L-Federal Aviation Administration. Department of Transportation. Subchapter F-Air Trattic and General Operatino Bules. Part 91 (14 CFR 91) U.S. National Archives and Record Administration, Washington, DC, January 1, 1995. 